DePartamento de Automática, Ingeniería ElÉCtrica Y ELECTRÓNICA E INFORMÁTICA INDUSTRIAL

ESCUELA TÉCNICA SUPERIOR DE INGENIEROS INDUSTRIALES

\title{
A Framework to Support Run-Time Adaptation in Reconfigurable Multi-Accelerator Systems
}

\author{
TESIS DOCTORAL
}

\author{
Autor: Alfonso Rodríguez Medina \\ Ingeniero Industrial por la Universidad Politécnica de Madrid \\ Máster Universitario en Electrónica Industrial por la Universidad Politécnica de Madrid \\ Director: Eduardo de la Torre Arnanz \\ Doctor Ingeniero Industrial por la Universidad Politécnica de Madrid
}





\section{Tribunal}

Tribunal nombrado por el Mgfco. y Excmo. Sr. Rector de la Universidad Politécnica de Madrid, el día de de

\section{Presidente:}

Vocales:

\section{Secretario:}

Suplentes:

Realizado el acto de lectura y defensa de la Tesis el día de

de

en la Escuela Técnica Superior de Ingenieros Industriales de la Universidad Politécnica de Madrid.

Calificación:

El Presidente

Los Vocales

El Secretario 

A mis padres

A Joaquín y a la Yiya 



\section{Agradecimientos}

Una Tesis doctoral es un camino largo, un camino lleno de retos y dificultades que llevan al límite (tanto para bien como para mal) la mente de una persona. Es un hecho irrefutable que una gran parte de dicho camino hay que recorrerla en solitario; cualquiera que lo niegue te estará dando, muy probablemente, una versión sesgada de la realidad. Esta soledad (necesaria, por otra parte) pone a su vez de manifiesto la importancia de poder contar con el apoyo y comprensión de los que te rodean, no sólo para superar las dudas y miedos que van surgiendo, sino también para crecer como persona. Por esta razón, me gustaría empezar esta memoria agradeciendo a todos aquellos que me han acompañado a lo largo de este viaje.

En primer lugar, quiero darle las gracias a Edu, mi director de Tesis pero también mi amigo, por haberme dado la oportunidad de llegar hasta aquí. A pesar de no haber trabajado previamente contigo, me acogiste desde el principio y me hiciste sentir como uno más del grupo. Gracias también por haberme enseñado muchas de las cosas que sé hoy en día, y por haberme hecho descubrir la docencia (jamás habría pensado que llegaría a ser profesor, y no te voy a negar que parte de la culpa de que haya cambiado de opinión es tuya). Espero que, aunque sigamos teniendo alguna que otra discusión y a veces nos guste jugar a ver quién es más cabezón de los dos (gano yo), podamos seguir aprendiendo cosas el uno del otro.

Gracias también a Tere y a Marcos, por haberme tratado siempre como si fuese de la familia y por haber estado siempre a mi lado, tanto desde el punto de vista académico como desde el punto de vista personal. Gracias por haber creído en mí desde el principio y por todos esos recuerdos juntos, aunque quizá algunos estén un poco borrosos (“iestamos secos?”).

Gracias a Jorge, por ser un gran amigo y por haberme puesto en el buen camino en muchos aspectos. Por esas noches infernales de consola hasta las mil, siempre con una buena cerveza al lado (o diez). Quiero aprovechar también para darle las gracias a Ya-Wen, primero por habernos aguantado en todas esas ocasiones, y luego por haberse preocupado siempre por mí.

No podría faltar también la mención al gallego pirado, Andrés, al que conocí cuando todavía era un joven doctorando al que le gustaba bañarse en lugares exóticos. Gracias por haberme enseñado cosas tan importantes como el rigor científico o la perseverancia, y por haberme ayudado con tus "interrogatorios" a encontrar respuestas. Es un orgullo y un placer poder trabajar contigo.

Yago, gracias por haberme enseñado que las cosas sin pasión no pueden llegar a ninguna parte, y que siempre hay que intentar ir un paso más allá. Por hacerme ver que el conocimiento técnico puede utilizarse para algo más que para resolver problemas de ingeniería, que quizá den de comer al cuerpo pero no tanto a la mente. 
Aunque haya tenido que exiliarse y ahora sea más difícil vernos, no me olvido del Ruben (sí, sin tilde y con artículo), al que quiero dar las gracias por haber estado cerca en algunos de los momentos más duros. Por saber dar siempre profundidad a todas las conversaciones, bien sean de trabajo o bien de temas más personales. Bueno, y por esos podcasts que nos marcamos a veces, que también se agradecen.

$\mathrm{Al}$ equipo $\mathrm{ARTICo}^{3}$ primigenio. Juanito, gracias por haberme cuidado y llevado de la mano cuando era un virgen absoluto. Quizá los detectores de flancos no eran lo tuyo, pero créeme cuando te digo que de ti he aprendido cosas muchísimo más valiosas. César, gracias por no haberme matado aquella víspera de congreso en la que borré la carpeta que no era. Y, ahora en serio, gracias por demostrarme que la validez de una persona no se debe medir por su edad, y que a veces está bien irse a un concierto aleatorio para desconectar de la rutina. Os echo de menos.

Mora. Qué más puedo decir que no te haya dicho ya. Gracias por haber sido mi vecino tanto tiempo, por las discusiones técnicas a última hora de la tarde, por esas sesiones de mirar a la ventana para buscar la inspiración (todavía sigo haciéndolo), y por haber sido capaz de explicarme siempre todo sin hacerme sentir imbécil, por mucho que mis preguntas lo fuesen. Espera, ¿oyes eso? Creo que te llaman de acción social para algo...

Gracias a Roi, uno de los últimos fichajes, por ser un gran compañero de encebollamientos y de mirar al abismo. Por intentar hacerme ver que siempre hay un punto de vista diferente, aunque no se esté de acuerdo con el mismo. Gracias por tus recomendaciones literarias y por tu idealismo, aunque a veces vaya acompañado de palabras barrocas. Muchas gracias también a tus padres, por haber tenido siempre buenas palabras y gestos hacia mí.

Me gustaría también expresar mi agradecimiento al resto de personas que han hecho o hacen que funcione el CEI día a día. Gracias a todos los profesores, en especial a Javier, Óscar, Pedro, Jesús, Miro, Regina, Airán, Gabriel, y Alberto (Málaga), por mantener en marcha esta maquinaria que permite generar conocimiento y que los alumnos consigan alcanzar sus metas. Gracias a los doctorandos que se fueron, en especial a Dani, Meneses, Chema y Víctor, porque de ellos aprendí desde cosas sencillas como leer un datasheet, hasta cosas más importantes como el valor de la amistad (aunque sea en la distancia). Gracias también a los doctorandos a los que aún les queda un poco para acabar, en especial a Rafa, Arturo, y Cristian, por haber aportado un punto de frescura al grupo con nuevas ideas y actitudes. ¡Ánimo, que ya os queda poco! Gracias a Yolanda y a Maite, por su apoyo imprescindible en las tareas menos agradecidas de nuestro trabajo.

Uno de los grandes activos de la actividad investigadora son las conexiones entre grupos. A este respecto, quiero dar las gracias a la gente del CITSEM, en especial a Edu Juárez y a Fernando Pescador, por haberme abierto sus puertas siempre que lo he necesitado. Gracias también a la gente del IUMA, en particular a Roberto y a Chano, por acogerme y ayudarme a expandir mis conocimientos técnicos. Gracias a Lucana y a Raúl, por aguantar mis infinitas preguntas y mis plazos absurdos (sí, estoy hablando de los papers). Samu, Himar, Abelardo, gracias por las birras y los buenos momentos. 
Big thanks to Marco Platzner, head of the Computer Engineering group at Universität Paderborn, for giving me the opportunity to spend 3 months with them. Thank you for making me feel part of the team since day one, and for all the knowledge that I was able to bring back to Spain. Shoutout also to Alex, Achim, and Kenter for showing me around and for always doing your best to make me feel at home.

Still on an international note, I would also like to say thanks to Francesca Palumbo. Thank you for your kind words, your patience, and your informal but always useful lessons. It has been a great honor to work with you and learn from your team.

Gracias a Juan Carlos, mi maestro de karate, por haberme enseñado el significado del esfuerzo y de la superación ("si la cabeza quiere, el cuerpo puede"). Gracias por haberme inculcado el amor por esa forma de vida que es el karate, tan útil en momentos duros como los asociados a una Tesis doctoral. Muchas gracias también al resto de compañeros de clase, en especial a Gonzalo, Pedro, y Dani, por estar siempre ahí y por ayudarme a mejorar como karateka y como persona.

Una de las mejores sorpresas que me ha dado la Tesis ha sido la de poder conocer grandes amigos: Dani, Raquel y Titti, muchas gracias por todos esos momentos juntos. Dani, gracias por tu saber estar envidiable, del que he intentado aprender todo lo posible. Sé que te cuesta verlo a veces, pero considero que eres una de las personas más válidas que conozco (prueba de ello es que me hayas adelantado en la última curva, algo por lo que te odiaré siempre, que lo sepas). Raquel, gracias por tu sinceridad, por no callarte nunca nada, y por haberme hecho ver siempre de buenas maneras cuándo estaba equivocado. A pesar de que vengas de una aldea, y de que no puedas bailar ballet, me alegro mucho de haberte conocido en aquel DCIS en el que te mofabas de mí. Titti, gracias por haberte convertido en mi mejor amiga y por haber estado siempre que te he necesitado, aunque tú estuvieses mal. Gracias por haberte esforzado por entenderme, escuchando mis movidas (normalmente en forma de podcast infinito) cuando las cosas no iban bien. Grazie di tutto.

Gracias a mis amigos del colegio, Alberto, Javi, y Paloma. Aunque últimamente no nos veamos tanto como en el pasado, seguís siendo parte muy importante de mi vida. Si he llegado hasta aquí, en parte también es gracias a vosotros. Gracias por estar en los buenos y, sobre todo, en los malos momentos.

Por último, quiero darle las gracias a mi familia. Gracias a mi primo Samuel, que ha escuchado pacientemente mis disertaciones sobre el barrio a las tantas de la madrugada, y otra serie de disquisiciones turbias que no reproduciré aquí. Gracias por ser como un hermano menor para mí. Gracias al tío, porque me lleva acompañando toda la vida, siempre dándome su apoyo incondicional en todas las cosas que he ido haciendo. Gracias también por haber hablado claramente cuando mi forma de actuar no era la más correcta. Gracias a la Yiya, que ha sido la que más ha presionado para que acabase esta Tesis. Gracias por haber estado siempre conmigo desde que era pequeño, y por creer ciegamente en mí desde el principio. Gracias a Sara, mi hermana, a la que quiero con locura a pesar de que a veces tengamos nuestros (grandes) desencuentros. Aunque quizá no hayas sido la primera en enterarte cuando había buenas o malas noticias, siempre has estado ahí para lo que he necesitado. Y, sobre todo, gracias a mis 
padres, porque sin ellos no estaría escribiendo estas líneas, ni me habría convertido en lo que soy hoy. Gracias por quererme, por haberme apoyado en todo lo que he hecho sin cuestionarme nunca nada. Simplemente, gracias. Os quiero. 


\section{Resumen en español}

Los sistemas embebidos de altas prestaciones han experimentado notables cambios tanto en la complejidad de las aplicaciones como en los requisitos de los propios sistemas. Para no quedarse atrás, las plataformas de cómputo tradicionales se han visto forzadas a evolucionar para poder adaptarse a estos nuevos escenarios. Así, a día de hoy es bastante habitual encontrar plataformas de cómputo paralelo (p.ej. CPUs multi-core, GPUs de propósito general, o FPGAs) en muchas soluciones embebidas de altas prestaciones. No obstante, y aunque el rendimiento sigue siendo uno de los factores decisivos de cara a elegir una plataforma u otra, el término "embebido" impone restricciones adicionales como la eficiencia energética o la tolerancia a fallos. En este contexto, se ha demostrado que el uso de dispositivos de lógica programable o FPGAs es altamente beneficioso, ya que juntan lo mejor del hardware (rendimiento, eficiencia energética) y del software (programabilidad, flexibilidad en la ejecución) gracias a sus capacidades de reconfiguración dinámica.

Sin embargo, y a pesar de sus buenas prestaciones, las FPGAs siguen siendo poco accesibles para el público general. De hecho, estos dispositivos exigen conocer con gran detalle la tecnología subyacente tanto para implementar algoritmos como para gestionarlos durante su ejecución. De cara a eliminar estas limitaciones, es un requisito indispensable que los sistemas de cómputo paralelo basado en FPGA cuenten con tres elementos fundamentales: arquitecturas de cómputo capaces de crear una infraestructura eficiente de aceleración, herramientas de soporte en tiempo de diseño con métodos de generación de aceleradores desde descripciones de alto nivel y mecanismos automatizados de implementación de sistemas reconfigurables, y herramientas de soporte en tiempo de ejecución para abstraer procesos complejos como la gestión de la propia reconfiguración hardware y de la ejecución de múltiples aceleradores en paralelo. El framework que se propone en esta Tesis integra estos tres componentes en una solución única.

El elemento central del framework es $\mathrm{ARTICo}^{3}$, una arquitectura hardware reconfigurable con múltiples aceleradores inspirada en las GPUs de propósito general y en los modelos de abstracción definidos en la especificación OpenCL. ARTICo ${ }^{3}$ usa reconfiguración dinámica y parcial (DPR) para desplegar paralelismo a nivel de tarea (cargando aceleradores con distinta funcionalidad) y de datos (cargando varias copias del mismo acelerador). Además, su datapath configurable puede ser modificado en tiempo de ejecución para alcanzar un compromiso entre rendimiento, eficiencia energética, y nivel de tolerancia a fallos. De hecho, tres modos de funcionamiento distintos pueden ser seleccionados cuando hay más de una copia del mismo acelerador en la FPGA: paralelo (cada acelerador recibe distintos datos de entrada y los procesa con un enfoque similar a SIMD), redundante (cada acelerador pertenece a un grupo TMR/DMR, recibe los mismos datos de entrada, y devuelve sus resultados 
a través de una unidad de votado), y reducción (parecido a paralelo, pero con una operación final de reducción antes de escribir los datos en la memoria principal). Para simplificar su programación, ARTICo ${ }^{3}$ virtualiza sus aceleradores usando mapas de memoria, y proporciona un conjunto reducido de registros de configuración. Por último, la arquitectura cuenta con una infraestructura de monitorización para poder extraer información relevante durante la ejecución de los aceleradores (p.ej. latencia, número de errores por región reconfigurable).

En paralelo a la arquitectura, el framework propuesto en esta Tesis incluye una herramienta para la generación automática de sistemas reconfigurables con múltiples aceleradores. Para simplificar el proceso de implementación de este tipo de sistemas, la herramienta soporta dos niveles de abstracción distintos para especificar la funcionalidad de los aceleradores: descripciones RTL de bajo nivel en VHDL/Verilog (para diseñadores con cierto conocimiento previo de diseño hardware), y descripciones algorítmicas de alto nivel en $\mathrm{C} / \mathrm{C}++$ (para usuarios sin experiencia previa en diseño hardware), que son implementadas usando un motor HLS comercial de manera automática y transparente. Los aceleradores $\mathrm{ARTICo}^{3}$ se generan mediante la instanciación de la lógica del usuario en un contenedor estándar. Dicho módulo incluye bancos configurables de memoria y registros, y una interfaz simplificada y fija para facilitar la integración con el resto del sistema. La herramienta ha sido implementada de manera modular, por lo que su funcionalidad puede ser fácilmente extendida mediante la creación de distintas plantillas de usuario.

El último componente del framework es una biblioteca de gestión de la ejecución que se basa en un modelo de paralellismo a nivel de datos similar al usado en OpenCL. Esta biblioteca es accesible desde las aplicaciones software del usuario gracias a una API que contiene las funciones necesarias para reconfigurar la FPGA y gestionar la ejecución de los aceleradores, cuya planificación se realiza de manera transparente usando un modelo de memoria compatible con DMA y un esquema de ejecución escalable. Como añadido, el framework incluye un modelo simplificado de la ejecución de los aceleradores que permite realizar estimaciones del rendimiento y el consumo energético durante el funcionamiento normal del sistema.

Para la validación del framework se han considerado tres escenarios distintos: usar aceleradores ad hoc descritos en VHDL, usar aceleradores ad hoc descritos en C, y usar un benchmark HLS con varias aplicaciones estándar. En el primer escenario, los distintos perfiles de ejecución de los aceleradores $\mathrm{ARTICo}^{3}$ han sido analizados, mostrando las diferencias entre aquellos limitados en memoria (el tiempo de transferencia de datos es mayor que el de procesamiento) y aquellos limitados en cómputo (el tiempo de procesamiento es mayor que el de transferencia de datos). En el segundo escenario se ha podido comprobar que las soluciones basadas en ARTICo $^{3}$ son mejores que sus alternativas software para ciertos algoritmos, llegando a obtener aceleraciones del orden de $14 \times \mathrm{y}$ ratios de eficiencia energética en torno a $10 \times$. Por último, la evaluación basada en benchmarks ha servido para identificar qué tipo de aplicaciones obtiene los mejores resultados cuando éstas son implementadas usando las propuestas de esta Tesis. Para ello, los distintos patrones 
de cómputo/comunicaciones han sido asociados a perfiles de ejecución limitados en memoria y en cómputo.

Tras la integración de los tres componentes previamente descritos, el framework permite implementar sistemas reconfigurables con múltiples aceleradores de manera accesible y transparente para muchos desarrolladores software. Sin embargo, también impone un único modelo de programación. Este modelo con paralelismo explícito a nivel de datos puede no ser el más adecuado en algunas aplicaciones, como se ha podido ver tras la validación basada en benchmarks. Además, limitar el lenguaje de especificación de alto nivel de los aceleradores a $\mathrm{C} / \mathrm{C}++$ va en contra de muchas corrientes actuales, en las que se busca incrementar el nivel de abstracción. Por ello, los modelos de programación y ejecución de ARTICo ${ }^{3}$ han sido extendidos para soportar la ejecución multihilo en hardware o en software por un lado, y el uso de especificaciones de alto nivel basadas en modelos de flujo de datos por otro.

La ejecución multihilo tanto en hardware como en software se ha conseguido mediante la integración directa con ReconOS, otro entorno de diseño y gestión de sistemas reconfigurables con múltiples aceleradores que incluye las extensiones necesarias a nivel arquitectural y de sistema operativo para permitir que los aceleradores se comporten como hilos software. Además, se ha implementado un sistema evolutivo basado en estructuras sistólicas, que permiten la adaptación a nivel de circuito mediante procesos de aprendizaje por imitación. Esta combinación genera un enfoque de reconfiguración multinivel, que combina DPR a nivel de región reconfigurable (grano grueso) con DPR a nivel de LUTs (grano fino).

Por otro lado, las extensiones orientadas a modelos de flujo de datos han sido introducidas mediante la integración directa con MDC, una herramienta capaz de generar aceleradores con sustratos CGR usando especificaciones escritas en CAPH. Esto permite un enfoque de reconfiguración híbrido en el se combina DPR (cambios funcionales completos, pero lentos) con CGR basada en registros de configuración (cambios paramétricos, pero muy rápidos). La integración de $\mathrm{ARTICo}^{3}$ con MDC habilita un mecanismo de generación de estructuras hardware muy optimizadas en las que se fomenta la reutilización de recursos lógicos, ya que MDC es capaz de identificar y unir unidades funcionales compartidas en la estructura interna de los aceleradores.

La última parte de este documento cubre la evaluación de las propuestas de la Tesis en una aplicación real para sistemas embebidos de altas prestaciones: el procesamiento de imágenes hiperespectrales a bordo. Las aplicaciones de sensado remoto que utilizan sensores hiperespectrales para la adquisición de imágenes generan grandes cantidades de datos que deben ser procesados para extraer la información que contienen. En este sentido, pueden darse dos posibles escenarios: que los datos sean adquiridos, comprimidos, y enviados a la Tierra para ser procesados, o que los datos sean directamente procesados a bordo. En esta Tesis se propone una implementación adaptativa de un algoritmo de compresión sin pérdidas basado en el estándar CCSDS 123. Dicha implementación usa un algoritmo de particionado de las imágenes de entrada para explotar el máximo paralelismo a nivel de datos posible. Los resultados obtenidos demuestran que la solución propuesta 
es competitiva con otras alternativas disponibles en la literatura. En esta Tesis también se ha cubierto el segundo escenario (procesamiento directamente a bordo, sin compresión), proponiendo una implementación adaptativa de una cadena de desmezclado espectral. Dicha implementación usa un algoritmo de particionado y reducción para explotar el paralelismo a nivel de datos, proporcionando una solución escalable en un contexto con múltiples FPGAs.

Las principales contribuciones de esta Tesis son las siguientes:

- [Arquitectura] Una arquitectura de procesamiento hardware para sistemas embebidos de altas prestaciones capaz de establecer compromisos, en tiempo de ejecución, entre rendimiento, eficiencia energética, y tolerancia a fallos.

- [Diseño] Una metodología automatizada de generación de sistemas reconfigurables con múltiples aceleradores desde descripciones RTL de bajo nivel o usando procesos HLS desde descripciones algorítmicas de alto nivel.

- [Ejecución] Una biblioteca para gestionar, de manera transparente, la reconfiguración y la gestión del cómputo en entornos con múltiples aceleradores.

- [Validación] Una estrategia de caracterización y validación del framework propuesto basada en benchmarks HLS.

- [Ejecución] Un enfoque multiparadigma para programar sistemas reconfigurables con múltiples aceleradores combinando ejecución paralela a nivel de datos con ejecución multihilo en hardware y software.

- [Diseño] Una flujo integrado de diseño para generar, de manera automática, sistemas reconfigurables con múltiples aceleradores desde especificaciones basadas en modelos de flujo de datos.

- [Aplicación] Una implementación adaptativa de un algoritmo de compresión hiperespectral basado en el estándar CCSDS 123.

- [Aplicación] Una implementación adaptativa de un algoritmo de desmezclado hiperespectral con múltiples dispositivos de tipo FPGA.

Este documento está organizado en 5 capítulos. El primero discute la motivación detrás del trabajo realizado, fijando los objetivos principales y proporcionando el marco tecnológico necesario. En el segundo capítulo, los tres componentes principales del framework (la arquitectura hardware, la herramienta de diseño automatizado y la biblioteca de gestión) son descritos en detalle y validados usando diseños ad hoc y benchmarks. Las extensiones multihilo y basadas en modelos de flujo de datos se cubren en el tercer capítulo, mientras que la evaluación de ARTICo ${ }^{3}$ en aplicaciones de procesado de imágenes hiperespectrales se presenta en el cuarto. Por último, el quinto capítulo cierra esta Tesis discutiendo las conclusiones, resumiendo las principales contribuciones, presentando una evaluación cuantitativa del impacto del trabajo realizado, y proponiendo líneas futuras de investigación. 


\section{Abstract}

High-performance embedded computing systems have experienced a surge in both application complexity and overall system requirements. In order to keep pace with these fast changes, traditional computing platforms and paradigms have been also forced to evolve and adapt themselves to unprecedented scenarios. As a consequence, it is fairly common nowadays to see parallel computing platforms (e.g., multi-core CPUs, general-purpose GPUs, or FPGAs) at the core of high-performance embedded computing solutions. However, and even though execution performance still plays an important role in the platform selection process, additional criteria, such as energy efficiency or fault tolerance, need to be taken into account as well. In this context, the use of run-time reconfigurable SRAM-based FPGAs as standalone processing elements has already proven to be the best option in multiple application scenarios, since they provide the best of both hardware (computing performance, energy efficiency) and software (programmability, execution flexibility) worlds.

However, the use of these devices presents several problems, the most relevant one still being their lack of accessibility for the general public. In most cases, developers are expected to have extensive low-level knowledge of the underlying technology, either to describe and implement their algorithms, or to execute and manage the generated hardware accelerators. In order to overcome these issues, it is mandatory for reconfigurable FPGA-based parallel computing frameworks to provide three key components: computing architectures to create a proper acceleration infrastructure, design-time support tools to enable high-level entry points for accelerator description and automated system implementation, and run-time support mechanisms to hide low-level reconfiguration and execution management details. The framework proposed in this Thesis is an integrated solution that covers all three components.

The framework is built around the ARTICo $^{3}$ architecture, a hardware-based reconfigurable multi-accelerator infrastructure inspired by general-purpose GPUs and OpenCL abstractions. As such, it is capable of using DPR to exploit both task- (load different application-specific hardware accelerators) and data-level (load multiple copies of the same application-specific hardware accelerator) parallelism. In addition, it features a configurable datapath that can be dynamically modified during application execution to enable run-time tradeoffs between computing performance, energy efficiency, and fault tolerance. In this regard, the architecture supports three operation modes when multiple copies of the same application-specific hardware accelerator are present on the FPGA fabric: parallel (each accelerator gets different input data and executes in SIMD-like fashion), redundant (each accelerator belongs to a TMR/DMR group, receives the same input data, and sends its results back through a configurable voter unit to enforce fault tolerance), and reduction-oriented (similar to parallel, but with a final reduction operation before writing results back 
to main memory). To simplify its programming, ARTICo ${ }^{3}$ uses a memory-mapped virtualization of the hardware accelerators, and provides a register-based interface for configuration purposes. The ARTICo ${ }^{3}$ architecture also features an embedded monitoring infrastructure to keep track of relevant execution metrics (e.g., accelerator latency, number of faults per reconfigurable slot).

The ARTICo ${ }^{3}$ framework also comes with a toolchain to automatically generate reconfigurable multi-accelerator systems with DPR-compatible floorplannings. In order to further simplify FPGA-based system implementation, two different entry points for hardware accelerator specification are supported: low-level RTL descriptions in VHDL/Verilog (for users with certain knowledge in hardware design) and high-level algorithmic descriptions in $\mathrm{C} / \mathrm{C}++$ (for users with no previous experience on hardware design), which are then implemented using a commercial HLS engine under the hood. Independently of the selected entry point, accelerators are integrated in the main $\mathrm{ARTICo}^{3}$ infrastructure by instantiating the input user logic in a standard wrapper, which features configurable memory and register banks, and a fixed interface to simplify the implementation of the static and reconfigurable partitions on the FPGA. The toolchain is implemented in a modular way, enabling users to extend its functionality by creating custom templates for multi-accelerator system deployment.

The last component of the ARTICo ${ }^{3}$ framework is a runtime library that enables a data-parallel execution model similar to the one present in the OpenCL specification. This library is accessible from user applications through a lightweight API that hides low-level reconfiguration and accelerator management details. In fact, accelerator scheduling is transparently performed using a DMA-friendly memory model and a scalable execution scheme. In parallel, the framework includes a lightweight model for hardware accelerator execution that can be used to perform run-time estimations of execution performance and power consumption.

In order to validate the proposed framework, three different approaches have been considered: using optimized custom VHDL-based hardware accelerators, using optimized custom HLS-based hardware accelerators, and using standard HLS-based benchmarks. In the first scenario, the different execution profiles in ARTICo ${ }^{3}$ have been assessed, showing the different behavior of memory-bounded applications (data transfers are larger than computing time) and computing-bounded applications (computing time is larger than data transfers). In the second scenario, it is shown that ARTICo ${ }^{3}$-based hardware acceleration does indeed outperform alternative highperformance embedded computing platforms, showing speedups of up to $14 \times$ and energy efficiency ratios of up to $10 \times$ when compared with a software-based implementation of the same algorithm. Finally, the benchmark evaluation has been used to identify which type of application can truly benefit from using the framework proposed in this Thesis, associating different computation/communication patterns to memory- and computing-bounded execution profiles.

Although the baseline ARTICo ${ }^{3}$ framework provides an accessible and transparent way for software programmers to implement reconfigurable multi-accelerator systems, it constrains them to use a single programming model. However, and as the 
benchmark-based validation has confirmed, the native data-parallel programming model of the ARTICo ${ }^{3}$ framework may not render proper acceleration values in certain application scenarios. Using plain $\mathrm{C} / \mathrm{C}++$ code for accelerator specification might be also a limitation, especially when considering that current programming trends tend to raise the level of abstraction. As a consequence, the ARTICo ${ }^{3}$ programming and execution models have been extended with transparent hardware/software multithreading capabilities and dataflow-based accelerator specification support.

Hardware/software multithreading has been enabled by a direct integration with ReconOS, another hardware-oriented framework for reconfigurable multi-accelerator systems that provides the required architectural and OS-level extensions to allow hardware accelerators to behave as regular software threads. Additionally, an evolvable SA system has been implemented as an ARTICo $^{3}$ accelerator to support circuit adaptation using learn-by-imitation procedures. This approach enables a multi-grain reconfiguration approach, combining the coarse-grained DPR used in ARTICo ${ }^{3}$ slots with a fine-grained DPR mechanism to change functional units at LUT level.

Dataflow-based extensions have been enabled by a direct integration with MDC, a tool to generate hardware accelerators with embedded CGR substrates using modelbased specifications written in CAPH. This enables a hybrid reconfiguration approach that combines DPR (to perform slow but complete functional changes) with registerbased CGR (to perform fast but parametric-only modifications in the accelerator logic). In addition, the integrated MDC-ARTICo ${ }^{3}$ toolchain allows designers to generate highly-optimized structures that heavily enforce resource reuse, since MDC identifies and merges common functional units in the internal accelerator datapath.

Finally, the ARTICo ${ }^{3}$ framework has been evaluated under a real-world application scenario for high-performance embedded computing: on-board hyperspectral data processing. Remote sensing applications that rely on hyperspectral imaging sensors produce large amounts of data that need to be further processed to extract relevant information. In this regard, there are two alternatives: on the one hand, data can be acquired, compressed on board, and sent to on-Earth processing facilities; on the other hand, data can be directly processed on board. In this Thesis, a run-time adaptive implementation of a lossless hyperspectral data compressor based on the CCSDS 123 standard has been proposed. A novel hardware-friendly data partitioning algorithm compatible with the standard has been applied to properly exploit datalevel parallelism. Experimental results show comparable performance an energy efficiency levels with alternative solutions available in the literature. The second scenario (i.e., on-board data processing without compression) has been also addressed in this Thesis, proposing a run-time adaptive implementation of a linear unmixing chain for hyperspectral images. This particular implementation relies on a novel hardware-friendly data partitioning and reduction algorithm to ensure proper datalevel parallelism and execution scalability, which has been addressed in both singleand multi-FPGA (using a small Ethernet-based computing cluster) contexts. 
The main contributions of this Thesis can be classified and summarized as follows:

- [Architecture] A hardware-based processing architecture for adaptive highperformance embedded computing based on run-time tradeoffs between computing performance, energy efficiency, and fault tolerance.

- [Design] An automated design methodology to generate custom reconfigurable multi-accelerator systems from either low-level RTL descriptions or high-level algorithmic descriptions and HLS.

- [Runtime] A runtime library to transparently manage FPGA reconfiguration and computation offloading in multi-accelerator scenarios.

- [Validation] A Dwarf-based characterization and validation strategy for the $\mathrm{ARTICo}^{3}$ framework based on HLS benchmarks.

- [Runtime] A multi-paradigm programming approach for reconfigurable multiaccelerator systems that combines SIMD-like data-parallel execution with transparent hardware/software multithreading.

- [Design] An integrated toolchain to automatically generate reconfigurable multiaccelerator systems from high-level dataflow descriptions.

- [Application] A run-time adaptive FPGA implementation of a low-complexity on-board CCSDS 123 lossless multispectral and hyperspectral compressor with selectable performance and energy efficiency levels.

- [Application] A run-time adaptive and multi-FPGA implementation of a lowcomplexity on-board linear hyperspectral unmixing chain with selectable performance and energy efficiency levels.

This Thesis is organized in 5 chapters. Chapter 1 discusses the motivation behind the developed work, establishes the main goals to be achieved, and presents a brief overview of the technology background (reconfigurable computing and parallel computing). In Chapter 2, the three main components of the ARTICo ${ }^{3}$ framework (hardware-based computing architecture, automated toolchain, and runtime library) are presented and validated using custom designs (i.e., in-house VHDL and HLSbased accelerators) and an HLS benchmark suite. The reference abstractions provided by the ARTICo ${ }^{3}$ framework are extended in Chapter 3 to also support transparent hardware/software multithreading using ReconOS and dataflow-oriented accelerator specification using MDC. Chapter 4 showcases the ARTICo ${ }^{3}$ framework in two stateof-the-art high-performance embedded computing applications: hyperspectral data compression and hyperspectral linear unmixing. Finally, Chapter 5 closes the Thesis, discussing the conclusions drawn from the developed work, summarizing its main contributions, analyzing its impact with quantitative measurements such as its related publications, and presenting the future lines of work. 


\section{Contents}

List of Figures $\quad$ xxi

$\begin{array}{ll}\text { List of Tables } & \text { XXv }\end{array}$

$\begin{array}{ll}\text { Acronyms } & \text { xxvii }\end{array}$

1 Introduction 1

1.1 Motivation . . . . . . . . . . . . . . . . . . . . 1

1.2 Main Goals of the Thesis . . . . . . . . . . . . . . . . . . 3

1.3 Technology Background . . . . . . . . . . . . . . . . 4

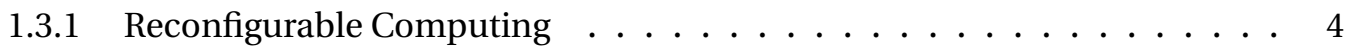

1.3.2 Parallel Computing . . . . . . . . . . . . . . . 8

1.4 Thesis Layout . . . . . . . . . . . . . . . . . . . . . . 13

2 The ARTICo ${ }^{3}$ Framework $\quad 15$

2.1 Framework Overview . . . . . . . . . . . . . . . . . . 16

2.2 State of the Art . . . . . . . . . . . . . . . . . . 18

2.2.1 Architectures for Reconfigurable Computing . . . . . . . . . . . 18

2.2.2 Toolchains for Reconfigurable Computing . . . . . . . . . . . . . . 32

2.2.3 Runtime Systems for Reconfigurable Computing . . . . . . . . . . . . . . 43

2.3 Processing Architecture . . . . . . . . . . . . . . . . . . . 53

2.3.1 Configurable Datapath . . . . . . . . . . . . . . . . . . 54

2.3.2 Monitoring Infrastructure . . . . . . . . . . . . . . . 56

2.3 .3 Operation Modes . . . . . . . . . . . . . . . . . . 57

2.3 .4 Programmer's Model . . . . . . . . . . . . . . . . . 62

2.4 Design-Time Support . . . . . . . . . . . . . . . . . . . . . . . 67

2.4 .1 Kernel Wrapper . . . . . . . . . . . . . . . . . . . 67

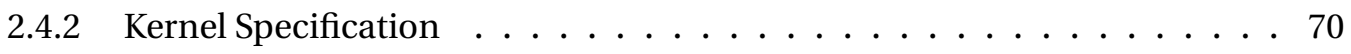

2.4 .3 Template-Based Toolchain . . . . . . . . . . . . . . . . 73

2.5 Run-Time Support . . . . . . . . . . . . . . . . . . . 78

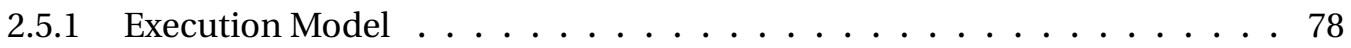

2.5 .2 Runtime Library . . . . . . . . . . . . . . . . . . . . 79

2.5 .3 Kernel Scheduling . . . . . . . . . . . . . . . . . . 81

2.5 .4 Kernel Modeling . . . . . . . . . . . . . . . . 86

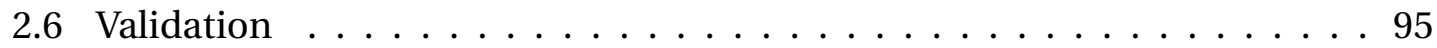

2.6.1 Scenario \#1: Image Filters and AES Block Cipher . . . . . . . . . . . . 96

2.6.2 Scenario \#2: Matrix Multiplication . . . . . . . . . . . . . . . . 99 
2.6.3 Benchmark Evaluation . . . . . . . . . . . . . . . . . . 105

3 Programming Models for Adaptive Hardware $\quad 111$

3.1 Motivation . . . . . . . . . . . . . . . . . . . . . 111

3.2 Multithreading Extensions . . . . . . . . . . . . . . . . . . . . . . 113

3.2.1 Additional Infrastructure . . . . . . . . . . . . . . . . . 116

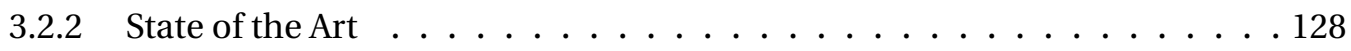

3.2 .3 Integration Details $\ldots \ldots \ldots 131$

3.2 .4 Validation . . . . . . . . . . . . . . . . . . . . . 134

3.3 Dataflow Extensions . . . . . . . . . . . . . . . . . . . . . . . 141

3.3.1 Additional Infrastructure . . . . . . . . . . . . . . . . . . 143

3.3 .2 State of the Art . . . . . . . . . . . . . . . . . . . 150

3.3.3 Integration Details . . . . . . . . . . . . . . . . . . 154

3.3.4 Validation . . . . . . . . . . . . . . . . . 156

4 Adaptive Hyperspectral Data Processing 163

4.1 Motivation . . . . . . . . . . . . . . . . . . . . . . . 163

4.2 Lossless Compression … . . . . . . . . . . . . . . . . . 165

4.2 .1 Algorithm Description . . . . . . . . . . . . . . . 166

4.2 .2 State of the Art . . . . . . . . . . . . . . . . . . 169

4.2 .3 Implementation Details $\ldots \ldots \ldots$. . . . . . . . . . . . . . . . .

4.2 .4 Validation . . . . . . . . . . . . . . . . . . 177

4.3 Linear Unmixing . . . . . . . . . . . . . . . . . . . . . . . 181

4.3.1 Algorithm Description . . . . . . . . . . . . . . . . . 183

4.3.2 State of the Art . . . . . . . . . . . . . . . . . 187

4.3.3 Implementation Details . . . . . . . . . . . . . . . . 188

4.3 .4 Validation . . . . . . . . . . . . . . . . . . 198

5 Conclusions, Impact and Future Lines of Work 203

5.1 Conclusions of the Thesis . . . . . . . . . . . . . . . . . . 203

5.2 Summary of Main Contributions . . . . . . . . . . . . . . . . . . . 204

5.3 Impact of the Thesis . . . . . . . . . . . . . . . . . . 205

5.3.1 Main Publications and Dissemination . . . . . . . . . . . . . . . 206

5.3.2 Additional Publications and Dissemination . . . . . . . . . . . . . . . 210

5.3 .3 Research Projects . . . . . . . . . . . . . . . . . . . . . . . 212

5.3 .4 Co-Supervised Works . . . . . . . . . . . . . . . . . . . . 213

5.3 .5 Collaborations . . . . . . . . . . . . . . . . . 214

5.3.6 Grants and Other Funding . . . . . . . . . . . . . . . . . . 215

5.4 Future Lines of Work ～. . . . . . . . . . . . . . . . . . . . 215

$\begin{array}{lr}\text { Bibliography } & 219\end{array}$ 


\section{List of Figures}

1-1 Generic FPGA architecture. . . . . . . . . . . . . . . . . 5

1-2 Accelerator coupling in reconfigurable systems [Compton'02]. . . . . . . 6

1-3 Flynn's Taxonomy. . . . . . . . . . . . . . . . . . . . . 9

1-4 Amdahl's Law. . . . . . . . . . . . . . . . . . . . . . . . 10

1-5 Gustafson's Law. . . . . . . . . . . . . . . . . . . . . . . . . . 11

1-6 Platform and memory models in OpenCL. . . . . . . . . . . . . . . 13

$2-1$ ARTICo ${ }^{3} \operatorname{logo} \ldots \ldots \ldots \ldots \ldots \ldots \ldots \ldots \ldots$

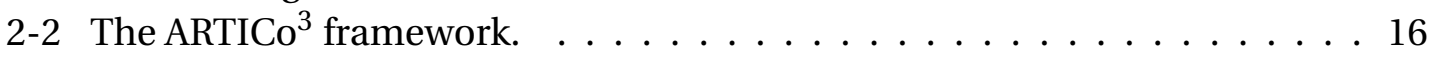

2-3 The ARTICo ${ }^{3}$ architecture. . . . . . . . . . . . . . . . . . . 54

2-4 Configurable datapath. . . . . . . . . . . . . . . . . . . . 55

2-5 Parallel operation mode. . . . . . . . . . . . . . . . . . . . . . . 58

2-6 Redundant operation mode. . . . . . . . . . . . . . . . . 58

2-7 Reduction-oriented operation mode. . . . . . . . . . . . . . . . . 59

2-8 Write burst data transfer in reduction-oriented and redundant mode. . . . 60

2-9 Read burst data transfer in reduction-oriented and redundant mode. . . . 60

2-10 Write burst data transfer in reduction-oriented and redundant mode (detail). . . . . . . . . . . . . . . . . . . . 61

2-11 Read burst data transfer in reduction-oriented and redundant mode

(detail) . . . . . . . . . . . . . . . . . . 662

2-12 Kernel addressing. . . . . . . . . . . . . . . . . . 63

2-13 ARTICo A $^{3}$ ernel wrapper. . . . . . . . . . . . . . . . . . 68

2-14 Control signal requirements. . . . . . . . . . . . . . . . . 70

2-15 ARTICo $^{3}$ floorplan in Spartan-6. . . . . . . . . . . . . . . . . . . . . . . . . . . . 75

2-16 ARTICo $^{3}$ floorplan in Kintex-7. . . . . . . . . . . . . . . . . . 75

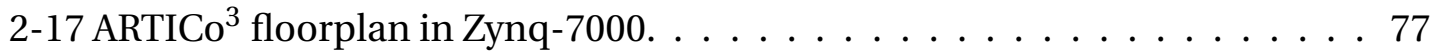

2-18 The ARTICo ${ }^{3}$ execution model. . . . . . . . . . . . . . . . . . . . . 79

2-19 ARTICo $^{3}$ kernel memory model. . . . . . . . . . . . . . . . . . . . . . . . . . . . . . . 84

2-20 Kernel scheduling in ARTICo $^{3}$. . . . . . . . . . . . . . . . . . . . . . . . . . . 84

2-21 Unpaired accelerator/round execution. . . . . . . . . . . . . . . . . . . 85

2-22 Kernel execution modeling in $\mathrm{ARTICo}^{3} \ldots \ldots$. . . . . . . . . . . . . 87

2-23 Kernel execution modeling in $\mathrm{ARTICo}^{3}$ with hardware redundancy. . . . . 89

2-24 Kernel execution modeling in ARTICo $^{3}$ with hardware multithreading. . . 89

2-25 Power consumption traces in Spartan-6. . . . . . . . . . . . . . . . . 90

2-26 Power consumption traces in Kintex-7. . . . . . . . . . . . . . . . . . . 91

2-27 Kernel model evaluation with multiple accelerators. . . . . . . . . . . . . 93

2-28 Static versus dynamic consumption in kernel models. . . . . . . . . . . . 93

2-29 Kernel model evaluation under different initial conditions. . . . . . . . . . 94 
2-30 Implementation methodology for validation. . . . . . . . . . . . . . . 95

2-31 Memory bank partitioning in single versus multithreaded accelerators. . . 97

2-32 Solution space exploration in the HiReCookie node [Valverde'12] . . . . . 98

2-33 Solution space exploration in the KC705 development board. . . . . . . . . 99

2-34 Transition from computing- to memory-bounded execution. . . . . . . . . 100

2-35 Block-based matrix multiplication. . . . . . . . . . . . . . . . . . 101

2-36 Power consumption profile in the custom Zynq-7000 board. . . . . . . . . 103

2-37 Energy consumption in the custom Zynq-7000 board during reconfigu-

ration. . . . . . . . . . . . . . . . . . . . . . . . . . 104

2-38 Energy consumption in the custom Zynq-7000 board during execution. . . 104

2-39 Benchmark evaluation: SDSoC (hardware) versus ARTICo ${ }^{3}$. . . . . . . . . . 109

2-40 Benchmark evaluation: ARTICo ${ }^{3}$ speedup. . . . . . . . . . . . . . . . . 109

2-41 Benchmark evaluation: SDSoC (software) versus ARTICo ${ }^{3}$. . . . . . . . 110

3-1 ReconOS architecture. . . . . . . . . . . . . . . . . . . . 117

3-2 ReconOs toolchain. . . . . . . . . . . . . . . . . . . . . . 119

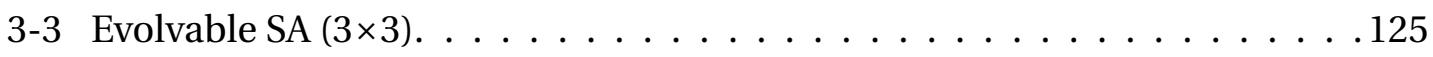

3-4 Internal components (per bit) of the evolvable SA. . . . . . . . . . . 126

3-5 Integrated ReconOS-ARTICo ${ }^{3}$ toolchain. . . . . . . . . . . . . . . 131

3-6 Integrated ReconOS-ARTICo ${ }^{3}$ architecture. . . . . . . . . . . . . . . . . . 132

3-7 ARTICo $^{3}$ kernel wrapper with an embedded evolvable SA core $(8 \times 8)$. . . 133

3-8 Fitness evaluation during evolution. . . . . . . . . . . . . . . . . 137

3-9 Evolution results for different filter types. . . . . . . . . . . . . . . . 138

3-10 Evaluation of the evolved filters for different input images. . . . . . . . . 139

3-11 Online training for environmental adaptation. . . . . . . . . . . . . . . 140

3-12 Generic CAL actor model. . . . . . . . . . . . . . . . . . . . . . . . . . . 142

3-13 MDC overview. . . . . . . . . . . . . . . . . . . . . . 146

3-14 Multi-dataflow merging and platform composition with MDC. . . . . . . . 147

3-15 Memory-mapped MDC coprocessor. . . . . . . . . . . . . . . . . . . 149

3-16 Integrated MDC-ARTICo ${ }^{3}$ flow. . . . . . . . . . . . . . . . . . . . . . . . . . 155

3-17 ARTICo $^{3}$ kernel wrapper with embedded MDC CGR core. . . . . . . . . . . 156

3-18 Simplified DFGs for the Sobel and Roberts image filters. . . . . . . . . . . 157

3-19 Sobel versus Roberts $(512 \times 512$ pixels $)$ using 4 accelerators. . . . . . . . . . 160

3-20 Sobel versus Roberts $(512 \times 512$ pixels) in software. . . . . . . . . . . . . . 161

3-21 CGR versus DPR when reconfiguring 4 accelerators. . . . . . . . . . . . . 162

4-1 Hyperspectral data cube. . . . . . . . . . . . . . . . . . . . . . . . 164

4-2 CCSDS 123 prediction neighborhood. . . . . . . . . . . . . . 167

4-3 CCSDS 123 flowchart. . . . . . . . . . . . . . . . . . . . . . . . 169

4-4 Block diagram of the HyLoC IP core. . . . . . . . . . . . . . . . . . 172

4-5 Hyperspectral image partitioning for compression. . . . . . . . . . . . 173

4-6 Compression rate with strip-based partitioning. . . . . . . . . . . . . . . 175

4-7 Compression rate with square-based partitioning. . . . . . . . . . . . 175

4-8 FPGA floorplan of the run-time adaptive hyperspectral compressor. . . . . 178 
4-9 Hyperspectral mixture. . . . . . . . . . . . . . . . . . . . . . . . 181

4-10 Hyperspectral unmixing: endmembers and abundances [Zhu'14] . . . . . 182

4-11 Linear and nonlinear hyperspectral mixture models. . . . . . . . . . . . . 183

4-12 Hyperspectral image partitioning for reduction-based unmixing. . . . . . . 190

4-13 Network setup of an ARTICo ${ }^{3}$-based computing cluster. . . . . . . . . . . 197

4-14 MPI-based communication overheads. . . . . . . . . . . . . . . . . . 198

4-15 ARTICo $^{3}$-based multi-FPGA computing cluster. . . . . . . . . . . . . . 200 



\section{List of Tables}

2-1 State of the Art-Architectures. . . . . . . . . . . . . . . . 31

2-2 State of the Art-Toolchains. . . . . . . . . . . . . . . . . . . 42

2-3 State of the Art - Runtime Systems. . . . . . . . . . . . . . . . . . . . 52

2-4 ARTICo $^{3}$ register map. . . . . . . . . . . . . . . . . . . . . . . 62

2-5 Signals in the ARTICo ${ }^{3}$ point-to-point interface. . . . . . . . . . . . . . 69

2-6 Port specification in HLS-based kernels. . . . . . . . . . . . . . . . . 72

2-7 Synthesizable helper functions for HLS-based kernels. . . . . . . . . . . . . 72

2-8 ARTICo ${ }^{3}$ toolchain commands. . . . . . . . . . . . . . . . . 73

2-9 ARTICo $^{3}$ runtime library API. . . . . . . . . . . . . . . . . . . 80

2-10 Model parameters for an AES-256 CTR kernel . . . . . . . . . . . . . . . 92

2-11 Model analysis for an AES-256 CTR kernel . . . . . . . . . . . . . . . . 92

2-12 Resource utilization of ARTICo ${ }^{3}$ kernels in Spartan-6 FPGAs. . . . . . . . 96

2-13 Resource utilization of ARTICo ${ }^{3}$ kernels in Kintex-7 FPGAs. . . . . . . . . 97

2-14 Resource utilization of ARTICo ${ }^{3}$ kernels in Zynq-7000 FPGAs. . . . . . . . . 101

2-15 Performance metrics in the matrix multiplication scenario. . . . . . . . . 102

2-16 C-based HLS-oriented benchmark suites. . . . . . . . . . . . . . . . . 107

2-17 MachSuite [Reagen'14] benchmarks. . . . . . . . . . . . . . . . . 107

3-1 Available OS functionality for ReconOS threads. . . . . . . . . . . . . . . . 118

3-2 Available PE functions in the evolvable SA . . . . . . . . . . . . . . . . . . 127

3-3 Standard EA configuration in the evolvable SA [Mora' 18]. . . . . . . . . . . 128

3-4 Resource utilization in the multithreading scenario. . . . . . . . . . . . . 134

3-5 Execution times in the multi-paradigm programming scenario. . . . . . . . 135

3-6 Evolution times using the ARTICo ${ }^{3}$ SA kernel. . . . . . . . . . . . . . . . . 136

3-7 Resource utilization in the dataflow-based hybrid reconfiguration scenario.158

3-8 Execution times in the dataflow-based hybrid reconfiguration scenario. $\quad .159$

3-9 Energy consumption in the dataflow-based hybrid reconfiguration scenario. . . . . . . . . . . . . . . . . . . . . . 159

3-10 Reconfiguration overheads in the dataflow-based hybrid reconfiguration scenario. . . . . . . . . . . . . . . . . . . . . 161

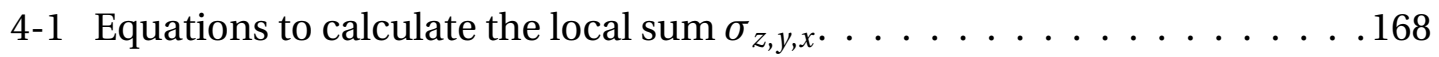

4-2 Equations to calculate the local differences vector $U_{z, y, x} \ldots \ldots \ldots \ldots$

4-3 CCSDS 123 compressor configuration. . . . . . . . . . . . . . 173

4-4 Compression rate with square-based partitioning over hyperspectral

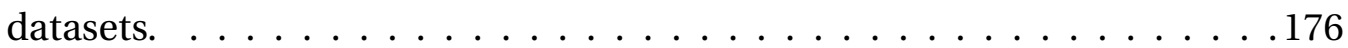

4-5 Resource utilization in the run-time adaptive hyperspectral compressor. . 177 
4-6 Estimated power consumption in the run-time adaptive hyperspectral

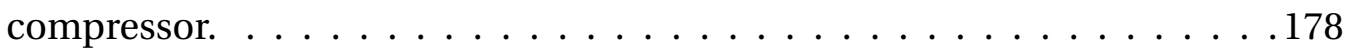

4-7 Compression throughput and energy efficiency (FPGA fabric @ $100 \mathrm{MHz}$ ). 179

4-8 Comparison of CCSDS 123 implementations. . . . . . . . . . . . . . 180

4-9 FUN profiling on Zynq-7000 (ARM Cortex-A9 core @ $666.67 \mathrm{MHz}$ ). . . . . . . 189

4-10 Endmember extraction accuracy with synthetic images. . . . . . . . . . . 193

4-11 Endmember extraction accuracy (real hyperspectral images with known endmembers). . . . . . . . . . . . . . . . . . . . . . 193

4-12 Impact of partitioning depth (i.e., pixel $_{\text {block }}$ ) on endmember extraction accuracy. . . . . . . . . . . . . . . . . . . . . . . 194

4-13 HLS-based FUN configuration. . . . . . . . . . . . . . . . . . . . . . . . 194

4-14 Execution latency in the HLS-based FUN accelerator. . . . . . . . . . . . . 195

4-15 Resource utilization of the HLS-based FUN accelerator. . . . . . . . . . . . 195

4-16 Resource utilization in the run-time adaptive endmember extraction. . . . 199

4-17 Execution performance and energy consumption in hyperspectral unmixing $\left(\right.$ standalone ARTICo $\left.{ }^{3}\right) \ldots$. . . . . . . . . . . . . . . 199

4-18 Execution performance in hyperspectral unmixing (networked ARTICo ${ }^{3}$ ). 201 4-19 Hardware versus software systems for networked hyperspectral unmixing. 201 


\section{Acronyms}

ADAS Advanced Driver Assistance Systems

AES Advanced Encryption Standard

ALU Arithmetic Logic Unit

AMBA Advanced Microcontroller Bus Architecture

API Application Programming Interface

ARTICo $^{3}$ Arquitectura Reconfigurable para el Tratamiento Inteligente de Cómputo, Consumo y Confiabilidad

ASIC Application-Specific Integrated Circuit

AS-Overlay Application-Specific FPGA Overlay

ASP Advanced Standard Profile

AST Abstract Syntax Tree

AVIRIS Airborne Visible InfraRed Imaging Spectrometer

AVIRIS-NG Airborne Visible InfraRed Imaging Spectrometer Next Generation

AXI

Advanced eXtensible Interface

BIL Band-Interleaved-by-Line

BIP Band-Interleaved-by-Pixel

BORPH Berkeley Operating system for ReProgrammable Hardware

bpppb bits per pixel per band

BRAM Block RAM

BSP Board Support Package

BSQ Band-Sequential

CAD Computer-Aided Design

CAL CAL Actor Language

CAPH CAPH just Ain't Plain HDL

CAP-OS Configuration Access Port Operating System

CCSDS Consultative Committee for Space Data Systems

CDFG Control/Data Flow Graph 


\begin{tabular}{ll} 
CGP & Cartesian Genetic Programming \\
CGR & Coarse-Grained Reconfiguration \\
CGRA & Coarse-Grained Reconfigurable Architecture \\
CLB & Configurable Logic Block \\
CPS & Cyber-Physical System \\
CPU & Central Processing Unit \\
CTR & Counter (block cipher operation mode) \\
CUDA & Compute Unified Device Architecture \\
DaCO & Dataflow Coprocessor Overlay \\
DAG & Directed Acyclic Graph \\
DAPR & Design Automation for Partial Reconfiguration \\
DFG & Dataflow Graph \\
DgS-NMF & Data-guided Sparse NMF \\
DMA & Direct Memory Access \\
DMR & Double Modular Redundancy \\
DPN & Dataflow Process Network \\
DPR & Dynamic and Partial Reconfiguration \\
DSE & Design Space Exploration \\
DSL & Domain-Specific Language \\
DSP & Digital Signal Processor \\
DWT & Discrete Wavelet Transform \\
DySER & Dynamically Specializing Execution Resources \\
\hline
\end{tabular}

EA Evolutionary Algorithm

eCos Embedded Configurable Operating System

EDA Electronic Design Automation

EDIF Electronic Design Interchange Format

EDPE Encapsulated Datapath Element

EEMBC Embedded Microprocessor Benchmark Consortium

EHW Evolvable Hardware

EP Evolutionary Programming

EPIC Explicitly Parallel Instruction Computing

ES Evolution Strategy 
ESA European Space Agency

FF Flip-Flop

FFT Fast Fourier Transform

FGPU FPGA general purpose GPU

FIFO First In, First Out

FISH FPA-Initiated Software-Handled

FL Fast Lossless

FlexGrip FLEXible GRaphIcs Processor for general-purpose computing

FOS FPGA Operating System

FPCA Fully Pipelined Composable Architecture

FPGA Field-Programmable Gate Array

FPU Floating Point Unit

FSM Finite State Machine

FUN Fast algorithm for linearly UNmixing hyperspectral images

FUSE Front-end USEr framework

GA Genetic Algorithm

GAL Generic Array Logic

GCU Group Computation Unit

GP Genetic Programming

GPU Graphics Processing Unit

GRVI Gray Research RISC-V RV*I

GUARD GUAranteed Reliability in Dynamically Reconfigurable Systems

GUI Graphical User Interface

HAL Hardware Abstraction Layer

HARP Hardware Accelerator Research Program

HARP $^{2}$ Homogeneous/Heterogeneous Accelerator-Rich Platform

HDL Hardware Description Language

HiPEAC High Performance and Embedded Architecture and Compilation

HLS High-Level Synthesis

HOpenCL Hybrid OpenCL-flavor

HPC High-Performance Computing 


$\begin{array}{ll}\text { HRE } & \text { Heterogeneous Reconfigurable Engine } \\ \text { Hthread } & \text { Hybrid Thread } \\ \text { HTI } & \text { Hardware Thread Interface } \\ \text { HW } & \text { Hardware } \\ \text { HWTI } & \text { Hardware Thread Interface } \\ \text { HyLoC } & \text { multispectral and Hyperspectral Lossless Compressor for space } \\ & \text { applications } \\ \text { ICAP } & \text { Internal Configuration Access Port } \\ \text { IDE } & \text { Integrated Development Environment } \\ \text { IEEE } & \text { Institute of Electrical and Electronics Engineers } \\ \text { ILA } & \text { Integrated Logic Analyzer } \\ \text { IoT } & \text { Internet of Things } \\ \text { IP } & \text { Intellectual Property } \\ \text { IPC } & \text { Interprocess Communication } \\ \text { IRQ } & \text { Interrupt Request } \\ \text { ISA } & \text { Instruction Set Architecture } \\ \text { ISE } & \text { Integrated Synthesis Environment } \\ \text { ISR } & \text { Interrupt Service Routine } \\ \text { ISRA } & \text { Image Space Reconstruction Algorithm } \\ \text { ISSCC } & \text { International Solid-State Circuits Conference } \\ \text { JIT } & \text { Just-In-Time } \\ \text { JTAG } & \text { Joint Test Action Group } \\ & \end{array}$

KAHRISMA KArlsruhe's Hypermorphic Reconfigurable-Instruction-Set Multi-grained-Array

KLT Karhunen-Loève Transform

KPN Kahn Process Network

LEAP Latency-insensitive Environment for Application Programming

LLVM Low-Level Virtual Machine (formerly)

LUT Look-Up Table

MAC Multiply-Accumulate 


\begin{tabular}{|c|c|}
\hline MDC & Multi-Dataflow Composer \\
\hline MEMIF & Memory Interface \\
\hline MIMD & Multiple Instruction, Multiple Data \\
\hline MIPS & Microprocessor without Interlocked Pipeline Stages \\
\hline MISD & Multiple Instruction, Single Data \\
\hline MITRACA & Many-core Interlinked Torus Reconfigurable ACcelerator Architecture \\
\hline MMU & Memory Management Unit \\
\hline MoC & Model of Computation \\
\hline MPEG & Moving Picture Experts Group \\
\hline MPI & Message Passing Interface \\
\hline NAS & NASA Advanced Supercomputing \\
\hline NASA & National Aeronautics and Space Administration \\
\hline NL & Network Language \\
\hline NMF & Nonnegative Matrix Factorization \\
\hline NoC & Network on Chip \\
\hline NPB & NAS Parallel Benchmarks \\
\hline OPAE & Open Programmable Acceleration Engine \\
\hline OpenCL & Open Computing Language \\
\hline OpenDF & Open Dataflow \\
\hline OpenMP & Open Multi-Processing \\
\hline OpenSPL & Open Spatial Programing Language \\
\hline ORCC & Open RVC-CAL Compiler \\
\hline OS & Operating System \\
\hline OSIF & Operating System Interface \\
\hline OTP & One-Time Programmable \\
\hline PAL & Programmable Array Logic \\
\hline PARSEC & Princeton Application Repository for Shared-Memory Computers \\
\hline PCAP & Processor Configuration Access Port \\
\hline PCIe & Peripheral Component Interconnect Express \\
\hline PE & Processing Element \\
\hline PLB & Processor Local Bus \\
\hline
\end{tabular}




$\begin{array}{ll}\text { PMC } & \text { Performance Monitoring Counter } \\ \text { pocl } & \text { Portable Computing Language } \\ \text { PolyPC } & \text { Polymorphic Parallel Computing } \\ \text { POSIX } & \text { Portable Operating System Interface } \\ \text { Pthread } & \text { POSIX thread } \\ \text { R3TOS } & \text { Reliable Reconfigurable Real-Time Operating System } \\ \text { RAM } & \text { Random-Access Memory } \\ \text { RAMPSoC } & \text { Runtime Adaptive Multi-Processor System on Chip } \\ \text { READY } & \text { REconfigurable Accelerator DeploY } \\ \text { ReconOS } & \text { Operating System for Reconfigurable Computing } \\ \text { RIFFA } & \text { Reusable Interface for FPGA Accelerators } \\ \text { RISC } & \text { Reduced Instruction Set Computer } \\ \text { RISP } & \text { Reconfigurable Instruction Set Processor } \\ \text { RSB } & \text { Reconfigurable Streaming Block } \\ \text { RTL } & \text { Register-Transfer Level } \\ \text { RTOS } & \text { Real-Time Operating System } \\ \text { RTSM } & \text { Run-Time System Manager } \\ \text { RVC-CAL } & \text { Reconfigurable Video Coding CAL }\end{array}$

SA Systolic Array

SA-C Single Assignment C

SAE Sum of Absolute Errors

SBox Switch Box

SDF Synchronous Data Flow

SDK Software Development Kit

SHA Secure Hash Algorithm

SHyLoC Hyperspectral Lossless Compressor for space applications

SIMD Single Instruction, Multiple Data

SISD Single Instruction, Single Data

SM Streaming Multiprocessor

SNR Signal-to-Noise Ratio

SoC System on Chip

SoPC System on Programmable Chip 
SPEC Standard Performance Evaluation Corporation

SPLASH Stanford Parallel Applications for Shared-Memory

SPREAD Streaming-Based Partially Reconfigurable Architecture and Unified Software/Hardware Multithreaded Programming Model

SRAM Static RAM

SW Software

TaPaSCo Task Parallel Systems Composer

Tcl Tool Command Language

TILT Thread and Instruction Level parallel Template architecture

TLB Translation Lookaside Buffer

TMR Triple Modular Redundancy

TOPPERS Toyohashi OPen Platform for Embedded Real-time Systems

USB Universal Serial Bus

USGS United States Geological Survey

VAPRES Virtual Architecture for Partially Reconfigurable Embedded Systems

VCA Vertex Component Analysis

VHDL Very High Speed Integrated Circuit Hardware Description Language

VLIW Very Long Instruction Word

VRC Virtual Reconfigurable Circuit

VSB VAPRES SoC Builder

WSN Wireless Sensor Network

XDF XML Dataflow Format

XDL Xilinx Design Language

XML eXtensible Markup Language

XRT Xilinx Runtime 



\section{Chapter}

\section{INTRODUCTION}

The introductory chapter of this Thesis constitutes the starting point for the rest of this document, providing the required problem formulation and reference context. As such, it opens with a discussion on the motivation and main goals to be achieved, followed by a brief overview of the technology surrounding the developed work. At the end, the document structure and a summary of each chapter are presented.

\subsection{Motivation}

Traditionally, embedded systems were application-specific electronic systems meant to execute algorithms to control the rest of the system to which they were attached. At the beginning, embedded computing faced relatively simple scenarios: household appliances (e.g., coffee machines or dishwashers) and personal items used on a regular basis (e.g., digital clocks or calculators) are some of the early examples. Hence, cost was the main limiting factor, even though additional requirements were also imposed sometimes (e.g., real-time responses in critical control loops).

Nowadays, embedded systems can be found in a much wider range of applications, including Advanced Driver Assistance Systems (ADAS), process automation, and even healthcare systems [Cardoso'17]. In fact, the integration of embedded systems within broader paradigms such as Cyber-Physical Systems (CPSs) or the Internet of Things (IoT) has caused the number of potential application scenarios to surge. CPSs [Lee'08] can be seen as the generalization of traditional embedded systems: computational elements (i.e., the cyber part) deeply intertwined with real-world processes (i.e., the physical part) by means of feedback-based sense-actuate interactions. The IoT approach $^{1}$ goes one step further, assuming that computational and physical actors are not only dependent on one another, but also connected to many other systems.

Independently of the underlying paradigm, the increase in application complexity (and therefore in computing requirements) is indisputable. Initially, this excessive computational burden was often assumed by High-Performance Computing (HPC) facilities located in the cloud. However, a recent trend advocates for moving computation back to where data to be processed are generated, with the idea of

\footnotetext{
${ }^{1}$ https://www.rfidjournal.com/that-internet-of-things-thing
} 
reducing network congestion and thus improving the overall processing performance. This paradigm is called edge computing [Shi'16], and makes the case for highperformance embedded computing, whether it is to be deployed on regular CPSs or on IoT nodes. It is worth noting that, as opposed to normal HPC deployments, where performance is still the main driving factor, high-performance embedded computing usually imposes equally stringent requirements for performance, energy consumption, fault tolerance, cost, and many other factors [Lentaris'18].

In parallel to the aforementioned growth in application complexity (and actually motivated by it), there has been a constant evolution in terms of computing platforms. At first, this process was driven by Moore's law [Moore'06], an empiric law that states that transistor count in electronic devices is nearly doubled every 18 months, and Dennard scaling [Dennard'74], another empiric law that states that power density in electronic devices stays constant as transistors get smaller. The combination of both principles allowed semiconductor manufacturers to increase the operating frequency of single-core processors without really affecting its overall power consumption.

Nevertheless, Dennard scaling does not hold true anymore, and increases in the operating frequency do not lead to affordable power consumption values. This effect is reflected in the International Solid-State Circuits Conference (ISSCC) trends report [ISSCC'20]. Notice, however, that Moore's law seems to remain valid (although there are voices nowadays claiming that it will soon cease to be that way). At this point, manufacturers changed their approach, relinquishing single-core processors with extremely high operating frequencies in favor of multi-core solutions [Parkhurst'06]. From this moment onwards, performance improvements would come from exploiting parallelism rather than from executing sequential operations faster and faster.

As a matter of fact, parallel computing is currently one of the most extended computing paradigms, and it has even permeated the field of embedded systems. There are multiple computing substrates capable of actually exploiting parallelism: multi-core Central Processing Units (CPUs) or Digital Signal Processors (DSPs), general-purpose Graphics Processing Units (GPUs), Application-Specific Integrated Circuits (ASICs), and Field-Programmable Gate Arrays (FPGAs). The software-based solutions (i.e., CPUs, DSPs, and GPUs) are usually more flexible and programmerfriendly, but the hardware-based alternatives (i.e., ASICs and FPGAs) render better performance because they favor domain-specific architectures [Hennessy'17]. If all the requirements of high-performance embedded systems are taken into account, FPGAs are usually the best implementation alternative in many application domains, mostly due to their unrivaled performance per watt ratios [Nurvitadhi'16, Qasaimeh'19].

In summary, FPGAs provide hardware-based parallel computing capabilities and execution performance (similar to ASICs, even though still worse), with significantly better energy efficiency than software solutions (especially when compared against general-purpose GPUs, whose architectures are parallel in nature and allow a fairer comparison in terms of computing performance). In addition, and depending on the underlying technology, these characteristics can be further extended. For instance, modern SRAM-based FPGAs support Dynamic and Partial Reconfiguration (DPR), a 
mechanism that enables changes in their internal logic at run time. This mechanism not only provides software-like flexibility, allowing developers to reprogram their devices even during normal system operation, but also favors the time-multiplexing of logic resources, which in turn reduces area overheads (i.e., the FPGA fabric only hosts the required acceleration logic at a given instant, avoiding dark silicon issues) and increases performance (i.e., since accelerators are no longer required to coexist on the FPGA fabric, their implementations can be highly parallel) [Koch'13].

Despite the benefits mentioned in previous paragraphs, high-performance embedded computing systems based on reconfigurable technology still face three main issues: lack of proper hardware architectures to deal with highly dynamic scenarios, lack of adequate design tools to isolate developers from low-level technology details, and lack of user-friendly runtime environments to manage application execution [Göhringer'14]. Regarding hardware architectures, most of the available solutions focus on optimizing computing performance and/or energy efficiency, but do not support run-time tradeoffs between them and other additional requirements (e.g., fault tolerance). Regarding design- and run-time support, there is still a huge gap that makes FPGAs inaccessible to developers with no background on hardware design, either because of the lack of software-friendly programming environments [Bacon'13], or because most DPR flows require extensive user intervention [Vipin'18].

\subsection{Main Goals of the Thesis}

This Thesis addresses the three main problems highlighted at the end of Section 1.1, and builds an integrated framework for reconfigurable multi-accelerator systems while pursuing the following objectives:

- Provide a hardware-based computing platform that adapts to changing scenarios of performance, energy consumption, and fault tolerance at run time.

- Provide the required infrastructure and companion tools to ease application development and reconfigurable hardware-based system implementation.

- Provide software-friendly entry points to hardware accelerator design for developers with no previous experience on hardware design.

- Provide lightweight software abstractions to hide low-level device reconfiguration and accelerator management from developers.

- Provide solid validation mechanisms to assess the developed technology, using a combination of benchmarks and real-world application scenarios. 


\subsection{Technology Background}

The work developed in this Thesis revolves around the use of run-time reconfigurable FPGA technology for adaptive parallel computing in the field of high-performance embedded systems. As a result, and in order to establish the context surrounding the Thesis itself, this section goes briefly over the basics of reconfigurable computing systems and some parallel computing concepts.

\subsubsection{Reconfigurable Computing}

Reconfigurable computing is an approach to customized computing that uses reconfigurable devices to offload computations onto application-specific hardware modules whose functionality may change at design time (i.e., configuration) or at run time (i.e., reconfiguration) [Bobda'07]. As opposed to traditional general-purpose processor execution, where instructions are executed sequentially and parallelism is exploited through pipelining and other fixed but hidden microarchitectural extensions, reconfigurable computing exploits parallelism by explicitly decomposing algorithms in spatially parallel, tiled, and application-specific pipelines [Gokhale'05].

Depending on their configuration granularity, reconfigurable devices can fall within two main categories:

- Fine-grained architectures: these devices are based on very basic logic primitives such as Look-Up Tables (LUTs) and Flip-Flops (FFs) and thus, they can be configured at bit level. The most important representative of this type of architectures are FPGAs. FPGAs appeared in the mid 1980's to deal with the increasing complexity of digital circuits at the time. Soon after, in the late 1980's, these devices grew in popularity due to their reprogramming capabilities, and actually became the foundation of the reconfigurable computing paradigm.

- Coarse-grained architectures: these devices use slightly more complex primitives such as Arithmetic Logic Units (ALUs) (usually distributed in arrays interconnected by a specific topology), which in turn forces them to be configured at word level. These reconfigurable devices, also known as CoarseGrained Reconfigurable Architectures (CGRAs), are usually faster to reconfigure than fine-grained architectures, but suffer from reduced implementation flexibility due to the nature of their Processing Elements (PEs).

Figure 1-1 shows an example of a generic FPGA architecture featuring not only Configurable Logic Blocks (CLBs), which contain basic logic blocks such as LUTs and FFs, but also dedicated on-chip memory storage and DSP blocks to perform optimized arithmetic operations. The physical distribution of programmable logic resources corresponds to an island-based FPGA architecture, the most common implementation 
nowadays [Chang'08]. Note that all the logic blocks are interconnected by programmable wires and switch matrices.

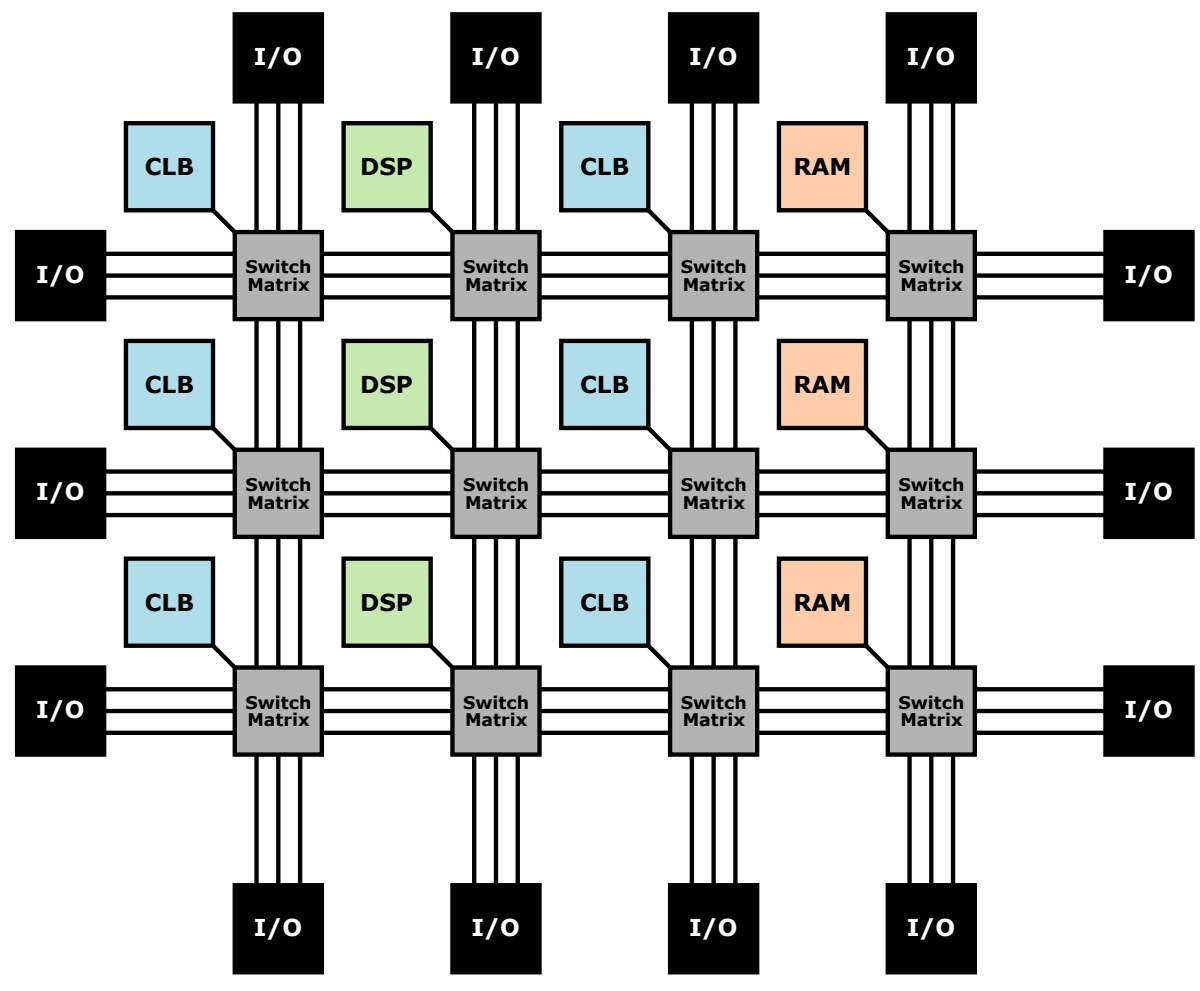

Figure 1-1: Generic FPGA architecture.

\section{SoPC and Accelerator Integration}

Reconfigurable devices render outstanding computing performance under massively parallel workloads, but tend to become inefficient when dealing with the purely sequential operations or control-oriented constructs (e.g., conditional branches) that are often found in most algorithms. Hence, a common trend is to couple reconfigurable devices with traditional microprocessors, and execute each task on the most suitable fabric of the resulting heterogeneous hardware/software platforms.

Depending on the adopted coupling strategy, reconfigurable devices can be classified in one of the following categories [Compton'02] (see Figure 1-2):

- Functional unit: the reconfigurable device is embedded in the datapath of the main processor. As such, the complexity and size of the implemented circuits are limited. This type of coupling is really useful to extend Instruction Set Architectures (ISAs) with custom instructions.

- Coprocessor: the reconfigurable device operates independently of the main processor, which only needs to initialize the accelerator, provide the data to be 
processed, and eventually wait for the results. Hence, this type of coupling allows processor and reconfigurable device to execute simultaneously.

- Attached processing unit: the reconfigurable device acts as an additional processor in the system. Therefore, communication with the main processor is achieved by standard multi-core input/output procedures. This type of coupling enables large computation offloading operations from software to hardware.

- Standalone processing unit: the reconfigurable device is almost completely independent of the main processor, which could not even be present in the system. Being the most loosely coupled approach, it enables complete independence between computing fabrics.

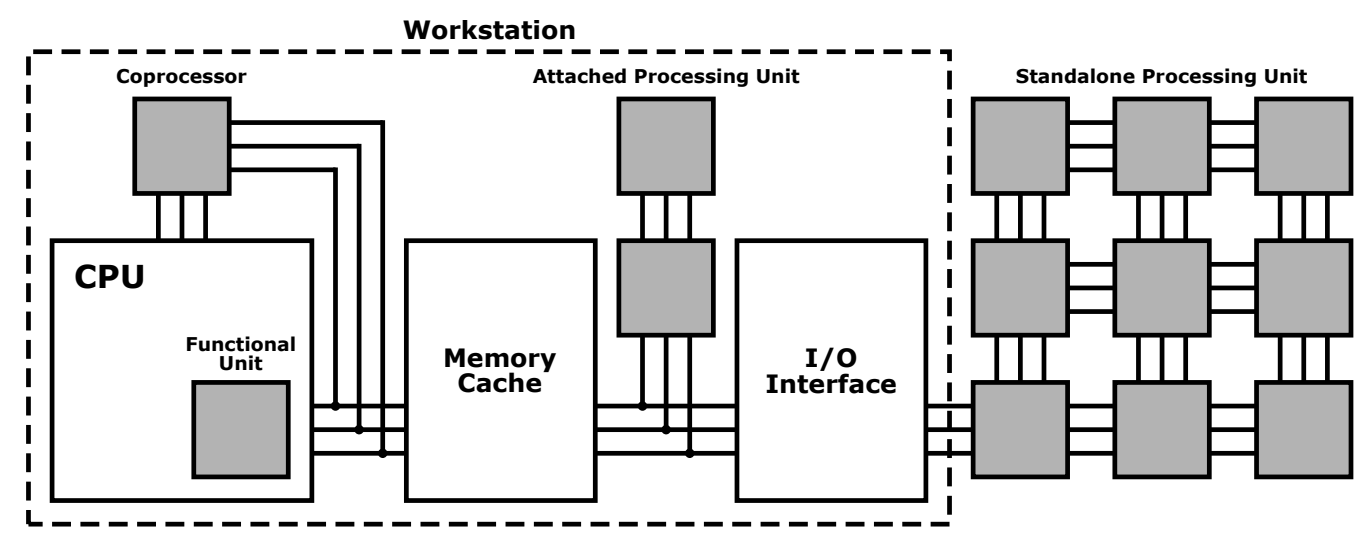

Figure 1-2: Accelerator coupling in reconfigurable systems [Compton'02].

A common and highly productive approach to implement these heterogeneous hardware/software computing platforms, almost independently of the coupling level, is to use System on Chip (SoC) methodologies, which allow system designers to build highly customized platforms using libraries of existing Intellectual Property (IP) cores [Saleh'06]. These IPs can be either soft cores, which are synthesizable RegisterTransfer Level (RTL) descriptions, or hard cores, which are layout-level designs (i.e., already implemented). When the SoC is implemented inside an FPGA device, it becomes a System on Programmable Chip (SoPC). Note that this approach favors the deployment of highly flexible and reconfigurable processor-accelerator systems.

\section{Dynamic and Partial Reconfiguration}

SRAM-based FPGAs provide better computing density (i.e., more logic resources) and performance than alternative implementation technologies (e.g., flash-based FPGAs) [Azarian'09], even though they still present several disadvantages (e.g., need to be reprogrammed every time they are powered on, larger power consumption). Nevertheless, the key feature of SRAM technology is that it enables higher implementation flexibility due to its capability to support infinite reconfiguration cycles. 
FPGA-based reconfiguration can be classified according to different criteria [Azarian'09]. A first classification can be made according to the amount of resources affected by the reconfiguration process:

- Full reconfiguration: the reconfiguration process affects the whole FPGA fabric (i.e., all its internal logic resources).

- Partial reconfiguration: the reconfiguration process affects only a region of the FPGA fabric (i.e., only part of its internal logic resources).

A second classification can be made based on the application-specific relationship between the reconfiguration process and the rest of the system:

- Static reconfiguration: the complete system needs to stop executing during the reconfiguration process. Usually, reconfiguration is performed prior to application execution.

- Dynamic reconfiguration: part of the system continues executing during the reconfiguration process. Usually, reconfiguration is performed at run time, overlapping with application execution.

The two previous classifications are not mutually exclusive. In fact, most reconfigurable systems nowadays present reconfiguration mechanisms that combine one category from each group (except for dynamic and full reconfiguration, which is actually not feasible). For instance, standard FPGA configuration (e.g., during power up) is a static and full reconfiguration procedure. Static and partial reconfiguration, on the other hand, has been used not only to change the functionality of the system, but also to enable energy-efficient FPGA power up cycles [Lombardo'12]. However, it is DPR what trully bridges the gap between software-like flexibility and hardware-like performance in FPGA-based computing systems [Koch'13].

\section{High-Level Synthesis}

Digital system design has been traditionally addressed from a low-level perspective, relying on RTL descriptions written in specific Hardware Description Languages (HDLs). Although this approach does in fact generate highly optimized circuits, it also requires a deep knowledge on the underlying technology to implement them properly. This usually leads to important programming challenges in FPGA-based reconfigurable systems, for they are potentially capable of combining the best of both hardware and software worlds, but lack software-friendly programming models and languages for developers outside the hardware world [Bacon'13].

In the last few years, High-Level Synthesis (HLS) has appeared as a suitable solution to address this issue. Basically, HLS is the process of translating high-level algorithmic descriptions into low-level RTL descriptions. The resulting architectures are usually application-specific datapaths managed by a Finite State Machine (FSM) [Cardoso' 16]. The HLS translation process consists of the following stages: 
- CDFG generation: the synthesizer front end transforms the input code in an intermediate representation such as an Abstract Syntax Tree (AST), applying both platform-independent (e.g., dead code removal) and hardware-specific (e.g., loop transformations) optimizations. Then, control (i.e., dependences between statements) and datapaths (i.e., implementations of statements) are analyzed independently, but combined in a Control/Data Flow Graph (CDFG).

- Scheduling: the synthesizer maps the operations of the CDFG into specific control steps (i.e., states of the FSM). Operations mapped into the same control step are executed in parallel (i.e., instruction-level parallelism), and operations mapped into different control steps may reuse functional units.

- Allocation: the synthesizer selects the number (limited by the amount of operations mapped per control step) and type (including arithmetic operators and storage registers) of hardware units required to implement the functionality.

- Binding: the synthesizer maps the scheduled operations into the allocated hardware resources. Notice that, for shared functional units, additional logic needs to be inserted to select the proper inputs at a given control step.

- Controller synthesis: finally, the synthesizer derives and implements the FSM that governs the transitions between control steps.

Most HLS tools allow developers to insert different optimization directives to actively alter the CDFG, making it possible to generate highly optimized hardware architectures. In the vast majority of scenarios, though, the performance of these implementations is still far from manually optimized HDL solutions. Nevertheless, the increased design productivity usually pays off these performance losses.

\subsubsection{Parallel Computing}

Parallel computing systems seek to improve execution performance by exploiting application parallelism at two different levels [Hennessy'17]:

- Task-level parallelism: appears when several independent operations (or tasks) can be executed at the same time.

- Data-level parallelism: appears when a single operation can be executed using different data at the same time.

Modern computer architectures address these two types of parallelism with specific mechanisms such as instruction-level parallelism (i.e., data-level parallelism via pipelining and speculative execution), vector architectures (i.e., data-level 
parallelism through applying a single instruction to multiple data), or threadlevel parallelism (i.e., task- and data-level parallelism using hardware-enabled multithreading) [Hennessy'17].

The parallel computing paradigm is a broad field of study, comprising diverse topics from computing architectures to programming models and languages. As such, this section will only provide the basic theoretical foundations followed by a brief discussion on modern abstraction models for parallel computing.

\section{Flynn's Taxonomy}

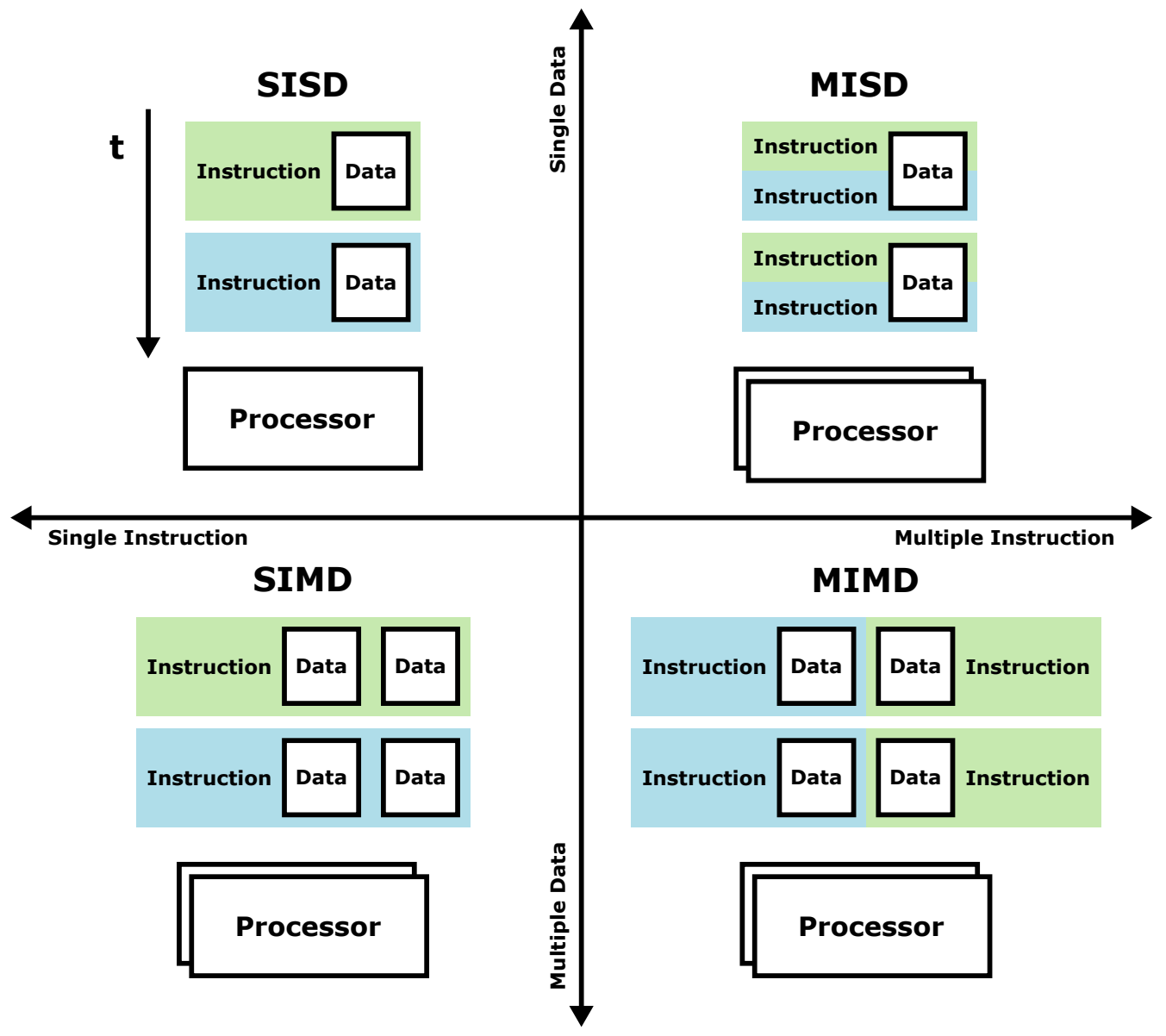

Figure 1-3: Flynn's Taxonomy.

When analyzing the behavior of a computer with regard to the associated instruction and data streams, its internal organization can fall within one of the following categories [Flynn'72] (see Figure 1-3):

- Single Instruction, Single Data (SISD): this computer organization favors sequential execution, leaving almost no room for parallelism. Example platforms include single-core processors. 
- Single Instruction, Multiple Data (SIMD): this computer organization favors data-level parallelism. Example platforms include vector processors and GPUs.

- Multiple Instruction, Single Data (MISD): although this computer organization exists within Flynn's taxonomy and some architectures may be close to it (e.g., systolic arrays), no real-world examples exist.

- Multiple Instruction, Multiple Data (MIMD): this computer organization favors task-level parallelism, even though data-level parallelism is also supported. Example platforms include multi-core processors.

Modern parallel computing architectures rely on SIMD when focusing on data-level parallelism, MIMD when focusing on task-level parallelism, or even a combination of both for enhanced parallelism exploitation.

\section{Amdahl's Law}

There is no such thing as a perfectly parallel application. Amdahl's law [Amdahl'67] provides an upper bound of the performance improvement when increasing the number of parallel computing resources, as shown in Equation 1-1, where $p$ is the parallel fraction of the application (i.e., the ratio of operations that can be completely parallelized) and $N$ is the number of parallel processors used.

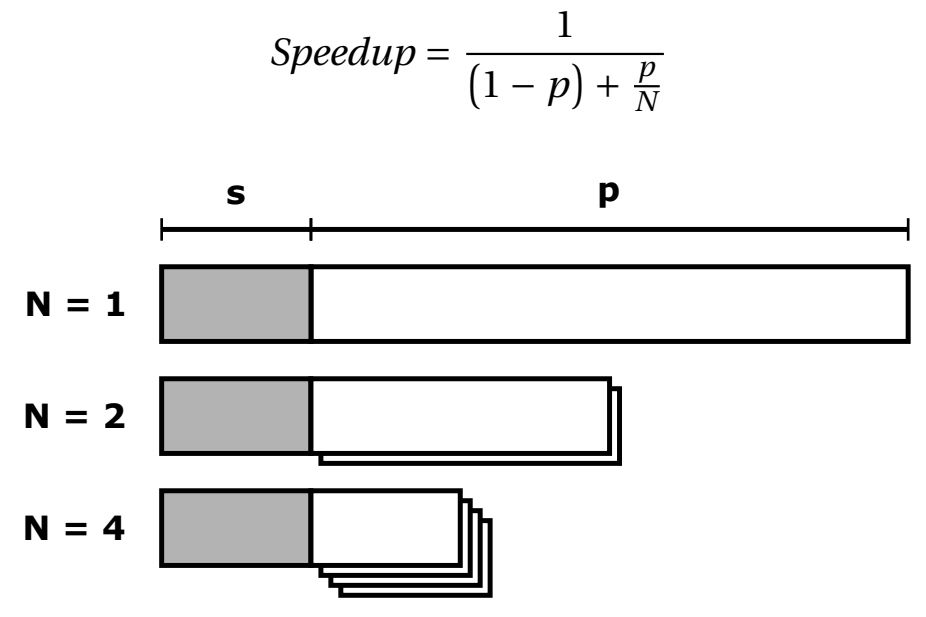

Figure 1-4: Amdahl's Law.

Figure 1-4 provides a graphical representation of Amdahl's law. As it can be seen, the basic principle is that the total workload remains constant, independently of the number of processors deployed, and hence the speedup limitation. 


\section{Gustafson's Law}

An alternative formulation to obtain the performance improvement in parallel computing systems is Gustafson's law [Gustafson'88], shown in Equation 1-2, where $N$ represents the number of parallel processors, whereas $s$ represents the sequential fraction of the application (i.e., the ratio of operations that cannot be parallelized).

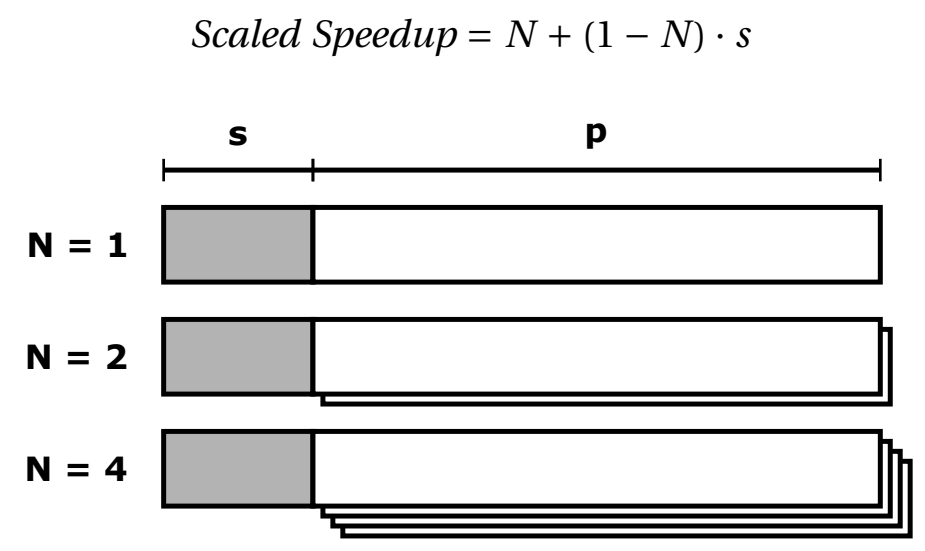

Figure 1-5: Gustafson's Law.

The graphical representation of Gustafson's law is shown in Figure 1-5. The main assumption here is that the total workload is no longer kept constant, since better computing platforms usually lead to scaled problem formulations. In particular, the parallel fraction is assumed to increase linearly with the number of processors available in the platform. Hence, the speedup is said to be also scaled and, despite being still bounded, provides a better approximation to real-world application scenarios.

\section{Abstraction Models}

Parallel computing platforms are highly heterogeneous, as they can be anything from multi-core processors, GPUs, or dedicated hardware accelerators running on FPGA fabrics. As a result, one of the most important problems in the parallel computing scene is how to easily develop applications for such a wide range of different devices.

Although several standard Application Programming Interfaces (APIs) such as POSIX threads (Pthreads), Open Multi-Processing (OpenMP), Message Passing Interface (MPI), or Compute Unified Device Architecture (CUDA) enable parallel execution, they are excessively device-dependent: if the target device is changed, it is highly likely that developers need to start a new implementation from scratch. In this context, the Open Computing Language (OpenCL) specification goes one step further to seamlessly support different heterogeneous computing systems, enabling functional portability across platforms (i.e., application code is guaranteed to run on any compliant device). ${ }^{2}$ To achieve this, four different abstraction models are defined:

\footnotetext{
${ }^{2}$ https://www.khronos.org/opencl/
} 
- Platform model: heavily inspired in general-purpose GPUs, the platform model assumes that computing systems have a host processor and one or more devices attached. To efficiently exploit task- and data-level parallelism, devices are further divided into one or more compute units, which are in turn further divided into processing elements (i.e., where computations actually take place).

- Execution model: applications are divided into host code (i.e., sequential, control-oriented code) and one or more kernels (i.e., data-parallel sections). Kernels are executed on devices using several groups (i.e., work-groups) of dataparallel execution threads (i.e., work-items). Work-groups, which are mapped into compute units, are assumed to be data-independent. Work-items from the same work-group can share data, and are mapped into processing elements. During kernel execution, work-groups and work-items are organized around a common abstraction called NDRange, which represents a N-dimensional grid.

- Memory model: memory is divided in host memory and device memory. This latter is further divided into global memory (i.e., accessible for all work-items in a kernel, independently of their corresponding work-group), local memory (i.e., accessible only for all work-items in a specific work-group), private memory (i.e., accessible only for a specific work-item), and constant memory (i.e., global memory that remains unchanged during kernel execution).

- Programming model: a $\mathrm{C} / \mathrm{C}++$ API is provided to write both host and kernel codes. Since this model is built on top of the previous ones, platform-specific dependencies are hidden from developers. However, and even though functional portability is guaranteed by the specification, developers may opt to include target-specific optimizations in their application descriptions (e.g., favor SIMD execution for GPUs, or enforce pipelined execution for FPGAs).

Figure 1-6 shows the hardware-oriented abstraction models of the OpenCL specification: platform and memory. These provide the required low-level support for scalable execution performance (i.e., according to Gustafson's law, increasing the number of computing devices leads to better execution performance, provided that the global workload is large enough) in heterogeneous parallel computing platforms. 


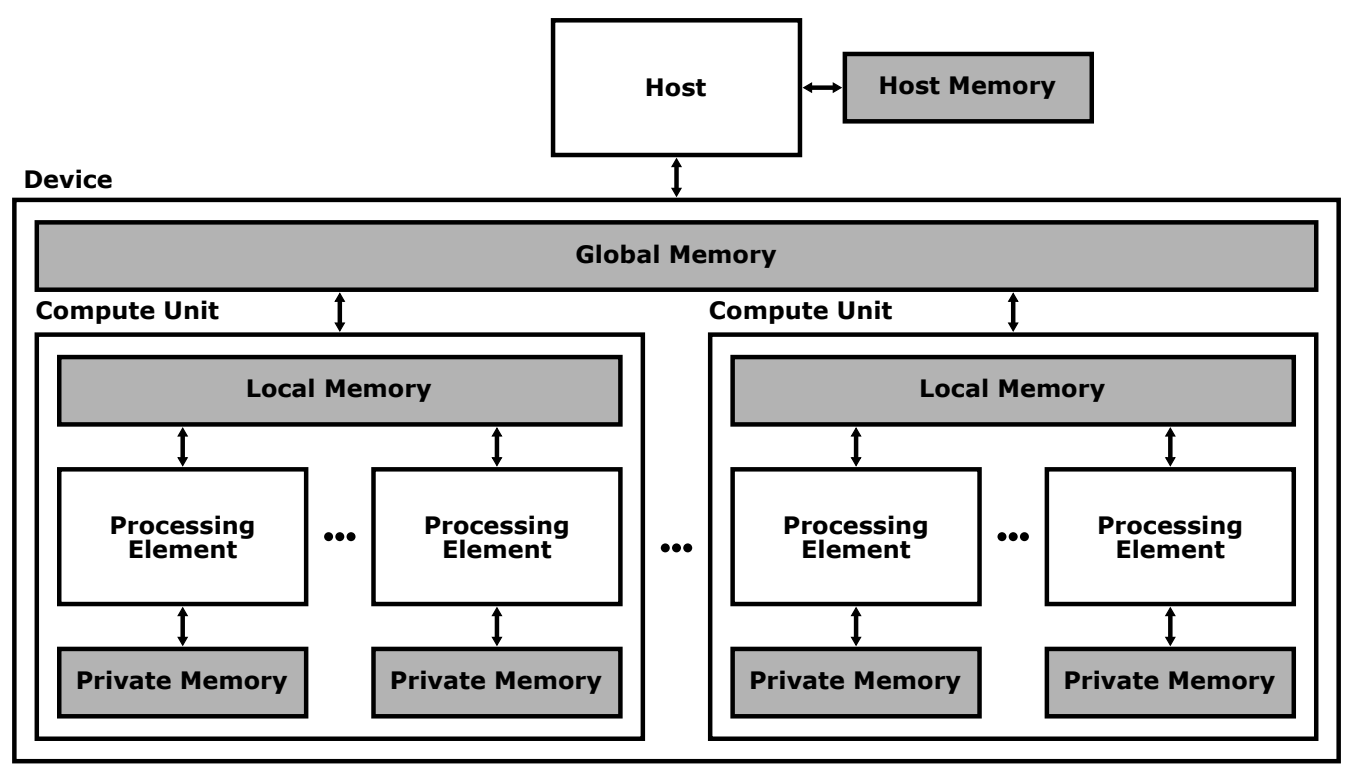

Figure 1-6: Platform and memory models in OpenCL.

\subsection{Thesis Layout}

This document is structured in five chapters, three of them (the central ones) being focused on technical aspects. The remaining two chapters provide the introductory and closing remarks, respectively. In the following, a brief description of the contents of each chapter is provided.

\section{Chapter 1 - Introduction}

This chapter. It includes a section to motivate the development of the framework proposed in this Thesis, and another one to identify the main goals to be achieved. Additionally, the chapter features an overview of the basic technology concepts and methodologies leveraged throughout the work.

\section{Chapter 2 - The ARTICo ${ }^{3}$ Framework}

The first and main technical chapter, in which the framework proposed in this Thesis is described and validated after an in-depth review of the state of the art. Separate sections are provided to present the main components of the framework: a hardwarebased computing architecture for adaptive multi-accelerator scenarios, an automated toolchain to transparently deploy reconfigurable multi-accelerator systems from either low- (i.e., HDL) or high-level (i.e., HLS-oriented $\mathrm{C} / \mathrm{C}++$ ) descriptions, and a runtime library to seamlessly manage device reconfiguration and multi-accelerator execution. Validation is addressed with optimized accelerators developed using both entry points, 
and further extended with several benchmarks to properly analyze the benefits of the framework.

\section{Chapter 3 - Programming Models for Adaptive Hardware}

The second technical chapter, in which the baseline data-parallel programming model proposed in this Thesis is extended to support alternative approaches. Two different extensions are discussed, implemented, and validated in this chapter: hardwarebased multithreading, and hardware-based dataflow execution. Complementary to programming models, the chapter also addresses framework extensions on hardware reconfiguration techniques.

\section{Chapter 4 - Adaptive Hyperspectral Data Processing}

The third and final technical chapter, in which the framework proposed in this Thesis is used in a representative real-world scenario for high-performance embedded computing systems: on-board processing of hyperspectral images. Two different algorithms are discussed, implemented, and evaluated in this chapter: lossless data compression, and linear unmixing.

\section{Chapter 5 - Conclusions, Impact and Future Lines of Work}

The final chapter of the Thesis, which starts by discussing the conclusions and summarizing the main contributions. A specific section is devoted to evaluate the impact of the developed work based on related publications, research projects, cosupervised works, and research collaborations. Finally, the chapter concludes with a discussion on the future lines of work derived from the results of this Thesis. 


\section{Chapter}

\section{THE ARTICo ${ }^{3}$ FRAMEWORK}

The framework to support run-time adaptation in reconfigurable multi-accelerator systems is presented in this chapter. The proposed framework builds around an FPGA-based processing architecture called ARTICo $^{3}$, which enables run-time tradeoffs between computing performance, energy consumption, and fault tolerance. The architecture is complemented at design time with an automated toolchain to map user-defined logic into reconfigurable multi-accelerator systems, and during operation with a runtime library to automatically manage hardware reconfiguration and computation offloading from applications to accelerators.

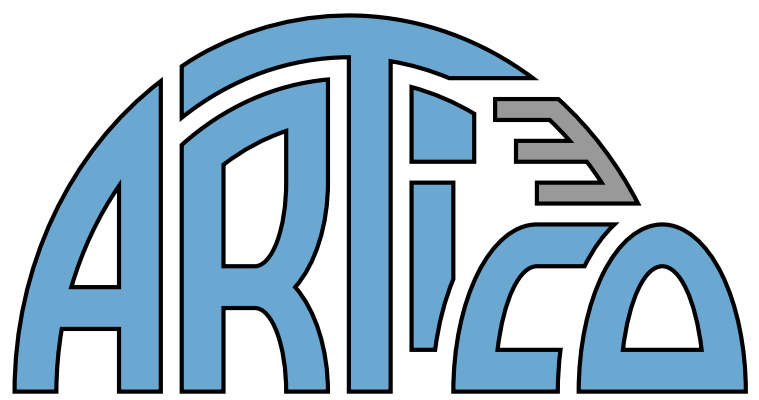

Figure 2-1: ARTICo $^{3}$ logo.

The chapter starts by providing a general introduction to the proposed framework, followed by a review of the state of the art. Then, each of the components of the framework is thoroughly analyzed, highlighting the main contributions of this Thesis at architectural, design-time, and run-time levels. The chapter concludes with the experimental validation of the framework, first using custom in-house designs and then moving to well-known benchmarks for hardware-accelerated computing. 


\subsection{Framework Overview}

The ARTICo ${ }^{3}$ framework is made of three different but complementary components: a hardware-based processing architecture, an automated toolchain to generate multiaccelerator systems, and a runtime management library to control their execution. A complete overview of the framework can be seen in Figure 2-2. Notice how it covers, from top to bottom, the following development stages for multi-accelerator systems: design, implementation, deployment, and run-time management.

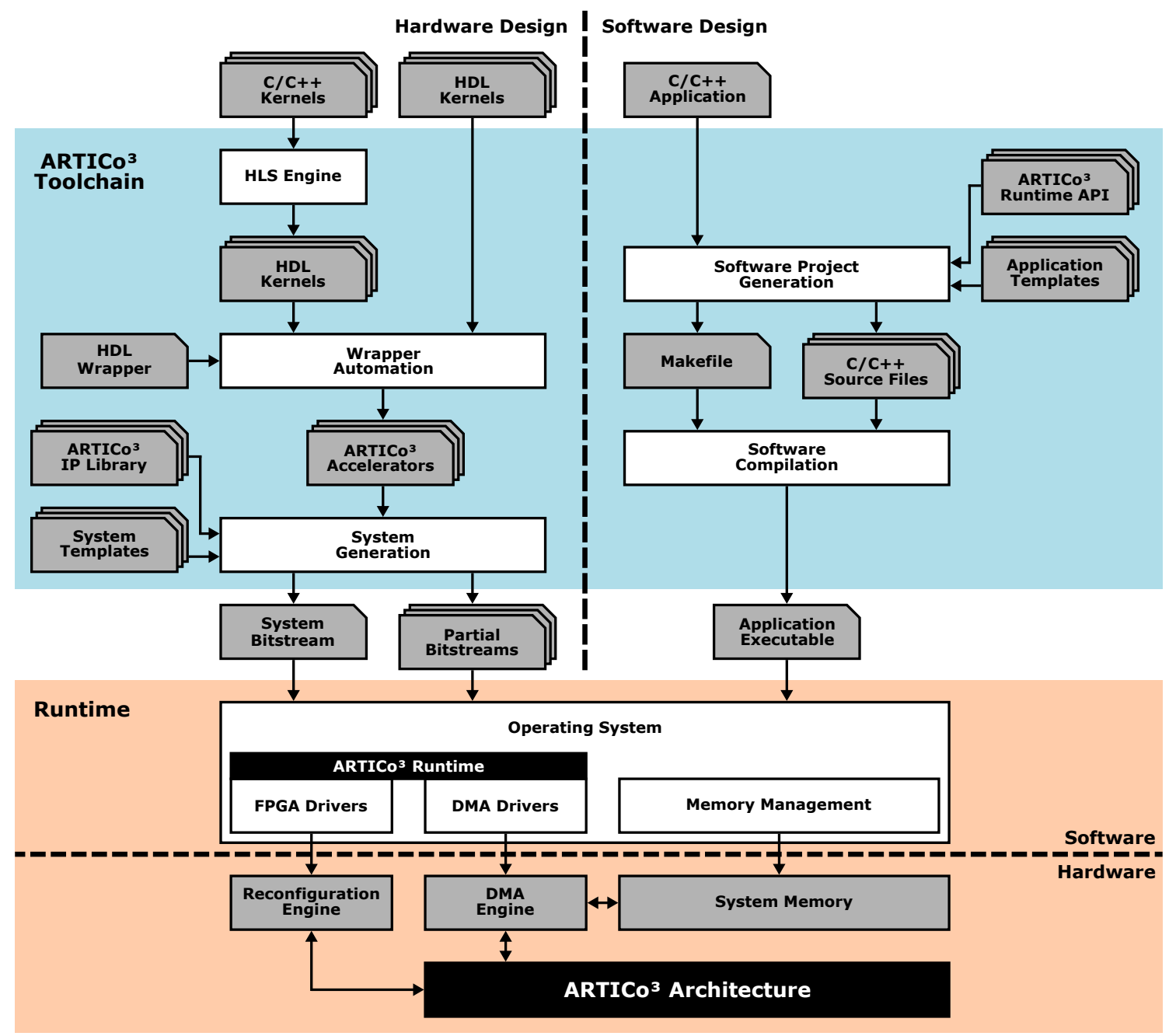

Figure 2-2: The ARTICo ${ }^{3}$ framework.

From all the wide range of scenarios in the field of high-performance embedded computing, the ARTICo ${ }^{3}$ framework addresses those in which applications are a combination of eminently sequential code, suitable for being executed on a microprocessor, and code sections with massive data-level parallelism. These sections, called kernels, are executed on one or multiple hardware accelerators 
inside a processing architecture that acts as an attached reconfigurable processing unit [Compton'02]. In ARTICo $^{3}$, each hardware accelerator is a kernel instance, i.e., an accelerator with a given kernel functionality. The use of DPR as key enabling technology makes it possible to either load several instances from the same kernel to properly exploit data-level parallelism, or instances from different kernels to also exploit task-level parallelism. Furthermore, on-demand hardware redundancy is supported for enhanced fault tolerance in the system.

Kernel functionality needs to be identified prior to implementing a multiaccelerator system, but the $\mathrm{ARTICo}^{3}$ framework does not perform any kind of hardware/software partitioning and thus, users are required to provide separate specifications for both software application and hardware kernel(s). Kernel functionality can be described in HDLs or in $\mathrm{C} / \mathrm{C}++$, since the framework leverages a commercial HLS engine. The automated ARTICo ${ }^{3}$ toolchain uses these input specifications to generate kernel instances (i.e., hardware accelerators) with standard interfaces, builds a multi-accelerator system, and produces the required configuration files for the FPGA (i.e., full and partial bitstreams).

The multi-accelerator system is managed at run time from the software application running on the main microprocessor. The source code for this application, which has to be provided by the user, makes use of a lightweight API to transparently manage device reconfiguration and kernel execution with multiple hardware accelerators. The application executable is also generated using the automated ARTICo $^{3}$ toolchain. The ARTICo ${ }^{3}$ runtime library, which relies on a Linux-based Operating System (OS) extended with proper FPGA and Direct Memory Access (DMA) drivers, performs memory management and multi-accelerator scheduling during kernel execution.

In summary, the main elements of the $\mathrm{ARTICo}^{3}$ framework are:

- A DPR-enabled processing architecture to exploit task- and data-level parallelism with adaptive performance, energy efficiency, and fault tolerance.

- An automated toolchain to generate reconfigurable multi-accelerator systems from low-level HDL or high-level C/C++ and HLS.

- A runtime library to transparently manage FPGA reconfiguration, memory management, and multi-accelerator scheduling.

The framework proposed in this Thesis is open source and is available online. ${ }^{1}$

Original contribution 2-1 An open source framework to support run-time adaptation in reconfigurable multi-accelerator systems.

\footnotetext{
${ }^{1}$ https://github.com/des-cei/artico3
} 


\subsection{State of the Art}

The analysis of the related work available in the literature has been structured according to the three main elements of the ARTICo ${ }^{3}$ framework: architectures, toolchains for design-time support, and runtime environments for run-time support. Each topic is addressed in an individual section that first goes over the main features of the most relevant historic and state-of-the-art solutions, and then finishes with a table that contains a qualitative comparison with the proposals of this Thesis.

\subsubsection{Architectures for Reconfigurable Computing}

\section{Warp Processors}

Warp processors [Lysecky'04] are SoCs implemented on ASIC technology that feature a host microprocessor, an embedded FPGA, an on-chip profiler, and an on-chip Computer-Aided Design (CAD) module. The embedded FPGA acts as a coprocessor, executing critical software sections (i.e., loops) in a mutually exclusive way (i.e., the main processor and the embedded FPGA cannot be executing at the same time). The internal architecture of the embedded FPGA includes a data address generator with dedicated loop control, 3 bidirectional input/output registers, a 32-bit MultiplyAccumulate (MAC) unit, and a reconfigurable fabric made of CLBs and switch matrices.

Critical software sections are identified at run time by the on-chip profiler, which uses a non-intrusive dynamic profiling approach to keep track of all branch operations in the instruction bus. This information is used by the on-chip CAD module (implemented as an additional ARM processor) to partition the application into hardware and software sections. In fact, the on-chip CAD module first decompiles the software binary to obtain its control and dataflow graphs, partitions the application and identifies its critical sections using the information from the on-chip profiler, and performs behavioral and register transfer synthesis to transform the control and dataflow graphs into a hardware netlist. Finally, the on-chip CAD module performs Just-In-Time (JIT) FPGA compilation (i.e., logic synthesis, technology mapping, placement, and routing), and modifies the original software binary to accelerate loop execution using the embedded FPGA and its new configuration. Please note that, even if the on-chip online hardware generation tools are extremely lightweight, accelerator synthesis and implementation takes 1.2 seconds (on average), a value that is at least one or two orders of magnitude larger than average DPR times. 


\section{RAMPSoC}

The Runtime Adaptive Multi-Processor System on Chip (RAMPSoC) [Göhringer'08] approach generates FPGA-based reconfigurable systems that act as standalone processing units. It is highly flexible, since it supports different types of processors and interconnection topologies. In fact, each processor node can be either a Reconfigurable Instruction Set Processor (RISP) or a standard soft-core processor coupled with one or more hardware accelerators acting as either coprocessors or attached processing units. Regarding interconnection topologies, RAMPSoC supports point-to-point, bus-based, or even NoC-based communication.

The main reconfiguration mechanism in RAMPSoC is DPR, which is extensively used for several purposes: to change the application binary to be executed by a processor node, to change the instruction set of a processor node (directly or by changing its associated hardware accelerators), to change the interconnection topology and/or the routing policy, and to add/remove/change processor nodes according to changing performance and energy efficiency requirements.

\section{Hthreads}

The Hybrid Threads (Hthreads) framework [Andrews'08] targets heterogeneous hardware/software multithreading and thus, its bus-based architecture features a CPU that acts as the main processor, and several application-specific hardware accelerators that act as attached processing units. Since Hthreads is built around OS concepts for reconfigurable computing, one of the key characteristics of its architecture is that it contains several hardware blocks used to manage accelerator execution at run time. Moreover, its POSIX-like multithreading programming model enables both task(i.e., each thread performs a different operation) and data-level (i.e., several threads perform the same operation on different data) parallelism, even though the former is favored over the latter. However, the framework does not report the use of any type of reconfiguration mechanism to enable system adaptation.

\section{Devaux et al.}

The work presented in [Devaux'09] introduces a processing architecture where several reconfigurable hardware accelerators act as a standalone processing unit. A softcore microprocessor, which is also instantiated on the same FPGA fabric, is used to control DPR. As a particular feature, the architecture uses a Fat-Tree interconnection topology, which is an indirect communication network based on a binary tree. The proposed topology places all tasks at the bottom of the tree, and provides bidirectional communication channels between the different tree levels. This approach favors an optimized device floorplanning in which the communication infrastructure is placed in the center of the FPGA, and all reconfigurable regions are placed around it so that tasks can have access to both the Fat-Tree and the input/output primitives. 


\section{VAPRES}

The Virtual Architecture for Partially Reconfigurable Embedded Systems (VAPRES) targets stream-oriented data processing applications [Jara-Berrocal'10]. In this architecture, the FPGA is divided into two parts: the controlling region and the data processing region. In the former, a soft-core processor is used for software-based processing and to execute support tasks (e.g., DPR management or run-time operation control). The latter, on the other hand, behaves as an attached processing unit.

In VAPRES, the data processing region hosts one or more Reconfigurable Streaming Blocks (RSBs), which are connected to the main processor using dedicated streaming channels. In turn, each RSB hosts one or more reconfigurable regions and input/output modules, which are interconnected using a linear array of custom switch boxes (i.e., multiplexers and FFs) [Jara-Berrocal'09]. Reconfigurability is achieved by means of DPR (to load different application-specific hardware accelerators into the reconfigurable regions of an RSB) and register-based dynamic communication channel creation (to adapt the interconnections between reconfigurable regions and input/output modules at run time). In fact, the latter is also combined with registerbased accelerator state save and restore techniques to perform hardware module switching in a seamless way (i.e., without stopping any data stream). Furthermore, and thanks to the automatic isolation provided by First In, First Out (FIFO) primitives, the operating frequency of each reconfigurable region is also configurable.

\section{KAHRISMA}

The KArlsruhe's Hypermorphic Reconfigurable-Instruction-Set Multi-grained-Array (KAHRISMA) [Koenig'10] is a software-programmable architecture that can be used as a standalone processing unit. Its main characteristic is that it can work with three different ISA types: Reduced Instruction Set Computer (RISC), Very Long Instruction Word (VLIW), and Explicitly Parallel Instruction Computing (EPIC) [Schlansker'00]. Moreover, it also supports custom instructions.

The KAHRISMA architecture is divided into four separate modules: an instruction predecoding stage (i.e., to handle variable instruction lengths and further preprocessing such as VLIW instruction isolation), a multi-grained reconfigurable array, a cache subsystem, and the processor control unit. The reconfigurable processing array is a network of fine- and coarse-grained Encapsulated Datapath Elements (EDPEs). A finegrained EDPE consists of a regular structure of CLBs connected by switch matrices (i.e., an embedded FPGA), whereas a coarse-grained EDPE is a small CPU that includes a local context memory to store operation-related code, a local sequencer to access this code, a DMA engine to move data to and from the caches, a register file, and additional instruction decoding and execution logic. It is worth noting that the multi-grained array can work in two different operation modes: processor mode, in which one or several coarse-grained EDPEs are used together with the instruction predecoding stage to replicate a RISC/VLIW/EPIC processor, and array mode, in which a variable number of fine- and coarse-grained EDPEs are used to implement custom instructions. 
KAHRISMA relies on a flexible software framework to generate system configurations from $\mathrm{C} / \mathrm{C}++$ code. This framework is based on LLVM, and automatically identifies and extracts custom instructions from the intermediate representation of an application, providing several implementation alternatives. Additionally, the framework also partitions the application into segments that are suitable for a given ISA variant, and generates the associated binaries (including the required array reconfiguration triggers). During operation, a runtime system handles these triggers and loads the proper configuration into the architecture: to execute normal ISA instructions, the runtime configures part of the array in processor mode; to execute custom instructions, the runtime analyzes the current state of the architecture, selects the most suitable implementation from all the available alternatives (generated at design time), and configures part of the array in array mode. Depending on these configurations, KAHRISMA can exploit parallelism at instruction level (i.e., VLIW and EPIC ISAs), at task level (i.e., multithreading based architecture configurations with more than one processor implemented), and at data level (i.e., custom instructions).

\section{Straka et al.}

The work presented in [Straka'10] shows several DPR-capable architectures for adaptive fault tolerance management. These architectures allow reconfigurable application-specific hardware accelerators to execute in either Double Modular Redundancy (DMR) or Triple Modular Redundancy (TMR) modes, with the comparator or the voter being placed in the static region of the FPGA. Additionally, fault recovery and device repair are also supported thanks to a dedicated ICAP-based reconfiguration controller that is triggered whenever the fault monitoring logic detects a fault.

\section{ReCoBus}

The ReCoBus-based computing architecture presented in [Oetken'10] is a reconfigurable multi-accelerator system with a hybrid communication infrastructure for its application-specific hardware accelerators: a bus connects them to the main processor as attached processing units, and dedicated point-to-point connections allow fast stream-based communication between contiguous hardware tasks.

ReCoBus implements accelerators using hard-macro templates and imposing stringent placement and routing constraints. As a result, a regular structure that eases DPR-based changes is generated in the FPGA fabric. In this structure, a fixedsize slot (i.e., width and height in terms of CLBs) is defined as the basic accelerator area to avoid run-time device fragmentation and dark silicon issues. The user logic can then use one or several of these basic blocks to implement all the required functionality. The physical regularity (once deployed on the FPGA) derived from this approach allows adjacent accelerators to have direct communication channels (i.e., input/output bars), and multiple ReCoBus instances to coexist in the same SoC implementation. While the input/output bars enable stream-based processing with 
no processor intervention, a PLB-to-ReCoBus bridge is required to provide a common access point for all reconfigurable accelerators to the main system bus, and to enable burst-based data transactions with the main memory.

\section{Hübner et al.}

The work presented in [Hübner'11] shows a bus-based reconfigurable processing system in which one or several virtual FPGAs are configured as attached processing units for a host microprocessor. A virtual FPGA is an overlay that behaves exactly as a regular FPGA, but allowing run-time reconfiguration in devices that do not support native DPR mechanisms or where DPR is not feasible (e.g., flash-based technology). In the proposed architecture, each virtual FPGA is made of multiple CLBs arranged in island-style and connected by switch matrices. Additionally, each CLB contains a 4-input LUT, a D-type FF, connection boxes, and glitch-free reconfiguration logic. Moreover, the proposal includes some CAD tools, such as a graphical configuration editor (to visualize the implemented designs) or a configuration bistream generator, to ease hardware development.

\section{FUSE}

The Front-end USEr framework (FUSE) [Ismail'11] is another approach that provides OS extensions to enable transparent hardware/software multithreading on FPGAs. The implementation made to test the framework uses a bus to connect task-specific hardware accelerators with a host microprocessor that runs a Linux-based OS.

Seamless hardware/software integration is enabled by a two-layer software stack that isolates application developers from hardware designers: on the software side, applications interact with the OS using a set of Portable Operating System Interface (POSIX) function calls; on the hardware side, accelerators and their particular interfaces are managed by low-level OS drivers. Thanks to these abstraction layers, FUSE can decide during normal system operation whether to use hardwareaccelerated versions of specific tasks or their default software-based implementations.

Although FUSE can theoretically support the use of DPR to dynamically change the hardware tasks instantiated on the FPGA fabric, the evaluation setup does not perform any test in this regard. 


\section{DySER}

The Dynamically Specializing Execution Resources (DySER) architecture extends the execution stage of a typical microprocessor pipeline with a custom functional unit to provide a tradeoff solution between functional specialization (i.e., applicationspecific hardware) and data-parallel specialization (i.e., software-programmable SIMD hardware) [Govindaraju'12].

Internally, DySER is arranged as a configurable array of basic arithmetic units (e.g., integer/floating-point adders, multipliers, or dividers) that are connected using a Network on Chip (NoC). In this regard, ISA extensions are provided to load a configuration into the array, read/write data from/to registers, and read/write data from/to memory. These additional instructions are automatically inserted by the compiler, which first performs profiling or static analysis to isolate memory regions from computation regions. Then, the compiler embeds the required instructions in the former and generates the corresponding array configuration for the latter.

As a matter of fact, the compiler manages not only functional specialization (i.e., array configuration generation) but also data-parallel specialization, even though the authors report only manual transformations in their original work. The first round of applied transformations depends upon the extracted Dataflow Graphs (DFGs) in computation regions: in case of insufficient data-level parallelism, the compiler uses loop unrolling (i.e., duplicate the body of a loop multiple times to avoid control overhead) and scalar expansion (i.e., transform a scalar variable into an array of variables to remove data dependencies); in case of excessive data-level parallelism, the compiler uses subgraph matching (i.e., merge common dataflow subgraphs) and fast configuration switching (i.e., store multiple contexts on array nodes and switches and use local FSMs to change configurations). Then, a second round of transformations such as strip mining (i.e., transform a single loop into several segments with SIMD encoding) are applied to enable vectorization.

\section{MORPHEUS}

MORPHEUS [Voros'13] is an ASIC-based embedded processing architecture that uses three different Heterogeneous Reconfigurable Engines (HREs) to support multiple reconfiguration granularities: the XPP-III CGRA [Schüler'09], the DREAM reconfigurable DSP [Campi'07], and the FlexEOS embedded FPGA [Pulini'09]. These HREs are meant to be used as attached processing units of an ARM-based host microprocessor. In MORPHEUS, data are exchanged using a DMA-powered NoC, whereas configuration is performed using bus-based communication.

The key characteristic of MORPHEUS is that both parallelism and reconfiguration can be exploited at different granularities. This can be seen when analyzing the internal architecture of each HRE: the XPP-III 16-bit processor features a datafloworiented ALU array and several VLIW cores, the DREAM engine features a 32-bit RISC CPU extended with a reconfigurable datapath that includes 4-input LUTs, 4-bit ALUs, 
and 4-bit multipliers, and the FlexEOS embedded FPGA features a basic logic block with a 4 -input LUT and a D-type FF.

Reconfiguration in MORPHEUS is software-based, since the DPR capabilities of the SRAM-based embedded FPGA are not used. Application development starts with an annotated C-based specification in which each code section is explicitly assigned to a particular HRE (using wrapper functions with pragma directives in the host code, and separate implementations for those functions in $\mathrm{C}$ code). The compiler infrastructure then extracts control and data information, and performs HLS to generate target-dependent code. Finally, a back-end compiler uses the targetdependent code to generate the configuration bitstreams for each fabric. At run time, overall system execution is managed by a software application running in the ARMbased microprocessor core under a modified version of the Embedded Configurable Operating System (eCos) Real-Time Operating System (RTOS).

\section{FlexGrip}

The FLEXible GRaphIcs Processor for general-purpose computing (FlexGrip) is a soft-core GPU for FPGA-based computing [Andryc'13]. When configured on the FPGA fabric, this architecture acts as an attached processing unit for a softcore microprocessor. FlexGrip is binary-compatible with a subset of the CUDA 1.0 specification, supporting up to 27 different integer instructions. As a result, time-consuming hardware synthesis is avoided in favor of a pure software-based compilation approach: once the soft core has been deployed, no hardware changes are required to execute different kernels on the FPGA fabric. It is worth noting that concurrent kernel execution (i.e., task-level parallelism) is not supported in CUDA 1.0 and thus, only instruction- and data-level parallelism can be exploited.

The soft GPU microarchitecture contains a configurable number of Streaming Multiprocessors (SMs), which are further divided into a configurable number of scalar execution units, and is written in Very High Speed Integrated Circuit Hardware Description Language (VHDL). In fact, most of the parts correspond to handwritten code, even though the scalar execution units have been automatically generated using model-based design (i.e., MATLAB/Simulink and Xilinx System Generator). Each SM in FlexGrip has a five-stage (i.e., fetch, decode, read, execute, and write) pipelined architecture controlled by a warp unit, which schedules warps (i.e., groups of threads that execute in SIMD fashion) using a round-robin algorithm. Scalar execution units are used during the execute stage to perform integer addition, subtraction, multiplication, MAC, data type conversion, shift operations, and bitwise logic operations. In addition, SMs in FlexGrip support hardware-assisted conditional branch execution and thread synchronization. 


\section{SPREAD}

The proposal of the Streaming-Based Partially Reconfigurable Architecture and Unified Software/Hardware Multithreaded Programming Model (SPREAD) [Wang' 13] builds around a unified hardware/software multithreading approach that exploits highly efficient point-to-point streaming communication channels. In fact, userdefined hardware accelerators are instantiated in a common wrapper structure called Hardware Thread Interface (HTI), which includes a bus-based control interface and two high-performance streaming interfaces for HW/HW and HW/SW communication. SPREAD, an attached processing unit, is complementary to a main microprocessor where software tasks execute.

SPREAD uses DPR to adapt point-to-point streaming interconnections between hardware accelerators, and to change the hardware accelerator type/number. As a result, it supports both task- and data-level parallelism. Run-time system management is enabled via OS extensions and a Pthread-compatible programming model that enables transparent hardware/software task switching.

\section{GUARD}

The GUAranteed Reliability in Dynamically Reconfigurable Systems (GUARD) method enables run-time reliability management in reconfigurable processing architectures [Zhang'14]. GUARD targets reconfigurable architectures where accelerators are connected to a main processor, which is assumed to have been previously hardened against radiation, using a bus-based communication infrastructure. Applications, which are expressed as DFGs, use one or several of these hardware accelerators, which behave as attached processing units. However, DFG mappings over reconfigurable regions and their associated scheduling are not unique, since they depend on the required performance and/or dependability levels and on FPGA resource availability.

The GUARD runtime system analyzes monitoring metrics to decide which accelerator variant (defined by its performance and number of resources) will be loaded, and which fault tolerance mechanism needs to be applied (including modular redundancy and configuration memory scrubbing techniques). Actually, decisions are made according to a combination of online soft error monitoring and reliability estimation during normal system operation. 


\section{HOpenCL}

In the Hybrid OpenCL-flavor (HOpenCL) framework [Ding'14], a two-level bus infrastructure is used to implement an FPGA-based OpenCL-compatible computing platform. The host processor (i.e., global host) is a soft-core MicroBlaze, whereas the compute device is constituted by a configurable number of Group Computation Units (GCUs). Each GCU includes another soft-core processor to manage data transfers and work-group execution (i.e., local host), and a configurable number of slave processors. These slave processors can be implemented either as additional softcore processors (for software-based kernel execution), or as HLS-generated cores (for hardware-accelerated kernel execution).

Communication inside GCUs is performed using a local bus, whereas data transfers between global host and GCUs are done using a global bus. Additional stream-based communication channels are also present in the HOpenCL hardware architecture to speed up operations that are not memory mapped, such as scheduling or synchronization. In this regard, HOpenCL also provides hardware-assisted barriers.

The framework does not support any kind of run-time reconfiguration mechanism (i.e., FPGA bitstream and application binaries need to be generated for each target setup), nor task-level parallelism, as it can be seen in the application/kernel code snippets that show how the HOpenCL API works.

\section{ReconOS}

The Operating System for Reconfigurable Computing (ReconOS) [Agne'14] implements a unified hardware/software multithreading programming model that enables both task- and data-level parallelism, and in which hardware accelerators are connected to a host microprocessor through a bus, acting as attached processing units. ReconOS supports DPR as a mechanism to modify the FPGA substrate, which is arranged in several reconfigurable slots.

ReconOS has been used in this Thesis to extend the ARTICo ${ }^{3}$ framework and thus, a detailed description of its hardware architecture can be found in Subsection 3.2.1.

\section{Backasch et al.}

The work presented in [Backasch'15] describes an architectural template to generate application-specific hardware accelerators using DPR for run-time functional composition of digital circuits for database processing, which replaces time-consuming hardware synthesis. The architecture features NoC-based communication, with each network node having a common interface (i.e., receive/transmit FSMs and FIFO channels) and a reconfigurable partition.

The architectural template is meant to be used as an attached processing unit, providing PCIe interfaces to this end. In fact, there are two different PCIe endpoints: 
one to enable data communication between the NoC entry point, and another to enable direct communication with the reconfiguration manager, which is a microprocessor embedded on the same FPGA device.

\section{HARP $^{2}$}

The Homogeneous/Heterogeneous Accelerator-Rich Platform (HARP ${ }^{2}$ ) [Hussain'16] is a standalone processing unit in which several software-programmable CGRAs are connected using a $3 \times 3$ DMA-capable NoC. A RISC processor is placed in the central node to manage CGRA contexts (i.e., functionality and interconnections). Each CGRA is built using a grid of 2D spatially distributed PEs made of 32-bit ALUs with support for both integer and floating point arithmetic. Spatially close and distant PEs exchange data using local and global interconnections, respectively. In addition, each CGRA features two local memories, accessible through specific input/output buffers, with as many banks as columns in the PE array.

HARP $^{2}$ supports both task- (i.e., each CGRA node accelerates a specific function) and data-level (i.e., several CGRA nodes accelerate the same function but operate on different input data) parallelism. The hardware architecture is defined in parameterizable VHDL code. This template can then be customized for applicationspecific scenarios, rendering homogeneous (i.e., occupied slave nodes in the NoC feature CGRAs with the same size) or heterogeneous (i.e., occupied slave nodes in the NoC feature CGRAs with different sizes) HARP $^{2}$ deployments.

\section{VThreads}

VThreads [Chouliaras'16] is a massively parallel and configurable multiprocessor system meant to be used as an attached processing unit of a host CPU embedded on the same FPGA or ASIC device. Internally, it consists of a configurable number of shared-memory multiprocessors connected through an AXI4 infrastructure. Due to these multiprocessors not being bus masters, an external DMA engine is required to perform data transactions.

Each shared-memory multiprocessor has a single data memory and features 256 contexts, with each context having a single instruction memory and being able to store 16 different architectural (i.e., process) states. Additionally, contexts feature 4 resource clusters that are used during the execute stages by the 16 architectural states using a vertical multithreading approach (i.e., only 1 thread uses the cluster at a given clock cycle). These resource clusters include multiple integer and floating-point ALUs, a multi-port load/store unit, and custom HLS-generated hardware accelerators for instruction set extension purposes.

As it can be seen, the VThreads microarchitecture, which is written in VHDL, is deeply hierarchical. In order to simplify the programming of such a complex system, a dedicated compilation toolchain is provided. Application developers can use this 
toolchain to easily implement highly optimized multithreading applications thanks to the hardware-accelerated Pthreads support of VThreads.

\section{Nyuzi}

The Nyuzi processor [Bush'16] is an open source soft GPU core written in SystemVerilog code. ${ }^{2}$ As opposed to mainstream GPU architectures, it follows a CPUlike approach with specific SIMD extensions (in fact, Nyuzi uses a 3-operand registerto-register RISC ISA), and operates as a standalone processing unit. An LLVM-based $\mathrm{C} / \mathrm{C}++$ compiler is provided with the architecture to ease application development.

The Nyuzi processor features a configurable number of pipelined cores with private L1 instruction and data caches, and a shared L2 cache. Each Nyuzi core includes a configurable number of hardware threads. From a software perspective, a hardware thread is an independent processor with its own program counter and register file. However, hardware threads share both $\mathrm{Ll}$ caches and the parallel execution pipelines: a single stage integer ALU and a 5-stage floating point ALU (both of them supporting scalar and 16-wide vector instructions), and a 2-stage load/store unit.

Nyuzi cores schedule hardware threads using a round-robin algorithm in the instruction fetch stages, selecting one of the available program counters. Then, they decode the associated instruction and place it on a per-thread queue that divides the processor pipeline in two isolated parts. In fact, a thread selection algorithm picks one of the enqueued instructions using a scoreboard, and ensures that the rest of the processor pipeline is kept busy even if a stall (e.g., a cache miss) occurs in the instruction fetch stages. Once an instruction has been selected from a queue and the required operands have been obtained from the register file, one of the parallel execution pipelines performs the required computation, and a write-back path is scheduled by the thread selection algorithm.

\section{FGPU}

The FPGA general purpose GPU (FGPU) [Al Kadi'18] is an open source soft GPU core for FPGAs written in VHDL code. ${ }^{3}$ It features an LLVM-based OpenCL compiler, and a 49-instruction ISA inspired by MIPS that supports both integer and single precision floating point arithmetic operations. As any other OpenCL-compatible device, it coexists with a host microprocessor as an attached processing unit with an AXI4-based control interface. Moreover, it connects to global memory using up to 4 parallel AXI4 interfaces.

The 32-bit deeply pipelined FGPU microarchitecture is configurable at design time, allowing users to instantiate a variable number of compute units. Each of these compute units includes an SIMD engine with 8 different PEs. A maximum of 512

\footnotetext{
${ }^{2}$ https://github.com/jbush001/NyuziProcessor

${ }^{3}$ https://github.com/malkadi/FGPU
} 
work-items can be assigned to a compute unit, which includes support for work-item divergence (i.e., conditional statements in kernel code). Individual PEs include a fixedpoint ALU and the private memory, which is implemented as a register file with 32 registers per work-item. Nevertheless, floating-point logic is placed outside PEs due to its higher execution latency.

Kernel execution is started after loading its executable binary into an instruction cache, and several runtime information (e.g., kernel parameter values, number of work-items to execute) into a link memory. Then, a work-group scheduler uses the content of the latter to initialize a runtime memory, which contains OpenCL-specific data that is retrieved using built-in kernel functions (e.g., get_global_id), and to distribute workload among compute units. Finally, work-item execution is bundled in wavefronts, which are groups of 64 work-items that share a program counter and execute the same instruction spatially (8 PEs) and temporally (8 clock cycles). Memory operations are handled using a hierarchical approach, with a local controller per compute unit and a global controller with embedded data cache support. As it can be seen from this description, FGPU does support data-level parallelism, but it does not allow concurrent kernel execution (i.e., task-level parallelism).

FGPU supports DPR to either completely change the soft GPU core before kernel execution (i.e., offline reconfiguration), or to change floating-point operators inside compute units during kernel execution (i.e., online reconfiguration). While both approaches are highly beneficial to reduce the area footprint of the FGPU instance, the latter incurs excessive performance reductions during normal operation.

\section{MITRACA}

The Many-core Interlinked Torus Reconfigurable ACcelerator Architecture (MITRACA) is a CGRA many-core overlay for FPGAs [Ben Abdelhamid'19]. It features a grid of $8 \times 8$ PEs interconnected using a $3 \mathrm{D}$ torus topology. Each PE is arranged as a 5-stage pipeline structure that includes a 64-bit integer ALU, a double precision Floating Point Unit (FPU), two separate 64-bit register files with 64 entries each, and a local memory. The architecture features an ISA with 27 instructions to handle not only integer and floating point operations, but also external memory transfers and local load/store operations.

Globally, the MITRACA architecture is managed by a processor-based controller, which includes a global memory controller, a DMA engine, and an instruction sequencer. This latter fetches instructions from program memory and configures all PEs to perform the same operation. Then, execution is carried out in SIMD fashion using data stored in the local memories. These local memories are read/written using a broadcast block that issues data transfers using masks associated with each PE. 


\section{READY}

The REconfigurable Accelerator DeploY (READY) framework [Silva'19] builds upon Intel Open Programmable Acceleration Engine (OPAE) ${ }^{4}$ and includes a reconfigurable processing architecture based on a CGRA overlay for FPGAs. A prototype implementation of the architecture is made on an Intel HARPv2, which combines a Xeon CPU with an Arria 10 FPGA in the same package. As a result, the CGRA architecture is connected to the main processor using PCle links, and behaves as an attached processing unit.

Apart from the PCIe interfaces and the OPAE logic, the hardware architecture features a $64 \mathrm{KiB}$ cache, several configurable PEs, a multi-stage global interconnection network, and a control unit. Each PE has 2 inputs and 2 outputs, an ALU that can execute up to 17 different instructions (i.e., add, sub, mult, abs, and, or, xor, not, beq, bne, slt, sgt, mux, min, max, shr, and shl), an instruction memory, and hardwarebased multithreading logic (i.e., several program counters and register files that are multiplexed in time using a thread counter, switching them every clock cycle).

The READY framework has a high-level dataflow entry point in which developers specify their applications using a C++ API. The dataflow graph described in the input code is then mapped into the target architecture, generating a CGRA configuration file that is sent to the FPGA fabric before execution. Finally, processing is carried out by the CGRA overlay in a pipelined way, overlapping input/output data streams with actual hardware execution to exploit task-level parallelism.

\footnotetext{
${ }^{4}$ https://01.org/OPAE
} 


\section{Qualitative Comparison}

Table 2-1 summarizes the most relevant features of the different architectures analyzed in this section. Additionally, the architectural component of the framework proposed in this Thesis has been also included in the last row for comparison purposes. Details regarding its main characteristics can be found in Section 2.3.

Table 2-1: State of the Art - Architectures.

\begin{tabular}{|c|c|c|c|c|c|c|}
\hline Architecture & Coupling & Topology & PES & Parallelism & Redundancy & Reconfiguration \\
\hline Warp Processors [Lysecky’04] & $\mathrm{C}$ & - & Embedded FPGA & - & - & JIT Compiler \\
\hline RAMPSoC [Göhringer'08] & SPU & $\mathrm{P} 2 \mathrm{P} / \mathrm{Bus} / \mathrm{NoC}$ & RISPs + AS-HWs & $\mathrm{T}+\mathrm{D}$ & - & DPR \\
\hline Hthreads [Andrews'08] & APU & Bus & AS-HWs & $\mathrm{T}+\mathrm{D}$ & - & - \\
\hline [Devaux'09] & SPU & Fat-Tree & AS-HWs & $\mathrm{T}$ & - & DPR \\
\hline VAPRES [Jara-Berrocal'10] & APU & Stream & AS-HWs & $\mathrm{T}$ & - & DPR \\
\hline KAHRISMA [Koenig'10] & SPU & NoC & $\begin{array}{c}\text { CPUs }+ \\
\text { Embedded FPGAs }\end{array}$ & $\mathrm{I}+\mathrm{T}+\mathrm{D}$ & - & SW \\
\hline [Straka'10] & SPU & - & AS-HWs & - & DMR/TMR & DPR \\
\hline ReCoBus [Oetken'10] & APU & $\mathrm{P} 2 \mathrm{P}+\mathrm{Bus}$ & AS-HWs & $\mathrm{T}$ & - & DPR \\
\hline [Hübner'11] & APU & Bus & Virtual FPGAs & - & - & VRC \\
\hline FUSE [Ismail'11] & APU & Bus & AS-HWs & $\mathrm{T}$ & - & DPR \\
\hline DySER [Govindaraju'12] & FU & NoC & $\begin{array}{c}\text { Arithmetic Units } \\
\text { CGRA + }\end{array}$ & $\mathrm{I}+\mathrm{D}$ & - & SW \\
\hline MORPHEUS [Voros'13] & APU & Bus + NoC & $\begin{array}{c}\text { DSP + } \\
\text { Embedded FPGA }\end{array}$ & $\mathrm{I}+\mathrm{T}+\mathrm{D}$ & - & SW \\
\hline FlexGrip [Andryc'13] & APU & Bus & Soft GPU & $\mathrm{I}+\mathrm{D}$ & - & SW \\
\hline SPREAD [Wang' 13] & APU & Stream + Bus & AS-HWs & $\mathrm{T}+\mathrm{D}$ & - & DPR \\
\hline GUARD [Zhang'14] & APU & Bus & AS-HWs & $\mathrm{T}$ & $\begin{array}{c}\text { Simplex + } \\
\text { DMR + TMR }\end{array}$ & DPR \\
\hline HOpenCL [Ding'14] & APU & Stream + Bus & CPUs/AS-HWs & $\mathrm{D}$ & - & - \\
\hline ReconOS [Agne'14] & APU & Bus & AS-HWs & $\mathrm{T}+\mathrm{D}$ & - & DPR \\
\hline [Backasch'15] & APU & NoC & AS-HWs & $\mathrm{T}$ & - & DPR \\
\hline HARP $^{2}$ [Hussain'16] & SPU & NoC & CGRAs & $\mathrm{T}+\mathrm{D}$ & - & SW \\
\hline VThreads [Chouliaras'16] & APU & Bus & $\begin{array}{l}\text { VLIW CPUs + } \\
\text { AS-HWs }\end{array}$ & $\mathrm{I}+\mathrm{T}+\mathrm{D}$ & - & SW \\
\hline Nyuzi [Bush’16] & SPU & Bus & Soft GPU & $\mathrm{I}+\mathrm{T}+\mathrm{D}$ & - & SW \\
\hline FGPU [Al Kadi'18] & APU & Bus & Soft GPU & $\mathrm{I}+\mathrm{D}$ & - & $\mathrm{SW}+\mathrm{DPR}$ \\
\hline MITRACA [Ben Abdelhamid'19] & SPU & 3D Torus & CPUs & $\mathrm{I}+\mathrm{D}$ & - & SW \\
\hline READY [Silva'19] & APU & Bus & CPUs & $\mathrm{T}$ & - & SW \\
\hline ARTICo $^{3}$ & APU & Bus & AS-HWs & $\mathrm{T}+\mathrm{D}$ & $\begin{array}{c}\text { Simplex + } \\
\text { DMR + TMR }\end{array}$ & DPR \\
\hline
\end{tabular}

N/A (-) | AND (+) | OR (/)

Coupling [Compton'02]

FU: Functional Unit

C: Coprocessor

APU: Attached Processing Unit

SPU: Standalone Processing Unit

Topology

P2P: Point-to-Point

PEs

AS-HWs: Application-Specific Hardware Accelerators

Parallelism

I: Instruction

T: Task

D: Data 


\subsubsection{Toolchains for Reconfigurable Computing}

\section{Xilinx Vitis}

Xilinx Vitis is the evolution of the previous SDx toolchains (i.e., SDAccel and SDSoC), and targets both PCIe-based accelerator boards and SoC-based embedded boards. The Vitis Unified Software Platform adopts a software-centric approach where the host application is written in $\mathrm{C} / \mathrm{C}++$ and uses the OpenCL API to interact with the different hardware accelerators instantiated on the FPGA fabric. Moreover, the entry point for these hardware-accelerated kernels is multiple, supporting low-level HDL (e.g., VHDL or Verilog), $\mathrm{C} / \mathrm{C}++$, and OpenCL descriptions.

Vitis uses predefined platforms (i.e., templates) to create hardware systems. These platforms are divided in two different parts: the static region and the dynamic region. The former contains clock and reset logic, a PCIe interface and a DMA engine (only in accelerator boards), and additional peripherals (if needed). The latter, on the other hand, is used to instantiate the kernel logic (generated by means of HLS), the required memory controllers, and an AXI-based interconnection network.

Once the system is implemented, Vitis generates a configuration bitstream for the dynamic region (i.e., a .xclbin file). During system operation, this partial bitstream is loaded into the FPGA fabric using the Xilinx Runtime (XRT) infrastructure.

\section{Intel FPGA SDK for OpenCL}

Intel (formerly Altera) provides a commercial toolchain to compile and run parallel applications on dedicated hardware resources using OpenCL as entry point. Although it features a Graphical User Interface (GUI) (mainly for host code development and compilation under Windows), this toolchain strongly relies on shell-based interactions with the user (host code compilation under Linux, and FPGA bitstream generation).

Hardware system generation involves several steps. First, one or several kernel specifications are transformed into hardware modules using HLS. Then, the compiler integrates these modules into a predefined Board Support Package (BSP) (i.e., a template) that contains a prebuilt netlist with all the peripherals required to communicate kernel logic with the host processor and the external (i.e., global) memory. This communication is based on memory-mapped Avalon interfaces. Finally, a binary file that contains a configuration bitstream is generated (i.e., a .aocx file).

The Intel OpenCL offline compiler supports DPR for both PCIe-based accelerator boards and embedded SoCs. At design time, developers decide how many kernels are to be placed in a single partial bitstream (i.e., a .aocx file) and how many different partial bitstreams are to be generated. During execution, the Intel OpenCL runtime performs DPR transparently whenever it is required. 


\section{ReCoBus-Builder}

ReCoBus-Builder [Koch'08] allows designers to create DPR-compatible systems in Xilinx FPGAs. This toolchain extends vendor-specific tools, even though it keeps most of their technology-dependent procedures (e.g., placement and routing).

In order to implement the target reconfigurable system, users interact with a GUI that enables proper configuration of the ReCoBus instance (i.e., protocol, data bus width, address bus width, number of interrupt lines, number of control signals, maximum number of master and slave elements) and the optional input/output bars (i.e., point-to-point interconnections between adjacent reconfigurable modules). In a first step, a simulation-oriented RTL model is generated to perform system validation. In addition, VHDL instantiation templates for the communication infrastructure are also generated.

The second step involves device floorplanning, which starts by synthesizing all the input VHDL codes (i.e., user-defined accelerators) to obtain estimated resource utilization reports. According to those preliminary reports, users perform floorplanning in the GUI, selecting the size (i.e., height and width in terms of CLBs) and the number of reconfigurable slots to be created. Then, the ReCoBus infrastructure and the input/output bars are automatically generated as hard macros (i.e., placed and routed components on the FPGA fabric) using Xilinx Design Language (XDL) descriptions.

The last step generates the configuration bitstreams required to program the target FPGA. ReCoBus-Builder provides separate generation of static and reconfigurable partitions (even though it performs a sort of bitstream composition called linking to obtain the initial bitstream for the static part), and supports module relocation. Common place and route issues are solved by instantiating blocker macros (i.e., dummy logic that prevents both placement and routing through specific components).

\section{VSB}

The VAPRES SoC Builder (VSB) [Jara-Berrocal'11a] is the companion toolchain to the VAPRES architecture [Jara-Berrocal'10], and comprises two separate modules: the base system design flow, and the application design flow. The former is used to generate a baseline VAPRES implementation from a BSP specification and userdefined parameters (e.g., number of reconfigurable and input/output modules, size of reconfigurable modules). A GUI assists developers in this process, whose output is a properly implemented system with several reconfigurable regions automatically allocated on the FPGA fabric.

The VSB application design flow, on the other hand, is an Integrated Development Environment (IDE) in which developers can write the code for both the host microprocessor $(\mathrm{C} / \mathrm{C}++)$ and the hardware accelerators (low-level VHDL or HLSoriented Impulse C). Since VSB does not perform automated hardware/software codesign, applications need to be already partitioned when entering the toolchain. 
The host code, apart from other software tasks, includes function calls to the VAPRES API to reconfigure the FPGA and manage hardware interconnections. The accelerator code, on the other hand, requires top-level streaming interfaces to enable seamless integration with the rest of the architecture. While in the VHDL-based accelerators this needs to be implemented manually, Impulse $\mathrm{C}$ templates are automatically generated (during base system creation) to ease the HLS-based design process.

It is worth noting that both the VSB base system and application design flows share a common Python-based implementation backend that issues the required technology-dependent commands to vendor-specific tools.

\section{GOAHEAD}

GOAHEAD [Beckhoff'12] is, to some extent, the successor of ReCoBus-Builder. As such, most of the design flow stages are similar. However, several improvements have been made to enable a more flexible DPR approach (e.g., scripting interface to automate reconfigurable system generation).

As a first step, system designers need to identify which parts will be placed in the static region, and which ones will be allocated into reconfigurable partitions. Resource utilization reports, which can be automatically generated synthesizing all input hardware blocks, are used to drive FPGA floorplanning. Hence, reconfigurable partitions can be generated either by direct user intervention (i.e., specifying them in the GUI) or automatically (i.e., letting GOAHEAD decide which is their optimal size and location). Additionally, GOAHEAD reads the input VHDL specifications of the modules to automatically generate custom interfaces between reconfigurable components.

GOAHEAD also isolates the implementation of the static and dynamic regions. However, the strategy is almost equal in both cases: once the floorplanning is finished, VHDL templates and physical implementation constraints are generated and attached to the input VHDL descriptions. Vendor-specific attributes are used to prevent vendor tools (i.e., the back end) from placing hardware primitives in forbidden areas, and blocker macros to prevent the same tools from using forbidden routing resources. Extended features of the GOAHEAD toolchain include hierarchical reconfiguration support (i.e., nested reconfigurable areas), automatic communication infrastructure synthesis (i.e., homogeneous communication primitives for slot- or grid-based reconfigurable layouts), and DPR simulation. Furthermore, it supports module relocation even if static routes are included in the reconfigurable partitions. 


\section{ReShape}

The ReShape methodology and tools [Neely'13] enable the automatic floorplanning of domain-specific FPGA-based DPR-capable systems. In fact, ReShape extends ShapeUp [Neely'10], a high-level design framework to automatically generate interconnected hardware systems from directed graph specifications.

The ReShape design flow has two main components. First, an element library of system components is generated by packaging their input RTL specification (e.g., VHDL or Verilog), a description of their interfaces (i.e., interface metadata), and additional information regarding resource utilization. In fact, these resource utilization metadata are obtained by synthesizing the input RTL code for each module. Afterwards, the main implementation flow uses the element library together with a high-level description of the application connectivity written in Click, a language to represent applications as directed graphs. After a validation step, ReShape builds the system RTL specification, connecting all the input modules, and performs automatic floorplanning of the FPGA fabric using a domain-specific algorithm (i.e., suitable for hardware-accelerated networking applications). Finally, full and partial bitstreams are generated using vendor-specific tools (i.e., Xilinx PlanAhead and ISE). One of the key aspects of the ReShape methodology is that, theoretically, it can be adapted to other application domains only by changing the floorplanning algorithm.

\section{ReconOS}

The ReconOS framework [Agne'14] features an automated toolchain for the automatic generation of hardware/software multithreading systems. This shell-based toolchain supports both low-level VHDL and HLS-oriented C/C++ accelerator descriptions, and automatically generates the target hardware system (i.e., modules + interconnections) using predefined templates (i.e., sets of IP cores and Tcl implementation scripts). Users select one of the available templates using a configuration file, which also includes the number and type of hardware threads to be instantiated on the FPGA fabric. Although DPR is supported, it requires extensive user intervention, since FPGA floorplanning and partial bitstream generation are not included among the processes automated by the toolchain. ${ }^{5}$

ReconOS has been used in this Thesis to extend the ARTICo ${ }^{3}$ framework and thus, a detailed description of its automated toolchain can be found in Subsection 3.2.1.

\footnotetext{
${ }^{5}$ http://www.reconos.de/gettingstarted/dynamic_reconfiguration/
} 


\section{ThreadPoolComposer}

The open source ThreadPoolComposer toolchain [Korinth'15] is built upon the idea of having a processor-centric system with a collection of different hardware accelerators that execute independent tasks (i.e., a thread pool). ${ }^{6}$ This toolchain features three different stages: behavioral synthesis (i.e., RTL accelerator code generation using HLS from $\mathrm{C} / \mathrm{C}++$ specifications), thread pool composition (i.e., accelerator instantiation and replication based on user preferences, as well as automatic insertion of the communication infrastructure), and system generation (i.e., device-dependent infrastructure composition and connection to the thread pool).

In ThreadPoolComposer, users provide simple key-value configuration files to select the appropriate template for the architecture (i.e., device-independent components) and platform (i.e., device-dependent components). Hence, accelerator interfaces and interconnections (e.g., AXI4) are customized with the former, whereas host processor implementation (e.g., external with PCIe link, internal with embedded processor) is configured with the latter. Additionally, an interactive shell allows users to generate the FPGA configuration bitstreams.

In parallel, users also need to provide the application code that is to be run on the host processor. A two-layer software stack is provided following the same architecture-platform principle: the ThreadPoolComposer API provides deviceindependent functionality (e.g., launching a job and delegating its scheduling to a dedicated hardware module in the platform), whereas the Platform API provides lowlevel device-dependent functionality (e.g., accessing hardware registers).

\section{Archborn}

Archborn [Ma'15b] is shell-based toolchain to automatically compose SoC systems on Xilinx FPGAs. It provides a Tcl API that acts as an intermediate abstraction layer between users and low-level Vivado commands for IP core instantiation and connection, as well as extended functions to implement predefined (i.e., templatebased) interconnection infrastructures.

Archborn is used to build bus-based SoC topologies (even though stream-based interfaces are also supported for point-to-point communication) in which the different PEs are provided within an IP library of vendor-specific and user-defined modules. Although not explicitly stated, these custom modules are assumed to have been previosly packaged from VHDL or Verilog sources, and require an AXI-compatible top-level interface to enable seamless integration with the rest of the system. Additionally, Archborn provides automated support for DPR-compatible module instantiation, which automatically generates the required floorplanning constraints for later placement of the specified module on the FPGA fabric.

As a proof of concept, Archborn is used to automatically implement two different hardware/software processing architectures, namely Hthreads and HOpenCL systems,

\footnotetext{
${ }^{6}$ https://git.esa.informatik.tu-darmstadt.de/REPARA/threadpoolcomposer
} 
providing additional DPR support to the latter. The easy-to-use system description approach of Archborn increases design productivity, favoring fast Design Space Exploration (DSE) in multi-accelerator systems.

\section{OOGen}

OOGen [Ding'16] extends Archborn to support object-oriented programming constructs to automate hardware system generation. Hence, predefined Java classes are used to describe templates, interconnections (e.g., AXI-based buses), and modules (e.g., MicroBlaze processors). However, developers can also implement additional classes to describe any other system interconnection or module. In fact, this approach is used to define reconfigurable hardware accelerators.

In OOGen, developers write system design programs that use standard and custom class libraries and generate an eXtensible Markup Language (XML) configuration file once executed. This file is fed to Archborn, which generates the required Tcl scripts for Vivado to actually build the target system using IP core libraries. Alternatively, application developers can also provide annotated host codes (i.e., using pragma directives) and use OOGen to automatically generate application-specific systems based on either the Hthreads or the HOpenCL programming models. OOGen supports memory optimizations (i.e., directives before DMA operations to automatically set the appropriate sizes for local memories), and thread optimizations (i.e., directives in Hthreads applications to automatically size the number of slave PEs required to execute a given number of threads). Finally, OOGen supports automatic DPR floorplanning and implementation as well.

\section{DAPR}

The Design Automation for Partial Reconfiguration (DAPR) flow [Yousuf'16] is a semiautomated methodology to perform both hardware/software partitioning and DPR-compatible floorplanning on top of the VAPRES reconfigurable architecture. It consists of three separate stages: analysis, partitioning, and floorplanning. In the analysis stage, every task in a given application is evaluated according to three quantitative metrics: resource utilization (obtained after running the Xilinx synthesis tool), reconfiguration time (approximated as the number of FPGA configuration frames to be written for a given resource utilization value), and execution time both in hardware and in software (estimated as the number of operators multiplied by their execution latency, which is obtained from vendor documentation files).

The next stage performs an exhaustive search of all possible hardware/software partitions (i.e., combinations of hardware and software tasks that implement the target application). It is assumed that the maximum number of tasks in DPR-capable systems is limited, which is what motivates the exhaustive search approach. Then, a Paretooptimal algorithm provides users with a set of candidate solutions from which one is manually selected. Finally, the last stage performs automatic device floorplanning and 
partition pin placement using simulated annealing to find the best solution in terms of clock frequency (i.e., maximum operating frequency once the system is deployed).

\section{PolyPC}

The Polymorphic Parallel Computing (PolyPC) framework [Ding' 17] further develops the line established with HOpenCL, ${ }^{7}$ providing a refurbished architecture with DPR support and a toolchain to automatically generate the bitstream for the static region, the executable binaries for software-based PEs, and the partial bitstreams for hardware-based PEs. Additionally, the PolyPC toolchain leverages the Xilinx PetaLinux infrastructure to generate a Linux-based OS.

To use this framework, users need to provide the following files as inputs: a hardware platform specification (i.e., the number of OpenCL-like compute units, and the number and type of PEs per OpenCL-like compute unit), the host application code, and all the required kernel codes for hardware and software implementations. In this regard, both hardware and software kernels are written in $\mathrm{C} / \mathrm{C}++$, even though their top function differs due to data addressing issues. Hence, PolyPC leverages a commercial HLS engine (i.e., Vivado HLS) to automatically generate hardware accelerators.

During system generation, the hardware platform specification is first transformed into a block diagram using all the required IP cores, and then implemented to generate a Vivado design checkpoint (i.e., a .dcp file). Another part of the toolchain takes this design checkpoint and those associated with each hardware kernel, which are synthesized independently, and generates the full and partial bitstreams. Note that this approach enables design reuse for the static partition, which does not need to be regenerated if new hardware kernels are to be deployed on the platform. Group schedulers and software-based PEs (i.e., both implemented as MicroBlaze processors) are placed in the static region, since the latter can be reconfigured by only changing the corresponding executable binary (which is also built with the toolchain). Hence, the scheduler program and a bootloader to enable such reconfiguration mechanism are compiled and embedded within the bitstream of the static region.

\section{PCIeHLS}

PCIeHLS [Vesper'17] is an OpenCL-based HLS alternative to commercial flows, and targets accelerator boards with PCIe interfaces. It features DPR support with relocation capabilities and thus, it divides the FPGA fabric in two separate parts. The static region is generated from HDL files, and contains a PCIe bridge, a module to control the FPGA Internal Configuration Access Port (ICAP), a clock manager to adapt the operating frequency of each accelerator, a module to control decoupling during DPR, memory controllers, and an AXI-based interconnection infrastructure. The reconfigurable region, on the other hand, is further divided into several homogeneous partitions.

\footnotetext{
${ }^{7}$ https://github.com/dhytxz/PolyPC
} 
Reconfigurable hardware accelerators generated from OpenCL specifications through Vivado HLS are placed in one or several of these partitions at run time, since PCIeHLS also supports slot-based (i.e., 1D) reconfiguration.

PCIeHLS can be used to generate the static partition, the reconfigurable modules, or both. In order to enable seamless connection between regions, hardware accelerators are forced to have two 32-bit AXI interfaces: one for control purposes (i.e., AXI4-Lite), and one for data transfers (i.e., AXI4-Full). Moreover, hard macros are placed in the border between static and reconfigurable regions to ensure proper interface connectivity. Although most of the toolchain relies on Xilinx tools (i.e., Vivado for logic synthesis and implementation, and Vivado HLS for accelerator generation), bitstream relocation is enabled and performed thanks to BITMAN [Pham'17], a Tclbased tool and API for bitstream manipulation.

\section{RapidWright}

RapidWright [Lavin'18] is an open source project aimed at extending the standard functionality of Xilinx tools (i.e., Vivado). ${ }^{8}$ In essence, RapidWright provides a gateway to the internal implementation back ends in Vivado, and is based on a 3-step design checkpoint (i.e., netlist) manipulation procedure: read design checkpoint, apply custom manipulation mechanisms, and write design checkpoint.

RapidWright is written in Java and can be integrated in Eclipse. As the natural evolution of RapidSmith [Lavin'11] for newer Xilinx tools and formats, it enables users to create custom CAD flows for FPGA development. Internally, RapidWright is organized around three different packages: device, which generates a database with internal FPGA information (e.g., clock regions, sites, or tiles), EDIF, which handles logical netlists, and design, which handles physical netlists (i.e., with place and route information). However, no DPR support is provided so far.

As example application, RapidWright is used to create a pre-implemented modular design flow that extends the out-of-context synthesis approach of Vivado to out-ofcontext placement and routing of each module. Composing a system using preimplemented modules renders better performance results (e.g., operating frequency). Additionally, RapidWright can increase productivity during hardware debug stages, since it allows designers to embed Integrated Logic Analyzer (ILA) cores in a placed and routed netlist, instead of doing so before full system placement and routing.

\footnotetext{
${ }^{8}$ https://github.com/Xilinx/RapidWright
} 


\section{TaPaSCo}

The Task Parallel Systems Composer (TaPaSCo) [Korinth'19] is an open source framework to automatically generate and deploy hardware-accelerated systems that exploit task-level parallelism. ${ }^{9}$ It is based on ThreadPoolComposer, but extends the generation of multi-accelerator systems using both HDL and HLS methodologies.

The TaPaSCo design flow also works with the concepts of architecture (i.e., device-independent components) and platform (i.e., device- or board-dependent components). However, a new approach is adopted: the T-model, which establishes predefined interfaces for PEs, processing clusters (i.e., groups of PEs with the same functionality), and architecture (i.e., groups of processing clusters). These interfaces are control (AXI4-Lite, left side of the T-model), data (AXI4-Full, right side of the T-model), and signaling (interrupt lines to notify host, bottom side of the Tmodel). Specific aggregators are placed at each hierarchical level to promote the signals/buses to the next layer (i.e., PEs to clusters, clusters to architecture). In addition, TaPaSCo supports several extensions by means of user-defined plugins (e.g., instantiate a TaPaSCo-compatible register interface between the automatically wired communication infrastructure and the user logic).

An additional feature of TaPaSCo is its capability of performing automated DSE according to a user-selectable combination of operating frequency, resource utilization, and PE variants (i.e., different implementations of the same functionality). Out-of-context synthesis is used to obtain the two first parameters.

\section{RTRLib}

RTRLib [Ivo'19] is a high-level modeling framework based on MATLAB/Simulink. In RTRLib, system specification is made using a model-based approach in which developers instantiate a top module that configures the target board (i.e., ZedBoard or Zybo) and the DPR mechanism (i.e., ICAP, PCAP, or JTAG). Then, users specify reconfigurable regions by manually instantiating specific blocks on the top module. Additionally, users specify whether these reconfigurable regions will be connected to the host processor using Advanced eXtensible Interface (AXI) interfaces or not. Finally, all the reconfigurable modules that can be placed in a reconfigurable region are instantiated inside their associated block. In this regard, RTRLib relies on predefined functions implemented as a library of IP blocks.

RTRLib also features a fault tolerant design methodology that optionally analyzes the reliability of the initial MATLAB/Simulink model and generates an equivalent one with embedded modular redundancy specifically tailored for each function. Finally, RTRLib generates the required Tcl scripts to build the system using Xilinx Vivado, even though manual floorplanning of the different reconfigurable regions is still needed.

\footnotetext{
${ }^{9}$ https://git.esa.informatik.tu-darmstadt.de/tapasco/tapasco
} 


\section{Pham-Quoc}

The work presented in [Pham-Quoc'19] comprises a toolchain to automatically generate custom hardware-accelerated systems with custom hybrid interconnections that are specifically tailored for a given application scenario. In fact, the main objective of the application-specific communication infrastructure is to reduce the on-chip data transfer overheads. This is achieved by ensuring that hardware accelerators are immediately provisioned with data (i.e., one hardware accelerator produces data, which are automatically sent to another hardware accelerator to be consumed).

The proposed toolchain first profiles the target application to identify computationally intensive segments (i.e., candidate sections for hardware acceleration) and communication patterns. Then, the profiling information is used to perform hardware/software partitioning. Afterwards, an application-specific interconnection infrastructure that minimizes communication overheads while keeping affordable resource overheads is generated. This infrastructure combines a NoC with shared local memories (i.e., point-to-point links between two hardware accelerators), and features minimal router/adapter utilization thanks to an adaptive mapping function. Finally, hardware accelerators are generated using HLS techniques, and integrated in the final system, which features a host microprocessor to execute software functions and manage hardware execution.

\section{AS-Overlays}

The Application-Specific FPGA Overlays (AS-Overlays) approach [Mbongue'19] uses the LLVM compilation infrastructure and RapidWright to efficiently generate FPGA overlays for dataflow-oriented applications. This framework targets systems in which computationally intensive functions are offloaded from a host processor to the overlay.

Users describe their applications on $\mathrm{C} / \mathrm{C}++$ code, and the LLVM front end is used to obtain an intermediate representation. Based on this representation, kernels (i.e., functions to be accelerated in hardware) are identified and optimized (i.e., unnecessary instructions are removed). Then, LLVM code injection is applied to insert the required functions to handle hardware acceleration (e.g., computation offloading) and generate a custom $\mathrm{C} / \mathrm{C}++$ code. The executable binary for the host processor is then compiled from this modified host code.

In parallel, the hardware substrate (i.e., the AS-Overlay) is generated by taking the LLVM intermediate representation of the kernels and using RapidWright to compose the associated circuit by stitching together several presynthesized modules. As a matter of fact, these modules (which correspond to basic operations in LLVM intermediate representations) are assumed to have been previously implemented by hand, using either HDL or HLS tools. During circuit composition, their netlists replace the black boxes that are embedded in a stream-oriented PE structure (i.e., an overlay template). Finally, a full FPGA bistream with the resulting AS-Overlay is generated. 


\section{Qualitative Comparison}

Table 2-2 summarizes the most relevant features of the different toolchains analyzed in this section. Additionally, the design-time support component of the framework proposed in this Thesis has been also included in the last row for comparison purposes. Details regarding its main characteristics can be found in Section 2.4.

Table 2-2: State of the Art - Toolchains.

\begin{tabular}{|c|c|c|c|c|c|c|c|}
\hline Toolchain & Type & Interface & Entry Point & Accelerators & Floorplanning & DPR & Relocation \\
\hline Xilinx Vitis & $\mathrm{C}$ & Shell + GUI & \begin{tabular}{|c} 
VHDL/Verilog \\
$\mathrm{C} / \mathrm{C}++/$ OpenCL
\end{tabular} & Template & Template & I & No \\
\hline Intel FPGA SDK for OpenCL & $\mathrm{C}$ & Shell + GUI & OpenCL & Template & Template & I & No \\
\hline ReCoBus-Builder [Koch’08] & A & GUI & VHDL & Template & User & S & Yes \\
\hline VSB [Jara-Berrocal'11a] & A & GUI & $\begin{array}{c}\text { VHDL } \\
\text { Impulse C }\end{array}$ & Template & Automatic & I & No \\
\hline GOAHEAD [Beckhoff'12] & A & Shell + GUI & VHDL & Custom & User/Automatic & $\mathrm{I}+\mathrm{S}+\mathrm{G}$ & Yes \\
\hline ReShape [Neely'13] & A & GUI & VHDL/Verilog & Custom & Automatic & I & No \\
\hline ReconOS [Agne'14] & A & Shell & $\begin{array}{l}\text { VHDL } \\
\mathrm{C} / \mathrm{C}++\end{array}$ & Template & Manual & I & No \\
\hline ThreadPoolComposer [Korinth'15] & A & Shell & $\mathrm{C} / \mathrm{C}++$ & Template & - & - & - \\
\hline Archborn [Ma'15b] & A & Shell & IPs & Template & Automatic & I & No \\
\hline OOGen [Ding'16] & A & Shell & IPs & Template & Automatic & I & No \\
\hline DAPR [Yousuf'16] & A & Shell & $\begin{array}{c}\text { VHDL } \\
\text { Impulse C }\end{array}$ & Template & Automatic & I & No \\
\hline PolyPC [Ding'17] & A & Shell & $\mathrm{C} / \mathrm{C}++$ & Template & Automatic & I & No \\
\hline PCIeHLS [Vesper'17] & A & Shell + GUI & OpenCL & Template & Automatic & S & Yes \\
\hline RapidWright [Lavin'18] & A & Shell + GUI & Netlists & Custom & User & - & - \\
\hline TaPaSCo [Korinth'19] & A & Shell & $\begin{array}{c}\text { VHDL/Verilog } \\
\text { C/C++ }\end{array}$ & Template & - & - & - \\
\hline RTRLib [Ivo'19] & A & GUI & Simulink & Template & Manual & I & No \\
\hline [Pham-Quoc'19] & A & Shell + GUI & $\mathrm{C} / \mathrm{C}++$ & Template & - & - & - \\
\hline AS-Overlays [Mbongue'19] & A & Shell + GUI & $\begin{array}{l}\text { VHDL/Verilog } \\
\text { C/C++ }\end{array}$ & Template & User & - & - \\
\hline ARTICo $^{3}$ & A & Shell & $\begin{array}{c}\text { VHDL/Verilog } \\
\text { C/C++ }\end{array}$ & Template & Template $^{1}$ & $\mathrm{I}^{1}$ & $\mathrm{No}^{1}$ \\
\hline
\end{tabular}

${ }^{1}$ Legacy flow: manual implementation + slot-based reconfiguration + module relocation.

N/A (-) | AND (+) | OR (/)

Type

C: Commercial

A: Academic

Accelerators

Template: wrapper/template with predefined interfaces

Custom: custom accelerator interfaces

DPR

I: Island

S: Slot (1D)

G: Grid (2D) 


\subsubsection{Runtime Systems for Reconfigurable Computing}

\section{ReConfigME}

ReConfigME [Wigley'06] is considered to be one of the first attempts to provide reconfigurable computing platforms with OS support. Conceived as a modular software architecture, it features three different components (called tiers) that communicate using standard network protocols. The platform tier contains a PCIebased FPGA board, which is interfaced using a Hardware Abstraction Layer (HAL) server written in Java. In fact, this HAL server provides a portable API to hide all platform-specific functions, enabling transparent support for different FPGA boards with common prototypes for bitstream loading, memory accesses, and clock management. Due to technology limitations, ReConfigME does not support DPR, but it is capable of simulating it with checkpointing mechanisms (i.e., save accelerator state, download new full bitstream, restore accelerator state).

The OS tier acts as an intermediate layer between the target platform (i.e., the HAL server) and the user, receiving the application specification and deciding (using an allocator and a partitioner) whether it can be placed on the FPGA fabric or not. If allocation is not possible, the application is put in a waiting queue until the required resources become available again. This module is also responsible for bitstream generation and downloading through direct communication with the HAL server.

Finally, the user tier is used to specify the applications, issue their execution, and check their FPGA layout. ReConfigME works with applications described as DFGs, where each node has an associated EDIF file (which can be generated from either VHDL or Handel-C, a high-level programming language), and node connectivity is specified using a Java description. The ReConfigME infrastructure automatically manages data transfers between user and platform tiers, and supports multiple concurrent applications and/or FPGA boards in a transparent and scalable way.

\section{Hthreads}

Hthreads [Andrews'08] implements its runtime management system (i.e., a thread manager, a thread scheduler, a mutex manager, and a CPU bypass interrupt scheduler) in hardware. As a result, not only transparent hardware/software multithreading is enabled, but also a more efficient software-based multithreading is supported. In fact, the Hthreads approach replaces complex software stacks by lightweight and atomic load/store operations, and removes speculative and variable execution in key system components (e.g., the scheduler). Moreover, the $\mathscr{O}(1)$ scheduler implementation has a fixed latency to decide which thread to run next (and it is therefore independent of the amount of threads already present in the ready queue), and makes those decisions in parallel with actual software execution on the CPU, thus avoiding unnecessary interrupts (e.g., scheduling is not always required whenever a mutex is released). Although the Hthreads framework provides a common API for hybrid 
hardware/software execution under a Pthreads-compatible programming model, it does not support DPR during normal system execution.

\section{BORPH}

The Berkeley Operating system for ReProgrammable Hardware (BORPH) [So'08] is an OS for reconfigurable computing systems. It is based on a modified Linux kernel, and assumes that software processes work together with hardware accelerators using a peer-to-peer approach rather than a master/slave one. In essence, BORPH enables hardware/software multithreading and is built around three key components: hardware processes, the IOREG interface, and the hardware file input/output interface.

Hardware processes are defined as running instances of hardware designs, and are created following UNIX conventions (i.e., using the fork and exec system calls). Moreover, each hardware process has its own memory space and execution domain. Hence, shared memory models are not enabled by default in BORPH. However, hardware processes are allowed to expose their internal memory maps through the IOREG interface. Finally, hardware processes can be terminated either using signals (e.g., SIGTERM or SIGKILL) or calling a function that is equivalent to exit.

The IOREG interface is a passive communication mechanism (i.e., software processes start data transfers) that provides access to different hardware constructs (i.e., registers, on-chip and off-chip memories, and FIFO channels) using a virtual file system (i.e., /proc/<pid $>/ \mathrm{hw} /$ ioreg). The file input/output interface, on the other hand, is an active communication mechanism that provides hardware processes with access to not only standard files (i.e., stdin, stdout, and stderr), which are used for debugging and interprocess data streaming, but also any other file in the system.

BORPH features an automated toolchain to generate hardware accelerators from MATLAB/Simulink specifications. In general, BORPH-compliant computing platforms have a main processor in which the OS runs, and one or more secondary processors that act as bridges between hardware and software domains. On the software side, BORPH significantly extends the Linux kernel to support custom executable files (which include the FPGA bitstreams of the associated hardware tasks and their corresponding IOREG information), load FPGA bistreams using a target-dependent configure function, communicate hardware and software components using a message-passing network, and manage process scheduling and signal handling. 


\section{CAP-OS}

The Configuration Access Port Operating System (CAP-OS) [Göhringer'10] is the main runtime management engine of the RAMPSoC platform. Written in C code, CAP-OS consists of several software threads running on top of Xilkernel, which is in turn deployed in one of the processors of the system. This processor needs to have direct access to the ICAP primitive, which is used to reconfigure the application-specific hardware accelerators, the soft-core processors, the executable binaries of the softcore processors (i.e., modifying the contents of their instruction memories with DPR), or even the clock configuration (i.e., accessing the on-chip clock generators).

CAP-OS works with applications defined as Directed Acyclic Graphs (DAGs) (with either soft or hard real-time constraints), and has three main functionalities: scheduling, resource allocation, and FPGA configuration. The scheduler thread analyzes the application graphs, sorts their tasks according to their priorities using a list scheduling algorithm, and selects tasks for execution whenever they are ready (i.e., tasks whose predecessors are already loaded on the FPGA or have finished execution). Soft real-time tasks are delayed whenever a hard real-time task is present, even if the latter has less priority than the former.

The resource allocation thread is executed after the scheduling stage. CAP-OS looks for an available processor core and assigns it the first task in the ready list. Two different scenarios can happen if this condition is not met: when there is no processor at all on the FPGA, a new one is configured; when there is at least one processor in the FPGA, CAP-OS checks whether it is busy or not. If the processor is busy, the resource allocation algorithm checks the remaining execution time and, if it is below a given threshold, the new task is assigned. If not, CAP-OS tries to configure a new processor on the FPGA fabric, blocking the task if there are not enough resources.

Finally, the configuration thread is used to modify the FPGA. CAP-OS supports preemptive reconfiguration for non-critical tasks (i.e., changing the software of an instantiated processor, or a hardware accelerator), stopping the DPR process whenever another task with a higher priority is scheduled for execution. After configuration, CAP-OS also sends configuration commands to establish the required communication channels between running tasks.

\section{VAPRES}

VAPRES features a dynamic runtime manager to automatically deploy reconfigurable stream processing systems (i.e., a set of hardware and software modules that accept an input data stream, process it, and produce an output data stream) as requested by user applications running on the host processor [Jara-Berrocal'11b]. This manager is implemented as a user-space application running on top a Linux-based OS.

During normal system execution, applications that want to exploit VAPRES for hardware-accelerated processing send a request to the dynamic runtime manager. These requests have three parameters: the process that is performing the petition, 
a netlist of the requested reconfigurable stream processing system (i.e., a file that describes which processing cores or input/output modules to load and how to connect them), and a flag to signal whether execution should start (i.e., implement system and execute) or finish (i.e., free resources). Once the request has been received, the manager performs resource allocation, checking whether there are enough reconfigurable slots to implement the requested functionality or not. If resource allocation is completed successfully, the manager performs module placement, enforcing hardware reuse (i.e., avoiding DPR if the module to be loaded is already loaded in one reconfigurable slot and not being used by any other application). Finally, the manager performs run-time assembly of the reconfigurable stream processing system by setting the specific configuration of the interconnection infrastructure.

\section{FUSE}

FUSE [Ismail'11] provides a user-space abstraction layer for hybrid hardware/software multithreading using accelerators under a Linux-based OS. The FUSE infrastructure provides a shared memory model for software and hardware components, and relies on a modified Pthreads programming model with a C-based API.

The software stack decides, automatically and upon each thread creation, whether to map it to a hardware accelerator (provided that it is instantiated on the FPGA and not being used by any other application) or to default to a software-based execution. Since no specific interface is forced on the hardware components, the stack also requires kernel-space functionality, which is implemented as target-dependent device drivers. These drivers provide standard filesystem procedures and system calls (i.e., open, close, read, write, ioctl, mmap) to enable hardware/software interaction.

Accelerator execution is managed using dedicated control and status registers on the FPGA fabric. An FSM ensures that each accelerator is set on the proper thread state (e.g., IDLE, RUNNING, BUSY), and uses these registers to receive commands from the low-level device drivers and to report the current hardware thread state.

\section{SPREAD}

SPREAD [Wang'13] extends a lightweight OS kernel (i.e., Xilkernel) to enable hybrid hardware/software multithreading for streaming applications. As other alternatives in the literature, it extends hardware accelerators with additional control and status logic to properly transform hardware threads in something more than mere system devices.

The proposed OS extensions provide hardware resource allocation and mapping, as well as thread and communication management services. Whenever a new hardware thread needs to be created, SPREAD looks for a previously configured instance of the requested accelerator that is in idle state. In other words, it provides a cached DPR mechanism to reduce the number of actual FPGA reconfigurations, since DPR is only used when no idle accelerator is found. During normal execution, hardware 
threads can be preempted by other tasks with higher priority. In this scenario, thread state is saved in several internal registers and sent back to the OS runtime. Finally, SPREAD also supports switchable threads (i.e., threads with dual hardware and software implementations), making it possible to migrate from one computing fabric to another in situations in which resource availability is limited.

SPREAD includes a Pthread-compatible programming model to transparently manage hardware thread creation and termination, inter-thread communication, and hardware/software switching for dual threads.

\section{Rainbow}

Rainbow [Jozwik'13] is another OS-based approach to enable seamless hardware/software task integration. It is built on top of the Toyohashi OPen Platform for Embedded Real-time Systems (TOPPERS) Advanced Standard Profile (ASP) RTOS kernel.

The Rainbow infrastructure consists of several software components, which can be either target-independent (i.e., task management and communication layer, task scheduling and placement layer) or target-dependent (i.e., task configuration layer), but it also includes dedicated (and target-dependent) hardware modules to support DPR, save and restore hardware task states, and enable clock management.

The task scheduling and placement layer uses a priority-based preemptive scheduler that can be accessed through low-overhead C API calls. However, preemption is only applied when the overall performance of the system will not be penalized, delaying FPGA configuration requests as needed. In order to support all these features, user-defined logic is instantiated in a common accelerator wrapper. The wrapper is divided in two different regions: static (i.e., independent of the application) and reconfigurable (i.e., user logic). It is worth noting that the latter can be specified using HDL or generated from $C$ code using an HLS engine.

\section{ReconOS}

ReconOS [Agne'14] extends the Linux kernel to enable hybrid hardware/software multithreading under a Pthreads-like programming model. Although specific scheduling approaches have been proposed to enable cooperative [Lübbers'09a] and preemptive [Happe'15] execution using DPR extensions, ReconOS hides hardwarespecific details behind delegate threads, which are software entities that are transparently managed by the Linux scheduler but receive petitions from the hardware accelerators and execute them on their behalf.

ReconOS has been used in this Thesis to extend the ARTICo ${ }^{3}$ framework and thus, a detailed description of its runtime infrastructure can be found in Subsection 3.2.1. 


\section{RIFFA}

The Reusable Interface for FPGA Accelerators (RIFFA) [Jacobsen'15] is an open source framework for PCIe-based hardware acceleration. ${ }^{10}$ One of the key aspects of RIFFA is its cross-platform support, allowing both Linux and Windows as host OS, and Xilinx and Intel FPGAs as target accelerators. RIFFA does not target DPR-capable systems, but focuses on high-performance CPU to FPGA communication. To this end, a maximum of 5 different FPGAs can be used in a single system, with up to 12 different hardware accelerators being used simultaneously per FPGA device.

Although RIFFA defines a hardware interface for accelerators to be integrated with the rest of the FPGA logic (e.g., the PCIe endpoint), the main component is still the software interface, which combines kernel-level functionality with a lightweight userlevel API. In fact, users are only provided with functions to list, open, close, and reset available FPGA devices. In addition, the API includes a function to send data to and another to receive data from a specific hardware accelerator (i.e., a channel). In the RIFFA implementation for Linux, these user-level functions end up using the ioct 1 system call to issue scatter-gather DMA transfers.

\section{R3TOS}

The Reliable Reconfigurable Real-Time Operating System (R3TOS) [Iturbe'15] is a runtime environment for reconfigurable computing systems that exploits DPR in multiple ways (i.e., to load/relocate hardware accelerators, to transfer data between hardware accelerators, to perform clock management, to save and restore the internal state of hardware accelerators, and to perform fault diagnosis and recovery). Being focused on fault-tolerant execution, R3TOS can be used to place and execute several instances of the same hardware task with either spatial or temporal redundancy, and even to isolate FPGA regions with permanent faults (i.e., relocating hardware accelerators to a different region). However, it can also support SIMD-like execution.

The main R3TOS functionality is implemented in a hardware microkernel, which mainly consists of three PicoBlaze processors (i.e., a scheduler, an allocator, and a configuration manager) and their associated logic (e.g., a timer to generate kernel ticks for the scheduler, feasibility and quality evaluation modules for the allocator, and an ICAP controller for the configuration manager). Moreover, each processor includes a dedicated data memory to store relevant information (e.g., process state in the scheduler, resource utilization in the allocator, and bitstream information in the configuration manager). Additionally, R3TOS provides specific hardware blocks to connect hardware accelerators to their input/output memory ports, as well as to connect ports from different hardware accelerators using disjoint memory resources. These blocks are used by the allocator to generate a continuous datapath on the FPGA fabric with direct connections (i.e., the output memory of the source accelerator is the input memory of the target accelerator), bridge-based connections (i.e., an

\footnotetext{
${ }^{10}$ https://github.com/KastnerRG/riffa
} 
intermediate module is placed between the output memory of the source accelerator and the input memory of the target accelerator), or DPR-based connections (i.e., data are transferred using the ICAP, as no physical route exists between source and target accelerator memories).

Complementary to the previous infrastructure is a software microkernel, which runs on a MicroBlaze processor and is based on FreeRTOS. Each hardware task has an associated high-priority software task (with preemption disabled), which uses a set of system calls to access the hardware microkernel functionality.

\section{RTSM}

The Run-Time System Manager (RTSM) [Charitopoulos'15] is a software-based runtime implemented as a bare-metal application that runs on top of a CPU. In order to properly place and schedule the different tasks, it requires certain information obtained at design time (i.e., FPGA fabric partitioning into reconfigurable regions and possible accelerator-to-region mappings, application graphs showing task order and dependencies, and task-related data such as execution and reconfiguration times).

During normal system operation, the RTSM engine makes scheduling decisions according to multiple criteria. For instance, it takes into account whether different implementations exist for a given task. If a task cannot be scheduled, it is automatically placed in a reservation queue until either a reconfigurable region or a software-based processing core becomes available (the latter being used only for tasks with software implementation). RTSM can mask reconfiguration times by means of module reuse (i.e., an accelerator loaded in a previous time step but idle at the time of scheduling is automatically assigned) and configuration prefetching (i.e., DPR is applied way before task execution start). Additional placement policies include module relocation (i.e., move a hardware accelerator from a reconfigurable region to another if possible), space fitness (i.e., modules with lower resource utilization in a reconfigurable region are considered worse), and time fitness (i.e., RTSM decides which strategy among reservation, reuse, and relocation is better in terms of task execution time).

\section{LEAP}

The Latency-insensitive Environment for Application Programming (LEAP) FPGA OS [Fleming'16] is another open source solution that tries to bridge the gap between software programming and hardware development. ${ }^{11}$ Its main abstraction mechanism is based on latency insensitive communication channels, which are reliable pointto-point links that specify certain communication rules but not their timing nor implementation details. In most implementation scenarios, though, these latency insensitive channels are implemented as RTL FIFOs.

LEAP programs are written in Bluespec System Verilog, and need to be specified as a set of latency insensitive modules. These modules, which can be RTL descriptions

\footnotetext{
${ }^{11}$ https://github.com/LEAP-FPGA
} 
but also normal software tasks, are the equivalent to software processes in regular OSs. Communication between different modules is made by direct instantiation of the FIFO-like primitives. In addition, LEAP provides private and shared memory primitives, as well as service libraries to implement common operations such as standard input/output. Service libraries can be extended to support any functionality specified by the user through the so-called SoftServices layer. Once the user has specified their target system, the LEAP compiler parses the high-level descriptions and generates a custom implementation according to low-level platform information. In this regard, most of the dynamic behavior present in software-oriented OSs is sacrified to avoid complex run-time management overheads.

\section{LinROS}

LinROS [Rettkowski'16] is a kernel-level extension of a Linux-based OS to ease accelerator reconfiguration and execution. It is organized around two main blocks: a scheduler, which relies on a priority queue in which tasks are ordered according to their deadlines, and an executor, which deals with low-level platform management (e.g., accelerator reconfiguration or MicroBlaze program uploading, task execution via DMA transfer start). In order to enable parallel task execution on the FPGA fabric, each node of the target NoC-based architecture is handled by an independent software thread.

LinROS provides three separate interfaces for user applications to communicate with the scheduler: a buffer interface to allocate memory for data transfers between user and kernel spaces, a task interface to specify task-specific memory constructs and regions, and a control interface to actually issue task execution. On the other side, LinROS features five different mechanisms to enable interaction between the executor and the actual hardware: a DMA module to transfer data from one domain to another, a module to program soft-core processors, a module to reconfigure hardware accelerators, a module to reset processing cores and put them in a known state, and a module to configure accelerator-specific registers.

\section{Janßen et al.}

The work presented in [Janßen'17] describes a type 0 hypervisor for reconfigurable computing systems. As opposed to type 1 (i.e., a bare-metal application running on the host processor) and type 2 (i.e., an application that runs on top of a host OS) hypervisors, which are based on software, the proposed alternative is implemented as part of the hardware architecture. Moreover, it focuses on task scheduling and system call handling, leaving out of scope the process of mapping accelerators to reconfigurable partitions.

In particular, the hypervisor implements a round-robin scheduling algorithm using a dedicated timer to generate the required periodic interrupts. These interrupts are issued to the main processor to trigger context switches. System calls, on the other hand, are requested to the hypervisor by means of standard input/output interrupts. 
Both processes are handled in the main processor by an intermediate software application that is executed on demand by triggering software-based interrupts. A dedicated Memory Management Unit (MMU) block is placed on the reconfigurable substrate to properly handle memory virtualization for the hardware accelerators.

\section{ZUCL}

ZUCL [Pham'19] is an OpenCL-based computing framework for heterogeneous CPU-FPGA systems. It features a script-based toolchain to automatically build reconfigurable systems that isolates the generation of static and dynamic regions. A standard AXI infrastructure is used in the static region, and protocol adapters are transparently inserted in the reconfigurable regions to connect accelerators with custom interfaces to the main CPU.

The ZUCL runtime environment features a cooperative scheduler that supports round-robin and resource elastic policies (i.e., hardware accelerators can have different implementations with tradeoffs between resource utilization and performance). Scheduling decisions are triggered using standard OpenCL constructs. In turn, a configuration controller is invoked by the scheduler to place the required hardware accelerators on the FPGA fabric. Placement information is read from a configuration XML file, even though the framework supports module relocation.

Although the first version of ZUCL relied on a single large consecutive physical memory region to enable communication between host CPU and hardware accelerators, ZUCL 2.0 includes a specific MMU for enhanced memory management and isolation. The complete software infrastructure of ZUCL is a combination of userspace libraries and kernel-space drivers in a Linux-based OS.

\section{FOS}

The FPGA Operating System (FOS) [Vaishnav'20] is an open source framework built on top of the ZUCL infrastructure. ${ }^{12}$ FOS supports three different types of execution profiles: static accelerators with single user, reconfigurable accelerators with single user, and reconfigurable accelerators with multiple users. The first two profiles are enabled by two acceleration interface libraries (i.e., Cynq, written in $\mathrm{C}++$, and Ponq, written in Python), which provide basic function calls to reconfigure the FPGA fabric with static and dynamic partitions, program hardware accelerators using generic drivers, access accelerator registers using a common interface, or allocate contiguous physical memory for hardware/software communication purposes.

The scenario with multiple users is covered thanks to the use of execution daemons, which are accessible through $\mathrm{C}++$ and Python constructs. It is worth noting that users are allowed to explicitly expose data-level parallelism in their applications, and this information is then used by the runtime system to execute tasks in parallel,

\footnotetext{
${ }^{12}$ https://github.com/khoapham/fos
} 
using resource-elastic accelerator variants, or even performing time-multiplexing as in traditional DPR-capable systems. FOS uses cooperative scheduling to execute acceleration requests from users, which are stored in request queues (one per user). Requests are assumed to be independent, which in turn means that they can be executed in parallel and in any order. Once a given request has been satisfied, the scheduler selects a new task from the next user in the user queue, and starts its execution. Accelerator instantiation is performed automatically by FOS (enforcing module reusability when possible to avoid excessive reconfiguration overheads).

\section{Qualitative Comparison}

Table 2-3 summarizes the most relevant features of the different runtime systems analyzed in this section. Additionally, the run-time support component of the framework proposed in this Thesis has been also included in the last row for comparison purposes. Details regarding its main characteristics can be found in Section 2.5.

Table 2-3: State of the Art - Runtime Systems.

\begin{tabular}{|c|c|c|c|c|c|c|c|}
\hline Runtime & Base OS & Domain & API & Mapping & Scheduling & Dual HW/SW & DPR \\
\hline ReConfigME [Wigley'06] & Windows XP & User & Java & Auto & Yes & No & No \\
\hline Hthreads [Andrews'08] & Custom & Kernel & $\mathrm{C}$ & No & Yes & Yes & No \\
\hline BORPH [So'08] & Linux & Kernel & Simulink & Auto & Yes & No & Yes \\
\hline CAP-OS [Göhringer'10] & Xilkernel & Kernel & - & Auto & Yes & No & Yes \\
\hline VAPRES [Jara-Berrocal'1lb] & Linux & User & $\mathrm{C}$ & Auto & Yes & No & Yes \\
\hline FUSE [Ismail'11] & Linux & Kernel + User & $\mathrm{C}$ & Auto & Yes & Yes & Yes \\
\hline SPREAD [Wang' 13] & Xilkernel & Kernel & $\mathrm{C}$ & Auto & Yes & Yes & Yes \\
\hline Rainbow [Jozwik'13] & TOPPERS/ASP & Kernel & $\mathrm{C}$ & Auto & Yes & No & Yes \\
\hline ReconOS [Agne'14] & Linux & Kernel + User & $\mathrm{C}$ & Manual & Yes & Yes & Yes \\
\hline RIFFA [Jacobsen'15] & $\begin{array}{l}\text { Windows } \\
\text { Linux }\end{array}$ & Kernel + User & \begin{tabular}{|c} 
C/C++ \\
Java \\
Python \\
MATLAB
\end{tabular} & No & No & No & No \\
\hline R3TOS [Iturbe'15] & FreeRTOS & Kernel & $\mathrm{C}$ & Auto & Yes & No & Yes \\
\hline RTSM [Charitopoulos'15] & - & - & - & Auto & Yes & Yes & Yes \\
\hline LEAP [Fleming'16] & - & - & Bluespec & No & No & No & No \\
\hline LinROS [Rettkowski'16] & Linux & Kernel & $\mathrm{C}$ & Manual & Yes & No & Yes \\
\hline [Janßen'17] & - & - & $\mathrm{C}$ & No & Yes & No & Yes \\
\hline ZUCL [Pham'19] & Linux & Kernel + User & OpenCL & Manual & Yes & No & Yes \\
\hline FOS [Vaishnav'20] & Linux & Kernel + User & $\begin{array}{c}\text { C++ } \\
\text { Python }\end{array}$ & Auto & Yes & No & Yes \\
\hline ARTICo $^{3}$ & Linux & Kernel + User & $\mathrm{C}$ & Manual & Yes & No & Yes \\
\hline
\end{tabular}

N/A (-) | AND (+) | OR (/) 


\subsection{Processing Architecture}

The ARTICo ${ }^{3}$ architecture is a hardware-based processing architecture for adaptive high-performance embedded computing. It relies on a dynamic pool of hardware accelerators that can be loaded and configured on demand to establish run-time tradeoffs between computing performance, energy efficiency, and fault tolerance. This is enabled by the use of DPR in modern SRAM-based FPGAs. ${ }^{13}$

$\mathrm{ARTICo}^{3}$, as many other reconfigurable computing architectures, divides the FPGA fabric in two virtual regions: static and dynamic (sometimes also referred to as reconfigurable). The static region contains all the logic resources that do not change during normal system execution. The reconfigurable region, on the other hand, hosts application-specific logic whose functionality may be completely changed throughout operation. In $\mathrm{ARTICo}^{3}$, the dynamic region is further partitioned in several subregions called reconfigurable slots.

The processing paradigm in ARTICo ${ }^{3}$ assumes that the architecture works as an attached reconfigurable processing unit [Compton'02]. Hence, applications running on a host microprocessor offload their computationally intensive tasks to the different hardware accelerators available in the reconfigurable slots. ${ }^{14}$ In this regard, two different levels of parallelism can be exploited in ARTICo ${ }^{3}:$ hardware accelerators with different application-specific functionality (i.e., different kernels) and different input data can be executed concurrently to achieve task-level parallelism, and hardware accelerators with the same application-specific functionality (i.e., same kernel) but different input data can be executed concurrently to achieve data-level parallelism.

In addition, the architecture also provides support for on-demand hardware redundancy, enabled by selectable Simplex (i.e., no redundancy), DMR, or TMR configurations. In both DMR and TMR scenarios, hardware accelerators with the same application-specific funcionality (i.e., same kernel) and the same input data are executed concurrently to achieve enhanced fault tolerance in the reconfigurable region thanks to a configurable voter unit.

A top-level block diagram of the ARTICo ${ }^{3}$ architecture can be seen in Figure 2-3. Notice that it follows a bus-based communication approach, and that the main infrastructure contains several building blocks. Section 2.3 describes the main components of the static part (i.e., voter unit, reduction engine, performance and fault monitors, and interconnection logic). For a complete description of the main components in the reconfigurable slots, please refer to Section 2.4.

\footnotetext{
${ }^{13}$ Despite being described in platform-independent VHDL code, the current version of the ARTICo ${ }^{3}$ architecture is only supported in Xilinx devices due to low-level technology restrictions in the DPR flow.

${ }^{14} \mathrm{ARTICo}^{3}$ does not impose neither the nature (i.e., it can be a hard or a soft core) nor the location (i.e., it can be inside or outside the FPGA) of the host microprocessor.
} 


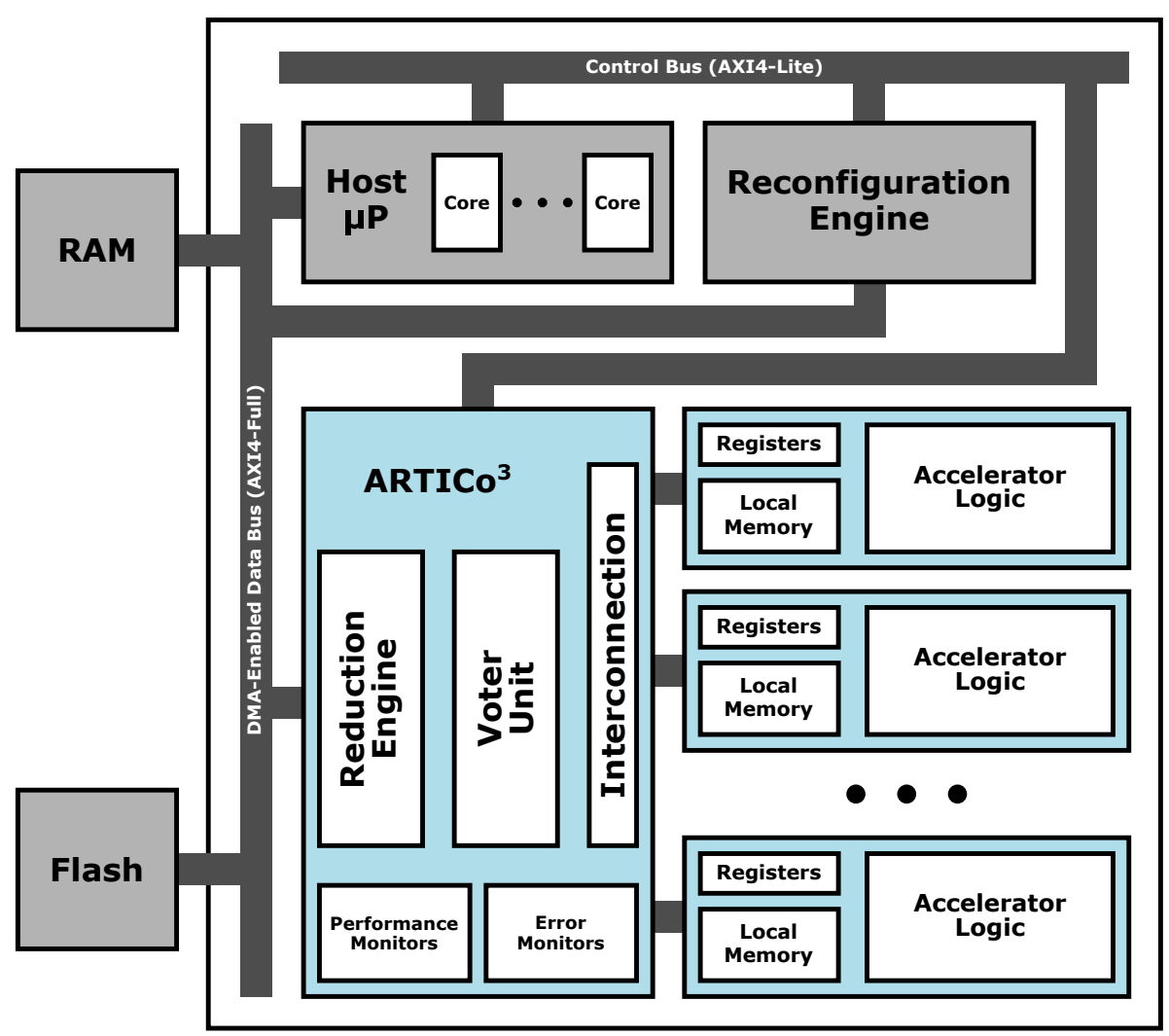

Figure 2-3: The ARTICo $^{3}$ architecture.

Original contribution 2-2 A hardware-based processing architecture for adaptive high-performance embedded computing based on run-time tradeoffs between computing performance, energy efficiency, and fault tolerance.

\subsubsection{Configurable Datapath}

The main ARTICo ${ }^{3}$ infrastructure acts as a dynamic communication gateway between the static region and the reconfigurable slots. The hardware-based processing architecture is attached to the rest of the static system by means of two standard bus-based interconnections. In fact, ARTICo $^{3}$ has an Advanced Microcontroller Bus Architecture (AMBA) AXI4-Lite slave interface for control purposes (i.e., register-based operations), and an AMBA AXI4-Full slave interface for DMA-enabled burst data transfers. On the dynamic region, ARTICo $^{3}$ features point-to-point communication links with each of the hardware accelerators hosted in the reconfigurable slots. ${ }^{15}$ The control/status logic in this dedicated interface is based on a start/ready signal pair. The architecture asserts the start control signal to launch accelerator execution, and each accelerator asserts its ready status signal to notify that its execution has finished.

\footnotetext{
${ }^{15}$ Please refer to Subsection 2.4.1 for the complete specification of this interface.
} 
From a system-wide perspective, the transparent bridging functionality of the ARTICo $^{3}$ architecture masks custom communication links behind standard interfaces. As a result, any component in the static region with master capabilities in the bus-based interconnection (e.g., host microprocessor, DMA engine) can access the hardware accelerators. It is worth noting that the proposed communication infrastructure constrains data transfers to be memory-mapped.

A highly configurable datapath is present in between the AXI4 interfaces and the point-to-point connections with the hardware accelerators. The internal structure of the ARTICo ${ }^{3}$ datapath contains a configurable interconnection crossbar, a voter unit, and a reduction engine, as it can be seen in Figure 2-4.

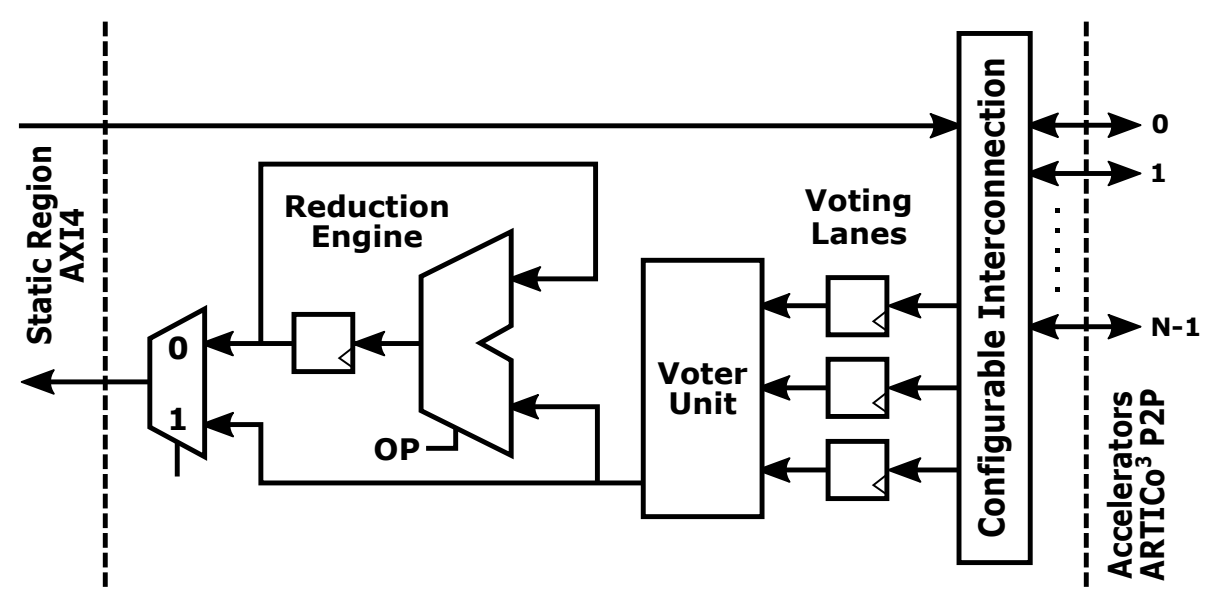

Figure 2-4: Configurable datapath.

The control logic of the architecture informs the configurable interconnection crossbar of which accelerators are involved in a data transaction at any given clock cycle. If the current transaction moves data from the static system to the accelerators, the interconnection crossbar reroutes input data and control signals to the target accelerator(s). On the contrary, if the current transaction moves data from the accelerator(s) to the static system, the interconnection crossbar connects the output data signals to the voting lanes. These are registers placed before the configurable voter unit. Depending on the hardware redundancy configuration, the behavior of the interconnection crossbar and the voter unit differs: in Simplex configuration, only one voting lane is used, and the voter unit acts as a bypass; in DMR configuration, two voting lanes are used, and the voter unit acts as a comparator; in TMR configuration, three voting lanes are used, and the voter unit acts as a full-fledged majority voter.

Finally, the reduction engine takes advantage of data serialization in transactions from accelerators to static system (imposed by the bus-based nature of the on-chip communication scheme), and performs selectable reduction-oriented operations on the returning burst transfers to produce a single datum as output. Currently, ARTICo ${ }^{3}$ supports three different reduction operations: ADD (i.e., accumulate all incoming data), MAX (i.e., extract the maximum value from incoming data), and MIN (i.e., extract the minimum value from incoming data). The internal structure of the accumulator-based 
reduction engine enables on-the-fly processing without incurring excessive overheads, neither in terms of latency nor in terms of circuit area.

Original contribution 2-3 A highly configurable pipelined datapath with simplified control logic that features an embedded interconnection crossbar, a voter unit, and a reduction engine for adaptive bus-based data processing.

\subsubsection{Monitoring Infrastructure}

The ARTICo ${ }^{3}$ architecture has been provided with different types of Performance Monitoring Counters (PMCs) to enable run-time tracing of the internal activity within the computing platform. The implemented monitors can be classified as performance, error, or power monitors, and further categorized as either built-in (i.e., part of the architecture) or external.

- Performance monitors: built-in PMCs to measure accelerator latency per slot.

- Error monitors: built-in PMCs to measure accumulated errors per slot.

- Power monitors: external PMCs to measure power consumption in the FPGA.

Performance monitors are implemented as hardware counters that measure the number of clock cycles needed for an accelerator to finish its execution. In order to achieve that, the start/ready signal pairs are connected to the control logic of the counters. As a result, performance PMCs reset their values and start counting when the start signal is asserted, and stop counting when the ready signal is asserted. Performance PMCs work in single-run fashion, storing only the number of clock cycles elapsed in the last execution of a given hardware accelerator in a given reconfigurable slot. Please note that data transfer times using the DMA engine are not measured with these monitors.

Error monitors are also implemented as hardware counters, but in this case they measure the amount of errors that have been detected by the configurable voter unit in each reconfigurable slot. Notice that, during burst data transfers, the voter unit operates on a datum basis (i.e., if there are $N$ data, the voter unit performs $N$ comparisons in DMR or $N$ majority votes in TMR), incrementing the corresponding counter(s) whenever an error is found. Error PMCs work in an accumulative manner (i.e., their value can be associated with more than one accelerator execution), but are reset after reading their value. It is important to highlight that, as opposed to the performance PMCs, these monitors are tightly coupled with the reconfigurable slots but not with any specific hardware accelerator. Hence, if an error is present in the FPGA fabric and cannot be fixed by applying DPR to load the same (i.e., configuration memory scrubbing) or a different hardware accelerator in a given position, the PMC 
will continue increasing its value. This is an important feature to detect and isolate permanent faults in the reconfigurable slots.

Since not all FPGA-based boards feature dedicated circuitry to measure their power consumption, power PMCs need to be implemented as external monitors. In $\mathrm{ARTICo}^{3}$, the universal FPGA power consumption meter developed in [Casado'19] can be used whenever collecting power traces is mandatory. Among its multiple functionalities, it supports measurements on the Universal Serial Bus (USB) power rail (useful for lowcost FPGA boards powered using USB connectors), or on any other power rail provided that a probe is connected to its shunt resistor.

The combination of both performance and power monitors provides a lightweight infrastructure to characterize accelerator execution in ARTICo $^{3}$ at run time. ${ }^{16}$

Original contribution 2-4 A lightweight monitoring infrastructure to perform run-time characterization of multiple hardware accelerators.

\subsubsection{Operation Modes}

The highly configurable datapath in $\mathrm{ARTICo}^{3}$ supports three different operation modes when performing data transfers between static and dynamic regions:

- Parallel operation mode: SIMD-like execution using different input data on several hardware accelerators with the same kernel functionality.

- Redundant operation mode: hardware redundant execution using the same input data on several hardware accelerators with the same kernel functionality, and enabling the configurable voter unit.

- Reduction-oriented operation mode: SIMD-like execution using different input data on several hardware accelerators with the same kernel functionality, and enabling the reduction engine.

The parallel operation mode, shown in Figure 2-5, is used to fully exploit datalevel parallelism. When operating in this mode, burst data transfers are split in as many blocks as hardware accelerators (i.e., kernel instances) during write transactions. During read transactions, partial burst data transfers coming from each hardware accelerator are merged before reaching the bus-based interconnection of the system. Both split and merge operations are performed on-the-fly in hardware without incurring additional latency overheads. In this mode, overlapping periods of data transfers and accelerator(s) execution occur, since the start control signal is asserted for each hardware accelerator once all its assigned input data have been delivered. ${ }^{17}$ Overlapped execution is due to the serial nature of the bus-based communication infrastructure, which forces the start signals to be asserted sequentially.

\footnotetext{
${ }^{16}$ The monitoring infrastructure is used in Subsection 2.5.4 to generate a run-time power model.

${ }^{17}$ The start control signal is always asserted when the required amount of input data has been written to a given accelerator, regardless of the operation mode.
} 

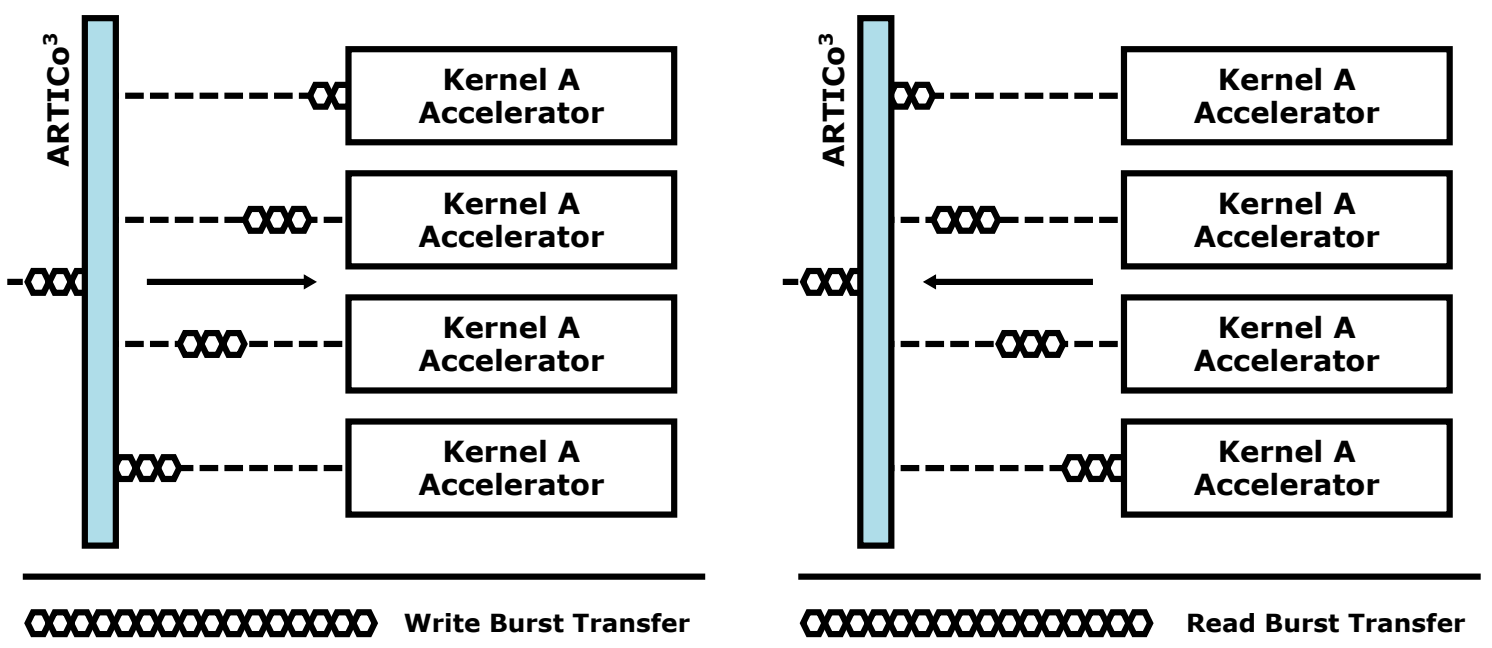

Figure 2-5: Parallel operation mode.

The redundant operation mode, shown in Figure 2-6, is used to enforce faulttolerant execution in the reconfigurable slots. As it has already been introduced, ARTICo $^{3}$ supports flexible DMR and TMR configurations. When operating in this mode, burst data transfers are issued in multicast fashion. During write transactions, all hardware accelerators belonging to the same redundant group receive their input data in parallel. Hence, processing in this operation mode occurs strictly in parallel (i.e., all start signals are asserted at the same time), as opposed to the overlapped execution in the parallel operation mode. During read transactions, data coming from the hardware accelerators are passed through the configurable voter unit, reducing from two (DMR) or three (TMR) data streams to one (bus-based interconnection).

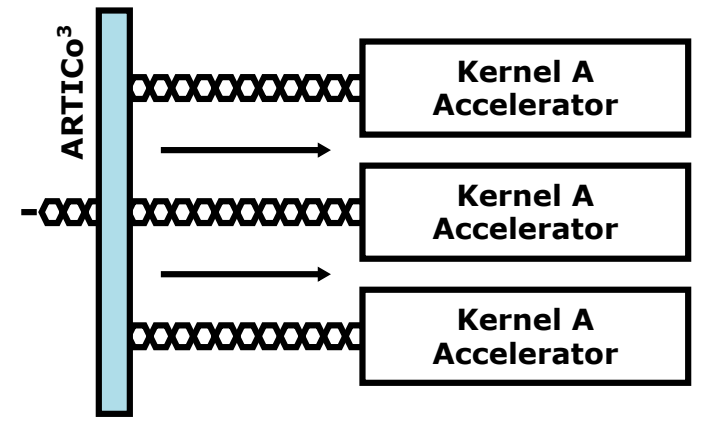

00000000000000 Write Burst Transfer

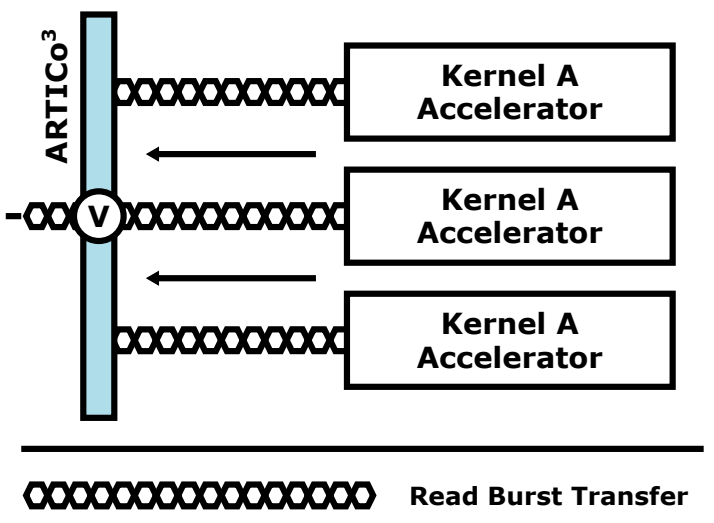

Figure 2-6: Redundant operation mode.

The reduction-oriented operation mode, shown in Figure 2-7, is an extension of the parallel operation mode. In fact, the only difference between both modes is that the reduction engine is used during read transactions to produce a datum as output instead of a whole burst data transfer. This operation mode is intended to perform reduction operations on partial results coming from the hardware accelerators, saving 
processing time in the host microprocessor. Example applications that could benefit from this built-in feature of the ARTICo ${ }^{3}$ architecture include, but are not limited to, distributed dot products and digital filters.
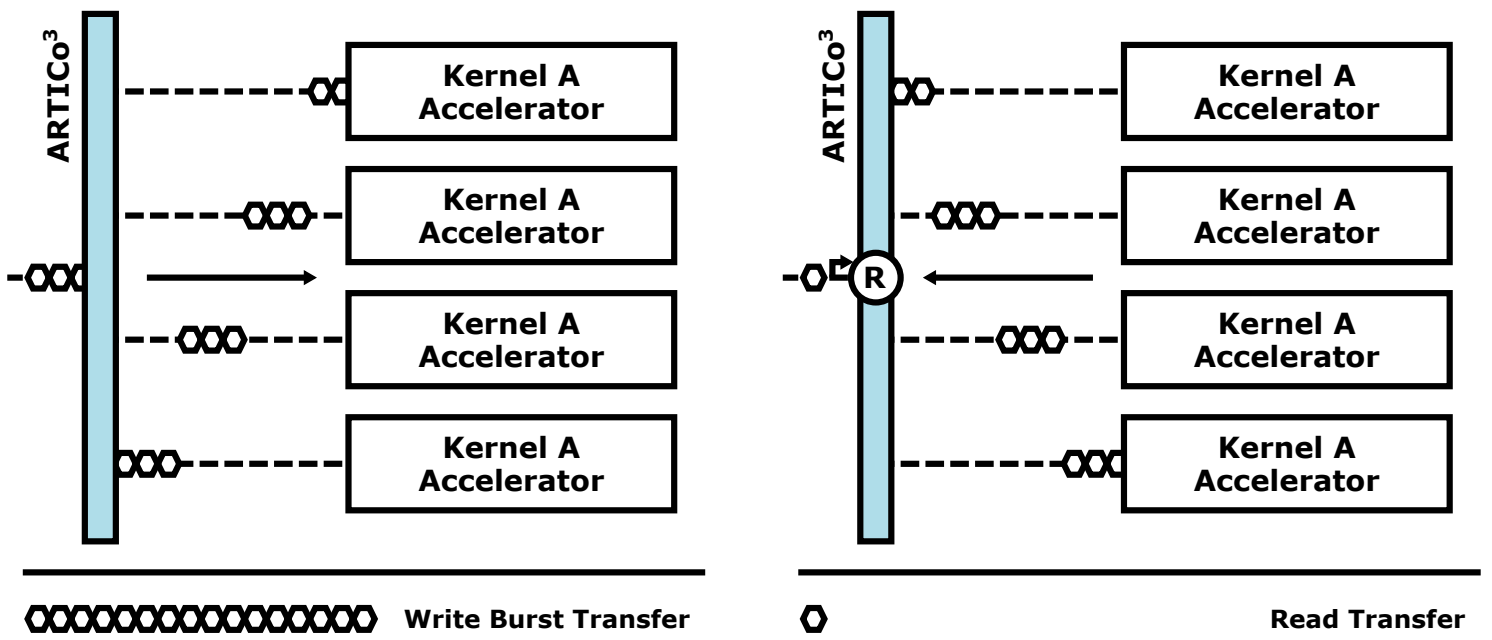

Figure 2-7: Reduction-oriented operation mode.

The ARTICo ${ }^{3}$ architecture not only provides these three different operation modes, but it also supports different combinations between them during system execution. As a result, the parallel and reduction-oriented operation modes can be combined with the redundant operation mode to enhance both computing performance and fault tolerance in the system. In these scenarios, effective accelerator instances are defined as abstractions that represent computing elements with the capability to process a certain workload on their own. Hence, an effective accelerator instance is either a single accelerator operating in any non-redundant mode, or a group of accelerators operating in redundant mode (i.e., 2 accelerators in DMR or 3 accelerators in TMR).

Consider a hypothetical scenario where the $\mathrm{ARTICo}^{3}$ architecture is configured to have 8 reconfigurable slots, and 6 hardware accelerators from the same kernel functionality are loaded. In addition, these hardware accelerators are configured to feature a TMR group (e.g., slots 0, 1, and 2), a DMR group (e.g., slots 4 and 5), and an additional accelerator in Simplex configuration (e.g., slot 7). Therefore, the setup features 3 different effective accelerator instances that will be used to perform an ADD reduction operation. Although this configuration does not really make sense in realworld scenarios, it will be used here for demonstration purposes.

Figure 2-8 showcases a write burst data transfer captured with an ILA. Notice that, from the AXI4-Full perspective, each datum (i.e., axi_wdata) is written when the channel handshake is produced (i.e., when axi_wvalid and axi_wready are both asserted). During the burst data transfer, each effective accelerator instance is enabled sequentially using the artico3_en signal. For accelerator groups, all elements are enabled in parallel. This is transparently done by the internal control logic of the architecture. Also notice that start signals (i.e., artico3_start) are asserted once all required input data have been provided to each effective accelerator instance. 

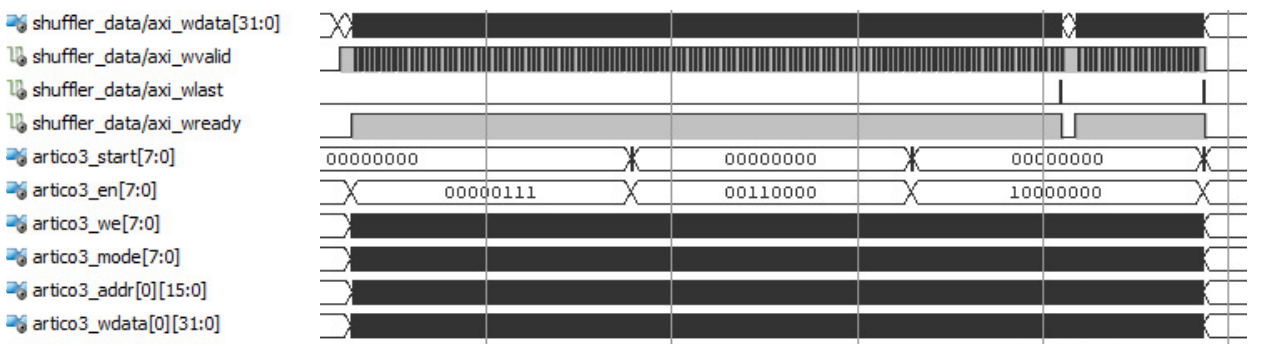

Figure 2-8: Write burst data transfer in reduction-oriented and redundant mode.

Figure 2-9 showcases a read burst data transfer captured with an ILA. Notice how the combination of operation modes retrieves data sequentially from each effective accelerator instance (i.e., artico3_rdata[0] is read before artico3_rdata[4], which in turn is read before artico3_rdata [7]), even though data come in parallel when hardware-redundant accelerator groups are used (e.g., artico3_rdata [4] and artico3_rdata[5]). Also note how the partial burst data transfers are merged in one line after the voter unit (i.e., voter_out), and how the output from the reduction engine (i.e., red_macreg) is transparently forwarded to the AXI4-Lite interface (i.e., axi_rdata) when the channel handshake happens (i.e., axi_rvalid and axi_rready are both asserted).
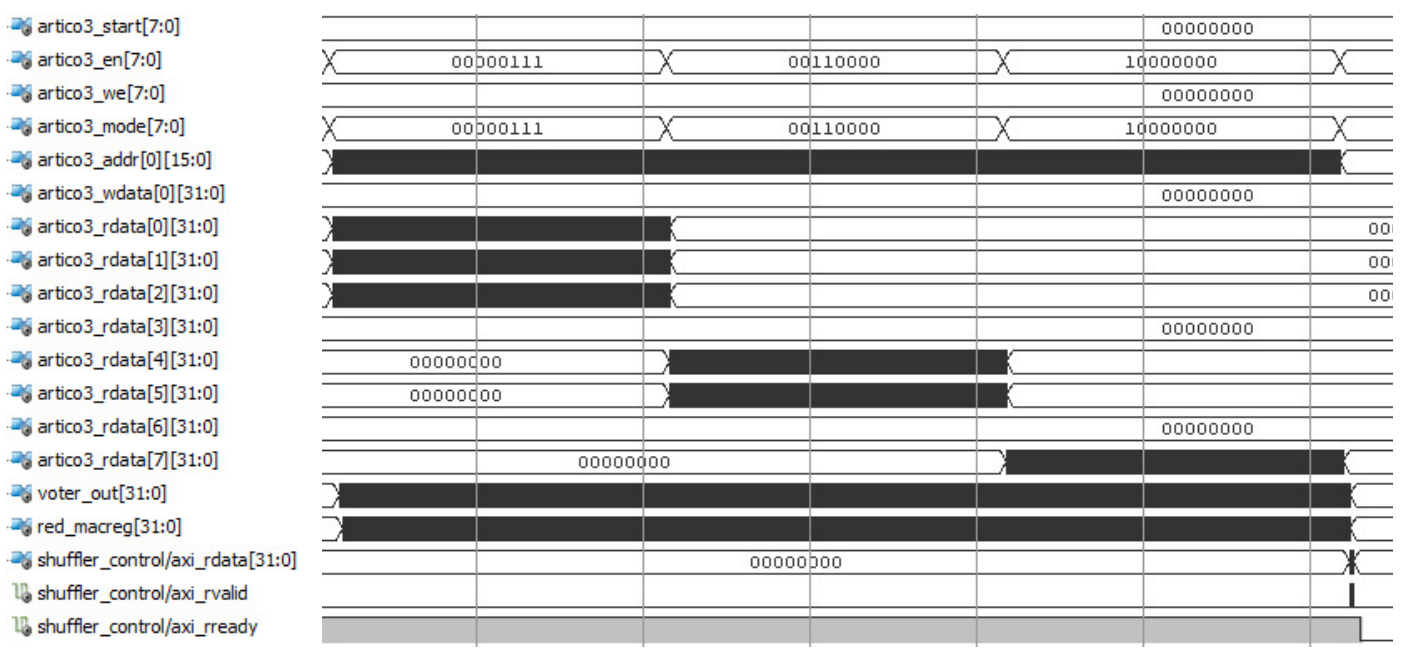

Figure 2-9: Read burst data transfer in reduction-oriented and redundant mode.

Figure 2-10 showcases a write burst data transfer with only 2 data per effective accelerator instance captured with an ILA. The borders between the memory-mapped AXI interfaces of the static region and the custom point-to-point interfaces with the accelerators have been highlighted with a dashed red line. Notice how addresses (i.e., artico3_addr) are automatically reset when switching from one effective accelerator group to the next one so that all data are written to the same memory positions in each accelerator. Also note that start signals (i.e., artico3_start) are asserted exactly one clock cycle after the last datum has been written to an effective accelerator instance, 
and that no additional latency is introduced in the AXI interface (i.e., axi_wready is always asserted). ${ }^{18}$
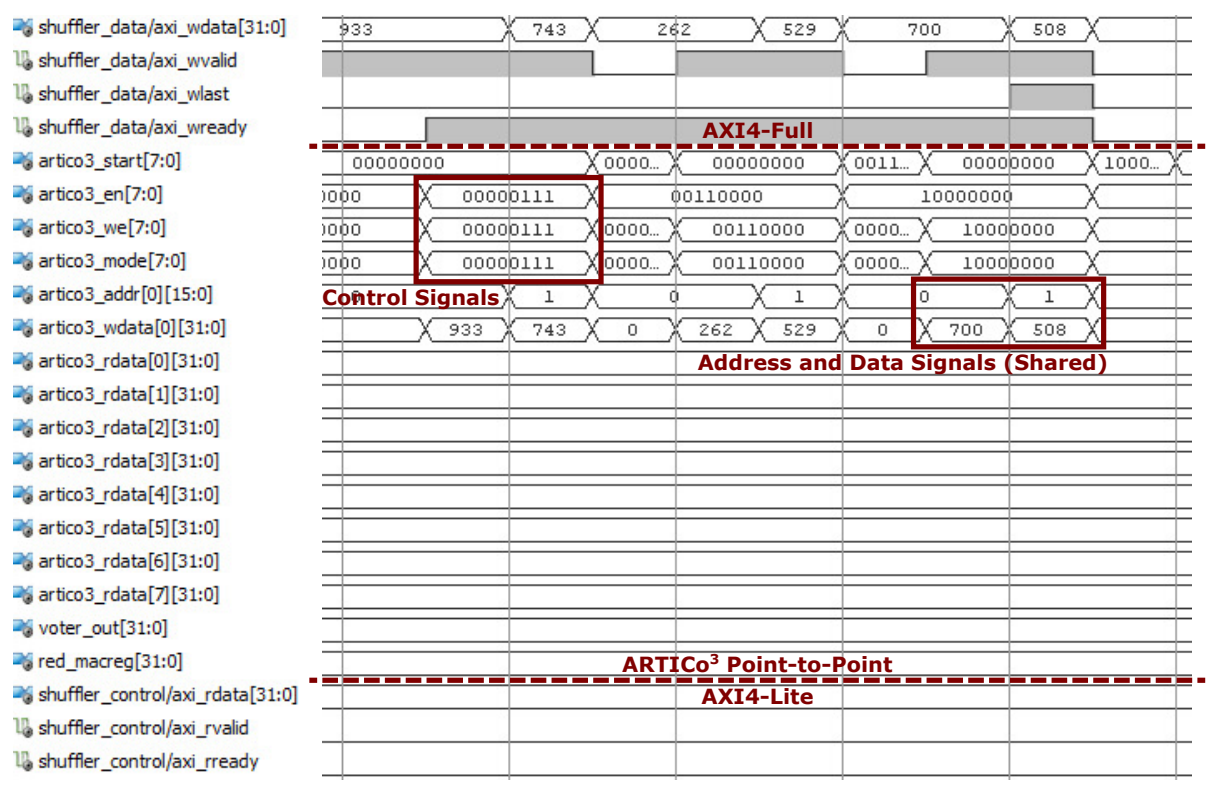

Figure 2-10: Write burst data transfer in reduction-oriented and redundant mode (detail).

Figure 2-11 showcases a read burst data transfer with only 2 data per effective accelerator instance captured with an ILA. Notice how addresses and control signals behave exactly as in the write burst data transfer. Since no functional errors have been injected in the hardware accelerators, all groups with hardware redundancy provide the same values in parallel. It takes two clock cycles for the output of an effective accelerator instance to reach the output of the voter unit, and one extra cycle to pass through the accumulator-based reduction engine. When the last datum has been reduced, the result is forwarded to the AXI interface with no additional latency.

Original contribution 2-5 A set of operation modes for DMA-enabled data transfers in multi-accelerator systems that enables combinations of concurrent execution with overlapped data-level parallelism, parallel execution with selectable hardware redundancy, and reduction-based execution in hardware.

\footnotetext{
${ }^{18}$ The shared address and write data lines, together with the channel selection part of the interface are described in Subsection 2.4.1.
} 

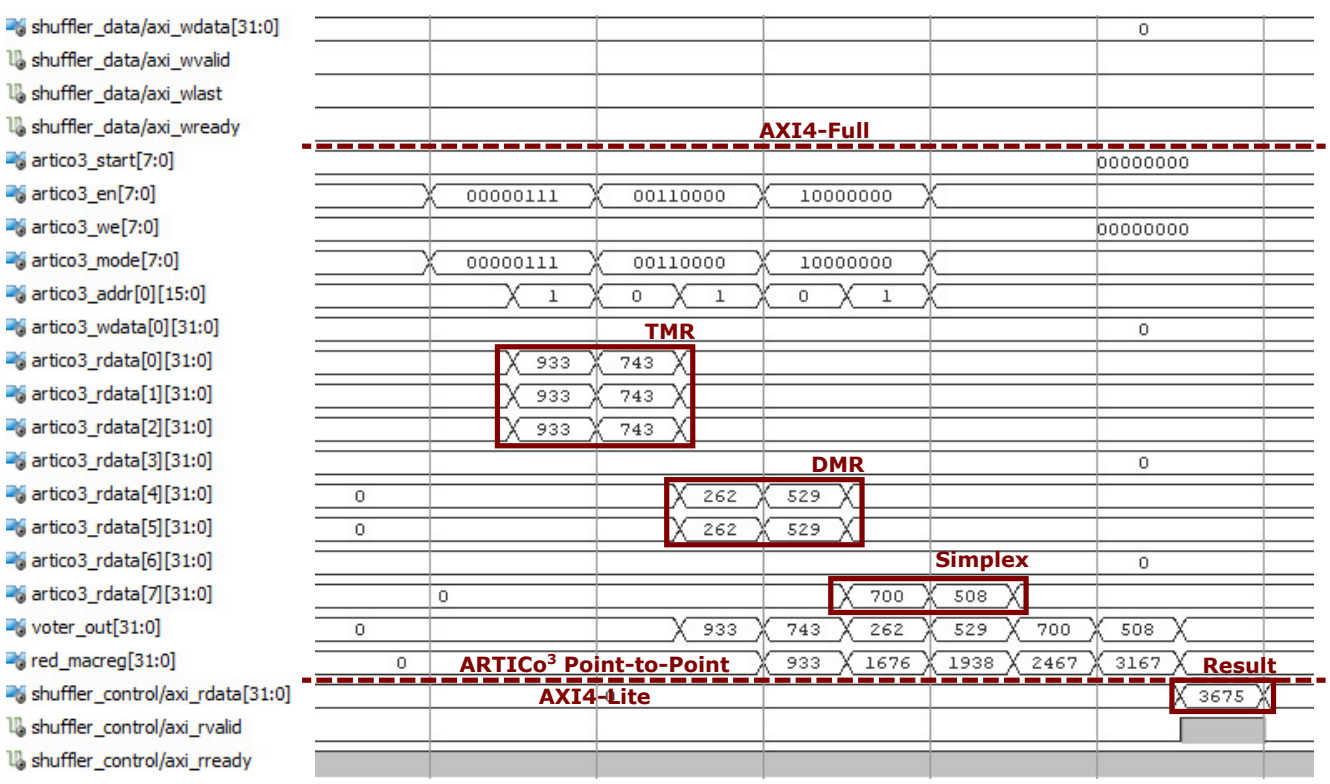

Figure 2-11: Read burst data transfer in reduction-oriented and redundant mode (detail).

\subsubsection{Programmer's Model}

A reduced set of configuration and status registers is available in the ARTICo ${ }^{3}$ architecture. These registers are used by the internal control logic to properly handle the configuration of the datapath, set the operation mode for the architecture, or configure how the DMA-enabled burst transfers are to be delivered to the accelerators, among other functionalities. The complete list of registers can be seen in Table 2-4. ${ }^{19}$

Table 2-4: ARTICo $^{3}$ register map.

\begin{tabular}{ccc}
\hline Register Name & Address Offset & Description \\
\hline A3_ID_REG_LOW & $0 \times 00$ & ID register (low) \\
A3_ID_REG_HIGH & $0 \times 04$ & ID register (high) \\
A3_TMR_REG_LOW & $0 \times 08$ & TMR register (low) \\
A3_TMR_REG_HIGH & $0 \times 0 c$ & TMR register (high) \\
A3_DMR_REG_LOW & $0 \times 10$ & DMR register (low) \\
A3_DMR_REG_HIGH & $0 \times 14$ & DMR register (high) \\
A3_BLOCK_SIZE_REG & $0 \times 18$ & Block size register \\
A3_CLOCK_GATE_REG & $0 \times 1 c$ & Clock gating register \\
A3_NSLOTS_REG & $0 \times 28$ & Firmware info $\left(n_{\text {slots }}\right)$ \\
A3_READY_REG & $0 \times 2 c$ & Ready register \\
A3_PMC_CYCLES_REG & $0 \times 30$ & Performance PMCs \\
A3_PMC_ERRORS_REG & $0 \times 30+0 \times 4 \cdot n_{\text {slots }}$ & Error PMCs
\end{tabular}

\footnotetext{
${ }^{19}$ The number of reconfigurable slots in ARTICo $^{3}$ is configurable, and depends on the implementation template used (see Subsection 2.4.3 for more information on system templates). This section assumes that $\mathrm{ARTICo}^{3}$ has been implemented using the maximum amount of reconfigurable slots (i.e., 16).
} 


\section{ID Register}

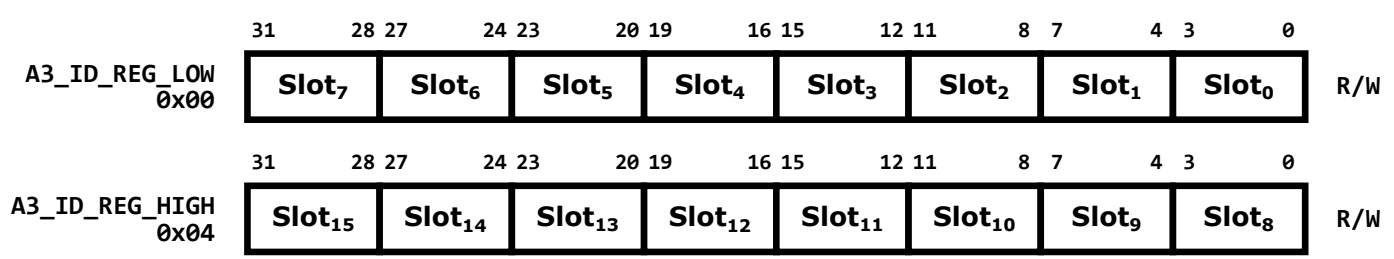

Whenever a hardware accelerator is loaded in the architecture, its kernel ID should be written into either A3_ID_REG_LOW or A3_DMR_REG_HIGH, depending on the reconfigurable slot. ARTICo ${ }^{3}$ currently supports up to 15 different kernel IDs using 4bit tags (i.e., $0 \times 1-0 \times F$ ), where there is an extra tag to represent empty slots (i.e., $0 \times 0$ ).

Kernel IDs are unique identifiers used to address all hardware accelerators that belong to the same kernel functionality. Taking advantage of the memory-mapped nature of the static region, kernel IDs are embedded in the AXI4 write address when sending data to the accelerators, and in the AXI4 read address when retrieving data from the accelerators. This process is performed for both register-based and DMAenabled burst data transfers (AXI4-Lite and AXI4-Full interfaces, respectively). The proposed addressing scheme can be seen in Figure 2-12.

AXI4 Memory-Mapped 32-bit Address

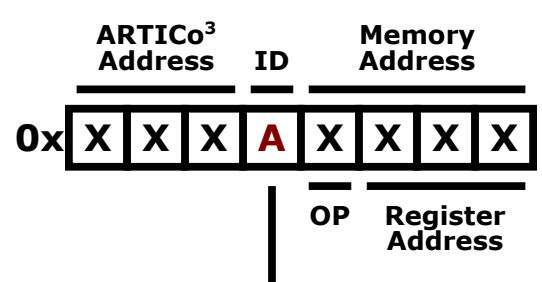

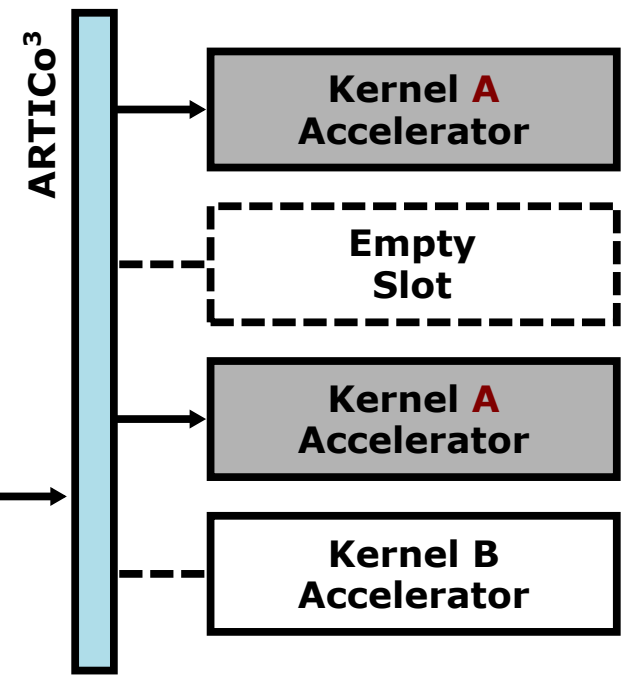

Figure 2-12: Kernel addressing.

Notice that the kernel addressing scheme also supports the encoding of specific operations into memory addresses for register-based data transactions (i.e., AXI4-Lite interconnect). Thus, multicast commands to all accelerators from a given kernel can be embedded in write operations (e.g., software reset), and configuration commands for the reduction engine can be embedded in read operations.

The main advantage of the proposed scheme is that addressing is made independent of the relative position of a given accelerator in the dynamic region. Virtual address subranges within the global memory map of the ARTICo ${ }^{3}$ architecture 
are used to access the internal memory maps of the hardware accelerators. These mappings are transparently managed by the architecture and the rest of the framework, making kernel design agnostic from this process. As a consequence, an additional support for fault tolerance is provided, enabling user-driven accelerator migration without compromising execution integrity in case a reconfigurable slot is permanently damaged.

Original contribution 2-6 An accelerator addressing scheme that isolates functionality (defined at design time) from location in the FPGA (defined at run time), and encodes target accelerator(s) and optional multicast commands in virtual subranges from system addresses.

TMR Register

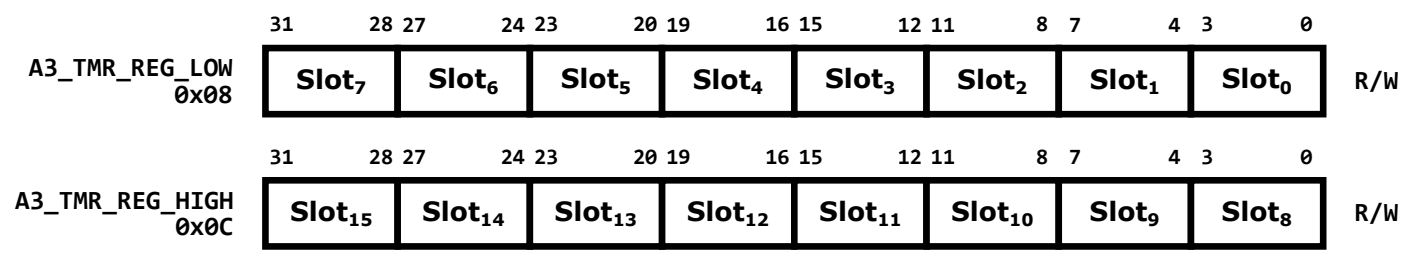

Hardware redundancy using three accelerators is enabled by writing the TMR group ID into either A3_TMR_REG_LOW or A3_TMR_REG_HIGH, depending on the reconfigurable slot. ARTICo ${ }^{3}$ currently supports up to 15 different TMR group IDs using 4-bit tags (i.e., $0 \mathrm{x} 1-0 \mathrm{xF}$ ), where there is an extra tag to represent accelerators that do not belong to any TMR group (i.e., $0 \times 0$ ).

The software or hardware manager in charge of configuring the hardware redundancy mode needs to make sure that each TMR group ID tag is written for either three reconfigurable slots or none at all. Furthermore, all reconfigurable slots that are configured to operate in TMR mode must not be also configured to operate in DMR. Simplex operation is enabled for a reconfigurable slot by setting both its TMR and DMR group IDs to $0 \times 0$.

\section{DMR Register}

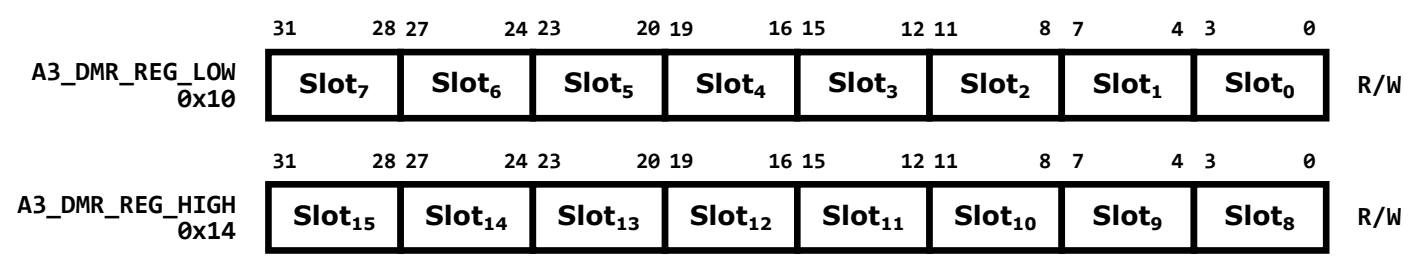

Hardware redundancy using two accelerators is enabled by writing the DMR group ID into either A3_DMR_REG_LOW or A3_DMR_REG_HIGH, depending on the 
reconfigurable slot. $\mathrm{ARTICo}^{3}$ currently supports up to 15 different DMR group IDs using 4-bit tags (i.e., $0 \mathrm{x} 1-0 \mathrm{xF}$ ), where there is an extra tag to represent accelerators that do not belong to any DMR group (i.e., $0 \times 0$ ).

The software or hardware manager in charge of configuring the hardware redundancy mode needs to make sure that each DMR group ID tag is written for either two reconfigurable slots or none at all. Furthermore, all reconfigurable slots that are configured to operate in DMR mode must not be also configured to operate in TMR. Simplex operation is enabled for a reconfigurable slot by setting both its TMR and DMR group IDs to $0 \mathrm{x} 0$.

\section{Block Size Register}

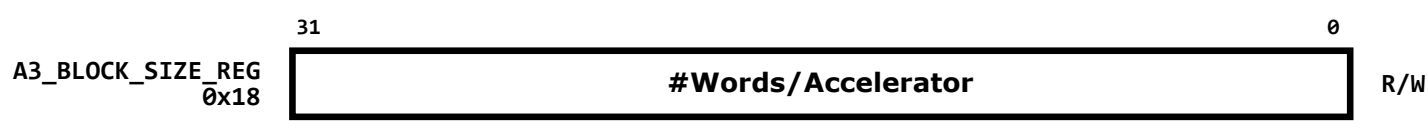

Before any burst data transfer is issued by the DMA engine, the total amount of 32bit words that are to be either written to or read from each hardware accelerator with the same kernel ID needs to be written into A3_BLOCK_SIZE_REG. The internal control logic of the ARTICo ${ }^{3}$ architecture keeps track of the amount of data already transfered by the DMA engine, and selectively enables the hardware accelerator(s) until all have received the number of data specified in this configuration register. Additionally, the start signal is asserted for each hardware accelerator one clock cycle after receiving the assigned amount of 32-bit words.

\section{Clock Gating Register}

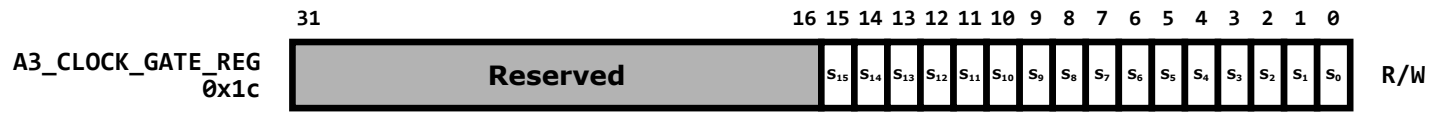

Each of the reconfigurable slots available in the dynamic region can have a dedicated clock buffer with an enable signal to perform selective clock gating. Whenever the clock signal of a given hardware accelerator needs to be enabled, a 1 has to be written to the bit corresponding to its reconfigurable slot in A3_CLOCK_GATE_REG. On the contrary, writing a 0 in the bit corresponding to a given reconfigurable slot disables its clock signal. This feature can be used to perform efficient power management in $\mathrm{ARTICo}^{3}$-based systems. 


\section{Firmware Info Register}

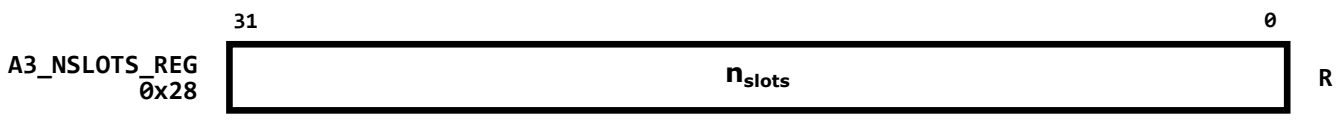

A read-only register is available in the register map of the architecture to access lowlevel information about the firmware loaded in the FPGA. Reading the A3_NSLOTS_REG register will provide the amount of reconfigurable slots available in that particular deployment.

\section{Ready Register}

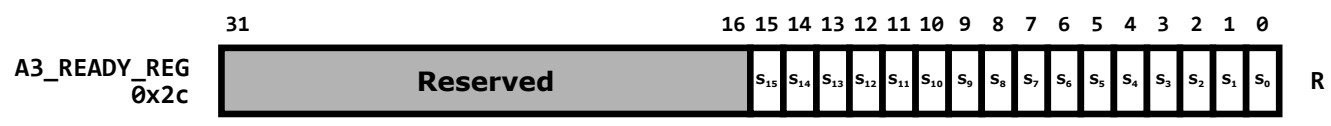

Whenever an ARTICo $^{3}$ accelerator finishes its execution, its ready signal is asserted. The A3_READY_REG status register provides a direct access to all the ready signals coming from the reconfigurable slots. This register can be used to implement a polling-based scheduling approach for accelerator execution, although the architecture also features a dedicated Interrupt Request (IRQ) line. This IRQ, which is to be treated as rising edge sensitive, is internally generated by the control logic whenever a change in any ready signal occurs.

\section{Performance PMC Registers}

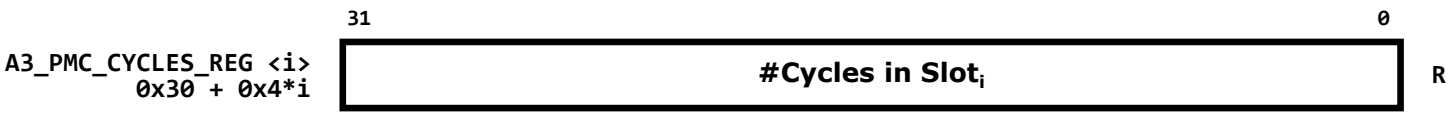

Each reconfigurable slot has a dedicated performance monitor (described in Subsection 2.3.2). Execution latencies from hardware accelerators (excluding DMA data transfers) can be read from these registers.

\section{Error PMC Registers}

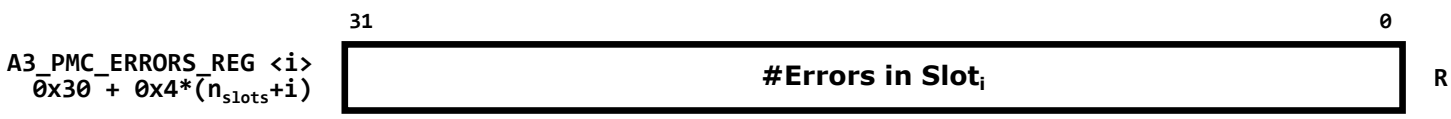

Each reconfigurable slot has a dedicated error monitor (described in Subsection 2.3.2). Errors occurred during accelerator execution can be read from these registers, which are reset after each read operation. 


\subsection{Design-Time Support}

Designing a flexible reconfigurable multi-accelerator system is a complex task. The use of DPR usually requires a deep knowledge of low-level technology details and a userguided and/or semi-automated methodology to generate the proper configuration files for the FPGA. The design-time support of the ARTICo ${ }^{3}$ framework includes a standard wrapper to ease the integration of user-defined logic in reconfigurable hardware accelerators, and an automated toolchain that applies predefined DPRcompatible templates and generates all the required FPGA configuration bitstreams. As opposed to commercial flows, where users play an active role in reconfigurable system deployment, the $\mathrm{ARTICo}^{3}$ toolchain makes it a transparent process: users only need to provide their kernel specifications, and the toolchain generates the reconfigurable multi-accelerator system automatically.

The ARTICo ${ }^{3}$ toolchain builds the application executable that runs in the host microprocessor as well. Predefined application templates are also applied to the input source files to generate the software part of the system automatically. These user-defined files must include calls to the ARTICo ${ }^{3}$ runtime API to manage FPGA reconfiguration and kernel execution. Due to its close relationship with the run-time support in the framework, this API is covered in Subsection 2.5.2.

\subsubsection{Kernel Wrapper}

Kernel functionality is implemented in ARTICo $^{3}$ as a hardware accelerator with custom user logic. The application-specific kernel descriptions provided by the user are instantiated in a standard wrapper that contains configurable local memory, a configurable register bank, and the required interconnection logic to attach the reconfigurable accelerator to the main ARTICo $^{3}$ infrastructure in a seamless way. Figure 2-13 shows the internal structure of the ARTICo ${ }^{3}$ kernel wrapper.

In ARTICo ${ }^{3}$-based reconfigurable multi-accelerator systems, the memory model is hierarchical to properly exploit data-level parallelism and data locality. Each hardware accelerator has its own local memory connected to the main memory of the system (i.e., the external RAM) by means of a DMA engine. This particular feature simplifies the control logic in both architecture and wrapper significantly, even though it prevents kernel logic from accessing the main memory directly. In other words, ARTICo ${ }^{3}$ accelerators act as virtual slave peripherals in the AXI infrastructure.

Local memory in the kernel wrapper is configurable in terms of total size and number of banks. Currently, up to $64 \mathrm{KiB}$ can be used inside each hardware 


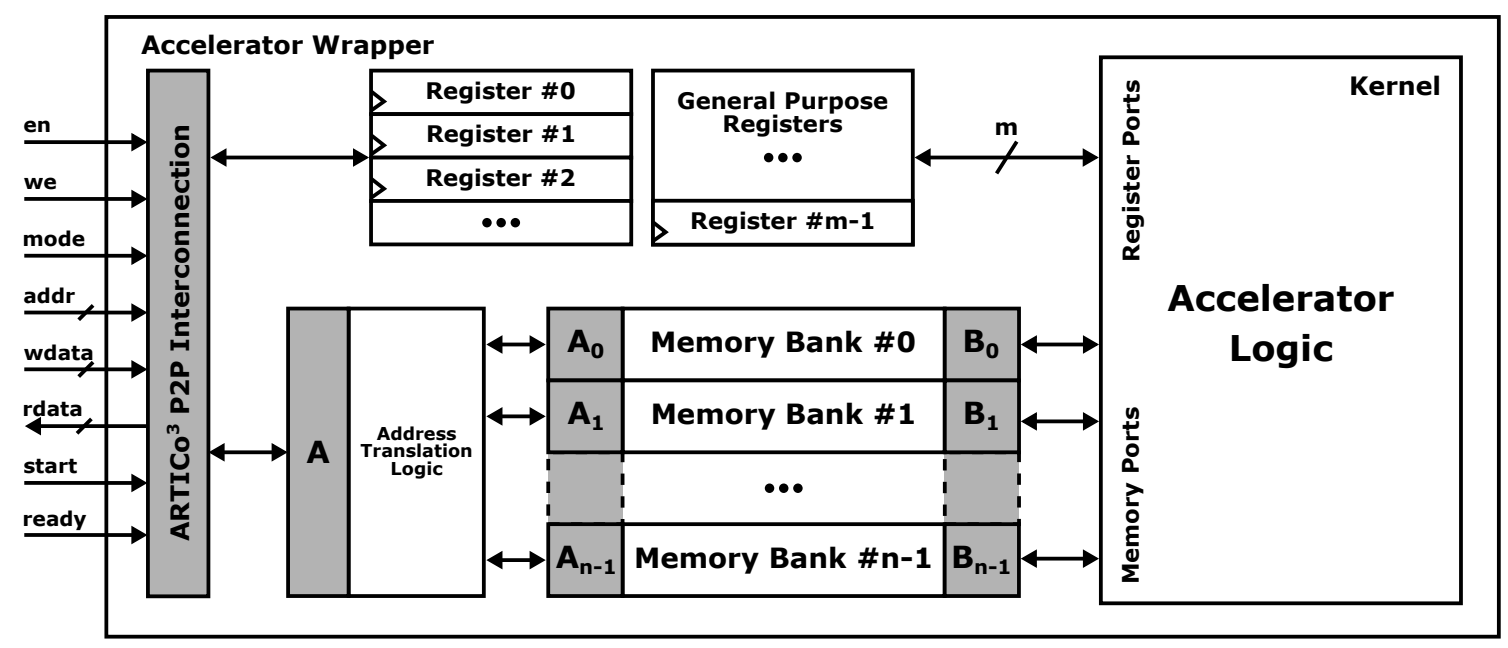

Figure 2-13: ARTICo $^{3}$ kernel wrapper.

accelerator. ${ }^{20}$ Moreover, once the size has been fixed, users can decide in how many banks will the local memory be divided. These memory banks are symmetrical, i.e., all of them have the same number of memory positions, since this simplifies the internal control logic in the accelerator wrapper.

For the static system in the FPGA, local memory is seen as a single memory map in the virtual address subrange of a given kernel. Dedicated address translation logic connects the signals coming from the $\mathrm{ARTICo}^{3}$ point-to-point interface to the appropriate memory bank. For the user logic, on the other hand, local memory is seen as a set of independent memory maps. This favors parallel accesses and increased memory bandwidth inside ARTICo ${ }^{3}$ accelerators.

ARTICo $^{3}$ kernels base their operation in data exchanges between main and local memories. As a result, each kernel instance is required to have at least one memory bank with at least one memory position. Notice that, in an ideal scenario, this is exactly the opposite of the desired setup that would process as many input data in parallel as possible. Configuration registers, on the contrary, are optional in the kernel wrapper. Its main purpose is either to store all the parameters required by the user logic to work properly (e.g., control registers), or to provide internal metrics of the user logic (e.g., status registers or internal monitors). Only a few configuration registers are expected to exist in each kernel and thus, they are accessed in parallel from the user logic. Nevertheless, the number of elements in the configurable register bank needs to be defined by the user, being the resources available in the reconfigurable slot the only limitation.

Both local memory and registers are connected to the main $\mathrm{ARTICo}^{3}$ infrastructure

\footnotetext{
${ }^{20}$ The maximum size of the local memory is limited by the ARTICo ${ }^{3}$ toolchain. However, the VHDL descriptions of both architecture and kernel wrapper already support larger memory maps in the accelerators, being the width of the system address bus the only real limitation. Nevertheless, $64 \mathrm{KiB}$ has proved to be a good tradeoff solution between memory size and area overhead, measured in terms of BRAM primitives and their impact on DPR-compatible floorplans.
} 
by means of a custom point-to-point interface. Table 2-5 shows all the signals in the interface, classified according to their main purpose.

Table 2-5: Signals in the ARTICo ${ }^{3}$ point-to-point interface.

\begin{tabular}{ccccc}
\hline Type & Port & Direction & Polarity & Description \\
\hline \multirow{2}{*}{ Clock/Reset } & aclk & in & - & Clock signal \\
& aresetn & in & Active low & Reset signal \\
\hline \multirow{2}{*}{ Control/Status } & start & in & Active high & Start execution \\
& ready & out & Active high & Execution finished \\
\hline \multirow{5}{*}{ Data } & en & in & Active high & Enable signal \\
& we & in & Active high & Write enable signal \\
& mode & in & - & Access mode \\
& addr & in & - & Address bus \\
& wdata & in & - & Write data bus \\
& rdata & out & - & Read data bus \\
\hline
\end{tabular}

Data transactions to either local memory banks or configuration registers are made when the enable signal en is asserted. In addition, the write enable signal we is also asserted for write operations. A mode control signal is used to detect whether the current transaction involves memory banks (1) or registers (0). The interface has a shared address bus (i.e., addr) that is used for both write and read operations, being wdata and rdata the corresponding data buses. Currently, the width of the address bus is fixed to 16 bits to support the $64 \mathrm{KiB}$ local memory map, and the width of the data buses is fixed to 32 bits. $^{21}$ The synchronous nature of the hardware accelerator is governed by the aclk and aresetn signals.

Accelerator execution is managed using the start/ready signal pair. ${ }^{22}$ The start control signal is asserted in the main ARTICo ${ }^{3}$ infrastructure for one clock cycle after all input data have been written to the local memory of the accelerator. At this point, the user logic inside the accelerator is expected to deassert the ready status signal, only to assert it again once all processing has been carried out. The run-time management system can then gather all data from the accelerator using the corresponding interrupt, or a polling-based policy using the A3_READY_REG register (see Subsection 2.3.4). The expected timing in the start/ready pair is shown in Figure 2-14.

Finally, the ARTICo ${ }^{3}$ kernel wrapper also features an internal data counter that can be used to track the amount of 32-bit words written to its local memory. This counter is only accessible from the user logic.

\footnotetext{
${ }^{21}$ The number of signals that cross the border between static and reconfigurable regions needs to be kept at a minimum to achieve an efficient DPR-compatible floorplan. Once more, this is limited by the toolchain, but the underlying VHDL allows both address and data buses to be extended as required.

${ }^{22}$ When designing the internal control logic of their accelerators, kernel designers need to clearly differentiate configuration parameters, which are written into registers, from input data, which are written into memory banks. In this regard, the ready signal can be generated taking into account the configuration registers, but must not depend upon the contents of the memory banks.
} 


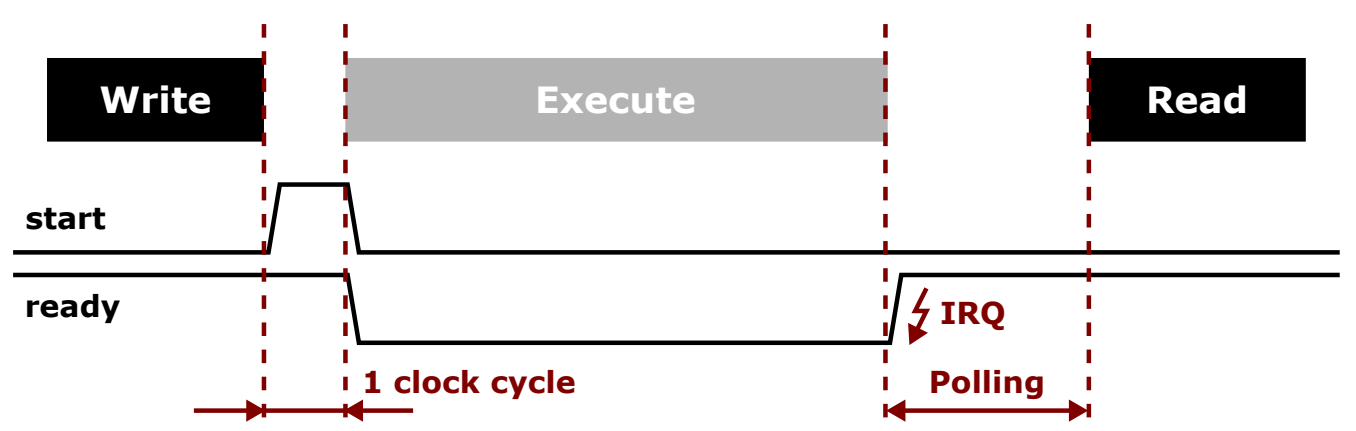

Figure 2-14: Control signal requirements.

Original contribution 2-7 A standard HDL wrapper for reconfigurable multiaccelerator systems with parallel memory banks, configuration registers, and a simplified DPR-friendly interface between static and reconfigurable regions.

\subsubsection{Kernel Specification}

The ARTICo ${ }^{3}$ toolchain supports two different approaches for kernel specification. Users with previous knowledge in hardware design can use low-level HDL descriptions of their algorithms written in either VHDL or Verilog. However, prior expertise on hardware design is not required to use $\mathrm{ARTICo}^{3}$, and high-level $\mathrm{C} / \mathrm{C}++$ input descriptions are also supported. In this case, the toolchain relies on HLS to generate the hardware accelerators. ${ }^{23}$

Listing 2-1 shows the definition of an ARTICo ${ }^{3}$ kernel in VHDL. Notice that the top module has to include, apart from the clk and reset signals, the start/ready signal pair. User logic is connected to the configuration registers using three signals: $i$ (data) to read from a register, o (data) and o_vld (data valid) to write to a register. Moreover, local memory banks are accessed using a standard Block RAM (BRAM) protocol: clk (clock), rst (reset), en (enable), we (write enable), addr (address), din (memory input), and dout (memory output). In addition, the internal data counter available in the kernel wrapper can be accessed using the values signal.

Listing 2-2 shows the definition of an $\mathrm{ARTICo}^{3}$ kernel in $\mathrm{C} / \mathrm{C}++$. Notice that kernel functionality is defined using the A3_KERNEL macro, and that each accelerator port is defined using one of the labels shown in Table 2-6. ${ }^{24}$

HLS-based ARTICo ${ }^{3}$ kernels need to include the artico3.h header file, which includes the port specification labels, the basic $\mathrm{ARTICo}^{3}$ data type (i.e., a3data_t, which currently is a 32-bit unsigned integer value), and some synthesizable helper

\footnotetext{
${ }^{23}$ It is highly advisable for users to have some background on hardware design and parallel processing, though. Optimization in HLS is usually a user-driven task, and accelerator performance can be significantly increased if the designer knows which knobs to adjust.

${ }^{24}$ The port specification labels in HLS-based kernels already include information from the local memory model shown in Subsection 2.5.3.
} 
Listing 2-1: VHDL-based ARTICo ${ }^{3}$ kernel.

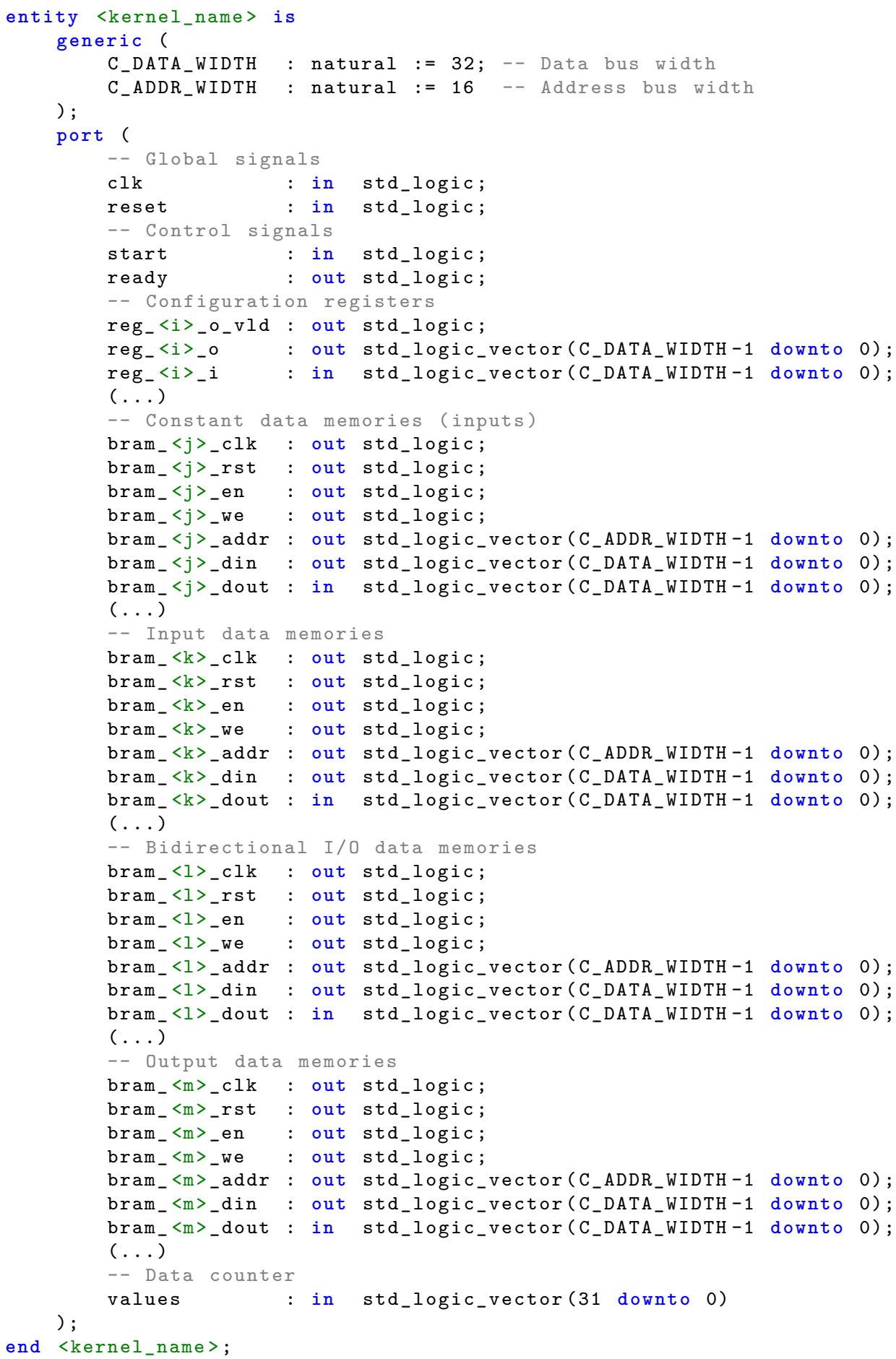


Listing 2-2: HLS-based ARTICo ${ }^{3}$ kernel.

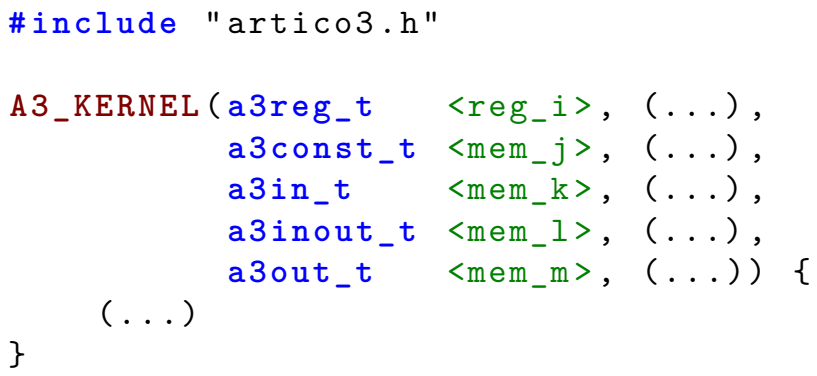

Table 2-6: Port specification in HLS-based kernels.

\begin{tabular}{cc}
\hline Label & Port Type \\
\hline a3reg_t & Configuration register \\
a3const_t & Constant input memory \\
a3in_t & Input memory \\
a3inout_t & Input/Output memory \\
a3out_t & Output memory \\
\hline
\end{tabular}

functions (shown in Table 2-7). Listing 2-3 shows how the a3reg_init function is used to initialize configuration registers in any HLS-based kernel.

Table 2-7: Synthesizable helper functions for HLS-based kernels.

\begin{tabular}{cc}
\hline Function & Description \\
\hline a3reg_init & Configuration register initialization \\
ftoa3 & float to a3data_t conversion \\
a3tof & a3data_t to float conversion \\
\hline
\end{tabular}

Listing 2-3: Register initialization in HLS-based kernels.

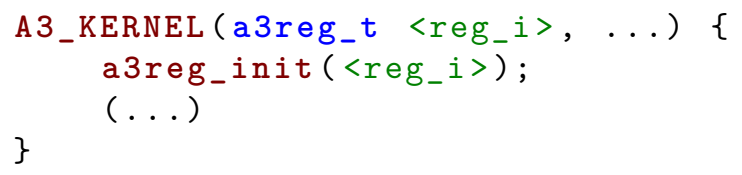

Original contribution 2-8 A method to describe reconfigurable accelerators in either HDL or HLS-oriented C/C++ using simplified interfaces and design automation with synthesizable helper macros and functions. 


\subsubsection{Template-Based Toolchain}

Design automation in the ARTICo ${ }^{3}$ framework is supported by means of a script-based toolchain. The toolchain is highly modular, featuring a core functionality that is target agnostic (i.e., it does not depend on the device nor on the OS present in the target platform), and target-specific templates for both hardware and software generation. The core functionality of the ARTICo ${ }^{3}$ toolchain is written in Python.

An interactive shell is enabled when the toolchain is executed. In this shell, a reduced set of commands can be executed to retrieve information from the current project, generate the reconfigurable multi-accelerator system and its associated bitstreams, generate the software application and its associated executable, or generate support files for a Linux-based OS. The complete command list can be found in Table 2-8.

Table 2-8: ARTICo $^{3}$ toolchain commands.

\begin{tabular}{ccc}
\hline Type & Command & Description \\
\hline General & info & Show current project information \\
\hline \multirow{3}{*}{ Hardware } & export_hw & Generate hardware project \\
& build_hw & Build hardware project \\
& clean_hw & Cleanup hardware project \\
\hline \multirow{3}{*}{ Software } & export_sw & Generate software project \\
& build_sw & Build software project \\
& clean_sw & Cleanup software project \\
\hline \multirow{2}{*}{ OS } & gen_driver & Generate Linux device driver \\
& gen_overlay & Generate Linux Device Tree overlay \\
& clean_os & Cleanup Linux-related files \\
\hline
\end{tabular}

The shell-based toolchain needs to be executed in an ARTICo $^{3}$ project folder. This folder contains two main elements: a configuration file (i.e., build.cfg) and a folder with all the source code (i.e., src). The project configuration file contains all the information needed to generate and execute the reconfigurable multi-accelerator system (e.g., target board, target FPGA device, or target OS). The source code folder is further organized in a subfolder for the software (i.e., application), and as many subfolders as kernels are to be implemented (i.e., a3_<kernel_i_name>). Please note that $\mathrm{ARTICo}^{3}$ kernels can be defined in $\mathrm{C} / \mathrm{C}++$, VHDL, or Verilog, placing the source code in the hls, vhdl, or verilog subfolders, respectively. Therefore, a generic template for any $\mathrm{ARTICo}^{3}$ project is as follows: 


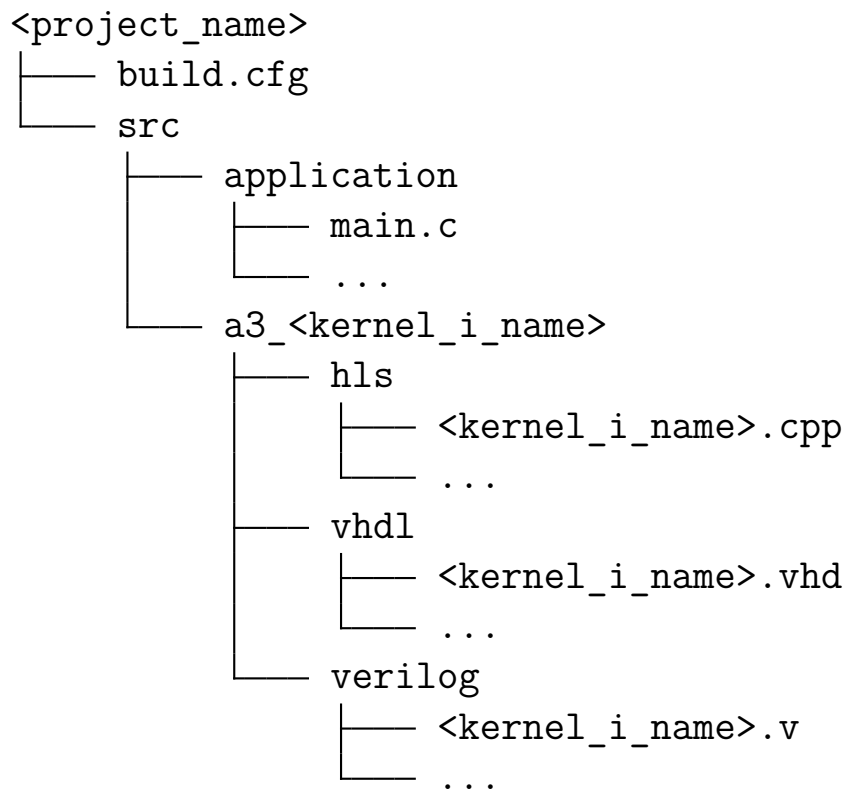

\section{Hardware Generation}

The use of commercial state-of-the-art CAD tools to implement DPR-compatible floorplans usually requires intensive user intervention, relying significantly on Tcl commands and/or scripts. In addition, they usually limit the amount of supported reconfiguration features (e.g., bistream relocation). Academic solutions try to overcome these limitations to provide flexible DPR capabilities, sometimes at the expense of design productivity.

The initial approach to FPGA floorplanning in $\mathrm{ARTICo}^{3}$ relied on a custom flow with certain non-automated parts. In fact, most of the implementation stage was performed using commercial tools, ${ }^{25}$ but manual adjustments were required to generate configuration bitstreams. Specifically, users had to inspect the placed and routed designs and modify them when required. Moreover, bus macros were used in the border between static and dynamic regions. This methodology rendered bad productivity results, but provided bitstream relocation capabilities and flexible FPGA partitioning (i.e., hardware accelerators could be hosted in more than one reconfigurable slot).

Figure 2-15 shows an ARTICo ${ }^{3}$-based system on a HiReCookie Wireless Sensor Network (WSN) node [Valverde'12], where the target FPGA is a Xilinx Spartan-6, ${ }^{26}$ implemented with the legacy flow. The reconfigurable partition is divided in 8 homogeneous reconfigurable slots to enable bitstream relocation (left). Two different setups are showcased: 8 small (center) and 4 large (right) accelerators.

Figure 2-16 shows an ARTICo ${ }^{3}$-based system on a KC705 development board,

\footnotetext{
${ }^{25}$ Xilinx ISE Design Suite.

${ }^{26}$ XC6SLX150-2FGG484.
} 

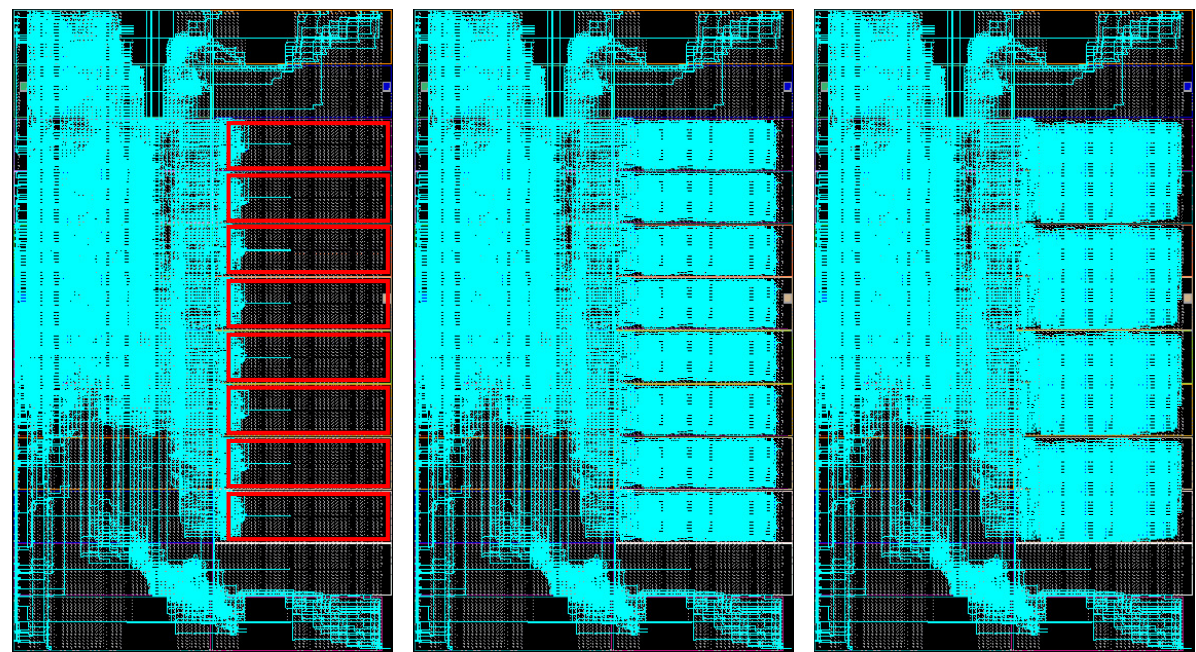

Figure 2-15: $\mathrm{ARTICo}^{3}$ floorplan in Spartan-6.

where the main FPGA is a Xilinx Kintex-7,27 implemented with the legacy flow. In this case, the reconfigurable partition is divided in 6 reconfigurable slots (left). Homogeneous FPGA resource distribution is present on the slots that are either on the right or on the left, ensuring that bitstream relocation is still supported in this setup (even though it is more limited than the one presented in the previous scenario). Two setups with 2 hardware accelerators of different size are showcased (center, right).
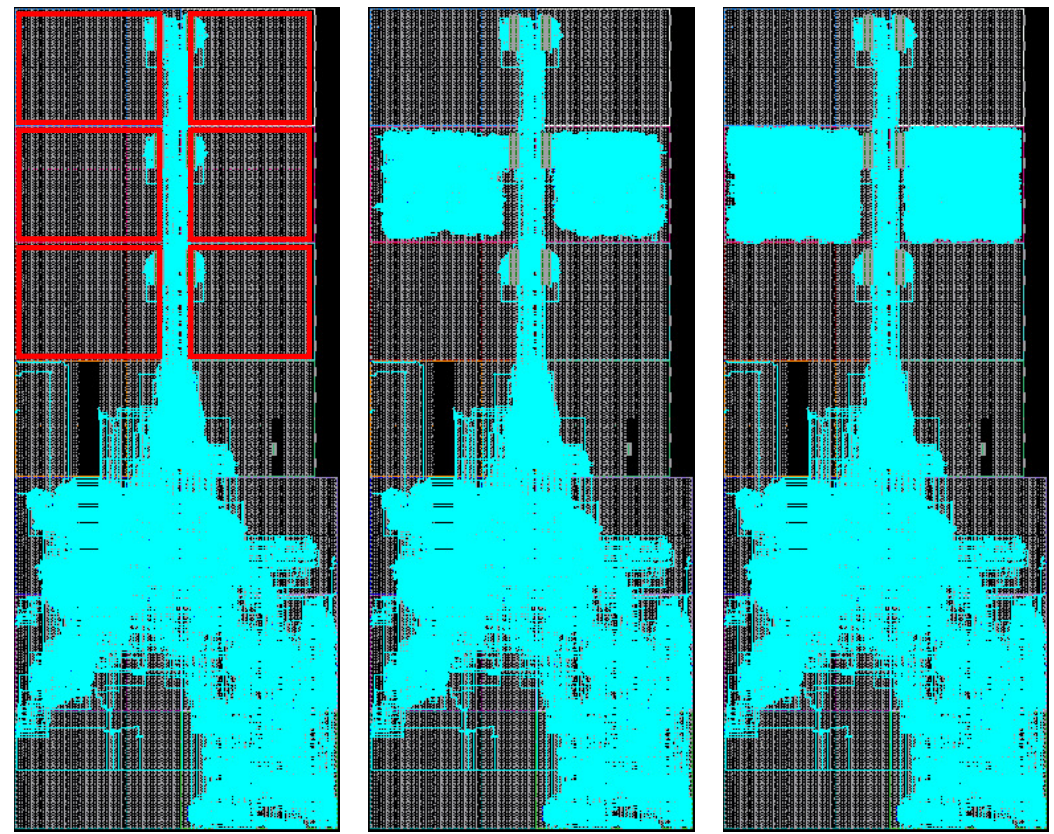

Figure 2-16: $\mathrm{ARTICo}^{3}$ floorplan in Kintex-7.

The natural evolution of both FPGA device families and their companion design

${ }^{27}$ XC7K325T-2FFG900. 
tools made it impossible to continue working with the implementation flow described above. As a consequence, the hardware generation part of the ARTICo ${ }^{3}$ toolchain was automated using Tcl-based templates that are used from the main Python core. In this regard, custom DPR methodologies were discarded in favor of standard vendor flows. Although certain features are no longer supported (e.g., bitstream relocation), the increased design productivity motivates the change. The structure of a hardware template is as follows:

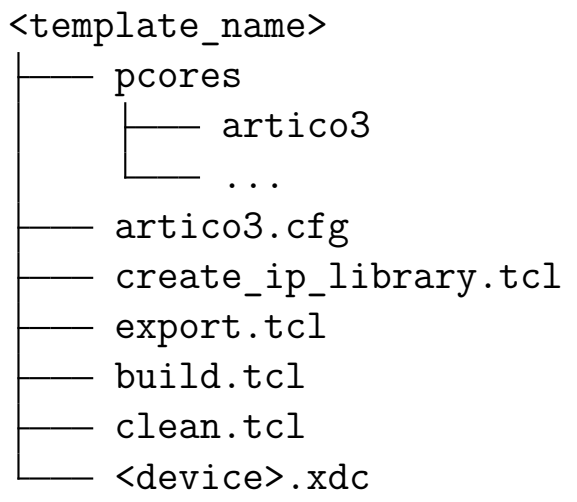

Currently, the system generation process is divided in several stages, each one supported by a given Tcl script. First, user-defined logic is instantiated in the kernel wrapper in create_ip_library.tcl, applying HLS if required. Then, the block diagram of the multi-accelerator system is generated in export.tcl, adding all the required kernels and IP cores from the pcores folder (e.g., the ARTICo ${ }^{3}$ architecture). In this step, a configuration file (i.e., artico3. $\mathrm{cfg}$ ) is used to setup the ARTICo $^{3}$ architecture (e.g., number of slots). Finally, the system is implemented in build.tcl applying the required low-level device constraints to support DPR, defined in <device $>$. xdc. This step produces both full and partial FPGA bitstreams.

Figure 2-17 shows an ARTICo ${ }^{3}$-based system on a Pynq development board, where the main FPGA is a Xilinx Zynq-7000, ${ }^{28}$ implemented with the current toolchain. Since relocation is not supported, a partial bitstream is generated for each kernel instantiated in each reconfigurable slot (highlighted in red). Notice that each hardware accelerator (blue logic) is connected to the ARTICo ${ }^{3}$ infrastructure in the static system (orange logic) using a fixed interface (gray areas).

Original contribution 2-9 An automated design methodology to generate custom reconfigurable multi-accelerator systems from either low-level RTL descriptions or high-level algorithmic descriptions and HLS.

${ }^{28}$ XC7Z020-1CLG400. 


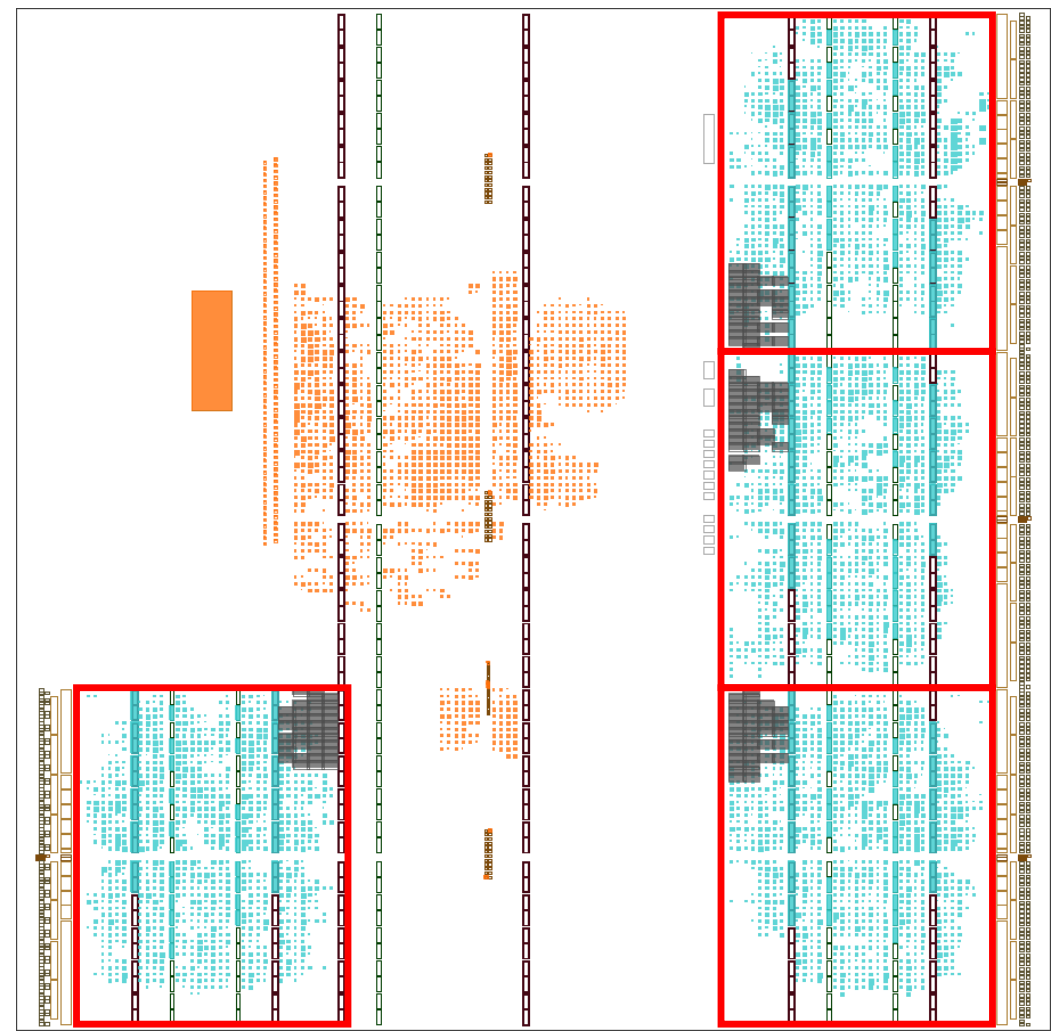

Figure 2-17: $\mathrm{ARTICo}^{3}$ floorplan in Zynq-7000.

\section{Software Generation}

The ARTICo ${ }^{3}$ toolchain also uses a template-based approach for software generation. Each software template includes all the required libraries and a Makef ile to automate the build process. Currently, ARTICo ${ }^{3}$-based applications have to be written in C code, and are executed under a Linux-based OS. Therefore, only one software template is available at the time of writing this Thesis.

In software templates, a runtime library is used to manage kernel execution and FPGA reconfiguration. This library is accessed using a lightweight API from the main application code. The complete specification of the ARTICo ${ }^{3}$ runtime API can be found in Subsection 2.5.2, providing detailed explanations on how the architecture is managed at run time. 


\subsection{Run-Time Support}

The run-time management of reconfigurable multi-accelerator systems is another major challenge in the field of reconfigurable computing. Parallel computation offloading to a dynamic pool of hardware accelerators and device reconfiguration are complex tasks even for experienced hardware designers. The ARTICo ${ }^{3}$ framework provides a software layer to address both issues and make them transparent for users. This layer is implemented as a runtime library that uses custom DMA and reconfiguration drivers under a Linux-based OS. The main functionality is accessed from user applications using a reduced API.

Moreover, a model has been also developed to characterize kernel execution in ARTICo $^{3}$. The model uses run-time measurements of both performance (i.e., execution time) and power, acquired using the architectural support of the lightweight monitoring infrastructure (see Subsection 2.3.2). In addition, the low-complexity of the model fosters its use to perform run-time estimations and online predictions.

\subsubsection{Execution Model}

Kernel execution in ARTICo ${ }^{3}$ follows a similar approach to the one used in modern general-purpose GPUs. However, as opposed to these devices, $\mathrm{ARTICo}^{3}$ can dynamically change the number of processing elements in the computing platform. Moreover, processing elements are more specialized and performance-oriented, since they are application-specific hardware accelerators instead of general-purpose SIMD processors.

The main idea behind the ARTICo ${ }^{3}$ execution model is exploiting the data-parallel nature of kernels using a pool of hardware accelerators that may continuosly change over system lifetime. In this scheme, two different types of workload are present: global and local. The global workload is the total amount of processing that a kernel requires. The local workload, on the other hand, is the amount of processing that one effective accelerator instance can perform. In order to finish the execution of a given kernel, the global workload is divided in as many execution rounds as needed, taking into account that the amount of processing equals the local workload in each round. Therefore, the relationship between global and local workload is defined by Equation 2-1.

$$
W_{\text {global }}=n_{\text {rounds }} \cdot W_{\text {local }}
$$

The ARTICo ${ }^{3}$ execution model assumes that processing rounds are data-independent. This assumption enables transparent scalability during kernel execution, which means that processing rounds can be performed concurrently, provided that the FPGA hosts several kernel instances. ${ }^{29}$ Figure 2-18 shows the ARTICo ${ }^{3}$ execution model in a

\footnotetext{
${ }^{29}$ Please, keep in mind that data serialization in the bus forces execution to be overlapped in the parallel operation mode of the ARTICo ${ }^{3}$ architecture.
} 
scenario where 4 processing rounds are executed using 1, 2 or 4 effective accelerator instances. Notice that, although the output results are exactly the same (data independence between execution rounds guarantees this), the execution times are reduced almost linearly and proportionally to the number of hardware accelerators.

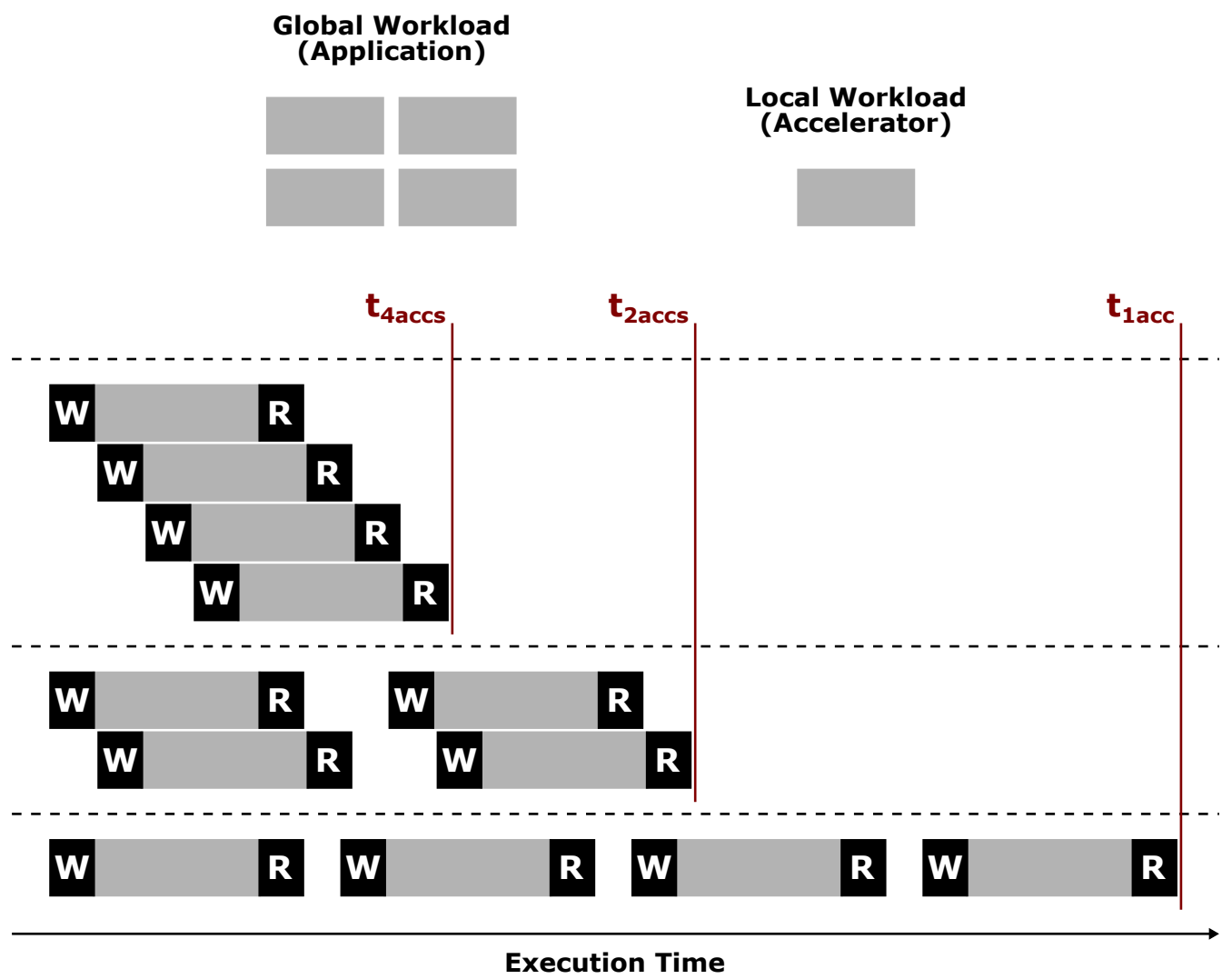

Figure 2-18: The ARTICo ${ }^{3}$ execution model.

\subsubsection{Runtime Library}

The ARTICo $^{3}$ runtime environment is implemented as a user-space extension of the application code, and relying on a Linux-based OS. In particular, the ARTICo ${ }^{3}$ management engine is a software library written in $C$ code. Its main purpose is to enable a common interface to link the automatically generated hardware platforms with user applications, providing an abstraction layer to handle FPGA reconfiguration and multi-accelerator execution (i.e., workload scheduling over a given number of accelerators using variable-size DMA transfers between external and local memories).

A minimal and developer-friendly API is available for users to write their host applications and use ARTICo ${ }^{3}$ transparently. Moreover, functions are grouped in the runtime library according to their class. The runtime library includes functions to initialize and clean the static system, perform DPR to load accelerators, change the working point of the architecture, manage shared memory buffers between application 
and accelerators, or run kernels with a given workload, among others. The complete list of functions in the API is shown in Table 2-9.

Table 2-9: ARTICo ${ }^{3}$ runtime library API.

\begin{tabular}{|c|c|c|}
\hline Type & Function & Description \\
\hline \multirow{2}{*}{ System } & artico3_init & Initialize runtime and load static system \\
\hline & artico3_exit & Release runtime and clean environment \\
\hline \multirow{2}{*}{ Slot } & artico3_load & Load kernel in slot and set configuration \\
\hline & artico3_unload & Remove previous slot configuration \\
\hline \multirow{2}{*}{ Memory } & artico3_alloc & Allocate DMA-capable shared memory buffer \\
\hline & artico3_free & Free DMA-capable shared memory buffer \\
\hline \multirow{7}{*}{ Kernel } & artico3_kernel_create & Register kernel in runtime (get kernel ID) \\
\hline & artico3_kernel_release & Remove kernel from runtime (free kernel ID) \\
\hline & artico3_kernel_execute & Execute kernel asynchronously (delegate thread) \\
\hline & artico3_kernel_wait & Wait for kernel completion \\
\hline & artico3_kernel_reset & Reset all kernel instances (accelerators) \\
\hline & artico3_kernel_wcfg & Write data to kernel registers \\
\hline & artico3_kernel_rcfg & Read data from kernel registers \\
\hline Monitor & artico3_hw_get_pmc_<type> & Get <type> PMC value \\
\hline \multirow{2}{*}{ Helper } & ftoa3 & float to a3data_t conversion \\
\hline & a3tof & a3data_t to float conversion \\
\hline
\end{tabular}

The ARTICo ${ }^{3}$ runtime library defines the basic data type a3data_t, which is a 32-bit unsigned integer. The management software uses this data type in all the transfers between static system and hardware accelerators. Hence, helper functions are provided to perform data conversion whenever floating point arithmetic is present in a kernel (i.e., ftoa3 and a3tof). These functions are the equivalent to the ones used in HLS-based accelerators (see Subsection 2.4.2). Therefore, both application code and accelerators can work with floating point arithmetic, even though data transfers are encoded as unsigned integers.

The FPGA is configured with its full bitstream in the artico3_init function. Then, users are expected to actively and explicitly decide how many kernel instances have to be loaded using the artico3_load function. This function is used to load a hardware accelerator using DPR in a certain slot, and set its specific configuration (e.g., DMR or TMR group ID). Shared memory allocation and deallocation is performed with the artico3_alloc and artico3_free functions, respectively. Once a given kernel is registered in the runtime using the artico3_kernel_create function, users can call artico3_kernel_execute to start its execution. This function takes advantage of the data-independent $\mathrm{ARTICo}^{3}$ execution model to automatically sequence all processing rounds over the available kernel instances. A delegate thread is generated for each kernel ID to perform concurrent scheduling in a multi-kernel context. Thus, the main application code needs to call artico3_kernel_wait to synchronize hardware and software execution, waiting for the outputs produced by the kernel.

Register-based access for configuration/monitoring purposes in kernel instances is performed using the artico3_kernel_wcfg function for write operations, and the artico3_kernel_rcfg for read operations. As opposed to those data transactions 
on the AXI4-Full interface, where a DMA engine moves several data in burst fashion, only one datum is transferred per AXI4-Lite transaction. Therefore, only one effective accelerator instance can be accessed at a time using that interface. Nonetheless, the runtime library automatically and transparently sequences these operations, generating as many independent transactions as effective accelerator instances are loaded in the FPGA. Reduced application buffers are used to store the data involved in these transfers. ${ }^{30}$

Finally, PMC values can be accessed using the artico3_hw_get_pmc_<type> functions, where <type> is either cycles for the performance monitors, or errors for the error monitors.

Original contribution 2-10 A runtime library to transparently manage FPGA reconfiguration and computation offloading in multi-accelerator scenarios.

\subsubsection{Kernel Scheduling}

Kernel execution in ARTICo ${ }^{3}$ follows several steps. First, kernels have to be registered in the runtime by calling artico3_kernel_create. This process takes four parameters as inputs (i.e., kernel name, local memory size, number of memory banks, and number of configuration registers), and assigns an unregistered ID to the kernel.

Communication between user application and hardware kernels is made through shared memory buffers. Linux does not support DMA management from user space and thus, the runtime library relies on a custom device driver that acts as a DMA proxy. The ARTICo ${ }^{3}$ runtime library sends its DMA requests to the device driver, and the kernel code performs the actual data transfers between local memory in the accelerators and physical memory buffers allocated by the driver. The device driver makes these physical memory buffers available to user-space applications through its mmap functionality. Nevertheless, using uncached physical memory directly in the user-space application code (i.e., the ARTICo ${ }^{3}$ runtime library) would penalize execution performance significantly. Hence, secondary memory buffers are also allocated with malloc, and the runtime copies data between them and the physical memory using standard memcpy calls. To simplify, these secondary memory buffers are referred to as shared memory buffers in the rest of this Thesis.

Each shared memory buffer obtained using the artico3_alloc function contains all data to be processed as global workload, and is associated with a specific memory bank in the local memory of ARTICo $^{3}$ accelerators. In ARTICo $^{3}$, local memory banks represent kernel ports. Therefore, during each kernel execution round, kernel ports contain the part of the shared memory buffers that corresponds to the local workload. Currently, the ARTICo ${ }^{3}$ runtime library supports four different types of

\footnotetext{
${ }^{30}$ Configuration buffers should have as many a3data_t elements as reconfigurable slots are in a given template, which is the maximum number of effective accelerator instances when all kernel instances are configured in Simplex mode.
} 
kernel ports, defined as an enumeration (i.e., a3pdir_t): constant memory (A3_P_C), input memory (A3_P_I), bidirectional input/output memory (A3_P_IO), and output memory (A3_P_O).

- Constant memory: input memory, common to all instances of a given kernel, that remains constant throughout kernel execution. It is meant to be initialized at the very beginning of the host application code, and it must not be changed after launching a kernel unless proper deallocation/allocation is performed.

- Input memory: memory used to store the data required for a given round during kernel execution. It is meant to be used as write-only memory from host application code and as read-only memory from kernel code.

- Bidirectional input/output memory: memory used to store both the data required for a given round during kernel execution (before the accelerator starts processing) and the results produced after the same round during kernel execution (after the accelerator asserts its ready signal). It is meant to be used as $\mathrm{read} / \mathrm{write}$ memory from host application and kernel code.

- Output memory: memory used to store the results produced after a given round during kernel execution. It is meant to be used as a read-only memory from host application code and as a write-only memory from kernel code.

Kernel ports are physically ordered inside hardware accelerators in such a way that maximizes DMA transfer efficiency, as it can be seen in Figure 2-19.

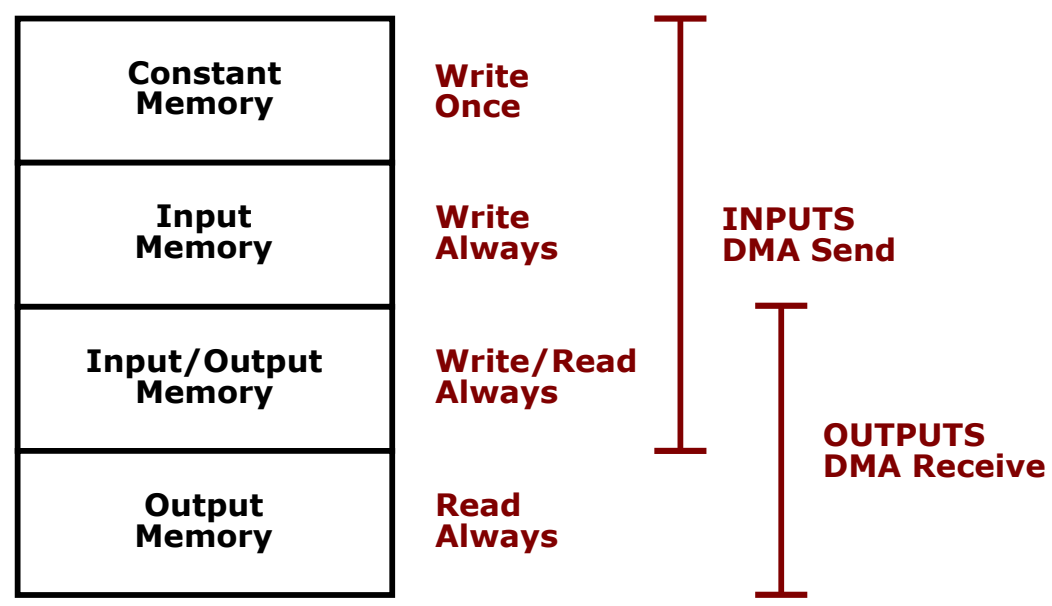

Figure 2-19: ARTICo $^{3}$ kernel memory model.

Kernel scheduling is performed in the delegate thread that is created inside the artico3_kernel_execute function, and consists of the sequencing of execution rounds using the effective accelerator instances available in the FPGA. Memory management is enforced in each round, copying data from the shared memory buffers to the DMA-capable physical memory buffers (write transactions), and vice versa (read 
transactions). The round sequencer waits until the ready signals of all instances of a given kernel are asserted before issuing a read transaction. Currently, this mechanism is implemented using interrupt-assisted polling on the A3_READY_REG register (using the poll system call in Linux), although an alternative implementation using busywait on the same register is also available. ${ }^{31}$

Figure 2-20 shows an in-depth view of the kernel scheduling procedure in a scenario with 4 execution rounds (i.e., global workload is 4 units, local wokload is 1 unit), 2 effective accelerator instances, and 4 kernel ports (1 constant memory port, 1 input memory port, 1 bidirectional input/output port, and 1 output port). First, the host application code allocates all shared memory buffers using artico3_alloc and fills all inputs with valid data. Then, the same code calls artico3_kernel_execute and creates a delegate software thread in which execution rounds are sequenced in a control loop. For each iteration of the loop, the runtime library copies data from the shared memory buffers associated with input ports to the DMA-capable physical buffer using memcpy (padding with zeros if the amount of input data is less than the local memory bank size), starts the write DMA transfer by issuing a petition to the proxy driver (ARTICo ${ }^{3}$ then triggers each accelerator by asserting its start signal), waits for accelerators to finish (when all ready signals are asserted), starts the read DMA transfer by issuing another petition to the proxy driver, and copies data from the DMA-capable physical buffer to the shared memory buffers associated with output ports using memcpy again. Notice that, for constant memory ports, the same values are written to all effective accelerator instances but only during the first iteration. Also note that the size of the DMA-capable physical buffers equals the total size of input/output memory banks in one accelerator multiplied by the number of effective accelerator instances during write/read operations, respectively. Finally, notice that several execution rounds can be processed concurrently in each iteration of the control loop. The host application code can only read the output buffers when kernel execution has finished (i.e., it has to call artico3_kernel_wait before reading from the output shared memory buffers).

The runtime library is prepared to face situations where the number of processing rounds is not an integer multiple of the number of effective accelerator instances. In these scenarios, kernel execution is handled exactly as already described, but the last loop iteration differs. To minimize the control part of the scheduling algorithm, the last loop iteration when there is an unpaired accelerator/round execution forces all kernel instances to execute, but only the results of those required to finish kernel execution are taken into account. In fact, the remaining effective accelerator instances are loaded with zeros to perform a dummy execution. ${ }^{32}$ Figure $2-21$ shows an unpaired accelerator/round execution scenario with the same configuration of the previous example, but increasing the global workload from 4 to 101 units.

\footnotetext{
${ }^{31}$ The management scheme using busy-wait should only be used in setups where kernels show short execution times that cause the IRQ signal to be asserted while its associated ISR has not finished processing the previous interrupt. These scenarios are easily recognizable, since the application stalls.

${ }^{32}$ Using the contents of memory banks to generate the ready signal can lead to malfunctioning in unpaired accelerator/round executions due to local memories being filled with zeros.
} 


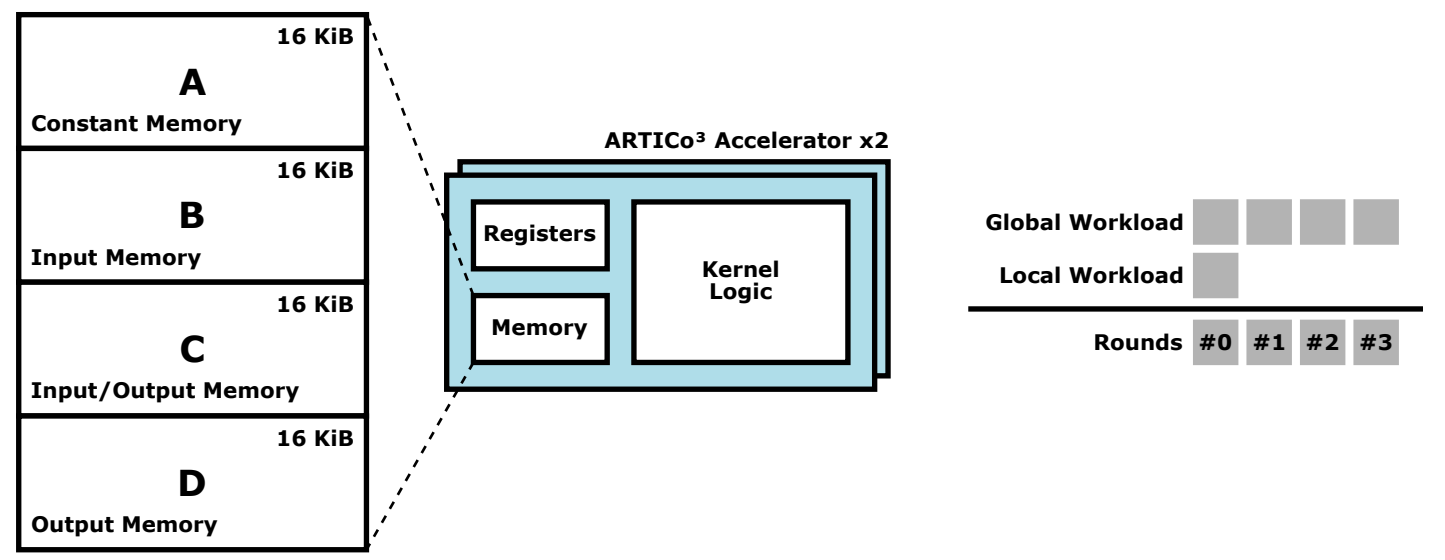

Application Buffers $\longleftarrow$ artico3_alloc()

A

\begin{tabular}{|l|l|l|l|}
\hline$B_{0}$ & $B_{1}$ & $B_{2}$ & $B_{3}$ \\
\hline
\end{tabular}

\begin{tabular}{|l|l|l|l|}
\hline $\mathrm{C}_{0}$ & $\mathrm{C}_{1}$ & $\mathrm{C}_{2}$ & $\mathrm{C}_{3}$ \\
\hline
\end{tabular}

$-------$

A

\begin{tabular}{|l|l|l|l|}
\hline$B_{0}$ & $B_{1}$ & $B_{2}$ & $B_{3}$ \\
\hline$C_{0}$ & $C_{1}$ & $C_{2}$ & $C_{3}$ \\
\hline
\end{tabular}

ready $\underset{\text { DMA }}{\longrightarrow}$

From Hardware

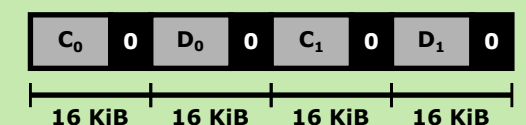

artico3_alloc( $) \longrightarrow$ Application Buffers

artico3_kernel_execute()

DMA Buffers

\begin{tabular}{|l|l|l|l|}
\hline$C_{0}$ & $C_{1}$ & $C_{2}$ & $C_{3}$ \\
\hline
\end{tabular}

\begin{tabular}{|l|l|l|l|}
\hline$D_{0}$ & $D_{1}$ & $D_{2}$ & $D_{3}$ \\
\hline
\end{tabular}
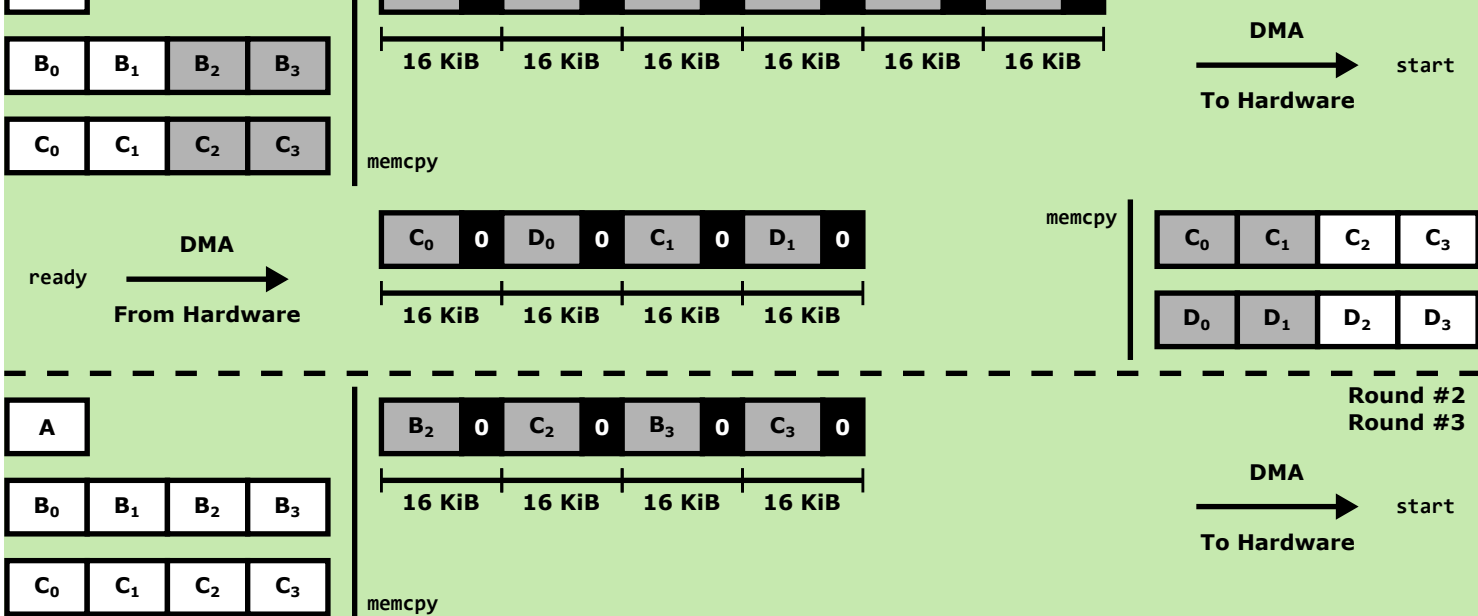

memcpy
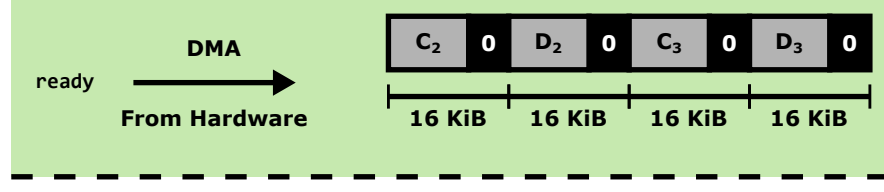

A

\begin{tabular}{|l|l|l|l|}
\hline$B_{0}$ & $B_{1}$ & $B_{2}$ & $B_{3}$ \\
\hline$C_{0}$ & $C_{1}$ & $C_{2}$ & $C_{3}$ \\
\hline
\end{tabular}

artico3_kernel_wait()

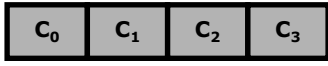

\begin{tabular}{|l|l|l|l|}
\hline$D_{0}$ & $D_{1}$ & $D_{2}$ & $D_{3}$ \\
\hline
\end{tabular}

Figure 2-20: Kernel scheduling in ARTICo ${ }^{3}$. 


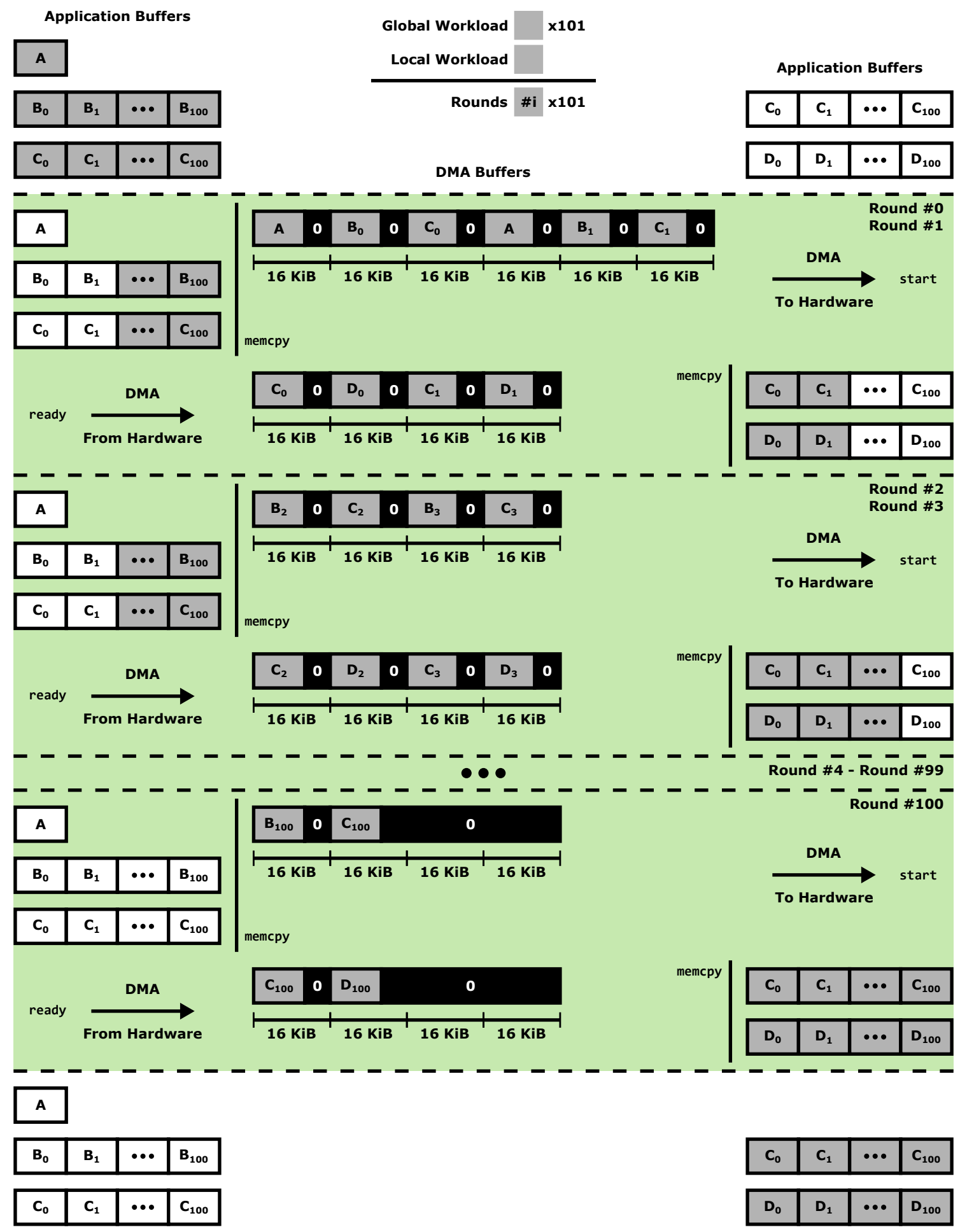

Figure 2-21: Unpaired accelerator/round execution. 
Original contribution 2-11 A DMA-friendly memory model and scheduling scheme for hardware accelerators that supports transparent multi-round execution in data-parallel scenarios.

\subsubsection{Kernel Modeling}

The ARTICo ${ }^{3}$ execution model shown in Subsection 2.5.1 depicts an ideal situation where kernel rounds show no idle processing times between execution and read transactions. This scenario is not always true, even if the round sequencer is configured to issue read transactions when the first accelerator asserts its ready signal, and the time it takes for the runtime library to realize that accelerators have finished execution and can be read (see Figure 2-14) is neglected. Actually, two different profiles can be identified for ARTICo $^{3}$ kernels based on the processing/transfer ratio: computing-bounded execution and memory-bounded execution.

- Computing-bounded execution: execution profile in kernels where accelerator processing takes significantly longer than data transfers. In this profile, the DMA engine becomes idle during accelerator execution.

- Memory-bounded execution: execution profile in kernels where accelerator processing takes less time than data transfers. In this profile, accelerators become idle during data transfers, and the DMA engine is always busy.

Ideally, kernels should show a computing-bounded profile in their round sequencing process, since the under-use of the DMA engine favors the execution of several kernels in parallel. Figure 2-22 shows the power consumption of the FPGA core and the external memory interface during one execution round of a single kernel in both memory- (top) and computing-bounded (bottom) profiles. Notice that these scenarios assume parallel operation mode and no hardware redundancy in the architecture, read transactions triggered by the first assertion of a ready signal in any accelerator (i.e., the amount of input data equals the amount of output data, and all kernel instances have the same execution time), and no overheads between this assertion and the start of the DMA-enabled read data transfer. Although these last two considerations might be seen as a simplification of the actual profile, they are still compatible with the current kernel scheduling implementation, which would only add extra idle times between the execution and read stages in each processing round.

In ARTICo $^{3}$, power consumption during kernel execution can be modeled using parametric equations. In fact, the power consumption in the FPGA core (i.e., $P_{\text {core }}$ ) can be modeled with Equation 2-2, where $P_{\text {base }}$ is the baseline power consumption in the platform, $P_{\text {dma }}$ represents the time-dependent contribution of the DMA-enabled data transfers, $n_{\mathrm{k}}$ is the total number of kernels used in the application, and $n_{\mathrm{ex}_{i}}$ and $P_{\mathrm{ex}_{i}}$ are the number of instances of kernel $i$ and their associated power consumption at a certain point in time $t$. 

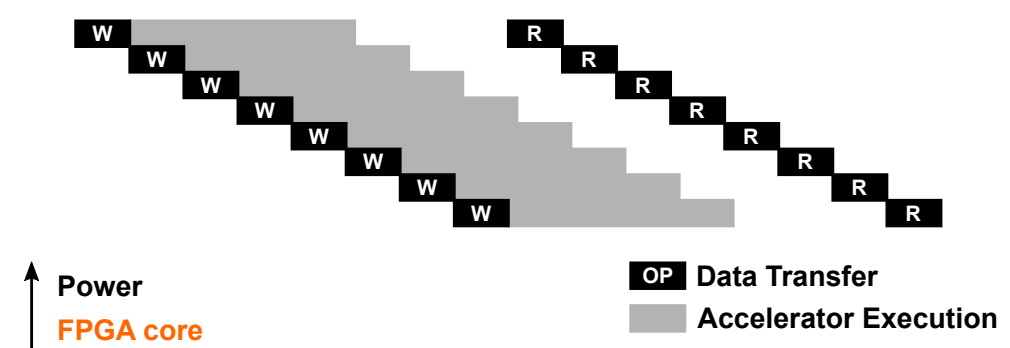

FPGA core

Accelerator Execution
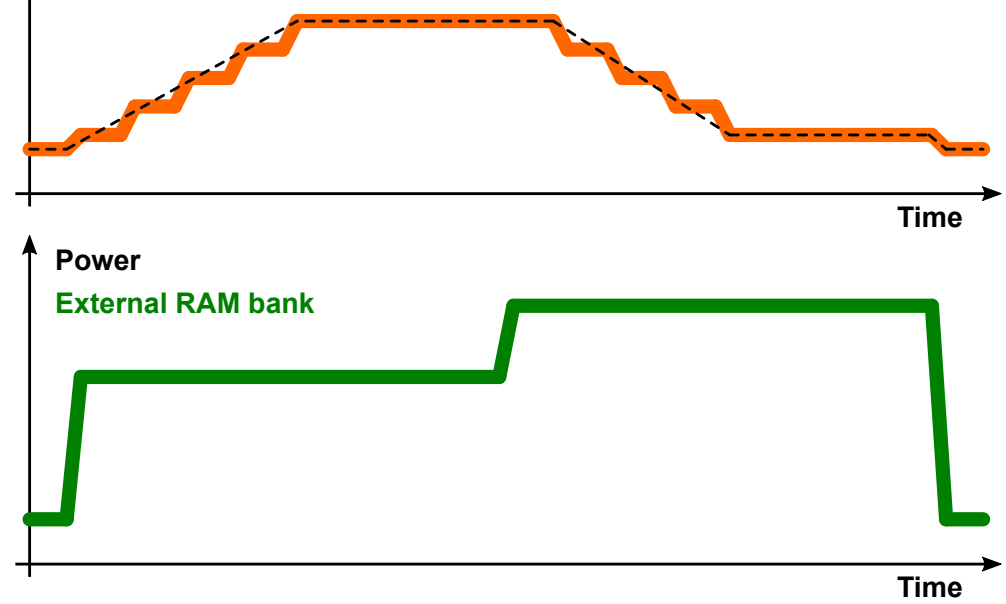

W
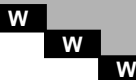

W
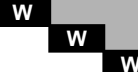

$\mathbf{R}$

$\mathbf{R}$
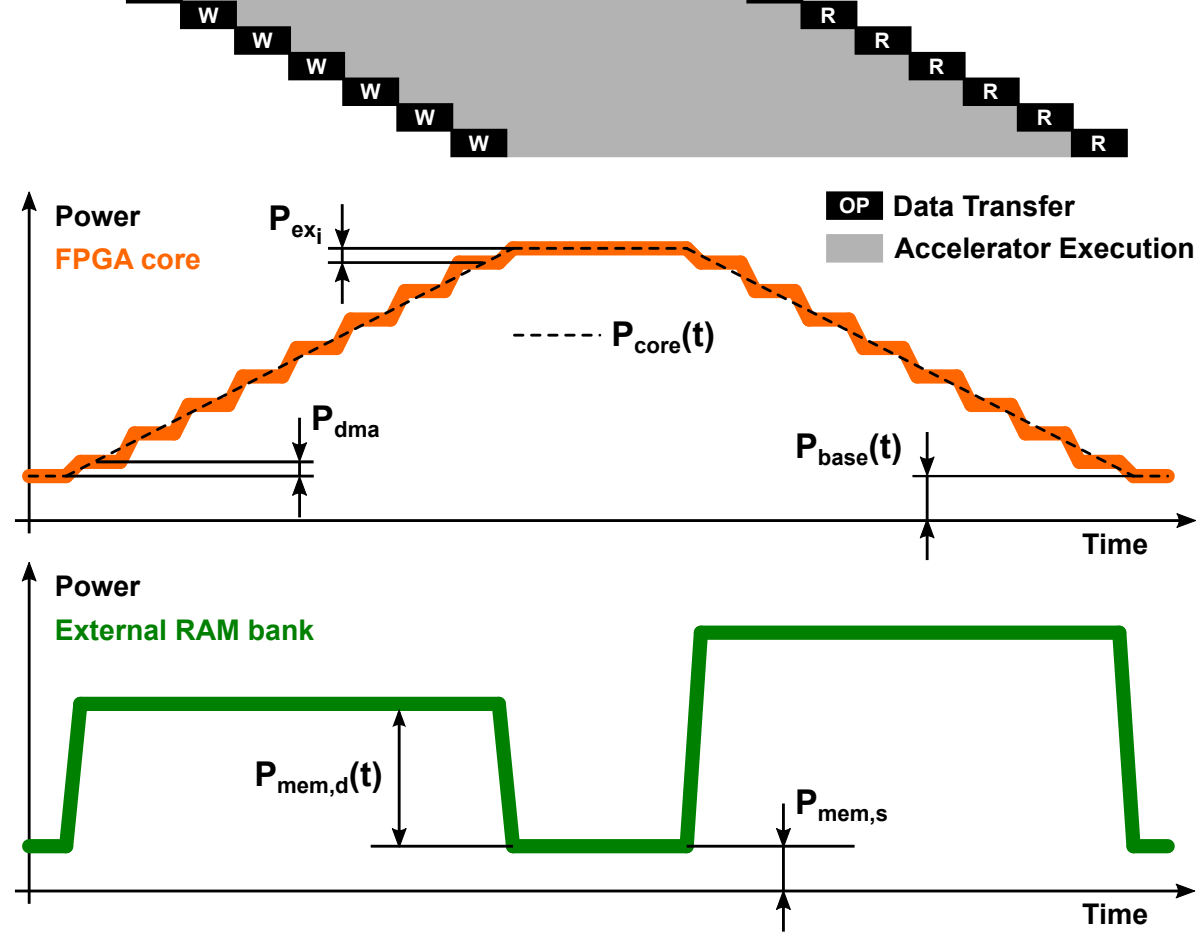

Figure 2-22: Kernel execution modeling in ARTICo ${ }^{3}$. 


$$
P_{\text {core }}(t)=P_{\text {base }}(t)+P_{\mathrm{dma}}(t)+\sum_{i=1}^{n_{\mathrm{k}}} n_{\mathrm{ex}_{i}}(t) \cdot P_{\mathrm{ex}_{i}}(t)
$$

$P_{\text {base }}$ can be computed using Equation 2-3, where $P_{\text {static }}$ is the time-dependent contribution of the static system, and $n_{i}$ and $P_{i}$ are the number of instances of kernel $i$ loaded in the reconfigurable slots and the power consumption of one of these instances in idle state at a certain instant $t$.

$$
P_{\text {base }}(t)=P_{\text {static }}(t)+\sum_{i=1}^{n_{\mathrm{k}}} n_{i}(t) \cdot P_{i}(t)
$$

Power consumption in the interface with the external memory (i.e., $P_{\text {mem }}$ ) can be modeled with Equation 2-4, where $P_{\text {mem,s }}$ is the time-dependent contribution in idle state, and $P_{\text {mem,d }}$ the time-dependent contribution during kernel execution.

$$
P_{\text {mem }}(t)=P_{\mathrm{mem}, \mathrm{s}}(t)+P_{\mathrm{mem}, \mathrm{d}}(t)
$$

Finally, the total power consumption in the platform can be approximated by the combination of these two power rails using Equation 2-5.

$$
P(t)=P_{\text {core }}(t)+P_{\text {mem }}(t)
$$

Up to this point, the power consumption model has only considered the parallel operation mode. Figure 2-23 shows how adding hardware redundancy, and therefore using the redundant operation mode in $\mathrm{ARTICo}^{3}$, affects kernel execution profiles. The reference profile corresponds to the Simplex mode and is shown on the left. Hardware-redundant profiles with DMR and TMR modes are shown in the center and on the right, respectively. As it can be seen, assuming that all accelerators have been previously loaded in the reconfigurable slots (i.e., $P_{\text {base }}$ does not change), using hardware redundancy only affects the $n_{\mathrm{ex}_{i}}$ parameter.

The kernel execution profile can be also altered when using hardware accelerators with more than one datapath executing in parallel (i.e., accelerators with several parallel threads). This scenario can be seen in Figure 2-24, where the reference profile using accelerators with one datapath is shown on the left. In addition, setups with two and three parallel datapaths inside each hardware accelerator are shown in the center and on the right, respectively. As it can be seen, potential energy savings are enabled thanks to the reduction in terms of execution time, even if power consumption is increased anyway.

The proposed model has been validated using actual kernel executions on different development boards. Power traces have been acquired using the power monitors in $\mathrm{ARTICo}^{3}$, and validated using external oscilloscopes. Figure 2-25 shows the power traces of a memory-bounded kernel (top) and a computing-bounded kernel (bottom) 


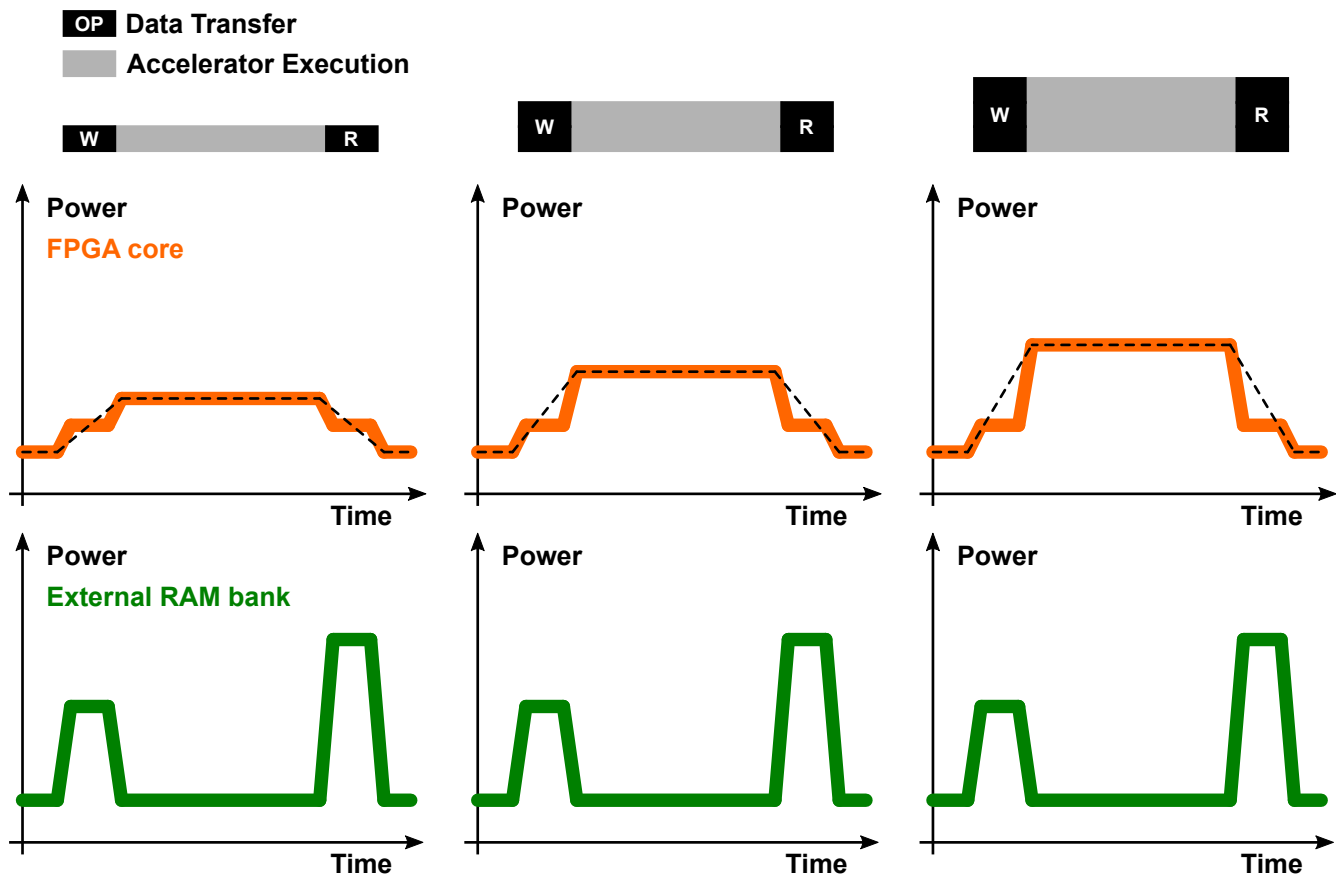

Figure 2-23: Kernel execution modeling in $\mathrm{ARTICo}^{3}$ with hardware redundancy.

OP Data Transfer

Accelerator Execution
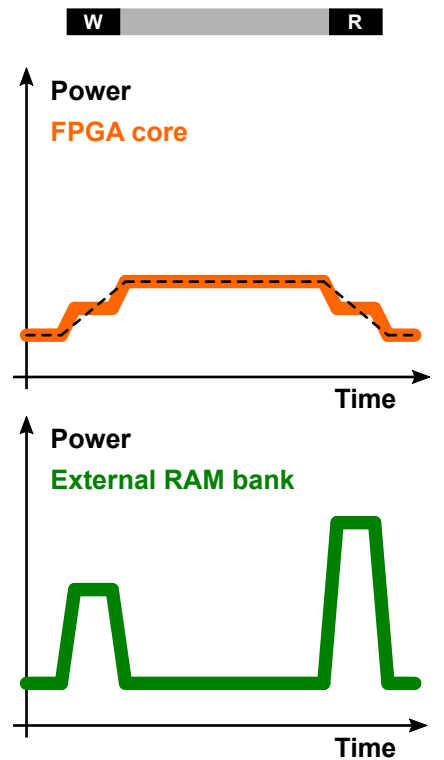

w
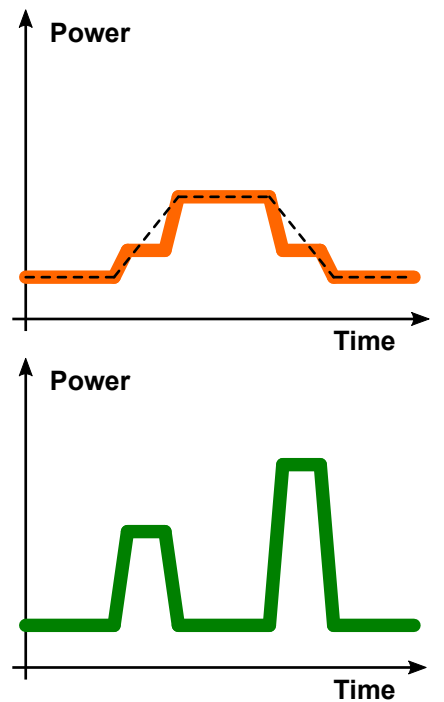
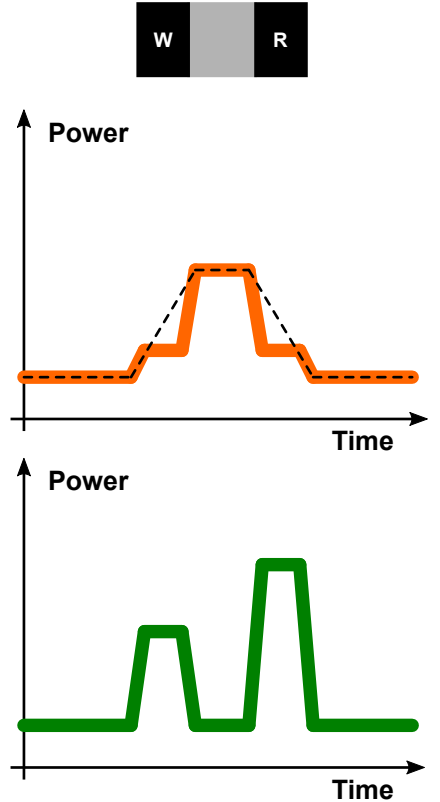

Figure 2-24: Kernel execution modeling in $\mathrm{ARTICo}^{3}$ with hardware multithreading. 
when executed on a HiReCookie WSN node [Valverde'12] with a Spartan-6 FPGA. ${ }^{33}$ Figure 2-26, on the other hand, shows the power traces of a computing-bounded kernel with one (top) or two (bottom) parallel datapaths inside each accelerator executed on a KC705 development board with a Kintex-7 FPGA. ${ }^{34}$
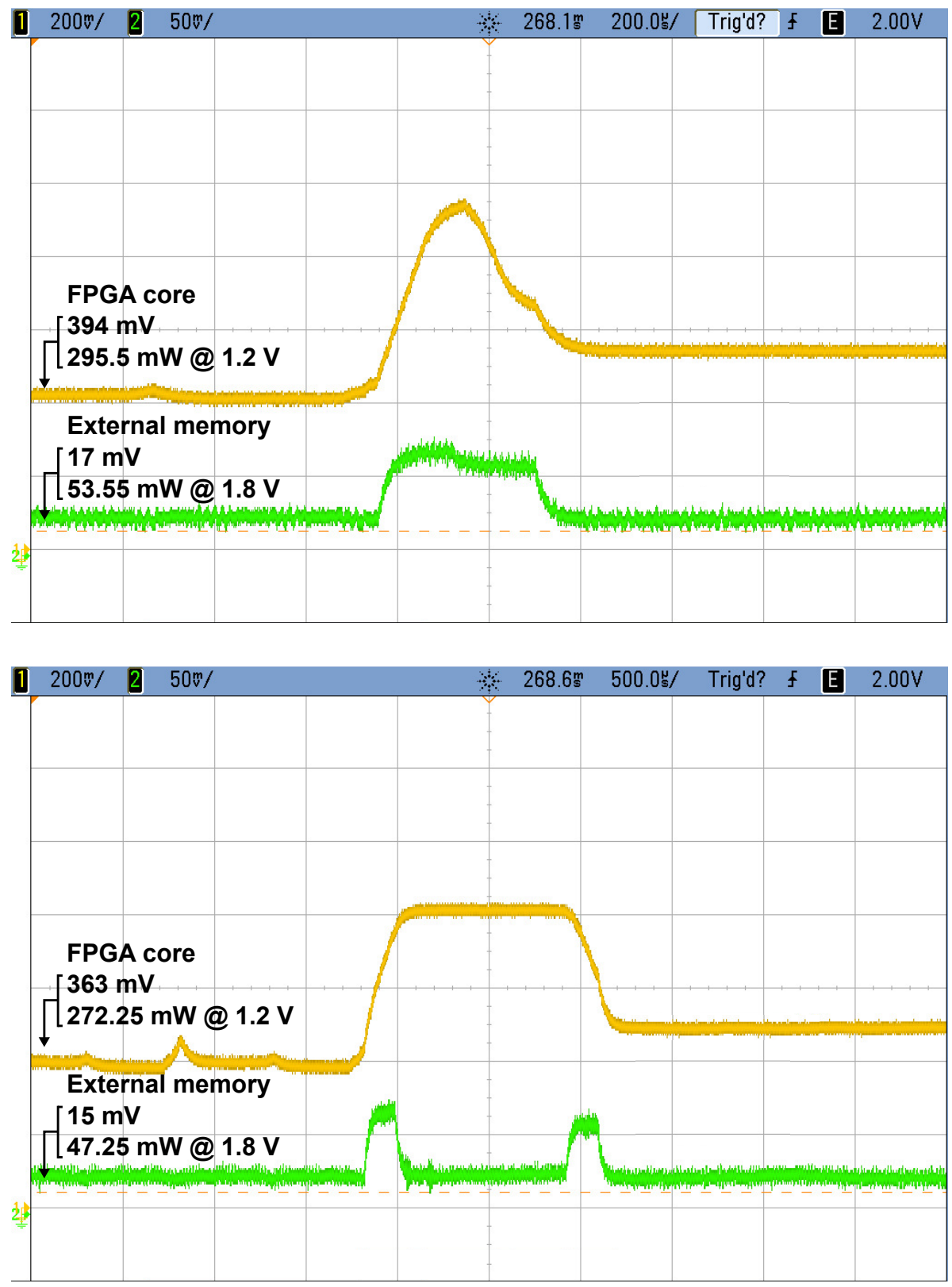

Figure 2-25: Power consumption traces in Spartan-6.

In the validation process, an AES-256 block cipher operating in Counter (CTR) mode has been used. Power traces have been collected using the available power PMCs

\footnotetext{
${ }^{33}$ XC6SLX150-2FGG484.

${ }^{34}$ XC7K325T-2FFG900.
} 

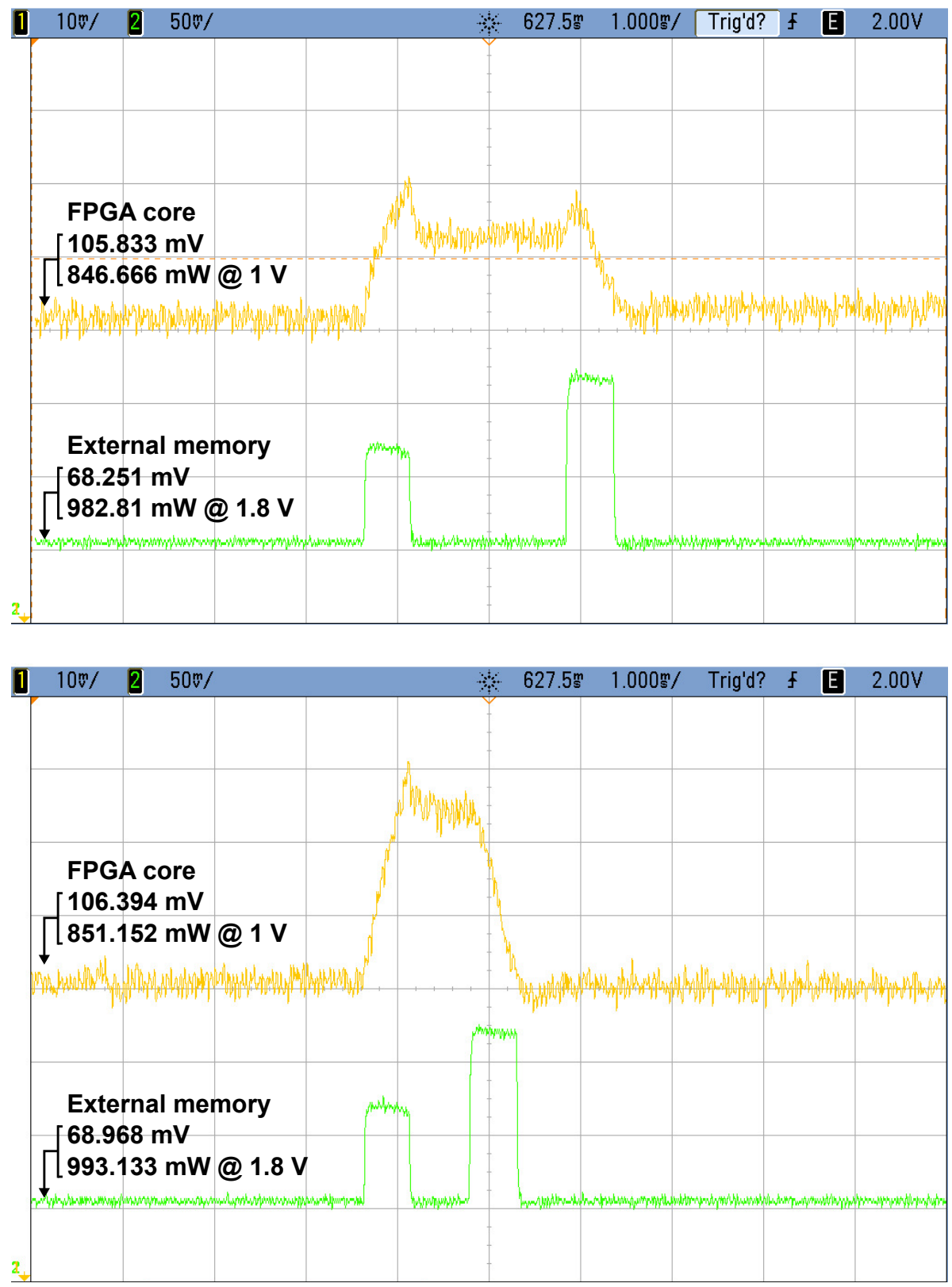

Figure 2-26: Power consumption traces in Kintex-7.

in ARTICo ${ }^{3}$. Table 2-10 shows the numeric values of the model parameters for each evaluation platform. Notice that all the parameters can be approximated to constant values, since there are no significant variations in the power consumption traces (this has been also confirmed using the external oscilloscope measurements).

The accuracy of the proposed power consumption model has been assessed using the correlation between the estimations/predictions obtained from it and the actual power traces. Table 2-11 shows these correlation results. Notice that the model provides significantly worse results when performing estimations/predictions in a 1accelerator scenario, due to the minimal contribution of a single accelerator to the total 
Table 2-10: Model parameters for an AES-256 CTR kernel

\begin{tabular}{ccc}
\hline Parameter & \multicolumn{2}{c}{ Value (mW) } \\
& KC705 & HiReCookie \\
\hline$P_{\text {static }}$ & 637.45 & 308.97 \\
$P_{\text {dma }}$ & 6.93 & 5 \\
$P_{i}$ & 38.66 & 44.55 \\
$P_{\text {ex }_{\mathrm{i}}}$ & 31.57 & 22.21 \\
$P_{\text {mem,s }}$ & 792 & 91.6 \\
$P_{\text {mem,d }}$ (read) & 768 & 133.4 \\
$P_{\text {mem,d }}$ (write) & 1368 & 101.25 \\
\hline
\end{tabular}

power consumption. However, results show a minimum 95\% accuracy when using at least 3 hardware accelerators.

Table 2-11: Model analysis for an AES-256 CTR kernel

\begin{tabular}{ccc}
\hline Accelerators & \multicolumn{2}{c}{$\begin{array}{c}\text { Correlation } \\
\text { KC705 }\end{array}$} \\
& HiReCookie \\
\hline 1 & 0.7177 & 0.4990 \\
2 & 0.9174 & 0.8971 \\
3 & 0.9644 & 0.9453 \\
4 & 0.9795 & 0.9628 \\
5 & 0.9790 & - \\
6 & 0.9860 & - \\
\hline
\end{tabular}

Figure 2-27 shows the graphical comparison between the proposed power model and actual measurements made with the $\mathrm{ARTICo}^{3}$ power PMCs in the KC705 development board. The global workload is modified according to the number of hardware accelerators, so that all of them process one round and the total execution time remains the same.

Figure 2-28 shows a full view of some of the previous scenarios, where it is possible to see that the power consumption in the static system (i.e., where $\mathrm{ARTICo}^{3}$ is not being used) is significantly larger than the individual contributions of the hardware accelerators. Notice that the use of additional accelerator instances for processing leads to more energy-efficient solutions, even though the overall power consumption is increased.

Finally, Figure 2-29 shows that the model is still accurate even if slight deviations are present in the baseline power consumption (i.e., $\left.P_{\text {base}}\right)$. However, the run-time profiling capabilities in the ARTICo ${ }^{3}$ architecture can be used to dynamically update and/or correct these deviations. Moreover, the proposed model is lightweight enough to provide online estimations/predictions (i.e., during system execution).

Original contribution 2-12 A lightweight and parametric power consumption model for multi-accelerator execution that can be embedded on the target platform to perform run-time estimations and predictions. 


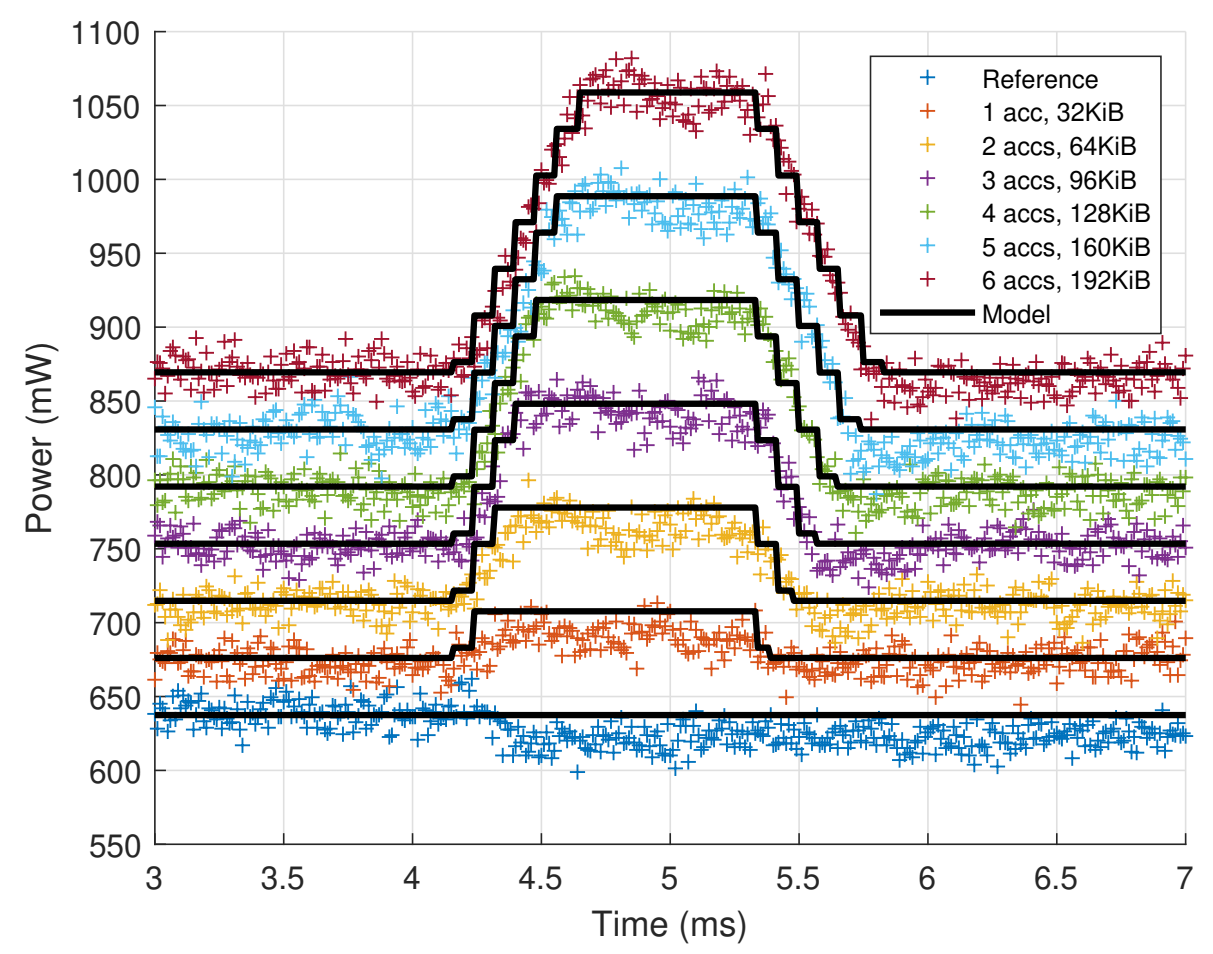

Figure 2-27: Kernel model evaluation with multiple accelerators.

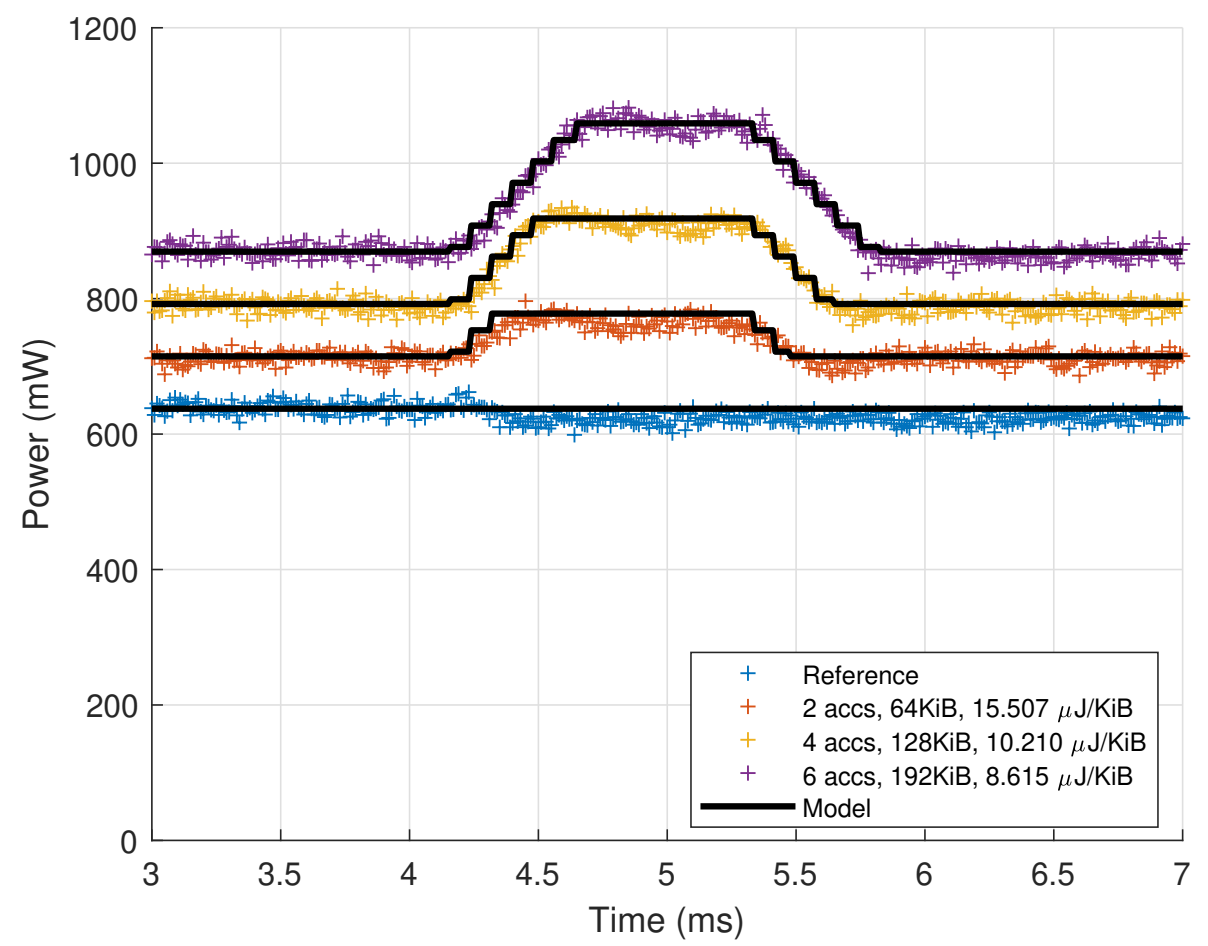

Figure 2-28: Static versus dynamic consumption in kernel models. 


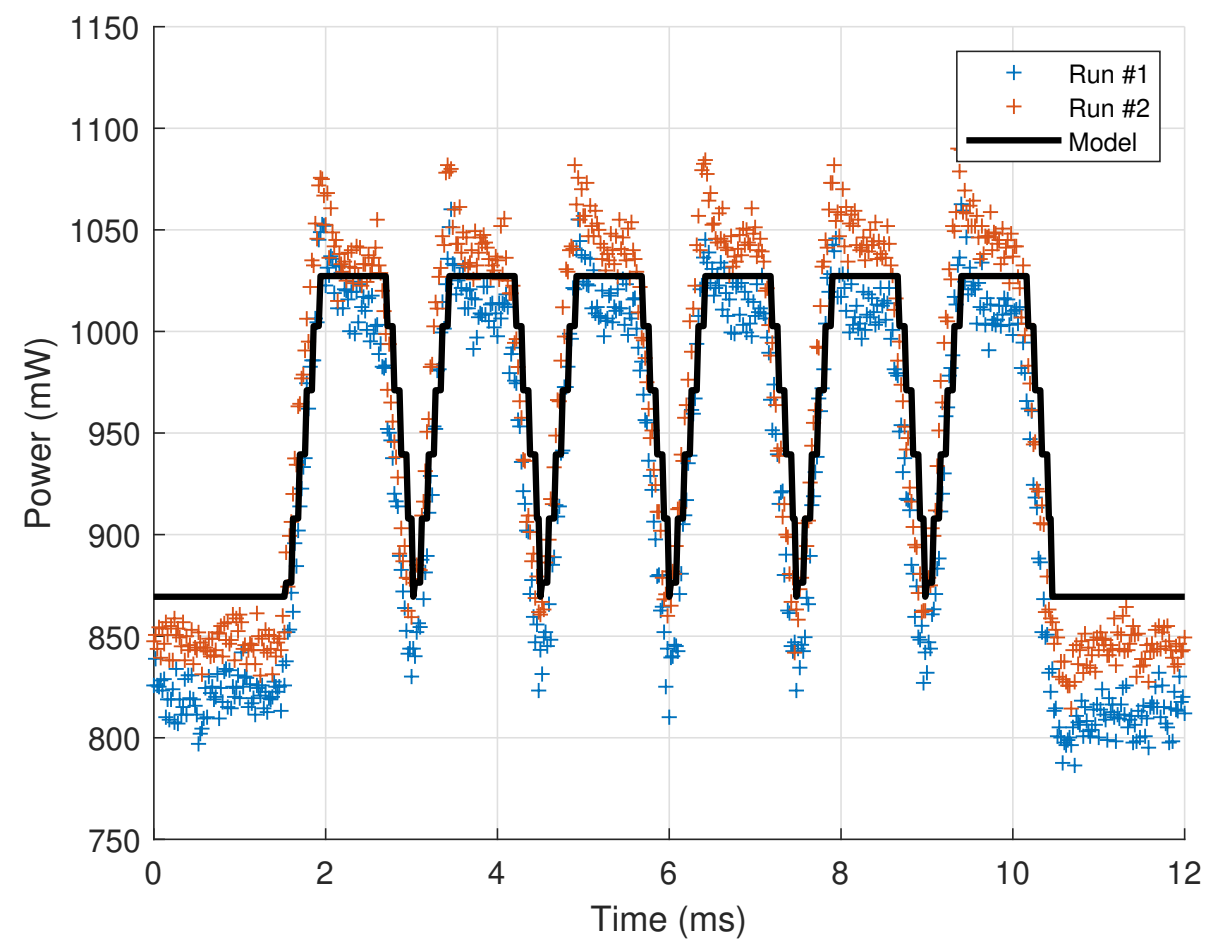

Figure 2-29: Kernel model evaluation under different initial conditions. 


\subsection{Validation}

The framework proposed in this Thesis has been validated usign three different scenarios. First, memory- and computing-bounded algorithms are analyzed to explore the ARTICo $^{3}$ run-time solution space, which includes all the possible configurations for a given kernel. Each configuration is defined by a performance value, an energy value, and a fault tolerance level. Second, the implementation of a computingintensive kernel is compared against a software-based alternative to evaluate the benefits in terms of computing performance and energy efficiency when using $\mathrm{ARTICo}^{3}$. Reconfiguration overheads are also analyzed in this scenario. Last, the ARTICo ${ }^{3}$-based implementations of several kernels from a well-known HLS benchmark suite are evaluated, showing the benefits of using reconfigurable multiaccelerator setups instead of standalone approaches.

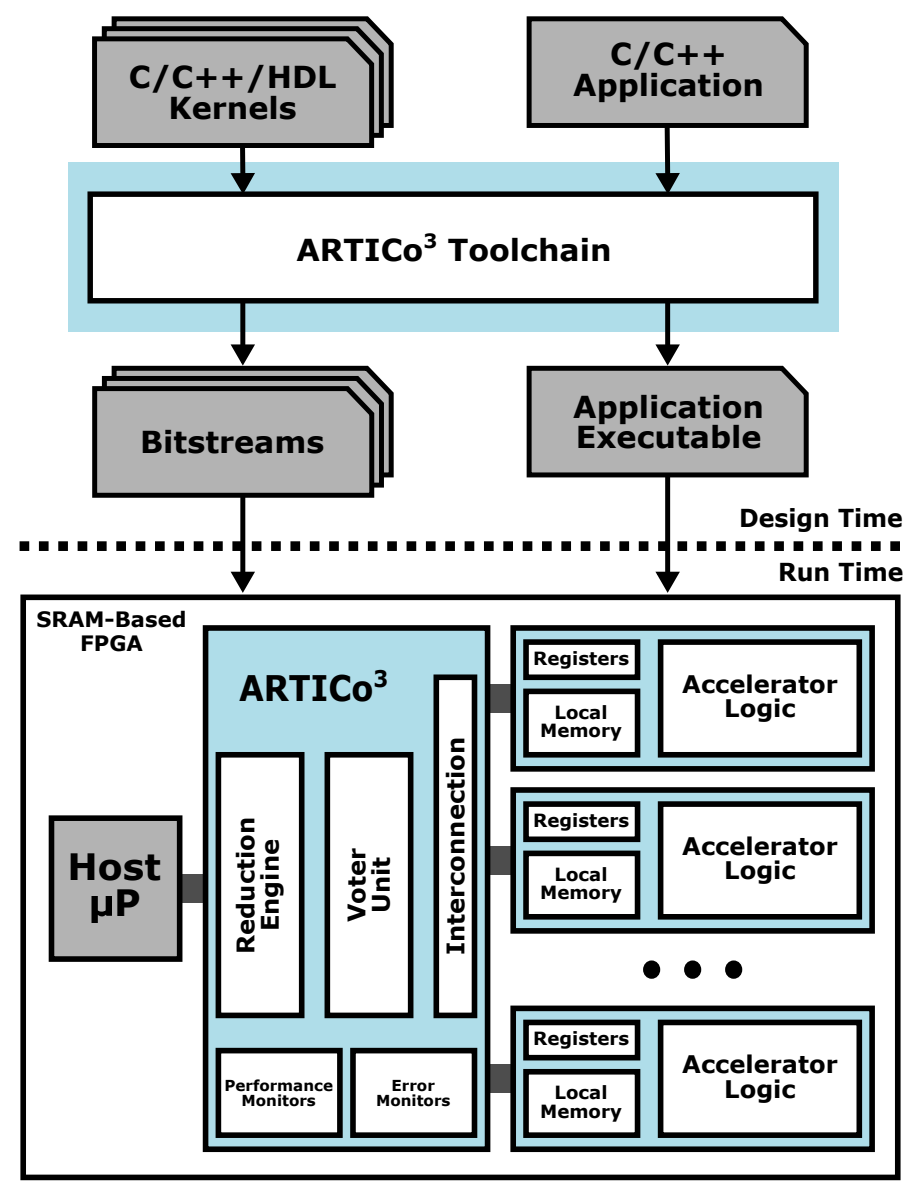

Figure 2-30: Implementation methodology for validation.

A standard implementation procedure, shown in Figure 2-30, is used in all the experimental setups: the descriptions of both application code and kernel functionality are fed to the ARTICo ${ }^{3}$ toolchain to generate the software executable and 
the FPGA bitstreams, which are used by the $\mathrm{ARTICo}^{3}$ runtime library to execute the hardware-accelerated applications on the ARTICo ${ }^{3}$ architecture.

\subsubsection{Scenario \# 1: Image Filters and AES Block Cipher}

The first validation setup uses VHDL kernels and showcases the ARTICo ${ }^{3}$ solution space, which is made from all the possible combinations of execution performance (i.e., execution time), energy consumption, and fault tolerance level (i.e., hardware redundancy mode). Two image filters (i.e., median and Sobel) and a block cipher (i.e., AES-256 in CTR mode, which enables parallelization during encryption) have been implemented in this scenario, since they cover both execution profiles available in ARTICo ${ }^{3}$ : image filters are memory-bounded kernels, whereas the block cipher is a computing-bounded kernel. It is worth noting that all implementations reported in this subsection have been made using the legacy flow. Moreover, an ad-hoc implementation of the runtime library that runs as a standalone application in a softcore microprocessor has been used to fully test the capabilities of the architecture.

Table 2-12 shows the resource utilization report of the different kernels when implemented on the HiReCookie WSN node [Valverde' 12] ${ }^{35}$ In this setup, the ARTICo ${ }^{3}$ architecture features 8 reconfigurable slots that can be filled with up to 8 instances of the median or Sobel kernels, or 4 instances of the AES-256 CTR kernel.

Table 2-12: Resource utilization of ARTICo ${ }^{3}$ kernels in Spartan-6 FPGAs.

\begin{tabular}{ccccc}
\hline & ARTICo $^{\mathbf{3}}$ & Median Filter & Sobel Filter & AES-256 CTR \\
\hline \multirow{2}{*}{ Info } & 8 slots & 16 KiB memory, 2 banks & 16 KiB memory, 2 banks & 32 KiB memory, 2 banks \\
& VHDL (ISE) & 8 registers & 8 registers & 8 registers \\
& Spartan-6 & VHDL (ISE) & VHDL (ISE) & VHDL (ISE) \\
& & Spartan-6 & Spartan-6 & Spartan-6 \\
\hline LUTs & 2400 & 1276 & 991 & 4855 \\
FFs & 1121 & 2975 & 2594 & 3453 \\
DSPs & 4 & - & - & - \\
BRAMs & - & 8 & 8 & 16 \\
\hline
\end{tabular}

Table 2-13, on the other hand, shows the resource utilization report of two different configurations of the AES-256 CTR kernel when implemented on a KC705 development board. ${ }^{36}$ Both configurations differ in the number of parallel datapaths available inside each kernel instance: the basic configuration uses one hardware thread per accelerator, whereas the parallel configuration uses two hardware threads per accelerator. In this scenario, the ARTICo ${ }^{3}$ architecture has 6 reconfigurable slots that can be filled with up to 6 kernel instances.

In order to implement two parallel datapaths inside an AES-256 CTR hardware accelerator, the partitioning of the local memory has been modified according to

\footnotetext{
${ }^{35}$ XC6S150-2FGG484.

${ }^{36}$ XC7K325T-2FFG900C.
} 
Table 2-13: Resource utilization of ARTICo ${ }^{3}$ kernels in Kintex-7 FPGAs.

\begin{tabular}{|c|c|c|c|}
\hline & ARTICo $^{3}$ & \multicolumn{2}{|c|}{ AES-256 CTR } \\
\hline \multirow{5}{*}{ Info } & & \multicolumn{2}{|c|}{$64 \mathrm{KiB}$ memory, 2 banks $64 \mathrm{KiB}$ memory, 4 banks } \\
\hline & 6 slots & 8 registers & 8 registers \\
\hline & VHDL (ISE) & VHDL (ISE) & VHDL (ISE) \\
\hline & Kintex-7 & Kintex-7 & Kintex-7 \\
\hline & & 1 thread & 2 threads \\
\hline LUTs & 2839 & 4388 & 8154 \\
\hline FFs & 1127 & 3456 & 6280 \\
\hline DSPs & 4 & - & - \\
\hline BRAMs & - & 16 & 16 \\
\hline
\end{tabular}

the scheme shown in Figure 2-31. Note that, although some address bits have been rearranged before driving port $\mathrm{A}$, the local memory map in each hardware accelerator is not altered from the point of view of the static system (i.e., it is still continuous). However, the number of parallel memory banks has been doubled to support additional input and output data streams for the second parallel thread inside the kernel instance. In addition, the configuration of the CTR core has been also adapted to fully support parallel encryption.

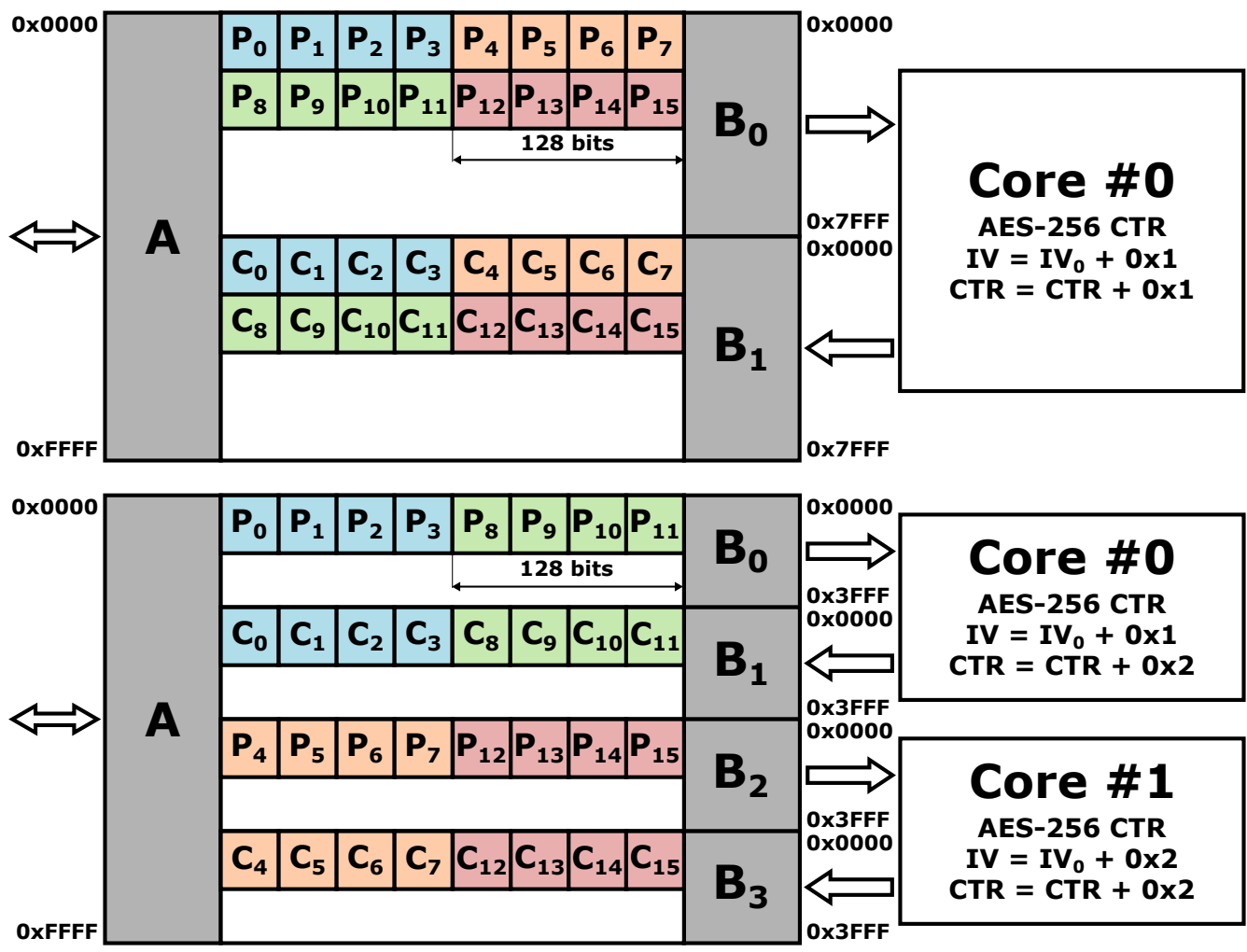

Figure 2-31: Memory bank partitioning in single versus multithreaded accelerators.

The solution space of the kernels implemented in the HiReCookie node [Valverde'12] is shown in Figure 2-32. As it can be seen, both image filters show operating points that 
are either computing- or memory-bounded. Performance scales almost linearly for the former (e.g., using 1 - 3 kernel instances), but gets stalled in the latter (e.g., using 4 8 kernel instances). Nevertheless, the prevailing behavior for these kernels is to have memory-bounded execution. The block cipher kernel, on the other hand, presents operating points that are always computing-bounded and thus, performance scales almost linearly regardless of the number of kernel instances. Notice that part of the graphs include dashed lines. These correspond to unfeasible solutions (i.e., scenarios where FPGA cannot host the required number of hardware accelerators) that have been estimated using the kernel modeling approach described in Subsection 2.5.4.
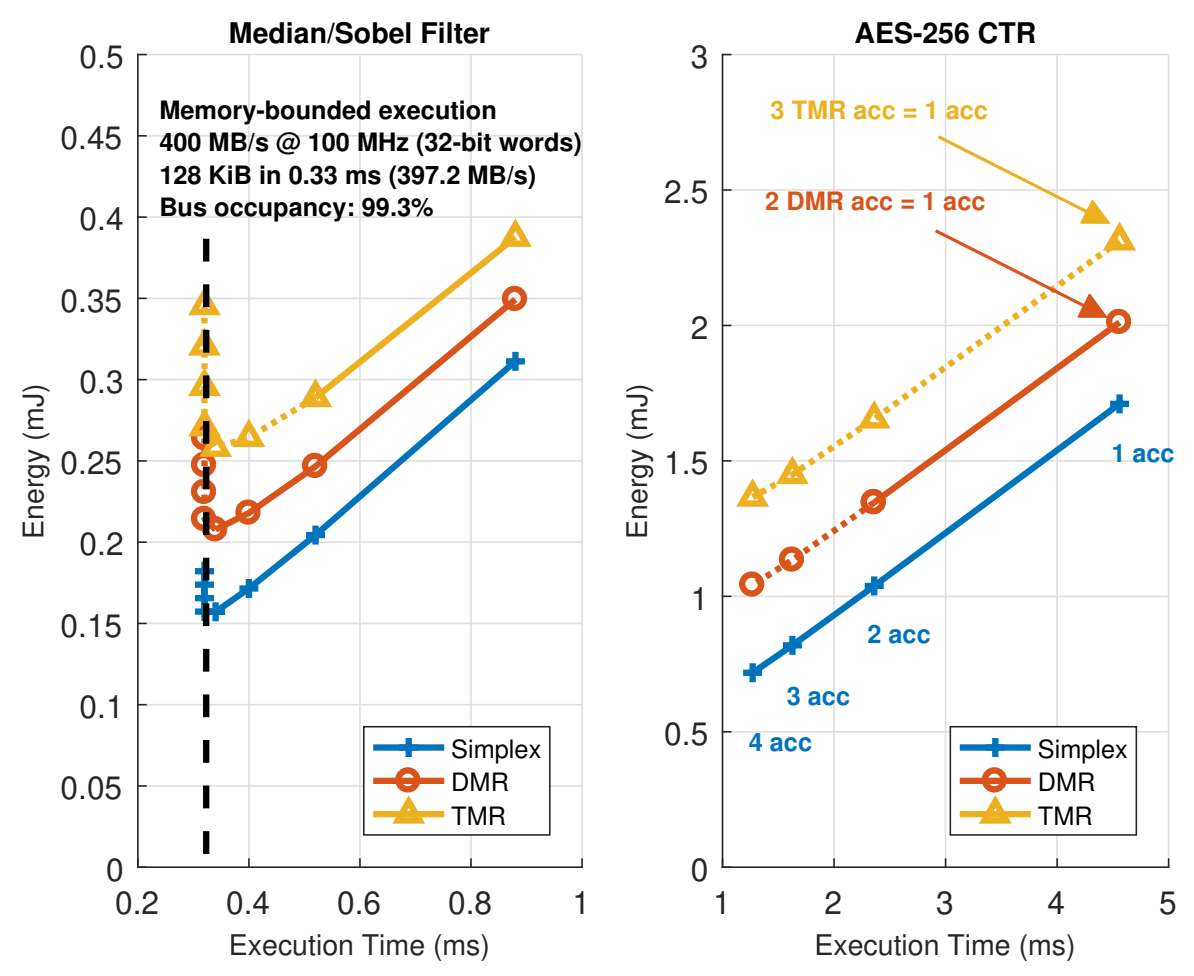

Figure 2-32: Solution space exploration in the HiReCookie node [Valverde'12].

The solution space of the AES-256 CTR kernel in the KC705 development board is shown in Figure 2-33. All the operating points of both configurations (i.e., one or two parallel threads inside each accelerator) are superimposed on the same graph. Results show that using two datapaths in one accelerator renders almost the same result as using two accelerators with a single datapath, with the former providing slightly less computing performance and the latter being slightly less energy efficient.

As opposed to the setups targeting Spartan-6 FPGAs, where all the operating points with Simplex configuration were feasible solutions and estimations were only provided for some configurations with hardware redundancy, the solution space in Kintex-7 FPGAs also includes unfeasible solutions in Simplex configuration to fully analyze the transition between computing- and memory-bounded execution in ARTICo ${ }^{3}$ kernels. This analysis is shown for both image filters and the block cipher in Figure 2-34, 
where dashed lines are used again to represent solutions that cannot fit in the FPGA. The graphs also show the bandwidth limit during memory-bounded execution, with ARTICo $^{3}$ rendering an almost perfect bus utilization ratio (i.e., above $99 \%$ for both Spartan-6 and Kintex-7 devices).

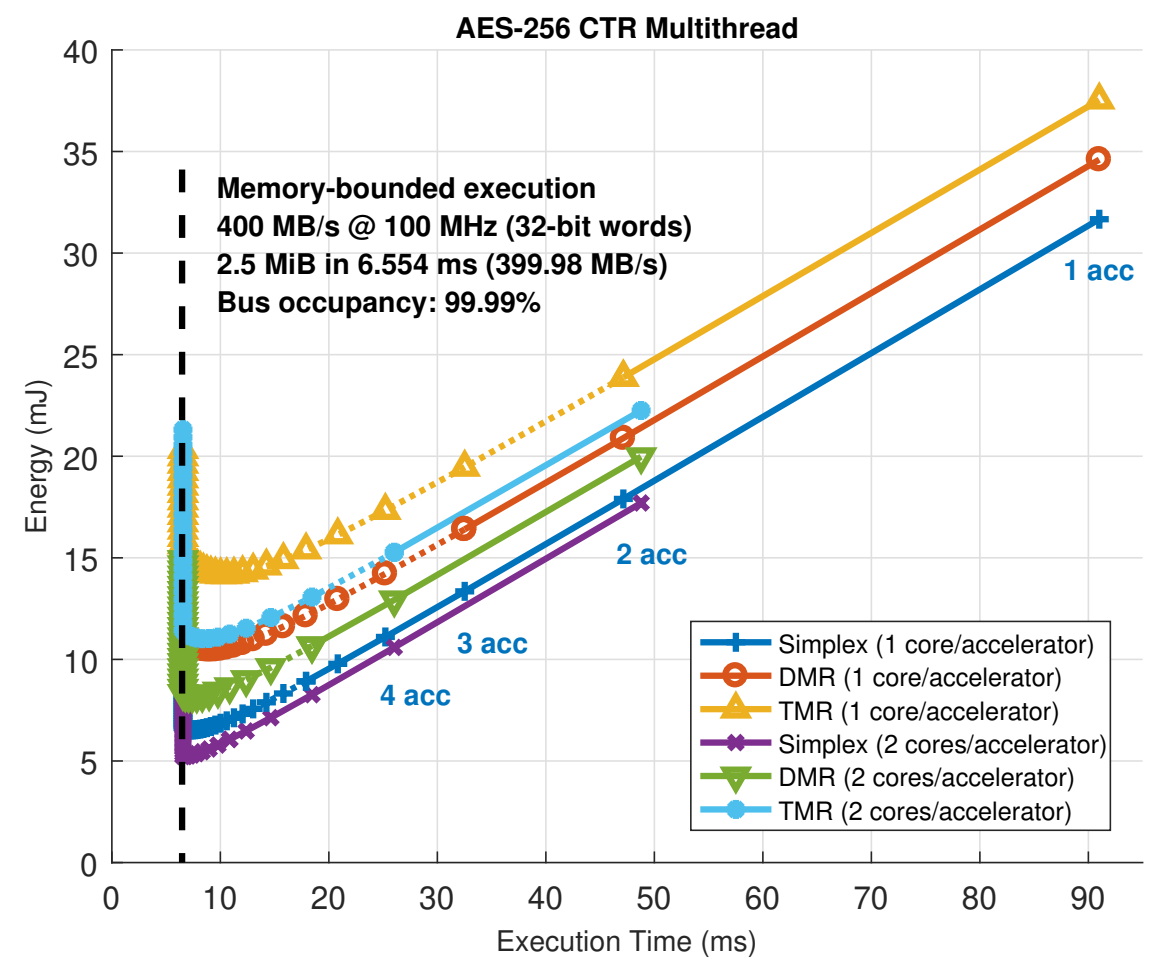

Figure 2-33: Solution space exploration in the KC705 development board.

\subsubsection{Scenario \#2: Matrix Multiplication}

The second validation setup evaluates a high-performance embedded computing scenario using a C-based kernel and HLS. In this case, the target application involves matrix multiplication, a computing-intensive task that is widely used as a reference for benchmarking parallel computing architectures. ${ }^{37}$ In fact, this second validation setup presents the benefits of using ARTICo ${ }^{3}$ as a high-performance embedded computing architecture instead of a more traditional software-based approach.

In order to fully exploit parallelism at both algorithm and accelerator levels, a nested block-based multiplication procedure with 2 identical layers has been implemented. This approach is used not only to support data-level paralellism with

\footnotetext{
${ }^{37}$ Matrix multiplication is a common operation in Dense Linear Algebra, which is known to be one of the Berkeley Dwarfs [Asanović'06]. A dwarf is an algorithm that captures a specific pattern of computation and communication and thus, its use is highly recommended when designing and evaluating parallel programming models and architectures.
} 

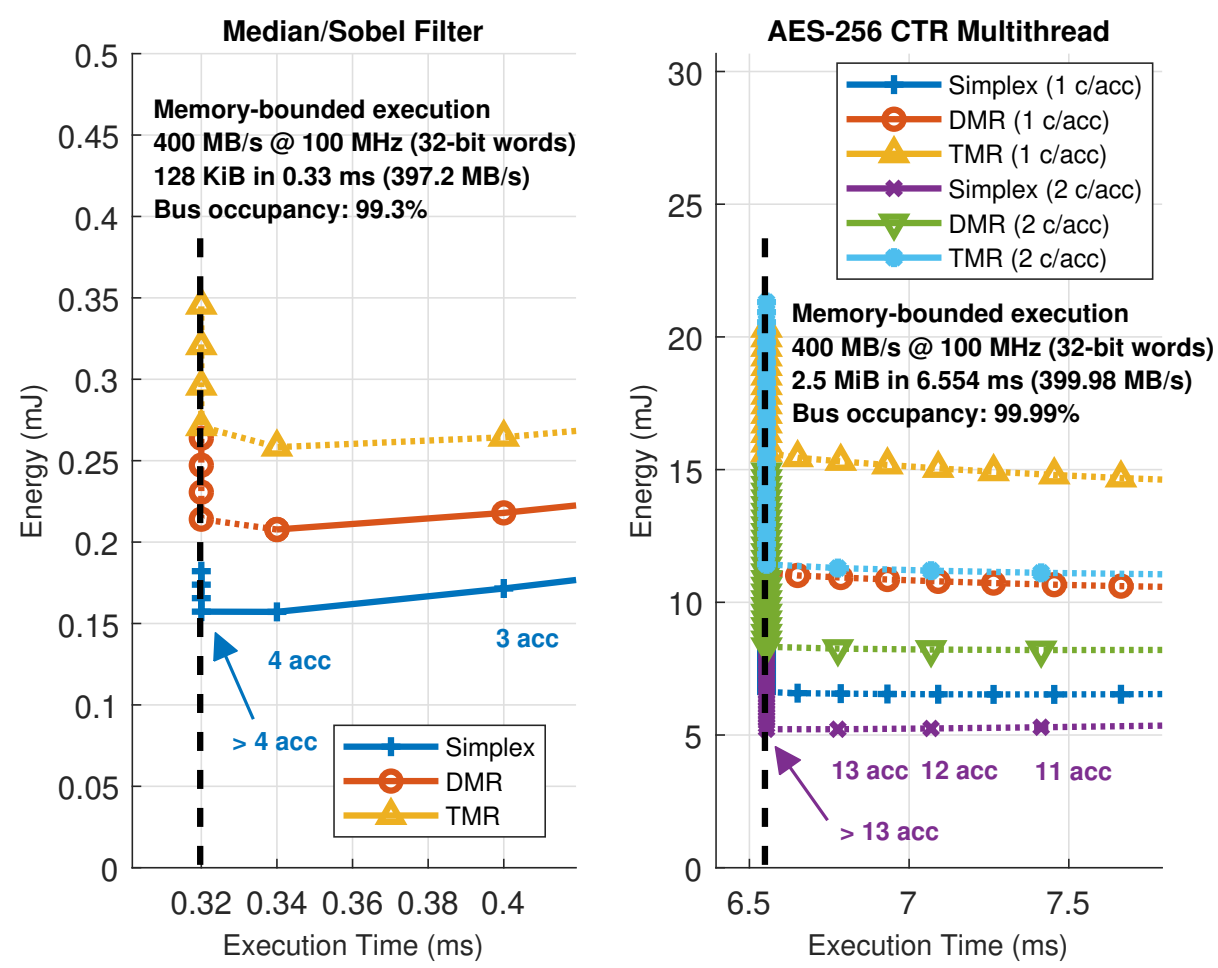

Figure 2-34: Transition from computing- to memory-bounded execution.

multiple accelerators, but also to enable mixed data-parallel and pipelined execution within each kernel instance. Figure 2-35 shows one layer of the block-based matrix multiplication algorithm used in both the software application and inside hardware accelerators.

The ARTICo ${ }^{3}$ kernel for matrix multiplication takes matrices of $64 \times 6432$-bit floating point values as inputs, and multiplies them using the aforementioned blockbased algorithm with a block size of $8 \times 832$-bit floating point values. The C-based description used to generate the accelerators with HLS has been annotated with pipelining, loop unrolling, and array partitioning optimization directives to maximize the performance/area ratio. Table 2-14 contains the resource utilization report of the matrix multiplication scenario when implemented on an in-house custom board that features a Zynq-7000 SoC. ${ }^{38}$

Apart from this first block-based scheme in the hardware accelerator, a second block-based multiplication layer has been also implemented in the software side. In this case, the application takes matrices of $512 \times 51232$-bit floating point values as inputs, and multiplies them using the same block-based algorithm with a block size of $64 \times 6432$-bit floating point values. In particular, each algorithm iteration on the

\footnotetext{
${ }^{38}$ XC7Z020-1CLG484.
} 


\section{$\mathbf{A} \times \mathbf{B}=\mathbf{R}$}
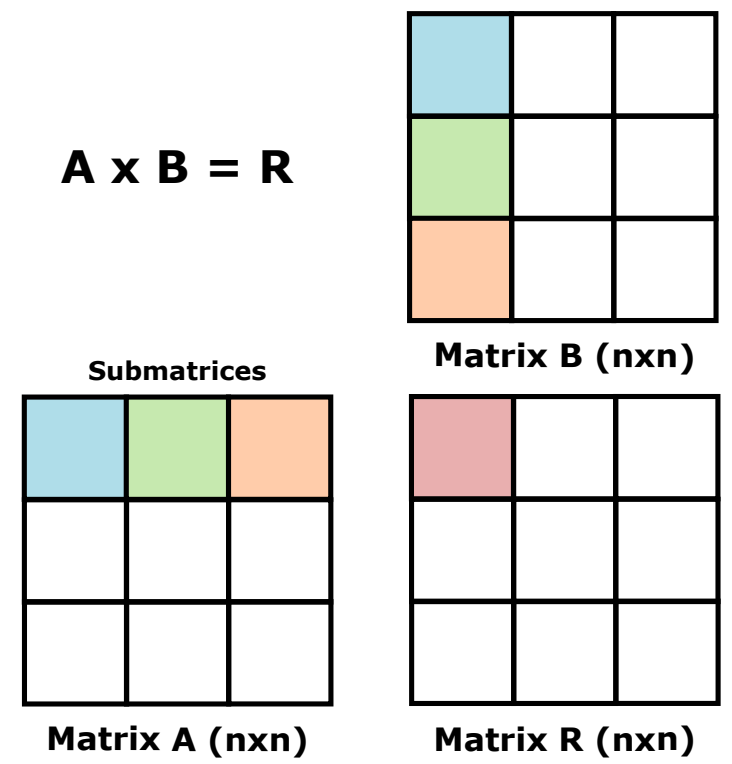

Matrix $B(\mathbf{n x n})$
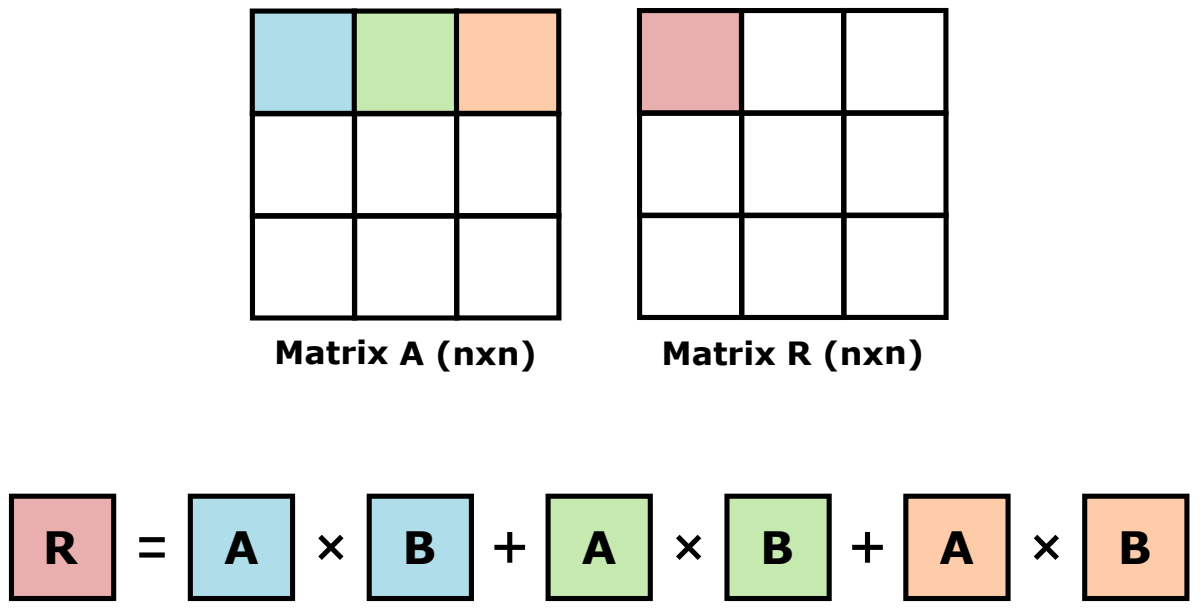

Figure 2-35: Block-based matrix multiplication.

Table 2-14: Resource utilization of ARTICo ${ }^{3}$ kernels in Zynq-7000 FPGAs.

\begin{tabular}{ccc}
\hline & ARTICo $^{\mathbf{3}}$ & Matrix Multiplier \\
\hline \multirow{2}{*}{ Info } & 4 slots & VHDL KiB memory, 3 banks \\
& Zynq-7000 & 0 registers \\
& & C + HLS (Vivado) \\
Zynq-7000
\end{tabular}

software side offloads 8 matrix multiplications onto the ARTICo ${ }^{3}$ computing fabric, ${ }^{39}$ and then gathers all the data-independent results to perform the accumulation operation in reduction-based fashion. Hence, several instances of the previously described matrix multiplication kernel can be used to adapt the loop unrolling depth at run time and thus, to exploit data-level parallelism in the block-based row-by-column operations, ranging from fully sequential (i.e., 1 accelerator) to fully parallel (i.e., 8 accelerators) execution.

The proposed algorithm has been evaluated in hardware, using a single-core

\footnotetext{
${ }^{39}$ The size of the shared buffers is $512 \times 6432$-bit floating point values, since they are used to store all the $64 \times 64$ blocks per required dimension (rows for the first input matrix, columns for the second).
} 
software-based implementation of the naive matrix multiplication as the baseline for comparisons. The experimental setup is based on a custom Zynq-7000 board that runs a Linux-based OS (dual core ARM Cortex-A9 @ 666.67 MHz, FPGA fabric @ $100 \mathrm{MHz}$ ). Table 2-15 shows the performance results in this scenario, including reconfiguration times, processing times, and overall speedup when compared with the baseline implementation.

Table 2-15: Performance metrics in the matrix multiplication scenario.

\begin{tabular}{ccccc}
\hline Implementation & Accelerators & Reconfiguration Time (ms) & Processing Time (s) & Speedup \\
\hline Software & - & - & 11.51 & 1 \\
\hline \multirow{2}{*}{ ARTICo $^{3}$} & 1 & 16.63 & 2.22 & 5.18 \\
& 2 & 35.15 & 1.31 & 8.79 \\
& 3 & 51.47 & 1.11 & 10.37 \\
& 4 & 66.45 & 0.86 & 13.38 \\
\hline
\end{tabular}

As it can be seen, the ARTICo ${ }^{3}$-based solution outperforms the baseline software implementation even when using only one hardware accelerator. However, performance scalability is not linear (e.g., 4 accelerators render roughly $2.5 \times$ the performance of 1 accelerator). Since the processing time is always reduced when increasing the number of accelerators, it is impossible for the matrix multiplication kernel to be operating on a memory-bounded profile. Actually, there is an additional execution overhead that limits performance scalability in kernels operating on a computing-bounded profile. This overhead is due to the non-deterministic behavior of the Linux-based OS on top of which the ARTICo ${ }^{3}$ runtime library (i.e., memory management and execution round sequencing) executes.

Power traces of the different configurations have been collected using the built-in power measurement circuitry available on the custom Zynq-7000 board. Figure 2-36 shows the power consumption in both the FPGA core (including the contribution of the dual core ARM Cortex-A9 system) and the external Random-Access Memory (RAM) memory rails. This isolation enables analyses based on the power used for processing with the former, and the power used to perform memory accesses with the latter. Note that the power consumption profiles in hardware-accelerated scenarios have been tagged and divided in three different stages: static system load with full FPGA reconfiguration (1), accelerator loads with DPR (2), and kernel execution (3).

Focusing only on the processing contribution, it is clear that using ARTICo ${ }^{3}$-based solutions leads to higher power consumption than the software-based alternative, especially taking into account that additional parts of the circuit need to be loaded in the FPGA using DPR. However, the optimized DMA-enabled communication infrastructure in ARTICo $^{3}$ provides higher power savings in memory accesses, especially when compared with the element-wise access patterns of the naive matrix multiplication algorithm.

Nevertheless, the true benefits of using $\mathrm{ARTICo}^{3}$ appear when analyzing the energy consumption, i.e., when combining the power consumption and the processing times. 

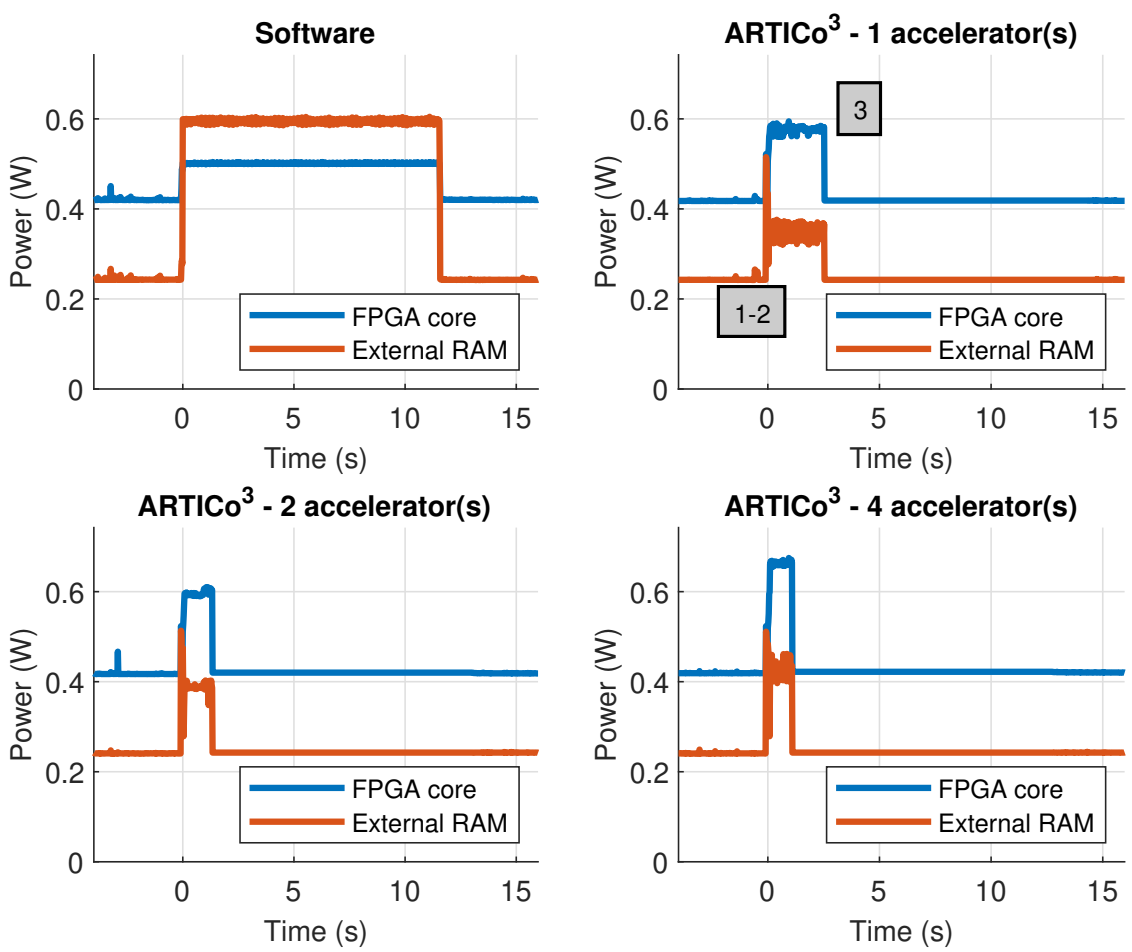

Figure 2-36: Power consumption profile in the custom Zynq-7000 board.

Figure 2-37 shows a closeup of the power trace (FPGA core + external RAM) during both full and partial reconfiguration stages, together with the energy consumption values. Note that the energy consumption due to the static system being loaded in the FPGA fabric remains almost constant for all configurations, and that DPR is slightly less energy efficient than full reconfiguration (there is a fixed overhead in the Linuxbased reconfiguration driver whenever a bitstream needs to be loaded, which is only incurred once for full FPGA reconfiguration and several times for DPR). However, it is also worth noticing that the impact of DPR in terms of energy is negligible for kernels with long execution times.

Finally, Figure 2-38 shows the power trace (FPGA core + external RAM) during the whole application execution cycle for different configurations, together with the energy consumption values. Note that the total power consumption is larger in the software-based implementation (due to its inefficient memory access patterns) than in any of the ARTICo ${ }^{3}$-accelerated ones, which also include reconfiguration overheads for completeness. As it can be seen, although the power consumption increases when the number of hardware accelerator rises, the overall processing time decreases even more significantly, leading to more energy-efficient solutions. As a result, the ARTICo ${ }^{3}$ implementation of the nested matrix multiplication algorithm is not only $13.38 \times$ better in terms of performance, but also $9.71 \times$ better in terms of energy efficiency than the single-core software-based naive matrix multiplication algorithm.

The code of the nested block-based matrix multiplication algorithm is also open 

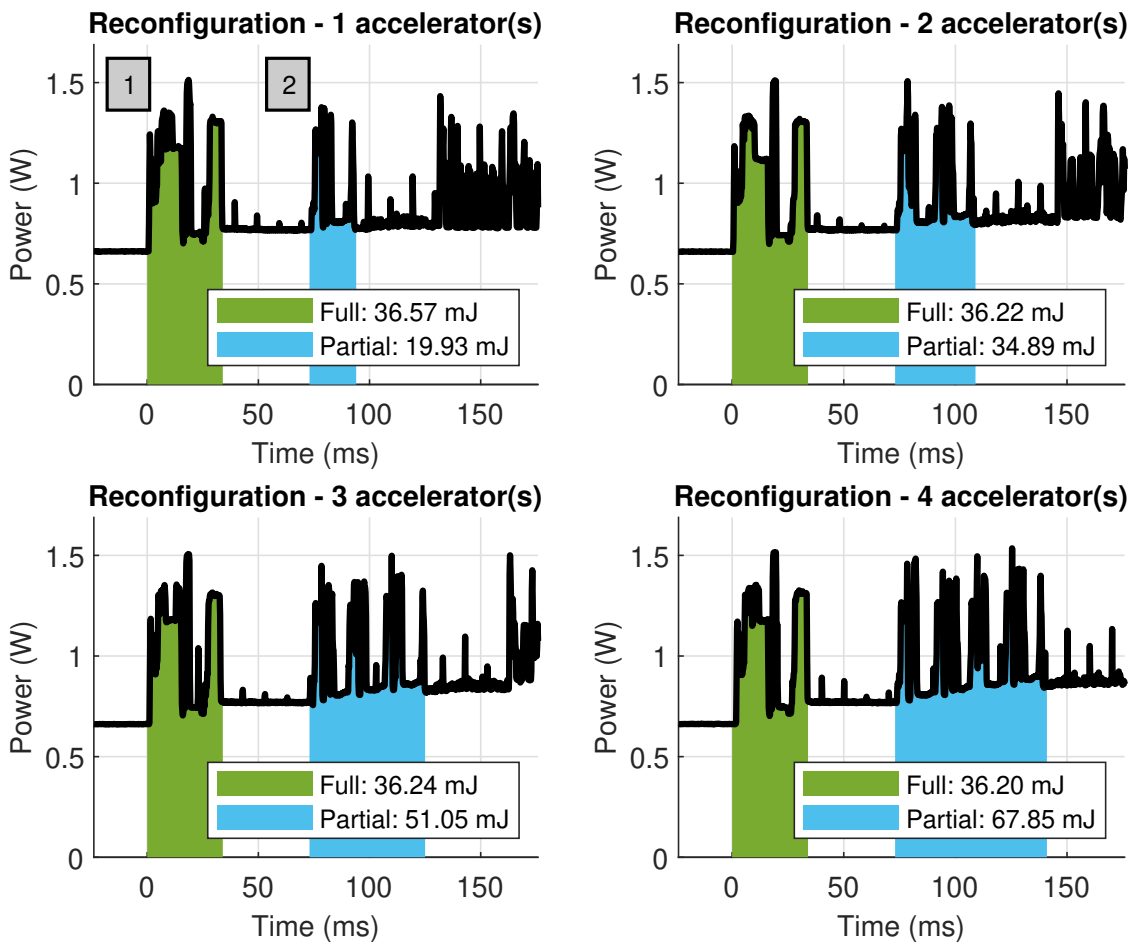

Figure 2-37: Energy consumption in the custom Zynq-7000 board during reconfiguration.
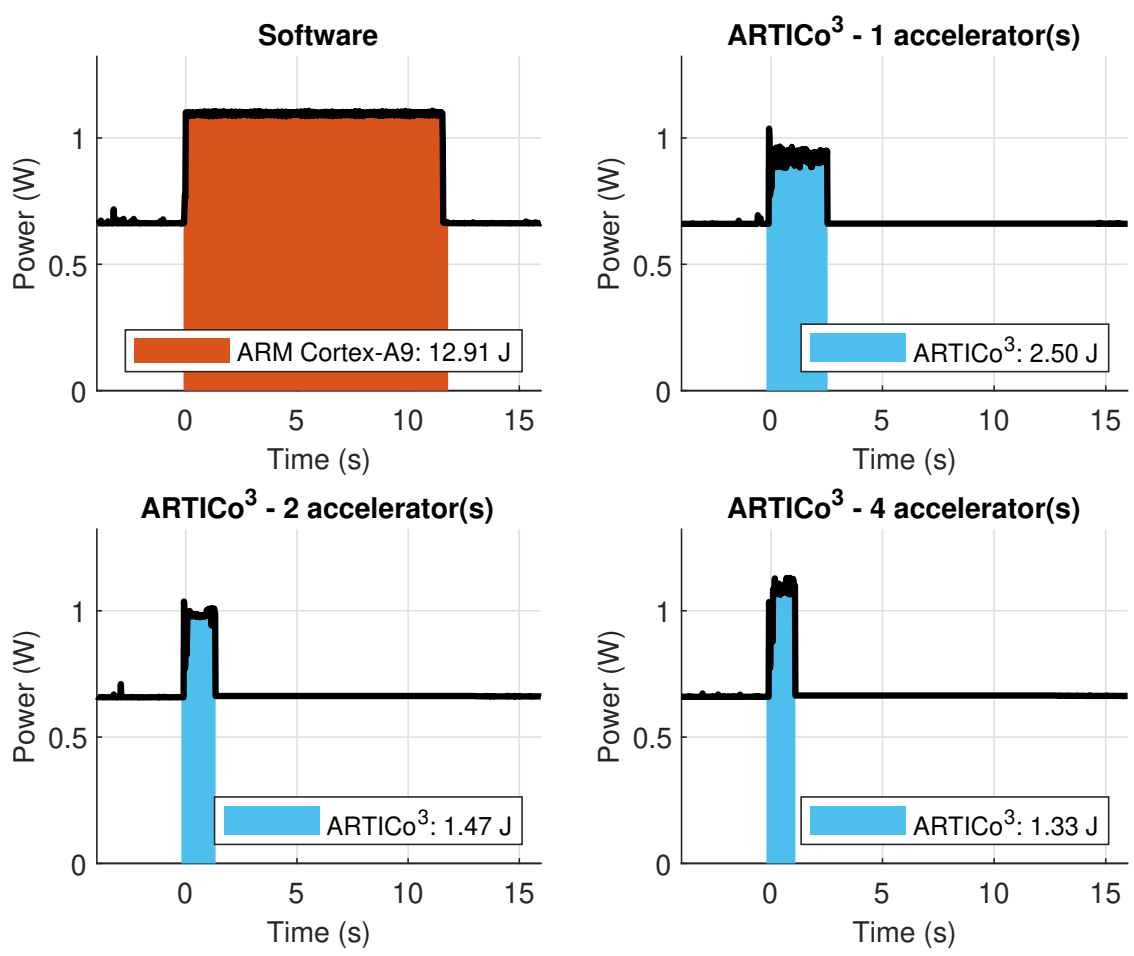

Figure 2-38: Energy consumption in the custom Zynq-7000 board during execution. 
source and available as a demo on the $\mathrm{ARTICo}^{3}$ repository. ${ }^{40}$

\subsubsection{Benchmark Evaluation}

The third and last validation setup relies on a C-based, HLS-oriented benchmarking suite. Benchmarks have been widely used throughout the literature to evaluate both programming models and computing architectures, and enable standardized comparisons with alternative solutions. Early examples of benchmarking suites are SPEC [SPEC], EEMBC [EEMBC], NPB [Bailey'91], SPLASH [Singh'92], PARSEC [Bienia'11], or MediaBench [Lee'97]. ${ }^{41}$

Traditionally, benchmarking suites consisted of a given set of applications, sometimes even optimized to run on specific platforms. However, with the rise of the parallel computing paradigm, the approach shifted towards the analysis of computation and communication patterns that capture the common requirements of different classes of application, rather than focusing on individual application implementations. One of the most relevant classifications of these computation/communication patters are the so-called Berkeley Dwarfs [Asanović'06], which can be summarized as follows:

- Dense linear algebra: dense matrix/vector operations with unit-stride/strided memory accesses.

- Sparse linear algebra: matrix/vector operations using datasets with many zero values, and relying on indexed load/store memory accesses.

- Spectral methods: multiple butterfly stages (multiply-add operations combined with specific patterns of data permutations) with either all-to-all or strictly local communication between stages.

- N-body methods: interactions between many discrete points (e.g., particleparticle or hierarchical particle methods) followed by all-to-all communication.

- Structured grids: regular grids where points are updated together either in place or using two grid copies, relying on high spatial locality.

- Unstructured grids: irregular grids where points, whose location and connectivity is explicitly defined, are updated together using multiple levels of memory reference indirection.

- MapReduce: multiple independent executions of a "map" function with no communication followed by global communication to aggregate results using a "reduce" function.

\footnotetext{
${ }^{40}$ https://github.com/des-cei/artico3

${ }^{41}$ Some of these benchmarks are still used nowadays and receive periodic updates and/or extensions.
} 
- Combinational logic: logical functions that exploit bit-level parallelism and stored state.

- Graph traversal: graphs whose nodes are visited and evaluated using multiple levels of memory indirection.

- Dynamic programming: complex problems that are solved by solving a set of overlapping problems of reduced complexity.

- Back-track and branch + bound: search and global optimization problems over large spaces that are solved by dividing those spaces in subregions and removing the suboptimal ones.

- Graphical models: graphs where variables are mapped into nodes and conditional probabilities into edges.

- Finite state machines: systems with behavior defined by states, transitions defined by inputs and current state, and outputs associated to either states or transitions.

Many modern benchmarking suites are designed to cover all the Berkeley Dwarfs, enabling more in-depth analyses of new computing architectures. In fact, there have been efforts to provide unified cross-platform and Dwarf-based benchmarks using OpenCL as the common entry point for CPUs, GPUs, and FPGAs [Krommydas'16]. However, this type of solution is not the best alternative when evaluating acceleratorcentric architectures in the context of high-performance embedded computing, since OpenCL provides functional portability but not performance portability. ${ }^{42}$ As a consequence, device-specific benchmarks have been also developed by the benchmarking community.

In this Thesis, only C-based and HLS-oriented benchmark suites have been considered. The purpose of this specific type of benchmarks is usually twofold: on the one hand, it allows the assessment and evaluation of HLS tools; on the other hand, it provides a common ground for architectural exploration and analysis in accelerator-centric designs. Leveraging on the latter, three different suites have been considered for implementation with the ARTICo ${ }^{3}$ framework: CHStone [Hara'08], MachSuite [Reagen'14], and Rosetta [Zhou'18]. Table 2-16 shows the comparison between these alternatives.

CHStone was one of the first HLS-oriented benchmark suites. It contains 12 different applications, whose complexity ranges from double-precision floating point operations (e.g., addition, multiplication, division) to several cryptographic primitives (e.g., AES, SHA). Rosetta, on the other hand, is one of the most recent benchmarking suites, and provides 6 highly complex applications (e.g., 3D rendering, face detection)

\footnotetext{
${ }^{42}$ OpenCL ensures that a given kernel code will run in any OpenCL-compatible device, but the inherent heterogeneity between computing fabrics makes it mandatory for designers to fine-tune their implementations in order to achieve the best performance on a given fabric.
} 
Table 2-16: C-based HLS-oriented benchmark suites.

\begin{tabular}{cccc}
\hline & CHStone [Hara'08] & MachSuite [Reagen'14] & Rosetta [Zhou'18] \\
\hline Benchmarks & 12 & 19 & 6 \\
Complexity & Low/Medium & Medium & High \\
Dwarf-based & No & Yes & No
\end{tabular}

to enable deep exploration of architectural and algorithmic optimizations in both computing- and memory-bounded scenarios. Finally, MachSuite provides 12 kernels, some of them with several implementations, for a total of 19 different Dwarf-oriented benchmarks. In this case, the complexity of the accelerators falls in between the other two benchmark suites.

Taking into account the execution model and the floorplanning restrictions in ARTICo $^{3}$, the most suitable candidate for evaluation in this Thesis is MachSuite, since it provides a good tradeoff between computing requirements, resource utilization, and architectural exploration coverage. Table 2-17 summarizes the different benchmarks that are shipped with MachSuite, highlighting the specific Berkeley Dwarf that is addressed in each of them.

Table 2-17: MachSuite [Reagen'14] benchmarks.

\begin{tabular}{ccc}
\hline Name & Description & Dwarf \\
\hline AES/AES & AES encryption & Combinational logic \\
BACKPROP/BACKPROP & Neural Network training & Unstructured grids \\
BFS/BULK & Breadth-first search & Graph traversal \\
BFS/QUEUE & Breadth-first search & Graph traversal \\
FFT/STRIDED & Fast Fourier Transform & Spectral methods \\
FFT/TRANSPOSE & Fast Fourier Transform & Spectral methods \\
GEMM/NCUBED & Matrix multiplication & Dense linear algebra \\
GEMM/BLOCKED & Matrix multiplication & Dense linear algebra \\
KMP/KMP & String matching & Finite state machines \\
MD/KNN & Molecular dynamics & N-body methods \\
MD/GRID & Molecular dynamics & N-body methods \\
NW/NW & DNA alignment & Dynamic programming \\
SORT/MERGE & Sorting & MapReduce \\
SORT/RADIX & Sorting & MapReduce \\
SPMV/CRS & Sparse matrix/vector multiplication & Sparse linear algebra \\
SPMV/ELLPACK & Sparse matrix/vector multiplication & Sparse linear algebra \\
STENCIL/STENCIL2D & Stencil computation & Structured grids \\
STENCIL/STENCIL3D & Stencil computation & Structured grids \\
VITERBI/VITERBI & Hidden Markov model estimation & Graphical models \\
\hline
\end{tabular}

In order to provide a thorough evaluation, together with an in-depth comparison with commercial alternatives to the framework proposed in this Thesis, the MachSuite benchmarks have been implemented in ARTICo $^{3}$ and SDSoC. ${ }^{43}$ Moreover, similar development boards have been used for experimental evaluation: a Pynq board for

\footnotetext{
${ }^{43}$ Tool versions: ARTICo ${ }^{3}$ with Vivado and Vivado HLS 2017.1, SDSoC 2017.1.
} 
ARTICo $^{3}$-based designs, ${ }^{44}$ and a ZedBoard for SDSoC-based designs. ${ }^{45}$ With these restrictions, two benchmarks (i.e., BACKPROP/BACKPROP and FFT/TRANSPOSE, marked in gray in Table 2-17) cannot be implemented due to resource limitations. For the remaining 17 benchmarks, three different setups have been generated:

- SDSoC-based bare-metal implementation with one accelerator per benchmark.

- ARTICo ${ }^{3}$-based implementation with one kernel instance per benchmark.

- ARTICo ${ }^{3}$-based implementation with four kernel instances per benchmark.

The validation strategy does not aim at providing results of highly optimized hardware accelerators and thus, no HLS-oriented optimization directives or code refactoring techniques have been applied to the original benchmark codes. In fact, in ARTICo ${ }^{3}$-based kernels, only minor modifications have been made to adapt the accelerator interface: use of 32-bit data types, port flattening (i.e., removing structbased ports, replacing them by their constituent elements), and port packing (i.e., grouping several small-sized ports in one for efficient DMA transfers). Simpler modifications have been made in SDSoC-based accelerators: port flattening, and AXIbased data and control accesses. In addition, some configuration parameters in a few benchmarks have been slightly modified in all three setups to comply with the local memory limitation of $64 \mathrm{KiB}$ per ARTICo ${ }^{3}$ accelerator. The code of the $\mathrm{ARTICo}^{3}$ and SDSoC implementations of the MachSuite benchmarks, together with the detailed list of modifications, is also open source and available online. ${ }^{46}$

Figure 2-39 shows the execution results of all 17 benchmarks in each setup. ${ }^{47}$ As it can be seen, even the ARTICo $^{3}$ setup that uses only one hardware accelerator outperforms the equivalent SDSoC implementation in almost one order of magnitude (in average). Although the hardware accelerators are essentially the same, the optimized communication infrastructure in ARTICo ${ }^{3}$ and the use of high-speed memories to perform local processing renders significantly better results than the SDSoC-based implementations, which are also DMA-enabled.

Regarding execution scalability, Figure 2-40 shows the speedup between both ARTICo $^{3}$-based setups. Ideally, using $4 \times$ more accelerators should lead to a speedup of $4 \times$. Computing-bounded scenarios render results that are close to the theoretical limit (e.g., AES/AES, GEMM/BLOCKED, or VITERBI/VITERBI), whereas memory-bounded scenarios only reach minimum speedup (e.g., BFS/BULK, KMP/KMP, or NW/NW). In addition, some benchmarks fall in between these corner cases (e.g., FFT/STRIDED, $\mathrm{MD} / \mathrm{KNN}$, or SORT/MERGE), reaching intermediate speedup values. According to these results, the use of ARTICo ${ }^{3}$-based solutions is beneficial in those applications whose corresponding Dwarf presents a high computation/communication ratio:

\footnotetext{
${ }^{44}$ XC7Z020-1CLG400.

${ }^{45}$ XC7Z020-1CLG484.

${ }^{46}$ https://github.com/des-cei/MachSuite

${ }^{47}$ Average execution time in 1024 processing rounds (FPGA fabric @ $100 \mathrm{MHz}$ ).
} 


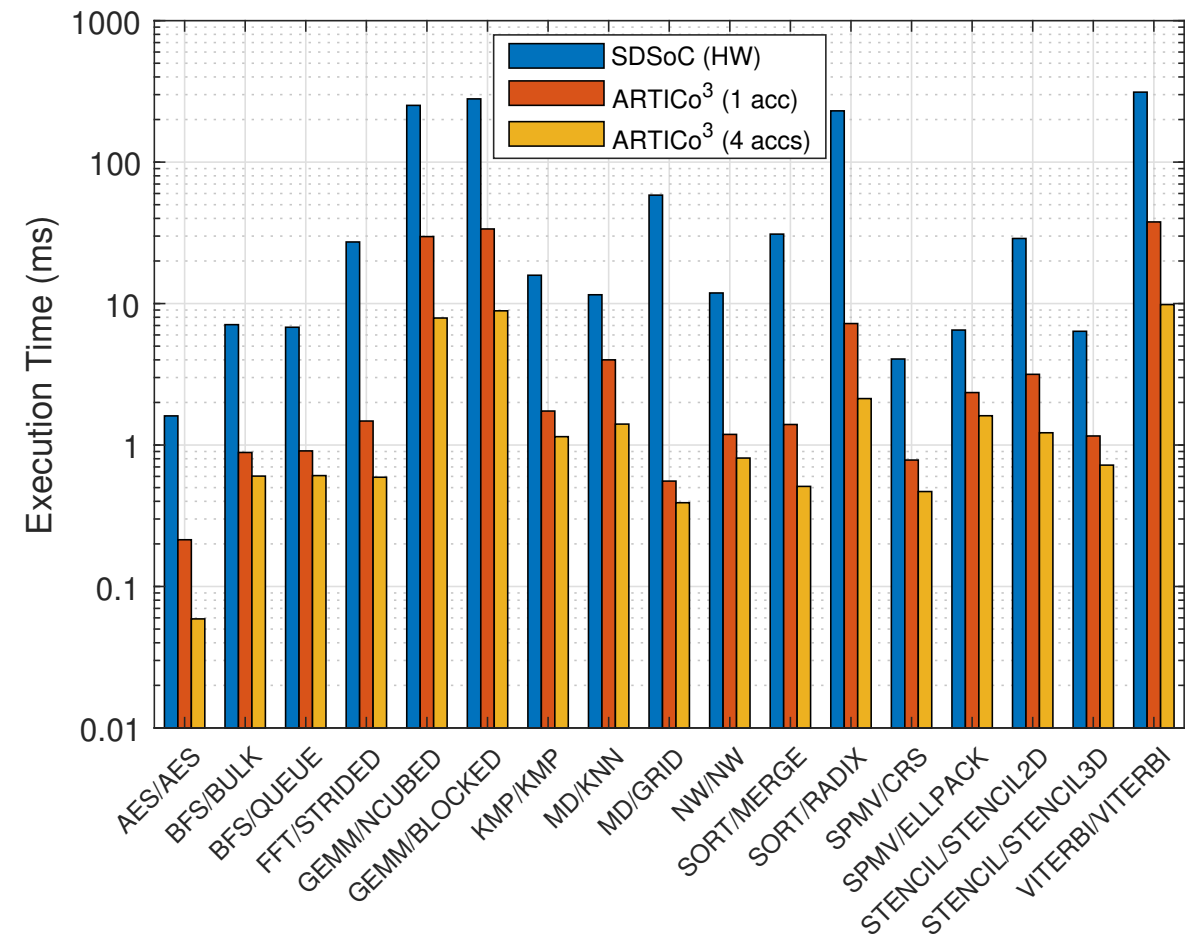

Figure 2-39: Benchmark evaluation: SDSoC (hardware) versus ARTICo ${ }^{3}$.

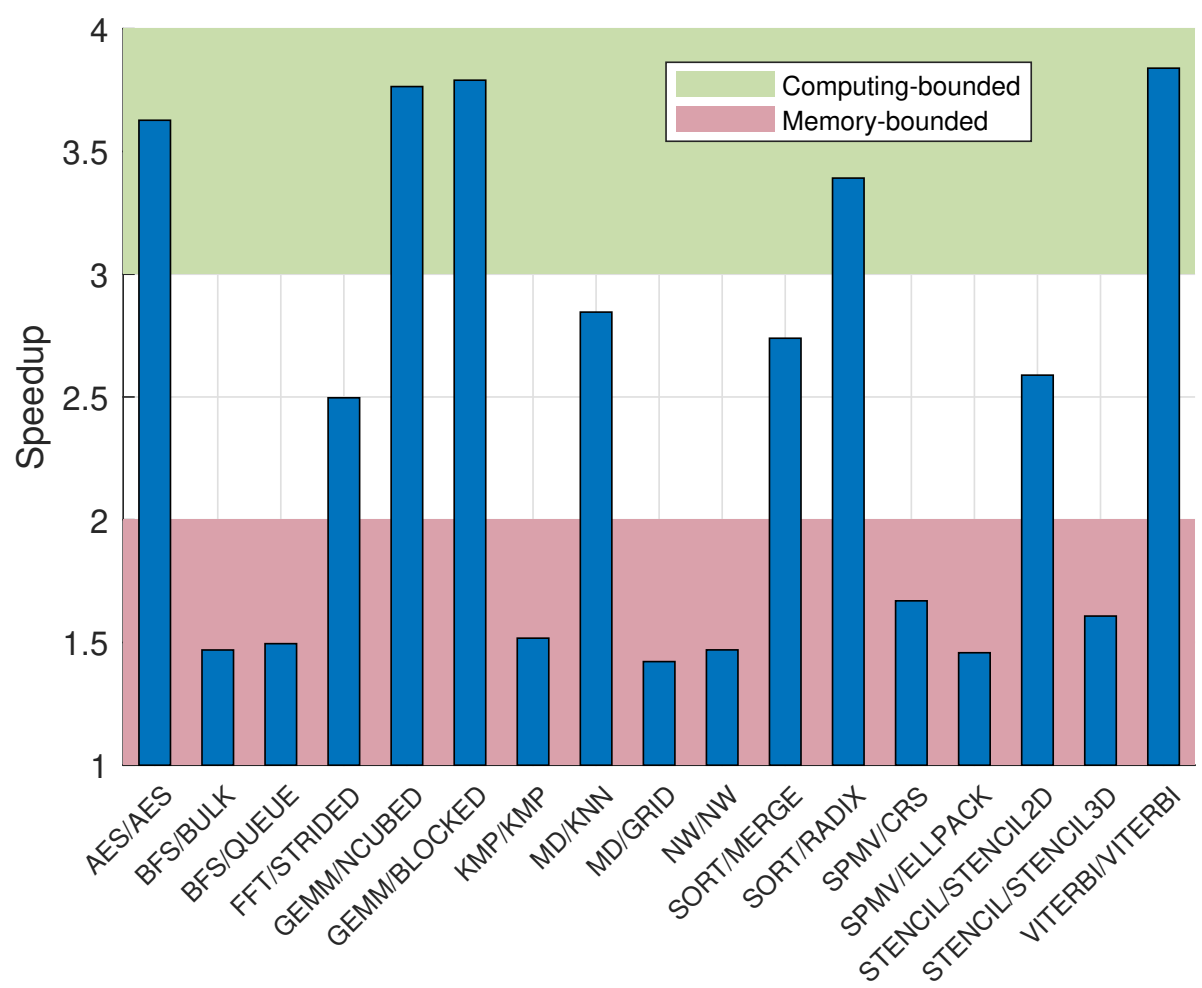

Figure 2-40: Benchmark evaluation: ARTICo $^{3}$ speedup. 
combinational logic, spectral methods, dense linear algebra, MapReduce, and graphical models.

Finally, Figure 2-41 shows an additional comparison between the ARTICo ${ }^{3}$-based implementation using one kernel instance per benchmark, and the software version of the SDSoC-based bare-metal implementation (i.e., the code used to generate the accelerator using HLS but running in one ARM Cortex-A9 core @ $666.67 \mathrm{MHz}$ ). Although the run-time management of $\mathrm{ARTICo}^{3}$-accelerated solutions generates additional overheads, the difference between the obtained results and the bare-metal software is below one order of magnitude in average. This is of special importance, since the previous comparison is biased against the proposal of this Thesis (i.e., the use of relatively small input data sizes benefits the software-based solution, especially when using accelerators generated with HLS and no optimizations).

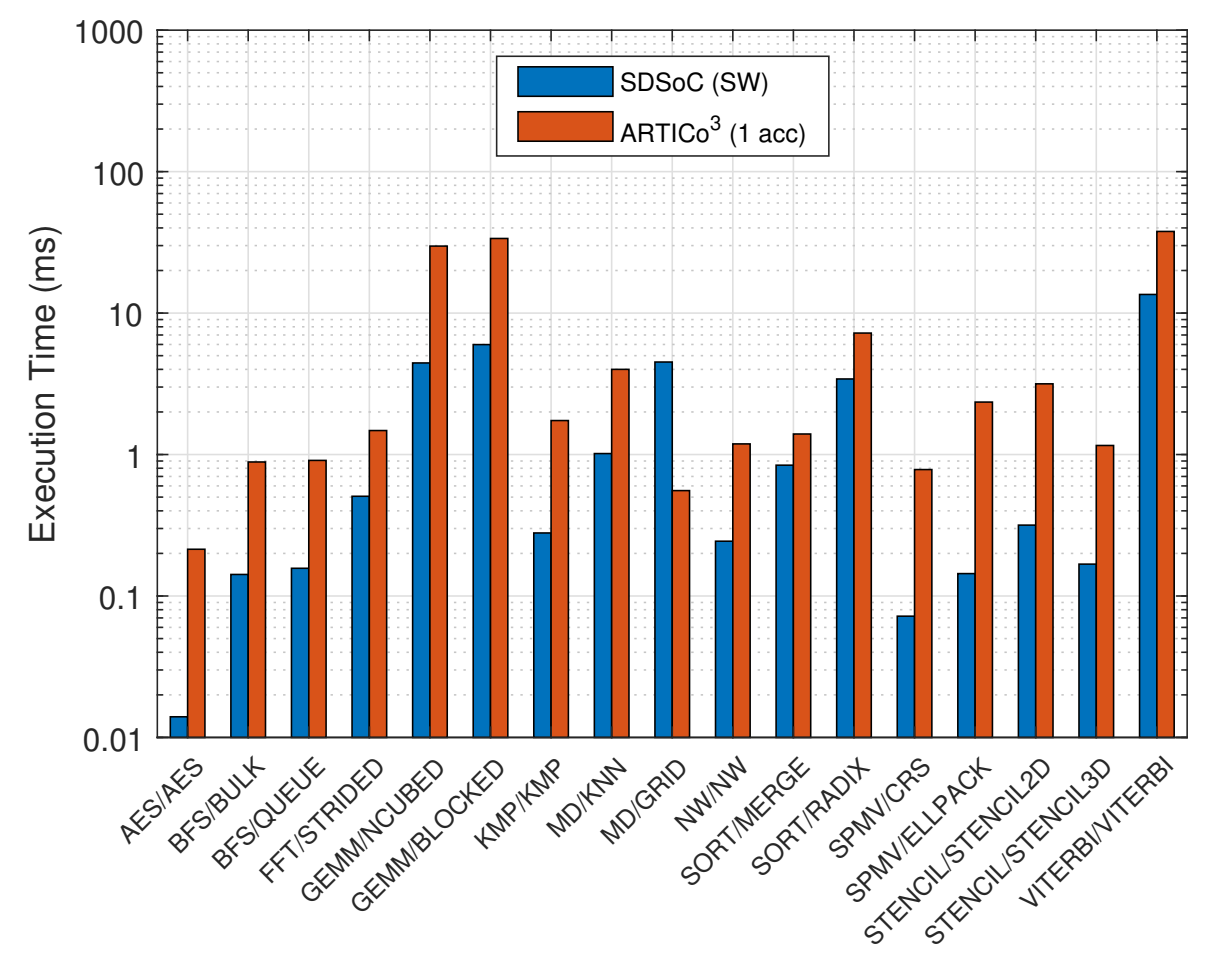

Figure 2-41: Benchmark evaluation: SDSoC (software) versus ARTICo ${ }^{3}$.

Original contribution 2-13 A Dwarf-based characterization and validation strategy for the ARTICo ${ }^{3}$ framework based on HLS benchmarks. 


\section{PROGRAMMING MODELS FOR ADAPTIVE HARDWARE}

The framework proposed in this Thesis is extended in this chapter to support two additional programming models for reconfigurable hardware-based computing platforms: software-like multithreading and dataflow-based processing. All the extensions presented in this chapter are supported by direct tool-to-tool integrations, and complement the native data-parallel programming model of the framework.

The chapter starts by providing a motivation on the use of different programming paradigms for hardware-accelerated embedded computing. Then, each extension is addressed separately but using a common structure: context and introduction, additional components required to support a new programming model in ARTICo ${ }^{3}$, state of the art and related work, contributions at integration level, and validation tests.

\subsection{Motivation}

Although it has been already proven that FPGAs have much better performance per watt ratios than other computing platforms such as CPUs or GPUs in certain application scenarios [Nurvitadhi'16], they have traditionally suffered from a generalized lack of acceptance in the software development community due to their associated programming challenges [Bacon'13].

FPGA development has been eminently associated with the use of HDLs to describe digital circuits at a very low level of abstraction (i.e., RTL). This approach is in clear contrast with standard software development flows, where the target computing architecture is fixed (e.g., CPUs or GPUs) and optimized compiler infrastructures can be used to translate high-level programming languages and models into executable binary files. HDLs have evolved throughout the years to incorporate new (or extend available) features in Electronic Design Automation (EDA) tools, but they still provide a restricted set of synthesizable constructs (i.e., code structures that can be translated into actual hardware). For instance, SystemVerilog features non-synthesizable objectoriented extensions for in-depth circuit verification, but it is still considerably similar to Verilog regarding synthesizable RTL hardware specifications.

HLS has emerged as a convenient and software-friendly alternative to HDL-based hardware design. In HLS-enabled EDA tools, high-level algorithmic descriptions 
are automatically translated into RTL code. The entry point for these descriptions is manyfold, but some of the most important approaches are either C-based, OpenCL-based, or model-based frameworks [Bacon'13]. C-based HLS frameworks are widely accepted because they provide a familiar programming interface for software developers. However, the lack of inherent parallelism in sequential C code hinders efficient hardware-based implementations [Edwards'06]. In these scenarios, parallelism is usually inferred by the compiler in an automatic or user-assisted way (e.g., with explicit optimization directives in the source code). An example of commercial C-based framework is Xilinx Vivado HLS.

OpenCL-based HLS frameworks rely on a standard and widespread parallel programming model for heterogeneous computing platforms that requires certain low-level knowledge of the underlying hardware architecture for developers to write highly efficient code. As a consequence, there is a smoother transition from software to hardware development, since OpenCL software developers are already capable of "thinking in parallel" to some extent. Notice, though, that there is still a huge difference between FPGAs (configurable hardware architecture generated by HLS mechanisms) and other accelerator platforms such as GPUs (fixed hardware architecture). An example of commercial OpenCL-based framework is the Intel FPGA Software Development Kit (SDK) for OpenCL. Provided that the required OS extensions are available, a similar approach (i.e., using a standard parallel programming model in heterogeneous hardware/software environments) can be applied to C-based hardware systems to complement their parallel processing capabilities, while at the same time making them more accessible to software developers [Andrews'04].

Finally, model-based HLS frameworks rise the level of abstraction even further, removing most of the burden associated to control-related structures and focusing on a more dataflow-oriented approach for algorithm specification (i.e., applications are described based on how data moves through different functional blocks). An example of commercial model-based framework is HDL Coder, embedded within MATLAB/Simulink. It is worth noting that model-based design has already been successfully applied to the design of reconfigurable systems [Desnos'19].

Another major issue in traditional FPGA development is implementation time. While software compilation is relatively fast, the translation of HDL code into a valid FPGA configuration file requires several time-consuming steps (i.e., logic synthesis, technology mapping, place and route, and bitstream generation). In this regard, FPGA virtualization appears as a mean to bridge the productivity gap between HLS frameworks and low-level FPGA architecture (i.e., logic resources) [Stitt'11]. As it will be discussed throughout this chapter, FPGA overlays, which provide devices with an intermediate programmability layer to enable fast circuit implementation and/or reconfiguration, are a key virtualization technique [Vaishnav'18].

In this Thesis, the native data-parallel programming model and HLS capabilities of the ARTICo $^{3}$ framework are complemented with two different mechanisms to make it more appealing for the software development community: OS extensions for unified hardware/software multithreading in C-based systems using ReconOS, 
and dataflow-based extensions for hardware accelerator specification using MDC. These extensions come together with additional reconfiguration mechanisms, which complement the native coarse-grained DPR approach (i.e., slot-based reconfiguration) with fine-grained LUT-based DPR and register-based Coarse-Grained Reconfiguration (CGR), to speed up functional changes in the reconfigurable logic.

\subsection{Multithreading Extensions}

The use of OSs in current computing platforms has become mainstream due to the increasing complexity of their target application scenarios. This is also true for software-based embedded systems, which have experienced an important evolution from their bare-metal origins to current full-fledged OS-based deployments. While it is true that some scenarios have hard timing deadlines and thus they require real-time OS responses, general-purpose alternatives are enough in most cases (e.g., Linux).

OSs serve two main purposes independently of their nature: from a top-down perspective, they provide an abstraction layer to hide the underlying hardware from user applications; from a bottom-up perspective, they implement resource management strategies to ensure proper system behavior (e.g., memory or I/O management) [Tanenbaum'15]. While the latter is usually transparent for the user, the former requires user applications to perform system calls to access the core OS functionality.

One of the key concepts in modern OSs is the process. A process is an abstraction of a running program [Tanenbaum'15]. In other words, a process is an instance of a program (i.e., an executable binary file) that has been loaded into memory and is being executed. The OS virtualizes the hardware resources and ensures that processes execute as if they had their own private CPU. To do so, it keeps track of relevant information for each running process, including the program counter, the CPU register contents, or the pointers to specific memory sections (e.g., text, data, or stack). According to this CPU virtualization scheme, a process can be in three different states: running (i.e., actually using the CPU), ready (i.e., potentially executable but kept away from the CPU to allow other processes to run), and blocked (i.e., waiting for some event to occur and thus not executable). ${ }^{1}$ In general, OSs play with these states and schedule processes to enable concurrent execution (i.e., all processes look as if they were being executed at the same time). In single-core processor systems, this is achieved by time-multiplexing the available hardware resources. However, in multicore processor systems, true parallelism can be also achieved (i.e., several processes are actually executed at the same time using different hardware resources).

\footnotetext{
${ }^{1}$ These are the three main process states. However, it is also possible to include additional states to represent process creation and termination [Silberschatz'18].
} 
Although processes can be completely independent, it is relatively easy to find them executing in a cooperative way, since this approach provides extended benefits such as information sharing (i.e., several processes may need the same data), computation speedup (i.e., dividing a task in several parallel subtasks can improve performance in multi-core systems), or modularity (i.e., isolating different functionalities in separate blocks fosters highly modular systems) [Silberschatz' 18]. In these scenarios, Interprocess Communication (IPC) mechanisms are required to allow data exchanges between processes. There are two basic approaches to IPC: shared memory and message passing [Silberschatz'18].

In the shared-memory IPC model, processes establish a common address space to share data using read and write operations. Data exchanges between processes are performed without the OS even noticing, although the creation and removal of shared memory regions does require system calls. The producer/consumer approach that is commonly used in the shared-memory model (i.e., one process produces data and another one consumes them) usually leads to race conditions when processes are executed concurrently. To properly address these issues, OSs provide different synchronization primitives such as semaphores or mutexes. Semaphores are integer variables that are accessed atomically (i.e., by a single process at a time) using the post (increments the semaphore value) and wait (decrements the semaphore value) functions. Whenever a process calling wait finds out that the semaphore equals 0, it blocks (before further decrementing its value) until another process calls post and increments the semaphore. If several processes are waiting for a specific semaphore, only one is awakened by the OS. Depending on the allowed range of values, semaphores can be binary (i.e., 0 or 1 ) or counting (i.e., any arbitrary range). Semaphores are commonly used to enforce sequential behavior in data-dependent processes that are executed concurrently. However, binary semaphores can also act as mutexes, which are synchronization primitives that prevent several processes from simultaneously executing the so-called critical sections (i.e., code regions that need to executed by only one process at a time, in a mutual exclusion context). Mutexes are also accessed using atomic operations, with a lock function to acquire the mutex and an unlock function to release it. Processes calling lock on an already locked mutex will block until another process calls unlock and releases the mutex. If several processes are waiting for a specific mutex, only one is awakened by the OS.

In the message-passing IPC model, on the other hand, processes send and receive messages whenever data needs to be exchanged. While this approach is useful for smaller data exchanges and avoids some of the synchronization problems present in the shared-memory model, it incurs additional overheads since a system call is usually required per message send/receive operation. Nevertheless, the messagepassing IPC model is still the most suitable solution for distributed-memory systems. In this model, processes call a send function to send data and a receive function to receive data. OSs often support two types of message-passing mechanisms: direct communication (i.e., processes need to specify the process to/from which they want to send/receive data) and indirect communication (i.e., processes send/receive data 
to/from an intermediate storage element sometimes referred to as mailbox or message queue, depending on its size) [Silberschatz'18].

It is worth noting that the process notion in modern OSs is built around two independent concepts: resource grouping and execution [Tanenbaum'15]. On the one hand, processes group related resources together (e.g., an address space with text and data sections, open files, or signals). On the other hand, processes have a thread of execution (i.e., the program counter, the CPU registers, and the stack), which is the base scheduling unit for the OS and therefore holds the process state (i.e., running, ready, or blocked). Multithreading appears when more than one thread is allowed to exist in a single process. Note that this scenario is different from one in which there are several concurrent processes in that threads inherently share all the available resources in their corresponding process (which significantly simplifies the programming model), whereas processes are independent by nature. Hence, all threads from a process exhibit an implicit shared-memory IPC model. Additionally, multithreading can be used to deploy two different types of parallelism: data-level (i.e., several threads perform the same operation on different data) and task-level (i.e., each thread performs a different operation) [Silberschatz'18].

In multithreading environments, threads are commonly managed using a dedicated library and API. In this regard, the IEEE POSIX standard specification [IEEE'18] defines a standard OS interface and environment that includes multithreading support. In particular, the Pthreads specification defines functions to create and terminate threads (e.g., pthread_create and pthread_exit), and to enable thread synchronization and IPC mechanisms (e.g., pthread_join, pthread_mutex_lock, and pthread_mutex_unlock). Since POSIX is an OS-wide specification, it not only contains thread-related constructs but also many others, including other IPC constructs such as semaphores and their related function calls (e.g., sem_post and sem_wait). It is important to highlight that the POSIX standard only specifies expected behavior and interfaces (i.e., function prototypes), but does not deal with actual library implementations, which are up to OS designers.

This section covers the extension of the ARTICo ${ }^{3}$ framework to support a hardwarebased multithreading programming model that complements its native data-parallel programming and execution schemes. This has been achieved by a direct tool-to-tool integration with an OS-based framework that enables transparent multithreading in mixed hardware/software environments. This section also covers the evaluation of the integrated framework using evolvable hardware kernels to increase system adaptivity. 


\subsubsection{Additional Infrastructure}

\section{ReconOS}

The multithreading programming model is a well-known and widely used approach for concurrent software development. However, the amount of parallelism that can be exploited with this model in software-based multi-core processing systems is somehow limited, since concurrency does not necessarily imply parallel execution. In fact, time-multiplexing mechanisms are still required when the number of application threads is larger than the number of available hardware resources (i.e., processing cores). In this context, the use of FPGA fabrics with dedicated application-specific hardware accelerators can significantly boost parallel execution while maintaining certain degree of flexibility, especially when using DPR techniques to enable timemultiplexing of resources also for hardware-based processing elements. However, hardware design is not as accessible as it should be for the vast majority of software developers, even though there have been several research initiatives aimed at bridging this gap (e.g., HLS techniques). One of these initiatives involves extending current OSs to transparently integrate custom-made hardware accelerators as standard software threads [Andrews'16], with the ReconOS framework as a key representative.

ReconOS [Agne'14] combines OS extensions and a modular hardware architecture to support a unified multithreading programming model for both hardware and software threads. To do so, it provides a hardware/software abstraction layer accessible through common POSIX-like function calls [Lübbers'08b], and portable across different OSs [Lübbers'08a]. This approach favors both DSE and rapid prototyping, and eases hardware/software codesign in FPGA-based embedded systems. Actually, a common development process with ReconOS would be as follows: first, developers write the target application using Pthreads on a Linux workstation and perform functional validation; then, developers port their application code to the target computing platform (using the POSIX-like abstraction layer on an embedded Linux) and profile the software-based execution to identify candidate threads for hardwarebased execution; finally, developers implement the identified threads as hardware accelerators and evaluate different hardware/software mappings.

The ReconOS modular hardware architecture is shown in Figure 3-1. Hardware threads are implemented as application-specific hardware accelerators on several reconfigurable slots available in the FPGA fabric. Each reconfigurable slot has two main communication channels with the rest of the system: the Operating System Interface (OSIF) and the Memory Interface (MEMIF). Hardware threads behave as bus masters thanks to the MEMIF and a hardware-based memory subsystem that includes arbitration logic to handle simultaneous memory accesses, a DMA controller to enable burst-based data transfers, and an MMU to provide a single virtual memory map for both hardware and software threads under Linux.

In ReconOS, the unified hardware/software multithreading model is enabled by the concept of delegate threads. A delegate thread is a software thread that receives OS-related petitions from a hardware thread through the OSIF, and executes them 


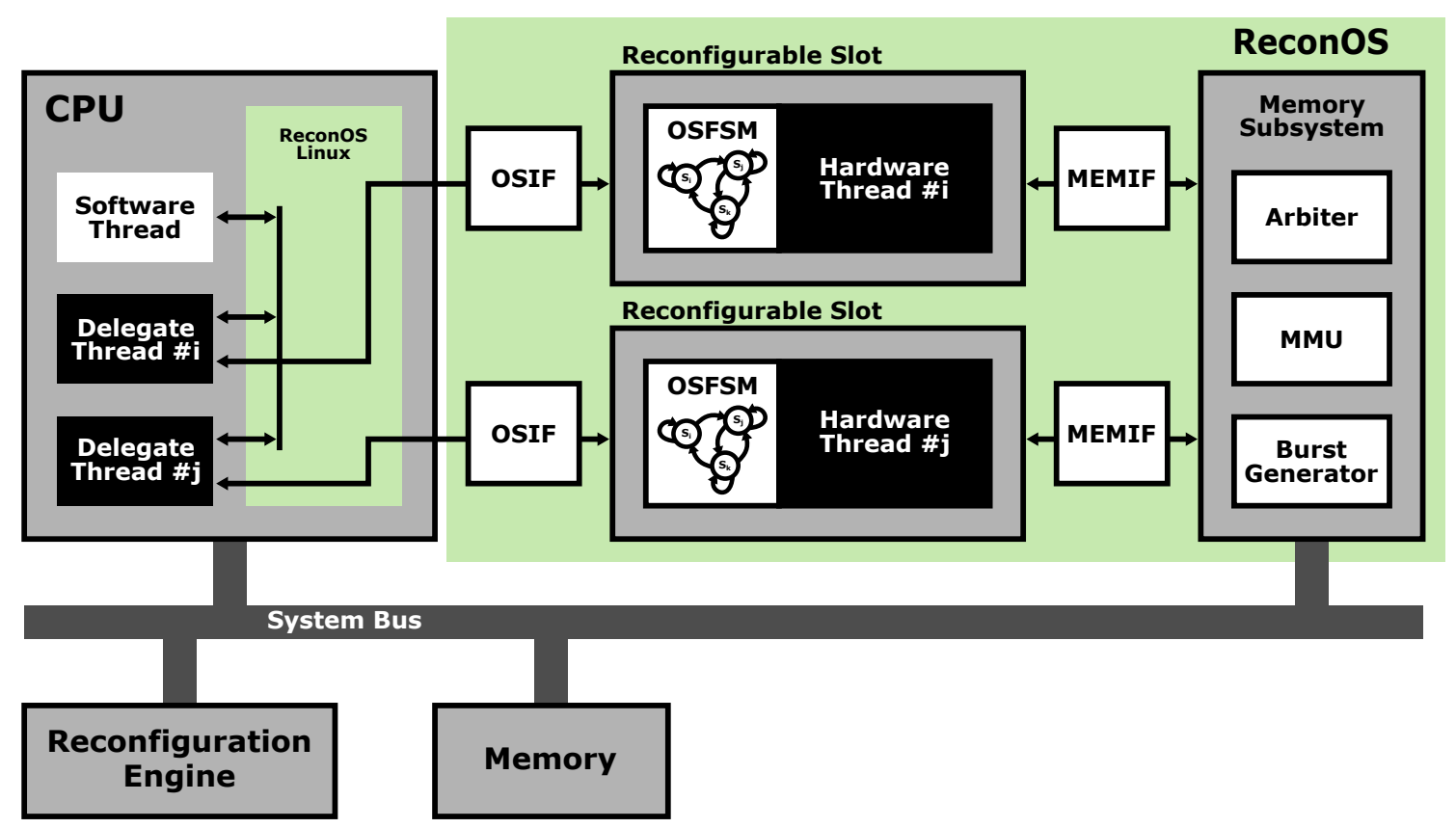

Figure 3-1: ReconOS architecture.

on its behalf. Hence, hardware threads are completely hidden from the OS, which effectively has to deal only with software threads. This particular feature is what enables OS portability, which is further supported by a common set of POSIX-like function calls for all ReconOS threads (independently of their nature) to access specific OS functionality, including semaphores, mutexes, condition variables (i.e., conditional thread blocking statements that are used together with mutexes), and mailboxes. ${ }^{2}$ The complete list of OS-related function calls supported in ReconOS and their equivalent POSIX specification (when available) can be seen in Table 3-1.

ReconOS supports hardware thread specification in both VHDL and HLScompatible $\mathrm{C} / \mathrm{C}++$ code. To ease the development process, a VHDL package and several $\mathrm{C}$ header files are provided for the former and latter entry points, respectively. These files contain the actual implementations of the OS-related function calls that use the OSIF (i.e., those shown in Table 3-1), but they also include additional function calls to perform memory accesses using the MEMIF. In this regard, it is worth noting that the hardware thread model in ReconOS requires accelerators to operate with data stored in local memory and thus, the MEMIF-related function calls allow developers to define local memory and to issue either one-word or burst-based data transfers between main system memory and accelerator local memory. To favor seamless development, software threads use equivalent function calls whose prototypes are identical to those used in HLS-based hardware threads. Listing 3-1 shows an example specification of a software thread or an HLS-based hardware thread that sorts a list of

\footnotetext{
${ }^{2}$ Mailboxes in ReconOS are implemented in user space using shared memory structures that are synchronized with semaphores and protected with mutexes.
} 
Table 3-1: Available OS functionality for ReconOS threads.

\begin{tabular}{ccc}
\hline Type & ReconOS Function & POSIX Specification \\
\hline \multirow{2}{*}{ Semaphore } & sem_post & sem_post \\
& sem_wait & sem_wait \\
\hline \multirow{2}{*}{ Mutex } & mutex_lock & pthread_mutex_lock \\
& mutex_trylock & pthread_mutex_trylock \\
mutex_unlock & pthread_mutex_unlock \\
\hline \multirow{3}{*}{ Condition Variables } & cond_signal & pthread_cond_signal \\
& cond_broadcast & pthread_cond_broadcast \\
& cond_wait & pthread_cond_wait \\
\hline \multirow{3}{*}{ Mailbox } & mbox_put & - \\
& mbox_tryput & - \\
& mbox_get & - \\
& mbox_tryget & - \\
\hline
\end{tabular}

1024 elements. ${ }^{3}$ The thread iteratively gets the address of the list from a mailbox, reads the list and stores it in local memory, sorts the local copy of the list, writes the result back to global memory, and puts the address of the ordered list in another mailbox.

Listing 3-1: C-based ReconOS thread.

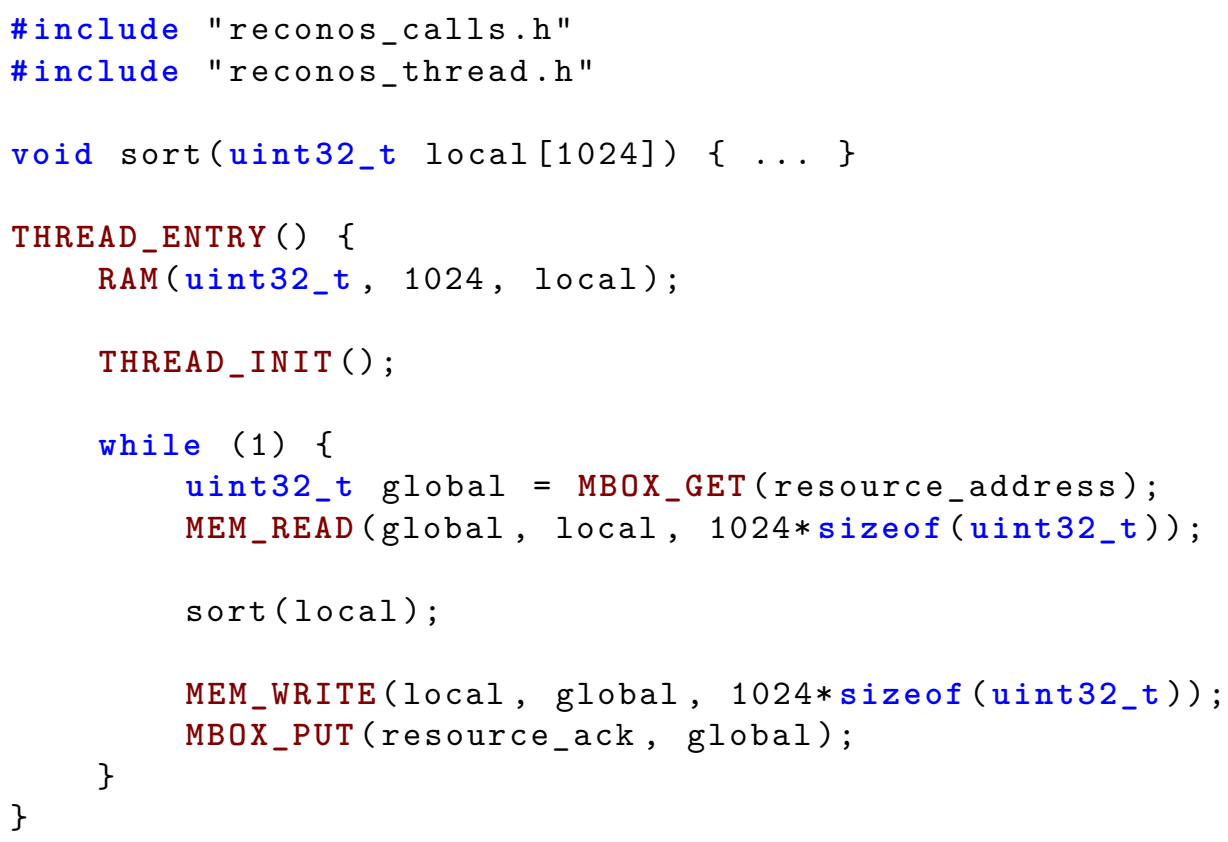

Although high-level descriptions of hardware threads in C code make ReconOS accessible to a wider range of embedded system designers, its main development flow is still focused on providing highly optimized low-level specifications in VHDL to truly exploit the benefits of hardware-based mutithreading on FPGAs. To do so, the internal

\footnotetext{
${ }^{3}$ Code adapted from the ReconOS sort demo available in https://github.com/ReconOS/reconos.
} 
architecture of a hardware thread should have two differentiated parts: a computingbounded processing core with an optimized datapath (i.e., massively parallel or deeply pipelined), and a synchronization FSM to interact with the OS sequentially. It is within this sequential block, called OSFSM, where the different VHDL procedures available in the ReconOS package need to be used. Each of these procedures implements yet another FSM to properly handle the interaction with the OS (including thread blocking) and therefore, the OSFSM is in reality a set of nested FSMs. Listing 3-2 shows an excerpt from the VHDL specification of the previous sorting thread where an example OSFSM can be seen. ${ }^{4}$ Notice how thread blocking is implemented in each state using the done flag from each ReconOS function call.

ReconOS provides not only a runtime environment to transparently manage hybrid hardware/software multithreading applications on FPGAs, but also an automated toolchain to generate and deploy them. The ReconOS toolchain, also known as the ReconOS Development Kit, is a modular set of Python (platform-agnostic) and Tcl (target-dependent) scripts that takes input thread specifications in any of the supported languages and generates the configuration bitstreams for the target FPGA. This process is driven by a configuration file that links a ReconOS project with a specific hardware template that defines the target system (e.g., FPGA device, OS, required hardware IPs). The ReconOS toolchain also uses this configuration file to generate a valid software project, build it, and produce the application executables to be run in the target system. Figure 3-2 shows an overview of the ReconOS toolchain.

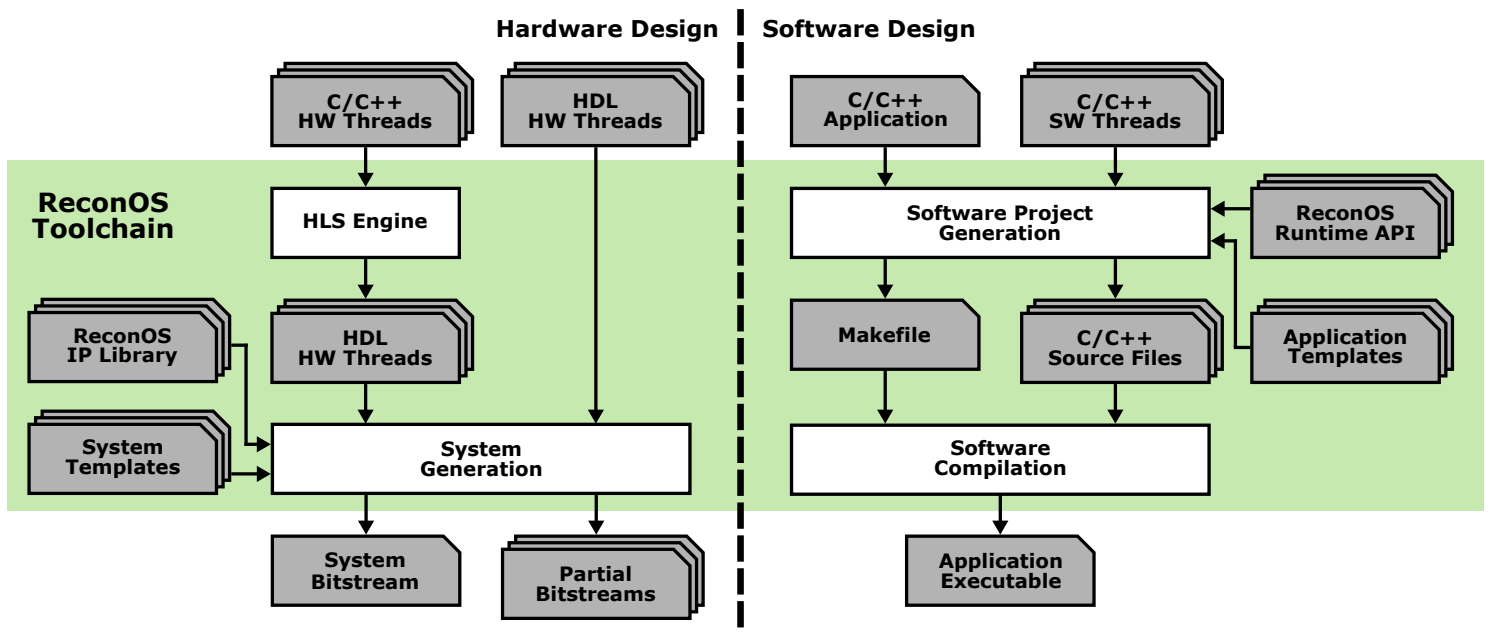

Figure 3-2: ReconOS toolchain.

As an open source research project, ${ }^{5}$ ReconOS has evolved throughout the years, a fact that has been reflected in its different releases. ReconOS v1.0 [Lübbers'07] was built on top of eCos, an open source RTOS for embedded systems, ${ }^{6}$ and targeted Xilinx Virtex-II Pro and Virtex-4 FPGAs with embedded PowerPC proces-

\footnotetext{
${ }^{4}$ Code adapted from the ReconOS sort demo available in https://github.com/ReconOS/reconos.

${ }^{5}$ https://github.com/ReconOS/reconos

${ }^{6}$ https://ecos.sourceware.org/
} 
Listing 3-2: VHDL-based ReconOS thread - OSFSM.

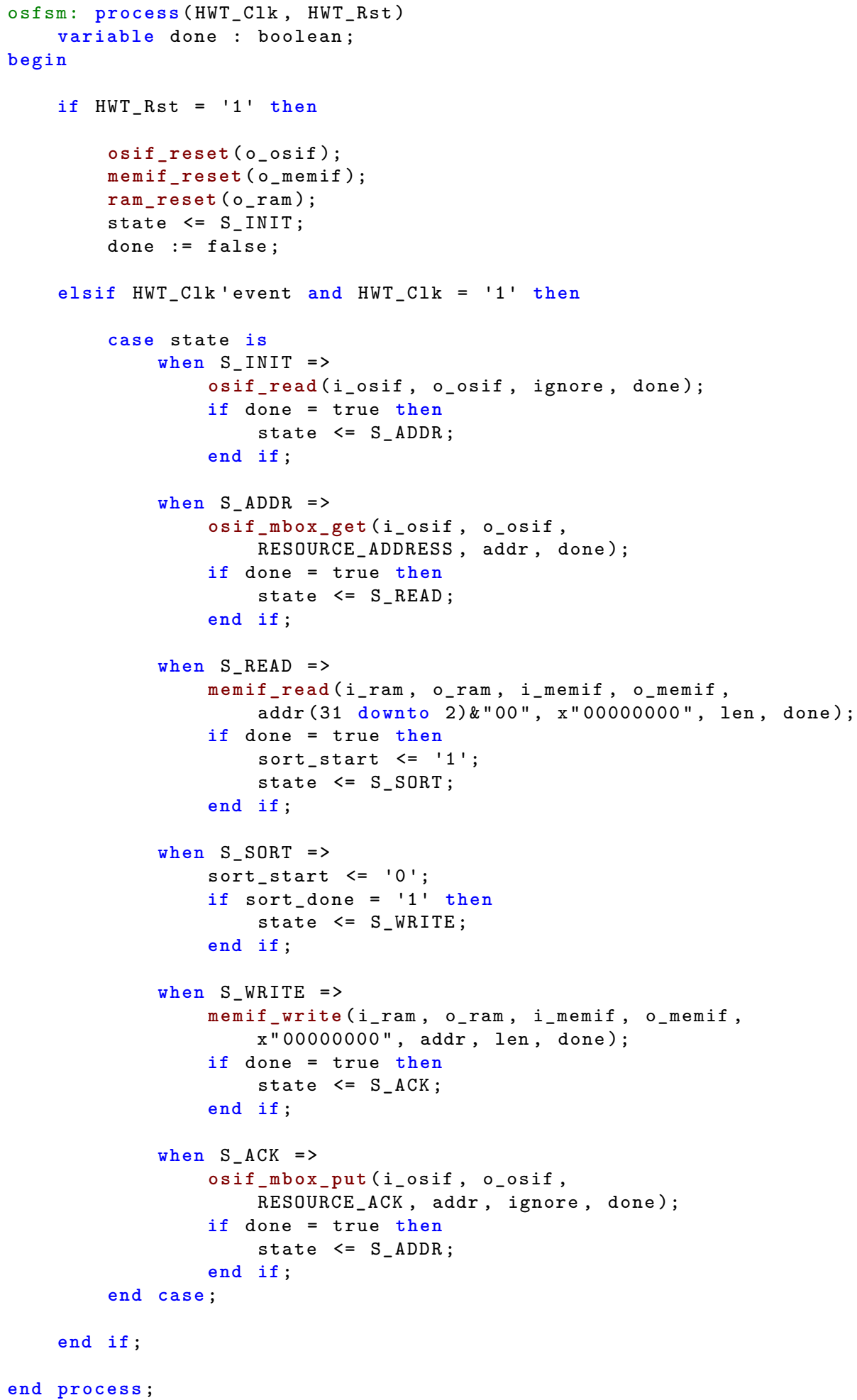


sors. The initial hardware/software abstraction layer was made fully portable for ReconOS v2.0 [Lübbers'09b], which supported eCos (for PowerPC processors) and Linux (for both PowerPC processors and MicroBlaze soft-core processors) in the same target FPGA devices. The initial Linux support relied on contiguous physical memory allocations to bypass virtual memory issues (i.e., hardware threads worked with physical memory addresses and software threads accessed the same physical memory using file mappings), but it was later extended to embed a custom MMU with a Translation Lookaside Buffer (TLB) in the hardware architecture, which effectively enabled a common virtual address space for both hardware and software threads [Agne'11]. ReconOS v2.0 also included dedicated FIFO channels on the FPGA fabric for optimized communication between hardware threads.

ReconOS v3.0 reshaped the hardware architecture to enable high modularity and supported both Linux and Xilinx Xilkernel running on soft-core MicroBlaze processors implemented on Xilinx Virtex-6 FPGAs. ${ }^{7}$ The ReconOS v3.1 revision [Agne'16] further modified the hardware architecture to simplify accelerator interfaces (e.g., removing FIFO channels for hardware threads) and to migrate the FPGA communication infrastructure from Processor Local Bus (PLB) to AXI. As a result, this release added support for Linux running on the ARM Cortex-A9 processors available in Xilinx Zynq7000 devices. ReconOS v3.1 also introduced a modular toolchain to ease hardware system generation from application and hardware/software thread specifications. Finally, ReconOS v4.0 migrated all its previous infrastructure to Xilinx Vivado, which adds support for HLS-driven hardware thread generation (previously only VHDL files were accepted as entry point for accelerator implementation). This is the most up-todate ReconOS release, and the one that has been used in this Thesis.

ReconOS has been also extended with DPR capabilities to support timemultiplexing of hardware tasks running on the FPGA fabric. Two different scheduling approaches for hardware threads have been analyzed and implemented in ReconOS: cooperative scheduling [Lübbers'09a] and preemptive scheduling [Happe'15]. ${ }^{8}$ Cooperative scheduling was implemented under eCos using a high-priority software thread as the hardware scheduler that, upon invocation, would schedule a hardware thread waiting for execution on an available reconfigurable slot (i.e., free or with a hardware thread that had previously called a yield function). In the event of no reconfigurable slots being available, the hardware scheduler would send a petition to yield to all hardware threads running on the FPGA. This cooperative scheduling mechanism avoided some expensive context save and restore operations in the reconfigurable hardware, since hardware threads called the yield function at points where state information was minimal. Notice, however, that all software threads (including the hardware scheduler) were still managed by the preemptive scheduler of the host OS.

\footnotetext{
${ }^{7}$ Xilinx Xilkernel was a highly customizable lightweight kernel with a POSIX API that was deprecated from software release v2017.1 onwards and replaced by FreeRTOS.

${ }^{8}$ In cooperative scheduling, threads voluntarily (i.e., without OS intervention) yield the CPU so that other threads can be executed. In preemptive scheduling, on the other hand, the OS actively interrupts threads in the running state to allow threads in the ready state to be executed.
} 
Preemptive scheduling, on the other hand, was implemented under Linux using a hybrid hardware/software thread as the scheduler. While the software component would be implemented as a user-space thread with a manually-defined scheduling policy, the hardware component would interface the FPGA ICAP to perform partial bitstream composition and to capture (task preemption) or restore (task resumption) the internal contents of all FFs and BRAMs present in a hardware thread (as opposed to the cooperative scheduling approach, in which each yielding thread would only use a few memory positions to save/restore its state). Due to its low-level technology dependencies (i.e., silicon primitives and configuration bitstream format), preemptive scheduling was only implemented on Xilinx Virtex-6 devices (as opposed to the cooperative scheduling approach, which is potentially portable across platforms).

\section{Evolvable Systolic Array}

Evolvable Hardware (EHW) stems from the combination of Evolutionary Algorithms (EAs) and reconfigurable hardware [Sekanina'04]. In particular, EHW applies evolutionary optimization techniques to electronic circuit design or even online circuit adaptation [Salvador'15]. Early works in the field can be found in [Higuchi'93], where a Generic Array Logic (GAL) device was used to obtain a 4-to-1 multiplexer through simulation-based evolution, ${ }^{9}$ and in [de Garis'93], which discussed the main technology limitations for EHW in its origins.

EAs are metaheuristic optimization algorithms with a population-based and stochastic nature based on Darwin's theory of evolution [Darwin'59]. In these algorithms, individuals from the actual problem/solution space (i.e., phenotype space) are encoded as chromosomes (i.e., genotype space), ${ }^{10}$ which are algorithm-friendly representations that can be further divided into genes. Evolution is guided by a fitness function (i.e., a quantitative measurement of how close an individual is to solving the problem at hand), and population diversity is enforced by means of basic genetic operations (i.e., recombination/crossover, which can create as many children as parents by randomly merging their genotypes, and mutation, which creates one child per parent by introducing random unbiased changes in its genotype). In general, EAs exhibit a common structure [Eiben'15]:

- Initialization: the algorithm generates an initial population of individuals and computes their fitness values. Both random and predefined individual values can be used to initialize the population.

- Iteration: the algorithm iteratively repeats several steps. Each algorithm iteration is called generation, and performs the following operations:

- Parent selection: several individuals are selected as parents, possibly based on their fitness value.

\footnotetext{
${ }^{9}$ GALs were developed by Lattice as an erasable and reprogrammable replacement for OTP PALs.

${ }^{10}$ The term "individual" is used interchangeably when referring to phenotype or genotype [Eiben'15].
} 
- Offspring generation: genetic operators are applied to these parents to obtain new individuals.

- Fitness evaluation: a quality measure is assigned to each genotype after evaluating its corresponding phenotype.

- Survivor selection: several individuals are promoted to the next generation, possibly based on their fitness value.

- Termination: the algorithm stops when an acceptable fitness value, a certain number of generations, or any other arbitrary termination condition is met.

There are four main types of EAs: Genetic Algorithms (GAs), Evolution Strategies (ESs), Evolutionary Programming (EP), and Genetic Programming (GP) [Eiben'15].

- GAs [Holland'92, De Jong'75] rely on binary encoded chromosomes, fitness proportional parent selection, generational survivor selection (i.e., all parents are replaced by their offspring), and use both recombination (1-point crossover) and mutation (bit flip), even though the former is favored over the latter.

- ESs [Rechenberg'73, Schwefel'93] rely on real-valued chromosomes, uniform random parent selection, deterministic survivor selection based on elitism (i.e., the fittest always survives), use both recombination (random gene selection or averaging) and mutation (Gaussian noise addition), and enable self-adaptation since algorithm parameters also evolve as part of the chromosome.

- A $(\mu+\lambda)$-ES uses $\mu$ parents to generate $\lambda$ children. Both parents and offspring are used in the survivor selection step.

- A $(\mu, \lambda)$-ES uses $\mu$ parents to generate $\lambda$ children. Only children are used in the survivor selection step.

- EP [Fogel'66] relies on real-valued chromosomes, deterministic parent selection (i.e., all individuals become parents), stochastic round-robin tournaments for survival selection $(\mu+\mu)$, and only uses mutation (Gaussian noise addition).

- GP [Koza'92] relies on tree structures that represent programs (usually written in functional languages) as chromosomes, fitness proportional parent selection, generational survivor selection, and use both recombination (subtree exchanges) and mutation (random changes in trees).

Independently of the specific EA used, circuit evolution in EHW systems can be either extrinsic or instrinsic [Sekanina'04]. In extrinsic evolution, individuals are evaluated using a model of the actual circuit, often within a simulation environment. Although this approach can be beneficial in scenarios where physical realizations of the electronic circuits are highly time-consuming and therefore unfeasible, extrinsic evolution can lead to unsatisfactory results if inaccurate models are used [Trefzer'15]. 
In intrinsic evolution, on the other hand, fitness evaluation is performed using physical hardware resources, which significantly improves evaluation times in most scenarios since actual hardware-based circuits are used instead of software-based models. However, intrinsic evolution is only affordable if circuit implementation is fast enough. In this regard, the use of DPR in SRAM-based FPGAs has been identified as a key enabling technology for intrinsic EHW systems [Mora'19a].

One of the most representative approaches in the area of digital circuit design using EHW techniques is Cartesian Genetic Programming (CGP) [Miller'99, Miller'00], which in fact appeared as a generalization of a digital circuit evolution method [Miller'98]. In CGP, individuals (i.e., programs) are expresed as DAGs whose computational nodes are structured in a 2D grid and usually perform simple operations. Chromosomes encode node functionality (i.e., function genes), node inputs (i.e., connection genes), and circuit outputs. CGP systems are configured using three user-defined parameters: the number of rows, the number of columns, and the maximum number of colums to the left from which a computational node can get its inputs. A key characteristic of CGP is that phenotypes can be of variable size, even though genotypes have a fixed length (a gene is still required to express an unused node/connection, even if that element is not present in the actual circuit represented by the chromosome).

Intrinsic evolution of CGP systems on FPGA devices has been already addressed in the past, and usually involves using Virtual Reconfigurable Circuits (VRCs) [Sekanina'11]. Although this implementation scheme provides really fast circuit evaluation, since all possible node functions and connections are always present on the reconfigurable fabric and can be easily modified using configuration registers, it does also increase area overheads while at the same time decreasing the maximum operating frequency of the circuit. Further developments have addressed these issues by using hybrid reconfiguration approaches with VRCs to change node connections and LUT-based DPR to change node functions [Dobai'15].

An alternative approach to implement EHW systems on FPGAs is to use Systolic Arrays (SAs) as the main architectural template [Salvador'11a]. SAs [Kung'78] are regular processing structures where each computational node has its outputs registered (hence the "systolic" nature) and is only connected to its direct neighbors. Although this last feature might seem restrictive, it actually solves most of the overhead problems of VRC-based CGP systems while at the same time providing pipelined processing for increased operation frequencies [Kung'84]. In addition, experimental evaluation has demonstrated that, for the same amount of FPGA resources and in the usual implementation range (i.e., $4 \times 8$ CGP [Dobai'15], $8 \times 8$ SA [Mora'18]), SAs provide better results than equivalent CGP structures [Mora'19b]. The main reason for this is that SAs are more resource efficient than CGP solutions and thus, more complex processing structures can be made using the same amount of logic resources.

In this Thesis, the improved SA-based EHW system from [Mora'18] has been used. This evolvable SA targets image processing applications, features intrinsic online evolution using fast LUT-based DPR, and proves some of the inherent features of EHW such as self-adaptation [Mora'13] and fault tolerance [Salvador'11b, Gallego' 13b, 
Gallego'13a]. Figure 3-3 shows a schematic view of a generic $3 \times 3$ implementation of the evolvable SA. Notice that, apart from the computational nodes marked as PEs, the architecture also features input/output data selectors and pipeline registers to achieve deterministic processing latency.

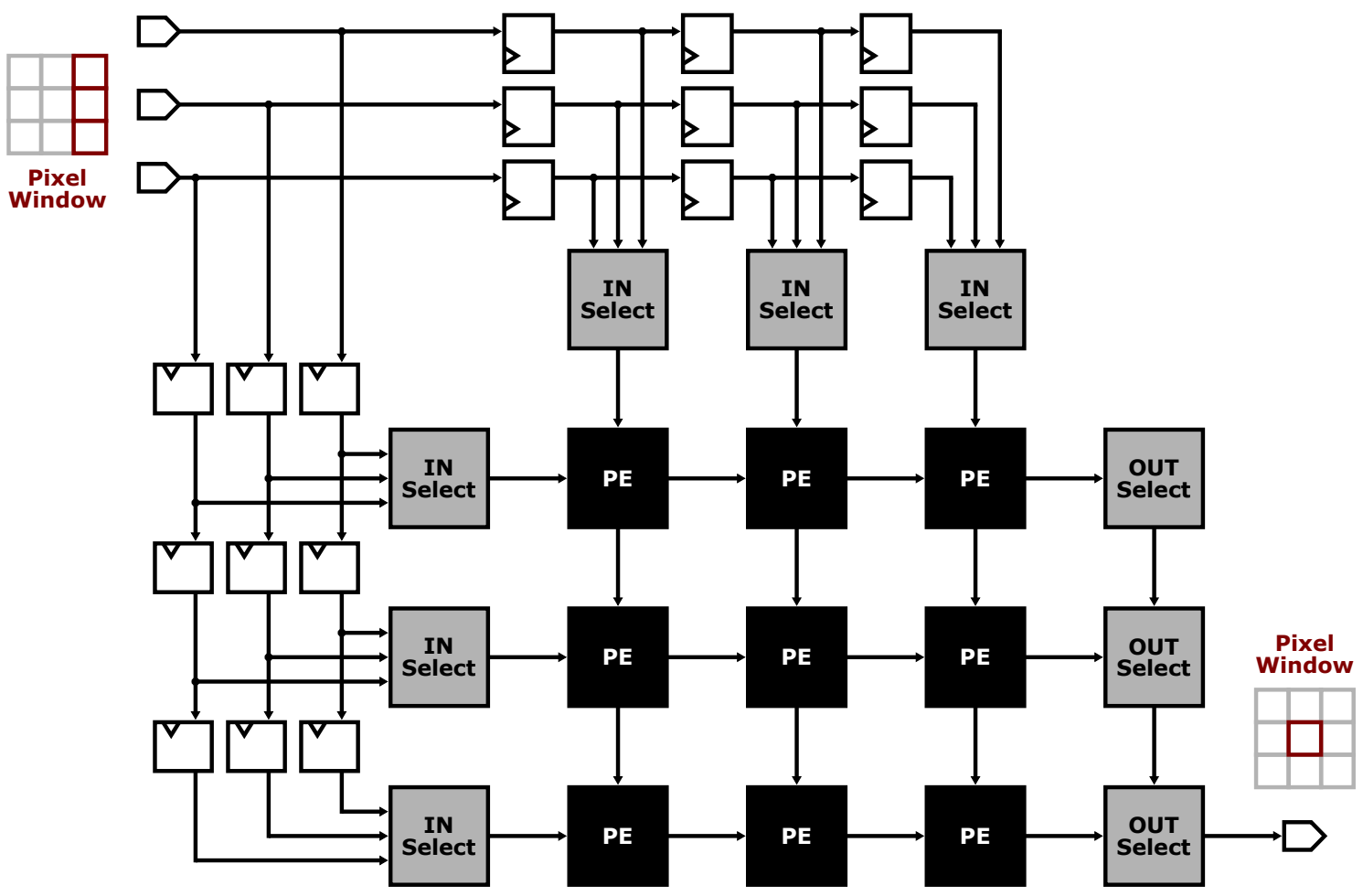

Figure 3-3: Evolvable SA $(3 \times 3)$.

The implementation of PEs and input/output selectors is highly technology dependent, since it relies on direct instantiation of FPGA primitives and annotation through VHDL attributes to ensure efficient DPR using specific placement constraints. In this regard, the evolvable SA system also includes an optimized reconfiguration engine to perform the LUT-based modifications that change both PE functionality and selector configuration. This generic reconfiguration engine for fine-grain DPR applications supports readback-free bitstream composition using the ICAP and BRAM-based storage [Mora'19a]. Figure 3-4 shows the internal structure (per bit) of PEs and input/output selectors, which include FFs to ensure systolic behavior.

Output selectors are implemented using 1 LUT per bit, and select the output from either the current SA row or from the previous one. The LUT behaves as a DPRcontrolled multiplexer. ${ }^{11}$ Input selectors, on the other hand, are implemented using 2 LUTs and 2 FFs per bit, and select one pixel from a $3 \times 3$ sliding window. Each LUT behaves as a DPR-controlled multiplexer, with the first one selecting the pixel row and the second one selecting the pixel column (using both FFs as a shift register or delay

\footnotetext{
${ }^{11}$ A DPR-controlled multiplexer has fixed inputs and its output is hardwired according to the contents of the configuration memory. In practice, it is not an actual multiplexer and thus it is called selector.
} 

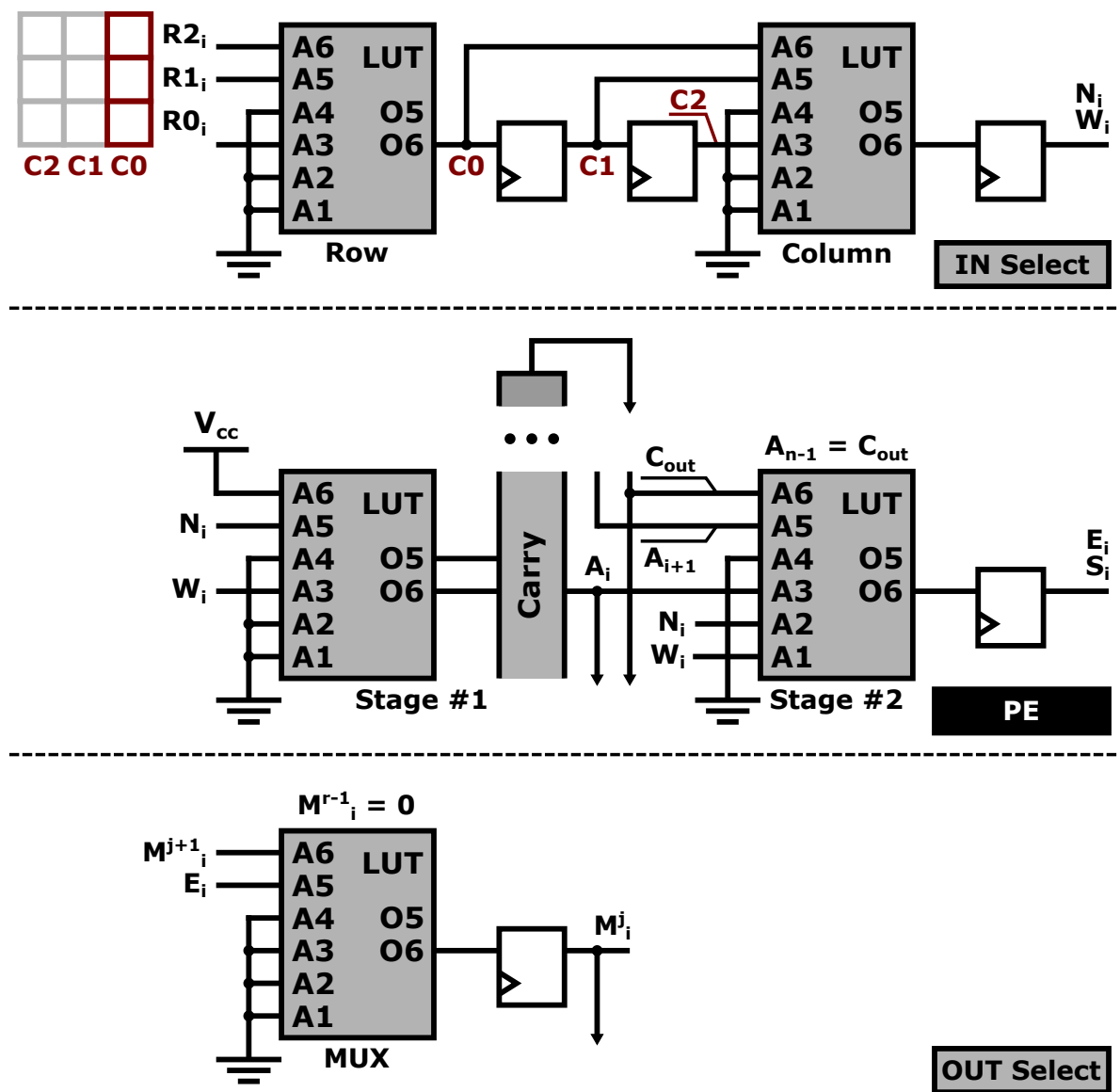

OUT Select

Figure 3-4: Internal components (per bit) of the evolvable SA.

line). Finally, PEs are implemented as a 2-stage structure using 2 LUTs and 1 shared carry chain per bit. In this case, the first stage (i.e., the first LUT and the carry chain) behaves as a 1-bit full adder with DPR-controlled inputs, ${ }^{12}$ whereas the second stage (i.e., the second LUT) behaves as a multiplexer with DPR-controlled inputs. ${ }^{13}$ For $n$ bit PEs, the first stage behaves as an $n$-bit full adder, and the most significant bit of its output (i.e., $n+1$ ) is used to control the multiplexer from the second stage. Please note that, being a common element, the output FF that ensures systolic behavior has been omitted from the previous descriptions. Table 3-2 shows the 2-stage PE structure with all its possible functions in the evolvable SA.

The SA architecture can be evolved using two custom GAs that do not support crossover. Hence, genetic diversity is introduced only by mutation. The mutation rate $K$ (i.e., random changes per chromosome) is configurable. In all cases, fitness proportional survivor selection is used, and evolution finishes when a configurable

\footnotetext{
${ }^{12}$ A full-adder with DPR-controlled inputs has a fixed structure and its inputs are hardwired according to the contents of the configuration memory.

${ }^{13}$ A multiplexer with DPR-controlled inputs has fixed control signals and its inputs are hardwired according to the contents of the configuration memory.
} 
Table 3-2: Available PE functions in the evolvable SA.

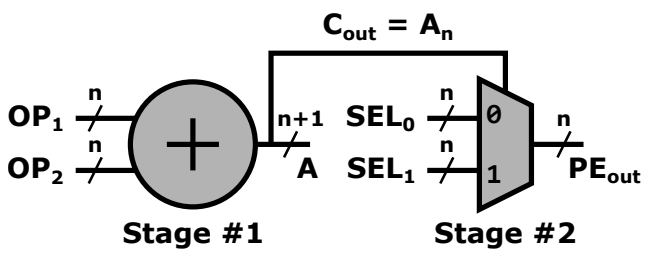

\begin{tabular}{|c|c|c|c|c|c|c|}
\hline$\#$ & Function & Description & $\mathbf{O P}_{1}$ & $\mathbf{O P}_{2}$ & $\mathrm{SEL}_{0}$ & SEL $_{1}$ \\
\hline 0 & $N+W \bmod 2^{n}$ & Addition (modulo $2^{n}$ ) & $N$ & $W$ & $A \bmod 2^{n}$ & $A \bmod 2^{n}$ \\
\hline 1 & $N+N \bmod 2^{n}$ & \multirow{2}{*}{ Multiply by 2 (modulo $2^{n}$ ) } & $N$ & $N$ & $A \bmod 2^{n}$ & $A \bmod 2^{n}$ \\
\hline 2 & $W+W \bmod 2^{n}$ & & $W$ & $W$ & $A \bmod 2^{n}$ & $A \bmod 2^{n}$ \\
\hline 3 & $\min \left(N+W, 2^{n}-1\right)$ & Addition (saturated to $2^{n}-1$ ) & $N$ & $W$ & $A \bmod 2^{n}$ & $\overline{0}$ \\
\hline 4 & $\min \left(N+N, 2^{n}-1\right)$ & \multirow{2}{*}{ Multiply by 2 (saturated to $2^{n}-1$ ) } & $N$ & $N$ & $A \bmod 2^{n}$ & $\overline{0}$ \\
\hline 5 & $\min \left(W+W, 2^{n}-1\right)$ & & $W$ & $W$ & $A \bmod 2^{n}$ & $\overline{0}$ \\
\hline 6 & $\left\lfloor\frac{N+W}{2}\right\rfloor$ & Average (rounded down) & $N$ & $W$ & $\frac{A}{2}$ & $\frac{A}{2}$ \\
\hline 7 & $2^{n}-1$ & Constant value & - & - & $\overline{0}$ & $\overline{0}$ \\
\hline 8 & $\left\lfloor\frac{N}{2}\right\rfloor$ & \multirow{2}{*}{ Divide by 2 (rounded down) } & $N$ & 0 & $\frac{A}{2}$ & - \\
\hline 9 & $\left\lfloor\frac{W}{2}\right\rfloor$ & & $W$ & 0 & $\frac{A}{2}$ & - \\
\hline 10 & $N$ & \multirow{2}{*}{ Identity (pass through) } & - & - & $N$ & $N$ \\
\hline 11 & $W$ & & - & - & $W$ & $W$ \\
\hline 12 & $\max (N, W)$ & Maximum & $N$ & $\bar{W}$ & $W$ & $N$ \\
\hline 13 & $\min (N, W)$ & Minimum & $N$ & $\overline{\bar{W}}$ & $N$ & $W$ \\
\hline 14 & $\max (N-W, 0)$ & \multirow{2}{*}{ Subtraction (saturated to 0 ) } & $\overline{\bar{N}}$ & $W$ & $\overline{A \bmod 2^{n}}$ & 0 \\
\hline 15 & $\max (W-N, 0)$ & & $\bar{W}$ & $N$ & $\overline{A \bmod 2^{n}}$ & 0 \\
\hline
\end{tabular}

number of circuit evaluations is reached. Upon termination, the individual with the best fitness value is selected as solution. The alternative EAs are:

- $(1+\lambda)$-GA: in each generation, $\lambda$ individuals are generated by mutating a single parent, and only the best individual among parent and offspring survives.

- (1+1)-GA with $\lambda$ parallel tribes: in each generation, 1 individual is generated by mutating a single parent, and only the best individual among parent and offspring survives. Optionally, a war mechanism can be triggered once every integer multiple of a configurable number of generations. This mechanism clones the best individual/tribe and kills the worst individual/tribe.

Circuit evaluation is performed using a memory-mapped accelerator that contains the evolvable SA architecture, local memory banks, and general-purpose registers. This 
accelerator is attached to the processor system in which the EA runs. During the circuit evaluation stage, LUT-based DPR is applied to translate a specific chromosome into its corresponding phenotype (i.e., input/output selector and PE configurations). Then, accelerator execution is triggered. This process reads an input image from local memory, generates a $3 \times 1$ window using line buffers, feeds the evolvable SA, and stores the processed image in local memory. In parallel, a reference image is also read from local memory and used together with the output of the evolvable SA to compute the fitness value as the Sum of Absolute Errors (SAE) (see Equation 3-1, where $h$ and $w$ represent the image height and width, respectively). Once computed, the fitness value is stored in a software-accessible general-purpose register. Additionally, another software-accessible register can be used to independently enable/disable certain accelerator features. For instance, storing the processed image in local memory can be disabled during evolution (only the fitness value is required), and computing the fitness value can be disabled during normal operation of an evolved circuit (the fitness value is no longer required after evolution).

$$
S A E=\sum_{i=0}^{h-1} \sum_{j=0}^{w-1}\left|o u t_{i, j}-r e f_{i, j}\right|
$$

Table 3-3 shows standard parameter values for the evolvable SA [Mora'18]. Optionally, mutations can be also restricted to a single column using another parameter, which significantly decreases reconfiguration times.

Table 3-3: Standard EA configuration in the evolvable SA [Mora'18].

\begin{tabular}{cc}
\hline Parameter & Value \\
\hline SA Rows & 8 \\
SA Columns & 8 \\
$K$ & 2 \\
$\lambda$ & 12 \\
Total Evaluations & 960000 \\
Generations/War & 1000 \\
\hline
\end{tabular}

\subsubsection{State of the Art}

Thread-based programming models for reconfigurable hardware have been widely studied in the literature. In fact, the multithreading programming model has been included even in some HLS tools as an additional entry point for hardware generation. For instance, LegUp [Canis'16] supports both Pthreads and OpenMP, and automatically generates hardware-based mutexes and barriers for concurrent thread synchronization. An additional feature of LegUp is that it allows designers to select between two different system generation flows: pure hardware and hybrid. In the pure hardware flow, the application code is transformed into a single hardware accelerator with a sequential module that triggers several parallel modules (i.e., one 
per thread) and eventually gathers their results. In the hybrid flow [Canis'13], on the other hand, LegUp uses the sequential parts of the application to generate software for an embedded hard- or soft-core processor, and only the parallel parts are synthesized into hardware modules (i.e., one per thread). In this scenario, the HLS engine automatically generates the required infrastructure for the target system as well, including interconnection modules for the hardware platform and application drivers to communicate the embedded software with the parallel hardware instances.

Despite these examples, research around HLS-based multithreading has been mainly focused on translating high-level input specifications (usually written in a software-oriented programming language) into hardware accelerators rather than on providing a unified programming model approach [Andrews'16]. Actually the optimal solution to bridge the gap between hardware and software multithreading is to complement the high-level syntax of a programming language, which eases hardware design in HLS-based environments, with the natural abstraction of hardware-specific details provided by a programming model [Andrews'04]. Apart from ReconOS, another representative framework that addresses programming model abstractions for reconfigurable computing is Hthreads [Andrews'08]. The Hthreads framework relies on a Pthread-compatible API, optional HLS-powered hardware generation, and an OS partially implemented in hardware (i.e., thread manager, thread scheduler, interrupt manager, mutexes, and condition variables are all implemented as hardware blocks on the FPGA fabric). An intermediate abstraction layer, called Hardware Thread Interface (HWTI), is placed between the system and the user-defined hardware thread. This additional hardware block allows accelerators to execute system calls (either directly or through a software thread if the operation is excesively complex), provides a load/store mechanism to access system memory, and features a local memory that can be used to implement a stack (with its associated push/pop operations) and a heap (with its associated malloc/free functions for dynamic memory management).

As it can be seen, a key aspect to implement a unified hardware/software programming model is to allow hardware accelerators to issue system calls as if they were regular software entities. In this regard, it is worth noting that this mechanism is not only available in Hthreads and ReconOS. For instance, the FPGAInitiated Software-Handled (FISH) framework [Nam'17] uses a Linux kernel module to communicate with different accelerators generated with LegUp. Since system calls (e.g., open, write, or close) are synthesized into empty Verilog modules, manual modifications are applied to include several control registers and the logic required to properly load them before notifying the Linux driver, which then reads those registers and executes the appropriate system call on behalf of the accelerator.

Additional examples of hybrid hardware/software multithreading approaches include FUSE [Ismail'11] and SPREAD [Wang'13]. Although both rely on Pthreadlike programming models under similar OSs, FUSE requires specific kernel drivers for each accelerator (as opposed to the unified and transparent abstraction provided by delegate threads in ReconOS) and SPREAD restricts the target space to streaming applications and does not support virtual memory (as opposed to the general-purpose 
multithreading approach provided by ReconOS). Moreover, none of them support rapid prototyping nor automated HLS-based hardware thread generation.

Regarding data-parallel programming models for FPGAs, OpenCL has become the de facto standard, and major FPGA vendors have promoted its usage by developing integrated frameworks with specific HLS support [Czajkowski'12]. As an example, both Intel (formerly Altera) and Xilinx provide OpenCL-based design flows currently. As most HLS tools, the Intel FPGA SDK for OpenCL and Xilinx Vitis (formerly SDAccel) use the LLVM compiler infrastructure [Lattner'04] to generate intermediate representations that are optimized with hardware-oriented directives prior to accelerator generation [Windh'15]. However, these commercial frameworks usually target HPC application scenarios [Zohouri'16, Muslim'17], which are out of the scope of this Thesis.

Nevertheless, OpenCL-related specifications have been also applied to the design and implementation of FPGA-based embedded systems. In particular, one of the most relevant examples is the HOpenCL parallel programmming model [Ding'14], which delves further into unified hardware/software programming models for reconfigurable computing. In HOpenCL, kernels can be executed either on software- (i.e., soft-core MicroBlaze processors) or hardware-based (i.e., HLS-based accelerators) compute units. Moreover, the associated on-chip architecture also provides support for hardware-assisted scheduling and synchronization. However, the programming model (which is similar to the one used in ARTICo $^{3}$ except for the explicit workitem management and a more complex API) is slightly different from the standard OpenCL specification, since it requires manual arrangements to execute kernels and only supports 2D data-parallel workloads.

In any case, the use of OpenCL in FPGA-based computing platforms is still a hot topic nowadays, and several works analyzing and discussing the best execution model (i.e., data-parallel versus deeply pipelined) for reconfigurable fabrics can be found in the most recent literature [Jiang'20].

As powerful as the multithreading and data-parallel programming models can be separately, their combination can significantly improve flexibility in the design stage and performance during the execution stage as well. This idea has been already evaluated in software-based computing systems that exploit multithreading programming for data preprocessing and data-parallel programming for core algorithm deployment [Díaz'12]. In addition, some OpenCL frameworks for software-based embedded computing systems rely on Pthread abstractions to implement the lowlevel layers of the compute devices (e.g., pocl [Jääskeläinen'15] or RuCL [Miyazaki'19]). However, at the moment of writing this Thesis and to the best of its author's knowledge, the combination of the multithreading (i.e., ReconOS) and data-parallel (i.e., ARTICo $^{3}$ ) programming models for reconfigurable hardware accelerators has never been addressed before. In fact, similar hardware-oriented frameworks such as Archborn [Ma'15b] or OOGen [Ding'16] force system designers to select either the multithreading programming model of Hthreads or the data-parallel programming model of HOpenCL. 


\subsubsection{Integration Details}

\section{ReconOS and ARTICo ${ }^{3}$}

Although their hardware architectures and programming models differ, the ReconOS and ARTICo $^{3}$ frameworks have a similar design methodology, since they both rely on a toolchain to automatically generate reconfigurable multi-accelerator systems. Therefore, the main integration effort has been focused on creating the common design-time support infrastructure shown in Figure 3-5.

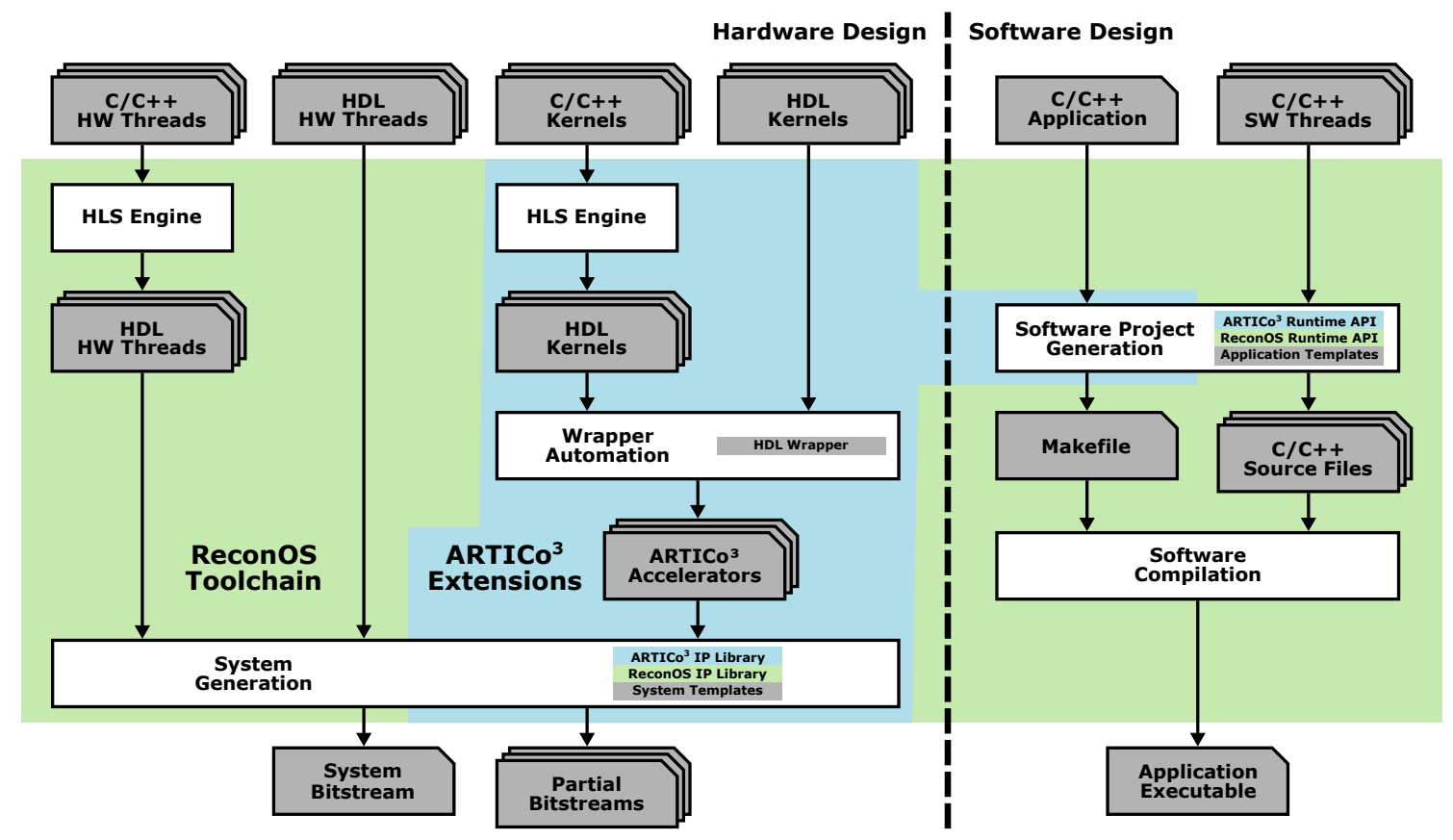

Figure 3-5: Integrated ReconOS-ARTICo ${ }^{3}$ toolchain.

To achieve such integration, two major modifications have been made to the original ReconOS toolchain. First, the platform-agnostic Python components have been extended to include the required functionality from the ARTICo ${ }^{3}$ toolchain, as well as all the associated software libraries (e.g., ARTICo ${ }^{3}$ runtime), IP repositories (e.g., ARTICo ${ }^{3}$ architecture), and design automation files (e.g., kernel wrapper, see Subsection 2.4.1). Second, a new custom hardware template has been also developed to include the target-dependent Tcl components that enable the generation of the integrated hardware/software architecture shown in Figure 3-6. As in any other ReconOS or ARTICo ${ }^{3}$ application, this template has to be selected in the project configuration file. In addition, the configuration file should also include the required information regarding ReconOS threads and ARTICo ${ }^{3}$ kernels.

Once the target hardware system has been implemented, the ReconOS and ARTICo $^{3}$ architectures coexist in the same FPGA fabric. To simplify the on-chip communication infrastructure, the ARTICo ${ }^{3}$ control interface is directly attached to the main ReconOS system bus. Notice that this approach does not affect the execution 


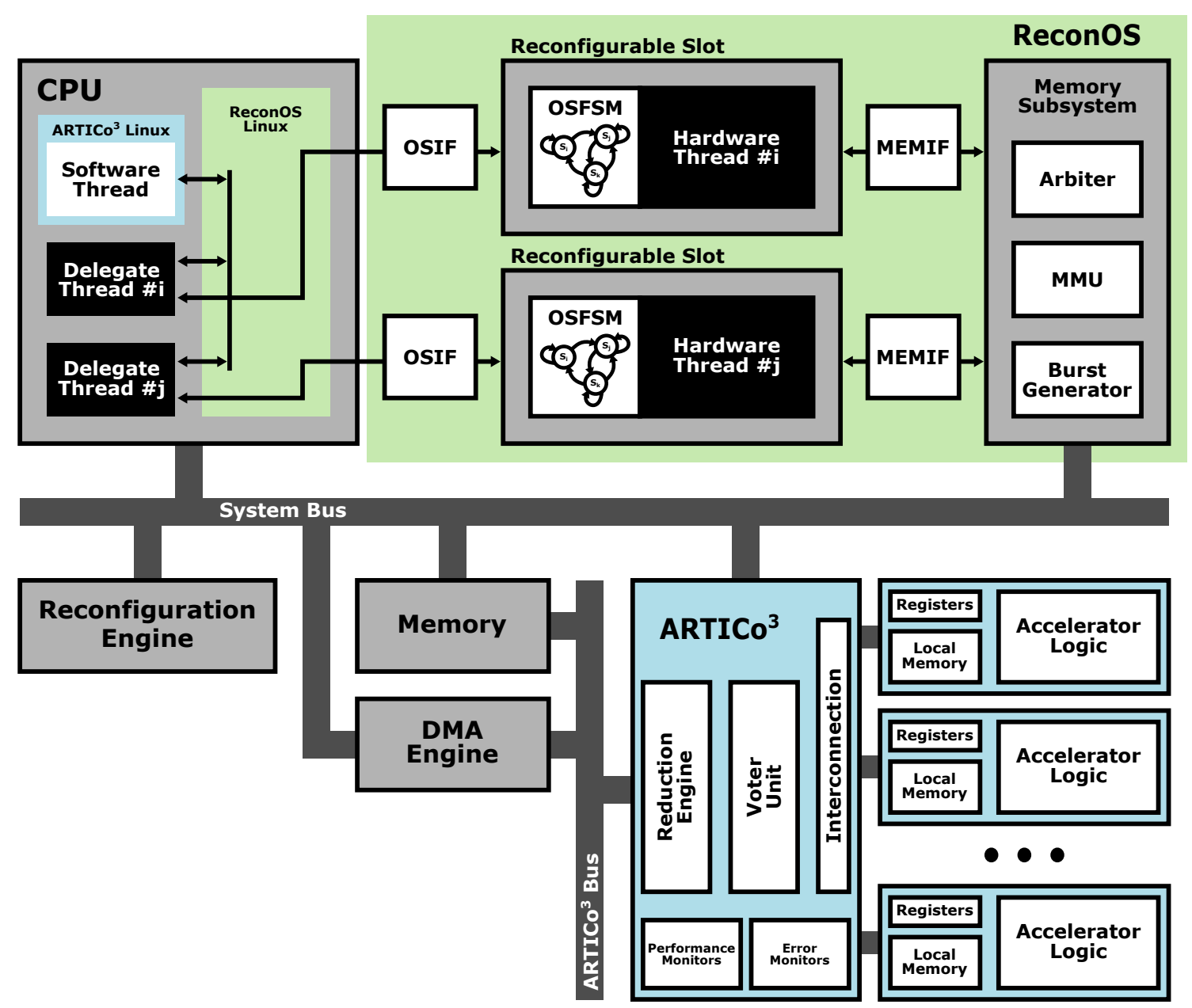

Figure 3-6: Integrated ReconOS-ARTICo ${ }^{3}$ architecture.

performance of hardware-based ReconOS threads, since the ARTICo ${ }^{3}$ control interface is only used to issue register-based configuration commands. ${ }^{14}$ However, an additional bus is still required to perform optimized burst-based data transfers between main memory and the different ARTICo ${ }^{3}$ kernel instances.

The integrated ReconOS-ARTICo ${ }^{3}$ infrastructure combines both runtime management libraries and APIs, and allows developers to deploy hardware-accelerated systems that exploit two different programming models: the data-parallel execution of $\mathrm{ARTICo}^{3}$ kernels, and the hardware/software multithreading of ReconOS accelerators.

Original contribution 3-1 A multi-paradigm programming approach for reconfigurable multi-accelerator systems that combines SIMD-like data-parallel execution with transparent hardware/software multithreading.

\footnotetext{
${ }^{14}$ In fact, ReconOS uses two separate buses (one to handle OSIF transactions and another to handle MEMIF transactions) when implemented on Zynq-7000 devices, due to their internal architecture. As a consequence, memory accesses from/to hardware threads are not affected at all in those setups.
} 


\section{Evolvable Systolic Array and ARTICo ${ }^{3}$}

The internal structure of the hardware accelerator used during the circuit evaluation stage in the evolvable SA system has a lot of elements in common with the ARTICo ${ }^{3}$ kernel wrapper. In fact, the integration of the SA core is straightforward and only requires minor modifications in the accelerator interfaces. Figure 3-7 shows an $8 \times 8$ evolvable SA implemented as an ARTICo $^{3}$ kernel instance. Notice that acceleratorspecific configuration (i.e., output image generation, fitness evaluation) and status (i.e., fitness value) information is accessible through the configurable register bank, and that image data are read from (i.e., input and reference images) or written to (i.e., output image) the configurable memory banks.

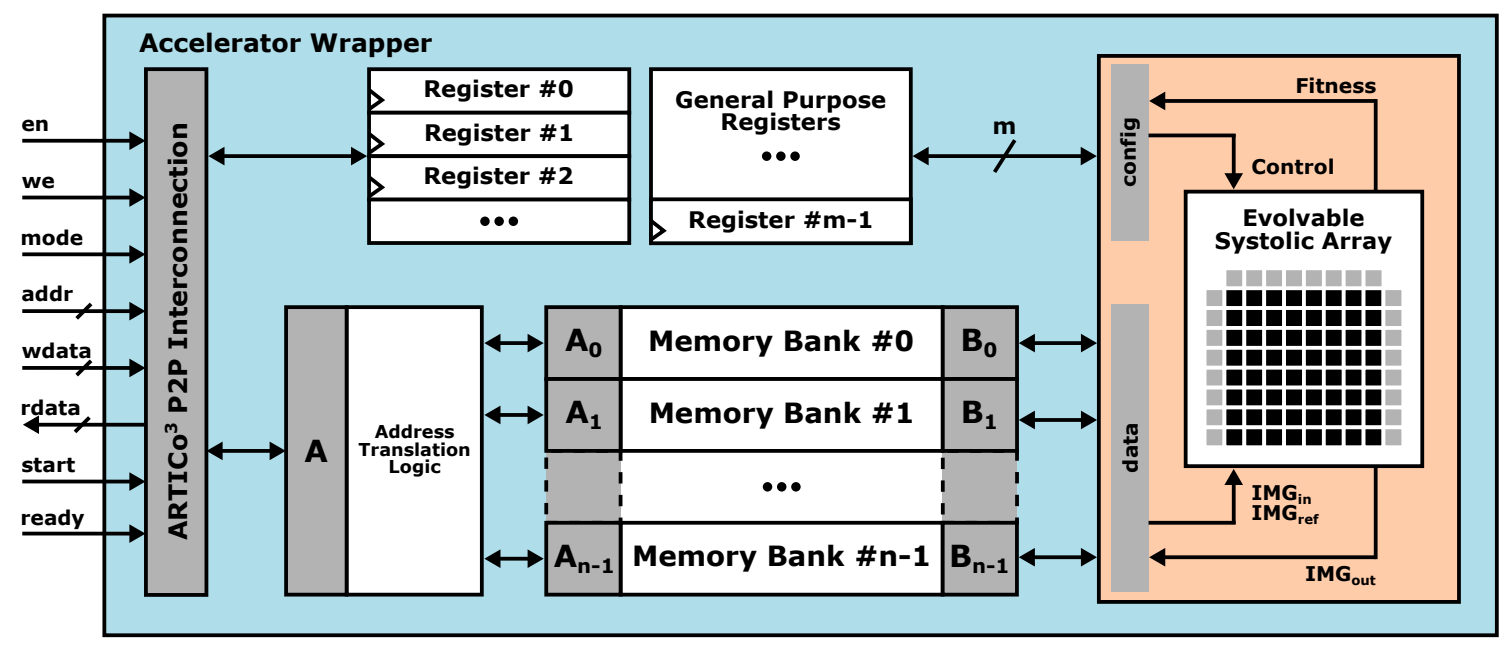

Figure 3-7: $\mathrm{ARTICo}^{3}$ kernel wrapper with an embedded evolvable SA core $(8 \times 8)$.

Nevertheless, the tight low-level restrictions of LUT-based reconfiguration represent a challenge at both design and run time. In this regard, a new custom hardware template for the ARTICo ${ }^{3}$ toolchain (see Subsection 2.4.3) has been developed. This template includes additional deployment scripts to perform SA relocation through the modification of the initial placement constraints (i.e., those specified with VHDL attributes), and an IP core repository with the optimized LUT-based reconfiguration engine from [Mora'19a]. Once deployed, the reconfigurable multi-accelerator system has access to two different FPGA configuration ports on Zynq-7000 devices (i.e., the PCAP for slot-based reconfiguration, and the ICAP for fast LUT-based reconfiguration) and thus, additional runtime library functions have been created to support timemultiplexed accesses to both.

Original contribution 3-2 A multi-grain reconfiguration mechanism for multi-accelerator systems that combines slot-based DPR for coarse-grained adaptation with LUT-based DPR for fine-grained adaptation. 


\subsubsection{Validation}

The integrated ReconOS-ARTICo ${ }^{3}$ framework has been tested in an image processing application scenario. The target algorithms are two image filters (a median filter for noise removal and a Sobel filter for edge detection), which are implemented as ReconOS threads using C-based descriptions (same code to generate both software and hardware threads, the latter using HLS). These descriptions manage filtering at image boundaries by replicating the required pixels (instead of performing zeropadding), and include several hardware-friendly components (e.g., line buffers) to produce more optimized accelerators. In parallel to the ReconOS threads, an evolvable SA is implemented as an ARTICo ${ }^{3}$ kernel and, even though it could be trained to potentially perform any arbitrary filtering operation, it is actually trained to replicate the behavior of the ReconOS-based filters (i.e., learn by imitation).

The experimental setup features 2 static hardware instances of the median filter thread, 2 static hardware instances of the Sobel filter thread, 4 reconfigurable ARTICo $^{3}$ slots to host an $8 \times 8$ SA each, and has been implemented on a Pynq board. ${ }^{15}$ Notice that each SA kernel instance can be used to learn and/or execute a given functionality independently (task-level parallelism) or cooperatively (data-level parallelism). Table 3-4 shows the resource utilization reports for the different system components.

Table 3-4: Resource utilization in the multithreading scenario.

\begin{tabular}{|c|c|c|c|}
\hline Componen & ReconOS & Median Thread & Sobel Thread \\
\hline Info & $\begin{array}{c}4 \text { threads } \\
\text { VHDL (Vivado) } \\
\text { Zynq-7000 }\end{array}$ & $\begin{array}{c}\text { C + HLS (Vivado) } \\
\text { Zynq-7000 }\end{array}$ & $\begin{array}{c}\text { C + HLS (Vivado) } \\
\text { Zynq-7000 }\end{array}$ \\
\hline LUTs & 3088 & 3645 & 2963 \\
\hline FFs & 2316 & 3362 & 2445 \\
\hline DSPs & - & - & - \\
\hline BRAMs & - & 8 & 8 \\
\hline Component & ARTICo $^{3}$ & ICAP Controller & $8 \times 8$ SA Kernel \\
\hline Info & $\begin{array}{c}4 \text { slots } \\
\text { VHDL (Vivado) } \\
\text { Zynq-7000 }\end{array}$ & $\begin{array}{c}\text { VHDL (Vivado) } \\
\text { Zynq-7000 }\end{array}$ & $\begin{array}{c}\text { KiB memory, } 3 \text { banks } \\
2 \text { registers } \\
\text { VHDL (Vivado) } \\
\text { Zynq-7000 }\end{array}$ \\
\hline LUTs & 4158 & 1020 & 6682 \\
\hline FFs & 2366 & 558 & 2436 \\
\hline DSPs & - & - & - \\
\hline BRAMs & - & 16 & 12 \\
\hline
\end{tabular}

This section will first evaluate the execution performance of the ReconOS threads versus the evolved ARTICo ${ }^{3}$-based solutions (which exhibit the same performance independently of the actual functionality configured in the SA), and then will analyze

\footnotetext{
${ }^{15}$ XC7Z020-1CLG400.
} 
the results of the learn-by-imitation process using the ARTICo ${ }^{3}$ SA kernel. In this regard, it is worth noting that the nature of the ReconOS thread does not affect the obtained results and therefore, both hardware- and software-based implementations can be used to generate the reference images for the evolutionary process. The same platform configuration has been used for all tests (dual core ARM Cortex-A9 @ 666.67 MHz, FPGA fabric @ $100 \mathrm{MHz}$ ). In addition, the EA configuration shown in Table 3-3 has been used for the evolution-related tests (unless stated otherwise).

\section{Performance Evaluation}

Table 3-5 shows the execution times of the filtering process using different configurations of accelerators and input image sizes. ReconOS threads have been configured to either process the whole image using one accelerator ("Full Image" configuration), or to exploit data-level parallelism using image partitioning in blocks of $128 \times 128$ pixels. Execution times of both hardware and software versions of the threads are provided. ARTICo ${ }^{3}$-based execution is always configured to exploit datalevel parallelism using the same partitioning approach as in ReconOS threads. As it can be seen, ReconOS threads are computing-bounded, and render better performance results when taking advantage of data-level parallelism (the differences when using a single thread are almost negligible when compared with the "Full Image" scenario). In fact, a speedup value of almost $2 \times$ is achieved when the number of threads is doubled. The SA kernel, on the other hand, has a marked memory-bounded behavior, showing no benefits when increasing the number of kernel instances. As a result, ARTICo $^{3}$ powered hardware redundancy can be added for free to enhance fault tolerance during filter execution. In addition, the SA kernel outperforms the hardware-based ReconOS threads for sufficiently large images, even when using a single instance.

Table 3-5: Execution times in the multi-paradigm programming scenario.

\begin{tabular}{|c|c|c|c|c|c|c|}
\hline \multirow{2}{*}{ Configuration } & \multirow{2}{*}{ Threads } & \multicolumn{5}{|c|}{ Execution Time (ms) - HW (SW) } \\
\hline & & $128 \times 128$ & $256 \times 256$ & $512 \times 512$ & $1024 \times 1024$ & $2048 \times 2048$ \\
\hline \multirow{3}{*}{ Median } & Full image & $6.66(12.13)$ & $8.21(26.04)$ & $31.94(103.23)$ & $126.01(415.45)$ & 499.65 (1711.35) \\
\hline & 1 & $2.18(6.55)$ & $8.55(25.83)$ & $34.6(103.12)$ & $137.97(411.68)$ & $551.73(1646.14)$ \\
\hline & 2 & $2.16(6.52)$ & $4.75(13.13)$ & $20.6(52.16)$ & $81.56(213.35)$ & $325.6(828.45)$ \\
\hline \multirow{3}{*}{ Sobel } & Full image & $1.7(0.9)$ & $6.18(3.48)$ & $24.11(14.7)$ & $94.5(62.66)$ & $373.7(291.36)$ \\
\hline & 1 & $1.62(0.88)$ & $6.56(3.52)$ & $26.67(14.02)$ & $106.24(55.46)$ & 424.09 (221.19) \\
\hline & 2 & $1.62(0.89)$ & $3.93(1.88)$ & $16.87(7.19)$ & $65.88(31.04)$ & 263.33 (116.39) \\
\hline \multirow{2}{*}{ SA } & 1 & 1.68 & 7.47 & 16.36 & 64.91 & 257.57 \\
\hline & 4 & 4.82 & 4.76 & 15.53 & 46.62 & 184.33 \\
\hline
\end{tabular}




\section{Online Learning Evaluation}

This section will cover the following issues: (i) performance scalability when changing the number of kernel instances during evolution, (ii) fitness evolution while performing online training using software-based reference algorithms, and (iii) system self-adaptation when changing the environmental conditions.

Table 3-6 shows the mean evolution times when changing the number of SA kernel instances used in parallel during the circuit evaluation stage and the total number of circuit evaluations. As it can be seen, the number of evaluations per second remains constant independently of the termination condition defined, but scales almost linearly with the number of parallel SAs used $(4 \times$ accelerators, $3 \times$ speedup). In a context where run-time adaptation is mandatory (e.g., the filtering algorithm is no longer valid, the noise level in the imaging sensor changes), the ARTICo ${ }^{3}$-based evolvable system can be used to automatically change the operation mode of the different SA kernel instances between "evolving" and "filtering", which will affect the speed of the evolutionary process but not the overall system functionality nor the ARTICo $^{3}$-based execution time (due to the memory-bounded nature of the SA kernel).

Table 3-6: Evolution times using the ARTICo ${ }^{3}$ SA kernel.

\begin{tabular}{ccc}
\hline \multirow{2}{*}{ SA Instances } & \multicolumn{2}{c}{ Evolution Time (s) } \\
& 96k Generations & 960k Generations \\
\hline 1 & $31.92(\sim 3 \mathrm{k}$ evals $/ \mathrm{s})$ & $318.32(\sim 3 \mathrm{k} \mathrm{evals} / \mathrm{s})$ \\
2 & $17.69(\sim 5.5 \mathrm{k} \mathrm{evals} / \mathrm{s})$ & $175.41(\sim 5.5 \mathrm{k} \mathrm{evals} / \mathrm{s})$ \\
4 & $10.72(\sim 9 \mathrm{k} \mathrm{evals} / \mathrm{s})$ & $103.44(\sim 9 \mathrm{k}$ evals $/ \mathrm{s})$ \\
\hline
\end{tabular}

Figure 3-8 reports the fitness values during the evolutionary process when learning by imitation from the median filter, the Sobel filter, and the chain of median filter plus Sobel filter. Evolution uses images of $128 \times 128$ pixels, $5 \%$ salt-and-pepper noise (whenever a median filter is involved), and 96k generations (top) or 960k generations (bottom). Although it is clear that a larger number of generations usually leads to better fitness values, a reduced number of generations can prove beneficial due to the good tradeoff between evolution time and filter accuracy, especially in systems where adaptation needs to comply with real-time requirements. Figure 3-9 shows the input and reference images (the reference being the output of the corresponding ReconOS threads) used during evolution, and the output image obtained after evolution.

Once trained, the evolved filters can be continuously used as long as the environmental conditions or the desired functionality remain unchanged. Figure 3-10 shows the results of applying the evolved filters to several images of multiple sizes (different from those used during the training process) using an image partitioning approach with blocks of $128 \times 128$ pixels. Note that, although the original ReconOS threads were capable of handling image boundaries, the evolvable SA kernel has some difficulties when processing those singular pixels. However, this is not a real problem, since it can be easily solved by applying software-based row/column replication prior to kernel execution (i.e., process overlapping image segments). It is also worth 

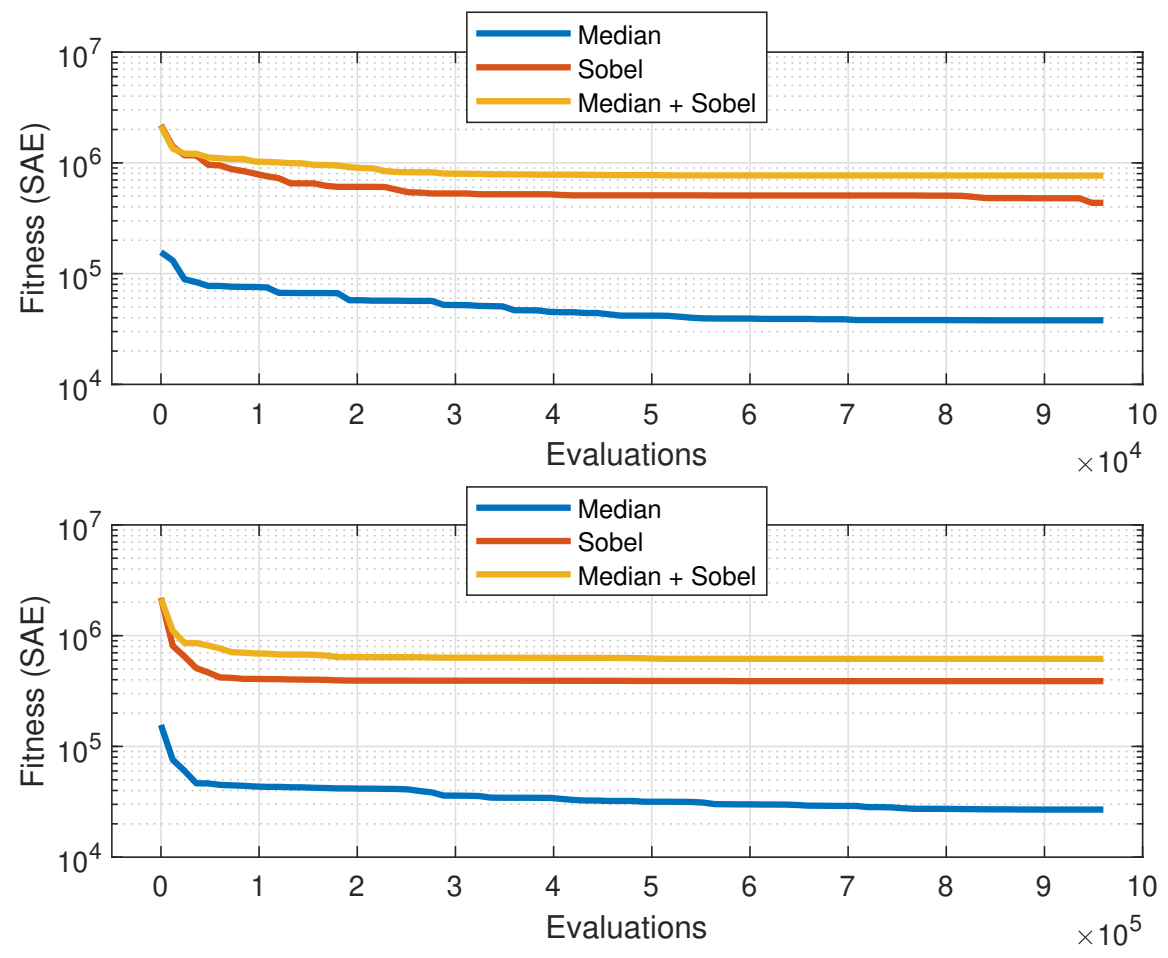

Figure 3-8: Fitness evaluation during evolution.

noting that, with the current configuration of the evolutionary process, the chain of median filter plus Sobel filter is harder to learn from than the isolated filters. However, the slightly worse results are compensated by the improved execution time (both operations are applied simultaneously in a hardware accelerator with better performance).

Finally, Figure 3-11 shows an scenario in which online retraining is issued after a sudden change in the environmental conditions. In particular, the application timeline starts with an initial training stage in which the evolvable SA learns from a perfect filter against 5\% salt-and-pepper noise using $4 \mathrm{ARTICo}^{3}$ kernel instances in roughly 10 seconds. Then, the evolved filter is loaded on a single kernel instance and executes for around 10 seconds. At this point, the input noise changes to a $15 \%$ burst noise, having a great impact in the fitness of the running filter. Hence, the remaining $3 \mathrm{SA}$ kernel instances are put to evolve from another perfect filter reference while the filter continues working on different input data, never actually stopping (i.e., the learning and execution stages are overlapped). After approximately 12 seconds (evolution time is larger since less SA kernel instances are used), the evolutionary process finishes, and the running filter is updated with the retrained filter. It is important to highlight that the same SA retraining scheme can be performed whenever a different filtering algorithm is required. 

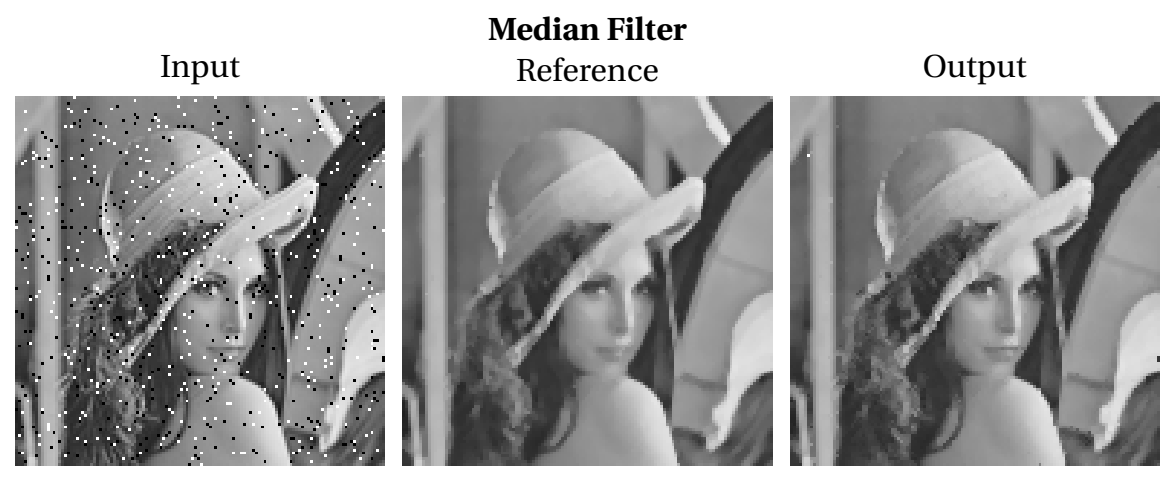

Input

Sobel Filter Reference
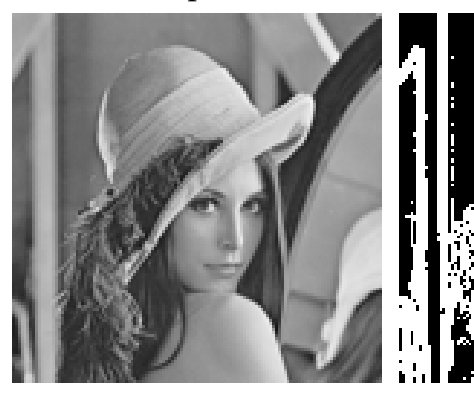

Output
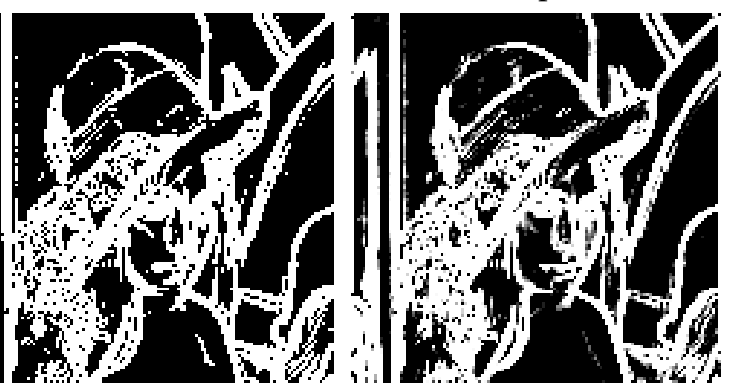

Median + Sobel Filter

Input

Reference

Output
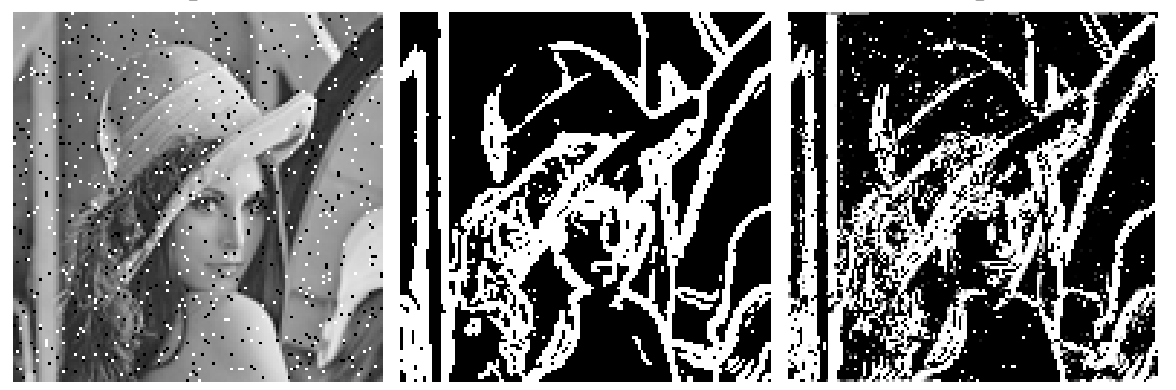

Figure 3-9: Evolution results for different filter types. 

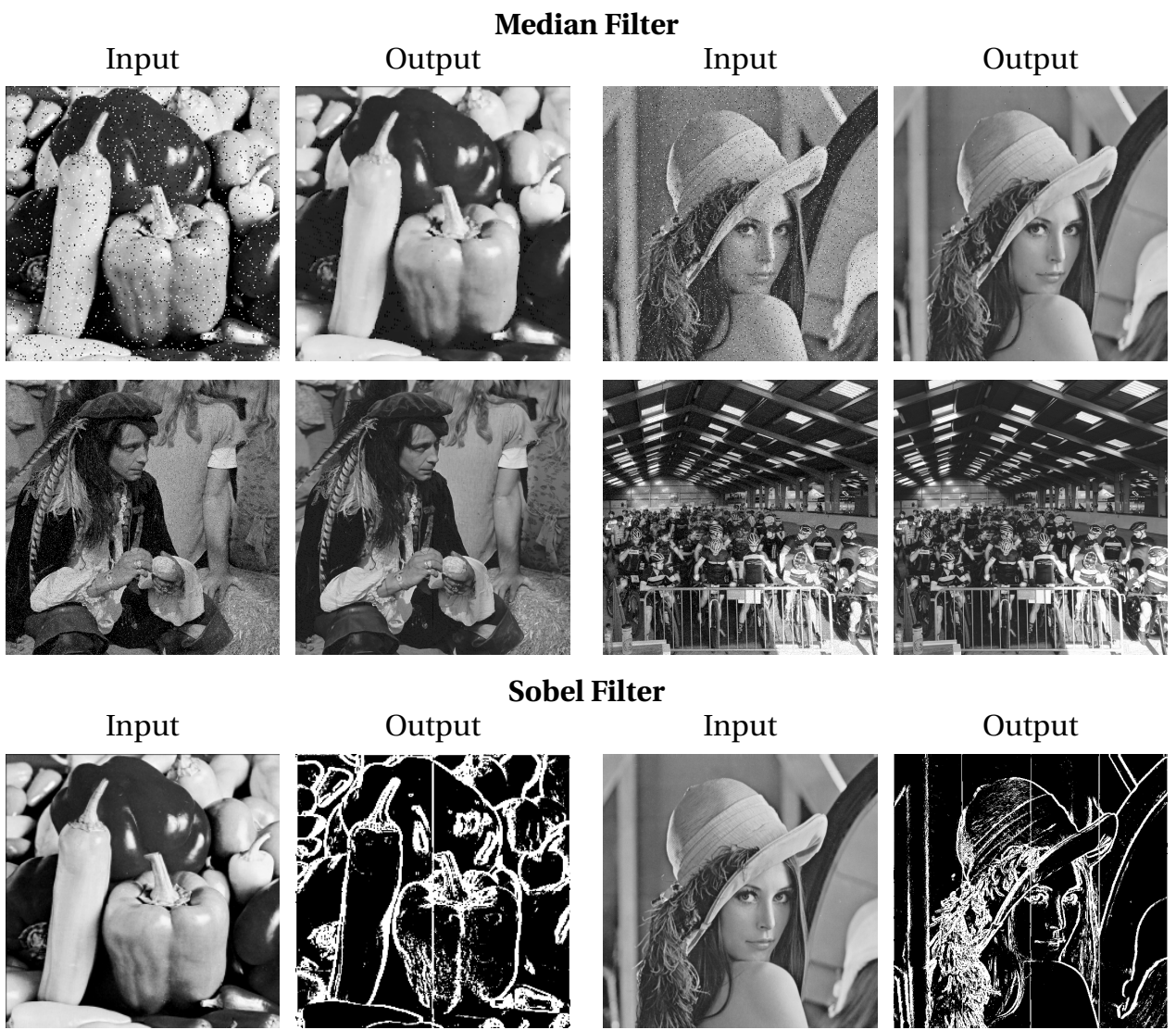

Sobel Filter

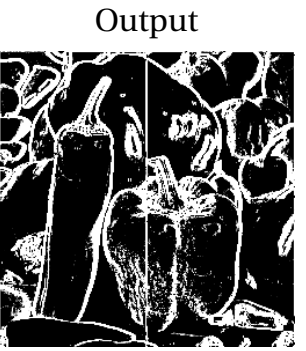

Input

Output
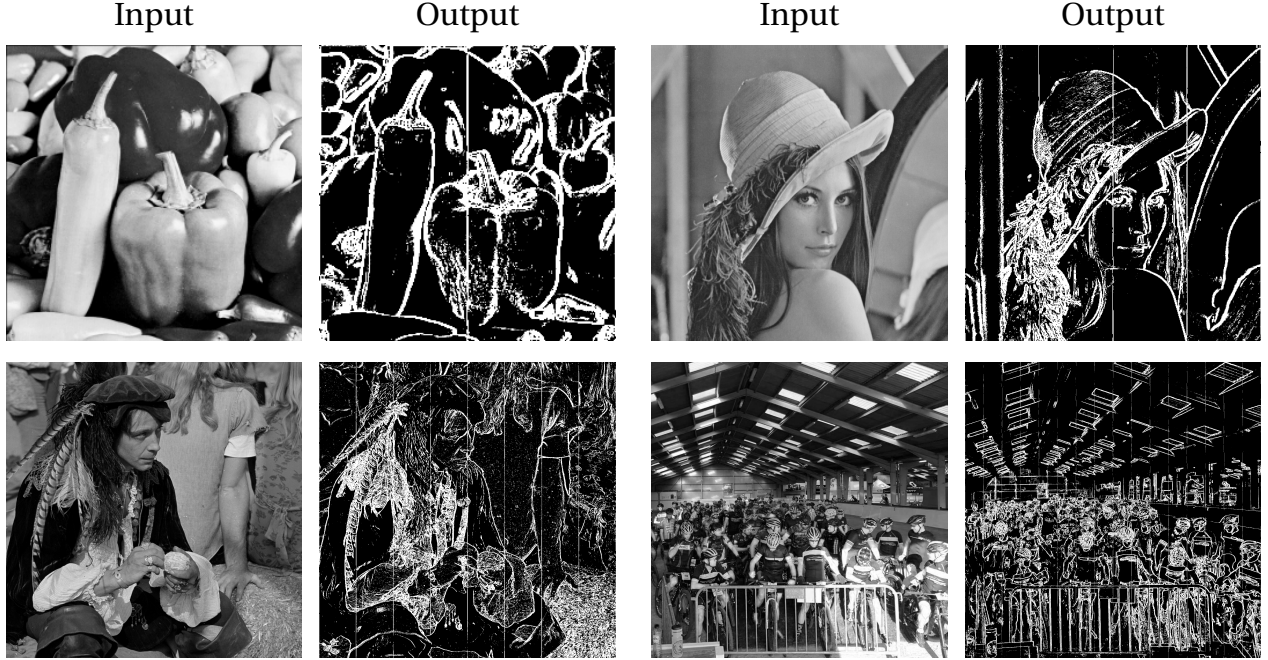

Input

Median + Sobel Filter

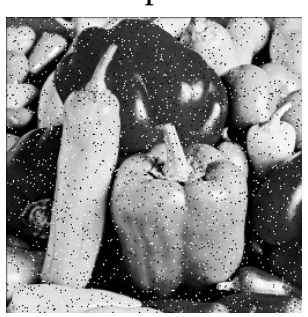
Output
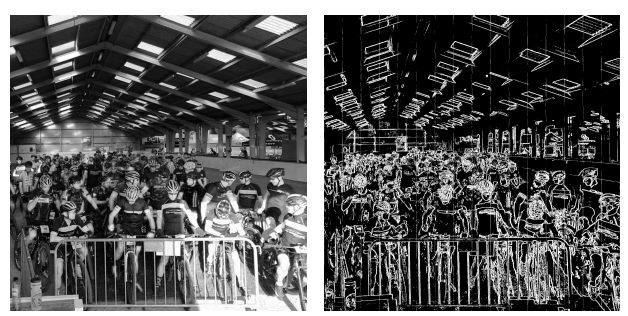

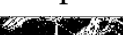

Input

Output
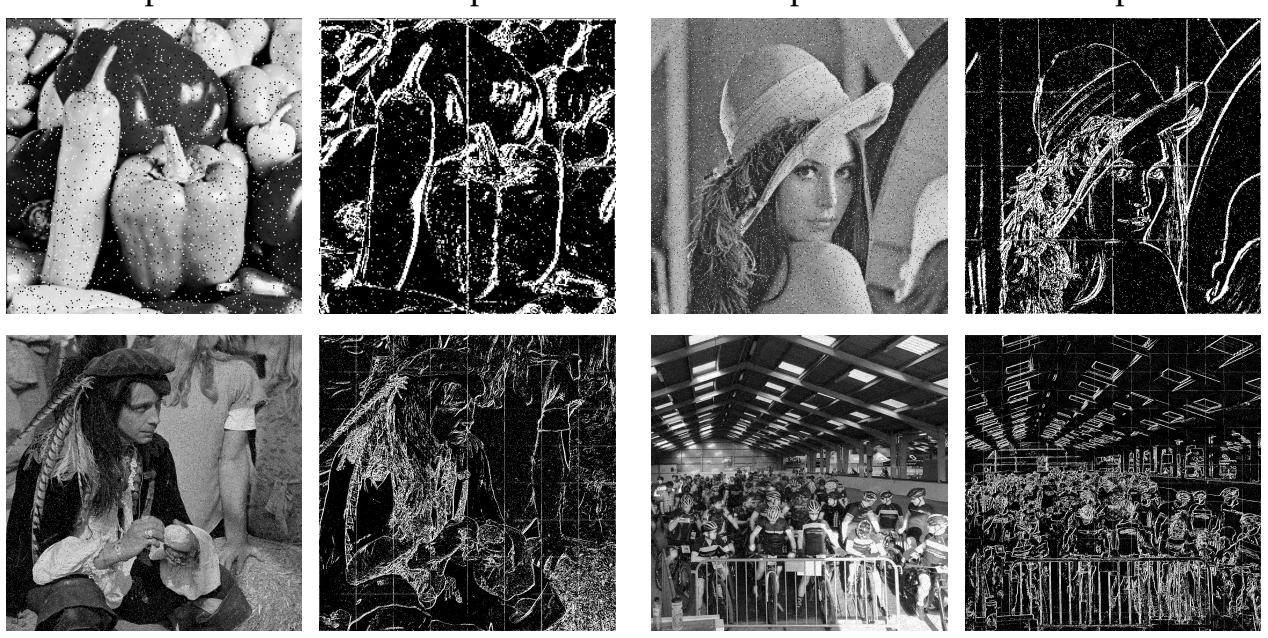

Figure 3-10: Evaluation of the evolved filters for different input images. 
5\% Salt \& Pepper

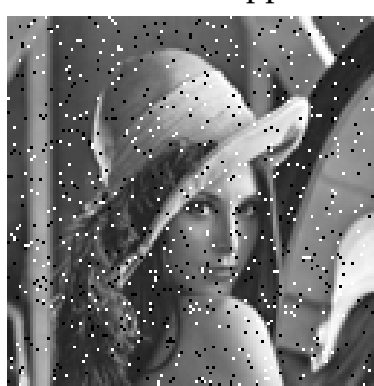

$15 \%$ Burst

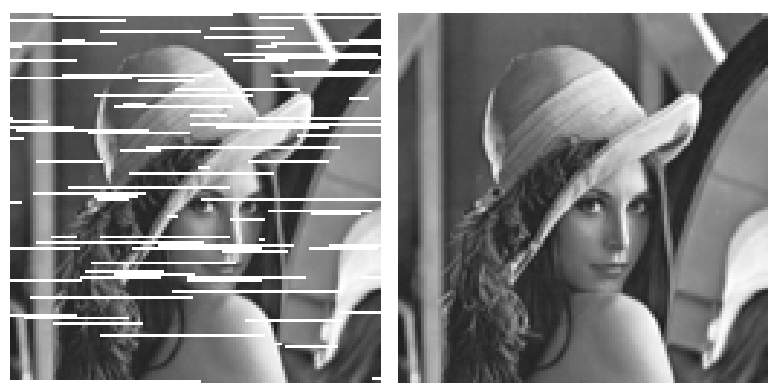

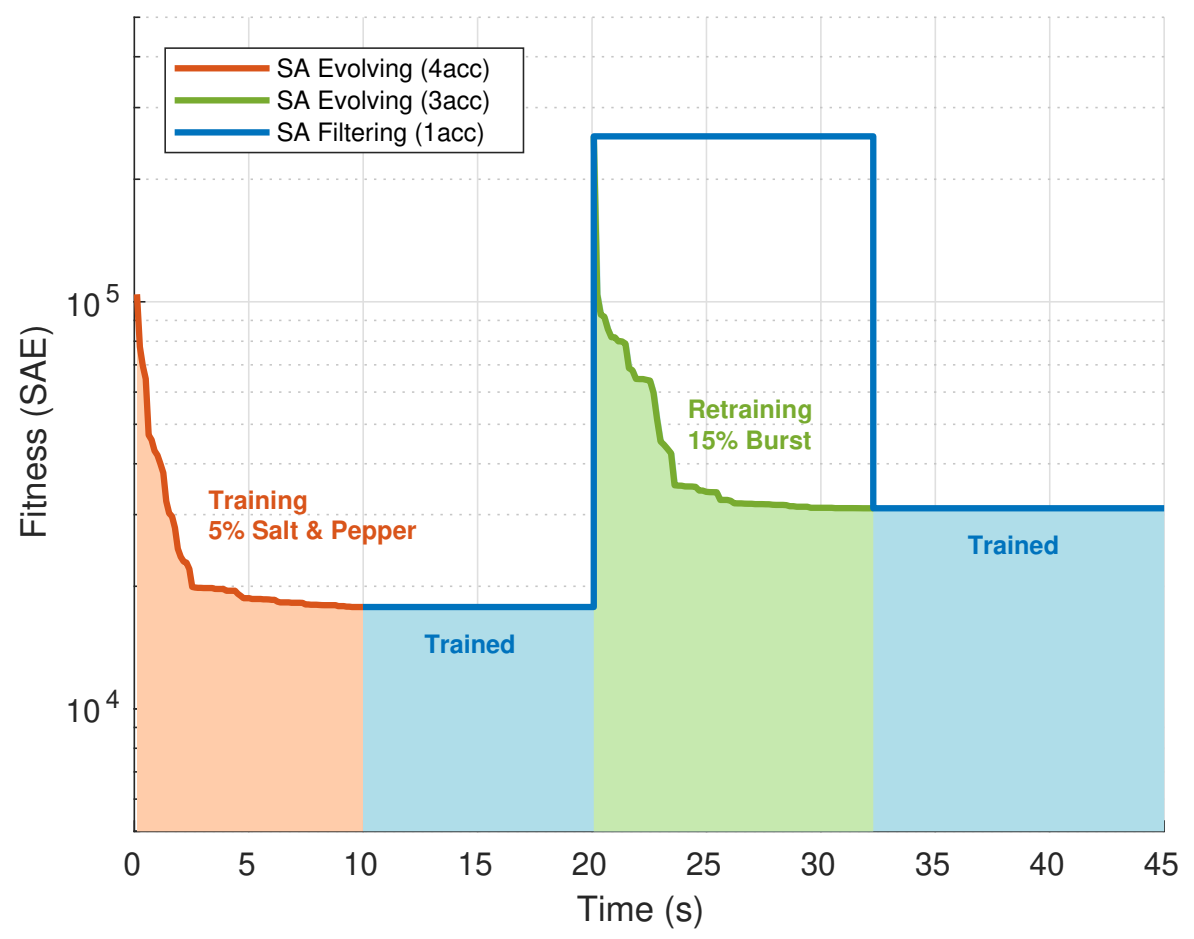

Figure 3-11: Online training for environmental adaptation. 


\subsection{Dataflow Extensions}

Dataflow systems fall within the data-driven computing paradigm, in which programs are executed according to input data availability rather than relying on controloriented constructs [Treleaven'82]. This approach has been around for a long time, and a lot of early works exploring the concept of dataflow-based programming and/or processing architectures can be found in the literature [Dennis'74, Dennis'80, Watson'82, Davis'82, Ackerman'82].

Dataflow programs are usually expressed as application graphs with nodes that represent functions/processes/tasks, and directional links between nodes that represent communication channels through which data items flow [Davis'82]. This approach is parallel in nature, since actors can be executed concurrently as long as their input data are available, even if their individual execution is sequential.

Several Models of Computation (MoCs) have been proposed throughout the years to describe dataflow systems. One of the early-stage examples are Kahn Process Networks (KPNs) [Kahn'74]. In KPNs, graph nodes are called processes. Processes execute sequentially and are connected using unbounded one-way FIFO channels. The model enables task-level parallelism by assuming that several actors might be executing simultaneously. In addition, it is also assumed that each data object is produced and consumed exactly once, with non-blocking write operations and blocking read operations (i.e., processes accessing an empty input channel stall until the communication link has enough data to perform the read operation), respectively.

Dataflow Process Networks (DPNs) [Lee'95], on the other hand, formalize several widely known definitions for dataflow-based computing. In DPNs, graph nodes are called actors (i.e., processes), and data items flowing through the graph are called tokens. Moreover, actor execution is called firing, and maps input tokens (i.e., consumed data) into output tokens (i.e., produced data). A set of conditions, called firing rules, controls whether an actor can be executed (i.e., fired) or not. In other words, firing rules establish which data need to be available for a given actor to execute. DPNs are considered to be a special case of KPNs under certain circumstances, ${ }^{16}$ but they also support non-determinism (i.e., several firing rules can be satisfied at the same time [Yviquel'14]).

There are other alternative MoCs to describe dataflow systems, but a significant number of them can be seen as simplifications of DPNs. For instance, Synchronous Data Flow (SDF) networks [Lee'87] assume that the number of tokens consumed and produced by all actors in a graph is constant in each firing. This feature enables the static scheduling (i.e., at compile time) of SDF application graphs, which are very extended in signal processing scenarios.

The use of dataflow MoCs and actor-oriented specifications can enable the model-

\footnotetext{
${ }^{16}$ Sequential firing rules (i.e., firing rules that can be tested sequentially using only blocking reads) and functional actors (i.e., actors whose output tokens only depend upon their input tokens) [Lee'95].
} 
ing, simulation, and design of concurrent real-time embedded systems, and several frameworks have been proposed in this regard, being the Ptolemy project [Lee'98, Eker'03] one of the most relevant examples. Moreover, some of these frameworks also provide support for system generation using reconfigurable hardware as a target. One example of such frameworks is Open Dataflow (OpenDF) [Bhattacharyya'09], which relies on a dataflow- or actor-oriented programming language called CAL Actor Language (CAL) [Lucarz'08] (also developed as part of the Ptolemy project).

CAL is used to describe algorithms using encapsulated dataflow components (i.e., actors), each of them having its own internal state. Since actor interaction in CAL is based on data flowing through input and output ports, the internal state of a given actor cannot be accessed nor changed by any other actor in the application graph. Moreover, the behavior of an actor in CAL is defined by a set of actions, which include operations such as reading from input ports (i.e., consuming tokens), changing the internal state of the actor, or writing to output ports (i.e., producing tokens). Although actors can be executed concurrently at graph level, actions are executed sequentially, provided that their firing conditions (i.e., availability and value of input tokens, internal state, action priority) are met. Figure 3-12 shows the generic structure of a CAL actor.

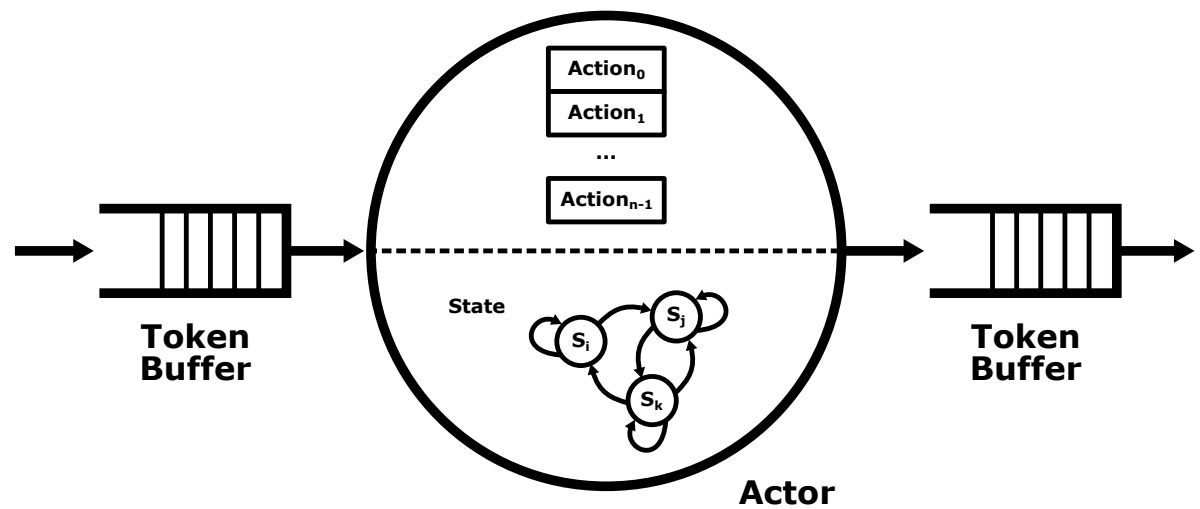

Figure 3-12: Generic CAL actor model.

As a final remark, it is important to highlight that CAL is not related to any specific dataflow MoC, although it essentially extends the concepts laid out by DPNs by providing internal state to actors, supporting multiple overlapping firing rules, and including priorities for firing rules.

This section covers the extension of the ARTICo ${ }^{3}$ framework to support datafloworiented accelerator descriptions. This has been achieved by a direct tool-to-tool integration with a framework that generates coarse-grained reconfigurable systems from dataflow specifications in several actor-oriented languages, including CAL. Hence, the integrated toolchain provides support not only for dataflow-oriented kernel specifications, but also for hybrid hardware reconfiguration techniques. 


\subsubsection{Additional Infrastructure}

Dataflow applications require optimized computing fabrics to fully exploit their inherent parallelism. Although many architectures can be used to this end, ranging from general-purpose multi-core CPUs to dedicated hardware accelerators on FPGAs, CGRAs are the most appropriate solution. After all, CGRAs are domain-specific computing fabrics that combine spatial and temporal computation using a datadriven execution model [Liu'19].

CGRAs are usually implemented as an array of multiple PEs that exchange data over a specific interconnection topology. Each PE can be as simple as a LUT, a more elaborate component such as an ALU, or even a custom-made programmable block. The communication infrastructure, on the other hand, can be based on dedicated point-to-point links (e.g., crossbar or mesh topologies), a bus, or even a NoC.

One of the most important traits of CGRAs when compared against other computing fabrics is their balanced tradeoff between execution flexibility and application reconfiguration, being able to deploy both spatial (i.e., parallel resources) and temporal (i.e., time-multiplexed resources) computation patterns. In contrast, general-purpose CPUs cannot deal with spatial computations, and FPGAs show an increased reconfiguration overhead when dealing with temporal computations.

However, the programming support in CGRA-based development frameworks is still highly limited from both hardware and software perspectives. In most cases, the compiler infrastructure that translates high-level application descriptions into valid configurations for the target CGRA provides results that are still far from manually optimized implementations [Liu'19]. Moreover, the domain-specific nature of CGRAs forces their associated design flows to be prominently application-driven [Liu'19].

In this context, the Multi-Dataflow Composer (MDC) tool [Palumbo'14] appears as an alternative solution for dataflow-oriented CGRA design. Instead of relying on a software compilation flow that maps applications into a generic architecture with configurable PEs and interconnections, MDC uses a multi-functional description of the target scenario (i.e., a set of applications) to generate an application-specific hardware accelerator whose internal datapath can be dynamically changed at run time using CGR. As a result, processing efficiency is highly increased, since PEs are specifically tailored for the functionality of their corresponding actors. Moreover, and in order to also optimize resource utilization, MDC identifies actors that are used in more than one DFG and merges their datapaths, generating a single shared actor instance in the CGR substrate. The MDC tool is open source, ${ }^{17}$ and features four different components:

- Baseline MDC core: performs the translation of multi-functional high-level dataflow specifications into CGR HDL code using datapath merging techniques.

- Structural profiler: performs DSE on multi-functional DFGs to find the optimal CGR substrate according to different design constraints.

\footnotetext{
${ }^{17}$ https://github.com/mdc-suite/mdc
} 
- Power manager: performs the analysis of multi-functional DFGs to implement efficient clock and power gating strategies in the CGR HDL code.

- Coprocessor generator: performs the integration of the generated CGR HDL code into a plug-and-play wrapper for easy SoC integration.

Please note that, although MDC targets CGRA design, the generated hardware accelerator is provided as HDL code, therefore making it possible to either design a custom ASIC with the application-specific CGRA, or to use it as a CGR overlay on an FPGA. The rest of this section provides an in-depth discussion on the key features of each component, focusing on those present in the integrated toolchain proposed in this Thesis, which uses CGR overlays in FPGA-based multi-accelerator systems.

\section{Baseline MDC Core}

The main functionality of MDC is to generate a CGR accelerator from an input set of dataflow specifications. To do so, the tool analyzes the multi-functional description of the target scenario (i.e., the different DFGs) and performs datapath merging to generate a reconfigurable substrate. This process is based on two user-selectable heuristic algorithms: empiric [Palumbo'14], which is better for non-recursive DFGs, and Moreano [Moreano'05], which is more optimized.

The baseline MDC core is further divided into two different elements: the front end (i.e., multi-dataflow generator) and the back end (i.e., platform composer). The front end takes the different application DFGs (i.e., network data) and performs the aforementioned datapath merging process, inserting low-overhead switching modules called Switch Boxes (SBoxes), ${ }^{18}$ and annotating the different datapaths and their associated SBox control codes in a configuration table. The back end then assembles the output CGR HDL code, fetching the required actor specifications from an external core repository (i.e., actor data), and applying a user-defined actor-to-actor communication protocol. This protocol consists of an HDL interface specification for actors, and another core repository with the required communication primitives (e.g., FIFOs). The approach followed in the back end increases the abstraction level, allowing MDC to treat dataflow networks as generic graphs, where communication is not restricted to FIFO channels (i.e., direct point-to-point actor connections are also supported), and where actors can be implemented as either sequential or even combinational logic blocks.

Since the MDC tool is under continuous development, different versions of the baseline core have been released throughout the years. The initial one [Palumbo'14] relied on Network Language (NL), an XML dialect based on the OpenDF format,

\footnotetext{
${ }^{18}$ SBoxes are currently implemented as combinational logic (i.e., 2-to-1 multiplexers and 1-to-2 demultiplexers), which prevents additional buffers from being instantiated and thus, from taking part in the well-known FIFO size optimization problem. Original actor buffers are placed after demultiplexers when SBoxes split the datapath, and before multiplexers when SBoxes merge the datapath.
} 
to specify the target application DFGs. The front end first flattened the networks to remove any existing actor hierarchy, and then performed the datapath merging stage. However, the whole implementation flow was significantly limited since no HLS support was available. Therefore, a pre-built HDL component library was required for the back end to generate the output CGR HDL code.

In order to increase design productivity, a modified version of MDC with support for HLS-powered HDL code generation from high-level dataflow specifications was developed [Nezan'12]. The proposed toolchain extended the previous one by using the Open RVC-CAL Compiler (ORCC) [Yviquel'13] and its VHDL back end to create the required HDL component library. ORCC is an open source compiler infrastructure that supports multi-target source code generation for dataflow applications specified using standard actor and network descriptions: ${ }^{19}$ actors are described using Reconfigurable Video Coding CAL (RVC-CAL), a subset of the original CAL language that has been standardized by the Moving Picture Experts Group (MPEG), and networks are described using the XML Dataflow Format (XDF), another XML dialect.

Unfortunately, the VHDL back end in ORCC was deprecated at some point [Sau'14b], forcing the development of another MDC release [Sau'16]. This version integrated several academic tools and featured three different stages: composition, optimization, and generation. In the composition stage, the ORCC front end was used to flatten network specifications and to generate intermediate representations of the input DFGs, which were then fed to the MDC front end to obtain the merged multi-functional datapath. In the optimization stage, TURNUS [Casale-Brunet'14], an open source DSE tool with an embedded dataflow profiler and performance estimation capabilities, ${ }^{20}$ was used to obtain the optimal FIFO sizes for all intermediate representations of the input DFGs. Afterwards, a worst-case analysis was performed to obtain the optimal FIFO sizes in the multi-dataflow datapath. Finally, in the generation stage, the XRONOS HLS engine [Bezati'13], an open source tool embedded within the ORCC project, ${ }^{21}$ was used to build the HDL core repository directly from RVC-CAL code.

One of the major disadvantages of the previous flows is their strong dependency on vendor-specific tools and devices. In fact, XRONOS eventually evolved into EXELIXI [Bezati'16], replacing the direct RVC-CAL to HDL code translation with an intermediate $\mathrm{C}++$ code generation stage that requires Vivado HLS to produce the ouput HDL descriptions. As a consequence, MDC was modified to become platformagnostic and HDL-independent (i.e., to support any type of HDL code as input). This modification is the most up-to-date version of MDC, and relies on a standard interface with the baseline core functionality based on XDF network descriptions and an HDL component library. The tool maintains its three-stage structure, even though minor changes are introduced to outsource tool- and/or device-specific processes.

With this idea in mind, an alternative entry point for MDC was developed using

\footnotetext{
${ }^{19}$ https://github.com/orcc/orcc

${ }^{20}$ https://github.com/turnus/turnus

${ }^{21}$ https://github.com/orcc/xronos
} 
the open source CAPH just Ain't Plain HDL (CAPH) suite [Sérot'13]. ${ }^{22}$ The CAPH language is a functional Domain-Specific Language (DSL) for complex dataflow network specification and implementation on reconfigurable hardware devices. The automated CAPH-based MDC design flow [Rubattu' 19] is platform-agnostic (i.e., valid for ASICs and FPGAs). In order to comply with the standard baseline core interface, an intermediate step is applied in the composition stage to translate from CAPH to RVC-CAL network specifications in XDF format. Additionally, the optimization stage is performed using the built-in SystemC simulation capabilities available in the CAPH suite. Finally, the HLS engine in the generation stage is replaced by the built-in CAPH HLS extension.

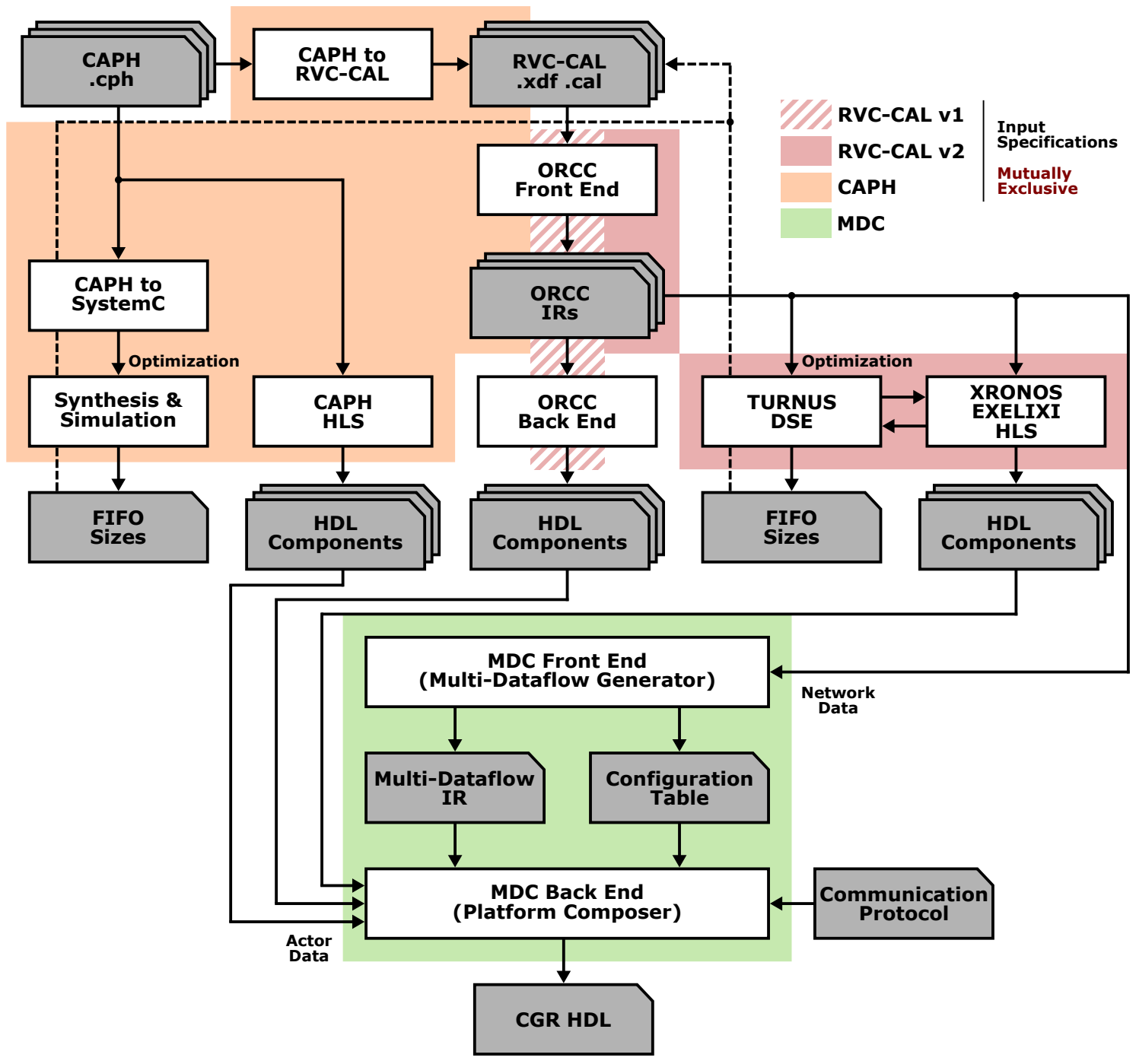

Figure 3-13: MDC overview.

Figure 3-13 shows all the automated CGR HDL code generation flows available in MDC according to the adopted high-level input specification format (i.e., RVC-CAL or

\footnotetext{
${ }^{22}$ https://github.com/jserot/caph
} 
CAPH). ${ }^{23}$ Please note that the flow that relies on the deprecated ORCC VHDL back end has been also included for the sake of completeness.

To finalize this section, an example run of the MDC tool using the empiric datapath merging algorithm is depicted in Figure 3-14. As it can be seen, there are three different input dataflow specifications that are assumed to have been previously flattened by the ORCC front end (i.e., $\alpha, \beta$ and $\gamma$ ). Then, the MDC front end (top) iteratively merges the datapaths of the input DFGs. The datapath merging process is performed by comparing two networks at a time and therefore, $N-1$ iterations are required to merge $N$ DFGs. It is worth noticing that a maximum of $N-1$ SBoxes can be required to access an actor (this is the worst-case scenario, with that particular actor shared among all the input dataflow networks), which in turn may significantly affect the maximum operating frequency of the output CGR accelerator. In the example, the output multidataflow is generated after 2 iterations (e.g., $\alpha$ and $\gamma$ first, the merged result and $\beta$ second). In addition, a configuration table containing the control information for the embedded SBoxes is also generated. Once the merging process has finished, the MDC back end (bottom) uses the HDL component library, generated by the appropriate HLS engine, and the selected communication protocol to create a CGR substrate, instantiating actors and communication primitives, establishing actor-toactor interfaces, and generating a configuration LUT to decode a specific dataflow network ID into the corresponding control bits for the SBoxes (e.g., the $\alpha$ and $\gamma$ network IDs would be decoded into 000 and 110 , respectively).
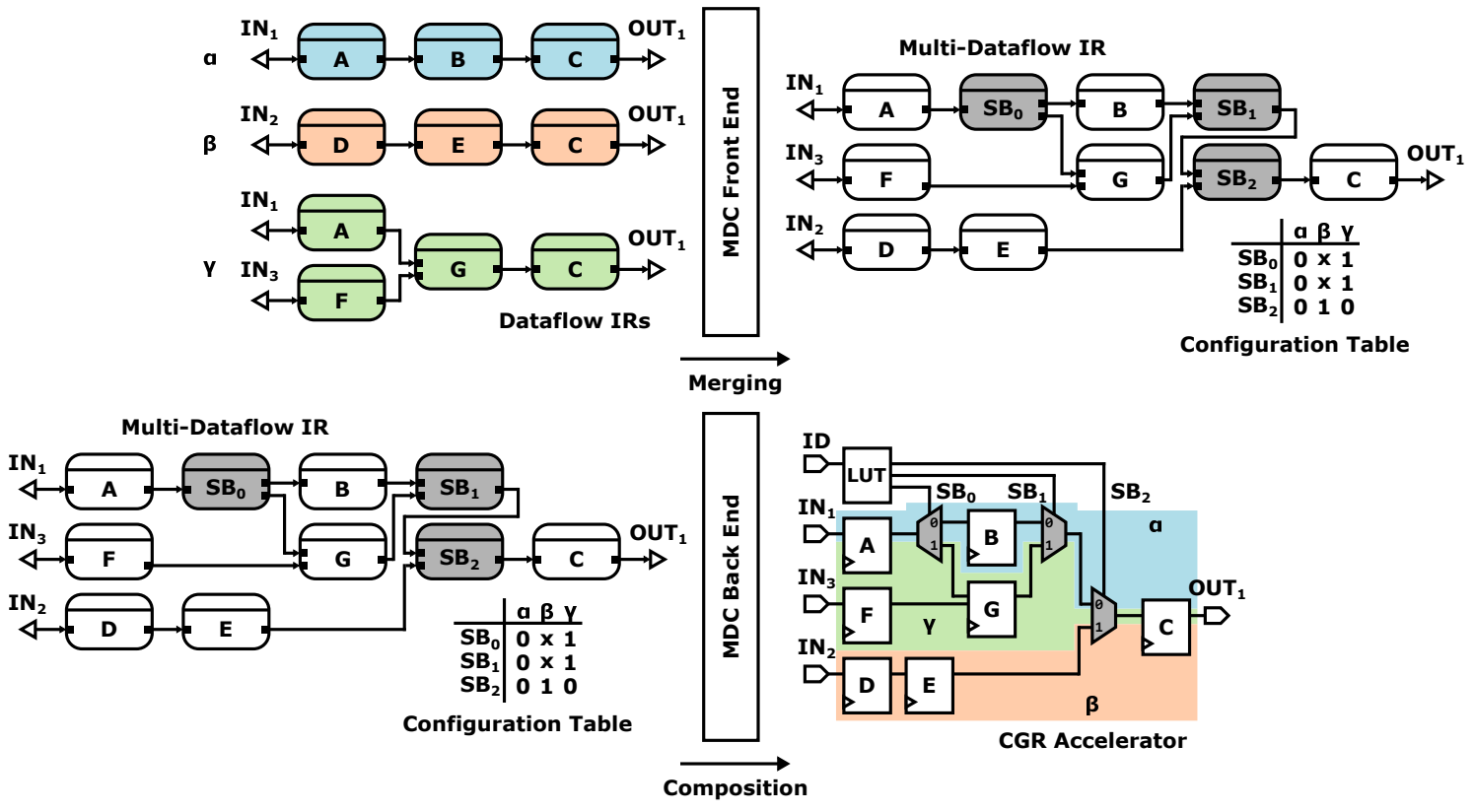

Figure 3-14: Multi-dataflow merging and platform composition with MDC.

\footnotetext{
${ }^{23} \mathrm{MDC}$ also supports semi-automated generation flows where users provide their network specifications in XDF format (for the ORCC front end to extract their intermediate representations) and a valid HDL component library (handwritten or generated using external HLS tools).
} 


\section{Structural Profiler}

As it has been already discussed, the worst-case scenario for the datapath merging process is to have one actor that is present in all the input dataflow specifications, since an excessive amount of combinational logic resources needs to be instantiated to generate the cascaded SBox structure. This not only increases the critical path in the merged accelerator, but also leads to larger area and power consumption overheads. Moreover, these problems can also arise in multi-functional dataflow specifications that are apparently far from the worst-case datapath merging scenario.

In order to deal with these implementation issues, MDC features an embedded DSE engine called structural profiler [Palumbo'13], which covers all possible datapath merging combinations (without repetition) and performs an exhaustive Pareto analysis. These combinations include one with completely independent datapaths (i.e., all input DFGs are left unmodified), one with a fully merged datapath (i.e., all input DFGs are merged into a single multi-functional network), and several with different depths of partially merged datapaths in between (i.e., some input DFGs are left unmodified, the rest are merged into a multi-functional network).

The structural profiler requires back-annotation in the HDL component library and computes three different values for each datapath merging combination: area, power consumption, and critical path. Then, it provides two implementation solutions as output: the datapath combination with sub-optimal power/area, and the one with sub-optimal operating frequency. The former usually corresponds to the fully merged DFG, whereas the latter is commonly found to be a partially merged network.

\section{Power Manager}

One of the characteristics of multi-functional CGR accelerators is that certain parts of their internal datapath remain idle while the rest of the elements are performing their computations. As a result, these architectures are highly suitable for implementing different power management strategies.

MDC features an embedded power manager that takes a multi-functional dataflow network (usually one of the sub-optimal solutions produced by the structural profiler) and automatically applies clock gating [Palumbo'15] or power gating [Palumbo'16] techniques. To do so, the merged DFG is analized and divided into non-overlaping logic regions with as many elements as possible. These logic regions include only actor logic when applying clock gating (SBoxes are combinational blocks), or actor and SBox logic when applying power gating. Individual control signals are assigned per logic region, and a decoding LUT is placed to assert or deassert those control signals according to the selected network ID.

Generally speaking, the MDC power manager strategy depends upon the target device: clock gating can be applied in either ASICs or FPGAs, whereas power gating is only supported for ASIC design. 


\section{Coprocessor Generator}

In order to ease the integration of CGR accelerators in processor-centric designs, the MDC tool also features a dedicated module that embeds the HDL code generated by the baseline core into standard coprocessor templates. In this regard, two different approaches are supported according to the classification in [Compton'02]: a memorymapped template wrapper to generate attached processing units (i.e., loosely coupled accelerators), and a stream-based template wrapper to generate coprocessors (i.e., tightly coupled accelerators) [Sau'15]. ${ }^{24}$

The MDC coprocessor generator enables seamless integration of CGR accelerators with ARM-based processing systems by using standard AXI interfaces [Fanni'19]. Although both memory-mapped and stream-based templates have an AXI4-Lite interface for register-based configuration, data transfer is performed using a single AXI4-Full interface (i.e., shared for all input/output ports) in the former and several AXI4-Stream interfaces (i.e., one per input/output port) in the latter.

Additional logic blocks are required inside the memory-mapped template wrapper to properly handle communications between the processor and the CGR core [Sau'14a]. These modules, called accelerator front and back ends, include address generation logic to read from or write to the local memory banks, data counters to keep track of the transferred data tokens between the local memories and the CGR core, and an FSM to sequence operations, generating the corresponding control signals. Figure 3-15 shows the generic structure of a memory-mapped MDC coprocessor. Notice that the network ID, which configures the internal CGR datapath of the accelerator, comes from the configuration data stored in the template registers.

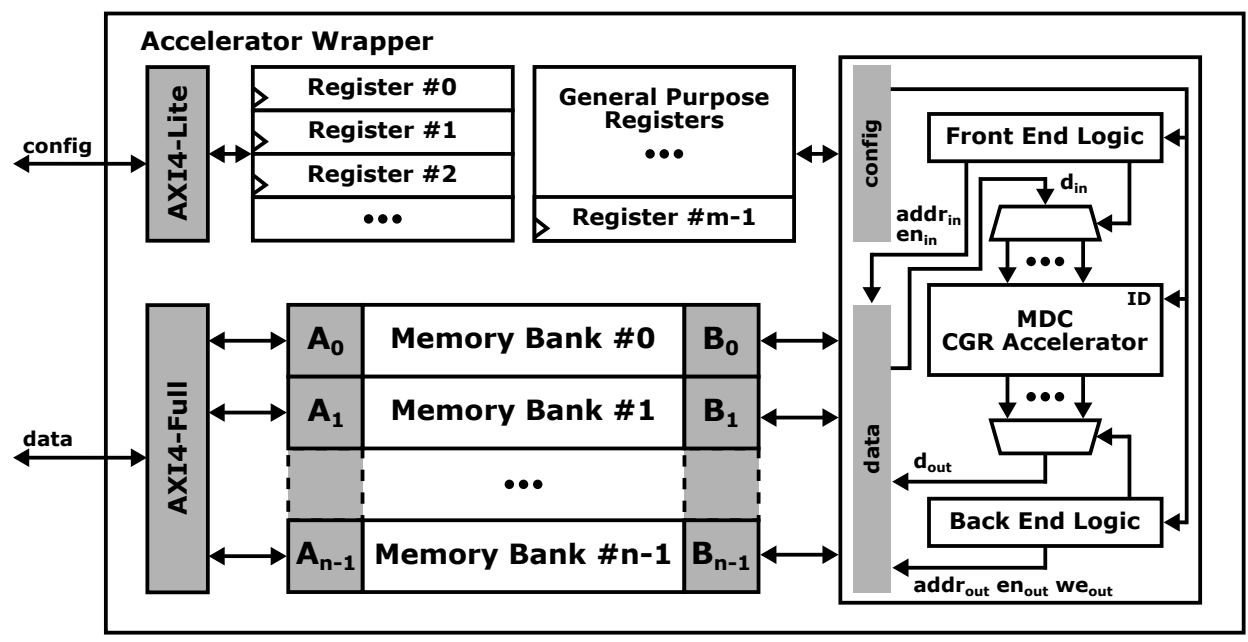

Figure 3-15: Memory-mapped MDC coprocessor.

\footnotetext{
${ }^{24} \mathrm{MDC}$ makes no distinction and uses the word "coprocessor" equally for both approaches.
} 


\subsubsection{State of the Art}

The integrated toolchain proposed in this Thesis exhibits two distinct characteristics: a dataflow-based entry point for the design and implementation of multi-accelerator systems, and a hybrid reconfiguration approach that combines CGR and DPR for enhanced run-time flexibility. As a result, the analysis of the literature has been split in two separate sections: dataflow-based hardware generation on the one hand, and hybrid reconfigurable architectures on the other hand.

\section{Dataflow-Based Hardware Generation}

The most common trend nowadays is to map dataflow applications into softwarebased computing fabrics [Bhattacharyya'19]. Nevertheless, several hardware generation flows from high-level dataflow descriptions can still be found throughout the literature. In fact, some of the first proposals were made to promote HLS design methodologies. For instance, a subset of the $\mathrm{C}$ programming language called Single Assignment C (SA-C) is used in [Rinker'01] to map DFGs into hardware. A specific SA-C compiler generates host processor code on the one hand, and a DFG representation on the other hand. This representation is then used by a DFG-to-VHDL translator to generate the hardware datapath with a library of predefined VHDL components.

Most dataflow-centric frameworks feature a dedicated extension for hardware system generation. In Ptolemy II, for example, actors are mapped into VHDL primitives that are fetched from a prebuilt library [Leung'08]. Some of the characteristics of these primitives are configurable at design time (i.e., using VHDL generics): bit width, fixed point arithmetic precision, integer overflow behavior, etc. In addition, nonsynthesizable primitives can be also generated to enable RTL simulation.

OpenDF was one of the first dataflow-centric frameworks to generate HDL code from high-level application specifications (written in CAL) using HLS techniques [Janneck'08]. ${ }^{25}$ The hardware generation flow first performs constant propagation to remove all parameters from actors, then isolates each action using an individual thread with static single assignments (i.e., an actor with $N$ actions produces $N+1$ threads, with the additional one being the action scheduler), and finally generates an RTL description in Verilog by synthesizing actors and network. Implementation results show that this solution outperforms hand-crafted VHDL code in terms of throughput and resource utilization when evaluated in a video decoding scenario.

Other CAL-based frameworks also feature hardware generation. One of the most relevant examples is ORCC, which initially featured a dedicated back end to generate VHDL code [Siret'10]. This back end worked with the intermediate representations produced by the ORCC front end, performing several transformations to obtain hardware-friendly versions, and printing actors and networks using a templatebased approach. In these templates, actors are implemented in two processes: a

\footnotetext{
${ }^{25}$ This hardware generation tool is also referred to as CAL2HDL [Bhattacharyya' 19].
} 
combinational one (with all actor inputs in the sensitivity list) for the firing rules, and a sequential one for the actions. As a result, each action can be executed in one clock cycle. Finally, networks are implemented using different component instances (e.g., actors, FIFOs). Once the ORCC VHDL back end was deprecated, XRONOS [Bezati'13] replaced it. XRONOS also uses the intermediate representations produced by the ORCC front end, performs several transformations, and feeds them to the CAL2HDL Verilog code generator from the OpenDF framework. Eventually, XRONOS evolved into EXELIXI [Bezati'16], which replaces the CAL2HDL code generator by a module that generates high-level $\mathrm{C}++$ code with embedded Vivado HLS optimization directives. EXELIXI has been recently extended to work with Zynq-7000 devices [Bezati'19].

The CAPH toolset [Sérot'13] is an alternative hardware generation method for dataflow-based systems. CAPH descriptions are first analyzed by a front end to generate an AST. Then, the CAPH compiler elaborates the AST, generating the network and the behavioral descriptions for the actors. While networks can be seen as a combination of black boxes (i.e., actor instances) and their interconnection wirings (i.e., FIFOs), behavioral descriptions are the translation of the actor firing rules and actions into an FSM. These target-independent representations are then transformed into VHDL code.

Most recently, several works have targeted hardware generation using dataflowbased descriptions. For instance, StreamDrive [Stoutchinin'19] applies simulationbased hardware/software codesign techniques to identify common domain-specific operations (e.g., convolution for image processing), and generates dedicated hardware blocks by manually translating $C$ code into RTL descriptions. In [Edwards'19], an assembler-like language is used to describe actors. These descriptions are then automatically compiled into small logic blocks written in SystemVerilog code, and complete networks are built by the composition of those logic blocks. Finally, Cal2Many [Savas'20] uses CAL descriptions to generate accelerator descriptions in Chisel [Bachrach'12], which are then integrated in a RISC-V Rocket Chip SoC [Asanović'16].

Dataflow-based hardware generation is not only limited to Academia. Maxeler toolchains [Pell'11] are a good example of commercial tools for dataflow-based programming on FPGAs using specialized languages such as Open Spatial Programing Language (OpenSPL) [Becker'16] ${ }^{26}$ Unfortunately, the main goal of Maxeler tools is HPC [Oriato'15], which prevents their applicability in embedded computing scenarios such as the ones targeted in this Thesis.

In this Thesis, CAPH-based hardware generation is used. The additional infrastructure (i.e., MDC and $\mathrm{ARTICo}^{3}$ ) extends its basic functionality to produce datafloworiented CGR accelerators in adaptive DPR-capable multi-accelerator systems.

\footnotetext{
${ }^{26}$ http://www.maxeler.com/
} 


\section{Hybrid Reconfigurable Architectures}

One of the most common methods to add a second reconfigurability layer in FPGAs is the use of overlays. FPGA overlays are virtual reconfigurable architectures deployed on top of the physical reconfigurable fabric, providing a virtualization layer between user applications and physical FPGA resources [So' 16]. In fact, overlays are a key FPGA virtualization mechanism at resource level [Vaishnav'18], since they offer three main benefits: reduced implementation times for increased productivity [Stitt'11], support for run-time compilation of hardware accelerators [Ma'15a, Ma'16], and functional portability across platforms/vendors [Brant'12].

According to the classification from [So'16], there are three different types of FPGA overlays: virtual FPGAs, CGRA overlays, and processor-like overlays. Virtual FPGAs implement platform-agnostic logic resources (e.g., LUTs, FFs, or multiplexers) using the technology-dependent primitives available in the target device. As a result, these virtual components (i.e., components that may not exist as physically independent entities) exhibit slightly larger configuration granularities than native components. ZUMA [Brant'12] is an example of such virtual FPGA that, after its integration with ReconOS [Wiersema'14], provides support for hybrid reconfiguration mechanisms.

CGRA overlays have been thoroughly covered in the literature. With the same architectural principles as standard CGRAs, these overlays can be further classified in two different groups depending on how their internal resources are used during task execution: time-multiplexed and spatially-configured [Jain'17]. Time-multiplexed CGRA overlays change the behavior of their PEs and interconnections while the offloaded computation is being executed, sometimes even on a clock cycle basis. In this scenario, PEs behave like standard software-based processor cores with dedicated instruction memories. Although this approach theoretically minimizes resource utilization by exploiting resource sharing, the truth is that additional overheads are incurred due to the internal instruction memories and the configurable routing structures [Li'19]. Examples of time-multiplexed CGRA overlays include the GRVI Phalanx [Gray'16], where each PE is a cluster of eight RISC-V cores with an optional tightly coupled accelerator core, and the work presented in [Li'16], where several DSP-based PEs are linearly connected to form a processing pipeline that executes feed-forward DFGs.

Spatially-configured CGRA overlays, on the other hand, rely on dedicated datapaths where both PEs and interconnections are left untouched while the offloaded computation is being executed. In this scenario, PEs execute a single arithmetic operation per task (even though that operation is still configurable and can change for a different task), and data are transferred over dedicated point-to-point communication links. With proper design considerations (e.g., deep pipelining), these architectures can provide high-throughput solutions. Examples of spatially-configured CGRA overlays include the Fully Pipelined Composable Architecture (FPCA) [Cong'14], an architecture that embeds 32-bit ALUs with on-chip memory and registers in its PEs and supports dynamic accelerator composition to increase parallelism, and the 
modified version of the DySER architecture [Govindaraju'12] presented in [Jain'16], where Xilinx DSP primitives are used to implement each PE.

Finally, processor-like overlays use the FPGA fabric to implement one or several soft-core processors. Although this approach leads to less hardware-centric solutions, most FPGA vendors provide custom soft-core processors to implement processorlike overlays (e.g., Xilinx MicroBlaze or Intel Nios II). More complex soft-core architectures have been also developed over the years, including VLIW processors (e.g., TILT [Rashid'15]), vector processors (e.g., VectorBlox MXP [Severance'13]), and even GPUs (e.g., FGPU [Al Kadi'18]). Notice that processor-like overlays are a perfect example of time-multiplexed FPGA overlays [Li'19], even though the internal architecture of their PEs is usually more complex than in time-multiplexed CGRA overlays.

The last developments in dataflow-based CGRA overlays include hardwareoriented frameworks like CGRA-ME [Chin'18], which can be used to describe CGRA architectures, implement them in HDL code, and map benchmark applications on the generated computing fabrics using LLVM-based compilation. Some of these frameworks also support the generation of application-specific overlays. For instance, the AS-Overlays framework [Mbongue'19] uses LLVM code inspection to implement the internal datapath of the different PEs, and relies on RapidWright [Lavin'18] to efficiently generate the output overlays. On the contrary, recent softwareoriented dataflow-based CGRA overlays include the Dataflow Coprocessor Overlay (DaCO) [Siddhartha'19], which uses custom soft-core dataflow processors as PEs, and the READY framework [Silva'19], which uses a DSL and JIT compilation to map dataflow applications onto software-programmable PEs with fixed structure.

It is also worth noting that the use of DPR in FPGA overlays has been recently identified as a key enabling technology [Vipin'18]. In this regard, there are two different approaches: on the one hand, medium- or coarse-grained DPR can be used to partially or completely change the overlay structure (i.e., hardware components and interconnections) [Omidian'19]; on the other hand, fine-grained DPR can be used to only change the overlay configuration (i.e., functional parameters) [Zamacola'19].

In this Thesis, DPR is used to change application-specific dataflow-based CGR overlays. As opposed to other solutions from the state of the art where finegrained DPR is applied to change CGRA configurations, accelerators generated with the integrated MDC-ARTICo ${ }^{3}$ toolchain use fast register-based operations to switch between the different configurations of a CGR overlay, relying on slower DPR mechanisms to swap between alternative spatially-configured CGR overlays. 


\subsubsection{Integration Details}

As it can be inferred from previous discussions, both MDC and ARTICo ${ }^{3}$ frameworks have a lot of things in common from an implementation point of view. For instance, accelerator IP cores are generated using standard templates in both cases. In addition, the internal structure of the memory-mapped MDC CGR accelerator template is almost identical to the ARTICo ${ }^{3}$ kernel wrapper.

Two main activities have been carried out to complete the toolchain integration process: on the one hand, MDC developers extended their coprocessor generator engine to generate an ARTICo ${ }^{3}$-compliant top module (as shown in Subsection 2.4.2) for the CGR HDL code [Fanni'19]; on the other hand, the author of this Thesis extended the $\mathrm{ARTICo}^{3}$ toolchain to support Verilog kernel specifications, ${ }^{27}$ and developed a custom hardware template (see Subsection 2.4.3) to provide, as a component library, all the communication primitives instantiated in the CGR HDL code.

The integrated toolchain, which is shown in Figure 3-16, relies on CAPH descriptions as the entry point for the multi-functional dataflow kernel specifications. Then, the CAPH HLS engine is used to generate the HDL component library, and the ORCC front end is used to manipulate the associated XDF networks (automatically generated by CAPH also from the input dataflow specifications), flattening all nonatomic actors (i.e., hierarchical actors with nested subnetworks), and generating the corresponding intermediate representations. The next step is performed by MDC, which takes both actor data (i.e., the HDL actor library) and network data (i.e., the intermediate representations of the networks) and generates a CGR HDL accelerator with an ARTICo ${ }^{3}$-compliant kernel interface. This accelerator code is provided as an input to the ARTICo ${ }^{3}$ toolchain to perform the hardware implementation of the multiaccelerator system. As it has been already mentioned, the custom hardware template used in this process includes the VHDL support library for the CAPH distribution. ${ }^{28}$ For the software generation part, the user provides a regular ARTICo ${ }^{3}$ application code.

In summary, users only need to provide as inputs the CAPH code for their dataflow networks, and the usual C/C++ software code to use $\mathrm{ARTICo}^{3}$ from a Linux application. The integrated toolchain automatically manages the implementation of the dataflowbased reconfigurable multi-accelerator system, and the ARTICo ${ }^{3}$ runtime library transparently manages its execution. In fact, no additional extensions are required in the ARTICo ${ }^{3}$ runtime API, since DPR is still managed by the artico3_load function, and CGR can be easily performed using the artico3_kernel_wcfg function to write a specific network ID in the configuration registers.

Original contribution 3-3 An integrated toolchain to automatically generate reconfigurable multi-accelerator systems from high-level dataflow descriptions.

\footnotetext{
${ }^{27}$ Verilog is the reference HDL used by the MDC code generator.

${ }^{28}$ https://github.com/jserot/caph/tree/master/lib/vhdl
} 


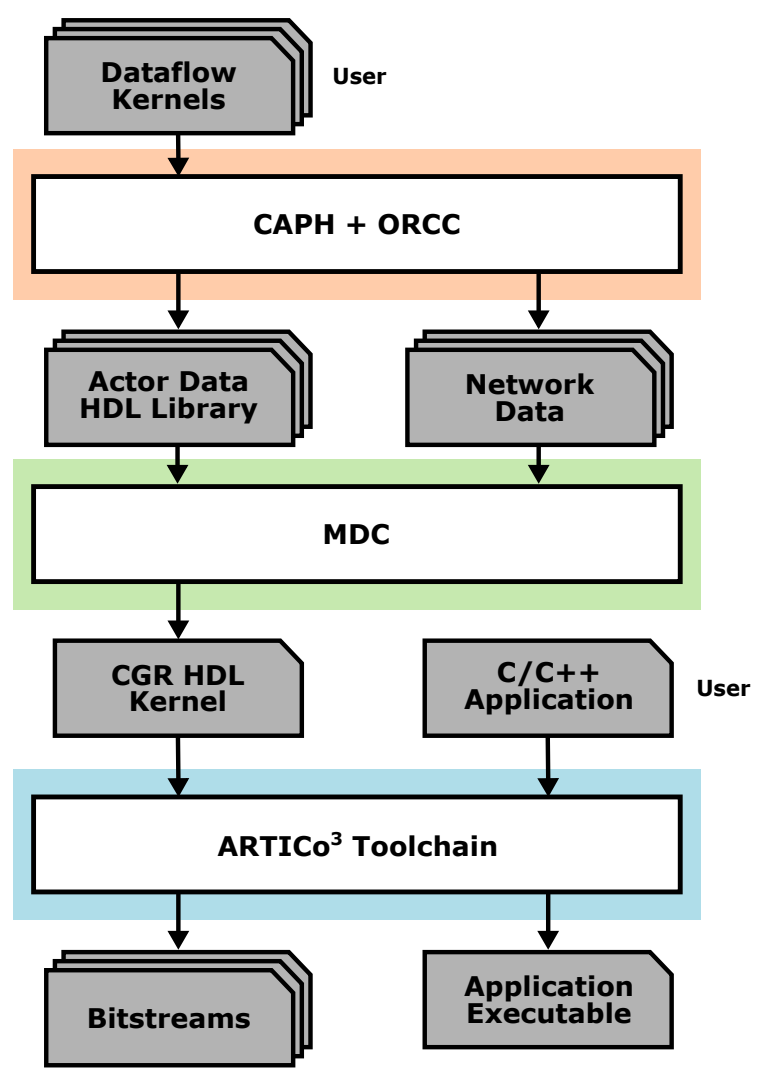

Figure 3-16: Integrated MDC-ARTICo ${ }^{3}$ flow.

After the deployment of the dataflow-based reconfigurable multi-accelerator system on the FPGA fabric, each kernel instance resembles the generic structure depicted in Figure 3-17. Thanks to its modified coprocessor generator, MDC provides a plug-and-play CGR HDL core for the ARTICo ${ }^{3}$ kernel wrapper. As a result, MDCgenerated ARTICo ${ }^{3}$ kernel instances exhibit a hybrid reconfiguration approach at run time, with an application-specific CGR overlay embedded in a DPR-capable computing fabric. This approach favors fast reconfiguration times when switching between prefixed functionalities (i.e., CGR), while still supporting complete functional changes at a lower reconfiguration speed (i.e., DPR).

Original contribution 3-4 A hybrid reconfiguration mechanism for multiaccelerator systems that embeds CGR-based application-specific overlays in DPR-capable FPGA partitions, allowing multiple reconfiguration speeds. 


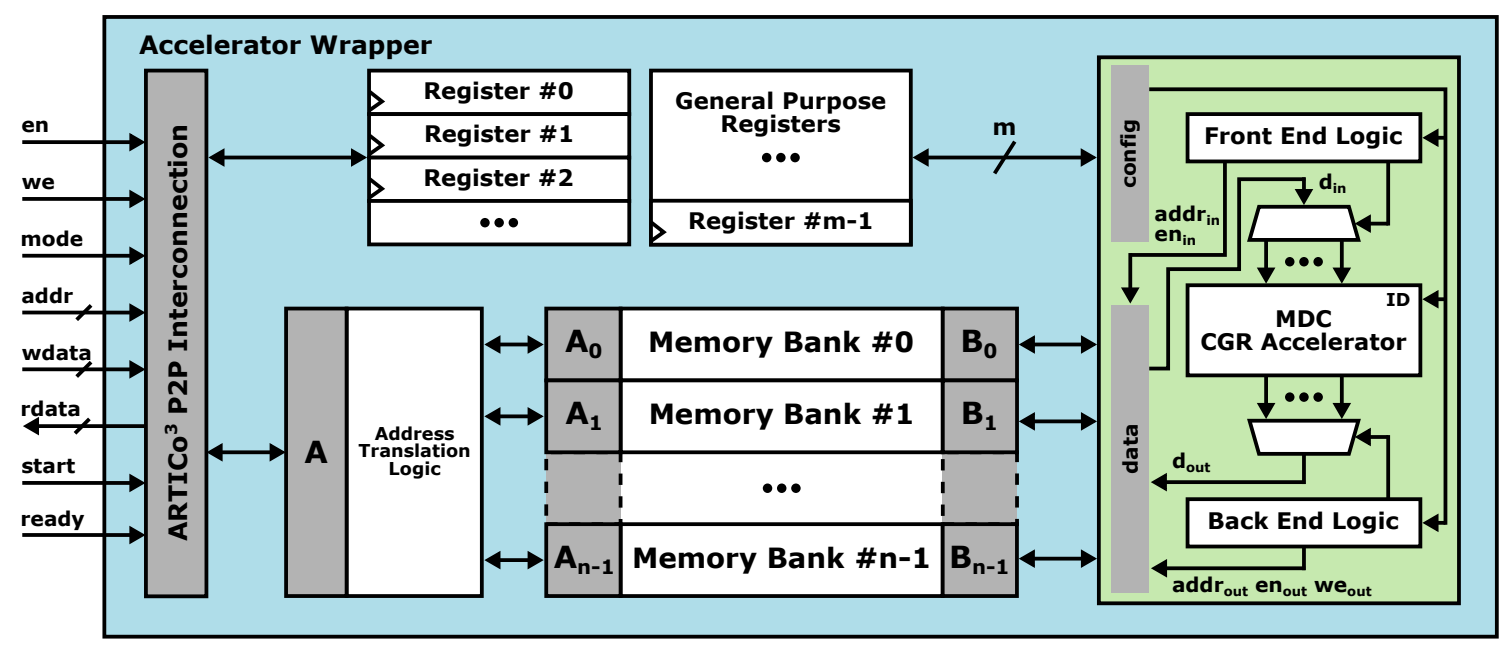

Figure 3-17: ARTICo $^{3}$ kernel wrapper with embedded MDC CGR core.

\subsubsection{Validation}

The integrated MDC-ARTICo ${ }^{3}$ toolchain has been tested using an image processing application scenario. In particular, two edge detection algorithms (i.e., Sobel and Roberts) have been selected as targets. These algorithms perform gradient-based edge detection in an image by applying a $2 \mathrm{D}$ convolution with two complementary kernels each: the $k_{x}$ kernel, which detects horizontal changes by computing an approximation of the vertical derivative, and the $k_{y}$ kernel, which detects vertical changes by computing an approximation of the horizontal derivative. Directional gradient images can be computed from the original image (i.e., $I$ ) using Equation 3-2.

$$
\begin{aligned}
G_{x} & =k_{x} * I \\
G_{y} & =k_{y} * I
\end{aligned}
$$

The main differences between both edge detection operators are the size of the convolution kernels and their coefficient values. Sobel implements $k_{x}$ and $k_{y}$ using $3 \times 3$ matrices, as shown in Equation 3-3. Roberts, on the other hand, implements $k_{x}$ and $k_{y}$ using $2 \times 2$ matrices, as shown in Equation 3-4.

$$
\begin{gathered}
k_{x}=\left[\begin{array}{ccc}
1 & 0 & -1 \\
2 & 0 & -2 \\
1 & 0 & -1
\end{array}\right], k_{y}=\left[\begin{array}{ccc}
1 & 2 & 1 \\
0 & 0 & 0 \\
-1 & -2 & -1
\end{array}\right] \\
k_{x}=\left[\begin{array}{cc}
1 & 0 \\
0 & -1
\end{array}\right], k_{y}=\left[\begin{array}{cc}
0 & 1 \\
-1 & 0
\end{array}\right]
\end{gathered}
$$

Despite these differences, both edge detection algorithms share a common structure (i.e., they are both based on 2D convolutions). Therefore, their dataflow descriptions are highly likely to have certain parts that can be merged, which 
makes this application scenario a suitable use case for the integrated MDC-ARTICo ${ }^{3}$ toolchain. Figure 3-18 shows the simplified DFGs for the Sobel (top) and Roberts (bottom) edge detectors. As it can be seen, additional actors have been inserted before and after the specific directional derivative kernels. As a matter of fact, these kernel actors are preceded by a combination of unit delays and line buffers to generate the appropriate pixel window for convolution, and followed by an actor that computes an approximation of the image gradient in a pixel, ${ }^{29}$ and another one that applies a certain threshold to produce a binary output value (i.e., edge or not edge). Note that potentially shareable actors in both DFGs have been highlighted in gray.
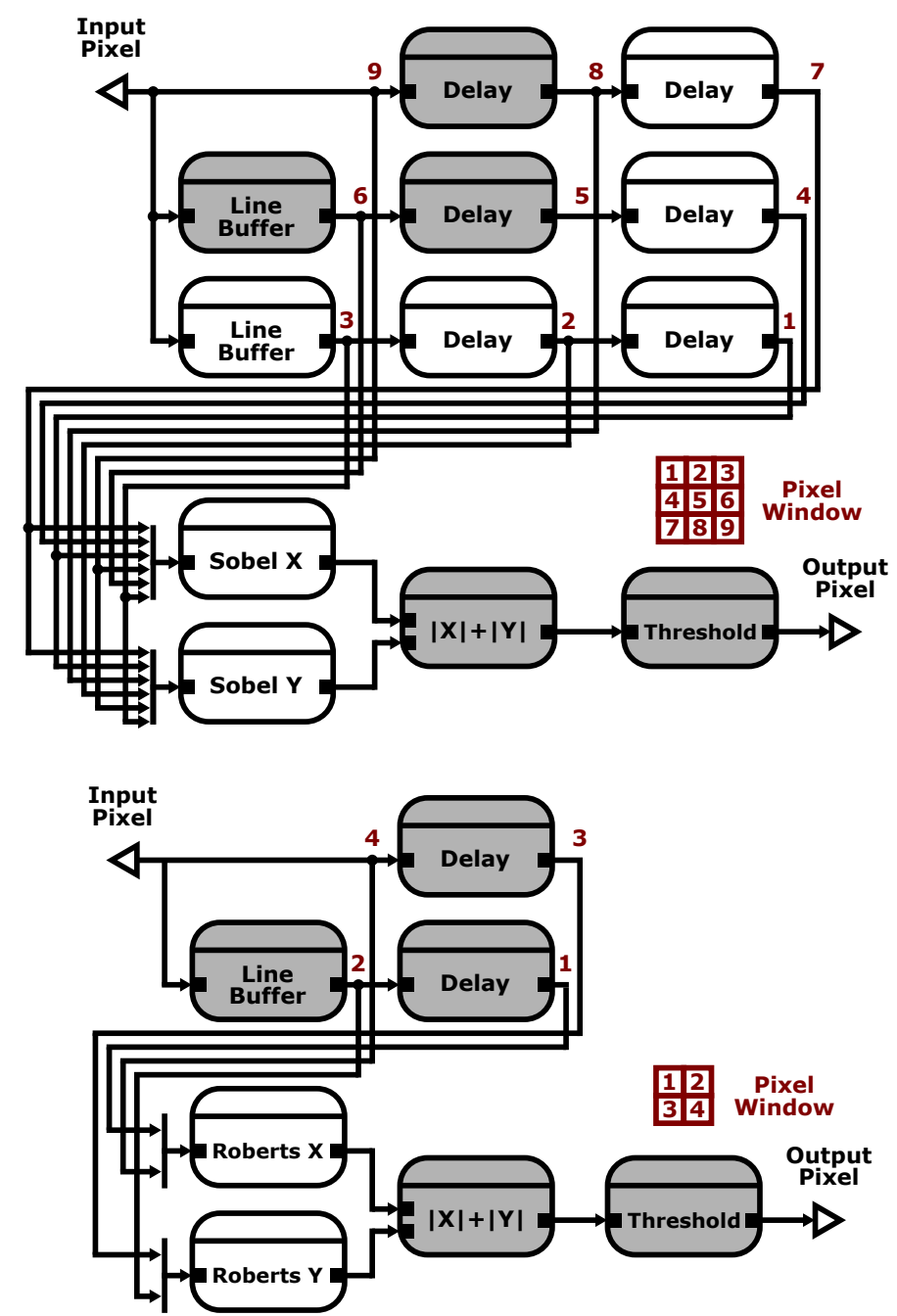

Figure 3-18: Simplified DFGs for the Sobel and Roberts image filters.

Three different ARTICo $^{3}$ kernels have been implemented in this evaluation scenario: a dataflow-based Sobel kernel, a dataflow-based Roberts kernel, and a dataflow-based CGR kernel. In other words, the CAPH descriptions for both dataflow

\footnotetext{
${ }^{29}$ It is usually reasonable to assume that, for hardware implementations, the image gradient can be approximated as $G=\sqrt{G_{x}^{2}+G_{y}^{2}} \approx\left|G_{x}\right|+\left|G_{y}\right|$.
} 
networks have been used in three different runs of the integrated toolchain, producing two separate accelerators (i.e., assuming no multi-functional specifications) first, and a single CGR accelerator later. The number of actors per accelerator is as follows: 13 actors in the standalone Sobel, 8 actors in the standalone Roberts, 16 actors in the CGR Sobel-Roberts (5 of them shared using a total of 14 SBoxes). Table 3-7 shows the resource utilization reports for all three runs when implemented on a custom board with a Zynq-7000 SoC. ${ }^{30}$ Notice that each ARTICo ${ }^{3}$ kernel has 3 local memory banks (one to store the size of the input/output images, one to store the input image, and one to store the output image) and 5 configuration registers (one to store the network ID, one per multi-dataflow graph port to store the amount of tokens to be read from or written to its corresponding memory bank, and one to enable fault injection ${ }^{31}$ ). It is also worth noting that, as expected and thanks to MDC, the resource utilization in the CGR kernel is less than in the other two kernels combined.

Table 3-7: Resource utilization in the dataflow-based hybrid reconfiguration scenario.

\begin{tabular}{|c|c|c|c|c|}
\hline Component & ARTICo $^{3}$ & Sobel Kernel & Roberts Kernel & CGR Kernel \\
\hline Info & $\begin{array}{c}4 \text { slots } \\
\text { VHDL (Vivado) } \\
\text { Zynq-7000 }\end{array}$ & $\begin{array}{c}48 \mathrm{KiB} \text { memory, } 3 \text { banks } \\
5 \text { registers } \\
\text { Verilog (Vivado) } \\
\text { Zynq-7000 }\end{array}$ & $\begin{array}{c}48 \mathrm{KiB} \text { memory, } 3 \text { banks } \\
5 \text { registers } \\
\text { Verilog (Vivado) } \\
\text { Zynq-7000 }\end{array}$ & $\begin{array}{c}48 \text { KiB memory, } 3 \text { banks } \\
5 \text { registers } \\
\text { Verilog (Vivado) } \\
\text { Zynq-7000 }\end{array}$ \\
\hline LUTs & 4158 & 2395 & 1497 & 2829 \\
\hline FFs & 2366 & 2488 & 1457 & 2769 \\
\hline DSPs & - & - & - & - \\
\hline BRAMs & - & 12 & 12 & 12 \\
\hline
\end{tabular}

The experimental setup relies on the same custom board used in Subsection 2.6.2 (dual core ARM Cortex-A9 @ 666.67 MHz, FPGA fabric@100 MHz), since it provides the required infrastructure to characterize the obtained multi-accelerator systems in terms of both performance and energy consumption. Table 3-8 and Table 3-9 summarize, respectively, the execution times and the energy consumption of the standalone accelerators, the CGR accelerator in both Sobel and Roberts configurations, and the software-based reference implementations of the algorithms when using input images of different sizes, with a local size of $32 \times 32$ pixels per kernel instance.

Results show that the software-based implementations always outperform the hardware-based ones. There are several reasons for this, including oversized memory banks in kernel wrappers (i.e., only a maximum of $32 \times 324$-byte pixels are used per 16-KiB-wide local memory bank) and memory-bounded behavior during ARTICo $^{3}$ kernel execution (as already identified in the ad-hoc HDL implementation evaluated in Subsection 2.6.1). In fact, the memory-bounded behavior can be also confirmed by analyzing the almost non-existent performance scalability (the best-case speedup value using up to 4 different kernel instances is around $1.3 \times$ ).

\footnotetext{
${ }^{30}$ XC7Z020-1CLG484.

${ }^{31}$ Fault injection can be emulated in $\mathrm{ARTICo}^{3}$ accelerators using a multiplexer to select, according to the value stored in one of the configuration registers, whether valid data or wrong values are written to an output memory bank. This mechanism can be used to test the voter unit and the error PMCs.
} 
Table 3-8: Execution times in the dataflow-based hybrid reconfiguration scenario.

\begin{tabular}{|c|c|c|c|c|c|}
\hline \multirow{2}{*}{ Configuration } & \multirow{2}{*}{ Accelerators } & \multicolumn{4}{|c|}{ Execution Time (ms) } \\
\hline & & $256 \times 256$ & $512 \times 512$ & $1024 \times 1024$ & $2048 \times 2048$ \\
\hline \multirow{4}{*}{ Sobel } & 1 & 49.78 & 201.51 & 806.01 & 3176.88 \\
\hline & 2 & 42.68 & 170.77 & 675.06 & 2709.36 \\
\hline & 3 & 41.57 & 161.17 & 642.7 & 2574.76 \\
\hline & 4 & 39.73 & 155.62 & 619.38 & 2499.97 \\
\hline \multirow{4}{*}{ Roberts } & 1 & 48.89 & 197.14 & 773.63 & 3108.91 \\
\hline & 2 & 41.62 & 165.12 & 657.4 & 2634.39 \\
\hline & 3 & 40.56 & 157.49 & 624.41 & 2502.06 \\
\hline & 4 & 39.46 & 152.86 & 607.05 & 2411.06 \\
\hline \multirow{4}{*}{ Sobel - CGR } & 1 & 49.87 & 198.73 & 791.97 & 3180.2 \\
\hline & 2 & 42.75 & 169.69 & 674.81 & 2712.07 \\
\hline & 3 & 41.67 & 161.7 & 648.25 & 2572.23 \\
\hline & 4 & 39.77 & 155.4 & 619.37 & 2482.98 \\
\hline \multirow{4}{*}{ Roberts - CGR } & 1 & 48.79 & 197.7 & 773.57 & 3107.48 \\
\hline & 2 & 41.88 & 165.22 & 659.29 & 2638.94 \\
\hline & 3 & 40.84 & 157.45 & 624.19 & 2502.62 \\
\hline & 4 & 38.71 & 151.51 & 601.25 & 2412.36 \\
\hline Sobel - SW & - & 22.42 & 90.09 & 366 & 1496.14 \\
\hline Roberts - SW & - & 9.56 & 39.52 & 157.99 & 655.41 \\
\hline
\end{tabular}

Table 3-9: Energy consumption in the dataflow-based hybrid reconfiguration scenario.

\begin{tabular}{|c|c|c|c|c|c|}
\hline \multirow{2}{*}{ Configuration } & \multirow{2}{*}{ Accelerators } & \multicolumn{4}{|c|}{ Energy Consumption (mJ) } \\
\hline & & $256 \times 256$ & $512 \times 512$ & $1024 \times 1024$ & $2048 \times 2048$ \\
\hline \multirow{4}{*}{ Sobel } & 1 & 33.47 & 130.32 & 532.13 & 2132.95 \\
\hline & 2 & 28.61 & 107.27 & 437.37 & 1756.8 \\
\hline & 3 & 26.76 & 102.38 & 406.44 & 1628.57 \\
\hline & 4 & 25.26 & 99.01 & 397.11 & 1562.94 \\
\hline \multirow{4}{*}{ Roberts } & 1 & 32.36 & 131.76 & 526.17 & 2106.31 \\
\hline & 2 & 27.23 & 106.58 & 429.57 & 1729.04 \\
\hline & 3 & 26.93 & 100.42 & 402.62 & 1603.06 \\
\hline & 4 & 25.29 & 96.08 & 387.19 & 1537 \\
\hline \multirow{4}{*}{ Sobel - CGR } & 1 & 33.04 & 133.66 & 529.74 & 2136.26 \\
\hline & 2 & 26.98 & 109.63 & 440.38 & 1751.99 \\
\hline & 3 & 27.82 & 102.26 & 412.56 & 1636.79 \\
\hline & 4 & 28.44 & 97.89 & 397.4 & 1578.09 \\
\hline \multirow{4}{*}{ Roberts - CGR } & 1 & 32.11 & 131.02 & 527.9 & 2128.85 \\
\hline & 2 & 27.01 & 107.24 & 435.99 & 1755.22 \\
\hline & 3 & 26.36 & 103.15 & 406.78 & 1631.55 \\
\hline & 4 & 25.42 & 97.22 & 399.73 & 1570.45 \\
\hline Sobel-SW & - & 18.72 & 75.82 & 296.39 & 1221.42 \\
\hline Roberts - SW & - & 8.84 & 33.97 & 130.43 & 524.6 \\
\hline
\end{tabular}


Detailed power traces have been acquired using the embedded ARTICo $^{3}$ monitoring infrastructure. Figure 3-19 showcases the different flavors of hardwarebased execution when processing input images of $512 \times 512$ pixels using 4 hardware accelerators working in parallel operation mode. As it can be seen, there is no significant difference between the energy consumed by the standalone dataflow kernels and its CGR variants. Figure 3-20, on the other hand, showcases the softwarebased execution under the same input image configuration. Although the energy consumption values are closer to the references than the execution times, the softwarebased implementations still outperform the dataflow-based hybrid reconfigurable multi-accelerator system.
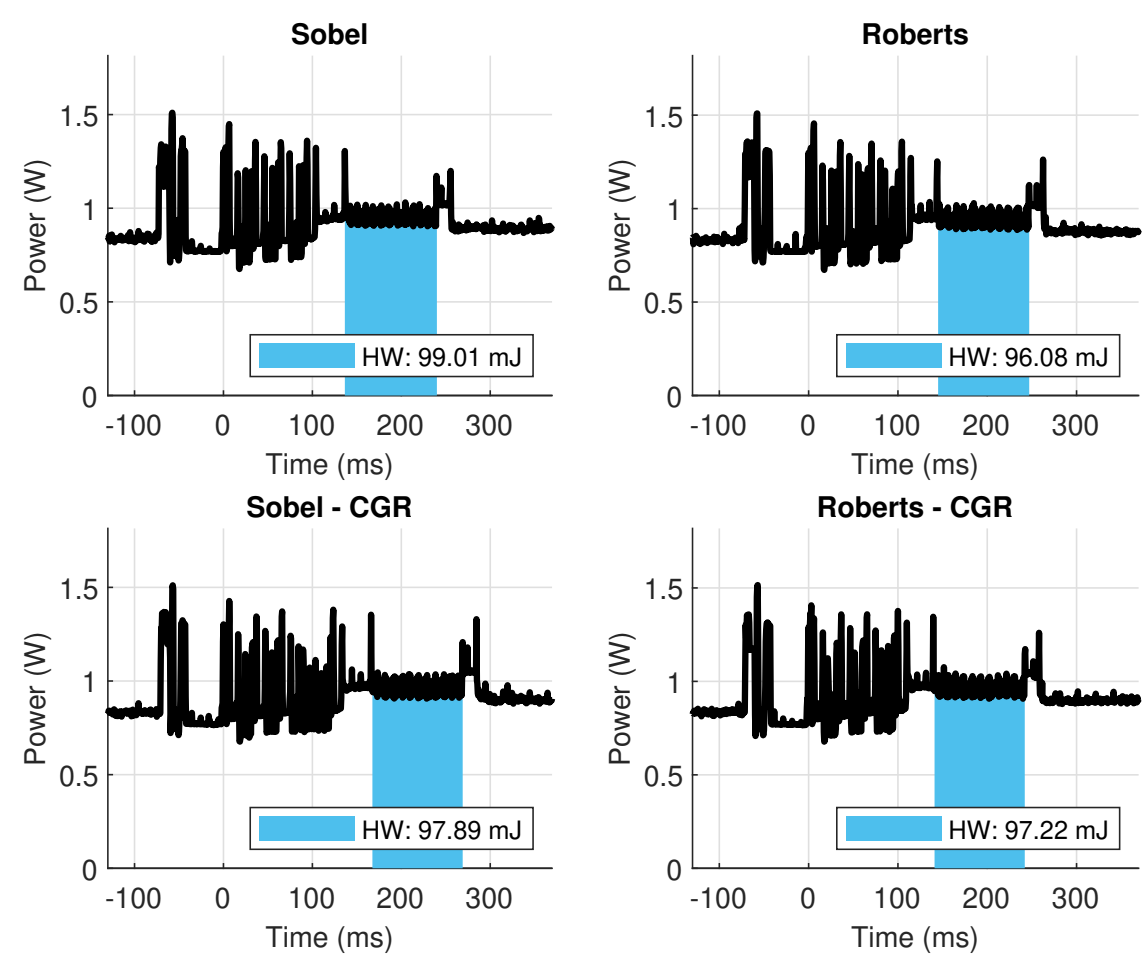

Figure 3-19: Sobel versus Roberts $(512 \times 512$ pixels $)$ using 4 accelerators.

Discouraging as the previous results may be, it is important to recall the main contributions of the MDC-ARTICo ${ }^{3}$ integration regarding reconfigurable multiaccelerator systems: a novel dataflow-based entry point for the design-time stage, and a hybrid reconfiguration mechanism based on embedding application-specific CGR overlays on DPR-capable substrates for the run-time stage. In this regard, the experimental setup has already proven the validity of the first contribution.

As a consequence, the last part of this section focuses on analyzing the different reconfiguration tradeoffs that can be achieved in the proposed hybrid reconfiguration scenario. Table 3-10 shows the time and energy overheads of both types of reconfiguration supported in the integrated framework. 


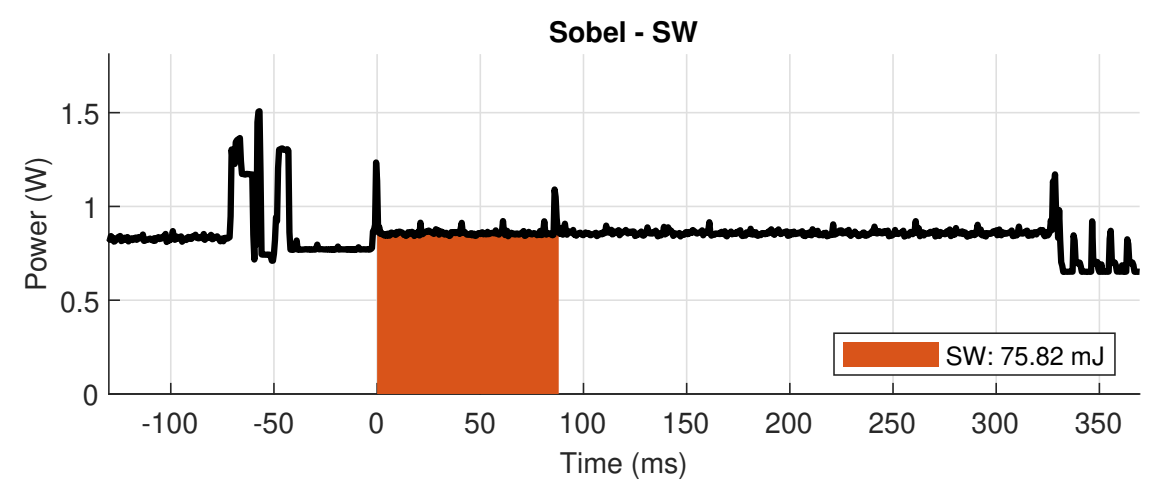

Roberts - SW

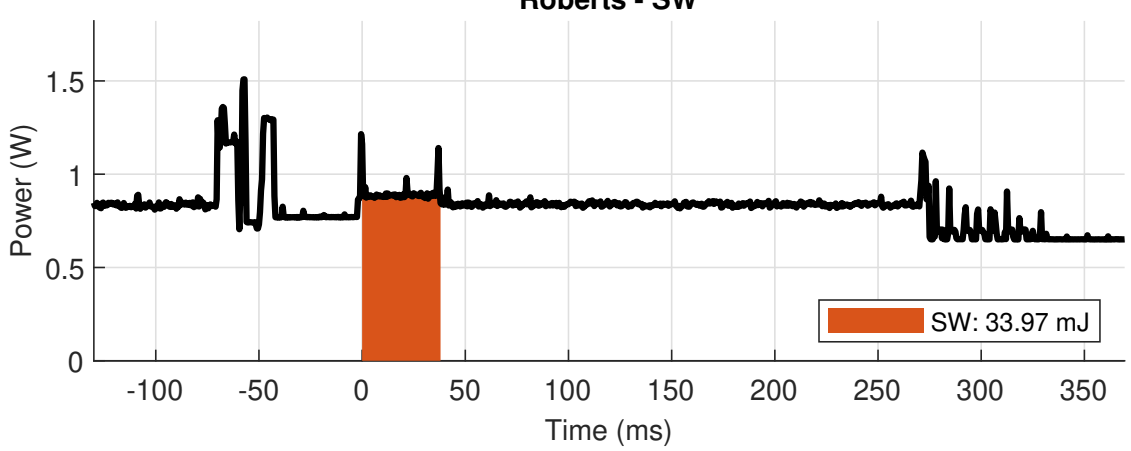

Figure 3-20: Sobel versus Roberts $(512 \times 512$ pixels $)$ in software.

Table 3-10: Reconfiguration overheads in the dataflow-based hybrid reconfiguration scenario.

\begin{tabular}{cccc}
\hline Type & Accelerators & Time (ms) & Energy (mJ) \\
\hline \multirow{4}{*}{ CGR } & 1 & 0.06 & 0.067 \\
& 2 & 0.07 & 0.082 \\
& 3 & 0.08 & 0.095 \\
& 4 & 0.09 & 0.109 \\
\multirow{4}{*}{ DPR } & 1 & 15.8 & 15.18 \\
& 2 & 46.4 & 41.91 \\
& 3 & 74.6 & 67.1 \\
& 4 & 104.4 & 94.11 \\
\hline
\end{tabular}

As it can be seen, CGR is significantly faster and more energy-efficient than DPR. The memory footprint of each reconfiguration strategy is also biased (in a positive way) towards CGR, which only requires a few bytes to modify the functionality instantiated on the FPGA, as opposed to the partial bitstream files (whose size is from three to six orders of magnitude larger in this evaluation scenario) used to perform DPR. However, it is important to highlight that the adopted CGR approach constrains the range of possible configurations to be contained in a reduced application set (i.e., the multifunctional dataflow specification used as input for MDC), whereas DPR still enables highly flexible functional changes in the reconfigurable device. Hence, the proposed CGR+DPR mechanism combines the best of both worlds, providing a fast, flexible, and adaptive hybrid reconfiguration solution for SRAM-based FPGAs. 
Finally, Figure 3-21 illustrates the power consumption traces acquired during system reconfiguration, where it is possible to clearly see the difference of three orders of magnitude between the energy spent to change the functionality of an ARTICo ${ }^{3}$ slot using CGR and DPR.

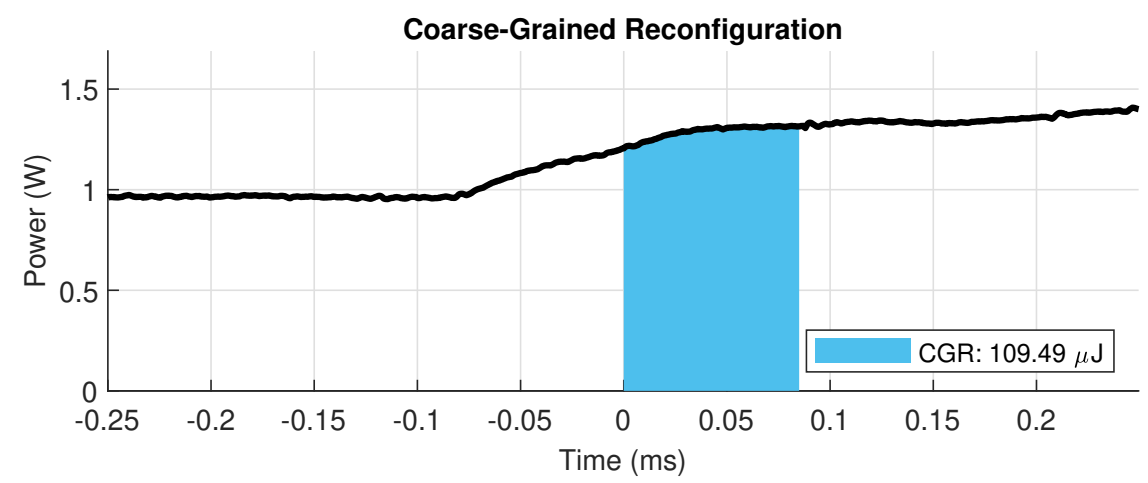

Dynamic and Partial Reconfiguration

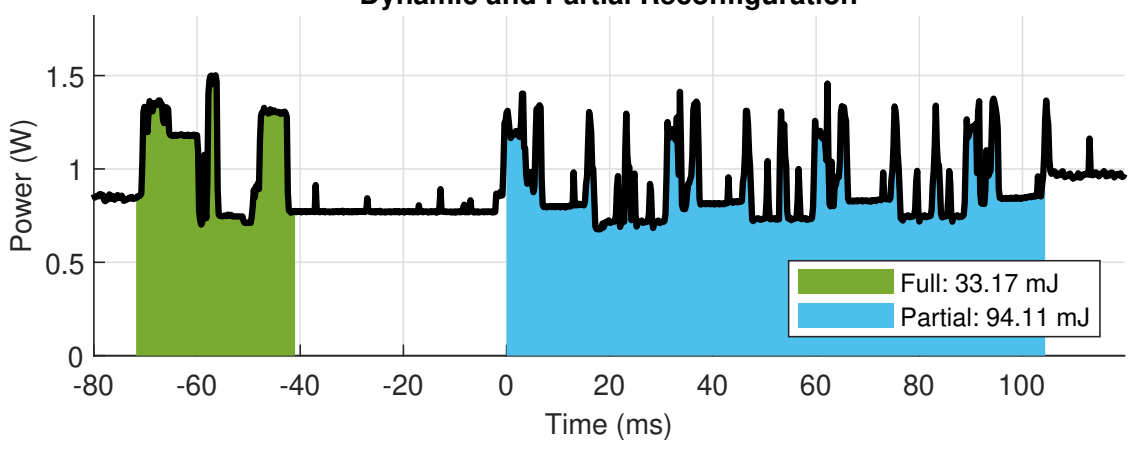

Figure 3-21: CGR versus DPR when reconfiguring 4 accelerators. 


\section{Chapter}

\section{ADAPTIVE HYPERSPECTRAL DATA PROCESSING}

The framework proposed in this Thesis is used in this chapter to deploy two hyperspectral data processing algorithms: lossless compression and linear unmixing. Hyperspectral data processing applications have highly demanding computing requirements and are usually performed on board, which makes them suitable use cases to evaluate the benefits of run-time adaptivity in reconfigurable highperformance embedded processing systems.

The chapter starts by giving a general overview and introduction to hyperspectral data processing, motivating the use of ARTICo ${ }^{3}$-based systems for that particular scenario. Then, each algorithm is addressed separately but using a common structure: context and introduction, description of the algorithm, state of the art and related work, contributions at implementation level, and validation tests.

\subsection{Motivation}

Hyperspectral imaging is considered nowadays a key technology in the field of remote sensing. As reported by the United States Geological Survey (USGS), the main objective of remote sensing is to analyze, detect or even classify the physical characteristics of a given area based on its radiation behavior (i.e., emission and reflection) when observed from a certain distance. ${ }^{1}$

The use of hyperspectral images for remote sensing applications was originally proposed in 1985 under the name of imaging spectrometry [Goetz'85]. With the introduction of this new technology, acquiring laboratory-like spectral samples from aircrafts or even spacecrafts became feasible, thus constituting a major breakthrough in the analysis and classification of remotely sensed data. From this moment onwards, there has been a constant evolution in tems of both data acquisition methods and data processing algorithms [Plaza'09, Bioucas-Dias'13, Ghamisi'17].

The main feature of hyperspectral images is that information is presented in several (usually a few hundred) contiguous spectral bands, where each band corresponds to the radiance of a surface at a certain wavelength [Bioucas-Dias'13]. Hence,

\footnotetext{
${ }^{1}$ https://www.usgs.gov
} 
hyperspectral images have two spatial dimensions (where columns are called samples and rows are called lines) and one spectral dimension. As a result, hyperspectral imaging sensors have two types of resolution: spatial and spectral. The former represents the physical distance between pixels, and the latter represents the distance between bands (i.e., wavelengths). Figure 4-1 shows a hyperspectral data cube.

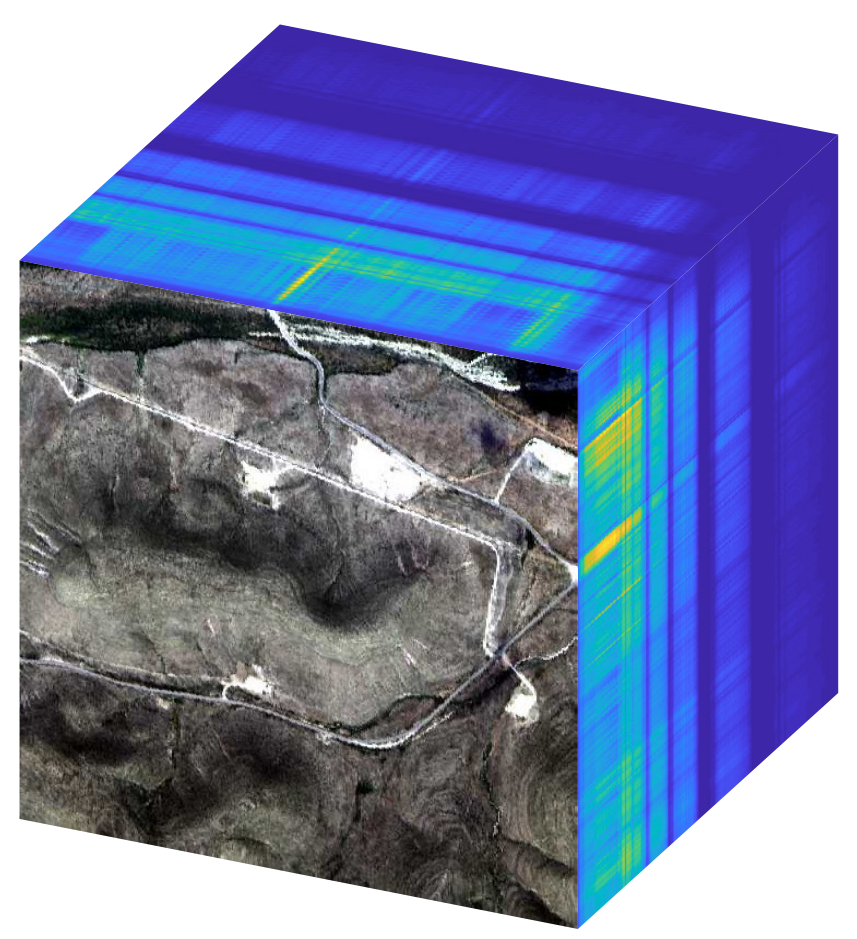

Figure 4-1: Hyperspectral data cube.

The vast amount of potential information contained in hyperspectral images has motivated the use of remotely sensed hyperspectral data in a wide range of application scenarios such as agriculture (e.g., vegetation classification or pest detection), geology, soil analysis, land cover, urban applications, water resource monitoring, or disaster prevention [Transon'18]. However, the use of hyperspectral images is not constrained to remote sensing applications anymore, as the number of new application fields where this technology has been successfully applied increases (e.g., medical applications [Fabelo'16, Fabelo'19]).

In any case, and although the benefits of using hyperspectral imaging technology are numerous, it also poses several challenges. Take for instance a hypothetical scenario where a satellite acquires remotely sensed hyperspectral images for geological classification. The amount of data generated by the on-board sensor system can be in the range of several GB of raw data per flight. In addition to these massive onboard storage requirements, the huge processing burden needed to extract relevant 
information from the captured data causes the computational requirements to surge as well [Bernabé'17]. As a result, energy-efficient yet high-performance computing systems are required to cope with these stringent requirements. Furthermore, these systems must be sometimes embedded. In fact, the traditional approach to hyperspectral data processing in remote sensing scenarios relies on a first stage where the acquired data is compressed on board [Santos'14], followed by decompression, preprocessing, and further processing stages in HPC facilities on Earth [Plaza'07].

In the last few years, there has been an increasing interest on using FPGAs to implement different on-board hyperspectral processing algorithms [López'13]. As opposed to other general-purpose computing platforms, such as CPUs or GPUs, FPGAs provide the required levels of computing power, energy efficiency, and size for those specific scenarios. In addition, some vendors (e.g., Xilinx or Microsemi) also provide radiation-hardened versions of their devices, making FPGAs the best implementation alternative for spaceborne hyperspectral applications.

In this Thesis, the use of $\mathrm{ARTICo}^{3}$ as a high-performance embedded computing platform for on-board hyperspectral data procesing in remote sensing applications is evaluated using two different use cases: lossless data compression and linear unmixing. The former is based on the traditional approach to remote sensing, exploiting the adaptive behavior of ARTICo ${ }^{3}$-based hardware acceleration. The latter, on the other hand, is barely performed on board. Nevertheless, the proposed implementation evaluates the benefits of moving certain parts of the hyperspectral processing chain to high-performance ARTICo ${ }^{3}$-enabled edge nodes in order to save data compression and communication overheads.

\subsection{Lossless Compression}

Data compression algorithms usually take advantage of information redundancy to reduce data volume. As opposed to traditional imaging sensors, where only spatial redundancies can be found, hyperspectral imaging sensors also present redundancies in the spectral domain. Hence, hyperspectral data compression algorithms can take advantage of both spatial and spectral redundancy to compress the acquired images. In addition, these algorithms can be designed to perform data compression without losing any of the information present in the input image (i.e., lossless compression), or sacrifice information integrity to achieve better compression efficiency (i.e., lossy compression). Hyperspectral image compression efficiency is measured in bits per pixel per band (bpppb), a metric that can be computed using Equation 4-1.

$$
\text { bppp } b=\frac{\text { Compressed image size (bits) }}{N_{\text {bands }} \cdot N_{\text {lines }} \cdot N_{\text {samples }}}
$$


The internal structure of a hyperspectral data compressor usually consists of a spatial and/or spectral decorrelator, a quantization stage, and an entropy coder [Santos'14, Guerra'17a]. The entropy coder analyzes the probability distribution of its input symbols and generates efficient encodings for them. As a result, shorter codewords are used for those symbols with higher probability values. Regarding the decorrelator, two main approaches can be used for its implementation: transformbased and prediction-based. Transform-based approaches couple a spectral transform with a spatial transform to decorrelate data. Example transforms that are commonly used include the Karhunen-Loève Transform (KLT) [Chang'00] and the Discrete Wavelet Transform (DWT) [Penna'06]. On the contrary, prediction-based approaches use a small three-dimensional neighborhood (i.e., spatial and spectral dimensions) to predict the value of a sample. Usually, transform-based solutions lead to more efficient lossy compression at the expense of increased computational complexity, whereas prediction-based solutions are usually preferred for lossless compression [Santos'14, Guerra'17a].

It is worth noting that lossless or near-lossless compression has been the preferred alternative for scientific missions. One of the main features in these scenarios is the need for computationally efficient algorithms to perform on-board processing. In this regard, the Consultative Committee for Space Data Systems (CCSDS), which is an organization constituted by the major space agencies in the world, has issued three reference standards (with their correspondent revisions) to address data compression in space: a universal lossless data compressor (i.e., CCSDS 121 [CCSDS'12a]), a twodimensional image compressor (i.e., CCSDS 122 [CCSDS'17]), and a low-complexity lossless and near-lossless multispectral and hyperspectral image compressor (i.e., CCSDS 123 [CCSDS'19]).

This section covers the implementation of a run-time adaptive lossless multispectral and hyperspectral compressor based on the CCSDS 123 standard using the ARTICo $^{3}$ framework. In the proposed solution, input hyperspectral images are partitioned in a variable number of fixed-size segments that are processed concurrently using a configurable number of low-complexity hardware accelerators, enabling run-time tradeoffs between compression throughput and energy efficiency.

\subsubsection{Algorithm Description}

The CCSDS 123 standard [CCSDS'12b] formalizes the definition of a lossless multispectral and hyperspectral compressor based on the Fast Lossless (FL) compressor [Klimesh'06]. ${ }^{2}$ The compressor has two adaptive functional stages: a predictor and an encoder. The former generates the prediction residuals (i.e., the difference between

\footnotetext{
${ }^{2} \mathrm{~A}$ new version of the reference standard is available from CCSDS [CCSDS'19]. This revision introduces changes to enable near-lossless compression by means of a closed-loop quantization scheme, and extends the reference entropy coding method to provide better compression for lowentropy data. However, the IP core used in this Thesis was designed according to the previous specification and thus, this section describes the compressor from the first version of the standard.
} 
the predicted and the actual samples), which are then mapped into unsigned integers, whereas the latter performs entropy coding of these mapped prediction residuals. The inherent adaptivity of the compressor forces the compressed bitstreams that are obtained as output to be of variable size.

The predictor uses an adaptive linear prediction method to predict the value of a sample based on a small three-dimensional neighborhood, as it can be seen in Figure 4-2. The nearby samples used for prediction are extracted from the current band and the $P$ previous bands, being $P$ a user-defined parameter that can range between 0 (i.e., no information from previous bands is used for prediction) and 15. It is important to highlight that the neighborhood is conveniently truncated when required (i.e., $y=0$, $x=0, x=N_{\text {samples }}-1$ or $\left.z<P\right) .^{3}$

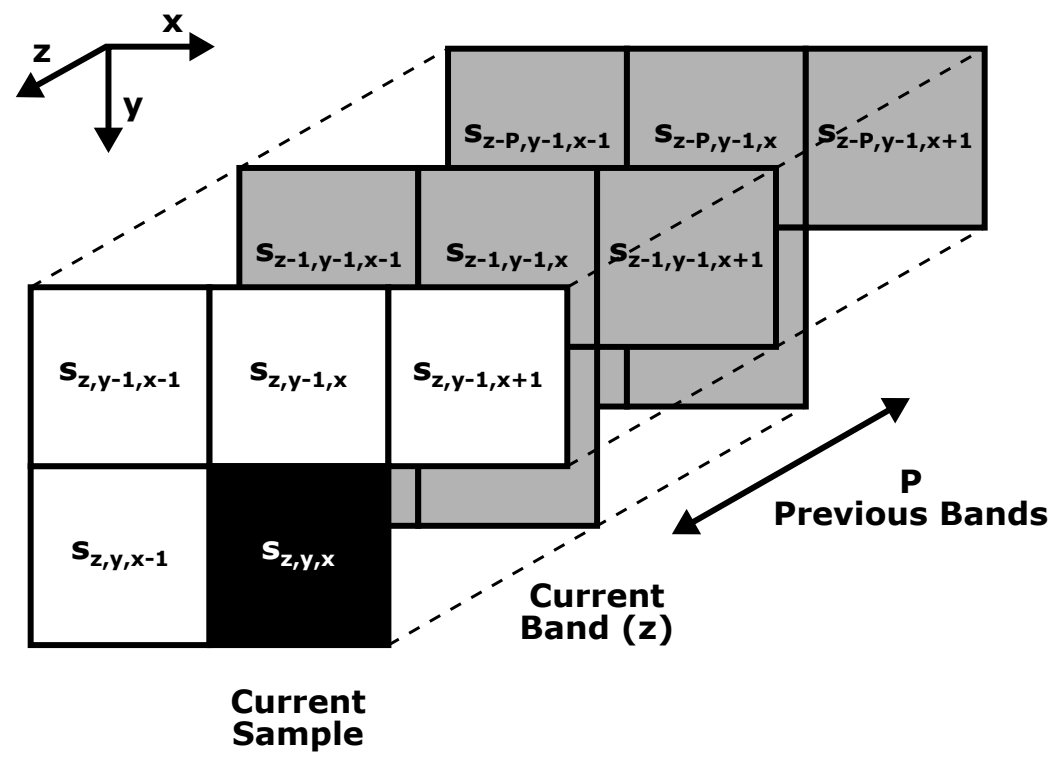

Figure 4-2: CCSDS 123 prediction neighborhood.

In order to provide the prediction (i.e., $\hat{s}_{z, y, x}$ ) of the current sample (i.e., $s_{z, y, x}$ ), the predictor first computes the local sums (i.e., $\sigma_{z, y, x}$ ), which are basically weighted sums of the adjacent pixels. The reference standard supports two alternatives for the predictor to calculate the local sums: neighbor-oriented and column-oriented. The neighbor-oriented local sum uses all nearby samples in the current band, whereas the column-oriented local sum only uses the sample located in the same position but in the previous line. Table 4-1 shows the equations for both cases. It is up to the user to decide which type of local sum is to be used in their implementation.

The next step that is performed by the predictor is to compute the local differences vector (i.e., $U_{z, y, x}$ ) using the local sums. Depending on the user preferences, the local differences vector will contain only the central local differences (i.e., $d_{z, y, x}$ ) when operating in reduced prediction mode, or both central local differences and directional

\footnotetext{
${ }^{3}$ In this document, all corner cases that appear when the neighborhood is truncated have not been considered in the equations. Please refer to the reference standard for more details.
} 
Table 4-1: Equations to calculate the local sum $\sigma_{z, y, x}$.

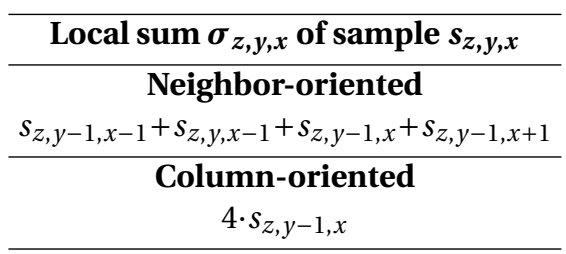

local differences (i.e., $d_{z, y, x}^{N}, d_{z, y, x}^{W}$ and $d_{z, y, x}^{N W}$ ) when operating in full prediction mode. The equations used to compute these values can be seen in Table 4-2.

Table 4-2: Equations to calculate the local differences vector $U_{z, y, x}$.

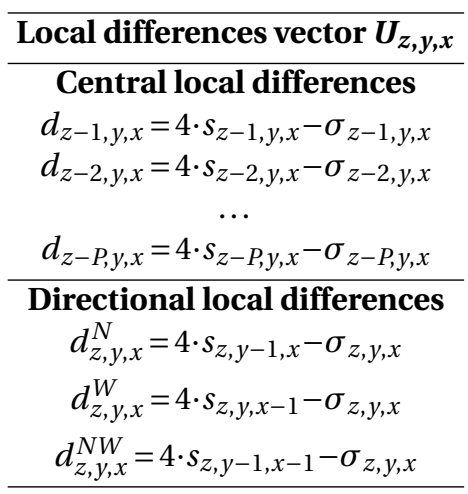

The predicted value is then computed using the local sums and a weighted sum of local difference values from the current and previous spectral bands. Moreover, the weights used in this calculation (i.e., $W_{z, y, x}$ ) are adaptively updated after each sample prediction taking into account the prediction error. The last step performed by the predictor is to compute the prediction residuals (i.e., $s_{z, y, x}-\hat{s}_{z, y, x}$ ) and map them into unsigned integer values (i.e., $\delta_{z, y, x}$ ).

The output of the predictor is fed to the encoder. The standard supports two different types of entropy coders: sample-adaptive and block-adaptive. The former uses a variable-length binary codeword to encode each mapped prediction residual, whereas the latter relies on the adaptive entropy coder defined in the CCSDS 121 reference standard [CCSDS'12a] to encode blocks of mapped prediction residuals using Rice's adaptive coding technique [Rice'93].

The complete flowchart of the CCSDS 123 lossless multispectral and hyperspectral compressor can be seen in Figure 4-3. 


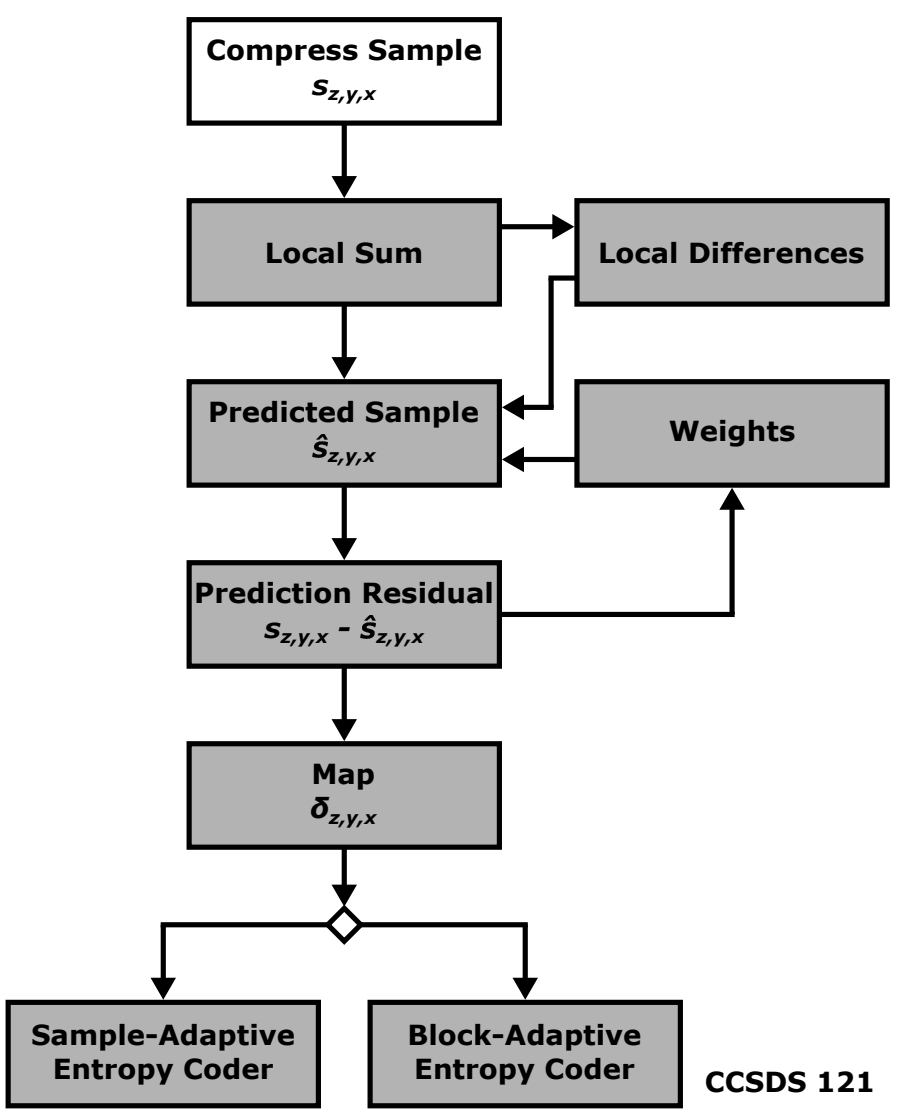

Figure 4-3: CCSDS 123 flowchart.

\subsubsection{State of the Art}

As it has been already discussed throughout the motivation in Section 4.1, the data compression stage is mandatory in remote sensing applications, especially in those based on hyperspectral imaging technology. Traditionally, hyperspectral data compression has been addressed from two different perspectives: algorithm- and implementation-wise [Santos'14]. The former focuses on taking advantage of the inherent properties of hyperspectral images in order to achieve better compression efficiency. The latter, on the other hand, focuses on developing high-performance, yet resource- and energy-efficient solutions to be deployed in on-board computers.

Algorithm-wise, Section 4.2 builds on top of the CCSDS 123 standard for lossless hyperspectral data compression [CCSDS'12b]. In this regard, a key contribution is the parallelization approach (i.e., the partitioning of input images in several segments that are then processed separately) to enable compatibility with the ARTICo ${ }^{3}$ framework, together with the analysis of its impact on the compression efficiency.

Using image partitioning to enable parallel processing in hyperspectral data compression algorithms is a common strategy. For instance, ICER-3D [Kiely'05] is a transform-based hyperspectral data compressor that partitions the input image in a user-defined number of segments that are later compressed independently, increasing 
the fault tolerance of the whole system as well (i.e., an error affecting one of the segments does not prevent the decompression of the rest of the original hyperspectral image). However, this algorithm is outperformed in terms of compression efficiency by the FL compressor [Klimesh'06], which was later promoted to the CCSDS 123 standard.

In any case, the main contribution of Section 4.2 is implementation-wise and thus, the following discussion focuses on different realizations of the CCSDS 123 hyperspectral data compression algorithm on several computing platforms.

The first implementations of the standard were made on CPUs [Sánchez'11] and GPUs [Hopson'12]. In fact, the former was done even before the official standard was released, and aimed at providing an open source tool (i.e., Emporda) to test and evaluate the algorithm under development. ${ }^{4}$ The latter, on the other hand, presented an in-depth analysis of the potential parallelism in the algorithm, as well as its implementation using both OpenMP in multi-core CPUs and CUDA in GPUs. Memory limitations in the target computing devices forced the partitioning of input images in several segments that were processed sequentially, since parallelism had been extracted from the algorithm on a per-stage basis. Although acceptable throughput values were obtained, the use of GPUs as the main computing platform discards this solution in actual space deployments, where devices need to comply with stringent requirements in terms of power consumption and radiation tolerance.

The limited execution performance of CPUs, and the aforementioned deployment problems of GPUs, motivated the use of dedicated hardware to perform on-board compression [López'13]. Precisely, these highly optimized on-board accelerators appeared in the shape of ASICs [Poupat'13] and radiation-hardened FPGAs. The first fully compliant implementation of the CCSDS 123 algorithm in an FPGA was HyLoC [Santos'16]. This hardware accelerator was designed with two main objectives: first, to provide better compression throughput than previous softwarebased implementations; second, to achieve low impact in terms of logic resources. As such, detailed DSE was performed to measure the ratio between compression efficiency and area overhead when modifying several parameters of the compression algorithm. Then, the resource utilization of the IP core was evaluated using a spacequalified device, showing less than $50 \%$ occupancy for the configuration with the highest complexity.

Current implementations of the CCSDS 123 standard in hardware diverge from the original goals of the HyLoC IP core, focusing on providing as much compression throughput as possible rather than on optimizing for size. SHyLoC [Santos'20], which is the evolution of the original HyLoC, is one example of this trend. This compressor, now part of the European Space Agency (ESA) repository, contains two different IP cores that are fully compliant with the CCSDS 121 [CCSDS'12a] and CCSDS 123 [CCSDS'12b] standards. Moreover, it is a highly configurable implementation where all the parameters of the internal compression cores can be changed either at design time (i.e., before the logic synthesis process) or at run time using a dedicated configuration interface.

\footnotetext{
${ }^{4}$ http://www.gici.uab.es
} 
The work presented in [Báscones'18] removes the need for outsourced storage by efficiently using the internal RAM memory in the FPGA rather than an external device and therefore, it is able to achieve real-time compression. This work is extended in [Báscones'17], where the internal architecture of the compressor is modified to enable parallel execution of the algorithm. In this case, data dependencies are handled by enabling direct path-to-path communication channels and intermediate buffers. The parallel implementation is compared against an OpenCL-based solution developed for GPUs in terms of compression throughput, energy-efficiency, and productivity.

In [Fjeldtvedt'18], the authors propose a deeply pipelined architecture to achieve high compression throughput. This implementation supports configuration tuning using the majority of parameters defined in the standard. An extended implementation is presented in [Orlandić'19], where several optimized pipelines are used in parallel to further increase the compression throughput. Communication with all the pipelined processing instances is performed using a specific routing infrastructure with delay modules to provide data when required.

Another high-performance implementation of the CCSDS 123 standard can be found in [Tsigkanos'18]. As some of the previously described solutions, it does not require outsourced memory, since all processing and storage is located inside the FPGA device. The main contribution of the work is the possibility of adjusting the pipeline depth of the compressor architecture at design time (i.e., applying reconfigurable pipelining). Lastly, the work presented in [Davidson'18] shows an upto-date implementation of the compression standard on a low-power embedded GPU. The implementation relies on input image partitioning in order to properly exploit data-level parallelism. Furthermore, its fault tolerance is thoroughly evaluated using error injection, as a first step towards GPU-based deployments in space applications.

The comparison between all these implementations and the solution proposed in this Thesis can be found in Table 4-8.

\subsubsection{Implementation Details}

The run-time adaptive lossless hyperspectral compressor presented in this Thesis is based on the multispectral and Hyperspectral Lossless Compressor for space applications (HyLoC) IP core [Santos'16]. HyLoC is a low-complexity HDL implementation of the CCSDS 123 standard. Since it targets resource-constrained devices (e.g., radiationhardened FPGAs or ASICs), it performs compression in Band-Sequential (BSQ) order and uses sample-adaptive entropy coding. Moreover, it serializes the computation of the local differences and weight vectors to reduce the complexity of its logic. Despite these restrictions, the IP core is fully compliant with the CCSDS 123 reference standard and supports parameter tuning at design time (i.e., before logic synthesis). The block diagram of the HyLoC compressor is shown in Figure 4-4.

A key aspect during the development process of the HyLoC IP core was the analysis of the different tradeoffs between compression efficiency and hardware complexity of 


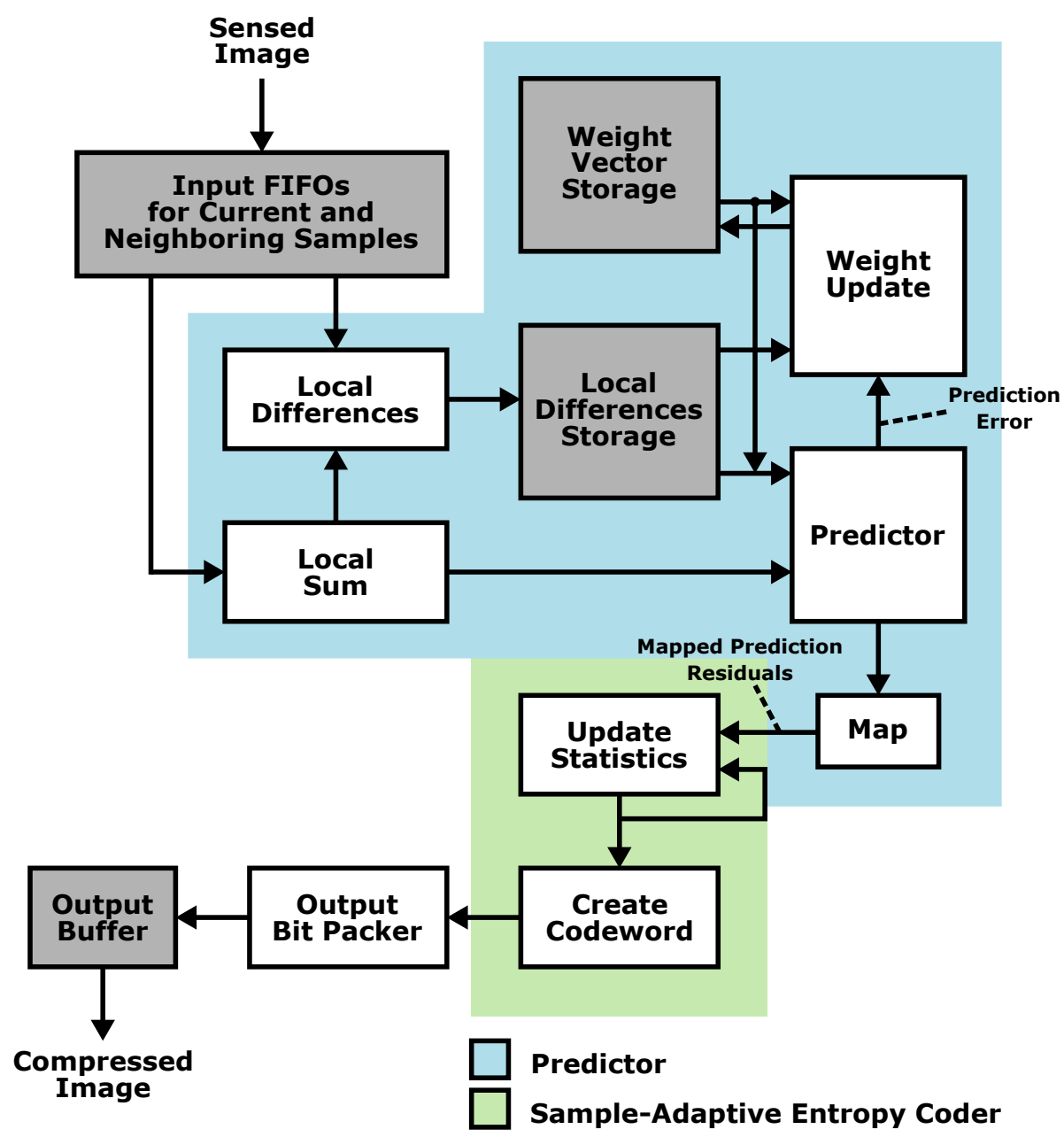

Figure 4-4: Block diagram of the HyLoC IP core.

the implementation. Hence, several scenarios were evaluated using variations in each of the user-defined parameters [Santos'16]. The resulting implementation enables the compression of any hyperspectral image in BSQ order without requiring external storage for the local differences vector, and with the smallest logic resource footprint in the prediction stage. In return, this leads to limitations in terms of computing performance, mainly due to operation serialization (e.g., in the dot product between the weights and the local differences vector).

Nevertheless, the excessive latency of the HyLoC IP core can be mitigated by instantiating more than one compressor in the target device, a feasible scenario due to the limited resource utilization of the hardware accelerator. Hence, the proposal of this Thesis is to use HyLoC together with the ARTICo ${ }^{3}$ framework to deploy a runtime adaptive CCSDS 123 lossless hyperspectral compressor configured as indicated in Table $4-3 .^{5}$

\footnotetext{
${ }^{5}$ The HyLoC IP core supports further parameter customization, but only the parameters shown in the table have an actual impact in terms of compression efficiency and area overhead [Santos'16].
} 
Table 4-3: CCSDS 123 compressor configuration.

\begin{tabular}{cc}
\hline Parameter & Value \\
\hline Dynamic Range & 16 \\
Encoding Order & BSQ \\
Bands for Prediction (P) & 3 \\
Local Sum Mode & Neighbor-Oriented \\
Prediction Mode & Full Prediction \\
\hline
\end{tabular}

Assuming normal operation, HyLoC should compress the whole hyperspectral image that is provided as input. However, this is incompatible with the data-parallel execution model of ARTICo ${ }^{3}$. Although a possible solution is to split the output of the hyperspectral imaging sensor in several subimages, this procedure might generate certain side effects: on the one hand, the resulting implementation could no longer be compatible with the reference standard; on the other hand, there could be an infinite number of schemes to partition the images.

Regarding the first issue, the reference standard states that "A user may choose to partition the output of an imaging instrument into smaller images that are separately compressed, e.g., to limit the impact of data loss or corruption on the communications channel, or to limit the maximum possible size of a compressed image. This Recommended Standard does not address such partitioning or the tradeoffs associated with selecting the size of images produced under such partitioning." [CCSDS'12b], and a complementary document also states that "The Recommended Standard does not directly address such image segmentation; the term 'segment' is not part of the standard. In the view of the Recommended Standard, each such image segment is simply a separate image." [CCSDS'15]. Hence, using any type of partitioning in the input hyperspectral image, such as the one depicted in Figure 4-5, is acceptable as long as each segment of the original image is addressed as a separate image. In fact, the scheme shown in Figure 4-5 produces image segments by partitioning the input along the y-axis, which is the sensible approach for imaging sensors that capture data in Band-Interleaved-byPixel (BIP) or Band-Interleaved-by-Line (BIL) order. Nevertheless, the CCSDS allows users to use other partitioning approaches [CCSDS'15].

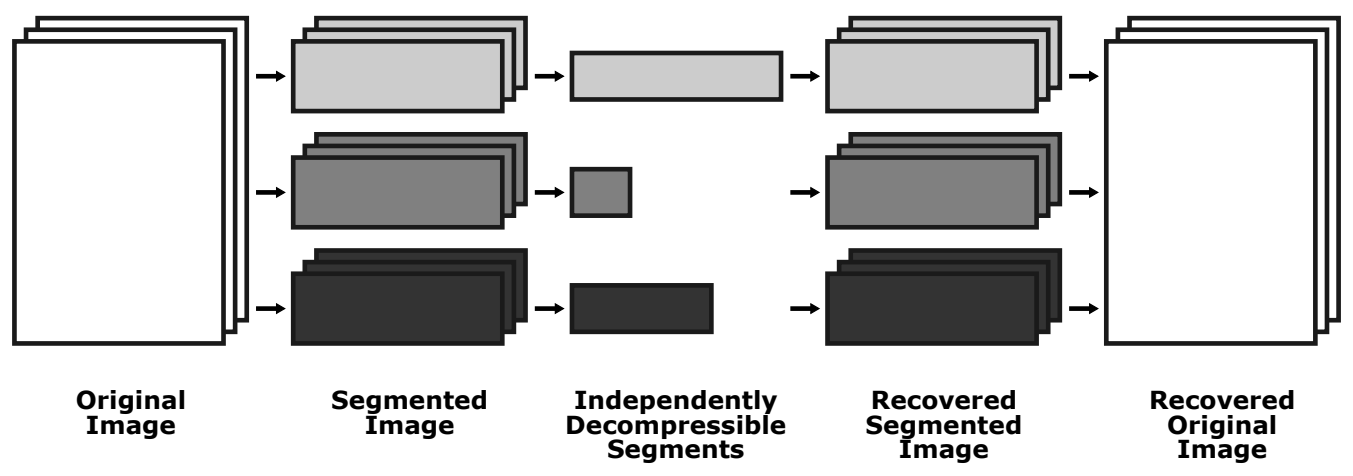

Figure 4-5: Hyperspectral image partitioning for compression. 
Note that the reference standard mentions certain tradeoffs that are to be established when applying partitioning to a hyperspectral image. Essentially, the main tradeoff is compression efficiency versus image segment size, which links to the second issue that was mentioned before. Generally speaking, partitioning any image renders worst compression efficiency for the following reasons:

- Each compressed segment has an independent image header (generated by the CCSDS 123 algorithm).

- The boundaries between segments are corner cases that limit prediction due to a reduced number of available neighbors.

- Small segments usually mean less data available in the predictor, which renders worst results for the first compressed samples.

Apart from these considerations, which are algorithm-specific, there are a few other things that need to be taken into account to enable an implementation that is fully compatible with the ARTICo ${ }^{3}$ execution model and its associated low-level restrictions (e.g., memory storage requirements). To this end, two different partitioning schemes have been analyzed and evaluated: on the one hand, a strip-based partitioning over the $y$-axis; on the other hand, a square-based partitioning over both $y$-axis and x-axis. ${ }^{6}$

The strip-based partitioning, whose results are presented in Figure 4-6, shows convergence in the compression efficiency provided that image segments are sufficiently large. Compression efficiency is far from optimal with small input images, especially if these images are further divided in smaller segments. In addition, this partitioning scheme is non-uniform, since the image segment size changes with the input image size.

The square-based partitioning, whose results are presented in Figure 4-7, shows regular but strong deviations in terms of compression efficiency when changing the size of the image segments. This is mainly due to data scarcity in relatively small image segments, as it was already foreseen. However, this partitioning has the advantage of being uniform (i.e., image segments have fixed size, regardless of the size of the actual input images), and renders acceptable deviations in the compression efficiency, provided that segments are sufficiently large. For instance, using segments of $8 \times 8$ pixels in images of $512 \times 512$ pixels (spatial dimensions), the compression rate goes from 4.022 to $5.015 \mathrm{bpppb}$ (an increment of around 25\%).

Although the best compression results seem to be obtained when using the stripbased partitioning approach, it is still incompatible with $\mathrm{ARTICo}^{3}$ for two reasons. First, the obtained results are not scalable, since they depend on the actual input image sizes. Second, most of the scenarios are not feasible to be implemented in hardware due to limited resource availability. In fact, the black dashed line

\footnotetext{
${ }^{6}$ Experimental validation has been performed using calibrated AVIRIS images with 224 bands and variable spatial dimensions. The base image used corresponds to flight f080927t01p00r10.
} 


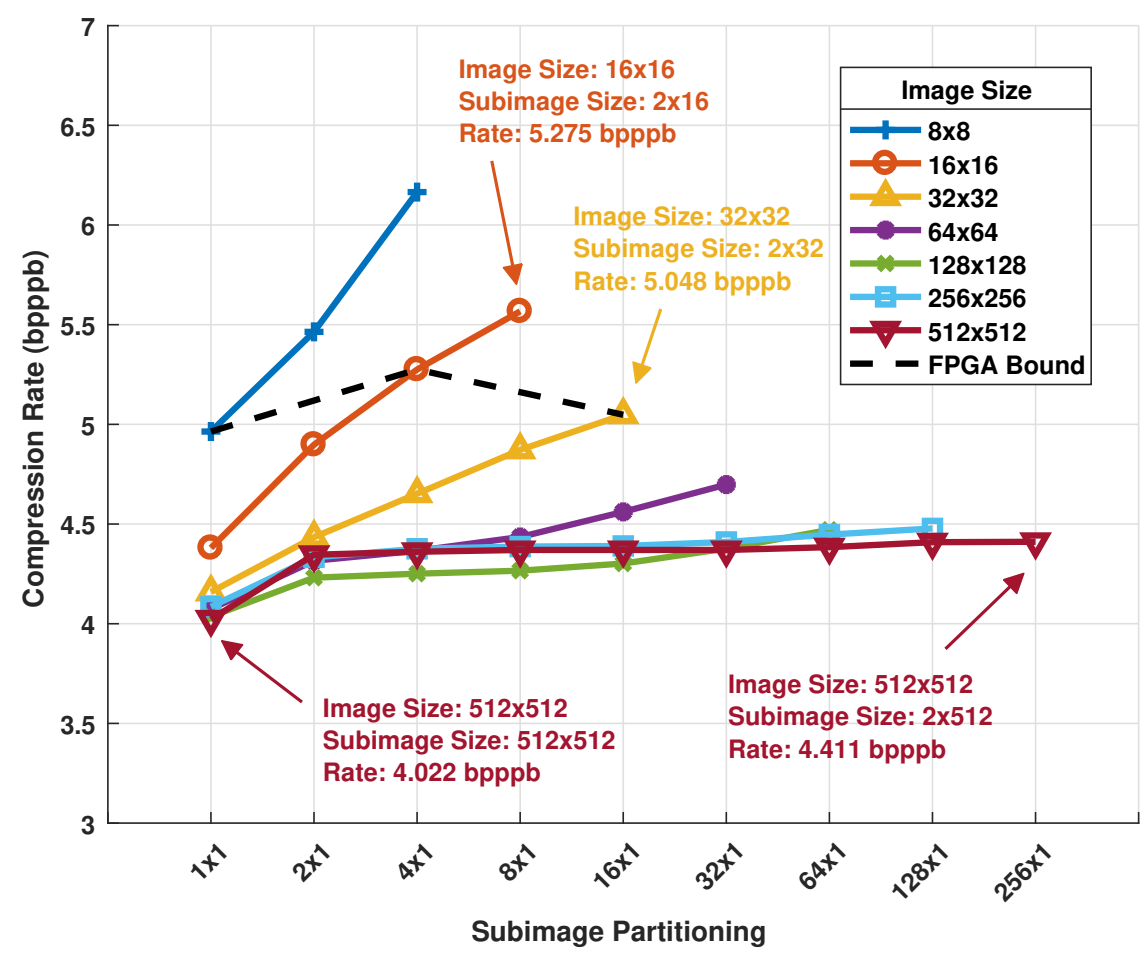

Figure 4-6: Compression rate with strip-based partitioning.

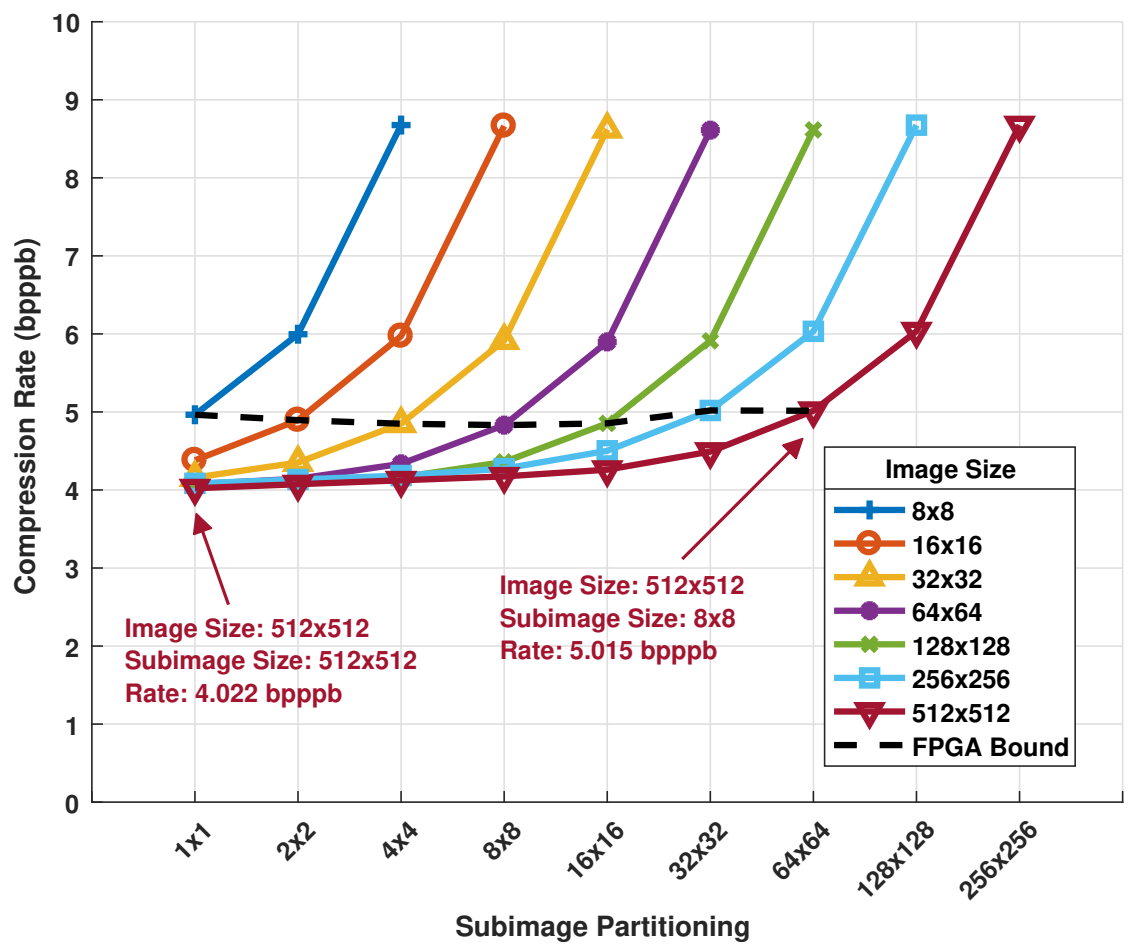

Figure 4-7: Compression rate with square-based partitioning. 
present in both Figure 4-6 and Figure 4-7 represents the low-level implementation restrictions imposed by the ARTICo ${ }^{3}$ toolchain, where feasible solutions are those placed on or above the line. ${ }^{7}$ Notice that, for the strip-based partitioning scheme, those configurations that provided better compression results fall out of the range of feasible solutions. As a result, the square-based partitioning, which renders bounded results regardless of the size of input images and thus favors scalable execution, has been implemented in this Thesis.

The selected hyperspectral image partitioning approach has been also tested with two of the most widely used hyperspectral datasets available: the Indian Pines scene [Baumgardner'15], and the Yellowstone scene collection [Kiely'09]. The results obtained by applying the proposed partitioning scheme to these input datasets can be seen in Table 4-4. Please note that the image segments used have 224 bands, 8 lines and 8 samples, and that the input datasets have been slightly augmented (Indian Pines) or reduced (Yellowstone scenes) to achieve correct partitioning with an integer number of segments. Results show that in the worst case there is only $28 \%$ overhead in terms of compression efficiency when compared against the full image compression process, a value that is consistent with previous tests.

Table 4-4: Compression rate with square-based partitioning over hyperspectral datasets.

\begin{tabular}{cccc}
\hline \multirow{2}{*}{ Image } & \multirow{2}{*}{$\mathbf{N z} \times \mathbf{N y} \times \mathbf{N x}$} & \multicolumn{2}{c}{ Rate (bpppb) } \\
& & Single & Parallel \\
\hline Indian Pines & $220 \times 152 \times 152$ & 6.3 & 7.07 \\
\hline Yellowstone SCN 0 & 3.98 & 4.98 \\
Yellowstone SCN 3 & & 3.87 & 4.86 \\
Yellowstone SCN 10 & $224 \times 512 \times 512$ & 3.37 & 4.28 \\
Yellowstone SCN 11 & 3.68 & 4.61 \\
Yellowstone SCN 18 & 3.97 & 5.09 \\
\hline
\end{tabular}

Although the analysis presented in this section has been particularized for $\mathrm{ARTICo}^{3}$, it is worth noting that the proposed parallelization and partitioning approach is platform-agnostic and therefore, holds valid for either CPUs, GPUs, and hardware accelerators implemented on FPGAs.

Original contribution 4-1 A scalable, hardware-friendly, and platformagnostic partitioning and parallelization strategy for lossless multispectral and hyperspectral compression based on the CCSDS 123 reference standard with limited impact on the compression efficiency.

\footnotetext{
${ }^{7}$ As stated in Subsection 2.4.1, ARTICo ${ }^{3}$ kernels are instantiated in a parametric wrapper to provide seamless intregration between user logic and the rest of the hardware infrastructure. Currently, the maximum amount of local memory available within this wrapper is $64 \mathrm{KiB}$.
} 


\subsubsection{Validation}

The run-time adaptive lossless multispectral and hyperspectral compressor based on the CCSDS 123 has been experimentally tested using a Zynq MMP development board. In this setup, the target device is a Zynq-7000 SoC with enough logic resources to support massive accelerator scalability. ${ }^{8}$ Actually, the template used in the ARTICo ${ }^{3}$ toolchain generates an overlay in the FPGA with 16 reconfigurable slots. Figure 4-8 shows the implemented design, where all reconfigurable regions (highlighted in red) are loaded with the HyLoC kernel (blue). Notice that each hardware accelerator is properly connected to the $\mathrm{ARTICo}^{3}$ architecture (orange) using the ARTICo ${ }^{3}$ point-topoint interface (gray).

Table 4-5 shows the resource utilization of the ARTICo ${ }^{3}$ architecture, one instance of the HyLoC kernel, and the original implementation of HyLoC [Santos' 16], which is used as baseline for comparison purposes. Although the logic synthesis tools and the target FPGAs are not exactly the same, the overhead introduced by the ARTICo ${ }^{3}$ kernel wrapper is almost negligible except for the number of BRAMs (used to implement the local memory banks). Moreover, the overhead of the ARTICo ${ }^{3}$ infrastructure also becomes less relevant when increasing the number of accelerator instances.

Table 4-5: Resource utilization in the run-time adaptive hyperspectral compressor.

\begin{tabular}{|c|c|c|c|}
\hline Component & ARTICo $^{3}$ & HyLoC Kernel & Baseline HyLoC [Santos' 16] \\
\hline Info & $\begin{array}{c}16 \text { slots } \\
\text { VHDL (Vivado) } \\
\text { Zynq-7000 }\end{array}$ & $\begin{array}{c}64 \text { KiB memory, } 2 \text { banks } \\
0 \text { registers } \\
\text { VHDL (Vivado) } \\
\text { Zynq-7000 }\end{array}$ & $\begin{array}{l}\text { VHDL (ISE) } \\
\text { Virtex-5 }\end{array}$ \\
\hline LUTs & 11260 & 2932 & 2342 \\
\hline FFs & 6648 & 1529 & 1535 \\
\hline DSPs & 2 & 1 & 1 \\
\hline BRAMs & - & 16 & - \\
\hline
\end{tabular}

Energy efficiency is also an important metric for on-board multispectral and hyperspectral compressors, since they often have to operate in resource-constrained scenarios. As a matter of fact, this is also addressed in the CCSDS reference standard, for the specification states that a compressor should be able to provide a compression throughput of $20 \mathrm{MSamples} / \mathrm{s}$ at less than $0.5 \mathrm{~W} /(\mathrm{MSamples} / \mathrm{s})$. The development board used in this particular setup does not include power monitoring capabilities and thus, estimations obtained using Xilinx Vivado have been used instead. The obtained values can be seen in Table 4-6. These estimations have been cross-checked and validated against other ARTICo $^{3}$ deployments where dedicated intrumentation circuitry was available.

The implemented design has been validated using a calibrated Airborne Visible InfraRed Imaging Spectrometer (AVIRIS) image with 224 bands, 512 lines and 512

\footnotetext{
${ }^{8}$ XC7Z100-2FFG900.
} 


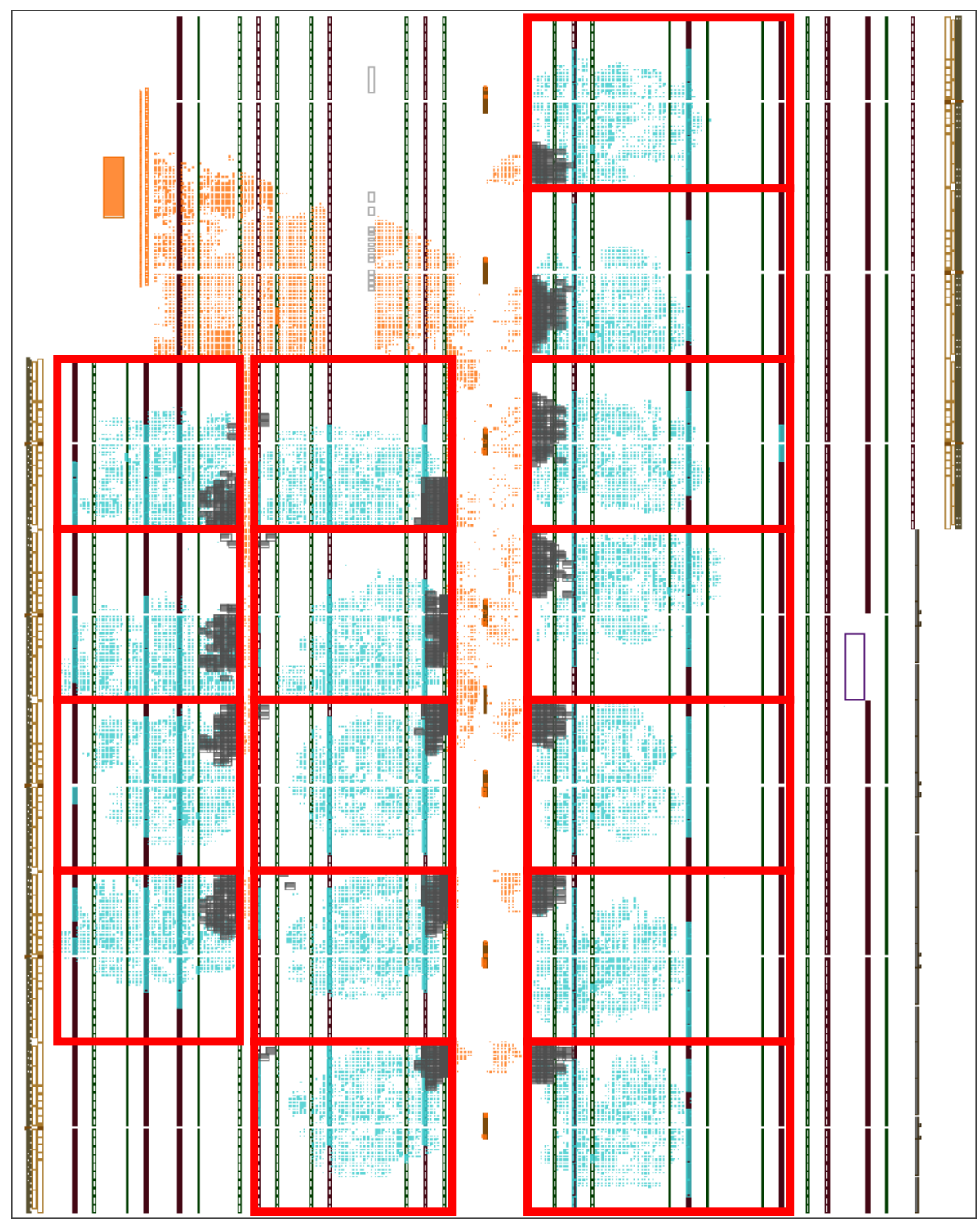

Figure 4-8: FPGA floorplan of the run-time adaptive hyperspectral compressor.

Table 4-6: Estimated power consumption in the run-time adaptive hyperspectral compressor.

\begin{tabular}{cc}
\hline & Power $(\mathbf{W})$ \\
\hline FPGA Static & 0.298 \\
Processing System & 1.578 \\
HyLoC & $0.04(\times 16)$ \\
ARTICo $^{3}$ & 0.08 \\
Additional Logic $^{-}$ & 0.021 \\
\hline Total & $\mathbf{2 . 6 1 7}$ \\
\hline
\end{tabular}


samples. ${ }^{9}$ Each instance of the HyLoC kernel has been configured to operate using the square-based partitioning with the maximum feasible image segment size (i.e., 224 bands, 8 lines and 8 samples), as discussed in Subsection 4.2.3. Results are presented in Table 4-7, and show values of theoretical throughput, actual throughput and energy efficiency in the run-time adaptive hyperspectral compressor. The theoretical throughput has been estimated assuming zero on-chip communication overhead, and knowing that each HyLoC kernel instance takes 185555 clock cycles (value measured with the embedded monitoring infrastructure) to produce a compressed bitstream from its input image segment. The actual throughput and the energy efficiency, on the other hand, have been measured using DPR to modify the number of accelerators available for processing in the FPGA fabric. Notice that these values have been obtained in a scenario where no hardware redundancy is enabled, even though the same performance can be obtained using the same number of effective accelerator instances.

Table 4-7: Compression throughput and energy efficiency (FPGA fabric @ $100 \mathrm{MHz}$ ).

\begin{tabular}{ccccc}
\hline Accelerators Iterations & $\begin{array}{c}\text { Theoretical Throughput } \\
\text { MSamples/s }\end{array}$ & $\begin{array}{c}\text { Actual Throughput } \\
\text { MSamples/s }\end{array}$ & $\begin{array}{c}\text { Energy Efficiency } \\
\text { mW/(MSamples/s) }\end{array}$ \\
\hline 1 & 4096 & 7.72 & 6.92 & 378.18 \\
2 & 2048 & 15.45 & 13.28 & 197.1 \\
4 & 1024 & 30.9 & 24.52 & 106.73 \\
8 & 512 & 61.81 & 42.48 & 61.61 \\
16 & 256 & 123.62 & 67.04 & 39.1 \\
\hline
\end{tabular}

By analyzing the obtained results, it is possible to appreciate that the run-time adaptive hyperspectral image compressor scales its performance linearly up to 8 hardware accelerators. This behavior, however, does not continue if the number of HyLoC kernel instances is increased. This can be seen in the scenario that uses 16 accelerators, where the speedup achieved is $9.7 \times$. Hence, the analyzed implementation goes from a computing-bounded operating point to a slightly memory-bounded one. Nonetheless, all the configurations presented in Table 4-7 are compliant with the reference standard requirements in terms of energy efficiency, even though some of them do not meet the expected values in terms of compression throughput. ${ }^{10}$

To conclude this section, Table 4-8 presents a comparison of the proposed run-time adaptive lossless multispectral and hyperspectral image compressor [Rodríguez'19] with several alternatives available in the literature. Moreover, reference values for the AVIRIS and Airborne Visible InfraRed Imaging Spectrometer Next Generation (AVIRIS-NG) imaging sensors, together with the specification of the CCSDS reference standard are also provided for the sake of completeness. In addition, both theoretical and actual values for both compression throughput and energy efficiency are provided

\footnotetext{
${ }^{9}$ The image used corresponds to flight f080927t01p00r10, but its spatial dimensions have been altered to enable partitioning with an integer number of image segments.

${ }^{10} 20 \mathrm{MSamples} / \mathrm{s}$ at less than $0.5 \mathrm{~W} /$ (MSamples/s).
} 
for the proposal of this Thesis, ${ }^{11}$ given the fact that some of the state-of-the-art solutions operate in ideal conditions (i.e., they do not consider communication and memory access overheads).

Table 4-8: Comparison of CCSDS 123 implementations.

\begin{tabular}{|c|c|c|c|c|c|c|}
\hline Implementation & Order & $\mathbf{P}$ & Device & $\begin{array}{l}\mathbf{f}_{\max } \\
\mathbf{M H z}\end{array}$ & $\begin{array}{l}\text { Throughput } \\
\text { MSamples/s }\end{array}$ & $\begin{array}{c}\text { Energy Efficiency } \\
\text { mW/(MSamples/s) }\end{array}$ \\
\hline AVIRIS $^{12}$ & - & - & Sensor & - & 1.7 & - \\
\hline AVIRIS-NG ${ }^{13}$ & - & - & Sensor & - & 42.29 & - \\
\hline CCSDS [CCSDS'15] & - & - & Reference standard & - & 20 & 500 \\
\hline Emporda $^{14}$ & All & $0-15$ & Software (i7-7500U) & - & 4.93 [Báscones'18] & - \\
\hline HyLoC [Santos'16] & BSQ & 3 & Virtex-5 & 134 & 11.3 & 207.52 \\
\hline SHyLoC [Santos'20] & BIP & 3 & Virtex-5 & 113.9 & 113.3 & - \\
\hline [Báscones'18] & All & $0-15$ & Virtex-7 & 50 & 47.62 & 9.45 \\
\hline [Báscones'17] & BIP & 3 & Virtex-5 & - & 179.7 & 16.92 \\
\hline [Báscones'17] & BIP & 3 & Virtex-7 & - & 219.4 & 24.16 \\
\hline [Fjeldtvedt'18] & BIP & $0-15$ & Zynq-7000 & 147 & 147 & 2 \\
\hline [Orlandić'19] & BIP & $0-15$ & Zynq-7000 & 150 & 750 & 0.69 \\
\hline [Tsigkanos'18] & BIP & 3 & Virtex-5 & 213 & 213 & 22.6 \\
\hline [Davidson'18] & BIP & 3 & Jetson TX1 & - & 116.2 & 86.06 \\
\hline [Rodríguez'19] & BSQ & 3 & Zynq-7000 & $100(134)$ & $67.04(165.65)$ & 39.1 (15.8) \\
\hline
\end{tabular}

The ARTICo ${ }^{3}$-based HyLoC compressor shows performance metrics in the range of the other implementations, being only $3.3 \times$ slower than the second fastest implementation [Báscones'17]. From all the reported alternatives, only the one shown in [Davidson'18] uses a partition scheme similar to the one proposed in this Thesis. However, the approach followed there is based on the strip-based partitioning, and the execution is performed using a fixed number of processing elements, since GPUs cannot dynamically change their computing resources. Thus, it is not possible to perform run-time adaptation of the compression performance as it can be done with the proposal of this Thesis. Regarding the other hardware-based implementations, it is possible to see that the main focus is always put on generating highly optimized hardware accelerators, either by combining task-level parallelism and a reconfigurable fine-grain pipeline to achieve one of the fastest implementations of the CCSDS 123 standard [Tsigkanos'18], or by exploiting several runs of logic synthesis at design time to perform fine tuning of the compressor parameters to the imaging sensor that is used [Báscones' 18]. The compressor proposed in this Thesis, on the other hand, relies on a low-complexity hardware accelerator that is replicated in the FPGA fabric several times to enable its run-time adaptive behavior.

Original contribution 4-2 A run-time adaptive FPGA implementation of a low-complexity on-board CCSDS 123 lossless multispectral and hyperspectral compressor with selectable performance and energy efficiency levels.

\footnotetext{
${ }^{11}$ In order to compute the theoretical compression throughput and energy efficiency figures, the maximum operating frequency of one HyLoC accelerator has been used. These values have been enclosed in parentheses for the sake of readability.

${ }^{12}$ https://aviris.jpl.nasa.gov

${ }^{13}$ https://aviris-ng.jpl.nasa.gov

${ }^{14}$ http://www.gici.uab.es
} 


\subsection{Linear Unmixing}

Remotely sensed hyperspectral images often present limited spatial resolution (e.g., $20 \mathrm{~m} /$ pixel for images captured with the AVIRIS sensor ${ }^{15}$ ). As a result, most of the captured pixels often contain spectral signatures from more than one material (see Figure 4-9). Hyperspectral unmixing refers to any procedure in which the pixel spectra from a hyperspectral image are separated into a collection of constituent spectral signatures (i.e., endmembers) and several sets of fractional abundances (i.e., one set per pixel) [Bioucas-Dias'12].

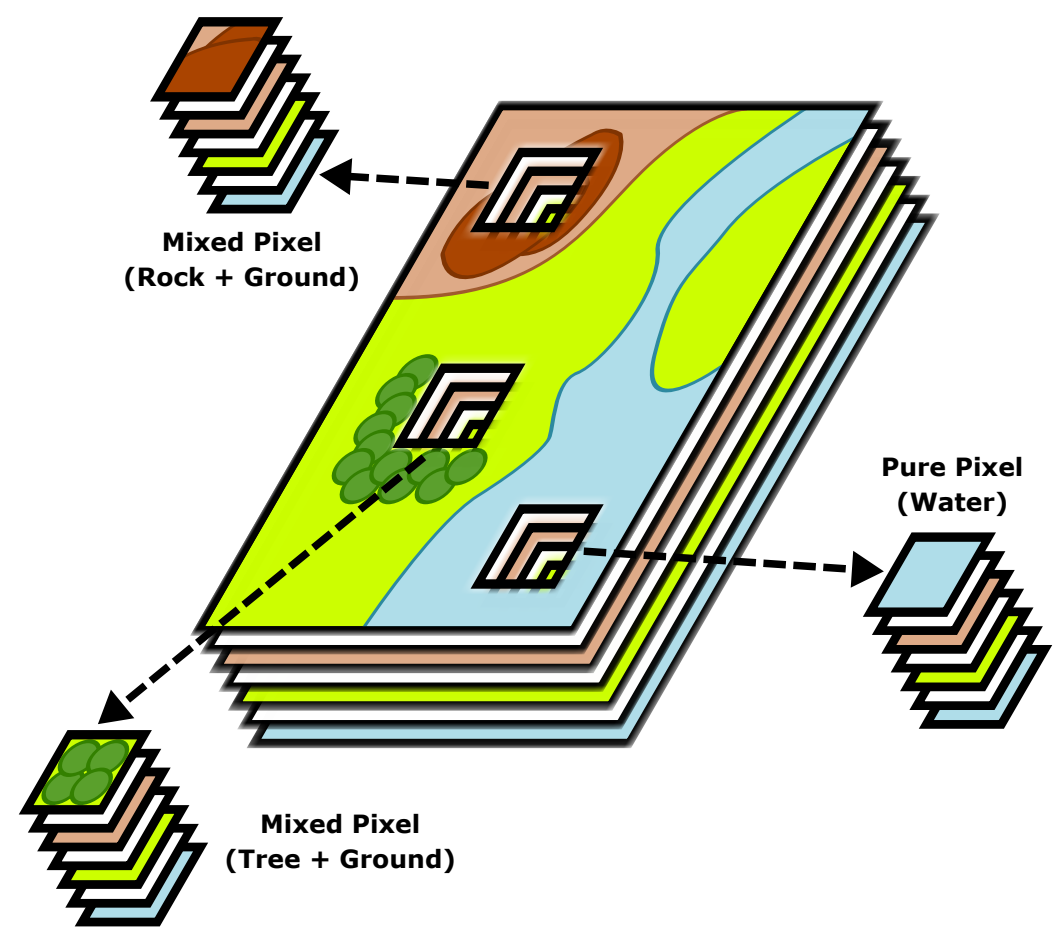

Figure 4-9: Hyperspectral mixture.

It is usually assumed that endmembers represent the pure materials present in the input image, whereas the abundances represent the amount of each endmember that is present in a given pixel (see Figure 4-10). Hence, the main objectives of the hyperspectral unmixing process are estimating the number of endmembers present in an image (i.e., $P$ ), extracting these endmembers from the image (i.e., $E=\left[e_{1} \ldots e_{P}\right]$ ), and calculating the abundance of each endmember in each pixel of the image (i.e., $\left.A=\left[a_{1} \ldots a_{\text {pixels }}\right]\right)$. Most of the works available in the literature use complex algorithms, usually a different one for each stage, that are forced to be executed sequentially in what is called a hyperspectral unmixing chain.

Unmixing algorithms rely on the type of mixture model that is selected to describe the radiance distribution in the captured data. Two main mixture models are currently

\footnotetext{
${ }^{15}$ https://aviris.jpl.nasa.gov/
} 

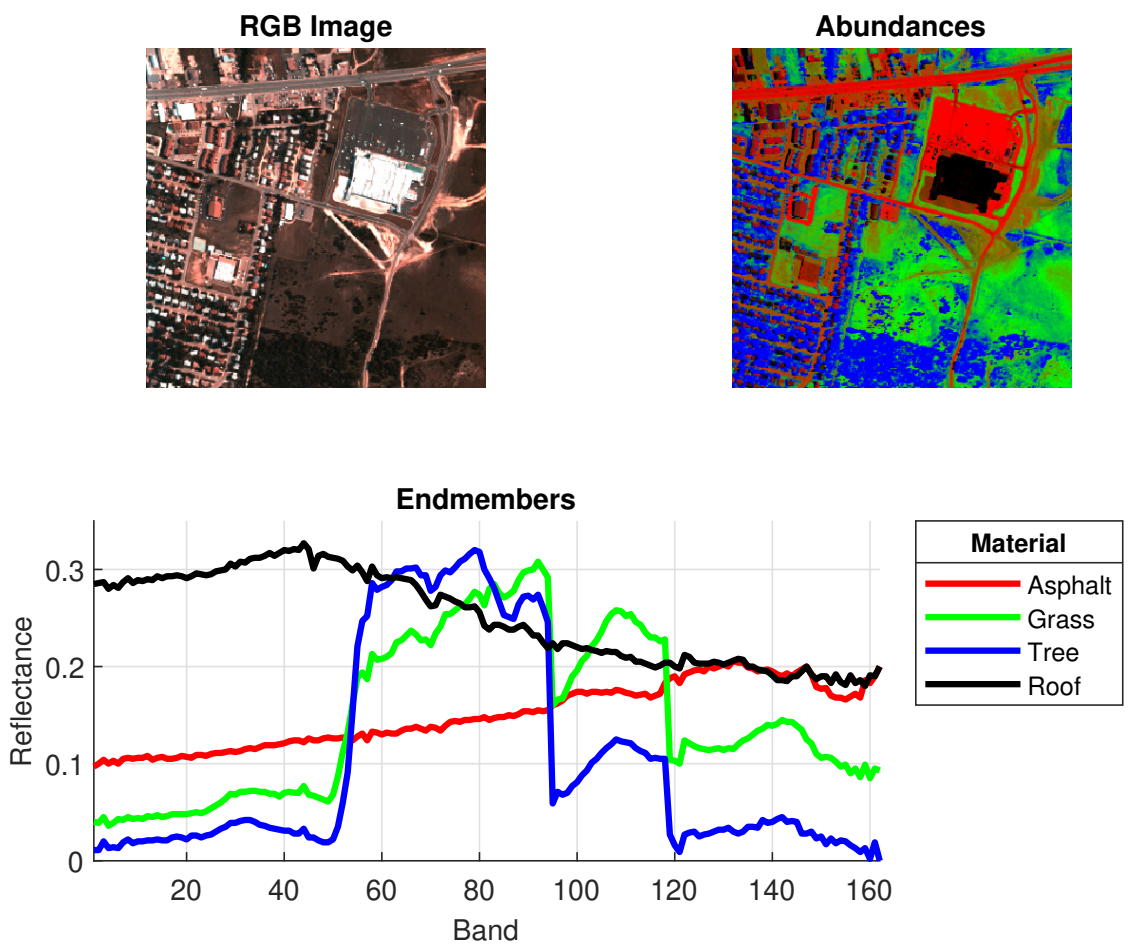

Figure 4-10: Hyperspectral unmixing: endmembers and abundances [Zhu'14].

used: linear and nonlinear [Bioucas-Dias'12]. Both models can be seen in Figure 4-11. In the linear mixture model, hyperspectral mixing is assumed to occur on the sensor itself, especially in those with limited resolution. Here, light coming from different materials is almost completely separated but gets mixed in the measuring device. In the nonlinear mixture model, on the other hand, light coming from different materials is assumed to physically interact before arriving to the measuring device. Although the nonlinear model might provide a more accurate description of the underlying physical phenomena, the linear mixture model holds valid in most realworld application scenarios [Bioucas-Dias'12]. As a consequence, the majority of hyperspectral unmixing algorithms available in the literature follow the linear mixture model, including the one presented in this Thesis.

The linear hyperspectral mixture model (shown in Equation 4-2) assumes that, given an input image (i.e., $M=\left[m_{1} \ldots m_{\text {pixels }}\right]$ ), each pixel (i.e., $\left.m_{i}\right)$ can be represented as a linear combination of endmembers (i.e., $e_{j}$ ). The contribution from each endmember to the spectral signature of the pixel is weighted using abundance values (i.e., $a_{i, j}$ ). In addition, the model also considers the existence of certain noise in each pixel (i.e., $n_{i}$ ).

$$
m_{i}=\sum_{j=1}^{P} a_{i, j} \cdot e_{j}+n_{i}
$$



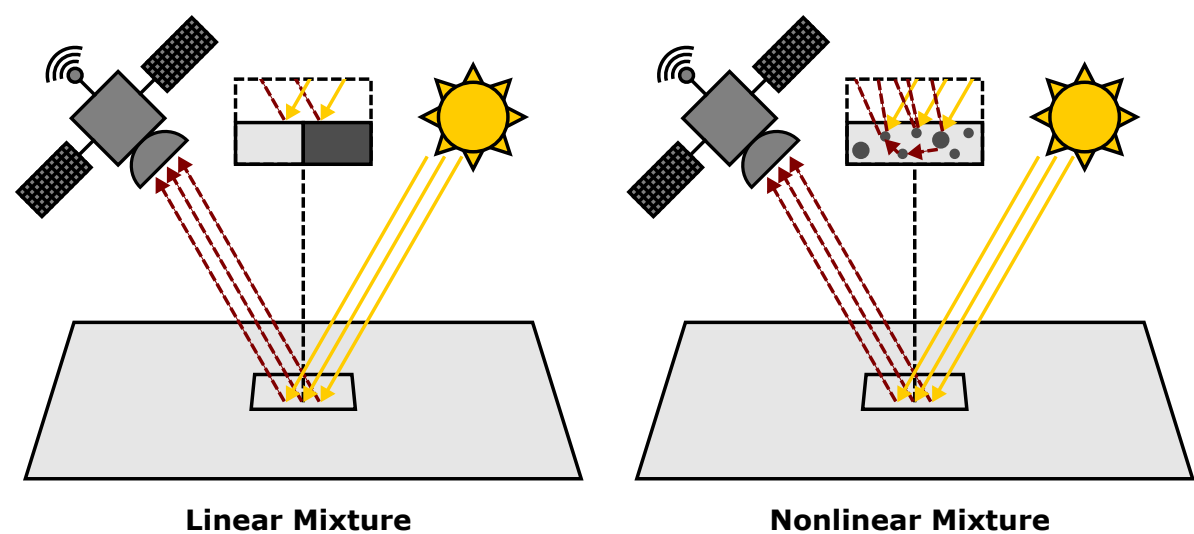

Figure 4-11: Linear and nonlinear hyperspectral mixture models.

Notice that, since abundances represent the fractional amount of a given endmember in a particular pixel, they have to comply with the nonnegativity and sumto-one constraints shown in Equation 4-3.

$$
\begin{aligned}
\text { Nonnegativity } & a_{i, j} \geq 0, \quad j=1, \ldots, P \\
\text { Sum-to-one } & \sum_{j=1}^{P} a_{i, j}=1
\end{aligned}
$$

This section covers the design and implementation of a run-time adaptive linear hyperspectral unmixing chain using the ARTICo ${ }^{3}$ framework in a multi-FPGA scenario. In the proposed solution, input hyperspectral images are partitioned in a variable number of fixed-size segments that are processed concurrently using a low-cost FPGA cluster. Following an iterative approach, data-level parallelism is exploited at two different levels using a configurable number of nodes and accelerators per node, and a reduction-based operation is applied afterwards to obtain the required results.

\subsubsection{Algorithm Description}

The run-time adaptive linear hyperspectral unmixing chain presented in this Thesis is based on the Fast algorithm for linearly UNmixing hyperspectral images (FUN) algorithm [Guerra'15]. ${ }^{16}$ According to the classification shown in [Chang'13], it is a sequential endmember extraction algorithm based on convexity geometry and, in particular, on orthogonal projections. However, a distinctive characteristic of the FUN algorithm is that it addresses all three stages of the linear unmixing process: estimation of the number of endmembers, endmember extraction, and abundance calculation.

In fact, most of the linear unmixing chains available in the literature use different algorithms for each of the three stages. This forces execution to be sequential

\footnotetext{
${ }^{16}$ The algorithm described in this section is a modification of the original FUN algorithm that enables massive parallelism and simplifies arithmetic operations, as shown in [Martel'17].
} 
and increases the probability of error propagation, with the corresponding loss of precision in the obtained results. Moreover, a vast majority of algorithms rely on complex mathematical operations (e.g., matrix inversion or eigenvalues/eigenvectors calculation) that increase the computational power required to solve the linear unmixing problem. Although FPGAs can be used to deliver the required levels of computing performance in this context, some of the aforementioned operations are nearly impossible to be implemented in hardware or provide execution times that are far from real-time processing constraints. The FUN algorithm not only covers all the stages in the linear unmixing chain, but it also uses simple operations in a highly parallelizable and regular manner.

The mathematical foundations of the FUN algorithm for both endmember extraction and abundance calculation lie on the Gram-Schmidt ortogonalization method. Moreover, it uses an initalization mechanism based on the centroid pixel. The centroid pixel is an artificially generated pixel that averages all the information contained in the input hyperspectral data cube. The initialization criterion in the FUN algorithm selects the pixel with larger orthogonal projection over the centroid pixel as the first endmember. In other words, the first endmember is the pixel with the largest amount of information not contained in the centroid pixel. Notice that this projection is also computed using the Gram-Schmidt method and thus, all the core functionality of the algorithm can be solved using the same mathematical operations.

The pseudocode of the endmember extraction part in the FUN algorithm can be seen in Algorithm 4-1. It is also in this part where the number of endmembers present in the input hyperspectral image is computed. To do so, the algorithm uses a user-defined input parameter (i.e., $\alpha$ ) as a threshold to determine whether the loss of information derived from not including additional endmembers in the pool of already extracted ones is acceptable or not.

The steps of the endmember extraction algorithm can be described as follows. First, all the required variables are initialized (lines 1 to 9). Notice that this part also includes the selection of the first endmember using the centroid pixel criterion (line 5). Then, the information represented by the last endmember that has been extracted is subtracted from each remaining pixel (line 12) and its resulting brightness is computed (line 13). Afterwards, the modified pixel (i.e., $x_{i}$ ) with the largest brightness value is selected (line 15), and the stop condition is evaluated (line 16). The stop condition uses the input parameter $\alpha$ and the brightness of the original pixel (i.e., $m_{i}$ ) to verify the potential information loss (i.e., how much remaining information cannot be represented with the already extracted endmembers). If the stop criterion is met, the algorithm finishes; otherwise, the number of endmembers found is increased (line 19) and a new candidate is extracted (line 20). The algorithm then updates the orthogonal vectors (lines 21 and 22) and keeps iterating until the termination condition is satisfied.

Once all the endmembers have been extracted, the FUN algorithm computes its abundances. Actually, this process is carried out by generating orthonormal sets of vectors using the extracted endmembers as starting point. The pseudocode for the abundance calculation in the FUN algorithm can be seen in Algorithm 4-2. 


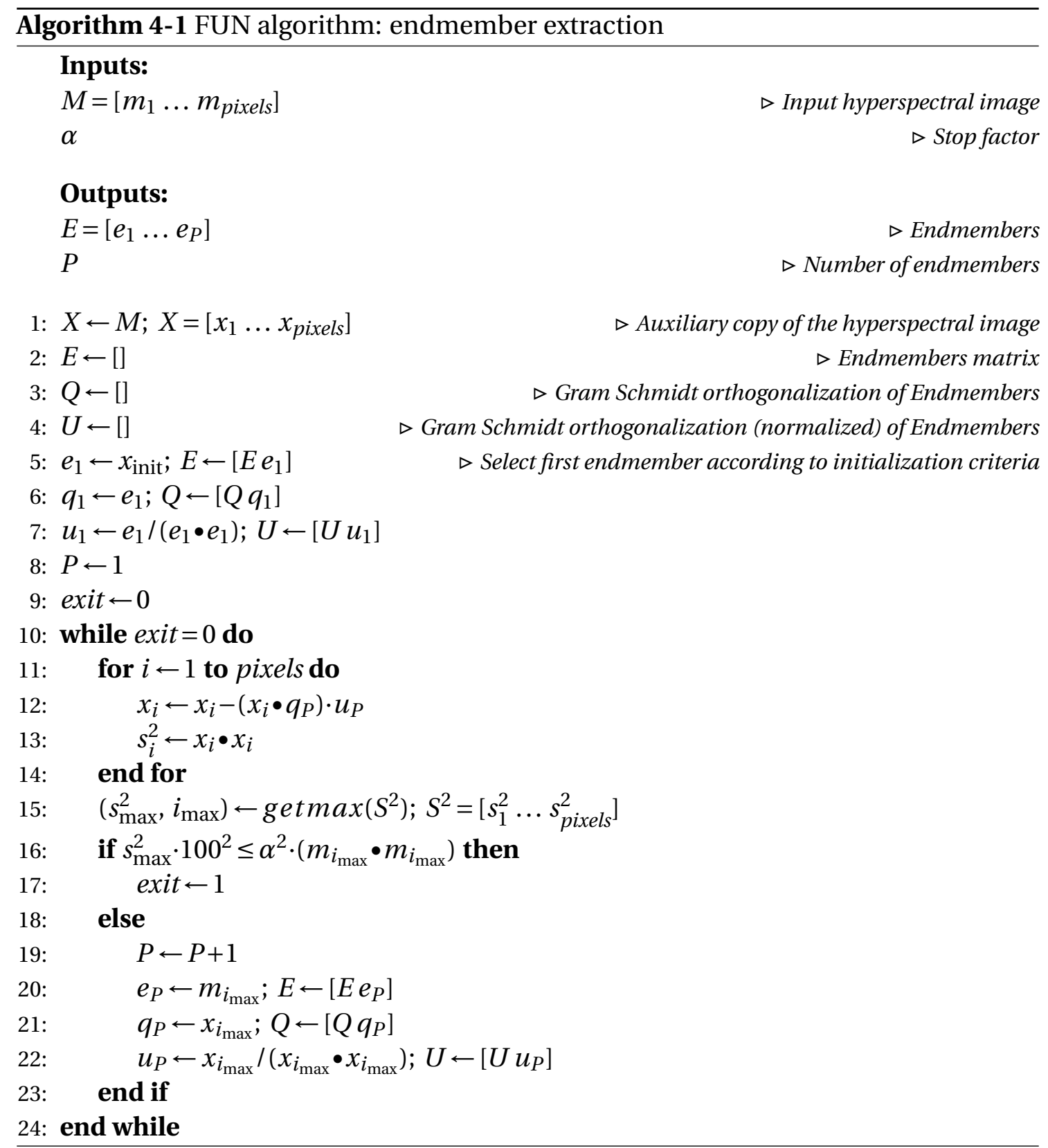

The steps of the abundance calculation algorithm can be described as follows. After initializing all the variables (lines 1 to 4), Gram-Schmidt ortogonalization is performed $P$ times (lines 5 to 19), i.e., as many times as endmembers have been extracted. Notice that the order in which this process is done changes between iterations (lines 6 and 7) and that only one component of the $U$ matrix is generated per iteration (line 16). Analyzing this procedure with more detail, it is possible to see that each iteration isolates the contribution of each endmember that cannot be represented by (i.e., is not contained in) the rest of endmembers (lines 6 to 15). Finally, the abundances are calculated performing the projection of the original image over the elements of the $U$ matrix (line 20). 


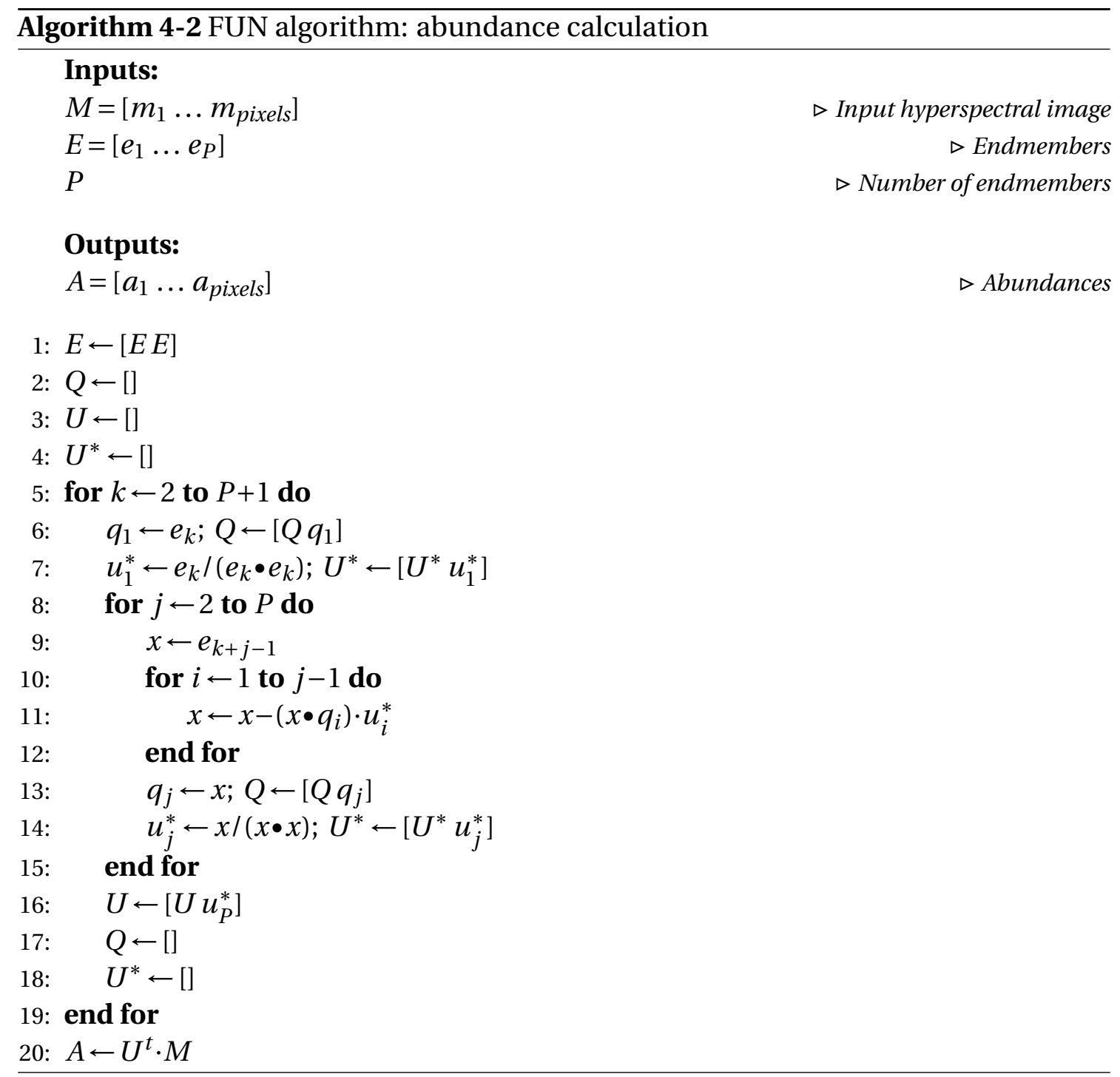

Before concluding this section, an additional remark must be made: the FUN algorithm does not impose the nonnegativity and sum-to-one contraints in the abundance calculation. However, experimental results obtained during the development of the algorithm showed that the deviations in the obtained abundances are almost negligible [Guerra'15]. 


\subsubsection{State of the Art}

At the beginning, research activities around hyperspectral unmixing were mostly focused on algorithm evaluation [Bioucas-Dias'12, Chang'13]. However, the complexity of the newly developed algorithms also imposed certain performance requirements that could only be satisfied by providing specialized and optimized implementations [Plaza'07]. As a matter of fact, the main contributions of Section 4.3 lay on the implementation side, even though there are some contributions at algorithmic level as well. Hence, the following discussion has been organized to present an implementation-wise review of the literature.

According to the traditional hyperspectral data processing chain described in Section 4.1, the unmixing stage is usually performed in HPC facilities, normally after first decompressing the data received from the satellite or airborne sensor, and then applying different preprocessing stages (e.g., atmospheric correction). In fact, almost all the works available in the literature target algorithm acceleration using datacenter GPU or FPGA boards [Ghamisi' 17].

Nevertheless, the sudden surge of FPGAs as the main processing element for onboard hyperspectral processing also brought some examples of high-performance embedded unmixing chains. For instance, in [López'13], the authors propose the use of DPR in a space-qualified FPGA to implement the endmember extraction and the abundance calculation steps of an on-board linear unmixing chain. The implementation uses two different hardware accelerators that have been optimized for performance and therefore, high resource utilization values are obtained (i.e., more than $90 \%$ in both cases). Actually, endmember extraction is performed with the $\mathrm{N}$ FINDR implementation presented in [González'12a], and the abundance calculation is performed with the Image Space Reconstruction Algorithm (ISRA) implementation presented in [González'12b]. With this setup, and using part of the AVIRIS Cuprite image (i.e., $350 \times 350$ pixels), ${ }^{17}$ endmember extraction takes 13.46 seconds, whereas abundance calculation takes 1.32 minutes. An additional overhead of 694 milliseconds is used to perform DPR and change between the two steps of the unmixing chain.

In [Nascimento'16], a hardware-based implementation of the Vertex Component Analysis (VCA) algorithm is made on a Zynq-7000 device. The authors propose an optimized architecture that operates with single precision floating point arithmetic. Moreover, and in order to comply with the inherent limitations of on-board processing scenarios, resource utilization is kept at a relatively low value by taking advantage of block reutilization (e.g., dot products are required during QR decomposition and during image projection). However, the size of the hardware accelerator varies significantly when modifying the maximum number of endmembers to be extracted. Experimental results show average times of around 1.2 seconds to process the same AVIRIS Cuprite image used in [López'13].

Unfortunately, and to the best of the author's knowledge, the list of academic works where on-board processing is explicitly targeted ends there. Although it is still possible

\footnotetext{
${ }^{17}$ Radiance file under flight number f080611t01p00r06.
} 
to find new algorithm implementations, the common trend is to use either GPUs or high-end FPGAs that are not radiation-hardened and thus, not suitable for space applications. Some works do mention the applicability of their results in on-board processing scenarios, but that statement is never actually demonstrated. For instance, a full hyperspectral unmixing chain is implemented on a GPU in [Sánchez'15]. In that implementation, all the steps are described in CUDA, achieving a total speedup of roughly $80-100 \times$ over the reference execution on CPU. Another example can be found in [Bernabé'17], where a real-time implementation of another full unmixing chain using OpenCL is presented.

In fact, the algorithm used in this Thesis, FUN [Guerra'15], has been also implemented on high-performance computing devices not suitable for on-board processing scenarios, such as GPUs [Martel'17] or FPGAs [Guerra'17b]. Both approaches use parallel programming languages (i.e., CUDA and OpenCL, respectively) to describe the different stages of the linear unmixing chain.

\subsubsection{Implementation Details}

The optimized implementation of the FUN algorithm over different types of accelerator boards has already been addressed in [Guerra'17b]. Using OpenCL as the common entry point, optimized implementations were deployed on both GPUs and FPGAs. ${ }^{18}$ The fact that OpenCL only provides functional portability and not performance portability made it mandatory to customize each implementation to better exploit parallelism in the target computing fabric. As a result, the specification targeting GPUs focused on exposing massive data-level parallelism to take advantage of SIMD execution, whereas the specification targeting FPGAs focused on generating a highly optimized processing pipeline to take advantage of instruction-level parallelism without significantly increasing the area overhead of the circuit.

All previous work with optimized FUN implementations focused on providing the best performance results when executing the linear unmixing chain in highperformance on-Earth facilities. However, the implementation presented in this Thesis differs, since it addresses on-board hyperspectral image processing. While it is true that high performance is still needed, leaving out data compression and transmission to on-Earth processing facilities increases the margin to generate slightly less optimized solutions that still meet the expected real-time processing requirements. This argument is in line with the resource-constrained scenarios in which on-board processing is performed. Hence, the proposal of this Thesis is to use low-complexity hardware accelerators and exploit the transparent performance scalability of the ARTICo ${ }^{3}$ framework to deploy a run-time adaptive on-board linear hyperspectral unmixing chain. The entry point for the design and implementation of the hardware accelerators is a high-level algorithmic description in $\mathrm{C}$ code that undergoes HLS.

\footnotetext{
${ }^{18}$ The FUN algorithm has also been implemented using CUDA [Martel'17].
} 
Following the same approach that was made when implementing the FUN algorithm in GPU and FPGA using OpenCL, an analysis of the algorithm is required to identify which is the best way to maximize execution performance in the target computing fabric. As a distinctive feature, the implementation over ARTICo ${ }^{3}$ requires optimizations suited for both GPUs (i.e., data-level parallelism) and FPGAs (i.e., pipelined datapath inside each hardware accelerator). More importantly, these optimizations need to be applied sequentially: first, an application code segment with potential data-level parallelism needs to be identified; second, the hardware accelerator onto which these data-parallel computations will be offloaded needs to have an optimized datapath. As a result, an initial profiling of the FUN algorithm was performed using a sequential C-based description on one of the ARM Cortex-A9 available in a Zynq-7000 board. The obtained results can be seen in Table 4-9. ${ }^{19}$

Table 4-9: FUN profiling on Zynq-7000 (ARM Cortex-A9 core @ 666.67 MHz).

\begin{tabular}{ccc}
\hline & Endmembers Extraction & Abundance Calculation \\
\hline Execution Time (ms) & 1723.31 & 552.44 \\
Execution Fraction (\%) & 75.4 & 24.6 \\
\hline
\end{tabular}

Analyzing these results, it can be deduced that the most time-consuming stage of the linear unmixing chain is the endmember extraction (around $75 \%$ of the total computation time). Hence, this part has been selected to be implemented in hardware. Therefore, the next steps are to devise a data-parallel processing strategy, generate an optimized datapath to fully exploit accelerator performance, and deploy the multiaccelerator system using the ARTICo ${ }^{3}$ framework.

\section{Parallelization Approach}

The reference $\mathrm{C}$ code for the FUN algorithm shows that most of the computations are performed sequentially inside nested loops, as already hinted by the pseudocode in Algorithm 4-1. From an HLS perspective, the simplest way to enable data-level parallelism in this algorithm consists of performing loop unrolling optimizations in the baseline code. As it has already been discussed, this is the more sensible approach when targeting GPUs. However, loop unrolling optimizations tend to generate excessive area overheads when using HLS and thus, loop pipelining is preferred to maintain a reasonable tradeoff between execution performance and resource utilization when targeting FPGAs.

Therefore, and assuming that only loop pipelining optimizations are to be applied to guide the HLS process, a different strategy to extract data-level parallelism in the original FUN algorithm is required. The method proposed in this Thesis explores the partitioning of the input hyperspectral image in multiple segments from which endmembers are extracted independently. An iterative reduction-based procedure

\footnotetext{
${ }^{19}$ Evaluation has been performed using an artificial hyperspectral image of 256 bands, 128 lines, and 128 samples with 10 endmembers.
} 
is applied to achieve dimensionality reduction. In other words, the proposed parallelization approach extracts endmembers from the pool of already existing endmembers until the actual ones are found. The reduction-based FUN algorithm for endmember extraction is shown in Figure 4-12.

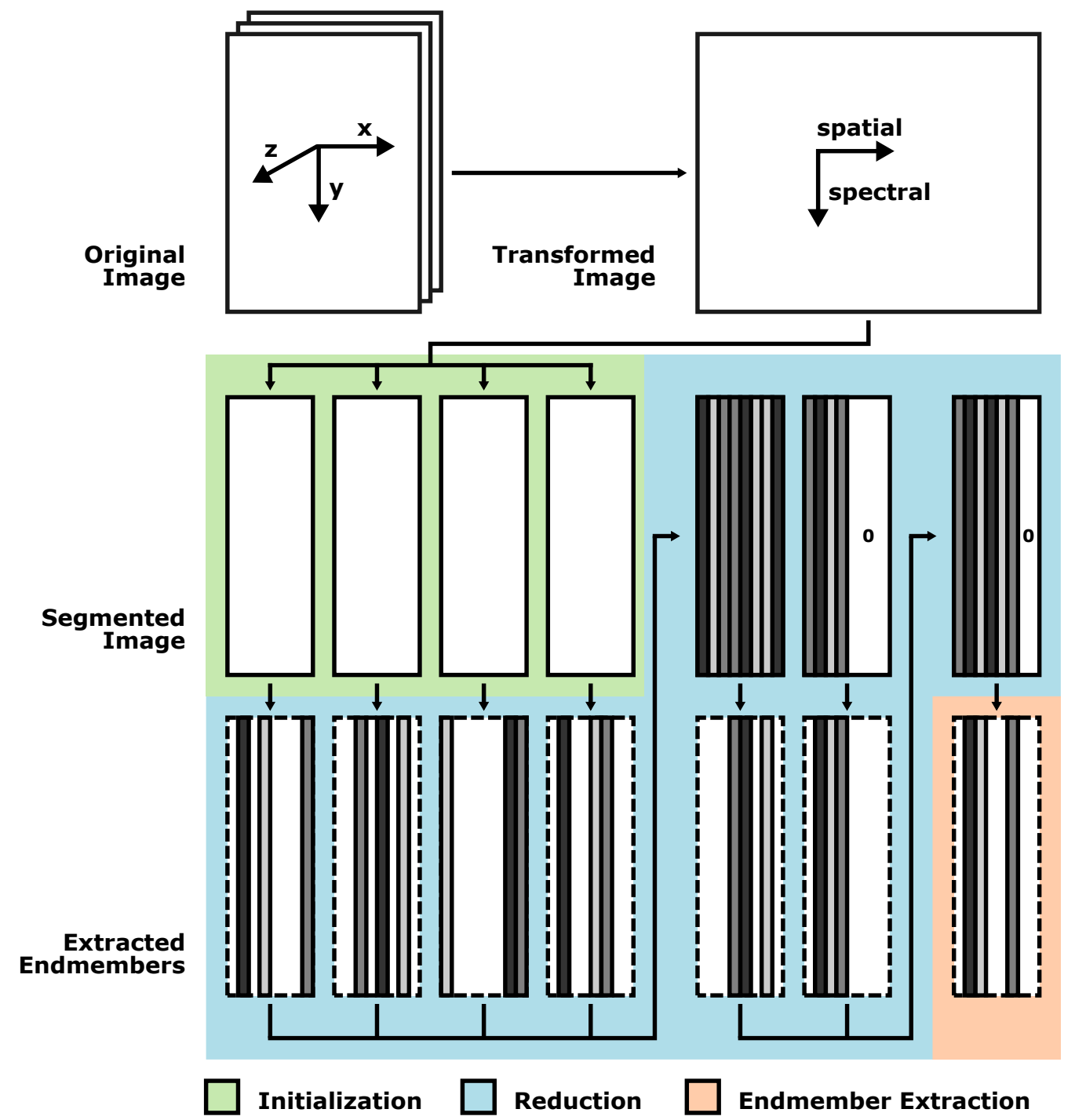

Figure 4-12: Hyperspectral image partitioning for reduction-based unmixing.

The modified algorithm starts by transforming the input hyperspectral data cube into a two-dimensional structure, where the new $\mathrm{x}$-axis represents the spatial dimensions (i.e., samples and lines) and the new y-axis represents the spectral dimension (i.e., bands). Then, the transformed image is split in an integer number of fixed-size segments, i.e., each segment contains a fixed number of pixels with all their spectral information. ${ }^{20}$ Zero-padding is applied in case a segment contains

\footnotetext{
${ }^{20}$ Fixed-size image segments are used to minimize memory requirements, since the maximum amount of local memory available within the $\mathrm{ARTICo}^{3}$ kernel wrapper is $64 \mathrm{KiB}$.
} 
less pixels than the rest. From this point onwards, the algorithm iteratively extracts endmembers by applying the FUN endmember extraction procedure to each segment independently, generates a new artificial image made from all the endmembers that have been extracted, and splits it in fixed-size segments again. This process is repeated until the artificial image fits inside one fixed-size segment. The endmembers extracted from this last image are the output of the modified FUN algorithm. As an example, the scenario depicted in Figure 4-12 shows how the three endmembers present in the input hyperspectral cube are extracted after two iterations of the reduction loop. ${ }^{21}$

The pseudocode to implement the reduction-based endmember extraction can be seen in Algorithm 4-3. A hardware-friendly version of the original FUN algorithm that allows users to specify the maximum number of endmembers to be extracted, and therefore limit the latency of the endmember extraction process, has been used (lines 6 and 17). In order to avoid information loss, since all the actual endmembers could be contained within one single image segment, the FUN algorithm is configured to allow all input pixels to be selected as candidate endmembers. Notice that an additional part has been included in the algorithm to avoid deadlocks during execution (lines 10 to 14). These deadlocks occur whenever no dimensionality reduction can be achieved between iterations, i.e., when the number of endmembers extracted from all image segments does not decrease from one iteration to the next one. Although the same (or similar) endmember may be present in two or more image segments and only one occurrence should be detected as endmember, the independent processing of these segments may prevent the algorithm from terminating properly. Hence, a failsafe mechanism has been implemented to ensure the algorithm converges: if there is no difference in the number of endmembers found in two consecutive iterations of the reduction loop, image segments of twice the spatial size are used (line 11) and dimensional reduction is forced since only a maximum of half the input pixels are allowed to be extracted as candidate endmembers (line 6).

The accuracy of the proposed algorithm has been evaluated using synthetic hyperspectral images with 256 bands, 128 lines, and 128 samples. Different parameters (e.g., amount of noise present in the input image, the number of endmembers, or the distribution used to generate the abundances) have been altered so that a significant amount of distinct scenarios could be generated. Table 4-10 shows the comparison between the original FUN algorithm and the reduction-based modification proposed in this Thesis. The precision of the endmember extraction method is evaluated according to the mean spectral angle, which measures the difference between the real endmembers that are known a priori and the endmembers extracted by a given algorithm. The spectral angle can be computed using Equation 4-4.

$$
\text { Spectral Angle }=\arccos \frac{e_{\text {real }} \cdot e_{\text {extracted }}}{\left\|e_{\text {real }}\right\| \cdot\left\|e_{\text {extracted }}\right\|}
$$

Perfect matches between extracted and real endmembers would render a spectral

\footnotetext{
${ }^{21}$ Endmember extraction when the artificially generated hyperspectral image fits within one fixed-size segment is performed out of the main reduction loop.
} 


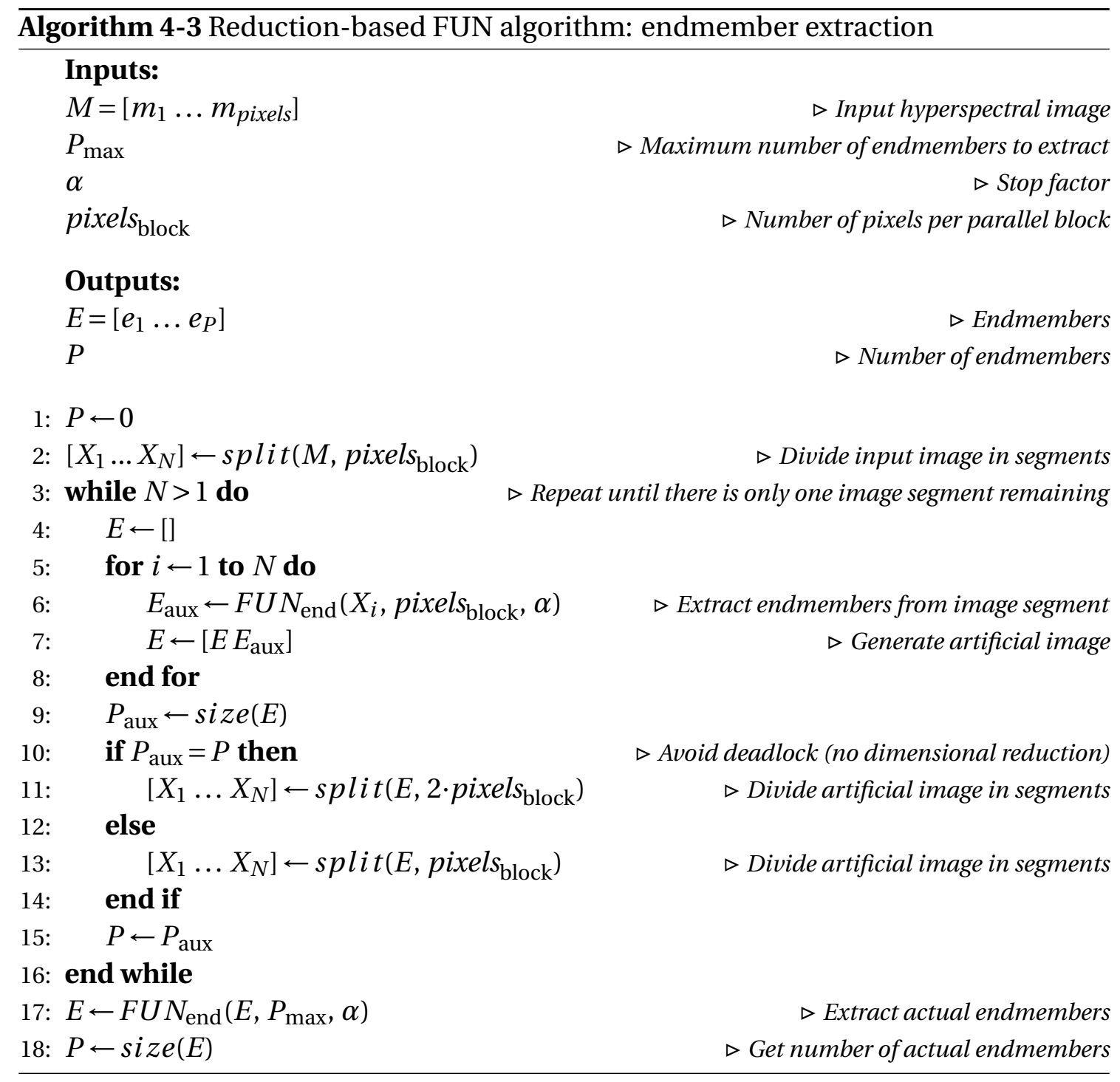

angle of $0^{\circ}$, whereas for completely unrelated pixels the spectral angle would tend to $90^{\circ}$. For images where the number of extracted endmembers (i.e., $P_{\text {extracted }}$ ) is larger than the actual number of endmembers (i.e., $P_{\text {real }}$ ), the spectral angle is computed as the minimum value from all possible endmember combinations.

The endmember extraction accuracy has also been evaluated using well-known hyperspectral datasets [Zhu'14]. Table 4-11 shows the obtained results, together with a comparison between the original FUN algorithm, its reduction-based modification, and several alternative algorithms available in the literature.

All the results from Table 4-10 and Table 4-11 have been obtained setting the pixels $s_{\text {block }}$ parameter to 32 . Regardless of the input image used for the evaluation, it is possible to see that the reduction-based FUN algorithm provides results that are in the range of its unmodified version. Moreover, the comparison with state-ofthe-art alternatives indicates that the proposed algorithm is objectively competitive, 
Table 4-10: Endmember extraction accuracy with synthetic images.

\begin{tabular}{|c|c|c|c|c|}
\hline \multicolumn{3}{|c|}{ Image Characteristics } & \multicolumn{2}{|c|}{ Mean Spectral Angle (o) } \\
\hline SNR (dB) & Endmembers & Abundances & FUN & Reduction-Based FUN \\
\hline \multirow{9}{*}{20} & \multirow{3}{*}{4} & Gauss Spheric & 1.5115 & 2.6709 \\
\hline & & Legendre Polynomial & 1.5433 & 1.4739 \\
\hline & & Dirichlet & 3.7219 & 2.0508 \\
\hline & \multirow{3}{*}{8} & Gauss Spheric & 2.8381 & 2.4991 \\
\hline & & Legendre Polynomial & 3.6176 & 3.8983 \\
\hline & & Dirichlet & 2.5348 & 2.7655 \\
\hline & \multirow{3}{*}{12} & Gauss Spheric & 3.7033 & 5.4387 \\
\hline & & Legendre Polynomial & 5.3731 & 5.4038 \\
\hline & & Dirichlet & 2.8855 & 4.3962 \\
\hline \multirow{9}{*}{40} & \multirow{3}{*}{4} & Gauss Spheric & 0.0798 & 0.2163 \\
\hline & & Legendre Polynomial & 0.3327 & 0.1521 \\
\hline & & Dirichlet & 0.1254 & 0.4491 \\
\hline & \multirow{3}{*}{8} & Gauss Spheric & 0.4679 & 0.3806 \\
\hline & & Legendre Polynomial & 0.6072 & 0.8048 \\
\hline & & Dirichlet & 0.3019 & 1.2459 \\
\hline & \multirow{3}{*}{12} & Gauss Spheric & 0.4252 & 1.1471 \\
\hline & & Legendre Polynomial & 0.3992 & 0.3853 \\
\hline & & Dirichlet & 0.3895 & 0.8428 \\
\hline \multirow{9}{*}{60} & \multirow{3}{*}{4} & Gauss Spheric & 0.0658 & 0.0726 \\
\hline & & Legendre Polynomial & 0.0367 & 0.2022 \\
\hline & & Dirichlet & 0.0682 & 0.0895 \\
\hline & \multirow{3}{*}{8} & Gauss Spheric & 0.0459 & 0.0936 \\
\hline & & Legendre Polynomial & 0.0737 & 0.0975 \\
\hline & & Dirichlet & 0.1463 & 0.1672 \\
\hline & \multirow{3}{*}{12} & Gauss Spheric & 0.0876 & 0.0860 \\
\hline & & Legendre Polynomial & 0.1705 & 0.1953 \\
\hline & & Dirichlet & 0.1384 & 0.2409 \\
\hline
\end{tabular}

Table 4-11: Endmember extraction accuracy (real hyperspectral images with known endmembers).

\begin{tabular}{|c|c|c|c|c|c|c|}
\hline \multirow{2}{*}{ Image } & \multirow{2}{*}{ Endmembers } & \multicolumn{5}{|c|}{ Mean Spectral Angle (o) } \\
\hline & & FUN & Reduction-Based FUN & VCA [Zhu' 14] & NMF [Zhu'14] & DgS-NMF [Zhu'14] \\
\hline Samson & 3 & 3.7004 & 2.6444 & 4.8301 & 5.1337 & 2.8934 \\
\hline Jasper Ridge & 4 & 7.5999 & 7.0384 & 19.8759 & 10.1069 & 3.0997 \\
\hline Urban & 4 & 4.4637 & 7.6292 & 23.2564 & 10.8633 & 4.8988 \\
\hline Cuprite & 12 & 5.9887 & 5.637 & - & 7.5401 & 5.9931 \\
\hline
\end{tabular}

either because it renders better precision results than some of the most widely used algorithms (e.g., VCA or NMF) or because of its hardware-friendly nature when compared with more complex alternatives (e.g., DgS-NMF).

Further analyses have been carried out by changing the partitioning depth (i.e., pixels $s_{\text {block }}$ ) while using synthetic hyperspectral images of 224 bands, 256 lines, and 256 samples with $60 \mathrm{~dB}$ of Signal-to-Noise Ratio (SNR). Results are provided in Table 4-12.

The experimental evaluation shows that the impact of the pixels $s_{\text {block }}$ parameter on the endmember extraction accuracy is negligible even when this parameter is set to a 
Table 4-12: Impact of partitioning depth (i.e., pixels $s_{\text {block }}$ ) on endmember extraction accuracy.

\begin{tabular}{ccccccccc}
\hline \multicolumn{2}{c}{ Image Characteristics } & & \multicolumn{8}{c}{ Mean Spectral Angle (o) } \\
Endmembers & Abundances & $\mathbf{4}$ & $\mathbf{8}$ & $\mathbf{1 6}$ & $\mathbf{3 2}$ & $\mathbf{6 4}$ & $\mathbf{1 2 8}$ & $\mathbf{2 5 6}$ \\
\hline \multirow{4}{*}{4} & Gauss Spheric & 0.2329 & 0.1933 & 0.1933 & 0.1933 & 0.1933 & 0.1933 & 0.1933 \\
& Legendre Polynomial & 3.3223 & 1.0612 & 0.1554 & 0.0682 & 0.0547 & 0.0546 & 0.0543 \\
& Dirichlet & 0.0595 & 0.0592 & 0.0592 & 0.0592 & 0.0592 & 0.0592 & 0.0592 \\
16 & Gauss Spheric & - & - & 0.0838 & 0.0871 & 0.1374 & 0.1374 & 0.0875 \\
& Legendre Polynomial & - & - & 0.7113 & 0.703 & 0.7154 & 0.7149 & 0.6541 \\
& Dirichlet & - & - & 0.0838 & 0.0852 & 0.0852 & 0.085 & 0.085 \\
\hline
\end{tabular}

relatively low value (i.e., no relevant variations are observed when pixels $s_{\text {block }} \geq 16$ ).

Original contribution 4-3 A reduction-based, hardware-friendly, and lowcomplexity linear unmixing algorithm for on-board hyperspectral image processing that exploits data-level parallelism without compromising endmember extraction accuracy.

\section{Hardware Tradeoffs}

The reduction-based algorithm presented in Algorithm 4-3 has a sequential component (i.e., the iterative reduction process) and a section with data-level parallelism (i.e., the extraction of endmembers from independent image segments). The hardware implementation proposed in this Thesis provides a mean to exploit this data-level parallelism by dynamically adjusting the loop unrolling depth of the for loop (lines 5 to 8 ) using the ARTICo ${ }^{3}$ framework. The configuration of the HLS-based hardware accelerator is shown in Table 4-13.

Table 4-13: HLS-based FUN configuration.

\begin{tabular}{ccc}
\hline Parameter & Description & Value \\
\hline pixels $s_{\text {block }}$ & Number of input pixels & 32 \\
$N_{\mathrm{z}}$ & Number of bands per pixel & 256 \\
$P_{\max }$ & Maximum number of endmembers to extract & 32 \\
$\alpha$ & Stop factor & 1.0 \\
\hline
\end{tabular}

The FUN algorithm operates with input images whose pixels are expressed as normalized (i.e., between 0 and 1) floating point numbers, which is one of the main reasons behind using a high-level description in C code and HLS instead of a highly optimized HDL specification, where floating point arithmetic is harder to implement. Two different accelerator alternatives have been evaluated according to their execution latency and area overhead. The first one uses single precision floating point arithmetic and relies on vendor-specific optimization directives. The second one, on the other hand, uses half precision floating point arithmetic and manual code refactoring 
together with the same vendor-specific optimization directives. ${ }^{22}$

Table 4-14 shows the estimated latency of both implementations when using Vivado HLS as the main implementation tool. Given the fact that the accelerator has a variable latency that depends on the number of endmembers inside the input image, results are presented in terms of the upper and lower boundaries. Table 4-15, on the other hand, shows the estimated resource utilization of both implementations also when using Vivado HLS.

Table 4-14: Execution latency in the HLS-based FUN accelerator.

\begin{tabular}{ccc}
\hline & Implementation \#1 & Implementation \#2 \\
\hline Precision & Single -32 bits & Half -16 bits \\
Optimization & Automatic (directives) & Automatic (directives) + Manual (code) \\
\hline \multirow{2}{*}{ Latency (cycles) } & $200331-1$ endmember & $50917-1$ endmember \\
& $1588512-32$ endmembers & $384509-32$ endmembers \\
\hline
\end{tabular}

Table 4-15: Resource utilization of the HLS-based FUN accelerator.

\begin{tabular}{ccc}
\hline & Implementation \#1 & Implementation \#2 \\
\hline Precision & Single -32 bits & Half - 16 bits \\
Optimization & Automatic (directives) & Automatic (directives) + Manual (code) \\
\hline LUTs & 3089 & 4296 \\
FFs & 2612 & 4172 \\
DSPs & 10 & 18 \\
BRAMs & 8.5 & 4 \\
\hline
\end{tabular}

Results show that the implementation based on half precision floating point arithmetic, manual code refactoring, and vendor-specific optimizations is better not only in terms of execution performance, but also when considering the area overheads. While it is true that the second implementation increases the amount of logic resources (except the amount of internal memory), both alternatives fit within standard $\mathrm{ARTICo}^{3}$ reconfigurable slots, and the slightly superior overhead is compensated by a significantly higher execution performance (roughly speaking, there is an increase of $4 \times$ in computing performance by just duplicating the amount of FPGA resources).

\footnotetext{
${ }^{22}$ Experimental evaluation has shown that reducing the precision in the floating point arithmetic cores does not affect endmember extraction accuracy.
} 


\section{Network Infrastructure}

As opposed to the traditional deployments in which the ARTICo ${ }^{3}$ framework has been used, a networked implementation of the run-time adaptive linear hyperspectral unmixing chain is proposed in this Thesis. The purpose of this approach is to evaluate the feasibility of using ARTICo $^{3}$-based systems in a multi-FPGA context, enabling adaptive processing at two different levels. By using a hardware-accelerated computing cluster, run-time adaptive processing is supported at node-level (by changing the number of accelerators and their configuration) and at network-level (by changing the number of nodes). In addition, network-level adaptivity also fosters the inclusion of heterogeneous computing nodes, leading to scenarios where highend FPGA devices can coexist with low-cost ones (i.e., cloud computing versus edge computing).

The use of a small multi-FPGA computing cluster using low-end devices is adopted in this Thesis. The cluster operates in master/slave(s) fashion and features one instance of the ARTICo $^{3}$ architecture in each node. Data distribution and synchronization is made using MPI, a well-known parallel-programming API used in high-performance systems with a distributed memory model. ${ }^{23}$ Figure 4-13 shows the structure of the proposed computing cluster and how the reduction-based FUN algorithm is deployed on it.

Data distribution in the multi-FPGA computing cluster has been analyzed under two complementary scenarios: one seeks to maximize parallelism whereas the other aims at minimizing data transactions. Both approaches start with the master node dividing the input hyperspectral image in as many segments as processing nodes are available, but they differ in the next steps.

In the maximum parallelization approach, each slave further divides its input segments in fixed-size subsegments that can be processed by the HLS-based FUN accelerators $\left(\right.$ pixels $_{\text {block }}=32$ ). Partial endmembers are extracted from these image subsegments using the original FUN algorithm and then sent to the master, which generates a new artificial image, splits it following the same approach used in the initialization, and sends it back to the slaves. This procedure is repeated until only one image segment remains and the actual endmembers are finally extracted. On the contrary, in the minimum data transactions approach, a two-level reduction process is implemented. Each slave performs endmember extraction using the reductionbased FUN algorithm and then sends the results to the master, which performs a final reduction round to extract the endmembers.

Figure 4-14 shows the communication overheads of both approaches in the multiFPGA computing cluster when using MPI to send images with 256 bands, 128 lines, and 128 samples to 4 computing nodes. ${ }^{24}$ The top graph shows maximum parallelization,

\footnotetext{
${ }^{23}$ MPI was used to speed up the deployment process. However, the communication strategies and the computation offloading approaches discussed in this Thesis are platform agnostic and thus, they are not limited by the communication primitives.

${ }^{24}$ The multi-FPGA cluster operates on a private network with a dedicated Ethernet switch @ 1 Gbps.
} 


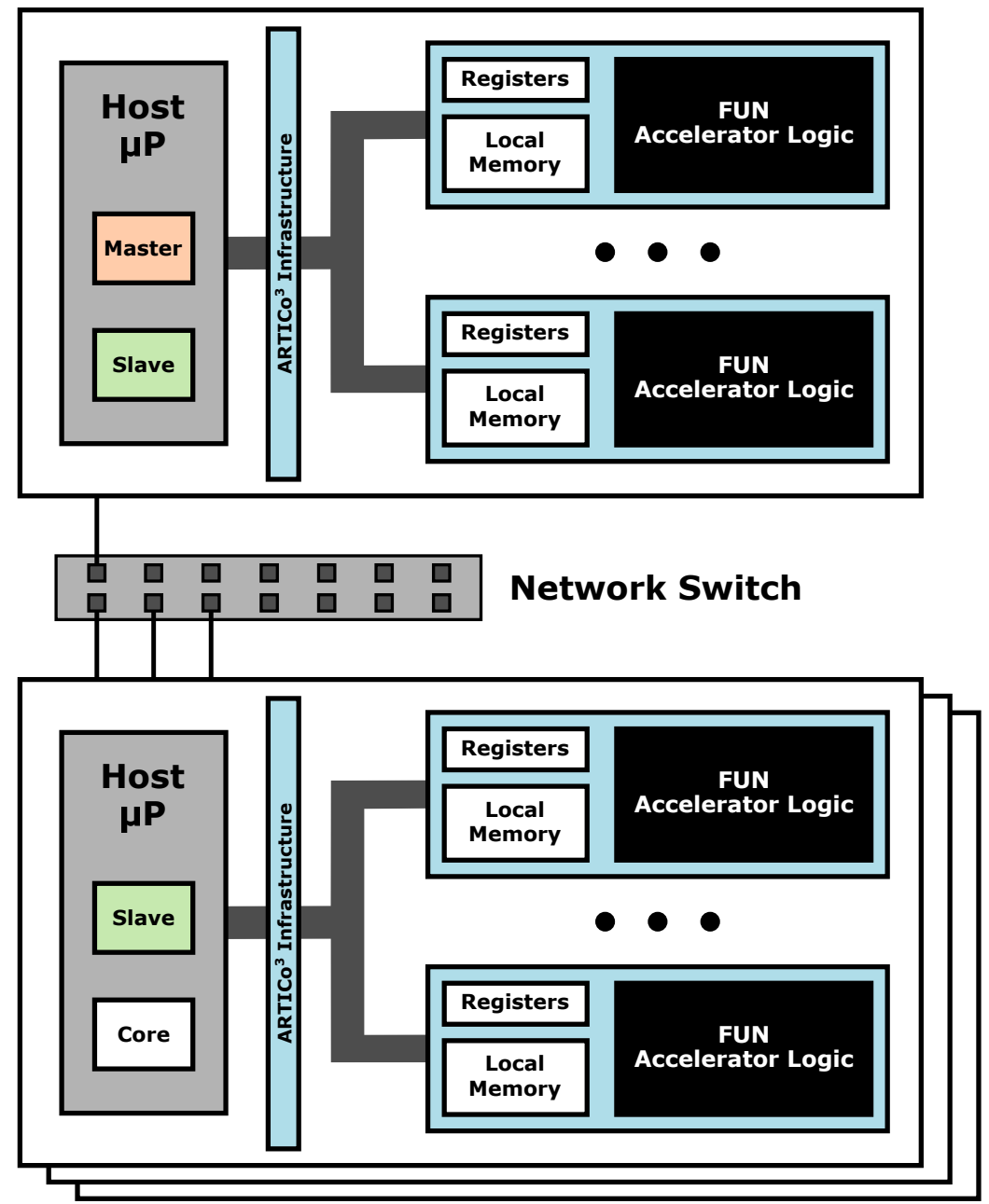

Figure 4-13: Network setup of an ARTICo ${ }^{3}$-based computing cluster.

whereas the bottom graph shows minimum data transactions.

Although the maximum parallelization approach should be, a priori, better than the minimum data transactions one, communication latency discourages its use. This is mainly due to the excessive data traffic over the dedicated network. Results confirm this, showing that minimizing data transactions between master and slave(s) results in faster communications (100 ms on average).$^{25}$

\footnotetext{
${ }^{25}$ All network experiments have been performed using the MPICH (https://www.mpich.org) implementation of the MPI API. This implementation provides better performance results than other alternatives such as OpenMPI (https://www.open-mpi.org) [Hablot'07].
} 

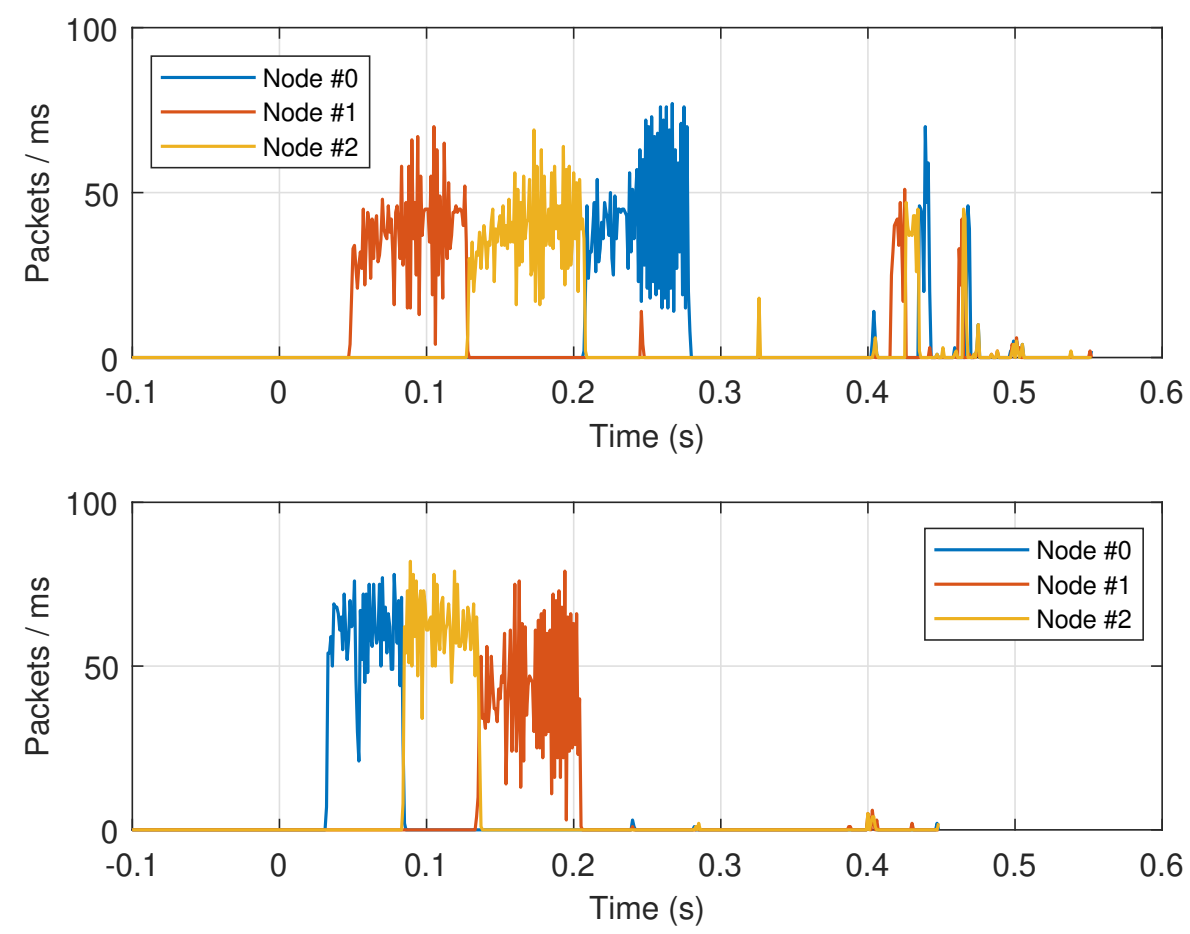

Figure 4-14: MPI-based communication overheads.

\subsubsection{Validation}

The run-time adaptive linear hyperspectral unmixing chain has been evaluated in two different setups. Single-node performance scalability and energy efficiency (i.e., the regular ARTICo ${ }^{3}$-based deployment) has been tested using a custom Zynq-7000 board that features an integrated power monitoring infrastructure. ${ }^{26}$ In parallel, multiFPGA performance scalability has been evaluated using a small Ethernet-based cluster with 8 MicroZed boards. ${ }^{27}$ The reduction-based FUN endmember extraction using hardware accelerators with half-precision floating point arithmetic has been used in both scenarios and, in the networked setup, the minimum data transactions approach.

Synthetic hyperspectral images have been used as inputs, since they allow for deep tuning of the evaluation scenarios (e.g., number of endmembers, spatial dimensions, etc.). The following configurations have been selected for testing purposes: 256 bands, 128 lines, and 128 samples with 10 endmembers; 256 bands, 256 lines, and 256 samples with 16 endmembers; and 256 bands, 512 lines, and 512 samples with 16 endmembers.

Table 4-16 shows the resource utilization of the ARTICo ${ }^{3}$ infrastructure (per node), as well as for the FUN kernel (includes user logic and wrapper). Since this kernel does not require any configuration register, the overhead of instantiating its user logic

\footnotetext{
${ }^{26}$ XC7Z020-1CLG484.

${ }^{27}$ XC7Z020-1CLG400.
} 
within an ARTICo ${ }^{3}$ wrapper is almost negligible in terms of LUTs and FFs, and there is only a significant contribution of the local memory in the number of BRAMs used.

Table 4-16: Resource utilization in the run-time adaptive endmember extraction.

\begin{tabular}{ccc}
\hline Component & ARTICo $^{\mathbf{3}}$ & FUN Kernel \\
\hline Info & $\begin{array}{c}4 \text { slots } \\
\text { VHDL (Vivado) } \\
\text { Zynq-7000 }\end{array}$ & $\begin{array}{c}\text { 64 KiB memory, 2 banks } \\
\text { 0 registers } \\
\text { C + HLS (Vivado) } \\
\text { Zynq-7000 }\end{array}$ \\
\hline LUTs & 4158 & 4502 \\
FFs & 2366 & 4207 \\
DSPs & - & 18 \\
BRAMs & - & 20 \\
\hline
\end{tabular}

\section{Standalone ARTICo ${ }^{3}$}

Although ARTICo ${ }^{3}$ enables dynamic tradeoffs between computing performance, energy efficiency, and fault tolerance during execution, in this section only the two first elements will be addressed. Table 4-17 shows the execution results of the algorithm running in ARTICo $^{3}$ (single node @ $166.67 \mathrm{MHz}$ ) versus a software-based implementation (single node, 1 ARM core @ $666.67 \mathrm{MHz}$ ).

Table 4-17: Execution performance and energy consumption in hyperspectral unmixing (standalone $\mathrm{ARTICo}^{3}$ ).

\begin{tabular}{ccccccccccc}
\hline \multirow{2}{*}{$\mathbf{N z} \times \mathbf{N y} \times \mathbf{N x}$} & \multicolumn{4}{c}{ Execution Time (s) } & \multicolumn{5}{c}{ Energy Consumption (J) } \\
& SW & $\mathbf{1}$ acc & $\mathbf{2}$ accs & $\mathbf{3}$ accs & $\mathbf{4}$ accs & SW & $\mathbf{1}$ acc & $\mathbf{2}$ accs & $\mathbf{3}$ accs & $\mathbf{4}$ accs \\
\hline $256 \times 128 \times 128$ & 1.05 & 1.05 & 0.75 & 0.66 & 0.58 & 1.44 & 0.98 & 0.79 & 0.76 & 0.75 \\
$256 \times 256 \times 256$ & 4.33 & 4.35 & 3.11 & 2.66 & 2.38 & 5.88 & 3.95 & 3.09 & 2.85 & 2.65 \\
$256 \times 512 \times 512$ & 69.44 & 46.4 & 29.12 & 22.97 & 19.75 & 92.99 & 38.42 & 27.17 & 23.16 & 21.2 \\
\hline
\end{tabular}

For small hyperspectral images, both software- and hardware-based (single accelerator) implementations are equivalent in terms of execution time, even though the former shows $50 \%$ better energy consumption. The hardware-accelerated implementation outperforms its software counterpart for large input images, even when using only one hardware accelerator in the FPGA. In addition, the energy consumption is kept at lower values.

Results also show that performance scalability is not linear. The main reasons behind this are kernels changing from computing- to memory-bounded operation on the one hand, and run-time memory management overheads in $\mathrm{ARTICo}^{3}$ on the other hand. However, the energy efficiency values and the possibility to add hardware redundancy on the fly still encourage the use of the hardware-based alternative. 


\section{Networked ARTICo ${ }^{3}$}

For this scenario, a small multi-FPGA computing cluster with 8 nodes has been used, where each node can host up to 4 ARTICo $^{3}$ accelerators. Figure 4-15 shows the Ethernet-based cluster.

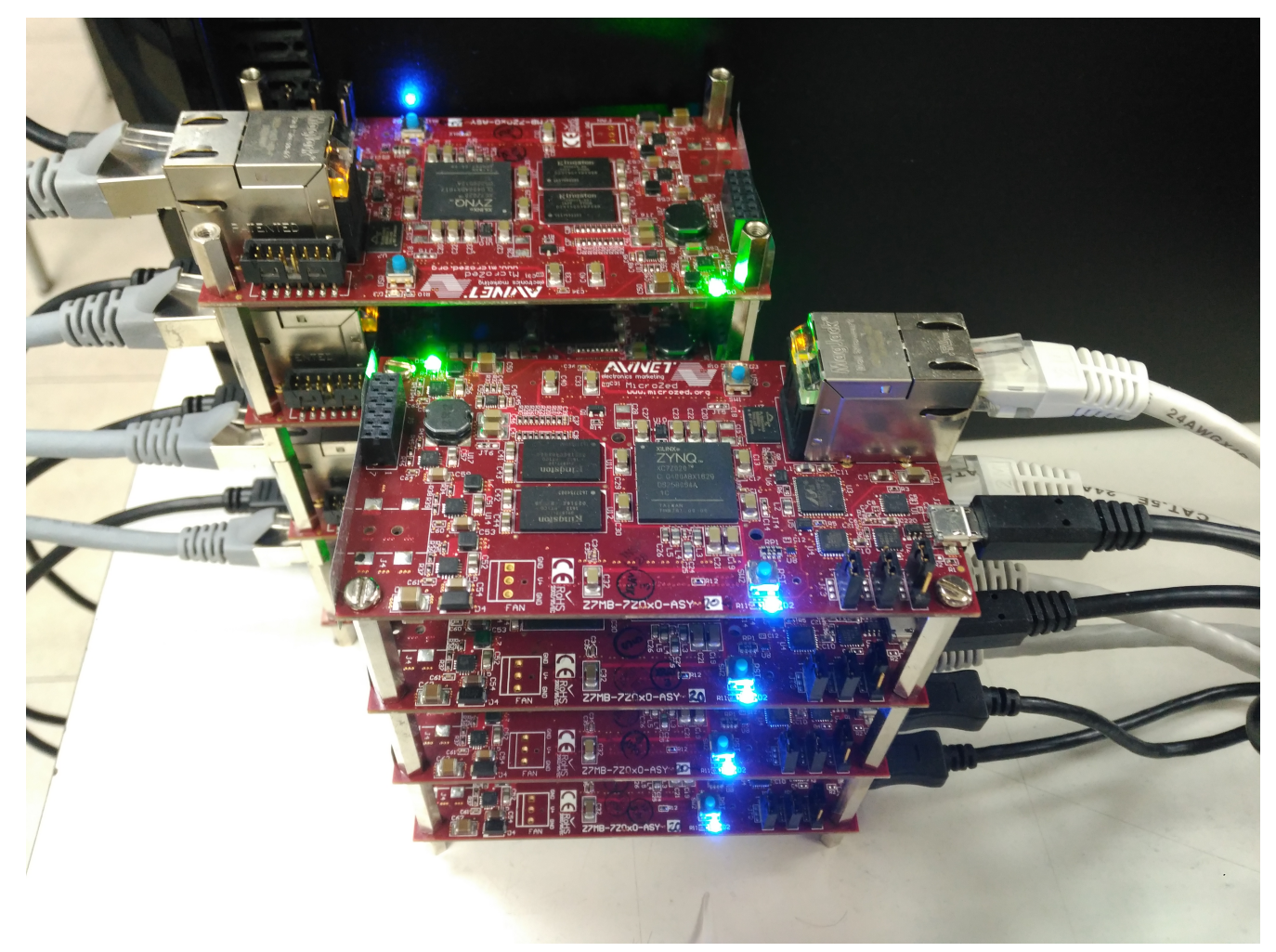

Figure 4-15: ARTICo ${ }^{3}$-based multi-FPGA computing cluster.

The first set of tests made with the networked implementation intend to evaluate the two-way scalability of the deployed system. Table 4-18 contains the results (multiple nodes @ $166.67 \mathrm{MHz}$ ) when changing the input image size, the number of nodes, and the number of accelerators per node. The overall improvement of each configuration with respect to the baseline solution (i.e., one node with one accelerator) is also provided.

Analyzing these results it is possible to verify that for the same amount of total hardware accelerators, it is advisable to scale the system in the number of nodes rather than in the number of accelerators per node. This is due to a memory management overhead per node slightly larger than the internode communication overhead. Please note that execution times when using one node are in the range of the ones presented in Table 4-17, with only a small increase generated by the communication API.

A second set of tests has been also performed to compare the proposed implementation (multiple nodes, 4 accelerators @ $166.67 \mathrm{MHz}$ per node) with a software-based alternative (multiple nodes, 2 ARM cores @ $666.67 \mathrm{MHz}$ per node), 
Table 4-18: Execution performance in hyperspectral unmixing (networked ARTICo ${ }^{3}$ ).

\begin{tabular}{cccccc}
\hline \multirow{2}{*}{$\mathbf{N z} \times \mathbf{N y} \times \mathbf{N x}$} & \multirow{2}{*}{ \# Nodes } & $\mathbf{1}$ acc & $\mathbf{2}$ accs & $\mathbf{3}$ accs & $\mathbf{4}$ accs \\
\hline & & $1.18(1 \times)$ & $0.84(1.4 \times)$ & $0.73(1.6 \times)$ & $0.67(1.8 \times)$ \\
$256 \times 128 \times 128$ & 2 & $0.72(1.6 \times)$ & $0.56(2.1 \times)$ & $0.49(2.4 \times)$ & $0.44(2.7 \times)$ \\
& 4 & $0.46(2.6 \times)$ & $0.41(2.9 \times)$ & $0.37(3.2 \times)$ & $0.34(3.5 \times)$ \\
& 8 & $0.38(3.1 \times)$ & $0.31(3.8 \times)$ & $0.29(4.1 \times)$ & $0.27(4.4 \times)$ \\
\hline \multirow{2}{*}{$256 \times 256 \times 256$} & 1 & $4.72(1 \times)$ & $3.35(1.4 \times)$ & $2.87(1.6 \times)$ & $2.66(1.8 \times)$ \\
& 2 & $2.83(1.7 \times)$ & $2.12(2.2 \times)$ & $1.88(2.5 \times)$ & $1.85(2.6 \times)$ \\
& 4 & $1.99(2.4 \times)$ & $1.51(3.1 \times)$ & $1.38(3.4 \times)$ & $1.45(3.3 \times)$ \\
& 8 & $1.4(3.4 \times)$ & $1.35(3.5 \times)$ & $1.15(4.1 \times)$ & $1.11(4.3 \times)$ \\
\hline \multirow{3}{*}{$256 \times 512 \times 512$} & 1 & $49.04(1 \times)$ & $30.67(1.6 \times)$ & $24.29(2 \times)$ & $21.14(2.3 \times)$ \\
& 2 & $25.88(1.9 \times)$ & $16.9(2.9 \times)$ & $14.17(3.5 \times)$ & $12.37(4 \times)$ \\
& 4 & $14.69(3.3 \times)$ & $10.18(4.8 \times)$ & $8.56(5.7 \times)$ & $7.8(6.3 \times)$ \\
& 8 & $9.12(5.4 \times)$ & $6.78(7.2 \times)$ & $6.52(7.5 \times)$ & $5.55(8.8 \times)$ \\
\hline
\end{tabular}

and to analyze the impacts of the communication primitives in the system. According to MPI terminology, the ARTICo ${ }^{3}$-based deployment uses one slave process and four hardware accelerators per node, whereas the software-based approach uses two slave processes per node to maximize the number of potential parallel processing elements. Note that the master process runs in one of the cores for both alternatives. Table 4-19 reports the obtained results.

Table 4-19: Hardware versus software systems for networked hyperspectral unmixing.

\begin{tabular}{|c|c|c|c|c|c|c|c|}
\hline \multirow{2}{*}{$\mathbf{N z} \times \mathbf{N y} \times \mathbf{N x}$} & \multirow{2}{*}{ \# Nodes } & \multicolumn{3}{|c|}{ ARTICo $^{3}$} & \multicolumn{3}{|c|}{ Software } \\
\hline & & Comm. (s) & Exec. (s) & Total (s) & Comm. (s) & Exec. (s) & Total (s) \\
\hline \multirow{4}{*}{$256 \times 128 \times 128$} & 1 & 0.06 & 0.59 & 0.65 & 0.08 & 0.35 & 0.43 \\
\hline & 2 & 0.18 & 0.27 & 0.46 & 0.15 & 0.21 & 0.36 \\
\hline & 4 & 0.29 & 0.14 & 0.34 & 0.22 & 0.09 & 0.31 \\
\hline & 8 & 0.27 & 0.07 & 0.28 & 0.24 & 0.04 & 0.28 \\
\hline \multirow{4}{*}{$256 \times 256 \times 256$} & 1 & 0.22 & 2.41 & 2.64 & 0.19 & 1.6 & 1.79 \\
\hline & 2 & 0.74 & 1.12 & 1.83 & 0.64 & 0.83 & 1.47 \\
\hline & 4 & 1.15 & 0.59 & 1.32 & 0.82 & 0.45 & 1.27 \\
\hline & 8 & 1.07 & 0.3 & 1.09 & 0.91 & 0.26 & 1.17 \\
\hline \multirow{4}{*}{$256 \times 512 \times 512$} & 1 & 0.89 & 19.36 & 21.25 & 0.44 & 25.93 & 26.37 \\
\hline & 2 & 2.87 & 9.77 & 12.36 & 1.4 & 13.8 & 15.2 \\
\hline & 4 & 4.52 & 4.94 & 7.87 & 3.69 & 6.63 & 10.32 \\
\hline & 8 & 5.51 & 2.42 & 5.58 & 3.45 & 3.45 & 6.9 \\
\hline
\end{tabular}

Since the computing infrastructure is different for each approach, total execution times equal communication plus processing time for software but not for hardware. This is due to the fact that $\mathrm{ARTICo}^{3}$ finishes its processing in each slave node while the master process is still sending or receiving data through the network. To some extent, this phenomenon resembles the memory-bounded execution behavior in singlenode deployments, where the memory bandwidth sets the maximum performance boundary for the hardware accelerators (i.e., the DMA-enabled data transfers take 
more time than the actual accelerator execution).

Results show that for small hyperspectral images, the hardware-based solution is worse than its software counterpart. However, this behavior is inverted for large input images, even though communication times are still larger in hardware than in software. If only execution time is taken into account, it can be seen that performance scales almost linearly for both hardware and software. As consequence, it is better to scale in the number of nodes than in the number of accelerators per node, especially when considering that more nodes also implies smaller image segments per node.

Original contribution 4-4 A run-time adaptive and multi-FPGA implementation of a low-complexity on-board linear hyperspectral unmixing chain with selectable performance and energy efficiency levels. 


\section{Chapter}

\section{CONCLUSIONS, IMPACT AND FUTURE LINES OF WORK}

This chapter presents the conclusions drawn from the work developed in this Thesis. A brief summary of its main contributions is also included afterwards. Then, a quantitative evaluation of the obtained results, measured in terms of publications, research projects, co-supervised works, and collaborations, is performed. Finally, a discussion on the future lines of work that lay beyond this Thesis closes the chapter.

\subsection{Conclusions of the Thesis}

FPGA-based reconfigurable multi-accelerator systems are complex parallel computing plaftorms that present challenges at three different levels: architectural, design-time support, and run-time support. The work developed in this Thesis has addressed all of them to provide a programmer-friendly approach to hardware-based highperformance embedded computing under dynamic operating conditions.

At architectural level, a DPR-capable computing substrate with a dynamically configurable datapath has been designed. The architecture provides three different operation modes to allow dynamic accelerator configurations that exploit data-level parallelism, redundant execution, or a combination of both. The inner workings of the architecture are hidden behind a memory-mapped abstraction model, in which groups of application-specific accelerators share virtual address spaces with specific subranges to identify local memory banks, registers, and built-in multicast operations.

At design-time support level, a toolchain to automate system implementation has been provided. A general-purpose interfacing mechanism to enable independent user logic developments has been proposed, using two separate mechanisms for accelerator descriptions: HDL and HLS.

At run-time support level, a runtime library to abstract DPR and accelerator scheduling has been developed. A data-parallel execution model to support transparent performance scalability when changing the number of hardware accelerators loaded on the FPGA has been devised and implemented.

Experimental results obtained during the validation of the baseline ARTICo ${ }^{3}$ framework show that the proposed reconfigurable multi-accelerator approach to run-time adaptivity in high-performance embedded systems renders better 
performance, energy efficiency, and fault tolerance than alternative computing platforms. Benchmark-based evaluation has highlighted the main scenarios where the proposed framework can become truly beneficial: applications with potential data-level parallelism where the computation/communication ratio is biased towards computation (i.e., computing-bounded applications). Considering the nature of current algorithms in high-performance embedded systems (e.g., machine learning), it is expected that the ARTICo ${ }^{3}$ framework plays an important role in the near future.

The proposed programming model extensions to the baseline framework open a new world of possibilities, from model-based design using dataflow specifications to adaptive multithreading using different accelerator granularities (i.e., SIMD-like versus MIMD-like). Furthermore, the associated reconfiguration techniques also expand the versatility of the framework, enabling multi-grained DPR approaches and applicationspecific CGR overlays on DPR-compatible partitions.

Finally, the real-world application examples shown at the end of the Thesis confirm that the proposed framework is not only valid under controlled laboratory experiments, but also in high-end scientific use cases with extremely stringent requirements. The inherent run-time adaptivity of ARTICo ${ }^{3}$-based deployments has made it possible to create implementations that are competitive with state-of-the-art alternatives, while at the same time offering novel and previously unexplored features.

\subsection{Summary of Main Contributions}

In this Thesis, an open source framework to support run-time adaptation in reconfigurable multi-accelerator systems has been developed and presented. Although all the original contributions have been highlighted throughout the document, this section summarizes the most relevant ones according to the goals established in Section 1.2:

- [Architecture] A hardware-based processing architecture for adaptive highperformance embedded computing based on run-time tradeoffs between computing performance, energy efficiency, and fault tolerance.

- [Design] An automated design methodology to generate custom reconfigurable multi-accelerator systems from either low-level RTL descriptions or high-level algorithmic descriptions and HLS.

- [Runtime] A runtime library to transparently manage FPGA reconfiguration and computation offloading in multi-accelerator scenarios.

- [Validation] A Dwarf-based characterization and validation strategy for the ARTICo $^{3}$ framework based on HLS benchmarks. 
- [Runtime] A multi-paradigm programming approach for reconfigurable multiaccelerator systems that combines SIMD-like data-parallel execution with transparent hardware/software multithreading.

- [Design] An integrated toolchain to automatically generate reconfigurable multiaccelerator systems from high-level dataflow descriptions.

- [Application] A run-time adaptive FPGA implementation of a low-complexity on-board CCSDS 123 lossless multispectral and hyperspectral compressor with selectable performance and energy efficiency levels.

- [Application] A run-time adaptive and multi-FPGA implementation of a lowcomplexity on-board linear hyperspectral unmixing chain with selectable performance and energy efficiency levels.

\subsection{Impact of the Thesis}

In this section, the quantitative results of this Thesis are presented. To do so, five different categories have been identified to classify dissemination activities with direct connection with this Thesis, rest of dissemination activities, research projects where this Thesis has been either developed in or applied to, co-supervised B.Sc. and M.Sc. theses that are related to this Thesis, and collaborations with other research entities.

All the results are arranged in lists, usually in subcategories. Please note that, when possible, list entries highlight joint effort activities with other research groups on the one hand, and describe their relationship with the rest of this document on the other hand, also providing links to the specific sections where their contents can be found. 


\subsubsection{Main Publications and Dissemination}

This subsection reports all the publications and dissemination activities with direct connection with the work developed in this Thesis. A direct connection implies work that generates an original contribution of this Thesis.

\section{Journal Publications}

[Rodríguez'19] A. Rodríguez, L. Santos, R. Sarmiento, E. de la Torre, "Scalable Hardware-Based On-Board Processing for Run-Time Adaptive Lossless Hyperspectral Compression", in IEEE Access, vol. 7, pp. $10644-10652$, 2019, ISSN 21693536, doi:10.1109/ACCESS.2019.2892308.

\section{JCR impact factor: 3.745 (Q1)}

Joint effort with Universidad de Las Palmas de Gran Canaria

This article presents and evaluates the run-time adaptive FPGA implementation of a low-complexity on-board CCSDS 123 lossless multispectral and hyperspectral compressor described in $\S 4.2$.

[Ortiz'18b] A. Ortiz, A. Rodríguez, R. Guerra, S. López, A. Otero, R. Sarmiento, E. de la Torre, "A Runtime-Scalable and Hardware-Accelerated Approach to On-Board Linear Unmixing of Hyperspectral Images”, in Remote Sensing, vol. 10, no. 11, 2018, ISSN 2072-4292, doi:10.3390/rs10111790.

\section{JCR impact factor: $4.118(Q 1)$}

Joint effort with Universidad de Las Palmas de Gran Canaria

This article presents and evaluates the run-time adaptive and multi-FPGA implementation of a low-complexity on-board linear hyperspectral unmixing chain described in $\$ 4.3$.

[Rodríguez'18b] A. Rodríguez, J. Valverde, J. Portilla, A. Otero, T. Riesgo, E. de la Torre, "FPGA-Based High-Performance Embedded Systems for Adaptive Edge Computing in Cyber-Physical Systems: The ARTICo ${ }^{3}$ Framework", in Sensors, vol. 18, no. 6, 2018, ISSN 1424-8220, doi:10.3390/s18061877.

\section{JCR impact factor: $3.031(Q 1)$}

This article presents the components of the $\mathrm{ARTICo}^{3}$ framework, including the processing architecture described in $\$ 2.3$, the design toolchain described in $\S 2.4$, the runtime management library described in $\$ 2.5$, and part of the validation tests described in $\$ 2.6$. 


\section{Conference Publications}

[Ortiz'19] A. Ortiz, A. Rodríguez, A. Otero, E. de la Torre, "Data Transfer Modeling and Optimization in Reconfigurable Multi-Accelerator Systems", in 2019 14th International Symposium on Reconfigurable Communication-centric Systems-on-Chip (ReCoSoC), pp. 20-26, July 2019, ISSN 2642-7230, doi: 10.1109/ReCoSoC48741.2019.9034940.

This article presents the implementation and evaluation of several benchmarks in multi-accelerator setups, which constitutes the basis of the validation scenario described in $\S 2.6 .3$.

[Rodríguez'18a] A. Rodríguez, T. Fanni, "DEMO: Multi-Grain Adaptivity in CyberPhysical Systems", in 2018 30th International Conference on Microelectronics (ICM), pp. 44-47, Dec 2018, doi:10.1109/ICM.2018.8704058.

Joint effort with Università degli Studi di Cagliari Joint effort with Università degli Studi di Sassari

This article presents a live demonstrator of the integrated dataflow-based framework with hybrid hardware reconfiguration capabilities described in $\S 3.3 .3$ using the application scenario described in $\$$ 3.3.4.

[Fanni'18] T. Fanni, A. Rodríguez, C. Sau, L. Suriano, F. Palumbo, L. Raffo, E. de la Torre, "Multi-Grain Reconfiguration for Advanced Adaptivity in CyberPhysical Systems", in 2018 International Conference on ReConFigurable Computing and FPGAs (ReConFig), pp. 1-8, Dec 2018, ISSN 2640-0472, doi: 10.1109/RECONFIG.2018.8641705.

Joint effort with Università degli Studi di Cagliari Joint effort with Università degli Studi di Sassari

This article presents the integrated dataflow-based framework with hybrid hardware reconfiguration capabilities described in $\$ 3.3 .3$, and includes its validation scenario as described in $\S$ 3.3.4.

[Rodríguez'17a] A. Rodríguez, C. Castañares, T. Riesgo, E. de la Torre, "Evaluating HighLevel Synthesis Techniques for Scalable Hardware-Accelerated Computing”, in 2017 32nd Conference on Design of Circuits and Integrated Systems (DCIS), pp. 1-8, Nov 2017.

This article presents a preliminary evaluation of HLS tools and their suitability to generate multi-accelerator systems from high-level algorithmic descriptions. The evaluation of different matrix multiplication cores is the basis of the validation scenario described in $\S 2.6 .2$.

[Rodríguez'15c] A. Rodríguez, J. Valverde, E. de la Torre, "Design of OpenCLcompatible multithreaded hardware accelerators with dynamic support for embedded FPGAs", in 2015 International Conference on ReConFigurable Computing and FPGAs (ReConFig), pp. 1-7, Dec 2015, doi:10.1109/ReConFig.2015.7393297. 
This article presents the execution model described in $\S$ 2.5.1. The initial OpenCL compatibility was later removed in favor of a more optimized runtime library, as shown in $\S 2.5 .2$ and $\S 2.5 .3$. The experimental evaluation explores the multithreaded accelerator approach also used in the validation scenario described in $\$ 2.6 .1$.

[Rodríguez'15a] A. Rodríguez, J. Valverde, C. Castañares, J. Portilla, E. de la Torre, T. Riesgo, "Execution modeling in self-aware FPGA-based architectures for efficient resource management", in 2015 10th International Symposium on Reconfigurable Communication-centric Systems-on-Chip (ReCoSoC), pp. 1-8, June 2015, doi:10.1109/ReCoSoC.2015.7238086.

This article presents the run-time kernel modeling described in $\$ 2.5 .4$, as well as a first version of the monitoring infrastructure described in $\S 2.3 .2$.

[Rodríguez'15b] A. Rodríguez, J. Valverde, C. Castañares, J. Portilla, E. de la Torre, T. Riesgo, "Live demonstration: A dynamically adaptable image processing application running in an FPGA-based WSN platform", in 2015 IEEE International Symposium on Circuits and Systems (ISCAS), pp. 1902-1902, May 2015, ISSN 0271-4302, doi:10.1109/ISCAS.2015.7169035.

This article presents a demonstrator based on the HiReCookie WSN node that uses some of the accelerators described in $\S 2.6 .1$.

[Valverde'14b] J. Valverde, A. Rodríguez, J. Mora, C. Castañares, J. Portilla, E. de la Torre, T. Riesgo, “A Dynamically Adaptable Image Processing Application Trading Off Between High Performance, Consumption and Dependability in Real Time”, in 2014 Conference on Design and Architectures for Signal and Image Processing (DASIP), pp. 1-2, Oct 2014.

This article presents a demonstrator based on the HiReCookie WSN node that uses some of the accelerators described in $\$ 2.6 .1$.

[Valverde'14a] J. Valverde, A. Rodríguez, J. Camarero, A. Otero, J. Portilla, E. de la Torre, T. Riesgo, "A dynamically adaptable bus architecture for trading-off among performance, consumption and dependability in Cyber-Physical Systems", in 2014 24th International Conference on Field Programmable Logic and Applications (FPL), pp. 1-4, Sep. 2014, ISSN 1946-147X, doi:10.1109/FPL.2014.6927394.

This article presents the first concepts of the ARTICo ${ }^{3}$ processing architecture described in $\$ 2.3$. The initial versions of the configurable datapath and addressing schemes were later streamlined to enable better performance with less overheads, as described in $\S 2.3 .1$ and $\S 2.3 .4$, respectively.

[Rodríguez'14] A. Rodríguez, J. Valverde, E. de la Torre, T. Riesgo, “Dynamic management of multikernel multithread accelerators using Dynamic Partial Reconfiguration”, in 2014 9th International Symposium on Reconfigurable and Communication-Centric Systems-on-Chip (ReCoSoC), pp. 1-7, May 2014, doi: 10.1109/ReCoSoC.2014.6861363. 
This article presents the first concepts of the ARTICo ${ }^{3}$ execution model described in $\S$ 2.5.1. The initial CUDA compatibility was first replaced by OpenCL compatibility, and later removed in favor of a more optimized runtime library, as shown in $\S 2.5 .2$ and $\S 2.5 .3$.

\section{Other Dissemination Activities}

The acquired knowledge on reconfigurable SoC-based systems has been applied to several subjects of a M.Sc. program, producing an education-related publication.

[Rodríguez'16b] A. Rodríguez, J. Portilla, E. de la Torre, T. Riesgo, “Teaching hybrid HW/SW embedded system design using FPGA-based devices", in 2016 Conference on Design of Circuits and Integrated Systems (DCIS), pp. 1-5, Nov 2016, doi:10.1109/DCIS.2016.7845372.

The integrated framework proposed in this Thesis has been used as the basis for two tutorials on hardware-based adaptivity in multi-accelerator systems.

Tutorial - "Multi-Grain Reconfiguration for Advanced Adaptivity in CPS", in CPS Summer School 2018: Designing Cyber-Physical Systems - From concepts to implementation, Alghero, Italy, September 2018.

Tutorial - "Coarse and Fine Grain Reconfiguration using the ARTICo ${ }^{3}$ Framework", in CPS Summer School 2017: Designing Cyber-Physical Systems - From concepts to implementation, Alghero, Italy, September 2017.

Intermediate results of this Thesis have been presented at multidisciplinary research forums, producing four separate contributions to dissemination booklets.

[Rodríguez'17b] A. Rodríguez, E. de la Torre, "Automatic Integration of HLS-Generated Hardware Accelerators in an Embedded Parallel Computing Architecture", in Industriales Research Meeting 2017, p. 66, Escuela Técnica Superior de Ingenieros Industriales, Universidad Politécnica de Madrid, 2017, ISBN 978-84-16397-58-7, http://oa.upm.es/46090.

[Ortiz'17] A. Ortiz, A. Rodríguez, E. de la Torre, "Resilient communications for a multi-FPGA, hardware-accelerated cluster", in Industriales Research Meeting 2017, p. 67, Escuela Técnica Superior de Ingenieros Industriales, Universidad Politécnica de Madrid, 2017, ISBN 978-84-16397-58-7, http://oa.upm.es/46090.

[Rodríguez'16a] A. Rodríguez, C. Castañares, L. Suriano, T. Riesgo, E. de la Torre, “Towards Safer and Predictable Hardware Acceleration in Distributed, Embedded and High Performance Systems", in Industriales Research Meeting 2016, p. 86, Escuela Técnica Superior de Ingenieros Industriales, Universidad Politécnica de Madrid, 2016, ISBN 978-84-16397-31-0, http://oa.upm.es/40073. 
[Castañares'16b] C. Castañares, A. Rodríguez, T. Riesgo, E. de la Torre, "Development of Hardware Accelerators Using OpenCL Methodologies for Single and Multiple FPGA Architectures", in Industriales Research Meeting 2016, p. 82, Escuela Técnica Superior de Ingenieros Industriales, Universidad Politécnica de Madrid, 2016, ISBN 978-84-16397-31-0, http://oa.upm.es/40073.

\subsubsection{Additional Publications and Dissemination}

This subsection reports all the publications and dissemination activities with indirect connection with the work developed in this Thesis. An indirect connection implies collaborative work where the framework developed in this Thesis is used.

\section{Journal Publications}

[Barrios'20] Y. Barrios, A. Rodríguez, A. Sánchez, A. Pérez, S. López, A. Otero, E. de la Torre, R. Sarmiento, "Lossy Hyperspectral Image Compression on a Reconfigurable and Fault-Tolerant FPGA-Based Adaptive Computing Platform", in Electronics, vol. 9, no. 10, 2020, ISSN 2079-9292, doi:10.3390/electronics9101576.

2019 JCR impact factor: 2.412 (Q2)

Joint effort with Universidad de Las Palmas de Gran Canaria

[Suriano'20] L. Suriano, A. Otero, A. Rodríguez, M. Sánchez-Renedo, E. de la Torre, "Exploiting Multi-Level Parallelism for Run-Time Adaptive Inverse Kinematics on Heterogeneous MPSoCs", in IEEE Access, vol. 8, pp. 118707-118724, 2020, ISSN 2169-3536, doi:10.1109/ACCESS.2020.3005202.

\section{JCR impact factor: 3.745 (Q1)}

[Pérez'20] A. Pérez, A. Rodríguez, A. Otero, D. González Arjona, A. Jiménez-Peralo, M. A. Verdugo, E. de la Torre, "Run-Time Reconfigurable MPSoC-Based On-Board Processor for Vision-Based Space Navigation”, in IEEE Access, vol. 8, pp. 5989159 905, 2020, ISSN 2169-3536, doi:10.1109/ACCESS.2020.2983308.

\section{JCR impact factor: 3.745 (Q1)}

[Suriano'19] L. Suriano, F. Arrestier, A. Rodríguez, J. Heulot, K. Desnos, M. Pelcat, E. de la Torre, "DAMHSE: Programming heterogeneous MPSoCs with hardware acceleration using dataflow-based design space exploration and automated rapid prototyping", in Microprocessors and Microsystems, vol. 71, p. 102 882, 2019, ISSN 0141-9331, doi:10.1016/j.micpro.2019.102882.

2019 JCR impact factor: 1.161 (Q3)

Joint effort with Institut National des Sciences Appliquées Rennes 


\section{Book Chapters}

[Armesto'20] L. Armesto, A. Rodríguez, A. Pérez, S. Sáez, J. Valls, Y. Barrios, A. Sánchez, D. González Arjona, Á. Jiménez-Peralo, F. Veljković, "Reconfigurable Video Processor for Space”, in A. Leitner, D. Watzenig, J. Ibanez-Guzman, editors, Validation and Verification of Automated Systems: Results of the ENABLE-S3 Project, pp. 231-249, Springer International Publishing, Cham, 2020, ISBN 9783-030-14628-3, doi:10.1007/978-3-030-14628-3_18.

\section{Conference Publications}

[Ortiz'20] A. Ortiz, R. Zamacola, A. Rodríguez, A. Otero, E. de la Torre, "Automated Toolchain for Enhanced Productivity in Reconfigurable Multi-accelerator Systems", in F. Rincón, J. Barba, H. K. H. So, P. Diniz, J. Caba, editors, Applied Reconfigurable Computing. Architectures, Tools, and Applications, pp. 45-60, Springer International Publishing, Cham, 2020, ISBN 978-3-030-44534-8, doi: 10.1007/978-3-030-44534-8_4.

[Palumbo'19b] F. Palumbo, T. Fanni, C. Sau, A. Rodríguez, D. Madroñal, K. Desnos, A. Morvan, M. Pelcat, C. Rubattu, R. Lazcano, L. Raffo, E. de la Torre, E. Juárez, C. Sanz, P. Sánchez de Rojas, "Hardware/Software Self-adaptation in CPS: The CERBERO Project Approach”, in D. N. Pnevmatikatos, M. Pelcat, M. Jung, editors, Embedded Computer Systems: Architectures, Modeling, and Simulation, pp. 416428, Springer International Publishing, Cham, 2019, ISBN 978-3-030-27562-4, doi:10.1007/978-3-030-27562-4_30.

[Palumbo'19a] F. Palumbo, T. Fanni, C. Sau, L. Pulina, L. Raffo, M. Masin, E. Shindin, P. S. de Rojas, K. Desnos, M. Pelcat, A. Rodríguez, E. Juárez, F. Regazzoni, G. Meloni, K. Zedda, H. Myrhaug, L. Kaliciak, J. Andriaanse, J. de Olivieria Filho, P. Muñoz, A. Toffetti, "CERBERO: Cross-layer modEl-based fRamework for multioBjective dEsign of Reconfigurable Systems in unceRtain hybRid envirOnments: Invited Paper: CERBERO Teams from UniSS, UniCA, IBM Research, TASE, INSARennes, UPM, USI, Abinsula, AmbieSense, TNO, S\&T, CRF”, in Proceedings of the 16th ACM International Conference on Computing Frontiers, CF '19, pp. 320-325, ACM, New York, NY, USA, 2019, ISBN 978-1-4503-6685-4, doi: $10.1145 / 3310273.3323436$.

[Suriano'18] L. Suriano, D. Madroñal, A. Rodríguez, E. Juárez, C. Sanz, E. de la Torre, "A Unified Hardware/Software Monitoring Method for Reconfigurable Computing Architectures Using PAPI", in 2018 13th International Symposium on Reconfigurable Communication-centric Systems-on-Chip (ReCoSoC), pp. 1-8, July 2018, doi:10.1109/ReCoSoC.2018.8449389.

[Suriano'17] L. Suriano, A. Rodríguez, K. Desnos, M. Pelcat, E. de la Torre, “Analysis of a heterogeneous multi-core, multi-hw-accelerator-based system designed using 
PREESM and SDSoC", in 2017 12th International Symposium on Reconfigurable Communication-centric Systems-on-Chip (ReCoSoC), pp. 1-7, July 2017, doi: 10.1109/ReCoSoC.2017.8016151.

\section{Other Dissemination Activities}

The combination of reconfigurable multi-accelerator systems with other types of computing fabrics in the field of adaptive CPSs constitutes the basis of two tutorials.

Tutorial - "Adaptation over Heterogeneous Embedded Computing Infrastructures", in High Performance and Embedded Architecture and Compilation Conference (HiPEAC 2020), Bologna, Italy, January 2020.

Tutorial - "Self-adaptation of Cyber Physical Systems", in High Performance and Embedded Architecture and Compilation Conference (HiPEAC 2019), Valencia, Spain, January 2019.

\subsubsection{Research Projects}

Some of the results described in this Thesis have been either obtained in or applied to several research projects. This subsection provides a list of those projects in which the author of this Thesis has been actively involved.

REBECCA - Resilient EmBedded Electronic systems for Controlling Cities under Atypical situations.

Spanish R\&D National Program (TEC2014-58036-C4-2-R)

In this project, the main infrastructure of the $\mathrm{ARTICo}^{3}$ framework, especially the hardware-based processing architecture, was developed. The use of HLS techniques as the entry point for designing reconfigurable multi-accelerator systems for smart cities was thoroughly evaluated. Initial tests with lossless hyperspectral image compression algorithms were also performed.

ENABLE-S3 - European Initiative to Enable Validation for Highly Automated Safe and Secure Systems.

H2020-ECSEL-2015-2-IA-two-stage (692455-2)

In this project, the $\mathrm{ARTICo}^{3}$ framework was used to generate reconfigurable multi-accelerator systems for space applications. Additional fault tolerance mechanisms were implemented on top of the configurable voter unit, including several types of configuration memory scrubbers. Experimental evaluation was carried out using lossless/lossy hyperspectral image compression and visionbased space navigation algorithms 
CERBERO - Cross-layer modEl-based fRamework for multi-oBjective dEsign of Reconfigurable systems in unceRtain hybRid envirOnments.

H2020-ICT-2016-1 (732105)

In this project, the main infrastructure of the $\mathrm{ARTICo}^{3}$ framework was refined and finalized. Additional extensions to support dataflow-based MoCs in reconfigurable multi-accelerator systems were also developed.

PLATINO - Plataforma HW/SW Distribuida para el Procesamiento Inteligente de Información Sensorial Heterogénea en Aplicaciones de Supervisión de Grandes Espacios Naturales.

Spanish R\&D National Program (TEC2017-86722-C4-2-R)

In this project, the ARTICo ${ }^{3}$ framework was used to implement reconfigurable multi-accelerator systems for smart farming scenarios. Experimental evaluation was carried out using linear unmixing algorithms for hyperspectral images.

\subsubsection{Co-Supervised Works}

This subsection reports all the B.Sc. and M.Sc. theses, co-supervised by the author of this Thesis, that are connected with the Thesis itself. All these documents have contributed in any of the following forms: supporting the development of the ARTICo ${ }^{3}$ framework, evaluating different implementation alternatives for some framework components, or providing the required infrastructure to develop experimental setups.

[Encinas'20] J. Encinas, Data Provisioning Infrastructure for Reconfigurable MultiAccelerator Systems, M.Sc. thesis, Universidad Politécnica de Madrid, Jun. 2020.

[Casado'19] P. Casado, Diseño e implementación de un sistema de auto-medida de consumo para FPGAs, B.Sc. thesis, Universidad Politécnica de Madrid, Feb. 2019, http://oa.upm.es/54152.

[Ortiz'18a] A. Ortiz, Diseño de una Plataforma Heterogénea Multi-FPGA para Cómputo Paralelo, Distribuido y Acelerado en Hardware sobre ARTICo ${ }^{3}$, M.Sc. thesis, Universidad Politécnica de Madrid, Oct. 2018.

[Paarup'17] L. Paarup, Aceleración Software y Hardware de Algoritmos de Montecarlo para Aplicaciones Financieras, B.Sc. thesis, Universidad Politécnica de Madrid, Feb. 2017.

[García'17] L. García, Desarrollo de un Motor de Reconfiguración HW para Plataformas Zynq, B.Sc. thesis, Universidad Politécnica de Madrid, Feb. 2017, http://oa.upm. es/45804.

[Vázquez'16] J. Vázquez, Alternativas de Comunicación Serie de Alta Velocidad en FPGAs, M.Sc. thesis, Universidad Politécnica de Madrid, Oct. 2016, http://oa. upm.es/44640. 
[Castañares'16a] C. Castañares, Desarrollo de aceleradores hardware con técnicas de High-Level Synthesis: exploración de alternativas con paralelismo explícito y ejecución escalable, M.Sc. thesis, Universidad Politécnica de Madrid, Oct. 2016, http://oa.upm.es/44649.

[Giménez'16] C. Giménez, Desarrollo de una Red de Comunicaciones para Sistemas de Cómputo Heterogéneos Distribuidos y Escalables, M.Sc. thesis, Universidad Politécnica de Madrid, Oct. 2016, http://oa.upm.es/44641.

[Cabrera'16] G. Cabrera, Smart Communication Device for Multi-FPGA High Performance Computing Platform, M.Sc. thesis, Universidad Politécnica de Madrid, Oct. 2016.

[Ortiz'16] A. Ortiz, Alternativas de implementación de comunicaciones TCP/IP/Ethernet en SoPCs, B.Sc. thesis, Universidad Politécnica de Madrid, Sep. 2016, http://oa. upm.es/43454.

[Gozalo'16] D. Gozalo, Comunicación mediante FIFOs para soporte de 'flujo de datos' en aceleradores HW, B.Sc. thesis, Universidad Politécnica de Madrid, Feb. 2016.

\subsubsection{Collaborations}

Some of the activities described in this Thesis are the result of close collaborations between the author and other researchers. This subsection briefly summarizes these activities, even though some of them have already been highlighted in the corresponding publications.

All the activity around hyperspectral data processing has been carried out together with the Instituto Universitario de Microelectrónica Aplicada at Universidad de Las Palmas de Gran Canaria. In particular, the work on lossless compression (developed in a short research stay at their research center) was supported by Lucana Santos, being the HyLoC compressor part of her Ph.D. thesis. The work on linear unmixing, on the other hand, was supported by Raúl Guerra, being the FUN algorithm and its HPC implementations part of his Ph.D. thesis.

The work on hybrid reconfiguration and dataflow-based MoCs has been carried out together with Università degli Studi di Cagliari and Università degli Studi di Sassari. In particular, the integration between the $\mathrm{ARTICo}^{3}$ framework and the MDC tool was developed together with Tiziana Fanni, and also constitutes part of her Ph.D. thesis.

The work on multithreaded programming models for hardware-based systems has been partly carried out in a 3-month research stay at Universität Paderborn. In particular, the integration between the $\mathrm{ARTICo}^{3}$ framework and ReconOS was developed under the supervision of Professor Marco Platzner.

Finally, the integration of the evolvable hardware system based on scalable systolic arrays was developed together with Javier Mora (another researcher from the author's group), and also constitutes part of his Ph.D. thesis. 


\subsubsection{Grants and Other Funding}

This Thesis has received funding from the Spanish Ministerio de Educación, Cultura y Deporte under the Formación de Profesorado Universitario program, with reference number FPU13/06007.

The 3-month research stay at Universität Paderborn was funded by the High Performance and Embedded Architecture and Compilation (HiPEAC) European network under the 2017 Collaboration Grant program.

\subsection{Future Lines of Work}

The framework proposed in this Thesis is a solid starting point to make hardwarebased high-performance embedded systems not only accessible but also appealing to a wider audience. In addition, its maturity has been demonstrated in relevant industrydriven applications. However, its main components still present some limitations. This section presents several lines of work to overcome them, as well as additional ideas to include novel and significant enhancements in future developments.

\section{Architectural Improvements}

Currently, the most important limitation of $\mathrm{ARTICo}^{3}$ framework is its excessive memory management overheads, which are derived from the Linux-based software stack that acts as runtime library. In particular, the overheads are caused by the existence of two separate memory buffers, one for the application (fast memory access, implemented as virtual memory obtained through malloc) and one for the hardware accelerators (slow memory access, implemented as non-cached contiguous physical memory obtained through mmap and the Linux DMA framework), and the need for memcpy operations among them. Experimental validation confirms these overheads, as it can be seen when comparing the results from Subsection 2.6.1, whose implementation relies on a bare-metal implementation of the runtime library, against the ones obtained in the rest of validation scenarios, whose implementations use the Linux-based runtime library described in Section 2.5.

The aforementioned problems could be addressed from two different perspectives: software and hardware. In the former, the current software stack would need to be changed to include either more optimized structures to avoid data copies (e.g., cached zero-copy buffers) or additional mechanisms to mask their impact (e.g., data prefetching and/or double buffering strategies). The author of this Thesis has collaborated in a preliminary evaluation of these software-based extensions [Ortiz'19]. From a hardware-based perspective, on the other hand, the approach should consist 
of adding specialized modules (e.g., soft-core processors) on the FPGA fabric to handle data transfers more efficiently. Preliminary evaluation of these architectural extensions has been carried out in a work co-supervised by the author of this Thesis [Encinas'20].

Apart from the memory management overheads, alternative architectural improvements could be also considered. Currently, the ARTICo ${ }^{3}$ framework assumes a memory-mapped slave nature for all hardware kernels. Although this approach has proven to be beneficial in multiple application scenarios, there are still cases in which it might not render proper acceleration. One such example is stream-based processing, in which the output of an accelerator is to be immediately processed by its neighboring modules. Since ARTICo ${ }^{3}$ kernels return their results to main memory before they can be used as input data for other accelerators, performance can be significantly affected in those applications. To mitigate this, a proposal would be to isolate the main architectural components of the processing architecture, generate a library of dynamically composable IP cores, and use those blocks to build application-specific datapaths with custom acceleration and fault tolerance extensions, instantiating only what is actually required. As a consequence, the architecture would move from a monolithic approach to a highly modular approach, which could be potentially exploited by the use of reconfigurable FPGA overlays.

\section{Design-Time Support Improvements}

The ARTICo ${ }^{3}$ toolchain constitutes an important step towards the user-friendliness of reconfigurable multi-accelerator systems, allowing software developers to transparently and efficiently use hardware acceleration. However, and even though the process is completely automated, the hardware implementation flow still exhibits long development cycles. In fact, the current system generation step requires both static and dynamic parts to be generated each time a change is made to any of these regions, thus having a huge impact on productivity.

As a consequence, a future line of work is to extend the current DPR flow, which is purely based on commercial solutions (i.e., Xilinx Vivado), to a more customized approach in which additional features (e.g., module relocation, independent staticdynamic region implementation) are supported. The author of this Thesis has collaborated in the preliminary evaluation of these design-time support extensions [Ortiz'20].

\section{Run-Time Support Improvements}

Although the ARTICo ${ }^{3}$ architecture enables run-time tradeoffs between computing performance, energy efficiency, and fault tolerance, it is still up to the developer to write the application code that triggers the required changes in the reconfigurable fabric. In a context where most high-performance embedded computing systems need to face highly dynamic application scenarios whose full solution space cannot be explored at design time, this approach is no longer valid. 
In this regard, the final line of work derived from this Thesis is the inclusion of embedded intelligence to enable autonomous system management. A proposal would be to provide the runtime with means to estimate/predict the evolution of the different working points of the architecture when changing the boundary conditions. This can be achieved using artificial intelligence or machine learning algorithms combined with the already existing monitoring infrastructure to extract data-driven models of the whole multi-accelerator system. With this information, a central management module would then be able to make informed decisions on which accelerators to load, how to configure them, and what scheduling policies would be more suitable to achieve the required performance, energy, and dependability goals. 



\section{Bibliography}

[Ackerman'82] W. B. Ackerman, “Data Flow Languages”, in Computer, vol. 15, no. 2, pp. 15-25, Feb 1982, ISSN 1558-0814, doi:10.1109/MC.1982.1653938. ${ }^{141}$

[Agne'11] A. Agne, M. Platzner, E. Lübbers, "Memory Virtualization for Multithreaded Reconfigurable Hardware”, in 2011 21st International Conference on Field Programmable Logic and Applications, pp. 185-188, Sep. 2011, ISSN 1946-1488, doi:10.1109/FPL.2011.42. 121

[Agne'14] A. Agne, M. Happe, A. Keller, E. Lübbers, B. Plattner, M. Platzner, C. Plessl, "ReconOS: An Operating System Approach for Reconfigurable Computing”, in IEEE Micro, vol. 34, no. 1, pp. 60-71, Jan 2014, ISSN 1937-4143, doi:10.1109/MM.2013.110. $26,31,35,42,47,52,116$

[Agne’16] A. Agne, M. Platzner, C. Plessl, M. Happe, E. Lübbers, “ReconOS”, in D. Koch, F. Hannig, D. Ziener, editors, FPGAs for Software Programmers, pp. 227-244, Springer International Publishing, Cham, 2016, ISBN 978-3-319-26408-0, doi:10.1007/978-3319-26408-0_13. 121

[Al Kadi'18] M. Al Kadi, B. Janssen, J. Yudi, M. Hübner, "General-Purpose Computing with Soft GPUs on FPGAs”, in ACM Trans. Reconfigurable Technol. Syst., vol. 11, no. 1, Jan. 2018, ISSN 1936-7406, doi:10.1145/3173548. ${ }^{28,31,153}$

[Amdahl'67] G. M. Amdahl, "Validity of the Single Processor Approach to Achieving Large Scale Computing Capabilities”, in Proceedings of the April 18-20, 1967, Spring Joint Computer Conference, AFIPS '67 (Spring), p. 483-485, Association for Computing Machinery, New York, NY, USA, 1967, ISBN 978-1-4503-7895-6, doi: 10.1145/1465482.1465560. ${ }^{10}$

[Andrews'04] D. Andrews, D. Niehaus, R. Jidin, M. Finley, W. Peck, M. Frisbie, J. Ortiz, Ed Komp, P. Ashenden, "Programming models for hybrid FPGA-CPU computational components: a missing link", in IEEE Micro, vol. 24, no. 4, pp. 42-53, July 2004, ISSN 1937-4143, doi:10.1109/MM.2004.36. 112, 129

[Andrews'08] D. Andrews, R. Sass, E. Anderson, J. Agron, W. Peck, J. Stevens, F. Baijot, E. Komp, "Achieving Programming Model Abstractions for Reconfigurable Computing", in IEEE Transactions on Very Large Scale Integration (VLSI) Systems, vol. 16, no. 1, pp. 34-44, Jan 2008, ISSN 1557-9999, doi:10.1109/TVLSI.2007.912106. 19, 31, 43, 52, 129

[Andrews'16] D. Andrews, M. Platzner, "Programming models for reconfigurable manycore systems", in 2016 11th International Symposium on Reconfigurable Communication-centric Systems-on-Chip (ReCoSoC), pp. 1-8, June 2016, doi: 10.1109/ReCoSoC.2016.7533897. 116, 129

[Andryc'13] K. Andryc, M. Merchant, R. Tessier, "FlexGrip: A soft GPGPU for FPGAs”, in 2013 International Conference on Field-Programmable Technology (FPT), pp. 230-237, Dec 2013, doi:10.1109/FPT.2013.6718358. 24, 31

[Armesto'20] L. Armesto, A. Rodríguez, A. Pérez, S. Sáez, J. Valls, Y. Barrios, A. Sánchez, D. González Arjona, Á. Jiménez-Peralo, F. Veljković, "Reconfigurable Video Processor for Space”, in A. Leitner, D. Watzenig, J. Ibanez-Guzman, editors, Validation and Verification of Automated Systems: Results of the ENABLE-S3 Project, pp. 231249, Springer International Publishing, Cham, 2020, ISBN 978-3-030-14628-3, doi: 10.1007/978-3-030-14628-3_18. ${ }^{211}$ 
[Asanović'06] K. Asanović, R. Bodik, B. C. Catanzaro, J. J. Gebis, P. Husbands, K. Keutzer, D. A. Patterson, W. L. Plishker, J. Shalf, S. W. Williams, K. A. Yelick, "The Landscape of Parallel Computing Research: A View from Berkeley”, Tech. Rep. UCB/EECS-2006183, EECS Department, University of California, Berkeley, Dec 2006, http://www2. eecs.berkeley.edu/Pubs/TechRpts/2006/EECS-2006-183.html. ${ }^{99,} 105$

[Asanović'16] K. Asanović, R. Avižienis, J. Bachrach, S. Beamer, D. Biancolin, C. Celio, H. Cook, D. Dabbelt, J. Hauser, A. Izraelevitz, S. Karandikar, B. Keller, D. Kim, J. Koenig, Y. Lee, E. Love, M. Maas, A. Magyar, H. Mao, M. Moreto, A. Ou, D. A. Patterson, B. Richards, C. Schmidt, S. Twigg, H. Vo, A. Waterman, “The Rocket Chip Generator”, Tech. Rep. UCB/EECS-2016-17, EECS Department, University of California, Berkeley, Apr 2016, http://www2.eecs.berkeley.edu/Pubs/TechRpts/2016/EECS-2016-17.html. ${ }^{151}$

[Azarian'09] A. Azarian, M. Ahmadi, “Reconfigurable computing architecture survey and introduction”, in 2009 2nd IEEE International Conference on Computer Science and Information Technology, pp. 269-274, Aug 2009, doi:10.1109/ICCSIT.2009.5234721. 6,7

[Bachrach'12] J. Bachrach, H. Vo, B. Richards, Y. Lee, A. Waterman, R. Avižienis, J. Wawrzynek, K. Asanović, “Chisel: Constructing hardware in a Scala embedded language”, in DAC Design Automation Conference 2012, pp. 1212-1221, June 2012, ISSN 0738-100X, doi: $10.1145 / 2228360.2228584 .^{151}$

[Backasch'15] R. Backasch, G. Hempel, C. Blochwitz, S. Werner, S. Groppe, T. Pionteck, “An architectural template for composing application specific datapaths at runtime", in 2015 International Conference on ReConFigurable Computing and FPGAs (ReConFig), pp. 1-6, Dec 2015, doi:10.1109/ReConFig.2015.7393300. ${ }^{26,31}$

[Bacon'13] D. F. Bacon, R. Rabbah, S. Shukla, "FPGA Programming for the Masses", in Commun. ACM, vol. 56, no. 4, p. 56-63, Apr. 2013, ISSN 0001-0782, doi: $10.1145 / 2436256.2436271 .^{3,7,111,112}$

[Bailey'91] D. Bailey, E. Barszcz, J. Barton, D. Browning, R. Carter, L. Dagum, R. Fatoohi, P. Frederickson, T. Lasinski, R. Schreiber, H. Simon, V. Venkatakrishnan, S. Weeratunga, "The NAS Parallel Benchmarks", in The International Journal of Supercomputing Applications, vol. 5, no. 3, pp. 63-73, 1991, doi:10.1177/109434209100500306. ${ }^{105}$

[Barrios'20] Y. Barrios, A. Rodríguez, A. Sánchez, A. Pérez, S. López, A. Otero, E. de la Torre, R. Sarmiento, "Lossy Hyperspectral Image Compression on a Reconfigurable and Fault-Tolerant FPGA-Based Adaptive Computing Platform”, in Electronics, vol. 9, no. 10, 2020, ISSN 2079-9292, doi:10.3390/electronics9101576. ${ }^{210}$

[Báscones'17] D. Báscones, C. González, D. Mozos, "Parallel Implementation of the CCSDS 1.2.3 Standard for Hyperspectral Lossless Compression”, in Remote Sensing, vol. 9, no. 10, 2017, ISSN 2072-4292, doi:10.3390/rs9100973. ${ }^{171,180}$

[Báscones'18] D. Báscones, C. González, D. Mozos, "FPGA Implementation of the CCSDS 1.2.3 Standard for Real-Time Hyperspectral Lossless Compression", in IEEE Journal of Selected Topics in Applied Earth Observations and Remote Sensing, vol. 11, no. 4, pp. 1158-1165, April 2018, ISSN 1939-1404, doi:10.1109/JSTARS.2017.2767680. 171, 180

[Baumgardner'15] M. F. Baumgardner, L. L. Biehl, D. A. Landgrebe, “220 Band AVIRIS Hyperspectral Image Data Set: June 12, 1992 Indian Pine Test Site 3”, Sep 2015, doi:10.4231/R7RX991C. 176

[Becker' 16] T. Becker, O. Mencer, G. Gaydadjiev, “Spatial Programming with OpenSPL”, in D. Koch, F. Hannig, D. Ziener, editors, FPGAs for Software Programmers, pp. 81-95, Springer International Publishing, Cham, 2016, ISBN 978-3-319-26408-0, doi:10.1007/978-3319-26408-0_5. 151 
[Beckhoff'12] C. Beckhoff, D. Koch, J. Torresen, "GoAHEAD: A Partial Reconfiguration Framework”, in 2012 IEEE 20th International Symposium on Field-Programmable Custom Computing Machines, pp. 37-44, April 2012, doi:10.1109/FCCM.2012.17. ${ }^{34,42}$

[Ben Abdelhamid'19] R. Ben Abdelhamid, Y. Yamaguchi, T. Boku, "MITRACA: A Next-Gen Heterogeneous Architecture", in 2019 IEEE 13th International Symposium on Embedded Multicore/Many-core Systems-on-Chip (MCSoC), pp. 304-311, Oct 2019, doi: 10.1109/MCSoC.2019.00050. ${ }^{29,31}$

[Bernabé'17] S. Bernabé, G. Botella, G. Martín, M. Prieto-Matias, A. Plaza, "Parallel Implementation of a Full Hyperspectral Unmixing Chain Using OpenCL”, in IEEE Journal of Selected Topics in Applied Earth Observations and Remote Sensing, vol. 10, no. 6, pp. 24522461, June 2017, ISSN 2151-1535, doi:10.1109/JSTARS.2017.2707541. 165, 188

[Bezati'13] E. Bezati, M. Mattavelli, J. W. Janneck, "High-level synthesis of dataflow programs for signal processing systems”, in 2013 8th International Symposium on Image and Signal Processing and Analysis (ISPA), pp. 750-754, Sep. 2013, ISSN 1845-5921, doi: 10.1109/ISPA.2013.6703837. 145, 151

[Bezati'16] E. Bezati, S. Casale-Brunet, M. Mattavelli, J. W. Janneck, "High-level system synthesis and optimization of dataflow programs for MPSoCs”, in 2016 50th Asilomar Conference on Signals, Systems and Computers, pp. 417-421, Nov 2016, doi: 10.1109/ACSSC.2016.7869072. ${ }^{145,151}$

[Bezati'19] E. Bezati, S. Casale-Brunet, R. Mosqueron, M. Mattavelli, "An Heterogeneous Compiler of Dataflow Programs for Zynq Platforms”, in ICASSP 2019 - 2019 IEEE International Conference on Acoustics, Speech and Signal Processing (ICASSP), pp. 1537-1541, May 2019, ISSN 2379-190X, doi:10.1109/ICASSP.2019.8682525. ${ }^{151}$

[Bhattacharyya'09] S. S. Bhattacharyya, G. Brebner, J. W. Janneck, J. Eker, C. von Platen, M. Mattavelli, M. Raulet, "OpenDF: A Dataflow Toolset for Reconfigurable Hardware and Multicore Systems”, in SIGARCH Comput. Archit. News, vol. 36, no. 5, p. 29-35, Jun. 2009, ISSN 0163-5964, doi:10.1145/1556444.1556449. ${ }^{142}$

[Bhattacharyya'19] S. S. Bhattacharyya, E. F. Deprettere, R. Leupers, J. Takala, Handbook of Signal Processing Systems, Springer International Publishing, 3rd ed., 2019, ISBN 978-3-31991734-4, doi:10.1007/978-3-319-91734-4. ${ }^{150}$

[Bienia'11] C. Bienia, Benchmarking Modern Multiprocessors, Ph.D. thesis, Princeton University, January 2011, https://parsec.cs.princeton.edu/publications/bienial lbenchmarking. pdf. 105

[Bioucas-Dias'12] J. M. Bioucas-Dias, A. Plaza, N. Dobigeon, M. Parente, Q. Du, P. Gader, J. Chanussot, "Hyperspectral Unmixing Overview: Geometrical, Statistical, and Sparse RegressionBased Approaches", in IEEE Journal of Selected Topics in Applied Earth Observations and Remote Sensing, vol. 5, no. 2, pp. 354-379, April 2012, ISSN 1939-1404, doi: 10.1109/JSTARS.2012.2194696. ${ }^{181,182,187}$

[Bioucas-Dias'13] J. M. Bioucas-Dias, A. Plaza, G. Camps-Valls, P. Scheunders, N. Nasrabadi, J. Chanussot, "Hyperspectral Remote Sensing Data Analysis and Future Challenges", in IEEE Geoscience and Remote Sensing Magazine, vol. 1, no. 2, pp. 6-36, June 2013, ISSN 2168-6831, doi:10.1109/MGRS.2013.2244672. ${ }^{163}$

[Bobda'07] C. Bobda, Introduction to Reconfigurable Computing-Architectures, Algorithms, and Applications, Springer Netherlands, 2007, ISBN 978-1-4020-6100-4, doi:10.1007/978$1-4020-6100-4{ }^{4}$

[Brant'12] A. Brant, G. G. F. Lemieux, “ZUMA: An Open FPGA Overlay Architecture”, in 2012 IEEE 20th International Symposium on Field-Programmable Custom Computing Machines, pp. 93-96, April 2012, doi:10.1109/FCCM.2012.25. ${ }^{152}$ 
[Bush'16] J. Bush, M. A. Khasawneh, K. Z. Mahmoud, T. N. Miller, "NyuziRaster: Optimizing rasterizer performance and energy in the Nyuzi open source GPU", in 2016 IEEE International Symposium on Performance Analysis of Systems and Software (ISPASS), pp. 204-213, April 2016, doi:10.1109/ISPASS.2016.7482095. ${ }^{28,31}$

[Cabrera'16] G. Cabrera, Smart Communication Device for Multi-FPGA High Performance Computing Platform, M.Sc. thesis, Universidad Politécnica de Madrid, Oct. 2016. ${ }^{214}$

[Campi'07] F. Campi, A. Deledda, M. Pizzotti, L. Ciccarelli, P. Rolandi, C. Mucci, A. Lodi, A. Vitkovski, L. Vanzolini, “A dynamically adaptive DSP for heterogeneous reconfigurable platforms", in 2007 Design, Automation \& Test in Europe Conference \& Exhibition, pp. 1-6, April 2007, ISSN 1558-1101, doi:10.1109/DATE.2007.364559. ${ }^{23}$

[Canis'13] A. Canis, J. Choi, M. Aldham, V. Zhang, A. Kammoona, T. Czajkowski, S. D. Brown, J. H. Anderson, "LegUp: An Open-Source High-Level Synthesis Tool for FPGA-Based Processor/Accelerator Systems”, in ACM Trans. Embed. Comput. Syst., vol. 13, no. 2, Sep. 2013, ISSN 1539-9087, doi:10.1145/2514740. ${ }^{129}$

[Canis'16] A. Canis, J. Choi, B. Fort, B. Syrowik, R. L. Lian, Y. T. Chen, H. Hsiao, J. Goeders, S. Brown, J. Anderson, “LegUp High-Level Synthesis”, in D. Koch, F. Hannig, D. Ziener, editors, FPGAs for Software Programmers, pp. 175-190, Springer International Publishing, Cham, 2016, ISBN 978-3-319-26408-0, doi:10.1007/978-3-319-264080_10. ${ }^{128}$

[Cardoso' 16] J. M. P. Cardoso, M. Weinhardt, High-Level Synthesis, pp. 23-47, Springer International Publishing, Cham, 2016, ISBN 978-3-319-26408-0, doi:10.1007/978-3-319-26408-0_2. 7

[Cardoso'17] J. M. P. Cardoso, J. G. F. Coutinho, P. C. Diniz, Embedded Computing for High Performance - Efficient Mapping of Computations Using Customization, Code Transformations and Compilation, Morgan Kaufmann, 2017, ISBN 978-0-12-8041895, doi:10.1016/C2015-0-00283-0. ${ }^{1}$

[Casado'19] P. Casado, Diseño e implementación de un sistema de auto-medida de consumo para FPGAs, B.Sc. thesis, Universidad Politécnica de Madrid, Feb. 2019, http://oa.upm.es/ 54152. 57,213

[Casale-Brunet'14] S. Casale-Brunet, M. Wiszniewska, E. Bezati, M. Mattavelli, J. W. Janneck, M. Canale, "TURNUS: An open-source design space exploration framework for dynamic stream programs", in Proceedings of the 2014 Conference on Design and Architectures for Signal and Image Processing, pp. 1-2, Oct 2014, doi:10.1109/DASIP.2014.7115614. ${ }^{145}$

[Castañares'16a] C. Castañares, Desarrollo de aceleradores hardware con técnicas de High-Level Synthesis: exploración de alternativas con paralelismo explícito y ejecución escalable, M.Sc. thesis, Universidad Politécnica de Madrid, Oct. 2016, http://oa.upm.es/44649. 214

[Castañares'16b] C. Castañares, A. Rodríguez, T. Riesgo, E. de la Torre, "Development of Hardware Accelerators Using OpenCL Methodologies for Single and Multiple FPGA Architectures", in Industriales Research Meeting 2016, p. 82, Escuela Técnica Superior de Ingenieros Industriales, Universidad Politécnica de Madrid, 2016, ISBN 978-84-16397-31-0, http: //oa.upm.es/40073. ${ }^{210}$

[CCSDS'12a] CCSDS, Lossless Data Compression Recommended Standard CCSDS 121.0-B-2, Apr 2012. $166,168,170$

[CCSDS'12b] CCSDS, Lossless Multispectral and Hyperspectral Image Compression Recommended Standard CCSDS 123.0-B-1, May 2012. 166, 169, 170, 173 
[CCSDS'15] CCSDS, Lossless Multispectral and Hyperspectral Image Compression Informational Report CCSDS 120.2-G-1, December 2015. 173, 180

[CCSDS'17] CCSDS, Image Data Compression Recommended Standard CCSDS 122.0-B-2, September 2017. ${ }^{166}$

[CCSDS'19] CCSDS, Low-Complexity Lossless and Near-Lossless Multispectral and Hyperspectral Image Compression Recommended Standard CCSDS 123.0-B-2, Feb 2019. ${ }^{166}$

[Chang'00] L. Chang, C.-M. Cheng, T.-C. Chen, “An efficient adaptive KLT for multispectral image compression", in 4th IEEE Southwest Symposium on Image Analysis and Interpretation, pp. 252-255, April 2000, doi:10.1109/IAI.2000.839610. ${ }^{166}$

[Chang'08] M. L. Chang, “Device Architecture”, in S. Hauck, A. Dehon, editors, Reconfigurable Computing - The Theory and Practice of FPGA-Based Computation, Systems on Silicon, pp. 3 - 27, Morgan Kaufmann, Burlington, 2008, doi:10.1016/B978012370522-8.50005-4. ${ }^{5}$

[Chang'13] C.-I. Chang, Hyperspectral data processing: algorithm design and analysis, John Wiley \& Sons, Apr 2013, ISBN 978-0-471-69056-6. 183, 187

[Charitopoulos'15] G. Charitopoulos, I. Koidis, K. Papadimitriou, D. Pnevmatikatos, "Hardware Task Scheduling for Partially Reconfigurable FPGAs”, in K. Sano, D. Soudris, M. Hübner, P. C. Diniz, editors, Applied Reconfigurable Computing, pp. 487-498, Springer International Publishing, Cham, 2015, ISBN 978-3-319-16214-0, doi:10.1007/978-3319-16214-0_45. 49, 52

[Chin'18] S. A. Chin, K. P. Niu, M. Walker, S. Yin, A. Mertens, J. Lee, J. H. Anderson, “Architecture Exploration of Standard-Cell and FPGA-Overlay CGRAs Using the Open-Source CGRA-ME Framework", in Proceedings of the 2018 International Symposium on Physical Design, ISPD '18, p. 48-55, Association for Computing Machinery, New York, NY, USA, 2018, ISBN 978-1-4503-5626-8, doi:10.1145/3177540.3177553. ${ }^{153}$

[Chouliaras'16] V. Chouliaras, D. Stevens, V. Dwyer, "VThreads: A novel VLIW chip multiprocessor with hardware-assisted PThreads", in Microprocessors and Microsystems, vol. 47, pp. 466 - 485, 2016, ISSN 0141-9331, doi:10.1016/j.micpro.2016.07.010. ${ }^{27,31}$

[Compton'02] K. Compton, S. Hauck, "Reconfigurable Computing: A Survey of Systems and Software”, in ACM Comput. Surv., vol. 34, no. 2, pp. 171-210, Jun. 2002, ISSN 03600300, doi:10.1145/508352.508353. xxi, 5, 6, 17,31, 53, 149

[Cong'14] J. Cong, H. Huang, C. Ma, B. Xiao, P. Zhou, “A Fully Pipelined and Dynamically Composable Architecture of CGRA", in 2014 IEEE 22nd Annual International Symposium on Field-Programmable Custom Computing Machines, pp. 9-16, May 2014, doi:10.1109/FCCM.2014.12. ${ }^{152}$

[Czajkowski'12] T. S. Czajkowski, U. Aydonat, D. Denisenko, J. Freeman, M. Kinsner, D. Neto, J. Wong, P. Yiannacouras, D. P. Singh, "From OpenCL to high-performance hardware on FPGAS”, in 22nd International Conference on Field Programmable Logic and Applications (FPL), pp. 531-534, Aug 2012, ISSN 1946-1488, doi:10.1109/FPL.2012.6339272. 130

[Darwin'59] C. Darwin, On the Origin of Species, John Murray, 1859. ${ }^{122}$

[Davidson'18] R. L. Davidson, C. P. Bridges, “Error Resilient GPU Accelerated Image Processing for Space Applications”, in IEEE Transactions on Parallel and Distributed Systems, vol. 29, no. 9, pp. 1990-2003, Sept 2018, ISSN 1045-9219, doi:10.1109/TPDS.2018.2812853. 171, 180

[Davis'82]

A. L. Davis, R. M. Keller, “Data Flow Program Graphs”, in Computer, vol. 15, no. 2, pp. 26-41, Feb 1982, ISSN 1558-0814, doi:10.1109/MC.1982.1653939. ${ }^{141}$ 
[de Garis'93] H. de Garis, "EVOLVABLE HARDWARE Genetic Programming of a Darwin Machine”, in R. F. Albrecht, C. R. Reeves, N. C. Steele, editors, Artificial Neural Nets and Genetic Algorithms, pp. 441-449, Springer Vienna, Vienna, 1993, ISBN 978-3-7091-7533-0, doi:10.1007/978-3-7091-7533-0_64. ${ }^{122}$

[De Jong'75] K. A. De Jong, Analysis of the behavior of a class of genetic adaptive systems, Ph.D. thesis, University of Michigan, Aug. 1975, http://hdl.handle.net/2027.42/4507. ${ }^{123}$

[Dennard'74] R. H. Dennard, F. H. Gaensslen, H. Yu, V. L. Rideout, E. Bassous, A. R. LeBlanc, "Design of ion-implanted MOSFET's with very small physical dimensions”, in IEEE Journal of Solid-State Circuits, vol. 9, no. 5, pp. 256-268, Oct 1974, ISSN 1558-173X, doi: 10.1109/JSSC.1974.1050511. ${ }^{2}$

[Dennis'74] J. B. Dennis, D. P. Misunas, “A Computer Architecture for Highly Parallel Signal Processing”, in Proceedings of the 1974 Annual ACM Conference - Volume 2, ACM '74, p. 402-409, Association for Computing Machinery, New York, NY, USA, 1974, ISBN 978-1-4503-7850-5, doi:10.1145/1408800.1408808. ${ }^{141}$

[Dennis'80] J. B. Dennis, “Data Flow Supercomputers”, in Computer, vol. 13, no. 11, pp. 48-56, Nov 1980, ISSN 1558-0814, doi:10.1109/MC.1980.1653418. ${ }^{141}$

[Desnos'19] K. Kesnos, F. Palumbo, “Dataflow Modeling for Reconfigurable Signal Processing Systems”, in S. S. Bhattacharyya, E. F. Deprettere, R. Leupers, J. Takala, editors, Handbook of Signal Processing Systems, pp. 787-824, Springer International Publishing, Cham, 2019, ISBN 978-3-319-91734-4, doi:10.1007/978-3-319-91734-4_22. ${ }^{112}$

[Devaux'09] L. Devaux, D. Chillet, S. Pillement, D. Demigny, "Flexible communication support for dynamically reconfigurable FPGAS", in 2009 5th Southern Conference on Programmable Logic (SPL), pp. 65-70, April 2009, doi:10.1109/SPL.2009.4914905. 19,31

[Díaz'12] J. Díaz, C. Muñoz-Caro, A. Niño, “A Survey of Parallel Programming Models and Tools in the Multi and Many-Core Era", in IEEE Transactions on Parallel and Distributed Systems, vol. 23, no. 8, pp. 1369-1386, Aug 2012, ISSN 1558-2183, doi: 10.1109/TPDS.2011.308. ${ }^{130}$

[Ding'14] H. Ding, M. Huang, "A unified OpenCL-flavor programming model with scalable hybrid hardware platform on FPGAs”, in 2014 International Conference on ReConFigurable Computing and FPGAs (ReConFig14), pp. 1-7, Dec 2014, ISSN 2325-6532, doi:10.1109/ReConFig.2014.7032563. ${ }^{26,31,130}$

[Ding' 16] H. Ding, S. Ma, M. Huang, D. Andrews, “OOGen: An Automated Generation Tool for Custom MPSoC Architectures Based on Object-Oriented Programming Methods”, in 2016 IEEE International Parallel and Distributed Processing Symposium Workshops (IPDPSW), pp. 233-240, May 2016, doi:10.1109/IPDPSW.2016.23. ${ }^{37,42,130}$

[Ding'17] H. Ding, M. Huang, "PolyPC: Polymorphic parallel computing framework on embedded reconfigurable system", in 2017 27th International Conference on Field Programmable Logic and Applications (FPL), pp. 1-8, Sep. 2017, ISSN 1946-1488, doi: 10.23919/FPL.2017.8056770. ${ }^{38,42}$

[Dobai'15] R. Robai, L. Sekanina, “Low-Level Flexible Architecture with Hybrid Reconfiguration for Evolvable Hardware”, in ACM Trans. Reconfigurable Technol. Syst., vol. 8, no. 3, May 2015, ISSN 1936-7406, doi:10.1145/2700414. ${ }^{124}$

[Edwards'06] S. A. Edwards, “The Challenges of Synthesizing Hardware from C-Like Languages”, in IEEE Design \& Test of Computers, vol. 23, no. 5, pp. 375-386, May 2006, ISSN 15581918, doi:10.1109/MDT.2006.134. ${ }^{112}$ 
[Edwards'19] S. A. Edwards, R. Townsend, M. Barker, M. A. Kim, "Compositional Dataflow Circuits", in ACM Trans. Embed. Comput. Syst., vol. 18, no. 1, Jan. 2019, ISSN 1539-9087, doi: 10.1145/3274280. ${ }^{151}$

[EEMBC] EEMBC, “Embedded Microprocessor Benchmark Consortium Benchmarks”, https:// www.eembc.org/. [Online] Accessed: October 13, 2020. ${ }^{105}$

[Eiben'15] A. Eiben, J. E. Smith, Introduction to Evolutionary Computing, Natural Computing Series, Springer-Verlag Berlin Heidelberg, 2nd ed., 2015, ISBN 978-3-662-44874-8, doi:10.1007/978-3-662-44874-8. 122, 123

[Eker'03] J. Eker, J. W. Janneck, E. A. Lee, Jie Liu, Xiaojun Liu, J. Ludvig, S. Neuendorffer, S. Sachs, Yuhong Xiong, "Taming heterogeneity - the Ptolemy approach", in Proceedings of the IEEE, vol. 91, no. 1, pp. 127-144, Jan 2003, ISSN 1558-2256, doi: 10.1109/JPROC.2002.805829. ${ }^{142}$

[Encinas'20] J. Encinas, Data Provisioning Infrastructure for Reconfigurable Multi-Accelerator Systems, M.Sc. thesis, Universidad Politécnica de Madrid, Jun. 2020. 213, 216

[Fabelo'16] H. Fabelo, S. Ortega, S. Kabwama, G. M. Callico, D. Bulters, A. Szolna, J. F. Pineiro, R. Sarmiento, "HELICoiD project: a new use of hyperspectral imaging for brain cancer detection in real-time during neurosurgical operations", in Proc. SPIE, Hyperspectral Imaging Sensors: Innovative Applications and Sensor Standards, vol. 9860, 2016, doi: 10.1117/12.2223075. ${ }^{164}$

[Fabelo'19] H. Fabelo, Contributions to the design and implementation of algorithms for the classification of hyperspectral images of brain tumors in real-time during surgical procedures, Ph.D. thesis, Universidad de Las Palmas de Gran Canaria, Apr. 2019, http://hdl.handle.net/10553/58355. ${ }^{164}$

[Fanni'18] T. Fanni, A. Rodríguez, C. Sau, L. Suriano, F. Palumbo, L. Raffo, E. de la Torre, "MultiGrain Reconfiguration for Advanced Adaptivity in Cyber-Physical Systems", in 2018 International Conference on ReConFigurable Computing and FPGAs (ReConFig), pp. 1-8, Dec 2018, ISSN 2640-0472, doi:10.1109/RECONFIG.2018.8641705. ${ }^{207}$

[Fanni'19] T. Fanni, Power and Energy Management in Coarse-Grained Reconfigurable Systems: methodologies, automation and assessments, Ph.D. thesis, Università degli Studi di Cagliari, Feb. 2019, http://hdl.handle.net/11584/260390. 149, 154

[Fjeldtvedt'18] J. Fjeldtvedt, M. Orlandić, T. A. Johansen, “An Efficient Real-Time FPGA Implementation of the CCSDS-123 Compression Standard for Hyperspectral Images”, in IEEE Journal of Selected Topics in Applied Earth Observations and Remote Sensing, vol. 11, no. 10, pp. 3841-3852, Oct 2018, ISSN 1939-1404, doi:10.1109/JSTARS.2018.2869697. 171,180

[Fleming'16] K. Fleming, M. Adler, “The LEAP FPGA Operating System”, in D. Koch, F. Hannig, D. Ziener, editors, FPGAs for Software Programmers, pp. 245-258, Springer International Publishing, Cham, 2016, ISBN 978-3-319-26408-0, doi:10.1007/978-3319-26408-0_14. 49, 52

[Flynn'72] M. J. Flynn, “Some Computer Organizations and Their Effectiveness", in IEEE Transactions on Computers, vol. C-21, no. 9, pp. 948-960, Sep. 1972, ISSN 1557-9956, doi:10.1109/TC.1972.5009071. ${ }^{9}$

[Fogel'66] L. Fogel, A. Owens, M. Walsh, Artificial Intelligence through Simulated Evolution, Wiley, Chichester, WS, UK, 1966, ISBN 978-0-471-26516-0. ${ }^{123}$

[Gallego'13a] A. Gallego, J. Mora, A. Otero, E. de la Torre, T. Riesgo, “A scalable evolvable hardware processing array", in 2013 International Conference on Reconfigurable Computing and FPGAs (ReConFig), pp. 1-7, Dec 2013, ISSN 2325-6532, doi: 10.1109/ReConFig.2013.6732266. ${ }^{125}$ 
[Gallego'13b] A. Gallego, J. Mora, A. Otero, R. Salvador, E. de la Torre, T. Riesgo, “A Novel FPGAbased Evolvable Hardware System Based on Multiple Processing Arrays", in 2013 IEEE International Symposium on Parallel Distributed Processing, Workshops and Phd Forum, pp. 182-191, May 2013, doi:10.1109/IPDPSW.2013.56. ${ }^{125}$

[García'17] L. García, Desarrollo de un Motor de Reconfiguración HW para Plataformas Zynq, B.Sc. thesis, Universidad Politécnica de Madrid, Feb. 2017, http://oa.upm.es/45804. ${ }^{213}$

[Ghamisi'17] P. Ghamisi, N. Yokoya, J. Li, W. Liao, S. Liu, J. Plaza, B. Rasti, A. Plaza, “Advances in Hyperspectral Image and Signal Processing: A Comprehensive Overview of the State of the Art", in IEEE Geoscience and Remote Sensing Magazine, vol. 5, no. 4, pp. 37-78, Dec 2017, ISSN 2168-6831, doi:10.1109/MGRS.2017.2762087. ${ }^{163,187}$

[Giménez'16] C. Giménez, Desarrollo de una Red de Comunicaciones para Sistemas de Cómputo Heterogéneos Distribuidos y Escalables, M.Sc. thesis, Universidad Politécnica de Madrid, Oct. 2016, http://oa.upm.es/44641. ${ }^{214}$

[Goetz'85] A. F. Goetz, G. Vane, J. E. Solomon, B. N. Rock, “Imaging Spectrometry for Earth Remote Sensing”, in Science, vol. 228, no. 4704, pp. 1147-1153, 1985, ISSN 0036-8075, doi:10.1126/science.228.4704.1147. ${ }^{163}$

[Göhringer'08] D. Göhringer, M. Hübner, V. Schatz, J. Becker, “Runtime adaptive multiprocessor system-on-chip: RAMPSoC”, in 2008 IEEE International Symposium on Parallel and Distributed Processing, pp. 1-7, April 2008, ISSN 1530-2075, doi: 10.1109/IPDPS.2008.4536503. ${ }^{19,31}$

[Göhringer'10] D. Göhringer, M. Hübner, E. N. Zeutebouo, J. Becker, “CAP-OS: Operating system for runtime scheduling, task mapping and resource management on reconfigurable multiprocessor architectures", in 2010 IEEE International Symposium on Parallel Distributed Processing, Workshops and Phd Forum (IPDPSW), pp. 1-8, April 2010, doi: 10.1109/IPDPSW.2010.5470732. ${ }^{45,52}$

[Göhringer'14] D. Göhringer, "Reconfigurable Multiprocessor Systems: Handling Hydras Heads - A Survey”, in SIGARCH Comput. Archit. News, vol. 42, no. 4, p. 39-44, Dec. 2014, ISSN 0163-5964, doi:10.1145/2693714.2693722. ${ }^{3}$

[Gokhale'05] M. B. Gokhale, P. S. Graham, Reconfigurable Computing - Accelerating Computation with Field-Programmable Gate Arrays, Springer US, 2005, ISBN 978-0-387-26106-5, doi:10.1007/b136834. ${ }^{4}$

[González’12a] C. González, D. Mozos, J. Resano, A. Plaza, “FPGA Implementation of the N-FINDR Algorithm for Remotely Sensed Hyperspectral Image Analysis", in IEEE Transactions on Geoscience and Remote Sensing, vol. 50, no. 2, pp. 374-388, Feb 2012, ISSN 15580644, doi:10.1109/TGRS.2011.2171693. ${ }^{187}$

[González'12b] C. González, J. Resano, A. Plaza, D. Mozos, "FPGA Implementation of Abundance Estimation for Spectral Unmixing of Hyperspectral Data Using the Image Space Reconstruction Algorithm", in IEEE Journal of Selected Topics in Applied Earth Observations and Remote Sensing, vol. 5, no. 1, pp. 248-261, Feb 2012, ISSN 21511535, doi:10.1109/JSTARS.2011.2171673. 187

[Govindaraju'12] V. Govindaraju, C. Ho, T. Nowatzki, J. Chhugani, N. Satish, K. Sankaralingam, C. Kim, "DySER: Unifying Functionality and Parallelism Specialization for Energy-Efficient Computing", in IEEE Micro, vol. 32, no. 5, pp. 38-51, Sep. 2012, ISSN 1937-4143, doi: 10.1109/MM.2012.51. ${ }^{23,31,153}$

[Gozalo'16] D. Gozalo, Comunicación mediante FIFOs para soporte de 'flujo de datos' en aceleradores HW, B.Sc. thesis, Universidad Politécnica de Madrid, Feb. 2016. ${ }^{214}$ 
[Gray'16] J. Gray, “GRVI Phalanx: A Massively Parallel RISC-V FPGA Accelerator Accelerator”, in 2016 IEEE 24th Annual International Symposium on Field-Programmable Custom Computing Machines (FCCM), pp. 17-20, May 2016, doi:10.1109/FCCM.2016.12. ${ }^{152}$

[Guerra'15] R. Guerra, L. Santos, S. López, R. Sarmiento, “A New Fast Algorithm for Linearly Unmixing Hyperspectral Images”, in IEEE Transactions on Geoscience and Remote Sensing, vol. 53, no. 12, pp. 6752-6765, Dec 2015, ISSN 0196-2892, doi: 10.1109/TGRS.2015.2447573. ${ }^{183,186,188}$

[Guerra'17a] R. Guerra, Towards the efficient processing of hyperspectral images: new hardwarefriendly algorithms and OpenCL-based implementations, Ph.D. thesis, Universidad de Las Palmas de Gran Canaria, May 2017, http://hdl.handle.net/10553/41780. ${ }^{166}$

[Guerra'17b] R. Guerra, E. Martel, J. Khan, S. López, P. Athanas, R. Sarmiento, “On the Evaluation of Different High-Performance Computing Platforms for Hyperspectral Imaging: An OpenCL-Based Approach", in IEEE Journal of Selected Topics in Applied Earth Observations and Remote Sensing, vol. 10, no. 11, pp. 4879-4897, Nov 2017, ISSN 19391404, doi:10.1109/JSTARS.2017.2737958. ${ }^{188}$

[Gustafson'88] J. L. Gustafson, “Reevaluating Amdahl's Law”, in Commun. ACM, vol. 31, no. 5, p. 532-533, May 1988, ISSN 0001-0782, doi:10.1145/42411.42415. ${ }^{11}$

[Hablot'07] L. Hablot, O. Gluck, J. Mignot, S. Genaud, P. V. Primet, "Comparison and tuning of MPI implementations in a grid context”, in 2007 IEEE International Conference on Cluster Computing, pp. 458-463, Sep. 2007, ISSN 1552-5244, doi: 10.1109/CLUSTR.2007.4629265. ${ }^{197}$

[Happe'15] M. Happe, A. Traber, A. Keller, "Preemptive Hardware Multitasking in ReconOS", in K. Sano, D. Soudris, M. Hübner, P. C. Diniz, editors, Applied Reconfigurable Computing, pp. 79-90, Springer International Publishing, Cham, 2015, ISBN 978-3319-16214-0, doi:10.1007/978-3-319-16214-0_7. 47, 121

[Hara'08] Y. Hara, H. Tomiyama, S. Honda, H. Takada, K. Ishii, “CHStone: A benchmark program suite for practical C-based high-level synthesis”, in 2008 IEEE International Symposium on Circuits and Systems, pp. 1192-1195, May 2008, ISSN 2158-1525, doi: 10.1109/ISCAS.2008.4541637. ${ }^{106,107}$

[Hennessy'17] J. L. Hennessy, D. A. Patterson, Computer Architecture - A Quantitative Approach, Morgan Kaufmann, 6th ed., 2017, ISBN 978-0-12-811905-1. 2, 8, 9

[Higuchi'93] T. Higuchi, T. Niwa, T. Tanaka, H. Iba, H. de Garis, T. Furuya, "Evolving Hardware with Genetic Learning: A First Step towards Building a Darwin Machine”, in Proceedings of the Second International Conference on From Animals to Animats 2: Simulation of Adaptive Behavior: Simulation of Adaptive Behavior, p. 417-424, MIT Press, Cambridge, MA, USA, 1993, ISBN 0-262-63149-0. ${ }^{122}$

[Holland'92] J. H. Holland, Adaptation in Natural and Artificial Systems: An Introductory Analysis with Applications to Biology, Control and Artificial Intelligence, MIT Press, Cambridge, MA, USA, 1992, ISBN 978-0-262-08213-6. ${ }^{123}$

[Hopson'12] B. Hopson, K. Benkrid, D. Keymeulen, N. Aranki, "Real-time CCSDS lossless adaptive hyperspectral image compression on parallel GPGPU multicore processor systems", in 2012 NASA/ESA Conference on Adaptive Hardware and Systems (AHS), pp. 107-114, June 2012, doi:10.1109/AHS.2012.6268637. ${ }^{170}$

[Hübner'11] M. Hübner, P. Figuli, R. Girardey, D. Soudris, K. Siozios, J. Becker, “A Heterogeneous Multicore System on Chip with Run-Time Reconfigurable Virtual FPGA Architecture", in 2011 IEEE International Symposium on Parallel and Distributed Processing Workshops and Phd Forum, pp. 143-149, May 2011, ISSN 1530-2075, doi: 10.1109/IPDPS.2011.135. ${ }^{22,31}$ 
[Hussain'16] W. Hussain, R. Airoldi, H. Hoffmann, T. Ahonen, J. Nurmi, "HARP²: An X-Scale Reconfigurable Accelerator-Rich Platform for Massively-Parallel Signal Processing Algorithms", in Journal of Signal Processing Systems, vol. 85, no. 3, pp. 341-353, Dec 2016, ISSN 1939-8115, doi:10.1007/s11265-015-1054-9. ${ }^{27,31}$

[IEEE'18] IEEE, “IEEE Standard for Information Technology-Portable Operating System Interface (POSIX(R)) Base Specifications, Issue 7”, in IEEE Std 1003.1-2017 (Revision of IEEE Std 1003.1-2008), pp. 1-3951, Jan 2018, doi:10.1109/IEEESTD.2018.8277153. 115

[Ismail'11] A. Ismail, L. Shannon, "FUSE: Front-End User Framework for O/S Abstraction of Hardware Accelerators", in 2011 IEEE 19th Annual International Symposium on Field-Programmable Custom Computing Machines, pp. 170-177, May 2011, doi: 10.1109/FCCM.2011.48. 22, 31, 46, 52, 129

[ISSCC'20] ISSCC, “ISSCC 2020 Trends”, http://isscc.org/wp-content/uploads/sites/17/2020/ 03/isscc2020.press_kit_final.pdf, 2020. [Online] Accessed: October 13, 2020. ${ }^{2}$

[Iturbe'15] X. Iturbe, K. Benkrid, C. Hong, A. Ebrahim, R. Torrego, T. Arslan, “Microkernel Architecture and Hardware Abstraction Layer of a Reliable Reconfigurable Real-Time Operating System (R3TOS)", in ACM Trans. Reconfigurable Technol. Syst., vol. 8, no. 1, Mar. 2015, ISSN 1936-7406, doi:10.1145/2629639. ${ }^{48,52}$

[Ivo'19] R. M. Ivo, D. M. Muñoz, "RTRLib: A High-Level Modeling Tool for the Implementation of Dynamically Partial Reconfigurable System-on-Chips”, in 2019 International Conference on ReConFigurable Computing and FPGAs (ReConFig), pp. 1-5, Dec 2019, ISSN 2640-0472, doi:10.1109/ReConFig48160.2019.8994779. ${ }^{40,42}$

[Jääskeläinen'15] P. Jääskeläinen, C. S. de La Lama, E. Schnetter, K. Raiskila, J. Takala, H. Berg, "pocl: A Performance-Portable OpenCL Implementation", in International Journal of Parallel Programming, vol. 43, no. 5, pp. 752-785, Oct 2015, ISSN 1573-7640, doi: 10.1007/s10766-014-0320-y. ${ }^{130}$

[Jacobsen'15] M. Jacobsen, D. Richmond, M. Hogains, R. Kastner, "RIFFA 2.1: A Reusable Integration Framework for FPGA Accelerators”, in ACM Trans. Reconfigurable Technol. Syst., vol. 8, no. 4, Sep. 2015, ISSN 1936-7406, doi:10.1145/2815631. ${ }^{48,52}$

[Jain'16] A. K. Jain, X. Li, S. A. Fahmy, D. L. Maskell, “Adapting the DySER Architecture with DSP Blocks as an Overlay for the Xilinx Zynq”, in SIGARCH Comput. Archit. News, vol. 43, no. 4, p. 28-33, Apr. 2016, ISSN 0163-5964, doi:10.1145/2927964.2927970. ${ }^{153}$

[Jain'17] A. K. Jain, Architecture Centric Coarse-Grained FPGA Overlays, Ph.D. thesis, Nanyang Technological University, Jan. 2017, http://hdl.handle.net/10356/69532. ${ }^{152}$

[Janneck'08] J. W. Janneck, I. D. Miller, D. B. Parlour, G. Roquier, M. Wipliez, M. Raulet, "Synthesizing hardware from dataflow programs: An MPEG-4 simple profile decoder case study”, in 2008 IEEE Workshop on Signal Processing Systems, pp. 287-292, Oct 2008, ISSN 2162-3570, doi:10.1109/SIPS.2008.4671777. ${ }^{150}$

[Janßen'17] B. Janßen, F. Korkmaz, H. Derya, M. Hübner, M. L. Ferreira, J. C. Ferreira, “Towards a type 0 hypervisor for dynamic reconfigurable systems", in 2017 International Conference on ReConFigurable Computing and FPGAs (ReConFig), pp. 1-7, Dec 2017, doi:10.1109/RECONFIG.2017.8279825. 50,52

[Jara-Berrocal'09] A. Jara-Berrocal, A. Gordon-Ross, "SCORES: A scalable and parametric streamsbased communication architecture for modular reconfigurable systems", in 2009 Design, Automation Test in Europe Conference Exhibition, pp. 268-273, April 2009, ISSN 1558-1101, doi:10.1109/DATE.2009.5090669. ${ }^{20}$ 
[Jara-Berrocal'10] A. Jara-Berrocal, A. Gordon-Ross, "VAPRES: A Virtual Architecture for Partially Reconfigurable Embedded Systems”, in 2010 Design, Automation \& Test in Europe Conference \& Exhibition (DATE 2010), pp. 837-842, March 2010, ISSN 1558-1101, doi: 10.1109/DATE.2010.5456934. ${ }^{20,31,33}$

[Jara-Berrocal'11a] A. Jara-Berrocal, A. Gordon-Ross, “An integrated development toolset and implementation methodology for partially reconfigurable system-on-chips”, in ASAP 2011 22nd IEEE International Conference on Application-specific Systems, Architectures and Processors, pp. 219-222, Sep. 2011, ISSN 2160-052X, doi:10.1109/ASAP.2011.6043272. 33,42

[Jara-Berrocal'11b] A. Jara-Berrocal, A. Gordon-Ross, "Hardware module reuse and runtime assembly for dynamic management of reconfigurable resources", in 2011 International Conference on Field-Programmable Technology, pp. 1-6, Dec 2011, doi: 10.1109/FPT.2011.6132721. ${ }^{45,52}$

[Jiang'20] J. Jiang, Z. Wang, X. Liu, J. Gómez-Luna, N. Guan, Q. Deng, W. Zhang, O. Mutlu, "Boyi: A Systematic Framework for Automatically Deciding the Right Execution Model of OpenCL Applications on FPGAs", in The 2020 ACM/SIGDA International Symposium on Field-Programmable Gate Arrays, FPGA '20, p. 299-309, Association for Computing Machinery, New York, NY, USA, 2020, ISBN 978-1-4503-7099-8, doi: 10.1145/3373087.3375313. ${ }^{130}$

[Jozwik'13] K. Jozwik, S. Honda, M. Edahiro, H. Tomiyama, H. Takada, "Rainbow: An Operating System for Software-Hardware Multitasking on Dynamically Partially Reconfigurable FPGAs", in International Journal of Reconfigurable Computing, vol. 2013, p. 789 134, Oct 2013, ISSN 1687-7195, doi:10.1155/2013/789134. ${ }^{47,52}$

[Kahn'74] G. Kahn, "The semantics of a simple language for parallel programming”, in Information processing, vol. 74, pp. 471-475, 1974. ${ }^{141}$

[Kiely'05] A. Kiely, M. Klimesh, H. Xie, N. Aranki, "ICER-3D: A Progressive Wavelet-Based Compressor for Hyperspectral Images”, Tech. Rep. IPN-PR-42-164, Jet Propulsion Laboratory, California Institute of Technology, February 2005, https://ntrs.nasa.gov/ archive/nasa/casi.ntrs.nasa.gov/20060008608.pdf. ${ }^{169}$

[Kiely'09] A. B. Kiely, M. A. Klimesh, "Exploiting Calibration-Induced Artifacts in Lossless Compression of Hyperspectral Imagery”, in IEEE Transactions on Geoscience and Remote Sensing, vol. 47, no. 8, pp. 2672-2678, Aug 2009, ISSN 0196-2892, doi: 10.1109/TGRS.2009.2015291. ${ }^{176}$

[Klimesh'06] M. Klimesh, “Low-complexity adaptive lossless compression of hyperspectral imagery”, in Proc. SPIE, Satellite Data Compression, Communications, and Archiving II, vol. 6300, 2006, doi:10.1117/12.682624. ${ }^{166,170}$

[Koch'08] D. Koch, C. Beckhoff, J. Teich, “ReCoBus-Builder - A novel tool and technique to build statically and dynamically reconfigurable systems for FPGAS”, in 2008 International Conference on Field Programmable Logic and Applications, pp. 119-124, Sep. 2008, ISSN 1946-1488, doi:10.1109/FPL.2008.4629918. ${ }^{33,42}$

[Koch'13] D. Koch, Partial Reconfiguration on FPGAs - Architectures, Tools and Applications, Springer-Verlag New York, 2013, ISBN 978-1-4614-1225-0, doi:10.1007/978-1-4614$1225-0 .{ }^{3,7}$

[Koenig'10] R. Koenig, L. Bauer, T. Stripf, M. Shafique, W. Ahmed, J. Becker, J. Henkel, "KAHRISMA: A Novel Hypermorphic Reconfigurable-Instruction-Set Multi-grainedArray Architecture", in 2010 Design, Automation \& Test in Europe Conference \& Exhibition (DATE 2010), pp. 819-824, March 2010, ISSN 1558-1101, doi: 10.1109/DATE.2010.5456939. ${ }^{20,31}$ 
[Korinth'15] J. Korinth, D. de la Chevallerie, A. Koch, "An Open-Source Tool Flow for the Composition of Reconfigurable Hardware Thread Pool Architectures", in 2015 IEEE 23rd Annual International Symposium on Field-Programmable Custom Computing Machines, pp. 195-198, May 2015, doi:10.1109/FCCM.2015.22. ${ }^{36,42}$

[Korinth'19] J. Korinth, J. Hofmann, C. Heinz, A. Koch, “The TaPaSCo Open-Source Toolflow for the Automated Composition of Task-Based Parallel Reconfigurable Computing Systems", in C. Hochberger, B. Nelson, A. Koch, R. Woods, P. Diniz, editors, Applied Reconfigurable Computing, pp. 214-229, Springer International Publishing, Cham, 2019, ISBN 978-3-030-17227-5, doi:10.1007/978-3-030-17227-5_16. 40, 42

[Koza'92] J. R. Koza, Genetic Programming: On the Programming of Computers by Means of Natural Selection, MIT Press, Cambridge, MA, USA, 1992, ISBN 978-0-262-11170-6. 123

[Krommydas'16] K. Krommydas, W.-c. Feng, C. D. Antonopoulos, N. Bellas, "OpenDwarfs: Characterization of Dwarf-Based Benchmarks on Fixed and Reconfigurable Architectures”, in Journal of Signal Processing Systems, vol. 85, no. 3, pp. 373-392, Dec 2016, ISSN 19398115, doi:10.1007/s11265-015-1051-z. ${ }^{106}$

[Kung'78] H. T. Kung, C. E. Leiserson, "Systolic Arrays for (VLSI)", Tech. Rep. CMU-CS-79103, Department of Computer Science, Carnegie-Mellon University, Apr 1978, https: //apps.dtic.mil/docs/citations/ADA066060. ${ }^{124}$

[Kung'84] S.-Y. Kung, “On supercomputing with systolic/wavefront array processors”, in Proceedings of the IEEE, vol. 72, no. 7, pp. 867-884, July 1984, ISSN 1558-2256, doi: 10.1109/PROC.1984.12944. ${ }^{124}$

[Lattner'04] C. Lattner, V. Adve, “LLVM: a compilation framework for lifelong program analysis \& transformation”, in International Symposium on Code Generation and Optimization, 2004. CGO 2004., pp. 75-86, March 2004, doi:10.1109/CGO.2004.1281665. ${ }^{130}$

[Lavin'11] C. Lavin, M. Padilla, J. Lamprecht, P. Lundrigan, B. Nelson, B. Hutchings, "RapidSmith: Do-It-Yourself CAD Tools for Xilinx FPGAs", in 2011 21st International Conference on Field Programmable Logic and Applications, pp. 349-355, Sep. 2011, ISSN 1946-1488, doi:10.1109/FPL.2011.69. ${ }^{39}$

[Lavin'18] C. Lavin, A. Kaviani, "RapidWright: Enabling Custom Crafted Implementations for FPGAs”, in 2018 IEEE 26th Annual International Symposium on Field-Programmable Custom Computing Machines (FCCM), pp. 133-140, April 2018, ISSN 2576-2621, doi: 10.1109/FCCM.2018.00030. 39, 42, 153

[Lee'87] E. A. Lee, D. G. Messerschmitt, "Synchronous data flow", in Proceedings of the IEEE, vol. 75, no. 9, pp. 1235-1245, Sep. 1987, ISSN 1558-2256, doi: 10.1109/PROC.1987.13876. ${ }^{141}$

[Lee'95] E. A. Lee, T. M. Parks, "Dataflow process networks", in Proceedings of the IEEE, vol. 83, no. 5, pp. 773-801, May 1995, ISSN 1558-2256, doi:10.1109/5.381846. ${ }^{141}$

[Lee'97] C. Lee, M. Potkonjak, W. H. Mangione-Smith, "MediaBench: a tool for evaluating and synthesizing multimedia and communications systems", in Proceedings of 30th Annual International Symposium on Microarchitecture, pp. 330-335, Dec 1997, ISSN 1072-4451, doi:10.1109/MICRO.1997.645830. ${ }^{105}$

[Lee'98] E. A. Lee, A. Sangiovanni-Vincentelli, “A framework for comparing models of computation”, in IEEE Transactions on Computer-Aided Design of Integrated Circuits and Systems, vol. 17, no. 12, pp. 1217-1229, Dec 1998, ISSN 1937-4151, doi: $10.1109 / 43.736561 .^{142}$ 
[Lee’08] E. A. Lee, “Cyber Physical Systems: Design Challenges”, in 2008 11th IEEE International Symposium on Object and Component-Oriented Real-Time Distributed Computing (ISORC), pp. 363-369, May 2008, ISSN 2375-5261, doi:10.1109/ISORC.2008.25.

[Lentaris'18] G. Lentaris, K. Maragos, I. Stratakos, L. Papadopoulos, O. Papanikolaou, D. Soudris, M. Lourakis, X. Zabulis, D. Gonzalez-Arjona, G. Furano, "High-Performance Embedded Computing in Space: Evaluation of Platforms for Vision-Based Navigation", in Journal of Aerospace Information Systems, vol. 15, no. 4, pp. 178-192, 2018, doi: $10.2514 / 1.1010555 .^{2}$

[Leung'08] M.-K. Leung, T. E. Filiba, V. Nagpal, "VHDL Code Generation in the Ptolemy II Environment”, Tech. Rep. UCB/EECS-2008-140, EECS Department, University of California, Berkeley, Oct 2008, http://www2.eecs.berkeley.edu/Pubs/TechRpts/2008/ EECS-2008-140.html. ${ }^{150}$

[Li'16] X. Li, A. K. Jain, D. L. Maskell, S. A. Fahmy, “An Area-Efficient FPGA Overlay using DSP Block based Time-multiplexed Functional Units”, in Proceedings of the 2nd International Workshop on Overlay Architectures for FPGAs (OLAF 2016), 2016, http: //arxiv.org/abs/1606.06460. ${ }^{152}$

[Li'19] X. Li, D. L. Maskell, “Time-Multiplexed FPGA Overlay Architectures: A Survey”, in ACM Trans. Des. Autom. Electron. Syst., vol. 24, no. 5, Jul. 2019, ISSN 1084-4309, doi: $10.1145 / 3339861 .{ }^{152,153}$

[Liu'19] L. Liu, J. Zhu, Z. Li, Y. Lu, Y. Deng, J. Han, S. Yin, S. Wei, “A Survey of Coarse-Grained Reconfigurable Architecture and Design: Taxonomy, Challenges, and Applications”, in ACM Comput. Surv., vol. 52, no. 6, Oct. 2019, ISSN 0360-0300, doi:10.1145/3357375. 143

[Lombardo'12] M. Lombardo, J. Camarero, J. Valverde, J. Portilla, E. de la Torre, T. Riesgo, “Power management techniques in an FPGA-based WSN node for high performance applications", in 7th International Workshop on Reconfigurable and Communication-Centric Systems-on-Chip (ReCoSoC), pp. 1-8, July 2012, doi:10.1109/ReCoSoC.2012.6322888. 7

[López'13] S. López, T. Vladimirova, C. González, J. Resano, D. Mozos, A. Plaza, “The Promise of Reconfigurable Computing for Hyperspectral Imaging Onboard Systems: A Review and Trends", in Proceedings of the IEEE, vol. 101, no. 3, pp. 698-722, March 2013, ISSN 1558-2256, doi:10.1109/JPROC.2012.2231391. 165, 170, 187

[Lübbers'07] E. Lübbers, M. Platzner, “ReconOS: An RTOS Supporting Hard-and Software Threads", in 2007 International Conference on Field Programmable Logic and Applications, pp. 441-446, Aug 2007, ISSN 1946-1488, doi:10.1109/FPL.2007.4380686. 119

[Lübbers'08a] E. Lübbers, M. Platzner, “A portable abstraction layer for hardware threads”, in 2008 International Conference on Field Programmable Logic and Applications, pp. 17-22, Sep. 2008, ISSN 1946-1488, doi:10.1109/FPL.2008.4629901. ${ }^{116}$

[Lübbers'08b] E. Lübbers, M. Platzner, “Communication and Synchronization in Multithreaded Reconfigurable Computing Systems”, in 8th International Conference on Engineering of Reconfigurable Systems and Algorithms, pp. 1-7, Jul 2008, http://www.reconos.de/ publications/luebbers08_ersa.pdf. 116

[Lübbers'09a] E. Lübbers, M. Platzner, “Cooperative multithreading in dynamically reconfigurable systems", in 2009 International Conference on Field Programmable Logic and Applications, pp. 551-554, Aug 2009, ISSN 1946-1488, doi:10.1109/FPL.2009.5272418. 47, 121 
[Lübbers'09b] E. Lübbers, M. Platzner, “ReconOS: Multithreaded Programming for Reconfigurable Computers”, in ACM Trans. Embed. Comput. Syst., vol. 9, no. 1, Oct. 2009, ISSN 15399087, doi:10.1145/1596532.1596540. ${ }^{121}$

[Lucarz’08] C. Lucarz, M. Mattavelli, M. Wipliez, G. Roquier, M. Raulet, J. W. Janneck, I. D. Miller, D. B. Parlour, "Dataflow/Actor-Oriented language for the design of complex signal processing systems", in Conference on Design and Architectures for Signal and Image Processing (DASIP 2008), pp. 1-8, Bruxelles, Belgium, Nov. 2008, https://hal. archives-ouvertes.fr/hal-00336520. ${ }^{142}$

[Lysecky'04] R. Lysecky, G. Stitt, F. Vahid, "Warp Processors", in ACM Trans. Des. Autom. Electron. Syst., vol. 11, no. 3, p. 659-681, Jun. 2004, ISSN 1084-4309, doi: 10.1145/1142980.1142986. ${ }^{18,31}$

[Ma'15a] S. Ma, Z. Aklah, D. Andrews, "A run time interpretation approach for creating custom accelerators”, in 2015 25th International Conference on Field Programmable Logic and Applications (FPL), pp. 1-4, Sep. 2015, ISSN 1946-1488, doi: 10.1109/FPL.2015.7293996. ${ }^{152}$

[Ma'15b] S. Ma, H. Ding, M. Huang, D. Andrews, "Archborn: an open source tool for automated generation of chip heterogeneous multiprocessor architectures”, in 2015 International Conference on ReConFigurable Computing and FPGAs (ReConFig), pp. 1-6, Dec 2015, doi:10.1109/ReConFig.2015.7393293. ${ }^{36,42,130}$

[Ma'16] S. Ma, Z. Aklah, D. Andrews, “Just In Time Assembly of Accelerators”, in Proceedings of the 2016 ACM/SIGDA International Symposium on Field-Programmable Gate Arrays, FPGA '16, p. 173-178, Association for Computing Machinery, New York, NY, USA, 2016, ISBN 978-1-4503-3856-1, doi:10.1145/2847263.2847341. ${ }^{152}$

[Martel'17] E. Martel, R. Guerra, S. López, R. Sarmiento, “A GPU-Based Processing Chain for Linearly Unmixing Hyperspectral Images”, in IEEE Journal of Selected Topics in Applied Earth Observations and Remote Sensing, vol. 10, no. 3, pp. 818-834, March 2017, ISSN 1939-1404, doi:10.1109/JSTARS.2016.2614842. ${ }^{183,188}$

[Mbongue'19] J. M. Mbongue, D. T. Kwadjo, C. Bobda, "Automatic Generation of ApplicationSpecific FPGA Overlays with RapidWright", in 2019 International Conference on Field-Programmable Technology (ICFPT), pp. 303-306, Dec 2019, doi: 10.1109/ICFPT47387.2019.00053. ${ }^{41,42,153}$

[Miller'98] J. F. Miller, P. Thomson, T. Fogarty, "Designing Electronic Circuits Using Evolutionary Algorithms. Arithmetic Circuits: A Case Study”, in D. Quagliarella, J. Périaux, C. Poloni, G. Winter, editors, Genetic Algorithms and Evolution Strategy in Engineering and Computer Science: Recent Advances and Industrial Applications, pp. 105-131, Wiley, 1998, ISBN 978-0-471-97710-0. ${ }^{124}$

[Miller'99] J. F. Miller, “An Empirical Study of the Efficiency of Learning Boolean Functions Using a Cartesian Genetic Programming Approach”, in Proceedings of the 1st Annual Conference on Genetic and Evolutionary Computation - Volume 2, GECCO'99, p. 1135-1142, Morgan Kaufmann Publishers Inc., San Francisco, CA, USA, 1999, ISBN 978-1-55860-611-1, https://dl.acm.org/doi/10.5555/2934046.2934074. ${ }^{124}$

[Miller'00] J. F. Miller, P. Thomson, “Cartesian Genetic Programming”, in R. Poli, W. Banzhaf, W. B. Langdon, J. Miller, P. Nordin, T. C. Fogarty, editors, Genetic Programming, pp. 121-132, Springer Berlin Heidelberg, Berlin, Heidelberg, 2000, ISBN 978-3-540-46239-2. ${ }^{124}$

[Miyazaki'19] T. Miyazaki, S. Takai, I. Taniguchi, H. Tomiyama, "An OpenCL-based Software Framework for a Heterogeneous Multicore Architecture on Zynq-7000 SoC”, in IPSJ Transactions on System LSI Design Methodology, vol. 12, pp. 46-49, 2019, doi: 10.2197/ipsjtsldm.12.46. ${ }^{130}$ 
[Moore'06] G. E. Moore, “Cramming more components onto integrated circuits, Reprinted from Electronics, volume 38, number 8, April 19, 1965, pp.114 ff.”, in IEEE Solid-State Circuits Society Newsletter, vol. 11, no. 3, pp. 33-35, Sep. 2006, ISSN 1098-4232, doi: 10.1109/N-SSC.2006.4785860. ${ }^{2}$

[Mora'13] J. Mora, A. Gallego, A. Otero, E. de la Torre, T. Riesgo, "Noise-agnostic adaptive image filtering without training references on an evolvable hardware platform", in 2013 Conference on Design and Architectures for Signal and Image Processing, pp. 182-189, Oct 2013. 124

[Mora'18] J. Mora, E. de la Torre, “Accelerating the evolution of a systolic array-based evolvable hardware system”, in Microprocessors and Microsystems, vol. 56, pp. 144 - 156, 2018, ISSN 0141-9331, doi:10.1016/j.micpro.2017.12.001. xxv, 124, 128

[Mora'19a] J. Mora, Architecture and methodology for automated development of evolvable and reconfigurable hardware applications, Ph.D. thesis, Universidad Politécnica de Madrid, Apr. 2019, http://oa.upm.es/54813. 124, 125, 133

[Mora'19b] J. Mora, R. Salvador, E. de la Torre, "On the scalability of evolvable hardware architectures: comparison of systolic array and Cartesian genetic programming”, in Genetic Programming and Evolvable Machines, vol. 20, no. 2, pp. 155-186, Jun 2019, ISSN 1573-7632, doi:10.1007/s10710-018-9340-5. ${ }^{124}$

[Moreano'05] N. Moreano, E. Borin, Cid de Souza, G. Araujo, "Efficient datapath merging for partially reconfigurable architectures", in IEEE Transactions on Computer-Aided Design of Integrated Circuits and Systems, vol. 24, no. 7, pp. 969-980, July 2005, ISSN 1937-4151, doi:10.1109/TCAD.2005.850844. ${ }^{144}$

[Muslim'17] F. B. Muslim, L. Ma, M. Roozmeh, L. Lavagno, "Efficient FPGA Implementation of OpenCL High-Performance Computing Applications via High-Level Synthesis", in IEEE Access, vol. 5, pp. 2747-2762, 2017, ISSN 2169-3536, doi: 10.1109/ACCESS.2017.2671881. ${ }^{130}$

[Nam'17] K. Nam, B. Fort, S. Brown, “FISH: Linux system calls for FPGA accelerators”, in 2017 27th International Conference on Field Programmable Logic and Applications (FPL), pp. 1-4, Sep. 2017, ISSN 1946-1488, doi:10.23919/FPL.2017.8056785. ${ }^{129}$

[Nascimento’16] J. M. P. Nascimento, M. Véstias, “System-on-chip field-programmable gate array design for onboard real-time hyperspectral unmixing”, in Journal of Applied Remote Sensing, vol. 10, no. 1, pp. 1 - 17, 2016, doi:10.1117/1.JRS.10.015004. ${ }^{187}$

[Neely'10] C. Neely, G. Brebner, W. Shang, "ShapeUp: A High-Level Design Approach to Simplify Module Interconnection on FPGAs", in 2010 18th IEEE Annual International Symposium on Field-Programmable Custom Computing Machines, pp. 141-148, May 2010, doi:10.1109/FCCM.2010.30. ${ }^{35}$

[Neely'13] C. E. Neely, G. Brebner, W. Shang, "ReShape: Towards a High-Level Approach to Design and Operation of Modular Reconfigurable Systems", in ACM Trans. Reconfigurable Technol. Syst., vol. 6, no. 1, May 2013, ISSN 1936-7406, doi: 10.1145/2457443.2457448. ${ }^{35,42}$

[Nezan'12] J. F. Nezan, N. Siret, M. Wipliez, F. Palumbo, L. Raffo, "Multi-purpose systems: A novel dataflow-based generation and mapping strategy", in 2012 IEEE International Symposium on Circuits and Systems (ISCAS), pp. 3073-3076, May 2012, ISSN 21581525, doi:10.1109/ISCAS.2012.6271969. ${ }^{145}$

[Nurvitadhi'16] E. Nurvitadhi, D. Sheffield, Jaewoong Sim, A. Mishra, G. Venkatesh, D. Marr, "Accelerating Binarized Neural Networks: Comparison of FPGA, CPU, GPU, and ASIC”, in 2016 International Conference on Field-Programmable Technology (FPT), pp. 77-84, Dec 2016, doi:10.1109/FPT.2016.7929192. ${ }^{2,111}$ 
[Oetken'10] A. Oetken, S. Wildermann, J. Teich, D. Koch, “A Bus-Based SoC Architecture for Flexible Module Placement on Reconfigurable FPGAs", in 2010 International Conference on Field Programmable Logic and Applications, pp. 234-239, Aug 2010, ISSN 1946-1488, doi:10.1109/FPL.2010.54. ${ }^{21,31}$

[Omidian'19] H. Omidian, G. G. Lemieux, "Software-Based Dynamic Overlays Require Fast, FineGrained Partial Reconfiguration”, in Proceedings of the 10th International Symposium on Highly-Efficient Accelerators and Reconfigurable Technologies, HEART 2019, Association for Computing Machinery, New York, NY, USA, 2019, ISBN 978-1-45037255-8, doi:10.1145/3337801.3337816. ${ }^{153}$

[Oriato'15] D. Oriato, S. Girdlestone, O. Mencer, "Dataflow Computing in Extreme Performance Conditions”, in A. R. Hurson, V. Milutinovic, editors, Dataflow Processing, vol. 96 of Advances in Computers, pp. 105 - 137, Elsevier, 2015, doi: 10.1016/bs.adcom.2014.11.002. ${ }^{151}$

[Orlandić'19] M. Orlandić, J. Fjeldtvedt, T. A. Johansen, “A Parallel FPGA Implementation of the CCSDS-123 Compression Algorithm”, in Remote Sensing, vol. 11, no. 6, 2019, ISSN 2072-4292, doi:10.3390/rs11060673. ${ }^{171,180}$

[Ortiz'16] A. Ortiz, Alternativas de implementación de comunicaciones TCP/IP/Ethernet en SoPCs, B.Sc. thesis, Universidad Politécnica de Madrid, Sep. 2016, http://oa.upm.es/ 43454. ${ }^{214}$

[Ortiz'17] A. Ortiz, A. Rodríguez, E. de la Torre, "Resilient communications for a multi-FPGA, hardware-accelerated cluster”, in Industriales Research Meeting 2017, p. 67, Escuela Técnica Superior de Ingenieros Industriales, Universidad Politécnica de Madrid, 2017, ISBN 978-84-16397-58-7, http:// oa.upm.es/46090. ${ }^{209}$

[Ortiz'18a] A. Ortiz, Diseño de una Plataforma Heterogénea Multi-FPGA para Cómputo Paralelo, Distribuido y Acelerado en Hardware sobre ARTICo ${ }^{3}$, M.Sc. thesis, Universidad Politécnica de Madrid, Oct. 2018. ${ }^{213}$

[Ortiz'18b] A. Ortiz, A. Rodríguez, R. Guerra, S. López, A. Otero, R. Sarmiento, E. de la Torre, "A Runtime-Scalable and Hardware-Accelerated Approach to On-Board Linear Unmixing of Hyperspectral Images”, in Remote Sensing, vol. 10, no. 11, 2018, ISSN 2072-4292, doi:10.3390/rs10111790. ${ }^{206}$

[Ortiz'19] A. Ortiz, A. Rodríguez, A. Otero, E. de la Torre, "Data Transfer Modeling and Optimization in Reconfigurable Multi-Accelerator Systems", in 2019 14th International Symposium on Reconfigurable Communication-centric Systems-on-Chip (ReCoSoC), pp. 20-26, July 2019, ISSN 2642-7230, doi:10.1109/ReCoSoC48741.2019.9034940. 207,215

[Ortiz'20] A. Ortiz, R. Zamacola, A. Rodríguez, A. Otero, E. de la Torre, “Automated Toolchain for Enhanced Productivity in Reconfigurable Multi-accelerator Systems”, in F. Rincón, J. Barba, H. K. H. So, P. Diniz, J. Caba, editors, Applied Reconfigurable Computing. Architectures, Tools, and Applications, pp. 45-60, Springer International Publishing, Cham, 2020, ISBN 978-3-030-44534-8, doi:10.1007/978-3-030-44534-8_4. 211, 216

[Paarup'17] L. Paarup, Aceleración Software y Hardware de Algoritmos de Montecarlo para Aplicaciones Financieras, B.Sc. thesis, Universidad Politécnica de Madrid, Feb. 2017. 213

[Palumbo'13] F. Palumbo, C. Sau, L. Raffo, “DSE and profiling of multi-context coarse-grained reconfigurable systems”, in 2013 8th International Symposium on Image and Signal Processing and Analysis (ISPA), pp. 744-749, Sep. 2013, ISSN 1845-5921, doi: 10.1109/ISPA.2013.6703836. ${ }^{148}$ 
[Palumbo'14] F. Palumbo, N. Carta, D. Pani, P. Meloni, L. Raffo, “The Multi-Dataflow Composer Tool: Generation of on-the-Fly Reconfigurable Platforms", in J. Real-Time Image Process., vol. 9, no. 1, p. 233-249, Mar. 2014, ISSN 1861-8200, doi:10.1007/s11554-012-0284-3. 143,144

[Palumbo'15] F. Palumbo, C. Sau, L. Raffo, “Coarse-grained reconfiguration: dataflow-based power management”, in IET Computers Digital Techniques, vol. 9, no. 1, pp. 36-48, 2015, ISSN 1751-861X, doi:10.1049/iet-cdt.2014.0089. ${ }^{148}$

[Palumbo'16] F. Palumbo, T. Fanni, C. Sau, P. Meloni, L. Raffo, "Modelling and Automated Implementation of Optimal Power Saving Strategies in Coarse-Grained Reconfigurable Architectures", in Journal of Electrical and Computer Engineering, vol. 2016, p. 5, Nov. 2016, ISSN 2090-0147, doi:10.1155/2016/4237350. ${ }^{148}$

[Palumbo'19a] F. Palumbo, T. Fanni, C. Sau, L. Pulina, L. Raffo, M. Masin, E. Shindin, P. S. de Rojas, K. Desnos, M. Pelcat, A. Rodríguez, E. Juárez, F. Regazzoni, G. Meloni, K. Zedda, H. Myrhaug, L. Kaliciak, J. Andriaanse, J. de Olivieria Filho, P. Muñoz, A. Toffetti, "CERBERO: Cross-layer modEl-based fRamework for multi-oBjective dEsign of Reconfigurable Systems in unceRtain hybRid envirOnments: Invited Paper: CERBERO Teams from UniSS, UniCA, IBM Research, TASE, INSA-Rennes, UPM, USI, Abinsula, AmbieSense, TNO, S\&T, CRF”, in Proceedings of the 16th ACM International Conference on Computing Frontiers, CF '19, pp. 320-325, ACM, New York, NY, USA, 2019, ISBN 978-1-4503-6685-4, doi:10.1145/3310273.3323436. ${ }^{211}$

[Palumbo'19b] F. Palumbo, T. Fanni, C. Sau, A. Rodríguez, D. Madroñal, K. Desnos, A. Morvan, M. Pelcat, C. Rubattu, R. Lazcano, L. Raffo, E. de la Torre, E. Juárez, C. Sanz, P. Sánchez de Rojas, "Hardware/Software Self-adaptation in CPS: The CERBERO Project Approach”, in D. N. Pnevmatikatos, M. Pelcat, M. Jung, editors, Embedded Computer Systems: Architectures, Modeling, and Simulation, pp. 416-428, Springer International Publishing, Cham, 2019, ISBN 978-3-030-27562-4, doi:10.1007/978-3030-27562-4_30. ${ }^{211}$

[Parkhurst'06] J. Parkhurst, J. Darringer, B. Grundmann, “From Single Core to Multi-Core: Preparing for a New Exponential”, in Proceedings of the 2006 IEEE/ACM International Conference on Computer-Aided Design, ICCAD '06, p. 67-72, Association for Computing Machinery, New York, NY, USA, 2006, ISBN 1-59593-389-1, doi:10.1145/1233501.1233516. ${ }^{2}$

[Pell'11] O. Pell, O. Mencer, "Surviving the End of Frequency Scaling with Reconfigurable Dataflow Computing”, in SIGARCH Comput. Archit. News, vol. 39, no. 4, p. 60-65, Dec. 2011, ISSN 0163-5964, doi:10.1145/2082156.2082172. ${ }^{151}$

[Penna'06] B. Penna, T. Tillo, E. Magli, G. Olmo, "Progressive 3-D coding of hyperspectral images based on JPEG 2000”, in IEEE Geoscience and Remote Sensing Letters, vol. 3, no. 1, pp. 125-129, Jan 2006, ISSN 1545-598X, doi:10.1109/LGRS.2005.859942. ${ }^{166}$

[Pérez'20] A. Pérez, A. Rodríguez, A. Otero, D. González Arjona, A. Jiménez-Peralo, M. A. Verdugo, E. de la Torre, "Run-Time Reconfigurable MPSoC-Based On-Board Processor for Vision-Based Space Navigation", in IEEE Access, vol. 8, pp. 59 891-59 905, 2020, ISSN 2169-3536, doi:10.1109/ACCESS.2020.2983308. ${ }^{210}$

[Pham-Quoc'19] C. Pham-Quoc, "Design Framework for FPGA-based Hardware Accelerators with Heterogeneous Interconnect", in 2019 6th NAFOSTED Conference on Information and Computer Science (NICS), pp. 148-153, Dec 2019, doi: 10.1109/NICS48868.2019.9023825. ${ }^{41,42}$

[Pham'17] K. D. Pham, E. Horta, D. Koch, "BITMAN: A tool and API for FPGA bitstream manipulations", in Design, Automation \& Test in Europe Conference \& Exhibition (DATE), 2017, pp. 894-897, March 2017, ISSN 1558-1101, doi:10.23919/DATE.2017.7927114. ${ }^{39}$ 
[Pham'19] K. D. Pham, K. Paraskevas, A. Vaishnav, A. Attwood, M. Vesper, D. Koch, "ZUCL 2.0: Virtualised Memory and Communication for ZYNQ UltraScale+ FPGAs”, in FSP Workshop 2019; Sixth International Workshop on FPGAs for Software Programmers, pp. 1-9, Sep. 2019, https://ieeexplore.ieee.org/document/8891818. ${ }^{51,52}$

[Plaza'07] A. J. Plaza, C.-I. Chang, High Performance Computing in Remote Sensing, Chapman \& Hall/CRC, 2007, ISBN 1-58488-662-5. 165, 187

[Plaza'09] A. Plaza, J. A. Benediktsson, J. W. Boardman, J. Brazile, L. Bruzzone, G. CampsValls, J. Chanussot, M. Fauvel, P. Gamba, A. Gualtieri, M. Marconcini, J. C. Tilton, G. Trianni, "Recent advances in techniques for hyperspectral image processing”, in Remote Sensing of Environment, vol. 113, pp. S110 - S122, 2009, ISSN 0034-4257, doi: 10.1016/j.rse.2007.07.028. Imaging Spectroscopy Special Issue. ${ }^{163}$

[Poupat'13] J.-L. Poupat, R. Vitulli, "CWICOM: A Highly Integrated \& Innovative CCSDS Image Compression ASIC”, in DASIA 2013 - DAta Systems In Aerospace, vol. 720 of ESA Special Publication, p. 62, Aug 2013. ${ }^{170}$

[Pulini'09] G. Pulini, D. Hulance, "FlexEOS Embedded FPGA Solution”, in N. S. Voros, A. Rosti, M. Hübner, editors, Dynamic System Reconfiguration in Heterogeneous Platforms: The MORPHEUS Approach, pp. 39-47, Springer Netherlands, Dordrecht, 2009, ISBN 978-90-481-2427-5, doi:10.1007/978-90-481-2427-5_4. ${ }^{23}$

[Qasaimeh'19] M. Qasaimeh, K. Denolf, J. Lo, K. Vissers, J. Zambreno, P. H. Jones, “Comparing Energy Efficiency of CPU, GPU and FPGA Implementations for Vision Kernels”, in 2019 IEEE International Conference on Embedded Software and Systems (ICESS), pp. 1-8, June 2019, doi:10.1109/ICESS.2019.8782524. ${ }^{2}$

[Rashid'15] R. Rashid, A Dual-Engine Fetch/Compute Overlay Processor for FPGAs, Ph.D. thesis, University of Toronto, Mar. 2015, http://hdl.handle.net/1807/69239. ${ }^{153}$

[Reagen'14] B. Reagen, R. Adolf, Y. S. Shao, G. Wei, D. Brooks, "MachSuite: Benchmarks for accelerator design and customized architectures", in 2014 IEEE International Symposium on Workload Characterization (IISWC), pp. 110-119, Oct 2014, doi: 10.1109/IISWC.2014.6983050. xxv, 106, 107

[Rechenberg'73] I. Rechenberg, Evolutionsstrategie - Optimierung technischer Systeme nach Prinzipien der biologischen Evolution, Frommann-Holzboog Verlag, Stuttgart, 1973. ${ }^{123}$

[Rettkowski'16] J. Rettkowski, P. Wehner, E. Cutiscev, D. Göhringer, "LinROS: A Linux-Based Runtime System for Reconfigurable MPSoCs”, in 2016 IEEE International Parallel and Distributed Processing Symposium Workshops (IPDPSW), pp. 208-216, May 2016, doi: 10.1109/IPDPSW.2016.156. ${ }^{50,52}$

[Rice'93] R. Rice, P.-S. Yeh, W. Miller, "Algorithms for high-speed universal noiseless coding", in 9th Computing in Aerospace Conference, American Institute of Aeronautics and Astronautics, Oct 1993, doi:10.2514/6.1993-4541. ${ }^{168}$

[Rinker'01] R. Rinker, M. Carter, A. Patel, M. Chawathe, C. Ross, J. Hammes, W. A. Najjar, W. Bohm, "An automated process for compiling dataflow graphs into reconfigurable hardware", in IEEE Transactions on Very Large Scale Integration (VLSI) Systems, vol. 9, no. 1, pp. 130-139, Feb 2001, ISSN 1557-9999, doi:10.1109/92.920828. ${ }^{150}$

[Rodríguez'14] A. Rodríguez, J. Valverde, E. de la Torre, T. Riesgo, "Dynamic management of multikernel multithread accelerators using Dynamic Partial Reconfiguration”, in 2014 9th International Symposium on Reconfigurable and Communication-Centric Systems-on-Chip (ReCoSoC), pp. 1-7, May 2014, doi:10.1109/ReCoSoC.2014.6861363. 208 
[Rodríguez'15a] A. Rodríguez, J. Valverde, C. Castañares, J. Portilla, E. de la Torre, T. Riesgo, "Execution modeling in self-aware FPGA-based architectures for efficient resource management”, in 2015 10th International Symposium on Reconfigurable Communication-centric Systems-on-Chip (ReCoSoC), pp. 1-8, June 2015, doi: 10.1109/ReCoSoC.2015.7238086. ${ }^{208}$

[Rodríguez'15b] A. Rodríguez, J. Valverde, C. Castañares, J. Portilla, E. de la Torre, T. Riesgo, "Live demonstration: A dynamically adaptable image processing application running in an FPGA-based WSN platform", in 2015 IEEE International Symposium on Circuits and Systems (ISCAS), pp. 1902-1902, May 2015, ISSN 0271-4302, doi: 10.1109/ISCAS.2015.7169035. ${ }^{208}$

[Rodríguez'15c] A. Rodríguez, J. Valverde, E. de la Torre, "Design of OpenCL-compatible multithreaded hardware accelerators with dynamic support for embedded FPGAs", in 2015 International Conference on ReConFigurable Computing and FPGAs (ReConFig), pp. 1-7, Dec 2015, doi:10.1109/ReConFig.2015.7393297. ${ }^{207}$

[Rodríguez'16a] A. Rodríguez, C. Castañares, L. Suriano, T. Riesgo, E. de la Torre, “Towards Safer and Predictable Hardware Acceleration in Distributed, Embedded and High Performance Systems", in Industriales Research Meeting 2016, p. 86, Escuela Técnica Superior de Ingenieros Industriales, Universidad Politécnica de Madrid, 2016, ISBN 978-8416397-31-0, http://oa.upm.es/40073. ${ }^{209}$

[Rodríguez'16b] A. Rodríguez, J. Portilla, E. de la Torre, T. Riesgo, “Teaching hybrid HW/SW embedded system design using FPGA-based devices”, in 2016 Conference on Design of Circuits and Integrated Systems (DCIS), pp. 1-5, Nov 2016, doi:10.1109/DCIS.2016.7845372. 209

[Rodríguez'17a] A. Rodríguez, C. Castañares, T. Riesgo, E. de la Torre, "Evaluating High-Level Synthesis Techniques for Scalable Hardware-Accelerated Computing”, in 2017 32nd Conference on Design of Circuits and Integrated Systems (DCIS), pp. 1-8, Nov 2017. ${ }^{207}$

[Rodríguez'17b] A. Rodríguez, E. de la Torre, "Automatic Integration of HLS-Generated Hardware Accelerators in an Embedded Parallel Computing Architecture", in Industriales Research Meeting 2017, p. 66, Escuela Técnica Superior de Ingenieros Industriales, Universidad Politécnica de Madrid, 2017, ISBN 978-84-16397-58-7, http://oa.upm. es/46090. ${ }^{209}$

[Rodríguez'18a] A. Rodríguez, T. Fanni, “DEMO: Multi-Grain Adaptivity in Cyber-Physical Systems”, in 2018 30th International Conference on Microelectronics (ICM), pp. 44-47, Dec 2018, doi:10.1109/ICM.2018.8704058. ${ }^{207}$

[Rodríguez'18b] A. Rodríguez, J. Valverde, J. Portilla, A. Otero, T. Riesgo, E. de la Torre, "FPGABased High-Performance Embedded Systems for Adaptive Edge Computing in CyberPhysical Systems: The ARTICo ${ }^{3}$ Framework", in Sensors, vol. 18, no. 6, 2018, ISSN 1424-8220, doi:10.3390/s18061877. ${ }^{206}$

[Rodríguez'19] A. Rodríguez, L. Santos, R. Sarmiento, E. de la Torre, "Scalable HardwareBased On-Board Processing for Run-Time Adaptive Lossless Hyperspectral Compression", in IEEE Access, vol. 7, pp. 10644-10652, 2019, ISSN 2169-3536, doi: 10.1109/ACCESS.2019.2892308. ${ }^{179,180,206}$

[Rubattu'19] C. Rubattu, F. Palumbo, C. Sau, R. Salvador, J. Sérot, K. Desnos, L. Raffo, M. Pelcat, "Dataflow-Functional High-Level Synthesis for Coarse-Grained Reconfigurable Accelerators", in IEEE Embedded Systems Letters, vol. 11, no. 3, pp. 69-72, Sep. 2019, ISSN 1943-0671, doi:10.1109/LES.2018.2882989. ${ }^{146}$ 
[Saleh'06] R. Saleh, S. Wilton, S. Mirabbasi, A. Hu, M. Greenstreet, G. Lemieux, P. P. Pande, C. Grecu, A. Ivanov, "System-on-Chip: Reuse and Integration", in Proceedings of the IEEE, vol. 94, no. 6, pp. 1050-1069, June 2006, ISSN 1558-2256, doi: 10.1109/JPROC.2006.873611. ${ }^{6}$

[Salvador'11a] R. Salvador, A. Otero, J. Mora, E. de la Torre, T. Riesgo, L. Sekanina, "Evolvable 2D computing matrix model for intrinsic evolution in commercial FPGAs with native reconfiguration support", in 2011 NASA/ESA Conference on Adaptive Hardware and Systems (AHS), pp. 184-191, June 2011, doi:10.1109/AHS.2011.5963934. ${ }^{124}$

[Salvador'11b] R. Salvador, A. Otero, J. Mora, E. de la Torre, L. Sekanina, T. Riesgo, "Fault Tolerance Analysis and Self-Healing Strategy of Autonomous, Evolvable Hardware Systems”, in 2011 International Conference on Reconfigurable Computing and FPGAs, pp. 164-169, Nov 2011, ISSN 2325-6532, doi:10.1109/ReConFig.2011.37. ${ }^{125}$

[Salvador'15] R. Salvador, Parametric and structural self-adaptation of embedded systems using evolvable hardware, Ph.D. thesis, Universidad Politécnica de Madrid, Dec. 2015, http: //oa.upm.es/39354. ${ }^{122}$

[Sánchez'11] J. E. Sánchez, E. Auge, J. Santalo, I. Blanes, J. Serra-Sagrista, A. Kiely, "Review and Implementation of the Emerging CCSDS Recommended Standard for Multispectral and Hyperspectral Lossless Image Coding”, in 2011 First International Conference on Data Compression, Communications and Processing, pp. 222-228, June 2011, doi: 10.1109/CCP.2011.17. ${ }^{170}$

[Sánchez'15] S. Sánchez, R. Ramalho, L. Sousa, A. Plaza, "Real-time implementation of remotely sensed hyperspectral image unmixing on GPUs", in Journal of Real-Time Image Processing, vol. 10, no. 3, pp. 469-483, 2015, ISSN 1861-8219, doi:10.1007/s11554-0120269-2. 188

[Santos'14] L. Santos, Hyperspectral image compression onboard next-generation satellites: implementation solutions on GPUs and FPGAs, Ph.D. thesis, Universidad de Las Palmas de Gran Canaria, Jul. 2014, http://hdl.handle.net/10553/13000. 165, 166, 169

[Santos'16] L. Santos, L. Berrojo, J. Moreno, J. F. López, R. Sarmiento, "Multispectral and Hyperspectral Lossless Compressor for Space Applications (HyLoC): A Low-Complexity FPGA Implementation of the CCSDS 123 Standard", in IEEE Journal of Selected Topics in Applied Earth Observations and Remote Sensing, vol. 9, no. 2, pp. 757-770, Feb 2016, ISSN 1939-1404, doi:10.1109/JSTARS.2015.2497163. 170, 171, 172, 177, 180

[Santos'20] L. Santos, A. Gómez, R. Sarmiento, “Implementation of CCSDS Standards for Lossless Multispectral and Hyperspectral Satellite Image Compression”, in IEEE Transactions on Aerospace and Electronic Systems, vol. 56, no. 2, pp. 1120-1138, April 2020, ISSN 1557-9603, doi:10.1109/TAES.2019.2929971. ${ }^{170,180}$

[Sau'14a] C. Sau, F. Palumbo, "Automatic generation of dataflow-based reconfigurable coprocessing units", in Proceedings of the 2014 Conference on Design and Architectures for Signal and Image Processing, pp. 1-8, Oct 2014, doi:10.1109/DASIP.2014.7115605.

[Sau'14b] C. Sau, L. Raffo, F. Palumbo, E. Bezati, S. Casale-Brunet, M. Mattavelli, "Automated design flow for coarse-grained reconfigurable platforms: An RVC-CAL multi-standard decoder use-case", in 2014 International Conference on Embedded Computer Systems: Architectures, Modeling, and Simulation (SAMOS XIV), pp. 59-66, July 2014, doi: 10.1109/SAMOS.2014.6893195. ${ }^{145}$

[Sau'15] C. Sau, L. Fanni, P. Meloni, L. Raffo, F. Palumbo, "Reconfigurable coprocessors synthesis in the MPEG-RVC domain”, in 2015 International Conference on ReConFigurable Computing and FPGAs (ReConFig), pp. 1-8, Dec 2015, doi: 10.1109/ReConFig.2015.7393351. ${ }^{149}$ 
[Sau'16] C. Sau, P. Meloni, L. Raffo, F. Palumbo, E. Bezati, S. Casale-Brunet, M. Mattavelli, "Automated Design Flow for Multi-Functional Dataflow-Based Platforms", in $J$. Signal Process. Syst., vol. 85, no. 1, p. 143-165, Oct. 2016, ISSN 1939-8018, doi: $10.1007 / \mathrm{s} 11265-015-1026-0 .{ }^{145}$

[Savas'20] S. Savas, Z. Ul-Abdin, T. Nordström, “A framework to generate domain-specific manycore architectures from dataflow programs”, in Microprocessors and Microsystems, vol. 72, p. 102 908, 2020, ISSN 0141-9331, doi:10.1016/j.micpro.2019.102908. ${ }^{151}$

[Schlansker'00] M. S. Schlansker, B. R. Rau, “EPIC: Explicitly Parallel Instruction Computing”, in Computer, vol. 33, no. 2, pp. 37-45, Feb 2000, ISSN 1558-0814, doi:10.1109/2.820037. 20

[Schüler'09] E. Schüler, M. Weinhardt, "XPP-III”, in N. S. Voros, A. Rosti, M. Hübner, editors, Dynamic System Reconfiguration in Heterogeneous Platforms: The MORPHEUS Approach, pp. 63-76, Springer Netherlands, Dordrecht, 2009, ISBN 978-90-481-24275, doi:10.1007/978-90-481-2427-5_6. ${ }^{23}$

[Schwefel'93] H.-P. Schwefel, Evolution and Optimum Seeking: The Sixth Generation, Wiley, USA, 1993, ISBN 978-0-471-57148-3. ${ }^{123}$

[Sekanina'04] L. Sekanina, Evolvable Components - From Theory to Hardware Implementations, Natural Computing Series, Springer-Verlag Berlin Heidelberg, 2004, ISBN 978-3-64218609-7, doi:10.1007/978-3-642-18609-7. 122, 123

[Sekanina'11] L. Sekanina, Z. Vašíček, “CGP Acceleration Using Field-Programmable Gate Arrays”, in J. F. Miller, editor, Cartesian Genetic Programming, pp. 217-230, Springer Berlin Heidelberg, Berlin, Heidelberg, 2011, ISBN 978-3-642-17310-3, doi:10.1007/978-3642-17310-3_7. 124

[Sérot'13] J. Sérot, F. Berry, S. Ahmed, “CAPH: A Language for Implementing Stream-Processing Applications on FPGAs”, in P. Athanas, D. Pnevmatikatos, N. Sklavos, editors, Embedded Systems Design with FPGAs, pp. 201-224, Springer New York, New York, NY, 2013, ISBN 978-1-4614-1362-2, doi:10.1007/978-1-4614-1362-2_9. 146, 151

[Severance'13] A. Severance, G. G. F. Lemieux, "Embedded supercomputing in FPGAs with the VectorBlox MXP Matrix Processor", in 2013 International Conference on Hardware/Software Codesign and System Synthesis (CODES+ISSS), pp. 1-10, Sep. 2013, doi: 10.1109/CODES-ISSS.2013.6658993. ${ }^{153}$

[Shi'16] W. Shi, S. Dustdar, “The Promise of Edge Computing”, in Computer, vol. 49, no. 5, pp. 78-81, May 2016, ISSN 1558-0814, doi:10.1109/MC.2016.145. ${ }^{2}$

[Siddhartha' 19] Siddhartha, Dataflow Optimized Overlays for FPGAs, Ph.D. thesis, Nanyang Technological University, Mar. 2019, http://hdl.handle.net/10220/47803. 153

[Silberschatz'18] A. Silberschatz, G. Gagne, P. B. Galvin, Operating System Concepts, Wiley, 10th ed., 2018, ISBN 978-1-119-32091-3. 113, 114, 115

[Silva'19] L. B. D. Silva, R. Ferreira, M. Canesche, M. M. Menezes, M. D. Vieira, J. Penha, P. Jamieson, J. A. M. Nacif, "READY: A Fine-Grained Multithreading Overlay Framework for Modern CPU-FPGA Dataflow Applications”, in ACM Trans. Embed. Comput. Syst., vol. 18, no. 5s, Oct. 2019, ISSN 1539-9087, doi:10.1145/3358187. $30,31,153$

[Singh'92] J. P. Singh, W.-D. Weber, A. Gupta, "SPLASH: Stanford Parallel Applications for SharedMemory”, in SIGARCH Comput. Archit. News, vol. 20, no. 1, p. 5-44, Mar. 1992, ISSN 0163-5964, doi:10.1145/130823.130824. ${ }^{105}$ 
[Siret'10] N. Siret, M. Wipliez, J. F. Nezan, A. Rhatay, "Hardware code generation from dataflow programs", in 2010 Conference on Design and Architectures for Signal and Image Processing (DASIP), pp. 113-120, Oct 2010, doi:10.1109/DASIP.2010.5706254. ${ }^{150}$

[So'08] H. K.-H. So, R. Brodersen, “A Unified Hardware/Software Runtime Environment for FPGA-Based Reconfigurable Computers Using BORPH”, in ACM Trans. Embed. Comput. Syst., vol. 7, no. 2, Jan. 2008, ISSN 1539-9087, doi:10.1145/1331331.1331338. 44,52

[So'16] H. K.-H. So, C. Liu, “FPGA Overlays", in D. Koch, F. Hannig, D. Ziener, editors, FPGAs for Software Programmers, pp. 285-305, Springer International Publishing, Cham, 2016, ISBN 978-3-319-26408-0, doi:10.1007/978-3-319-26408-0_16. ${ }^{152}$

[SPEC] SPEC, "Standard Performance Evaluation Corporation Benchmarks", http://www. spec.org/. [Online] Accessed: October 13, 2020. ${ }^{105}$

[Stitt'11] G. Stitt, J. Coole, "Intermediate Fabrics: Virtual Architectures for Near-Instant FPGA Compilation”, in IEEE Embedded Systems Letters, vol. 3, no. 3, pp. 81-84, Sep. 2011, ISSN 1943-0671, doi:10.1109/LES.2011.2167713. ${ }^{112,152}$

[Stoutchinin'19] A. Stoutchinin, L. Benini, "StreamDrive: A Dynamic Dataflow Framework for Clustered Embedded Architectures”, in J. Signal Process. Syst., vol. 91, no. 3-4, p. 275-301, Mar. 2019, ISSN 1939-8018, doi:10.1007/s11265-018-1351-1. ${ }^{151}$

[Straka'10] M. Straka, J. Kastil, Z. Kotasek, "Modern fault tolerant architectures based on partial dynamic reconfiguration in FPGAs", in 13th IEEE Symposium on Design and Diagnostics of Electronic Circuits and Systems, pp. 173-176, April 2010, doi: 10.1109/DDECS.2010.5491793. ${ }^{21,31}$

[Suriano'17] L. Suriano, A. Rodríguez, K. Desnos, M. Pelcat, E. de la Torre, "Analysis of a heterogeneous multi-core, multi-hw-accelerator-based system designed using PREESM and SDSoC", in 2017 12th International Symposium on Reconfigurable Communication-centric Systems-on-Chip (ReCoSoC), pp. 1-7, July 2017, doi: 10.1109/ReCoSoC.2017.8016151. ${ }^{211}$

[Suriano'18] L. Suriano, D. Madroñal, A. Rodríguez, E. Juárez, C. Sanz, E. de la Torre, “A Unified Hardware/Software Monitoring Method for Reconfigurable Computing Architectures Using PAPI", in 2018 13th International Symposium on Reconfigurable Communication-centric Systems-on-Chip (ReCoSoC), pp. 1-8, July 2018, doi: 10.1109/ReCoSoC.2018.8449389. ${ }^{211}$

[Suriano'19] L. Suriano, F. Arrestier, A. Rodríguez, J. Heulot, K. Desnos, M. Pelcat, E. de la Torre, "DAMHSE: Programming heterogeneous MPSoCs with hardware acceleration using dataflow-based design space exploration and automated rapid prototyping", in Microprocessors and Microsystems, vol. 71, p. 102 882, 2019, ISSN 0141-9331, doi: 10.1016/j.micpro.2019.102882. ${ }^{210}$

[Suriano'20] L. Suriano, A. Otero, A. Rodríguez, M. Sánchez-Renedo, E. de la Torre, “Exploiting Multi-Level Parallelism for Run-Time Adaptive Inverse Kinematics on Heterogeneous MPSoCs", in IEEE Access, vol. 8, pp. 118707-118724, 2020, ISSN 2169-3536, doi: 10.1109/ACCESS.2020.3005202. ${ }^{210}$

[Tanenbaum'15] A. S. Tanenbaum, H. Bos, Modern Operating Systems, Pearson, 4th ed., 2015, ISBN 978-0-13-359162-0. ${ }^{113,115}$

[Transon'18] J. Transon, R. D’Andrimont, A. Maugnard, P. Defourny, “Survey of Hyperspectral Earth Observation Applications from Space in the Sentinel-2 Context", in Remote Sensing, vol. 10, no. 2, 2018, ISSN 2072-4292, doi:10.3390/rs10020157. ${ }^{164}$ 
[Trefzer'15] M. A. Trefzer, A. M. Tyrrell, Evolvable Hardware-From Practice to Application, Natural Computing Series, Springer-Verlag Berlin Heidelberg, 2015, ISBN 978-3-662-44616-4, doi:10.1007/978-3-662-44616-4. ${ }^{123}$

[Treleaven'82] P. C. Treleaven, D. R. Brownbridge, R. P. Hopkins, “Data-Driven and Demand-Driven Computer Architecture”, in ACM Comput. Surv., vol. 14, no. 1, p. 93-143, Mar. 1982, ISSN 0360-0300, doi:10.1145/356869.356873. ${ }^{141}$

[Tsigkanos’18] A. Tsigkanos, N. Kranitis, G. A. Theodorou, A. Paschalis, “A 3.3 Gbps CCSDS 123.0-B-1 Multispectral \& Hyperspectral Image Compression Hardware Accelerator on a SpaceGrade SRAM FPGA", in IEEE Transactions on Emerging Topics in Computing, pp. 1-1, 2018, ISSN 2168-6750, doi:10.1109/TETC.2018.2854412. 171, 180

[Vaishnav'18] A. Vaishnav, K. D. Pham, D. Koch, “A Survey on FPGA Virtualization”, in 2018 28th International Conference on Field Programmable Logic and Applications (FPL), pp. 131-1317, Aug 2018, ISSN 1946-1488, doi:10.1109/FPL.2018.00031. 112, 152

[Vaishnav'20] A. Vaishnav, K. D. Pham, J. Powell, D. Koch, "FOS: A Modular FPGA Operating System for Dynamic Workloads”, in ACM Trans. Reconfigurable Technol. Syst., vol. 13, no. 4, Sep. 2020, ISSN 1936-7406, doi:10.1145/3405794. ${ }^{51,52}$

[Valverde'12] J. Valverde, A. Otero, M. López, J. Portilla, E. de la Torre, T. Riesgo, “Using SRAM Based FPGAs for Power-Aware High Performance Wireless Sensor Networks”, in Sensors, vol. 12, no. 3, p. 2667-2692, Feb 2012, ISSN 1424-8220, doi:10.3390/s120302667. xxii, 74, 90, 96, 97, 98

[Valverde'14a] J. Valverde, A. Rodríguez, J. Camarero, A. Otero, J. Portilla, E. de la Torre, T. Riesgo, "A dynamically adaptable bus architecture for trading-off among performance, consumption and dependability in Cyber-Physical Systems", in 2014 24th International Conference on Field Programmable Logic and Applications (FPL), pp. 1-4, Sep. 2014, ISSN 1946-147X, doi:10.1109/FPL.2014.6927394. ${ }^{208}$

[Valverde'14b] J. Valverde, A. Rodríguez, J. Mora, C. Castañares, J. Portilla, E. de la Torre, T. Riesgo, "A Dynamically Adaptable Image Processing Application Trading Off Between High Performance, Consumption and Dependability in Real Time”, in 2014 Conference on Design and Architectures for Signal and Image Processing (DASIP), pp. 1-2, Oct 2014.
208

[Vázquez'16] J. Vázquez, Alternativas de Comunicación Serie de Alta Velocidad en FPGAs, M.Sc. thesis, Universidad Politécnica de Madrid, Oct. 2016, http://oa.upm.es/44640. ${ }^{213}$

[Vesper'17] M. Vesper, D. Koch, K. D. Pham, "PCIeHLS: an OpenCL HLS framework”, in FSP 2017; Fourth International Workshop on FPGAs for Software Programmers, pp. 1-6, Sep. 2017, https://ieeexplore.ieee.org/document/8084547. ${ }^{38,42}$

[Vipin'18] K. Vipin, S. A. Fahmy, "FPGA Dynamic and Partial Reconfiguration: A Survey of Architectures, Methods, and Applications”, in ACM Comput. Surv., vol. 51, no. 4, Jul. 2018, ISSN 0360-0300, doi:10.1145/3193827. 3, 153

[Voros'13] N. S. Voros, M. Hübner, J. Becker, M. Kühnle, F. Thomaitiv, A. Grasset, P. Brelet, P. Bonnot, F. Campi, E. Schüler, H. Sahlbach, S. Whitty, R. Ernst, E. Billich, C. Tischendorf, U. Heinkel, F. Ieromnimon, D. Kritharidis, A. Schneider, J. Knaeblein, W. Putzke-Röming, "MORPHEUS: A Heterogeneous Dynamically Reconfigurable Platform for Designing Highly Complex Embedded Systems”, in ACM Trans. Embed. Comput. Syst., vol. 12, no. 3, Apr. 2013, ISSN 1539-9087, doi:10.1145/2442116.2442120. 23, 31

[Wang'13] Y. Wang, X. Zhou, L. Wang, J. Yan, W. Luk, C. Peng, J. Tong, "SPREAD: A StreamingBased Partially Reconfigurable Architecture and Programming Model”, in IEEE Transactions on Very Large Scale Integration (VLSI) Systems, vol. 21, no. 12, pp. 21792192, Dec 2013, ISSN 1557-9999, doi:10.1109/TVLSI.2012.2231101. 25, 31, 46, 52, 129 
[Watson'82] I. Watson, J. Gurd, “A Practical Data Flow Computer”, in Computer, vol. 15, no. 2, pp. 51-57, Feb 1982, ISSN 1558-0814, doi:10.1109/MC.1982.1653941. ${ }^{141}$

[Wiersema'14] T. Wiersema, A. Bockhorn, M. Platzner, "Embedding FPGA overlays into configurable Systems-on-Chip: ReconOS meets ZUMA", in 2014 International Conference on ReConFigurable Computing and FPGAs (ReConFig14), pp. 1-6, Dec 2014, ISSN 23256532, doi:10.1109/ReConFig.2014.7032514. ${ }^{152}$

[Wigley'06] G. Wigley, D. Kearney, M. Jasiunas, "ReConfigME: a detailed implementation of an operating system for reconfigurable computing", in Proceedings 20th IEEE International Parallel Distributed Processing Symposium, pp. 8 pp.-, April 2006, ISSN 1530-2075, doi:10.1109/IPDPS.2006.1639475. ${ }^{43,52}$

[Windh'15] S. Windh, X. Ma, R. J. Halstead, P. Budhkar, Z. Luna, O. Hussaini, W. A. Najjar, "High-Level Language Tools for Reconfigurable Computing", in Proceedings of the IEEE, vol. 103, no. 3, pp. 390-408, March 2015, ISSN 1558-2256, doi: 10.1109/JPROC.2015.2399275. ${ }^{130}$

[Yousuf'16] S. Yousuf, A. Gordon-Ross, “An Automated Hardware/Software Co-Design Flow for Partially Reconfigurable FPGAs”, in 2016 IEEE Computer Society Annual Symposium on VLSI (ISVLSI), pp. 30-35, July 2016, ISSN 2159-3477, doi:10.1109/ISVLSI.2016.73. 37,42

[Yviquel'13] H. Yviquel, A. Lorence, K. Jerbi, G. Cocherel, A. Sanchez, M. Raulet, “Orcc: Multimedia Development Made Easy”, in Proceedings of the 21st ACM International Conference on Multimedia, MM '13, p. 863-866, Association for Computing Machinery, New York, NY, USA, 2013, ISBN 978-1-4503-2404-5, doi:10.1145/2502081.2502231. ${ }^{145}$

[Yviquel'14] H. Yviquel, E. Casseau, M. Wipliez, J. Gorin, M. Raulet, “Classification-Based Optimization of Dynamic Dataflow Programs”, in Advancing Embedded Systems and Real-Time Communications with Emerging Technologies, pp. 282-301, IGI Global, 2014, doi:10.4018/978-1-4666-6034-2.ch012. ${ }^{141}$

[Zamacola'19] R. Zamacola, A. García Martínez, J. Mora, A. Otero, E. de la Torre, “Automated Tool and Runtime Support for Fine-Grain Reconfiguration in Highly Flexible Reconfigurable Systems", in 2019 IEEE 27th Annual International Symposium on Field-Programmable Custom Computing Machines (FCCM), pp. 307-307, April 2019, ISSN 2576-2621, doi: 10.1109/FCCM.2019.00048. ${ }^{153}$

[Zhang'14] H. Zhang, M. A. Kochte, M. E. Imhof, L. Bauer, H. J. Wunderlich, J. Henkel, "GUARD: GUAranteed reliability in dynamically reconfigurable systems", in 201451 st ACM/EDAC/IEEE Design Automation Conference (DAC), pp. 1-6, June 2014, ISSN 0738100X, doi:10.1145/2593069.2593146. ${ }^{25,31}$

[Zhou'18] Y. Zhou, U. Gupta, S. Dai, R. Zhao, N. Srivastava, H. Jin, J. Featherston, Y.-H. Lai, G. Liu, G. A. Velasquez, et al., "Rosetta: A Realistic High-Level Synthesis Benchmark Suite for Software Programmable FPGAs", in Proceedings of the 2018 ACM/SIGDA International Symposium on Field-Programmable Gate Arrays, FPGA '18, p. 269-278, Association for Computing Machinery, New York, NY, USA, 2018, ISBN 978-1-4503-5614-5, doi: 10.1145/3174243.3174255. ${ }^{106,107}$

[Zhu'14] F. Zhu, Y. Wang, B. Fan, S. Xiang, G. Meng, C. Pan, "Spectral Unmixing via Data-Guided Sparsity”, in IEEE Transactions on Image Processing, vol. 23, no. 12, pp. 5412-5427, Dec 2014, ISSN 1057-7149, doi:10.1109/TIP.2014.2363423. xxiii, 182, 192, 193

[Zohouri'16] H. R. Zohouri, N. Maruyama, A. Smith, M. Matsuda, S. Matsuoka, "Evaluating and Optimizing OpenCL Kernels for High Performance Computing with FPGAs”, in SC '16: Proceedings of the International Conference for High Performance Computing, Networking, Storage and Analysis, pp. 409-420, Nov 2016, ISSN 2167-4337, doi: 10.1109/SC.2016.34. ${ }^{130}$ 